\title{
2018 International Conference on Multidisciplinary Research (MyRes)
}

\author{
$22^{\text {nd }}$ and $23^{\text {rd }}$ June 2018
}

\section{2 myressnet $\mathrm{S}|\mathrm{R}| \mathbf{A}|\mathrm{K}| \mathrm{M} \quad$ LeMERIDIEN}

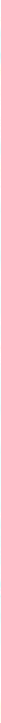

P R O C E E D I N G S

LE MERIDIEN HOTEL,

POINTE AUX PIMENTS, MAURITIUS 


\title{
2018 International Conference on Multidisciplinary Research (MyRes)
}

22-23 June 2018, Le Meridien Hotel, Mauritius

ISBN：978-99949-0-384-9

pISSN: 1694-3597

eISSN: 1694-3600

\section{MyRes Conference Proceedings}

\author{
2018 International Conference on Multidisciplinary Research (MyRes)
}

ISBN：978-99949-0-384-9

https://doi.org/10.26803/MyRes.18.06.22

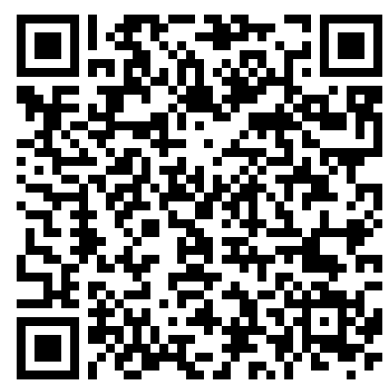

This work is subject to copyright. All rights are reserved by the Publisher, whether the whole or part of the material is concerned, specifically those of translation, reprinting, re-use of illustrations, broadcasting, reproduction by photocopying machines or similar means, and storage in data banks.

\section{$\mathrm{S}|\mathrm{R}| \mathrm{A}|\mathrm{K}| \mathrm{M}$}

Society for Research and Knowledge Management 


\section{Message from the General Chair}

Research is one way to discover, re-discover and obtain new knowledge by blending the wisdom of the past, the knowledge of the present and the uncertainties of the future. Research would not be useful if it is not shared, presented and published. With this vision in mind, the Society of Information Technologists and Entrepreneurs is organizing the first International Conference on Multidisciplinary Research (MyRes2018) on 22-23 June 2018. The event is to be held at Le Meridien, Pamplemousses, Mauritius.

The 2018 International Conference on Multidisciplinary Research (MyRes) is an exceptional platform for researchers, academics, students, innovators, scientists and industry practitioners to discuss and share current research, innovations and bestpractices which is shaping a new world society. Many topics of a multi-disciplinary nature as a response to everyday challenges faced in both developing and developed world contexts will be discussed. Submissions of cross-disciplinary nature cutting across many disciplines/subject-areas were encouraged.

For its first edition, we are pleased to inform you that this event has attracted 101 submissions from 162 authors coming from 31 countries and 5 different continents. The STEM track with 32 submissions is the most popular track. The Education track has received 23 submissions, the Arts, Humanities and Social Sciences track 23 submissions and the Business track has got 18 submissions. Submissions included full-length articles, case studies, extended abstracts, abstract and innovative solutions to problems faced by researchers from all over the world.

A rigorous peer-review process was followed for all submissions and the mean acceptance rate was less than 50\%. Thus, only 49 papers were accepted and 43 of them will be presented at the conference. The papers are published in a conference proceedings with an ISBN number. An ISSN number has also been allocated to this publication as future editions are also on their way.

We are forever grateful to our two keynote speakers for their support, the members of the International Programme Committee for the advice and guidance, the reviewers for their feedback and the authors for contributing high-quality articles to this conference. Our thanks also go to all the volunteers for their endless and selfless help and support during the organisation of this conference. This international event would not have been possible without the collaboration, support, trust and confidence of all of you. Thank you.

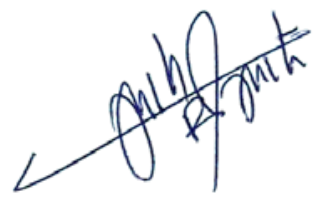

Dr R. P. Gunputh, General Chair 


\section{Prof. Rajendra Parsad Gunputh, University of Mauritius, Mauritius}

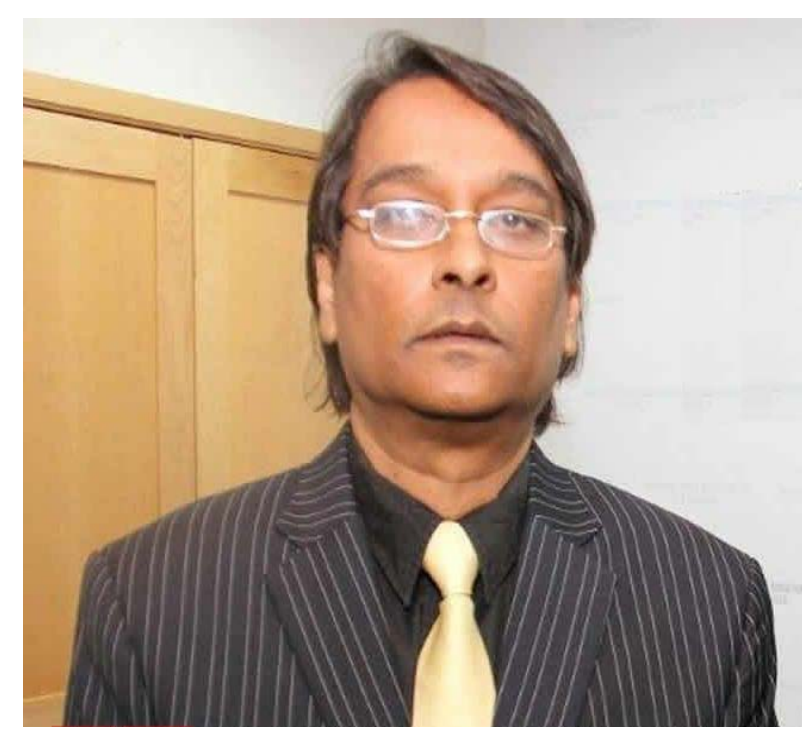

Prof. (Dr. Dr.) Rajendra Parsad Gunputh is the holder of two PhDs in Law, one in Public Law and another in Private Law. $\mathrm{He}$ is a Honorary Professor of the University of Xiangtan in China. He is the Editor-in-Chief of the Just Africa Journal (South Africa). He is also an editorial board member and reviewer for many reputable international law journals. At national level, he has been a member of the following organisations and committees: National Coordination Committee on issues of Human Rights,

Environmental and Land Use Appeal Tribunal, Council for Legal Education, Institute of Judicial and Legal Studies, Mauritius Business Law Review, Programme Accreditation for the Tertiary Education Council, International Humanitarian Law Committee, Mauritius Marine Conservation Society, etc. He received the Bhagwati Memorial Shield award from the Bharati Vidyapeeth University in 2014. He has been a Visiting Professor for the Gujarat National Law University in 2011 \& 2013.

Prof. Gunputh has published several books at both national and international levels. He has more than 130 publications. He is regularly invited to deliver keynote speeches in both national and international conferences. He is currently the Principal Investigator of a project funded by the Mauritius Research Council (MRC) - A Legal Information Retrieval System for Mauritius. 


\section{Keynote Speakers}

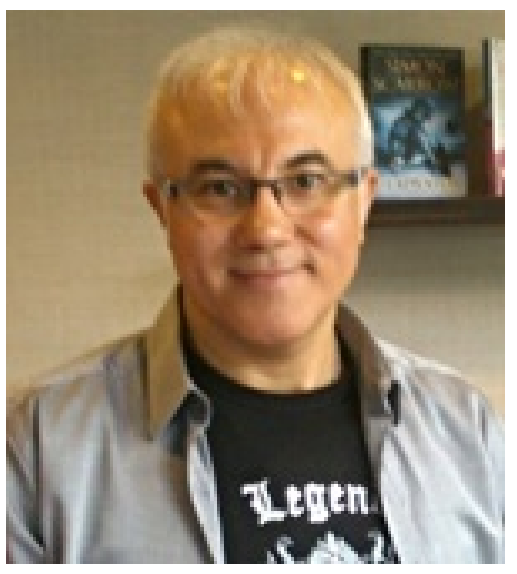

Prof. Mustafa Zülküf Altan is a Professor in ELT at the Department of Foreign Languages Education at Erciyes University, Turkey. He is the author of four books including the 2015 best educational research award winning book, "Educational Gridlock of Turkey: Entrepreneurial Teaching Entrepreneurial Teacher". His research interests include teacher education, teacher development, individual differences in foreign language learning, alternative assessment, intercultural communication entrepreneurial teaching and innovation \& education.

\section{How to Create a Culture of Entrepreneurship and Innovation?}

We live in a time of unrivaled social, economic, technological and environmental problems and desperately are in need of change and improvement in many different areas on a global scale. These concerns and ever increasing need of change are placing pressure on education systems and calling into question many of the traditional models, content and processes of education leading to new thinking about the nature of education, what learners should know and what they should be able to do as a result of their education in order to be able to come up with innovative solutions to these concerns and meet the needs of nations and the globe in general.

With the above picture in mind, educating young people becomes the key to future innovation and entrepreneurship. So it is a good and critical idea to ask ourselves if we are on the right track. Innovation and entrepreneurial initiatives start with the power of human thought. Ideas, inspiration and creativity all come from humans, not machines. Since the technology is the product of human innovation, the greatest technology cannot reproduce what the human mind can do. It is obvious that current educational models, assumptions about learning and approaches to learning and teaching are unable to equip individuals for these new and unexpected challenges since present education systems all over the world are almost exclusively based on a world of yesterday! Therefore, as educators we need to create alternatives. That is, we desperately need for an educational paradigm shift to educate more individuals with entrepreneurial mindset to create innovation in each and every area of life.

Entrepreneurship and Innovation are usually thought as a notion of business, capital, commerce and growth and this way of thinking usually misleads us drastically to understand the real power of entrepreneurship and innovation and their vital relationship with education. The globe needs individuals who are more creative, innovative, and able to come up with solutions to both present and future concerns in every sector of the societies they live in. What is education for? What kind of people do we wish to develop? Why do we need more innovation and innovative individuals? How can this vision be accomplished? Who can bring this vision to come true? This keynote speech will highlight the importance of implementing an entrepreneurial education carried out by entrepreneurial teachers to foster the right mindset to create a real entrepreneurial culture in order to live in a better and peaceful world. 


\section{Keynote Speakers}

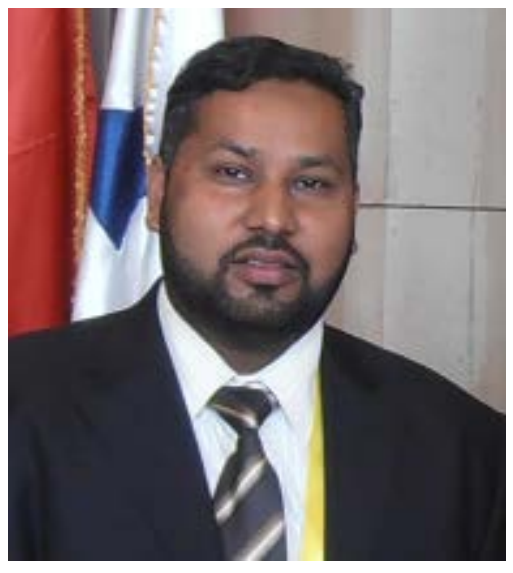

Dr. Mahomoodally, Fawzi Mohamad is an Associate Professor and former Head of Department at the Faculty of Science, University of Mauritius. Fawzi is an alumni of the Harvard University, USA, and recipient of several awards. He has authored 214 scientific publications (133 full scientific papers in ISSN/impact factor journals, edited 4 academic books, 36 book chapters, and 41 abstracts in international/national conferences). He is recipient of $>40$ fellowships/travel grants. In 2011, he was invited as a key speaker at the $14^{\text {th }}$ Asian Chemical Congress (Thailand); ASM-GM (Boston, USA), the ICAAC meeting in Colorado, USA and 6 strategic meetings in Washington DC, USA. In 2018, he was an invited speaker for at the ISE 2018, Dhaka and plenary speaker for Creative Educational Society's College of Pharmacy, India. He was invited to the commonwealth conference (Bangalore) by the Royal Society, UK, the Young leader session with Nobel Laureates at the STS forum, Japan and Fellow of African Science Leadership Programme, South Africa. He is presently the PI/co-PI of 6 research grants/consortium (total of 13 million MUR). He has organized/instructed $>15$ international/national workshops/training courses. Fawzi works in the field of health sciences, with a deep-rooted interest towards documenting/validating the use of complementary/alternative medicine and indigenous/traditional knowledge pertaining to the use of natural products (medicinal herbs/spices/food plants/animal products/practices). He endeavours to develop therapeutic bio-products from medicinal herbs/food plants to address global health, wellness, and food security issues. He is presently collaborating with private companies to translate his research into commercial therapeutic bioproducts.

\section{Bioprospecting Nature and Traditions for New Therapies}

Nature has always been an inspiration for the discovery of new therapies to alleviate human suffering and buffer the mortality and morbidity associated with both communicable and non-communicable diseases. With the recent award of a Nobel Prize in Physiology or Medicine to Youyou Tu, for her discovery of the anti-malarial drug Artemisinin, drug discovery from nature and traditional medicines has been intensified. This has subesequently resulted in a paradigm shift geared towards the translation of traditional medicine into clinical setting. Interestingly, the past decades have witnessed a renewed interest in validating traditional claims surrounding the use of herbs to manage and/or treat plethora of human ailments.This presentation will highlight the importance and contribution of traditional medicines, particualy herbal medicines, in the drug development process and will overview recent work conducted in this area from the tropical island of Mauritius. 


\section{International Programme Committee}

\section{Track 1: STEM (Science, Technology, Engineering \& Mathematics)}

Adel Abdulrahman, Sana'a University, Yemen

Andriy Peleshchyshyn, Lviv Polytechnic National University, Ukraine

Bazlar Rashid, Geological Survey of Bangladesh

Chevonne Dancer, Jackson State University, Mississippi, USA

Elżbieta Macioszek, Silesian University of Technology, Poland

Gregory Woodrow, Putney High School, United Kingdom

Hlaing Khaung Tin, University of Computer Studies, Yangon

Mahfoudh Al-Musli, Qassim University, Saudi Arabia

Mohammad Arif Kamal, Aligarh Muslim University, India

Mohammad Rasoul Momeni, Golpayegan University of Technology, Iran

Mueen Uddin, Effat University, Saudi Arabia

Muhammad Jahangir Khan, Bahria University, Pakistan

Mursalin, S.Pd., M.Pd, Universitas Malikussaleh, Indonesia

Parameshachari B D, GSSS Institute of Engineering and Technology for Women, India

Prof. Dr. Eng. Hamid Ali Abed AL-Asadi, Basra University, Iraq

R. S. Ajin, GeoVin Solutions, Kerala, India

Rose Nyambura, Laikipia University, Kenya

Saber Iraji, Payame Noor University, Iran

Sathishkumar Karupusamy, Bluecrest College, Ghana

Shaleen Bhatnagar, Presidency University, Bangalore, India

Somkait Udomhunsakul, Rajamangala University of Technology Suvarnabhumi, Thailand

Sridhar Subramanian, S. A. Engineering College, Chennai, India

Venkatesh Sharma, Sri Indu College of Engineering and Technology, India

VPS Naidu, CSIR - National Aerospace Laboratories, Bangalore, India

Wasal Khan, Director of Technology, Sarhad University of Science \& IT, Pakistan

Yuriy Syerov, Lviv Polytechnic National University, Ukraine

\section{Track 2: Arts, Humanities and Social Sciences}

Aslina Ahmad, University Pendidikan Sultan Idris, Malaysia

Cynthia Jorgenson, Academic Vice-Principal, United Arab Emirates (UAE)

Eva Tuckyta, Universitas Padjadjaran, Indonesia

Isaak M. Papadopoulos, Aristotle University of Thessaloniki, Greece

Lyndal Bullock, University of North Texas, USA

Pineteh Angu, University of Pretoria, South Africa

Rosanna Tammaro, University of Salerno, Italy

Shweta Verma, Jamia Millia Islamia Central University, India

Solomiia Fedushko, Lviv Polytechnic National University, Ukraine

Syed Rashid Ali, Abdul Wali Khan University, Pakistan

Wakwoya Olani, Addis Ababa University, Ethiopia

Yoones Tavoosy, Foreign Languages Department, Tehran Technical Complex, Iran 


\section{Track 3: Business, Management \& Accounting}

Alexander Franco, Stamford International University, Thailand

Cecilia Junio Sabio, University of the Philippines, Philippines

Diana Martinez, Stamford International University, Thailand

Edwige Kamitewoko, Marien Ngouabi University, Republic of Congo

GK Palem, Chief Research Adviser \& Board Member, Cenacle Research India Pvt Ltd

Joshua Ayeni, Adekunle Ajasin University, Nigeria

Kalu Ema, The Federal University, Ndufu Alike Ikwo, Nigeria

Maxim Vlasov, Ural Federal University, Russia

Peter Mutia, United States International University-Africa, Kenya

Tariq Tawfeeq Yousif Alabdullah, University of Basrah, Iraq

Temesgen Teshome, Wachemo University, Ethiopia

Thesara Jayawardane, University of Moratuwa, Sri Lanka

Wei-Bin Zhang, Ritsumeikan Asia Pacific University, Japan

\section{Track 4: Education}

Adamu Babikkoi, Federal College of Education Nigeria

Aina Kola, College of Education (Technical) Lafigi, Nigeria

Ali Shafaei, University of Urmia, Iran

Antonio Silva Sprock, Director of Central Archive, Universidad Central de Venezuela

Asma Belkhir, University of Tlemcen, Algeria

Eija Valanne, University of Lapland, Finland

Eshetu Mandefro, Hawassa University, Ethiopia

Jerry Moreno, Northeastern Illinois University, Chicago, USA

Julia Chere, National University of Lesotho, Lesotho

Mukhtar Wani, Department of School Education (Govt), India

Mustafa Zülküf Altan, Erciyes University, Turkey

Pauline Machika, Director: Academic Development, Vaal University of Technology, SA

Ray Thompson, Texas A\&M University, USA

Riina Kylmalahti, Al Raqiah School, United Arab Emirates (UAE)

Robert Gable, Old Dominion University, Virginia, USA

Saras Krishnan, INTI International University, Malaysia

Staci Zolkoski, University of Texas at Tyler, USA

Tommi Eranpalo, Ministry of Education, United Arab Emirates (UAE)

Zerf Mohammed, University of Mostaganem, Algeria 


\section{Table of Contents}

\section{Track 1: STEM (Science, Technology, Engineering \& Mathematics)}

Paper 69: Integrating Digital Health Services: An Open Platform Approach for Resource-

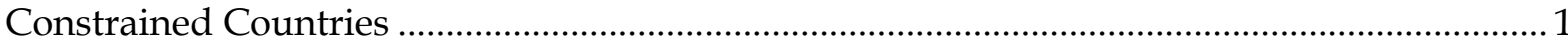

Karl A. Stroetmann

Paper 70: Implementation of Kanban Practices with a Medical Device Software Development

Lifecycle.

Juergen Wieland

Paper 8: Non-Complex Cost-Effective Energy Monitoring using the Internet of Things 31

Pierre Hertzog and Arthur James Swart

Paper 62: A Gender Identification of Russian Text Author on Base of Multigenre Data-

Driven Approach using Machine Learning Models ....

Alexandr Sboev, Ivan Moloshnikov, Dmitry Gudovskikh and Roman Rybka

Paper 77: A Fuzzy Approach to Prioritisation of Security Requirements in a Constrained Agile Software Development Environment.

Nalindren Naicker and Manoj Maharaj

Paper 85: Challenges of Big Data from a Philosophical Perspective

Sunil Choenni, Niels Netten, Mortaza S. Bargh and Rochelle Choenni

Paper 97: Reliability of a Directly Cooled PV/T System.

Busiso Mtunzi and Edson Meyer

Paper 5: Analyzing the Performance of Identical PV Modules in a Semi-Arid Region over a

2-year Period.

Arthur James Swart

Paper 100: Integrating 5S Principles as a Strategy for Improving Clothing Manufacture .... 109 Kemlall Ramdass and Mothibeli Pita

Paper 96: Design and Implementation of an Automatic Electrical Motor Protection 120 Busiso Mtunzi,Tinashe Shelton Gavhu, Zedekia Madumbu Nyathi, Reginald Gonye and Fidelis Nhenga Mugarsanwa

Paper 29: Customer satisfaction: A South African Case 135

Kemlall Ramsaroop Ramdass

Paper 79: Poor Road User Practices: Colombo Urban Roads a Case Study 157 Kolita Weerasekera

Paper 94: From Sustainable Engineering Education to Knowledge Transfer: A Preview.... 164

Nadiir Bheekhun and Abd Rahim Abu Talib

Paper 28: Five Lessons Learned using Water-Scrum-Fall in South Africa 175

Laurie Butgereit 


\section{Track 2: Arts, Humanities \& Social Sciences}

Paper 32: Herding Behaviour and Investment Decisions in Individuals with Fraudulent Microfinance Institutions in Ghana

Anselm Komla Abotsi and Theophilus Edward Richardson

Paper 59: Martha Nussbaum's Theory of Emotion and Death Issues 200

Vasil Gluchman and Marta Gluchmanová

Paper 84: Radio Listening Clubs in Malawi and How they Contribute to Expanding Ordinary People's Capabilities

Peter Mhagama

Paper 95: The Pass-Through of Global Food Price Inflation to Domestic Prices Inflation:

Empirical Evidence from South Asia

Selliah Sivarajasingham and Navaratnam Balamurali

Paper 101: Inflation Rate and Poverty: Does Poor Become Poorer with Inflation?

Mahua Paul and Pooja Sharma

\section{Track 3: Business, Management \& Accounting}

Paper 83: Assessment of Final Year Accountancy Students' Knowledge of the Elements of

Financial Statements

Stephen Chimtengo, Eric Chimpesa and Rabiya Hanif

\section{Track 4: Education}

Paper 38: Epistemic Injustice: Barrier to Articulation Management between Higher Education Institutions in South Africa

Darren Brendan Lortan and Savathrie Maistry

Paper 40: Community Based Participatory Research: Exploring the value of Indigenous Knowledge for Early Childhood Development.

Kanya Padayachee, Savathrie Maistry and Darren Lortan

Paper 60: ResearchGate: Investigating Altmetric and Bibliometric Relationships for Environmental Science Researchers at Unisa.

Leslie S. Adriaanse and Chris Rensleigh

Paper 61: mooKIT - A MOOC Platform for Developing Countries

Tadinada V. Prabhakar, Balaji Venkataraman and Revathy K. T.

Paper 34: A New Approach to Teacher Assessment: Joys and Sorrows of its Introduction.

(Case study).

Zdenka Gadusova, Eva Svarbova and Timea Sipkai 
Paper 33: Instructional Resources and Teacher Effectiveness in Government-aided Secondary Schools in Uganda

Paul Netalisile Malunda and Juliet Atwebembeire

Paper 56: Assessing Senior Engineering Student's Ability to Identify Graduate Attributes..

Maraka Lefera and Arthur James Swart

Paper 27: Gendered Influence of Artisanal and Small Scale Mining on Primary School Pupils' Learning Achievements in Western Tanzania 381

Boaz Kamugisha, Theodora A.L. Bali and Abich Dah Omollo

Paper 24: The Relationship between Contingencies of Self-Worth and Happiness with Academic Achievement of High School Male Students 396

Firouzeh Sepehrianazar, Abolfazl Heidari and Roshank Fallah

Paper 68: Gender and Differences in School Performance in Reading and in Mathematics in Italian School 403

Francesca Sartori and Carlo Buzzi

Paper 7: Final Pass Rate Fluctuations of Power Engineering Students May Be Related to Load Shedding, and not Load Shifting....

Arthur James Swart

Paper 44: Learning Hypothesis Test the Flipped Way: What Do Students Feel? 425

Saras Krishnan

\section{Abstracts}

\section{Track 1: STEM (Science, Technology, Engineering \& Mathematics) - Abstract}

Paper 98: Mathematical Model Reduction using Inertial Manifold Concept Applied to Digitalization of Laser Processing Wolfgang Schulz

\section{Track 2: Arts, Humanities \& Social Sciences - Abstract}

Paper 63: Interpreting Zigi from the Perspective of Anku's Set Theory: An Analytical Inquiry Moses Adzei and Michael Kwasi Adomina

Paper 99: Sensationalism in Argumentation: A Case of the Zimbabwean Parliament Debates Jakaza Ernest

Paper 36: Cross-Cultural Differences in Attitudes to Workplace Relationships in Russia and in the United States

Olga Tararukhina and Svetlana Gurieva

\section{Track 3: Business, Management \& Accounting - Abstract}

Paper 21: Creating a Knowledge Economy: What Can Mauritius Learn from U.S. Models of Innovation and Entrepreneurship?

Rajeev Sooreea and Jaydoyal Sooreea

Paper 43: The Microfinance Institutions Development in Morocco

Oxana Wieland 
Paper 41: Economic Growth and the Linkage Effects Between Foreign Direct Investment and Domestic Investment: The Case of Mauritius

Brinda Sooreea-Bheemul

Paper 86: The Effect of Organisational Culture and Leadership on Employees' Job Satisfaction at a Private Commercial Bank in Mauritius

Roshan Oodally

Paper 22: Are Small Businesses Being Sustainable? Is Cash Management the Key to Profitability and Sustainability?

Avika Mungal

\section{Track 4: Education - Abstract}

Paper 89: Factors Affecting the Participation of Rural Male Students in Fashion and Fabrics and Food and Nutrition in Secondary Schools in Zimbabwe

Anna Gudyanga, Josephine Gora and Lydia Moyo

Paper 66: English or Communication: Engineering Students' Dilemma

Faiza Haddam-Bouabdallah

Paper 23: Integrated Assessments the Key to Developing Critical Thinking Skills of FirstYear University Students

Melanie Cloete 


\section{Track 1: STEM}

\section{(Science, Technology, Engineering \& Mathematics)}

Engineering disciplines (Civil, Electrical, Mechanical, Ocean, etc) Green and Renewable Energy Design \& Technology Image Processing \& Remote Sensing in Geography Information and Communication Technologies Artificial Intelligence \& Robotics Innovations in Mathematics \& Statistics Climate Change and Environmental Issues Agriculture, Forestry \& Food Science Diversity in STEM STEM Education 


\title{
Integrating Digital Health Services: An Open Platform Approach for Resource-Constrained Countries
}

\author{
Karl A. Stroetmann \\ Empirica Communication \& Technology Research \\ Bonn, Germany \\ School of Health Information Science, University of Victoria \\ Victoria, BC, Canada
}

\begin{abstract}
Better health enables greater wealth. And digital health enables better healthcare. Also across resource-constraint countries digital health applications are becoming more prevalent. To establish a resilient national or district Digital Health Ecosystem, not only a holistic strategy - based on health policy priorities - is mandatory, but it must also be followed by a realistic roadmap and its comprehensive implementation, taking into account the success factors needed for its long-term sustainability and growth.

A key challenge in this context is that deployed eHealth systems are usually in silos, such that no system or application is integrated with another. It is the missing interoperability of the many siloed systems which constitutes a core barrier towards reaping greater benefits from digital health. To successfully transform the provision of quality healthcare services it is mandatory to put into place an open digital health platform that comprehensively integrates eHealth services across all healthcare facilities in a timely, efficient and seamless manner.

The open platform concept and its technical approach are developed, and core elements and aspects are critically explored. A constituent complement of such an open approach is a detailed interoperability framework which must be adhered to by all services and applications coordinated via the platform. The key question in this context of how to determine interoperability requirements is briefly discussed. This is complemented by identifying leading open source eHealth software products available and being applied in emerging market and developing economies around the world.

Digital health is different from almost any other sector. By identifying the challenges encountered in healthcare the discussion reviews how the open platform approach helps to overcome these barriers, and identifies important pitfalls to be avoided.
\end{abstract}

Keywords: Digital health, open platform, open source, integration, interoperability. 


\section{Context}

\section{Health, wealth, and growth}

Investment in health system infrastructures and services benefits the people, and thereby improves productivity and wealth creation (WHO, 2001). Wealth means more resources are available for investing - amongst other things - in better health of the population. And it will allow to not only grow material products, but to also invest in education, security and governance - basic building blocks for a stable society and a growing economy. This gave free European cities and counties already in medieval times a significant competitive advantage (Cantoni, 2015), and modern development economists have proven this relationship for many countries (Robinson \& Acemoglu, 2012). A 'good civic order', striving for principles of solidarity, legality, equality will provide a fertile ground also for a sustainable health services environment.

\section{Digital Health}

Digital Health services and applications will deliver an additional dimension to support national health services and enable better healthcare by individual providers. We define such a system as follows:

A 'Digital Health Ecosystem' is the holistic application of information and communications technologies to support and improve healthcare delivery, its coordination and integration across providers.

Substantial investment in digital technology solutions for improved health services has occurred in recent years in Africa. But the need is growing further, and substantial opportunities to support the scale-up of digital health in the region remain. African leaders who gathered at the African Ministerial Dialogue on Digital Health Leadership at the May 2017 World Health Assembly affirmed their commitment to digital health and identified the pathway to achieving strong digital health systems in their countries (Long, 2017). This presents ample opportunities for industry, donor organisations and agencies for international cooperation to constructively support, facilitate and participate in the further development of digital health ecosystems in districts, countries and regions of Africa - and elsewhere.

\section{Objectives}

The goal of this paper is not to cover the complex field of developing a consistent, holistic digital health strategy for an individual country, analyse its roadmap towards implementation, and explore the success factors needed for its long-term sustainability and growth. Rather, after briefly exploring the benefits from digital health experienced around the globe, and contrasting this with key problems and barriers encountered towards realisation, a core ingredient of any district or national eHealth infrastructure and its enabling power for delivering digital health service provision will be identified and explored - the open digital health platform. This will be complemented by identifying leading open source software products available and being applied in emerging market and developing economies (EMDE) around the world. 


\section{Methods}

This paper is based on own research and experience as well as on critically reviewing research reports, papers, and literature. It builds upon work performed in the context of Africa-related studies undertaken by the author. This concerns, inter alia, the "Interoperable eSystems for Africa Enhanced by Satellites" (ISAES) Study for the European Space Agency's (ESA) eHealth for Sub-Saharan Africa (eHSA) Programme. There an interoperability approach and roadmap towards designing and implementing national eHealth platforms in Sub-Saharan Africa (SSA) countries was developed (ISAES Consortium, 2014). Part of the work involved collecting detailed statistical and health system data on 48 SSA countries to assess and evaluate their eHealth readiness. Other input was derived from detailed case studies of national or district eHealth platforms and electronic health record (EHR) systems covering eight countries on five continents. Similar information on seven more platforms and national eHealth strategies was reviewed.

Key knowledge has also been derived from research on „Digital Health Ecosystems for African countries - Integrated framework and approach" which was undertaken for the Strategic Partnership Digital Africa (SPDA), an initiative of the German Federal Government and industry supported by the German Society for International Cooperation (GIZ) (German Federal Ministry for Economic Cooperation and Development , 2017).

\section{Results}

\section{Global experience}

Across the globe, developing and successfully implementing a digital health ecosystem is undertaken in order to improve patient safety, healthcare delivery and economic efficiency of the health sector. Invariably, this turns out to constitute a complex task requiring enthusiasm, political power, skilled people as well as many other resources and ingredients. The benefits, opportunities and challenges of creating such an ecosystem will briefly be traced - identifying the motivational base to realise such an ecosystem also in resource-constrained national or district contexts. It requires a dedicated, focused healthcare policy, the setting of priorities for digital health support, a realistic implementation plan, and the provision of adequate governance and technology infrastructures. No one-size-fits-all approach will be useful here - rather the identification and exploration of the respective core national health policy priorities guiding the development of a successful Digital Health Ecosystem.

\section{The 'Why' of digital health}

Progress in clinical knowledge, in medical technologies and devices, in management and administration of healthcare services will allow to

$>$ Improve prevention, diagnosis and treatment

$>$ Advance patient safety

$>$ Foster public health

$>$ Raise the cost effectiveness of healthcare 
Digital Health systems and applications enable achievement of these goals through the provision of powerful tools for:

$\checkmark$ Data/information gathering, exchange, access and analysis

$\checkmark$ Communication and collaboration in health service processes across patients and their family members, healthcare professionals, provider organisations, administrators/managers, and Public Health services

$\checkmark$ Medical knowledge generation and support of clinical decision making. As a long-term vision, this will lead towards a learning healthcare system designed to generate and apply the best evidence for the collaborative healthcare choices of each patient and provider, and allow optimising resource allocation and efficiency. It will drive the process of discovery as a natural outgrowth of patient care and thereby ensure innovation, quality, safety, and value in health care (Institute of Medicine, 2012).

\section{Benefits from Digital Health}

Already during the '90s significant efforts towards the establishment of district or national digital health infrastructures emerged. They brought mixed results: some were quite successful - like those in some district health systems of northern European countries, in Scotland, or in Andalucía in the south of Spain (Stroetmann, Jones, Dobrev \& Stroetmann, 2006). Others had mixed results like the NHS in England, or Australia, Canada, Germany. On the other hand, several developing countries or districts in such countries have been quite successful in investing in digital infrastructures and eHealth services (Stroetmann, 2014).

\section{Opportunities}

Digital Health provides for proven, often highly beneficial applications in many different areas of health systems. It can support the urgently needed transformation of healthcare delivery, and its potential is seemingly boundless. A few examples may illustrate this:

$>$ Faster, more effective and efficient care provision

Digital health enables healthcare teams to access core or even complete patient health data and information, which saves time, reduces duplicate tests, and leads to better patient care decisions. It may connect electronic patient or medical records with measuring devices and mobile phones (mHealth), allow patients to be informed or even input their own data.

For example, a path-setting application platform has been developed by the mHealth4Afrika consortium - a collaboration between African and European companies. It provides an open source, multilingual digital health platform improving the quality of community based maternal and newborn healthcare delivery in districts of Ethiopia, Kenya, Malawi, and South Africa. It combines electronic medical records to store patient history, associated test results derived from sensors to capture the data of a range of standardised tests, with analytical and visualisation tools as well as speech synthesis to address literacy deficits.

$>$ Telemedicine, virtual visits

Telemedicine applications allow connecting with patients, health workers, nurses, and doctors remotely, which renders healthcare accessible to hitherto unserved 
people, reduces the need to travel, and makes specialist knowledge and experience available on the spot.

In Tanzania, e.g., teleconsultation equipment to support obstetric emergency care in rural and outmost areas was installed in ten upgraded rural health centres, four rural district hospitals and one regional hospital.

$>$ mHealth

Making use of cell and smart phones/mobile devices is a very promising application field in Africa due to the relatively ubiquitous mobile telecommunication connectivity when compared to other modes of telecommunications. Smart phones have been engineered to serve as local hubs or platforms to connect sensors and electronic measurement devices, printers etc. at the local level, and to connect to more complex systems at community centres and district hospitals.

Worldwide known applications focus on pregnant women, like the UNICEFsupported MomConnect service in South Africa which links pregnant women and mothers to healthcare centres. Ghana and Nigeria have introduced forms and checklists to be used on smart phones for regulatory process support of pharmacies or for consultations of patients.

$>$ Administration and infrastructure services

This is an often neglected application domain, but in urgent need of attention where larger patient populations are concerned.

Triggered by the need to reduce endless waiting times for patients, in South Africa the Western Cape's Primary Health Care Information System (PHCIS) is an operational system for managing patient throughput in primary care clinics through electronically drawing information on past clinic visits, creating electronic appointments, and providing patient and facility management tools for reporting purposes.

$>$ Public Health surveillance, disaster preparedness

Health surveillance is the systematic collection and analysis of health-related data needed for the planning, implementation, and evaluation of Public Health practice. Electronic collection of such data can fundamentally strengthen national health information systems and decision making. Resulting benefits can be further improved by integrating into such a platform service a geographic information system (GIS) and early warning system (EWS), and combining them with mHealth devices which may serve not only as display devices and dashboard, but also for early warnings to citizens.

$>$ eLearning

This is an underused resource with considerable potential. The Tanzanian Training Centre for International Health uses an audio teleconferencing model; and an online eLearning platform to teach health workers and nurses in maternal and perinatal healthcare in rural areas. In Gilgit-Baltistan, the thinly populated north of Pakistan, a multimedia platform based on open source software facilitates the continuing professional development of nurses and other healthcare workers through case based collaboration and a bulletin board system (BBS) for sharing and exchanging messages and files. 


\section{A key challenge: Integration}

In spite of these promising applications and the concrete benefits demonstrated by them, there remain many serious challenges and implementation problems which need to be considered to assure long-term success. Perhaps the most serious challenge relates to coordination. Here the core problem in many countries is that various eHealth systems are not integrated, but are run by different independent organisations. It regularly happens that donors, charities/churches, governments, and (other) implementing partners work in the same district, without coordination, on similar health issues. This phenomenon was recently succinctly pointed at by $\mathrm{Mr}$. Kumalija, Ministry of Health, Tanzania: “Existing of parallel routine reporting systems such as IVD, HIV, PEPFAR - DATIM is a challenge. Uncoordinated national surveys such as by SPA (USAD), SARA (Global Fund), SDI (World Bank) collect almost the same indicators" (Kumalija, 2017).

And in a recent Request for Expression of Interest on "Enhancement of Rwanda National Digital Health Care System 'Smart Health'” it was noted: “The deployed [eHealth] systems are in silos and there is no system that is integrated with another. There is no timely information for easy and quick decision making; there is no ability to track service levels across the whole health sector. Due to the silos of systems patient records are only limited to the health facility visited. ... The Ministry of Health would like to transform the provision of quality healthcare services by putting in place an integrated, comprehensive information system across all the healthcare facilities nationwide in a timely, efficient and seamless manner." (Ministry of Health of Rwanda, 2018). It is here where open digital health platforms will provide an innovative solution.

\section{Open digital health platform}

Comprehensive digital health systems have often been provided by commercial companies applying proprietary architectures and standards. This renders it difficult, sometimes impossible, and usually expensive to integrate new applications, extract and transfer patient data to other, often legacy applications, or even change the software supplier completely.

Open Digital Health Ecosystems implemented at the national or district level will help to overcome this core challenge. Such an approach also makes it easier to develop and flexibly integrate any time innovative third-party applications that support safe, high quality healthcare. It allows apps and services from multiple vendors to work together such that there is a many-to-many substitutability between applications and services. An application requiring access to an infrastructure service, e.g. the master-patient index (MPI), can use other infrastructure services as well as patient data provided by other applications via common, open and standardised data models and application programming interfaces (APIs). In this way, open platforms liberate both data and applications making them portable and interoperable across different platform implementations.

Figure 1 briefly describes core design principles of such an open digital health platform. 


\section{Core Design Principles}

The Core Design Principles relating to this open platform approach are the key 'non-tangible' objectives that provide an overarching guide in the development and deployment of this new open platform. They are critical to conveying the purpose and goals. Once agreed with key stakeholders, these Core Design Principles will be translated into expected outcomes and design controls to be used to assess success going forward.

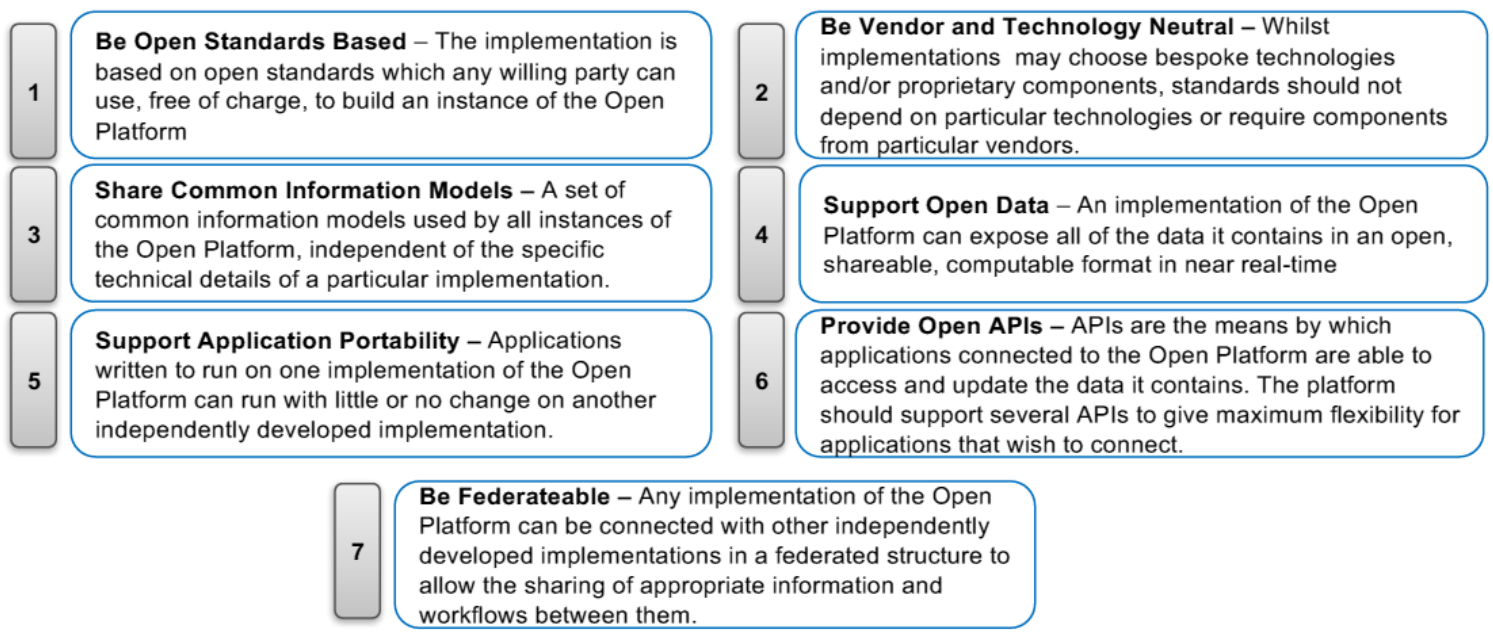

Source: Maidment (2017)

Figure 1 - Core Design Principles of an Open Digital Health Platform

Such an ecosystem is vendor and technology neutral and eliminates the expensive and much-dreaded vendor lock-in. It thereby facilitates innovation also by smaller companies and start-ups facing lower barriers to market entry, and any of their applications built for such an open platform will operate on any other open platform applying the same standards. It forces vendors to compete solely on quality, value, and service.

Figure 2 illustrates the generic architecture of such an open Digital Health Ecosystem. It is taken from the openEHR Foundation - an open domain-driven platform for developing flexible eHealth systems (openEHR, 2018). It shows how the different digital healthcare applications are separated from platform tools and services. Their integration is ensured via an enterprise service bus (ESB), the integration engine. For its overall functionality, it is mandatory that every software provider implements the agreed-upon and openly available standards and data models, like those developed by the openEHR (open electronic health record) community, the Health Level Seven International (HL7) standards developing organisation, the International Standards organisation (ISO) and many others. And that their software has been tested and certified as compliant with these requirements. 


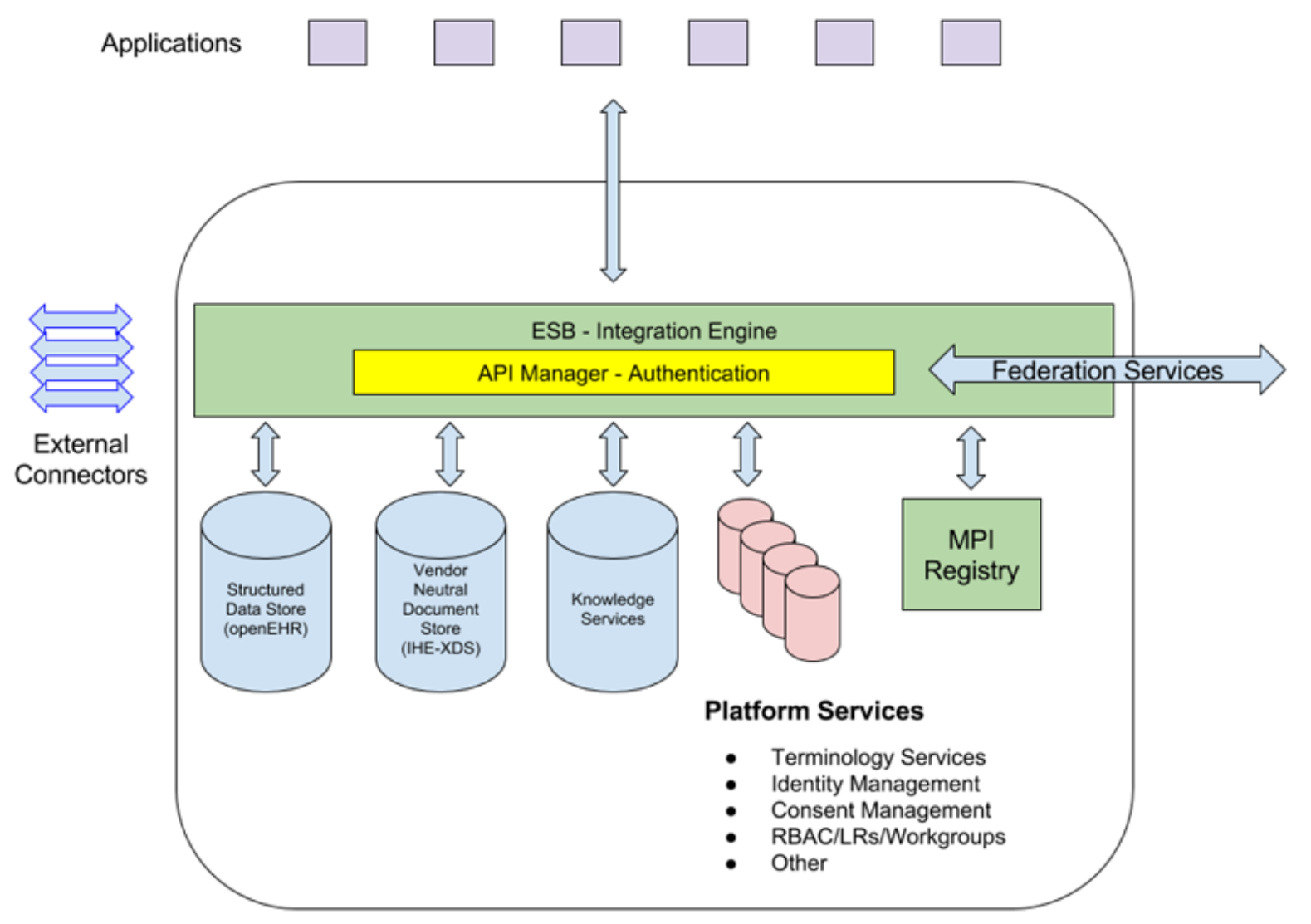

Source: openEHR Foundation

Figure 2 - Open Digital Health Ecosystem platform architecture

For data that need to become available in a highly structured, quantified form, commonly agreed detailed clinical models must be available. Such models and their elements provide an unambiguous description of a piece of information, its structure and parameters, and how they are measured or represented. This may apply to key elements of a basic patient or emergency summary (like a diagnosis coded by ICD 10 - the 10th version of the WHO International Classification of Diseases), to quantifiable elements of an electronic prescription, or patient data needed as input for an electronic clinical decision support system.

For other information needs an electronic copy of a paper document like a referral letter or a lab report may suffice, or a consistently structured document complying with agreed-upon document standards like the clinical document architecture (CDA) of HL7 (Health Level 7) or message profiles as specified by IHE (Integrating the Healthcare Enterprise).

And data and information extracted from the respective data stores can be consolidated into a clinical data warehouse (CDW) or clinical data repository (CDR). This may be used to obtain a comprehensive overview of a single patient. Or it may be applied to gain data for medical research, epidemiological analyses, or public health purposes. If an Open Data approach is pursued and the required patient consent, data protection, anonymisation and security services have been 
implemented, such data may also be exposed to other users for purposes as sanctioned e.g. by regulation.

Whether such an open Digital Health Ecosystem is managed and maintained by a public institute, a cooperative of healthcare provider organisations, or a private entity will depend on the respective circumstances. For IT companies to stay involved, it may require to engage in new business models based on delivering cloud services, software as a service (SaaS), licensing agreements etc. rather than selling hardware and software.

Worldwide, there exists already a growing community of cooperating commercial suppliers of software, cloud and other platform services, of infrastructure tools, implementation and management support. This is complemented by open source and proprietary application providers offering a wide spectrum of electronic healthcare tools and systems, all able to connect to an open platform and to exchange data in open standards and specifications.

\section{Determining interoperability requirements}

However, for such a platform to be successful, it is mandatory to solve the related interoperability challenges. It is a strategic necessity for any Digital Health Ecosystem; it requires the full attention of all stakeholders. Globally, all national digital health strategies talk about the need to enable interoperability, but most of them failed here due to a lack of precision and translating this generic concept into a strategic perspective first, and from there into concrete actions meeting the real needs of stakeholders.

Interoperability must always be seen and analysed in the wider context of establishing a Digital Health Ecosystem. This implies that interoperability requirements cannot be identified ex ante and as such, but rather firstly need to reflect the respective data access and exchange needs of health system actors to be supported by the electronic tools and applications to be implemented. And these in turn will be driven by the overall health policy goals and the specific health system/health services domain in question - which may be the overall health system, but perhaps only primary care, public health, administration and billing, or any other of the earlier discussed specific implementation fields - or a combination of them.

Furthermore, the geographic context within which interoperability is to be achieved can have a considerable impact, e.g. the languages spoken and the languages in which health records are maintained, the health care structures and resulting process and information sharing needs for cooperation between rural health stations, community centres, district hospitals, laboratories, and others - whatever the local situation may be.

\section{Leveraging the "open" approach}

Globally, support and engagement for "openSource" software, "openData" access, "openStandard" availability and "openPlatform" approaches has gained great momentum, both in industrialised and resource-constraint environments. This "open" movement is now ubiquitous, recognized across public and private entities as a fundamental course of action towards building interoperable, easy to use 
infrastructure components, as well as a critical factor for driving innovation in 'vertical' markets. The source code of software and tools developed by the open source community is not proprietary, but can be freely copied, modified and distributed; it is managed and continuously improved by engaged participants. Even some of the biggest IT companies in the world like Google and Facebook have released software to this community to allow for it to evolve through community support and feedback to improve their own services.

Also in the health sector it is by now a well-established procedure to work together at global scale to improve both interoperability and economic efficiency of digital health systems and applications. Thereby it is fostering competition across suppliers, triggering new business models and markets, and easing market entry barriers for small, innovative companies. At the same time, vendor lock-in can be avoided. Some exemplar applications and tools are as follows:

$>$ Open Medical Record System (OpenMRS)

is an open source medical record platform. It allows designing a customized medical records system with little programming skills. Its features include a central concept dictionary, modular architecture and standards support. Instead of just releasing a generic enterprise-grade platform and leaving it up to each implementation to configure, the new OpenMRS2 includes more functionality 'out of the box' like patient summary, visit view for data clerks, vital signs capture, diagnosis capture, support for multiple wards/health services.

$>$ Open domain-driven platform for developing flexible eHealth systems (openEHR)

is a virtual community working on means of turning health data from the physical form into electronic form and ensuring universal interoperability among all forms of electronic data. The primary focus is on electronic health records (eHR) and related systems. Components and systems conforming to openEHR are 'open' in terms of data, models and APIs. They share adaptability, due to the archetypes being external to the software, and significant parts of the software being machine-derived from the archetypes. The archetype specification is now an ISO standard (ISO 13606-2). These are used by several national governments to specify national eHealth information standards.

$>$ District Health Information System 2 (DHIS 2)

is a free software tool for the collection, validation, analysis, and presentation of aggregate and transactional data, tailored to integrated health information management activities. It is being used at various levels in about 50 countries.

$>$ Open Health Insurance Management Information System (openIMIS)

is an initiative for providing a comprehensive system linking patient, provider and payer data. The system is designed to manage any health insurance scheme, from enrolling patients to transmitting and processing claims, and calculating reimbursements. The initiative has created a community of practice for software developers and users, and provides capacity-building services. 


\section{$>$ OpenHIE}

is a global mission-driven community of practice to promote interoperability in the health sector. It has developed a Health Information Exchange architecture and freely available standards-based approaches and reference technologies which leverage on existing Health Information standards like HL7, DICOM...

$>$ Open Health Information Mediator (OpenHIM) is a middleware component designed to facilitate secure communications and data governance support between disparate clinical information systems as well as support for routing, orchestrating and translating requests. It also supports sharing of information among infrastructure tools and applications.

> Magpi

is an OpenSource tool that can be used for any kind of mobile data collection, e.g. as input into an electronic health record system or for a medical research project. The community started with applications in global health, but it is now applied also in many other domains. Critical patient data values may be identified to trigger alerts, to-do items, or other actions.

$>$ openeLearning

There exist various software platforms and tools to support eLearning. E.g. iPath, an open source web application service, is providing a free platform for "case based collaboration", especially designed for medical applications (telemedicine, etc). The iPath-Server package provides a medical bulletin board system (BBS) to discuss/consult cases online. A BBS is an application dedicated to the sharing or exchanging of messages or other files on a network.

\section{Discussion}

\section{Digital health is different}

The results presented have to be seen against the often disappointing and sometimes disastrous results of attempting to develop and implement national Digital Health Ecosystems. Why do other service sectors like banking, retail, insurances and others succeed - and health systems not? And how can an open platform approach help to overcome the barriers experienced?

A few observations may help to explain this earlier experienced situation - and they can allow, at the same time, to better understand why the approach towards Digital Health Ecosystems presented may help to improve such experience in future:

$\checkmark$ Clinical \& care data is idiosyncratic, textual - and not easily interoperable (data complexity)

Clinical terminology is often ill defined - the same word may have different meaning in different contexts, and for the same 'fact', like a diagnosis, different terms may be in use. The details with which something is 'measured' will depend on the clinical situation - a brain surgeon needs much more detail than a general practitioner. For a liver transplant patient, it may be mandatory to not only have the latest lab analyses, but to also have information on which lab has measured them with which technical equipment.

$\checkmark$ Wide variety of actors involved (cultural complexity) 
In health systems, a wide variety of actors have to work together - general practitioners, specialists, nurses, health workers, administrators, managers, public health specialists, and many others - all with their own interests, 'language' and behaviour.

$\checkmark$ Decision processes with countless options and interactions which may need to be fast (decision complexity)

For, e.g., multi-morbid patients, decision options may not be well defined or countless, no empirical evidence is often available to support the treating professional.

$\checkmark$ Non-determination (no simple workflow rules) of healthcare processes (process complexity)

In spite, e.g., of clinical guidelines and defined clinical pathways, the respective pathway may be different in concrete detail for each patient (personalised medicine).

$\checkmark$ Information governance/personal data, safety, cyber-security (regulatory complexity)

Patient health data are very special data - there are patients who do not want even their treating physicians to have access to all their earlier recoded information.

$\checkmark$ Monolithic, proprietary, non-interoperable software systems (vendor lock-in IT complexity)

Often hospitals or regional structures have invested in basic proprietary information systems, which in themselves are already technologically highly complex. They may consist of up to 30 or more different modules, need to connect and exchange data with sometimes hundreds of other (proprietary or bespoke) systems used in different divisions and wards of a hospital.

An open platform approach will not by itself solve these challenges, but it surely presents a more promising solution to obtain better outcomes in future and fundamentally help to overcome the identified barriers.

\section{Pitfalls to be avoided}

To indeed achieve the improvements promised by digital health systems and applications through an open platform approach, a few further observations on pitfalls to be avoided in such an endeavour will be helpful:

\section{$>$ Technology push versus user focus}

"A lot of solutions have come from technologists and engineers who are excited by the technology, but at times, they are not starting with the true need. If you first start with the need, then consider the technologies to apply as well as the overall business model and ecosystem, the solution might be very different. End-users must be central to the design... The problem with African countries is that e-health systems are not integrated and are instead run by different independent organisations." (Ligami, 2014)

\section{Pilotitis}

The digital health landscape in Africa (and elsewhere) is plagued by 'pilotitis', the expansion of numerous pilot projects that rarely reach scale and fail to connect to a national strategy. Of the many interesting projects that have been taken to the field in Africa, most are neither sustainable nor scalable. Very few are expected to scale up 
into sustainable enterprises with most remaining speculative pilots (Long, 2017). "In Kenya, for example, out of the 183 innovative health care programs, only 44 per cent have been implemented and are working efficiently." (Ligami, 2014)

Even worse seems to be the success rate of mHealth services. The USAid "African strategies for health - The mHealth Compendium Series" identified 167 profiles of mobile health programmes. It remarked that "while many of the pilot interventions featured in the series volumes have since phased out, some scaled up. Ten scaled mHealth programs are presented with in-depth profiles in the mHealth Compendium Special Edition 2016." (USAid, 2016)

Human resources and absorption capacity

The ability to successfully invest in eHealth solutions is dependent on adequate human resources -health professionals/experts with ICT knowledge within healthcare provider organisations, and external IT support and infrastructure service providers. In a small survey of healthcare workers and professionals in Tanzania, it was noted that demand for local technical capacity dramatically exceeds availability in fields like software development, digital health program implementation, health informatics and enterprise architecture (Busagala \& Kawono, 2013).

Governance, legal and regulatory base

Healthcare provision is laden with complex situations that involve confidentiality and ethical decisions to be made. Privacy of patient data is mandatory to avoid abuse by unauthorised persons or the government. On the other hand, security and access issues must not impede proper patient care. They must be non-disruptive; and the system, with the proper safeguards in place, must be easily accessible. In principle, the technology is available to solve these issues, but the governance context must make sure that patient data are not shared with anyone the patient has not consented to.

When analysing these and related legal, regulatory and governance aspects across 48 Sub-Sahara countries, only four countries scored high with respect to their "regulatory readiness", six more were assessed as ready, and the remaining 38 countries - achieving on average only $50 \%$ of the sores of the "ready" countries were judged as not ready (Greenfield, 2013).

\section{Conclusion and outlook}

Substantial investment in digital solutions for improved health services has occurred in many countries around the world in recent years. However, it has turned out that investing in isolated eHealth applications does not meet the needs of today's health systems, because this will not enable the urgently needed transformation towards better coordinated, integrated and sustained healthcare. Usually, proprietary architectures and software have been implemented, which render it difficult, sometimes impossible, and usually expensive to integrate new applications, extract and transfer patient data to other applications, or change the software.

Rather, a comprehensive approach is needed building on a well-founded health policy, setting clear priorities for healthcare and how digital applications can support it. Do not go for all the potential eHealth benefits at once; rather focus initially on most pressing policy needs, and develop from there. To implement 
successfully a Digital Health Ecosystem, both the success factors and the challenges identified should be taken into account. A core building block of a holistic approach should be an open infrastructure platform and digital ecosystem implemented at the national or district level. This allows apps and services from multiple vendors to work together, facilitates innovation also by smaller companies and start-ups facing lower barriers to market entry, and forces vendors to compete solely on quality, value, and service.

This is all the more important because, when considering investments in digital health infrastructures and systems in years to come, the overall economic situation in African countries indicates a promising perspective. Inflow of foreign direct investment into Africa was at $\$ 14$ billion in 2004, and is expected to grow to about $\$ 65$ billion in 2017 (UNCTAD, 2017).

\section{Acknowledgements}

Part of this research was financially supported by the German Gesellschaft für Internationale Zusammenarbeit (GIZ) for the Strategic Partnership Digital Africa.

The work was undertaken in the context of the author's affiliation with empirica $\mathrm{GmbH}$. Critical review and input by external experts and colleagues are gratefully acknowledged.

\section{References}

Busagala, L. S. P., \& Kawono, G. C. (2013). Underlying Challenges of E-Health Adoption in Tanzania. International Journal of Information and Communication Technology Research, 3, 34-41.

Cantoni, D. (2015). The economic effects of the Protestant Reformation: testing the Weber hypothesis in the German lands. Journal of the European Economic Association, 13, 561-598.

German Federal Ministry for Economic Cooperation and Development (BMZ) (2017). Strategic Partnership Digital Africa - Harnessing Digitalisation Opportunities for Africa's Development. Berlin: BMZ.

Greenfield (2013). Study on Regulatory Aspects - eHealth Regulatory Readiness Ranking. A Critical Review of the Existing Regulatory Environment in SubSaharan Africa and its Readiness to Support eHealth Services. Technical Note 4. Kimberly, SA: Greenfield. Retrieved from http://www.greenfield.org.za/downloads/eHSA\%20Reg\%20Study\%20Sum mary\%20Report.pdf

Institute of Medicine (IOM) (2012). Best Care at Lower Cost: The Path to Continuously Learning Health Care in America. Washington, DC: IOM

ISAES Consortium (2014). ISAES - Interoperable e-Systems for Africa Enhanced by Satellites - Final Report. European Space Agency (ESA) eHealth for SubSaharan Africa Program - eHSA. Barcelona: INDRA.

Kumalija, C. J. (2017). Update of M\&E Strengthening Initiatives (M\&E SI) - A Tanzanian Platform for Health Information and Accountability. Presentation at Tanzania Health Data Collaborative (THDC) Launch Meeting, Dar es 
Salaam, 11- $12 \quad$ September. Retrieved from https://www.healthdatacollaborative.org/fileadmin/uploads/hdc/Docume nts/Country_documents/Tanzania_M_ESI_presentation_11Sept2017.pdf

Ligami, C. (2014, March 29). E-health solutions must have end-user as focal point. The East African. Retrieved from http://www.theeastafrican.co.ke/news/Ehealth-solutions-must-have-end-user-as-focal-point/2558-2262332$12 \mathrm{gn} 67 \mathrm{kz} /$ index.html

Long, L.-E. (2017, May 24). The power and promise of digital health for Africa. PATHblog - Stories of innovation and Impact. Retrieved from https:/ / blog.path.org/2017/05/the-power-and-promise-of-digital-health-forafrica/

Maidment, D. (2017, Nov. 22). Leeds Person Held Record - building on an open platform. ICT Strategy, Architecture \& Commissioning, Leeds City Council: Presentation at openEHR Day in London, UK.

Ministry of Health of Rwanda (2018). Request for Expression of Interest Enhancement of Rwanda National Digital Health Care System "Smart Health". Kigali: MoH.

openEHR (2018). What is openEHR? Retrieved from http://openehr.org/what_is_openehr

Robinson, J. A., \& Acemoglu, D. (2012). Why nations fail: The origins of power, prosperity and poverty. New York, NY: Crown Business.

Stroetmann, K., Jones, T., Dobrev, A., \& Stroetmann, V. N (2006). eHealth is Worth it - The economic benefits of implemented eHealth solutions at ten European sites. Luxembourg: Office for Official Publications of the European Communities.

Stroetmann, K. (2014, May). Scoping global good eHealth platforms: Implications for Sub-Saharan Africa. In IST Africa Conference Proceedings, 2014. IEEE. Retrieved from https://www.researchgate.net/profile/Karl_Stroetmann/publication/27154 6180_Scoping_global_good_ehealth_platforms_Implications_for_subSaharan_Africa/links/573c757e08ae9ace840fd6fe/Scoping-global-goodehealth-platforms-Implications-for-sub-Saharan-Africa.pdf

United Nations Conference on Trade and Development (UNCTAD) (2017). World Invgrestment Report - Investment and the Digital Economy. Geneva, Switzerland: United Nations.

USAid (2016). African strategies for health. The mHealth Compendium Series. Retrieved from http://www.africanstrategies4health.org/resources/mhealth

WHO Commission on Macroeconomics and Health (2001). Macroeconomics and health: Investing in health for economic development. Geneva, Switzerland: World Health Organization.

World Economic Forum (2016). 3 reasons things are looking up for African economies. Lausanne: WEF. Retrieved from https://www.weforum.org/agenda/2016/05/what-s-the-future-ofeconomic-growth-in-africa. 


\title{
Implementation of Kanban Practices with a Medical Device Software Development Lifecycle
}

\author{
Juergen Wieland \\ University of Gloucestershire, School of business and management \\ Cheltenham GL50 2RH, United Kingdom
}

\begin{abstract}
Objective. The objective of this study is to provide a better understanding of the challenges faced by software developers when developing safety-critical medical device software by the use of a plan-driven SDLC and to resolve these shortcomings by the proposition of a mixed method SDLC (KV-model) to the development of software as medical device.
\end{abstract}

Methods. The proposed mixed method SDLC was developed on the foundation of a formal development process (v-model) and adds the benefits of lean-Kanban practices. The proposed KV-model includes the principles and recommendations of IEC 60601-1 (v-model) to accomplish regulatory requirements for the development of safe and reliable high quality medical software according to IEC 62344. To provide a better understanding of the challenges faced by software developers, a survey of a sample of 18 German medical software developers was performed. Surveying at least two members of each company, five central development stages with ten Kanban practices were identified. The lean-Kanban practices were then assessed for consistency using IEC 62304 and unified to a hybrid approach.

Results. The following results were discovered: 1.$) 72 \%$ of the surveyed companies use v-modelling as SDLC; 2.) $28 \%$ of the survey companies already implement leanKanban practices within their SDLC; 3 ) challenges faced by software developers can be solved by integrating lean-Kanban practices as an iterative and continuously improving process within a plan-driven SDLC 4.) identification of ten lean-Kanban practices to be implemented within a plan-driven SDLC; 5 .) the development of a hybrid SDLC combining lean Kanban practices within a plan-driven v-model (KVmodel).

\section{Keywords}

Kanban practices, ISO 62304, software development lifecycle (SDLC), software as medical device (SaMD). 


\section{Introduction}

Lean management practices have become highly prevalent since the lean-Kanban manifesto emerged in early 2011 as a reaction to the ineffectiveness of current development methods in a fast-changing software development environment. Kanban software development practices apparently solve the problems of a plandriven development lifecycle. The Kanban approach is claimed to be "the next wave of software process" 1 , offering improved software quality at lower development costs and saving resources. Regardless of the shift from plan software development methods to Kanban software development, only a low rate of publicly available information is available which suggest a widespread implementation of Kanban practices for the development of software as medical device. Using Kanban methods, the software developer is directed in a way that enables to resolve programming errors in minimum time frame possible, but on the other hand ensures the use of standardized practices when developing quality- and safety-critical medical device software. It is apparent that medical device software development projects might benefit from using Kanban practices, whilst still keeping the discipline associated with the following plan-driven development lifecycle (Pekar et al., 2016). The purposes of this paper, consequently, are:

- To provide a better understanding of the challenges faced by software developers when developing safety-critical medical device software by the use of a plan-driven SDLC.

- And to show how shortcomings in both Kanban and plan-driven approaches are resolved by the use of a mixed method approach to the development of software as medical device.

To answer these research objectives, a survey with 18 Germany-based manufacturers of medical software has been performed. As a study result, this paper proposes a hybrid SDLC combining both lean-Kanban and plan-driven practices to develop regulatory-compliant medical software. In examining current software development practices, the v-model appears to be the SDLC most widely used by medical device software organisations (Polgár \& Kazinci, 2014). This study identifies Kanban practices to be implemented into the v-model and proposes the Kanban v-model (KV-model) as a SDLC for use in the medical device industry. The paper aims to provide developers of medical software with the structure of a plan-driven SDLC whilst reaping the benefits available through the use of Kanban practices.

\section{International regulation}

Developers of medical device software must comply with region-specific quality and regulatory requirements, depending on the country where the software as medical device is intended to be marketed. A study carried out by Markets \& Markets (2016) see USA and Europe as the major markets for medical device software until the year 2022. Accordingly, medical device software must comply with FDA requirements for marketization within the USA and with the medical device directive (MDR) and the in-vitro diagnostic directive (IVDR) for marketization in Europe.

\footnotetext{
${ }^{1}$ http://atlanta2010.leanssc.org/
} 
USA

Software as medical device intended to be marketed in the United States of America must comply with regulations specified by the FDA. The software developer must ensure that the software has been designed, developed, coded, validated and verified consistent with these regulations in order to be both safe and reliable according to its intended use. The software developer can use guidance documents issued by the FDA, helping in the interpretation of these regulations:

- General Principles of Software Validation (GPSV) - Final Guidance for Industry and FDA Staff

- Guidance for OTS Software Use in Medical Devices - FDA

- Cybersecurity for Networked Medical Devices Containing Off-the-Shelf (OTS) Software - Guidance for Industry

- Mobile Medical Applications - Guidance for Industry and Food and Drug Administration Staff

- Postmarket Management of Cybersecurity in Medical Devices - Guidance for Industry

- Software as a Medical Device (SAMD): Clinical Evaluation - Guidance for Industry

- Medical Device Data Systems - Guidance for Industry

The FDA's general principles of software validation enforce the software developer to document the requirements, based on the intended use, prior to implementation and testing. Documentation of requirements appears to be a challenge when introducing Kanban practices into the software development lifecycle, as one of the main principles of the lean-Kanban manifesto is "no documentation, ever, which can't make sense". The "guidance for content of premarket submission for software contained in medical devices" forces the manufacturer to use a plan-driven software development lifecycle. This appears to be another challenge for the adoption of Kanban practices within the software development process, clearly favouring the implementation of requirements by the use of a fluid set of practices and in small iterative steps through intuitive abilities. Unclearly defined requirements before initiating a software development process are difficult to trace, as required by the FDA. Furthermore, the software developer must analyse the potential risk of each defined requirement for criticality and probability, placing the intended user at risk. Pekar et al. (2016) emphasise that a unified risk management approach, as required by the FDA, represents another challenge in the adoption of Kanban practices. Guidance on the implementation of risk management activities is almost absent in current development methods based on Kanban practices.

Another hindrance in the adoption of Kanban practices is that developers may perceive software development based on Kanban practices to be of lower quality compared to a plan-driven software development lifecycle (Özcan-Top \& McCaffery, 2017). The Kanban manifesto clearly underlines quality as the first principle to be followed, with a clear focus on "quality systems [...] and principles that guide the understanding of practices in which we [the software developer] can trust". Nevertheless, the linkage to quality systems when using Kanban practices with a 
medical device software development lifecycle is almost absent in academic literature.

\section{Europe}

The European parliament amended the existing medical- and in-vitro diagnostic device regulation in 2017 with a transition period of three and five years, respectively. Both directives, the MDR and the IVDR, are mandatory for software as medical device to be marketed within the European Union (Clarke et al., 2017; EU, 2017). These regulations introduce further rules, including specific requirements addressing the classification of software. Accordingly, a notified body certifies conformance with the MDR and the IVDR and then the software as medical device can be marketed within the European Union. For software as medical device, the latest amendment to the MDR and the IVDR introduced several changes, including:

- specific requirements addressing software classification into diagnostic, therapeutic and physiological devices

- the classification of software as active therapeutic medical device, prior classified solely as active medical devices

- identification of eHealth and mHealth software as mobile medical application. Once a mobile medical application is classified as a medical device, the MDR requirements must be considered.

With these changes, the European Parliament placed great emphasis on the international standards to be followed when developing software as medical device. For Europe, the programmer is recommended to develop the software according to IEC 62304 and IEC 60601-1 and its aligned guidance documents. The standard for IEC 62304:2006 Medical device software - Software life-cycle processes emphasises the management of software development to be realised by one independent person having the overall responsibility. Kanban practices in contrast favour the selforganisation of development teams. Software development through self-organising teams hinders a plan lifecycle, removes centrality and decision-making powers from one independent person. This appears to be a challenge, resulting in a loss of management control. Further organisational support might be necessary for Kanban practices to successfully develop medical device software (McHugh, McCaffery, \& Casey, 2014).

\section{Lean-Kanban software development}

The terms "lean" and "lean-Kanban" are poorly defined in literature on software development (Anand, Chandrashekar, \& Narayanamurthy, 2014). The authors Wang, Conboy, and Cawley (2012, p. 3) emphasise that the terms are often "poorly considered, multi-dimensional, ambiguous and inconsistent". The first step in developing a mixed method approach to the development of software as medical device is a clear concept and explicit corresponding Kanban practices. Corresponding to established lean-Kanban concepts and practices, this paper considers that the development of medical software also involves two main elements: the basic lean-Kanban concept with its corresponding practices: 


\section{Lean-Kanban concept}

The Kanban concept is not a term uniquely used in software development. The roots of the lean-Kanban concept can be traced back to other disciplines, with most literature dating the origin back to the 1940s and the production system implemented by Toyota (Müller, Tolujew, \& Kienzle, 2014). The lean-Kanban practice itself has not been well-known in academic literature since a study carried out by the Massachusetts Institute of Technology (MIT), researching huge differences in quality and productivity between car manufacturers in the USA and Japan. A study carried out by Sugimori, Kusunoki, Cho, and Uchikawa (1977), former Vice Presidents of Toyota Motor Company, developing a guidance system for implementing a continuous improvement system for high quality products in the right quantity, with developers displaying their full capabilities through active participation in improving their own work. The lean-Kanban concept is a development method enabling a company to programme high quality software, create knowledge, defer commitment, deliver fast, respect people, optimize the process, and only create the documentation needed (Poppendieck \& Poppendieck, 2007). The lean-Kanban concept interlinks five inherent values underling the Kanban approach:

- Quality systems: Implementing principles that guide the understanding of development practices which the developer trusts;

- Value stream: Mapping the steps of the development process identifying the value it adds;

- Flow: Implementing a continuously flowing development process;

- Perfection: Striving for perfection in the development process by identifying and removing waste in a continuous way;

- Small steps: Implementing changes through small steps driven by continuous improvement.

\section{Lean-Kanban practices}

The primary focus and overall practice of lean-Kanban is the identification and implementation of what to develop, when to develop, and in which way to develop (Münch, 2016) with respect to product quality and customer value. Kanban practice is Japanese for "visual sign" and, through a visual framework, classifies work into categories: "work to do", "work being done", "work done". By mapping the SDLC into manageable chunks, "quality adding" and "risk eliminating" activities can be identified and prioritised accordingly. Another main focus of lean-Kanban is implementation of practices in small, iterative steps into a current SDLC. Münch (2016) emphasises that the implementation of Kanban practices can be done by adding "Kanban on top of other existing workflows", such as a plan-driven SDLC.

The contemporary understanding of lean-Kanban development is primarily driven by practitioners' writings (Cawley, Wang, \& Richardson, 2012; Klespitz, Bíró, \& Kovács, 2015; Mc Hugh, McCaffery, \& Casey, 2017; Müller et al., 2014; Münch, 2016; Wang et al., 2012). To maintain the core intent of lean-Kanban, several lean-Kanban 
practices have been established and are steadily evolving. For the categorisation of the empirical data on lean-Kanban practices, Table 1 outlines what is considered to be lean-Kanban specific practices necessary for the development of medical software. As certain lean-Kanban practices are overlapping with a plan-driven SDLC and agile practices, this paper limits lean-Kanban practices to those practices which were found to be less represented in academic writing on Kanban software development but were recurring issues in terms of a lean-Kanban context. It is noted that the identification and categorisation of lean-Kanban practices is somewhat subjective. Nevertheless, the list is agreed upon by consensus among software developers and research, offering a perfect starting point for a mixed method approach to the development of software as medical device.

Table 1: Lean-Kanban practices for medical device software development

\begin{tabular}{|l|l|}
\hline Lean-Kanban practice & Reference \\
\hline Address bottlenecks & $\begin{array}{l}\text { (Mc Hugh et al., 2017; Wang et al., } \\
\text { 2012) }\end{array}$ \\
\hline Automatic verification and validation & (Reinertsten, 2009) \\
\hline Avoid waste & (Münch, 2016) \\
\hline $\begin{array}{l}\text { Clear conceptualization of software development } \\
\text { process }\end{array}$ & (Münch, 2016) \\
\hline $\begin{array}{l}\text { Continuous improvement through iterative steps } \\
\text { to establish a smoother flow }\end{array}$ & (Hibbs, Jewett, \& Sullivan, 2009) \\
\hline $\begin{array}{l}\text { Create only necessary documentation } \\
\text { Establish software documentation planning to } \\
\text { deliver necessary documentation to notified } \\
\text { bodies }\end{array}$ & $\begin{array}{l}\text { (Graziotin \& Jedlitschka, 2013; } \\
\text { Shalloway, 2018) }\end{array}$ \\
\hline $\begin{array}{l}\text { Establish group performance, no heroic } \\
\text { individuals }\end{array}$ & (Stellman \& Greene, 2014) \\
\hline $\begin{array}{l}\text { Implementation of Kanban approach within } \\
\text { quality system }\end{array}$ & (Shalloway, 2018) \\
\hline Implementation of virtual Kanban board & (Münch, 2016; Shalloway, 2018) \\
\hline $\begin{array}{l}\text { Link voice of customers to user and usability } \\
\text { requirements }\end{array}$ & $\begin{array}{l}\text { (Cawley et al., 2012; Raffo, Mehta, } \\
\text { Anderson, \& Harmon, 2010) }\end{array}$ \\
\hline Manage pair programming & $\begin{array}{l}\text { (Al-Baik \& Miller, 2015; Cawley et } \\
\text { al., 2012; Mc Hugh et al., 2017) }\end{array}$ \\
\hline Optimise the whole process and provide feedback & $\begin{array}{l}\text { (Cawley et al., 2012; Poppendieck \& } \\
\text { Poppendieck, 2007) }\end{array}$ \\
\hline PDCA (plan do check act) cycle & (Deming, 1986) \\
\hline Radical improvement within a limited time & (Lester, 2008; Wang et al., 2012) \\
\hline Visualise the workflow & (Poppendieck \& Poppendieck, 2007) \\
\hline
\end{tabular}

\section{Mixed method Kanban SDLC}

American and European guidelines clearly outline the tailoring of a software development lifecycle in order to standardize development practices and to achieve maximum impact (Zeegers, 2018). This paper focuses on the implementation of Kanban practices within a plan-driven medical device software development 
lifecycle. Implementing Kanban practices within a plan-driven SDLC has to primarily aim at developing medical device software with the highest quality possible in order to gain regulatory approval, while still reaping the benefits associated with utilising Kanban practices (Mitasiunas, Rout, O'Connor, \& Dorling, 2017). Accordingly, the foundation of such a mixed method approach is provided by a plan-driven SDLC, providing a comprehensive approach guiding through all necessary development stages mandatory for the development of safety-critical medical software (EU, 2017; Kim, Park, Lee, \& Lee, 2017). Özcan-Top and McCaffery (2017) emphasise the development of safe and reliable medical software particularly through a combination of Kanban practices with plan-driven methods. For the development of a mixed method Kanban SDLC, the v-model was chosen as a foundation, being a widely accepted approach for medical software developers (Mc Hugh et al., 2017). The mixed method approach particularly benefits from choosing a v-model as foundation in that it provides guidance for all necessary stages for developing medical device software with the highest quality possible in order to gain regulatory approval (Trektere, McCaffery, \& Lepmets, 2016). Academic research on the implementation of Kanban practices with a plan-driven SDLC clearly emphasise the use of the v-model as foundation, as it provides guidance on how to perform the necessary development activities (Kropp \& Meier, 2014; Pekar et al., 2016; Roßik \& Schwarcz, 2015). Several studies outline the v-model as a widely accepted approach to medical software (Cicotti, 2017; Mc Hugh et al., 2017; Polgár \& Kazinci, 2014). Furthermore, regulations such as IEC 60601-1 propose the use of a vmodel when developing medical devices. In selecting the v-model as foundation for a mixed method SDLC, the v-model for certification, as proposed by Ge, Paige, and McDermid (2010) is used. This v-model differs from the typical v-model, as it also includes a development stage for "usability specification and risk analysis" and the requirements outlined by IEC 60601-1 to obtain "regulatory certification". This model consists of five development phases, integrating the 1.) user requirements, 2.) usability specification and risk analysis, 3.) architectural design, 4.) module design specification, and 5.) module coding. These phases are consistent with the main software development activities specified in the EN 62304:2006: understand and specify the intended use and user requirements, identify possible risks and specify usability requirements, identify design solutions to meet user requirements, code and test designs against requirements. This mixed method approach was used as a basis for the survey, fulfilling both American and European requirements in order to gain regulatory approval when filing a software as medical device to the regulatory agency.

\section{Research methodology}

In order to gain an understanding of how shortcomings in both Kanban and plandriven approaches are resolved by the use of a mixed method Kanban SDLC, a questionnaire-based survey with organisations developing medical device software within Germany was carried out. A literature review was performed, examining the current SDLCs used for the development of safety-critical software within the invitro-diagnostics and medical device industry. The results of the literature review, 
particularly examining the use of a mixed method Kanban SDLC are outlined in section 1. Several papers on the use of lean-Kanban practices when developing safety-critical software were identified, as outlined in Table 2.

In the year 2016, approximately 1200 organisations produced medical devices in Germany, with only 12 \% developing medical device software (Flemming, 2016). Sufficient sample size was determined as eighteen companies, using sample size equation. The organisations surveyed ranged from small to medium-sized companies to large organisations, producing Class I to Class III medical device software. 2 The goal of the survey was to provide a better understanding of the challenges faced by software developers when developing safety-critical medical device software by the use of a plan-driven SDLC. The insights gained through the surveys then helped in the development of a mixed method Kanban SDLC approach. Participants surveyed included all members of a development team, regulatory affairs and senior management, whereas at least two persons from each of the eighteen companies identified had have been surveyed.

The survey was pursued through open-ended questions, being in several areas supplemented by closed-ended questions. The closed-ended questions were also designed through simple multiple-choice answers, in order to gain an understanding of the challenges faced by software developers when developing safety-critical medical software. Open-ended questions where used to permit respondents to freely choose how to answer (Patten, 2002). The open-ended questions encourage a continued discussion to provide a richer source of information than closed-ended questions, necessary to answer the second research purposes (Özcan-Top \& McCaffery, 2017; Patten, 2002).

In a first part, the responded was asked about the actual role in the medical device industry, followed by the years of experience with medical device software. The quality of the responses were validated through asking several persons within the same company.

The second part of the survey, revealing significantly relevant information, was concerned with the question which SDLC the organisation actually follows. The questionnaire then was structured according to the steps of the v-model for certification as proposed by Ge et al. (2010). The third part of the survey was structured according to the five development phases proposed by Ge et al. (2010), asking the respondents to provide recommendations as to how the adoption of Kanban practices can resolve the challenges associated with the current SDLC applied. Based on the five development phases proposed by Ge et al. (2010), the respondents were provided with a set of activities mandatory to be fulfilled when developing safety-critical medical software. For each activity, the responded rated the significance of the activity pertaining to one of the five phases. In particular, the respondents were asked to provide further insights and proposals for the

\footnotetext{
${ }^{2}$ The safety classification of the software as medical device is based on the European MDR classification system 2017/745/EU. This regulation defines Class I medical device software, which possesses a relatively small risk to the intended user, and Class III medical device software, possessing a potentially lifethreatening risk to the intended user.
} 
implementation of these activities. The objective of this questions was to establish which Kanban practices would be merged with the v-model l. The respondents then were asked how they would rate the effectiveness of their organisation in performing these activities. The objective of this question was to provide an understanding which area of the development of safety-critical medical software are performed most efficiently. For each of the five phases pertaining to the v-model of Ge et al. (2010), information is collected identifying which phases of the SDLC are the ones most challenging to software developers. This information is used to answer the first purpose.

In the last part of the survey the respondents were asked to provide information pertaining to the implementation of Kanban practices within a SDLC. The respondents further were asked in which way the adoption of Kanban practices has to occur. The aim of these questions was to evaluate how to implement the Kanban practices within the v-model.

\section{Results of the survey}

One of the objectives of the survey was to provide a better understanding of the challenges faced by medical device software developers. The insights provided by the respondents were also used to analyse the findings of the literature review in this context. All of the organisations surveyed develop and market medical software for Europe. Furthermore, $58 \%$ of these organisations develop software as medical device for use in the USA. $72 \%$ of the eighteen companies surveyed develop their medical software products based on the v-model. Furthermore, $28 \%$ of these companies use lean-Kanban practices for the development of their medical software products or combine the v-model with lean-Kanban practices. As part of the survey, the respondents were asked what, in their view, was a challenge to the adoption of Kanban practices. The survey showed that $35 \%$ of the respondents see "regulatory certification" as a challenge to the adoption of Kanban practices. Furthermore, $20 \%$ of the respondents' report "traceability", whilst $18 \%$ of the respondents report "implementation of risk management activities", $18 \%$ report "quality compliance issues", and $9 \%$ report "documentation" as challenges when implementing Kanban practices. The challenge of "regulatory implementation" is consistent with the findings of the literature review, while all other challenges were not reported in the literature reviewed. Finally, the respondents were asked to report on the implementation of Kanban practices within a v-model. The respondents provided ten essential Kanban practices to be implemented within the v-model. The respondents mentioned that "use cases", "user stories", "Kanban cards" and "collocation of team members" are important practices to be implemented within the first stage, that is, the user requirements stage, of the v-model. For the second stage, the respondents did not mention any further practice to be included. For the third stage of the v-model, the respondents mentioned "limit work in progress" and "use of stories" as important Kanban practices to be implemented. For the fourth stage, the module detailed design, no Kanban practices were proposed by the respondents. The respondents mentioned that in the fifth stage, "pair programming", "continuous coding and integration", "continuous improvement through automated testing", 
and "continuous feedback" are important Kanban practices to be implemented. Two respondents added that the Kanban practices mentioned in the fifth stage also have to take place when integration and integration testing occur. When asking the respondents how to implement the Kanban practices within the v-model 1, $84 \%$ of them answered that an iterative development approach should be used for stages three to five.

\section{Integration of Kanban practices within a plan-driven SDLC}

As part of the survey, the respondents were asked on their organisations SDLC actually used and implemented, to develop medical device software. The survey revealed that most companies use the v-model for the development of software of use in the USA and Europe. This result is consistent with the survey carried out by Ryan (2016), emphasising that software development in the medical device industry is traditionally reflected in a v-model standard. The answers of the respondents lead to the decision to select the v-model as foundation for a plan-driven SDLC for the integration of Kanban practices. The answers show that most companies are very familiar with the use and application of the v-model. The respondents further stated that based on a familiar foundation, the later integration of new practices seems to be much easier. In applying structures and phases already known by the German medical device companies, they are much more willing to adopt lean-Kanban practices within the SDLC they use. An insight not yet described in academic literature is that six companies see the implementation of regulatory requirements as a hindrance in adapting a completely new approach, rather than applying new structures to an existent v-model already implemented. In fact, applying a new SDLC would force companies to re-apply for regulatory approval (Mc Hugh et al., 2017). Application for regulatory approval because of the implementation and application of an approach seems to be challenging and therefore not applicable in the introduction of lean-Kanban practices for medical software development. This is another reason for the use of a v-model as foundation, where lean-Kanban practices are implemented, to create a hybrid SDLC.

Another new insight, not yet described in the literature, is that $28 \%$ of these German medical device organisations use lean-Kanban practices within their SDLC. Actual standards and requirements do not force medical device software developers to use a v-model. Nevertheless, it appears to guide the companies through the development process. Furthermore, the iterative nature of a Kanban approach seems to be one of the main reasons for its implementation, particularly because of the iterative nature of software development projects with several prototypes, as noted by three respondents. It was pointed out by the respondents that the structures of the v-model help to produce the necessary documents for gaining regulatory approval. Two respondents commented that this is the main reason for trying to adopt lean-Kanban practices within their SDLC. The respondents further commented that for the organisations it is unclear at which stages iterative leanKanban practices are best implemented.

In answering this question, the integration of lean-Kanban practices occurred on the basis of a plan-driven SDLC, the v-model as proposed by Ge et al. (2010). The survey 
revealed that, when integrating Kanban practices, iterative steps in the development of medical software are particularly the stages "architectural design", "module design specification" and "module coding". Accordingly, within these development stages, the lean-Kanban practices are implemented as an iterative approach. This is also confirmed by literature, as Graziotin and Jedlitschka (2013) point out that this iterative approach addresses "the issue of managing a constant flow of requirements". The integration of the iterative approach within the v-model is illustrated in Figure 1.

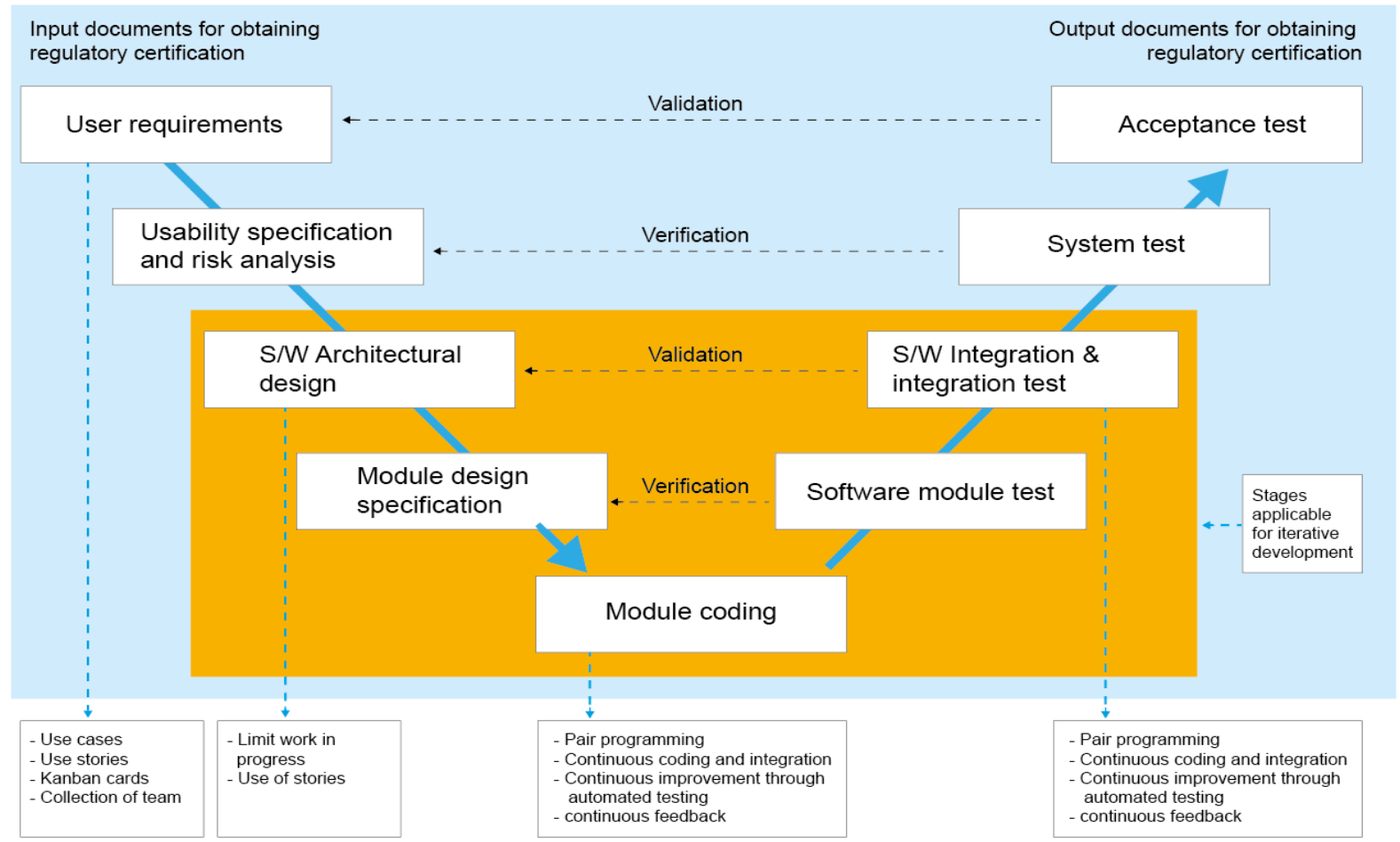

Figure 1: The Kanban V-model (KV-model)

As part of the survey, the respondents were asked what they regard as challenges to the adoption of Kanban practices. In particular, "regulatory implementation" and "traceability" were reported as challenges to the adoption of Kanban practices. The aspect of the implementation of regulatory practices is the challenge consistent with the findings of the literature review (Mc Hugh et al., 2017). An important aspect emerging from the research is the way in which these challenges can be successfully overcome and managed when using Kanban practices. The American, European and Canadian regulatory environment requires the developers of medical software, on the one hand, to document the requirements before beginning with the software development. Afterwards, the requirements are then translated into architectural and modular specifications and required to be traceable during the entire SDLC. Kanban practices require a continuous and fluid set of actions and improvements throughout the entire software project. This does not seem to be contradicting, as a fluid set of actions and continuous improvements is to be implemented in an iterative way within the SDLC, being traceable at any time (Pekar et al., 2016). Much more, the FDA outlines in its guideline for industry on the "General Principles of Software Validation" that: 
"Most software development models will be iterative. This is likely to result in several versions of both the software requirement specification and the software design specification. All approved versions should be archived and controlled in accordance with established configuration management procedures".

Accordingly, after identifying and documenting the user requirements, each of them is broken down into manageable chunks (Kim et al., 2017) and traced during the entire development project. Thus, the requirements to, on the one hand, enable "regulatory implementation", and to ensure, on the other hand, "documentation" right from the beginning, are also reported as being challenges in the implementation of Kanban practices. "Traceability" is another example of how challenges to the implementation of Kanban practices into a plan-driven SDLC can be overcome.

Furthermore, a set of Kanban practices were identified to be implemented within each of the five stages pertaining to the v-model proposed by Ge et al. (2010). This set of Kanban practices is based on the comments provided by the respondents and based on the practices identified during the literature review. The literature revealed several activities consistent with the insights provided by the respondents. Nevertheless, nowhere did the literature provide information as to at which stage of the development the Kanban practices should be incorporated. The Kanban practices proposed by the respondents were implemented to the stages proposed. Furthermore, a cross-checking with the activities defined by IEC 62304:2006 was performed to proof consistency of the Kanban practices proposed by the respondents, as tabulated in Table 2.

Table 2: Proposed Kanban activities and cross-reference to IEC 62304:2006

\begin{tabular}{|c|c|c|}
\hline Development stage & Respondents' insights & IEC 62304:2006 \\
\hline User requirements & $\begin{array}{ll}\text { - } & \text { Use cases } \\
\text { - } & \text { Use stories } \\
\text { - } & \text { Kanban cards } \\
\text { - } & \text { Collection of team } \\
& \text { members } \\
\end{array}$ & - $\quad$ No reference \\
\hline $\begin{array}{l}\text { Usability specification and } \\
\text { risk analysis }\end{array}$ & - $\quad$ No propositions made & - $\quad$ No reference \\
\hline Architectural design & $\begin{array}{ll}\text { - } & \text { Limit work in progress } \\
\text { - } & \text { Use of stories }\end{array}$ & $\begin{array}{l}\text { Section 5.3: } \\
-\quad \text { Stories } \\
\end{array}$ \\
\hline Module design specification & - $\quad$ No propositions made & - $\quad$ No reference \\
\hline Module coding & 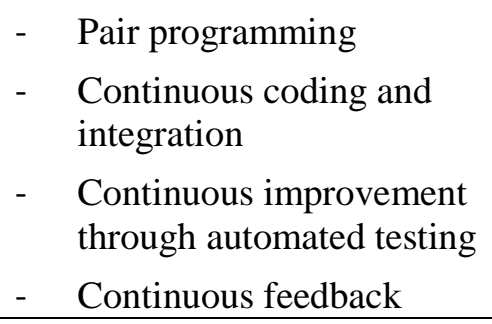 & $\begin{array}{ll}\text { Section 5.5.5: } \\
\text { - } & \text { Pair programming } \\
\text { - } & \text { Continuous integration } \\
\text { - } & \text { Continuous automated } \\
& \text { testing }\end{array}$ \\
\hline
\end{tabular}




\section{Conclusions}

A number of challenges to the adoption of lean-Kanban practices when developing software as medical device has been identified during the literature review. The identified challenges have been confirmed by the respondents during the survey, and challenges not yet described in literature were identified. Key requirement is that the software developer must develop the software according to international regulations and regulatory requirements, in order to obtain regulatory approval. Regulatory bodies do not dictate to the software developer the use of a specific development approach, but generally recommend to the developer the implementation of iterative life cycling. Streamlining the SDLC to gain regulatory conformance, implementing the required stages with design control activities to produce the necessary documentation, and implementation of iterative lifecycle management are perceived as challenges when implementing a lean-Kanban SDLC. The proposed hybrid SDLC (KV-model) is categorised into five development activities, each producing the regulatory deliverables which device manufacturers must deliver to the regulatory bodies. The KV-model proposed in this research is based on a mixed method Kanban SDLC and identifies Kanban practices pertaining to each of the five development stages. The lean-Kanban practices are founded on the comments provided by German software as medical device developers. Selectively choosing appropriate lean-Kanban practices and integrating them within a plan-driven lifecycle reaps the advantages of employing Kanban practices whilst still producing the mandatory regulatory deliverables.

\section{References}

Al-Baik, O., \& Miller, J. (2015). The kanban approach, between agility and leanness: a systematic review. Empirical Software Engineering, 20(6), 1861-1897.

Anand, G., Chandrashekar, A., \& Narayanamurthy, G. (2014). Business process reengineering through lean thinking: a case study. Journal of Enterprise Transformation, 4(2), 123-150.

Cawley, O., Wang, X., \& Richardson, I. (2012). Regulated software development-an onerous transformation. Paper presented at the International Symposium on Foundations of Health Informatics Engineering and Systems.

Cicotti, G. (2017). An evidence-based risk-oriented V-model methodology to develop ambient intelligent medical software. Journal of Reliable Intelligent Environments, 3(1), 41-53.

Clarke, P., Lepmets, M., McCaffery, F., Finnegan, A., Dorling, A., \& Eagles, S. (2017). Characteristics of a medical device software development framework.

Deming, W. (1986). Out of the crisisMIT Center for Advanced Engineering. Cambridge, $M A$.

EU. (2017). Medical device regulation. Official Journal of the European Union(2017/745 ) .

Flemming, G. (2016). The medical technology industry in Germany. In G. t. a. invest (Ed.). Nürnberg. 
Ge, X., Paige, R. F., \& McDermid, J. A. (2010). An iterative approach for development of safety-critical software and safety arguments. Paper presented at the AGILE Conference, 2010.

Graziotin, D., \& Jedlitschka, A. (2013). Recent developments in product-focused software process improvement: PROFES 2013 conference report. ACM SIGSOFT Software Engineering Notes, 38(6), 29-34.

Hibbs, C., Jewett, S., \& Sullivan, M. (2009). The art of lean software development: $a$ practical and incremental approach: "O'Reilly Media, Inc.".

Kim, D., Park, Y.-S., Lee, B., \& Lee, J.-W. (2017). Association-Based Process Integration for Compliance with Core Standards in Development of Medical Software Advances in Computer Science and Ubiquitous Computing (pp. 12201226): Springer.

Klespitz, J., Bíró, M., \& Kovács, L. (2015). Aspects of improvement of software development lifecycle management. Paper presented at the Computational Intelligence and Informatics (CINTI), 2015 16th IEEE International Symposium on.

Kropp, M., \& Meier, A. (2014). Swiss Agile Study 2012. Agile Software-Entwicklung in der Schweiz. Zürcher Hochschule für Angewandte Wissenschaften.

Lester, R. I. (2008). Lean Thinking: Banish Waste and Create Wealth in Your Corporation. Air \& Space Power Journal, 22(2), 103-105.

Mc Hugh, M., McCaffery, F., \& Casey, V. (2017). Barriers to using agile software development practices within the medical device industry.

McHugh, M., McCaffery, F., \& Casey, V. (2014). Adopting agile practices when developing software for use in the medical domain. Journal of Software: Evolution and Process, 26(5), 504-512.

Mitasiunas, A., Rout, T., O'Connor, R. V., \& Dorling, A. (2017). Software Process Improvement and Capability Determination. Communications in Computer and Information Science, 477.

Müller, E., Tolujew, J., \& Kienzle, F. (2014). Push-Kanban-a kanban-based production control concept for job shops. Production Planning $\mathcal{E}$ Control, 25(5), 401-413.

Münch, P. (2016). Using Smartsheet with Kanban. Retrieved 24.08.2018, 2018, from https:/ / www.smartsheet.com/agile-vs-scrum-vs-waterfall-vs-kanban

Özcan-Top, Ö., \& McCaffery, F. (2017). A hybrid assessment approach for medical device software development companies. Journal of Software: Evolution and Process.

Patten, D. M. (2002). The relation between environmental performance and environmental disclosure: a research note. Accounting, organizations and Society, 27(8), 763-773.

Pekar, V., Felderer, M., Breu, R., Nickl, F., Roßik, C., \& Schwarcz, F. (2016). Integrating a lightweight risk assessment approach in to an industrial development process. Paper presented at the International Conference on Software Quality.

Polgár, P. B., \& Kazinci, F. (2014). Process communication in medical software development. Journal of Software: Evolution and Process, 26(3), 313-320.

Poppendieck, M., \& Poppendieck, T. (2007). Implementing lean software development: From concept to cash: Pearson Education. 
Raffo, D., Mehta, M., Anderson, D. J., \& Harmon, R. (2010). Integrating Lean principles with value based software engineering. Paper presented at the Technology Management for Global Economic Growth (PICMET), 2010 Proceedings of PICMET'10:.

Reinertsten, D. G. (2009). The principles of product development flow: second generation lean product development: Celeritas.

Roßik, C., \& Schwarcz, F. (2015). Integrating a Lightweight Risk Assessment Approach into an Industrial Development Process. Paper presented at the Software Quality. The Future of Systems-and Software Development: 8th International Conference, SWQD 2016, Vienna, Austria, January 18-21, 2016, Proceedings.

Ryan, K. T. (2016). Software processes for a changing world. Journal of Software: Evolution and Process, 28(4), 236-240.

Shalloway, A. (2018). Lean-Kanban manifesto. Retrieved 24.03.2018, 2018, from http:/ / www.netobjectives.com/blogs/lean-kanban-manifesto

Stellman, A., \& Greene, J. (2014). Learning agile: Understanding scrum, XP, lean, and kanban: " O'Reilly Media, Inc.".

Sugimori, Y., Kusunoki, K., Cho, F., \& Uchikawa, S. (1977). Toyota production system and kanban system materialization of just-in-time and respect-forhuman system. The International Journal of Production Research, 15(6), 553-564.

Trektere, K., McCaffery, F., \& Lepmets, M. (2016). Development of a software process framework to assist organizations developing mobile medical apps. Paper presented at the Biomedical and Health Informatics (BHI), 2016 IEEE-EMBS International Conference on.

Wang, X., Conboy, K., \& Cawley, O. (2012). “Leagile” software development: An experience report analysis of the application of lean approaches in agile software development. Journal of Systems and Software, 85(6), 1287-1299.

Zeegers, R. (2018). Information Security Management Professional based on ISO/IEC 27001 Courseware-English: Van Haren. 


\title{
Non-Complex Cost-Effective Energy Monitoring using the Internet of Things
}

\author{
Pierre E Hertzog and Arthur J Swart \\ Central University of Technology \\ Bloemfontein, South Africa
}

\begin{abstract}
Energy monitoring is vital to identifying ways of reducing energy consumption. This may lead to a reduction in the use of non-renewable energy sources (such as coal), increasing the lifespan of current energy storage systems (such as batteries) and reducing financial costs (such as electrical utility costs). Current commercial monitoring systems may prove too complex or too expensive for the average homeowner to install and use. How can the internet of things, therefore, be used to identify sources of energy consumption? The purpose of this paper is to describe the design and setup of a non-complex cost-effective energy monitoring system for a residential home environment. This may enable homeowners to identify unwanted energy consumption and thereby reduce their electrical utility bills. The system incorporates an Arduino Uno Board, ESP8266 WiFi module and a cloud server called ThingSpeak. No physical wiring connections are needed, as only a current transformer sensor needs to be hooked around the main AC supply line from the electrical utility provider. No PC data storage is required, as the data is stored on the cloud server. The only key requirement is internet connectivity so that the WiFi module may connect directly to the cloud server where all data is captured and presented.
\end{abstract}

Keywords: Arduino, internet of things, energy.

\section{Introduction}

Energy monitoring is essential for understanding the sources of consumption inside a building and to take appropriate measures to save energy (Peng \& Qian, 2014). The importance of understanding the sources of energy consumption may lead to identifying ways of reducing energy consumption. This is vital as a global drive exists in decreasing the use of non-renewable energy sources and in reducing carbon footprints. This is even more critical within the boundaries of South Africa (SA), that has felt the effects of load shedding and massive electricity price hikes over the past decade. 
Load shedding is defined as coordinated sets of controls that decrease the electric loads in the system so as to restore the system to its normal operating condition (Mageshvaran \& Jayabarathi, 2015). It is often manifested by switching off the electrical energy supply to pre-selected neighborhoods within a city or town for up to 2 hours per day. This may have a serious impact on many small medium and micro-sized enterprises (SMME) (such as food stores, laundry services and internet café's), causing them to lose revenue or valuable stock items. Reducing the occurrence of load shedding is of primary importance which each homeowner may contribute to by taking responsibility for the electrical energy usage in their homes. Helping homeowners to identify unwanted energy consumption in their homes may empower them to better fulfill their responsibility.

Massive electrical energy price increases have hit SA home owners over the past decade. The National Energy Regulator of South Africa (NERSA) approved an 8\% average electrical energy price increase per annum for the period 2013 through 2017 (NERSA, 2013). Currently, homeowners pay around R1 000 for $555 \mathrm{kWh}$ of electrical energy (or $0.12 \$$ per $\mathrm{kWh}$ ) that is almost sufficient to cover the energy consumption of an average sized residential household (consists of 3 people) in SA for one month. Many commercial energy monitoring systems exist that may be used to identify unwanted energy consumption (P.E. Hertzog \& Swart, 2015; Swart, Pienaar, \& Schoeman, 2013). Stand-alone systems may cost from $\$ 150$ to $\$ 1000$, often requiring specialised PC software to analyse the data. One innovative way of by-passing these systems involves the use of the internet of things (IoT). IoT is demarcated as a huge network that merge with the Internet through sensors to collect information such as temperature, electricity, light intensity and position to name a few (Fan \& Liu, 2014). How can the IoT though be used to identify sources of energy consumption?

The purpose of this paper is to describe the design and setup of a non-complex costeffective energy monitoring system for a residential home environment. Firstly, a short overview of the Arduino UNO board, the ESP8266 WiFi module and the ThingSpeak cloud server will be given. The design of the energy monitoring system will follow. Lastly, a few test results are provided to highlight the data presentation formats of the system.

\section{Arduino}

Arduino originated from Italy and was designed in 2005 to teach Interaction Design. This design discipline puts prototyping at the heart of its process (Junior et al., 2013). The Arduino users consist of scientists and people from all walks of life. These may include, artists, DIY hobbyists and students from almost all disciplines (Jamieson, 2010). The concept of sharing designs on the web gives these users entree to a vast knowledge base (Jamieson, 2010).

The Arduino platform is very adaptable, and it is also an open-source platform which makes it very accessible (Aguilar-Acevedo \& Alejo, 2013). The system offers a variety of digital and analog inputs and outputs, serial interface and Pulse-widthmodulation (PWM) outputs (Pierre E Hertzog \& Swart, 2016). The user code can easily be changed and updated via the USB port on a PC. The communication to the USB occurs by the use of standard serial protocol. The hardware is inexpensive, and the software is freely available for students (Kumar, Bindu, Sneha, \& Sravani, 2013). 


\section{ESP8266}

The ESP8266 is a very cheap WiFi chip with a full TCP/IP stack and an onboard microcontroller (Di Nisio, Di Noia, Carducci, \& Spadavecchia, 2015). In August 2014, the ESP-01 module was launched by a manufacturer with the name AI-Thinker (Bohora, Maharjan, \& Shrestha, 2016). The ESP-01 module (shown in Figure 1) incorporates the ESP8266 chip and allows a microcontroller to connect to a WiFi network and make simple TCP/IP connections. In this paper, the ESP-01 module is used to communicate data from an Arduino UNO board to the ThingSpeak cloud server. The Arduino board serves to interface a current transformer (CT) sensor that is hooked around the main AC supply line from the electrical energy utility.

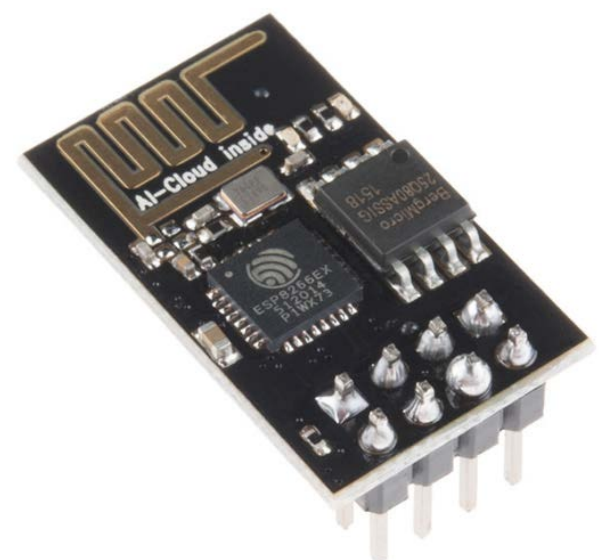

Figure 1- ESP-01 Wi-Fi module

\section{ThingSpeak}

ThingSpeak was launched in 2010 with the aim of supporting IoT applications (Montori, Bedogni, \& Bononi, 2016). As part of the service, ThingSpeak has integrated support from MATLAB through Mathworks (Malagi, 2017). This gives ThingSpeak users access to tools to analyse and visualize uploaded data without the need for a software license for MATLAB.

The data captured on ThingSpeak is stored in channels (Acharya \& Kuzhalvaimozhi, 2015). Each channel includes eight fields that can hold measured data, three fields for location data and one field for status data. Once data is collected in a channel, ThingSpeak can be used to analyse and visualize it. Recorded data can also be exported in a spreadsheet format (Abo-Zahhad, Ahmed, Farrag, Ahmed, \& Ali, 2015). Channel settings include the following:

1. Channel Name: The user can enter a unique channel name.

2. Description: The user can describe the ThingSpeak channel.

3. Field\#: Here the user can name and enable up to 8 data fields per channel.

4. Metadata: The user can enter information about channel data.

5. Tags: User identified tags.

6. Latitude: The latitude where the sensor is located.

7. Longitude: The longitude where the sensor is located.

8. Elevation: Specify the height position of the sensor.

9. Make Public: A checkbox to make channel public or to keep it private.

10. URL: If the user has a website that contains information about the channel, it can be specified here. 
11. Video ID: If the user has a YouTube ${ }^{\mathrm{TM}}$ or Vimeo® video that displays the channel information, it can also be specified.

\section{Design}

In this section, the design of the energy monitoring system is presented. Firstly, an overview of the whole system will be given, where after the CT sensor and signal conditioning circuit will be described. The next step will be to explain the software and calculations performed by the Arduino microcontroller. The WiFi module will be described next, where after the setup of the cloud server (ThingSpeak) will be discussed. Lastly, the collection of data by the researcher from the cloud server will be outlined.

The complete block diagram of the energy monitoring system is shown in Figure 2. In SA, the single-phase AC mains supply from the electrical energy utility to residential houses is $230 \mathrm{~V}$. As a non-complex cost-effective system was envisioned, only the mains current was measured and not the mains voltage, which is known. Data from the CT in block one (see Figure 2) is passed to the Arduino microcontroller in the next block. The RMS current is measured every one second, with a one-second delay between samples, where the average current for ten measurements is used to calculate the power. This data is then passed to the ESP8266 WiFi module that is set to connect to a specified WiFi network to enable it to upload data to a cloud server, named ThingSpeak. The researcher can access the cloud server at any time and from anywhere on the planet, to simply view the data or download it for further analysis.

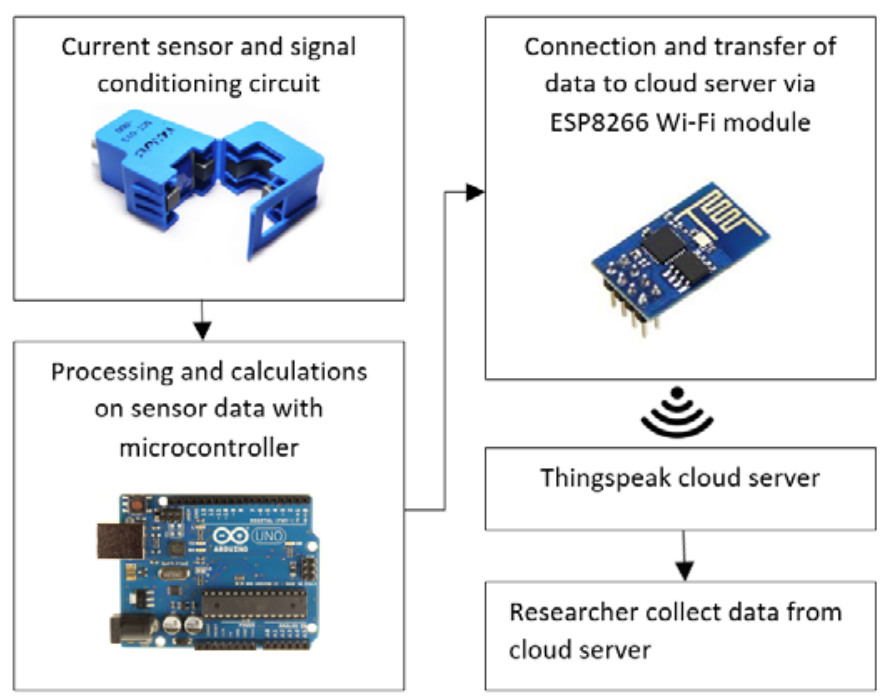

Figure 2- Block diagram of non-complex energy monitoring system

\section{The current transformer sensor}

The CT sensor that was chosen is rated for 100 A input, giving a corresponding 50 $\mathrm{mA}$ output, having a split core (SCT013). This enables easy installation of the CT sensor around the main AC supply line as shown in Figure 3 (within the dotted circle). Subsequently, the user does not need to disconnect any wires or modify any connections within the distribution board of the house. A multimeter is also visible in Figure 3 (AC-DC MS2600) that was used to validate the captured data on the 
cloud server. The circuit that was used for signal conditioning is shown in Figure 4, which is connected between the CT sensor and the Arduino microcontroller.

The purpose of the signal conditioning circuit is to convert the current reading from the CT to a voltage below $2.5 \mathrm{~V}$ that can be sampled by the analog to digital converter of the Arduino microcontroller. Calculations concerning component values and calibration will follow.

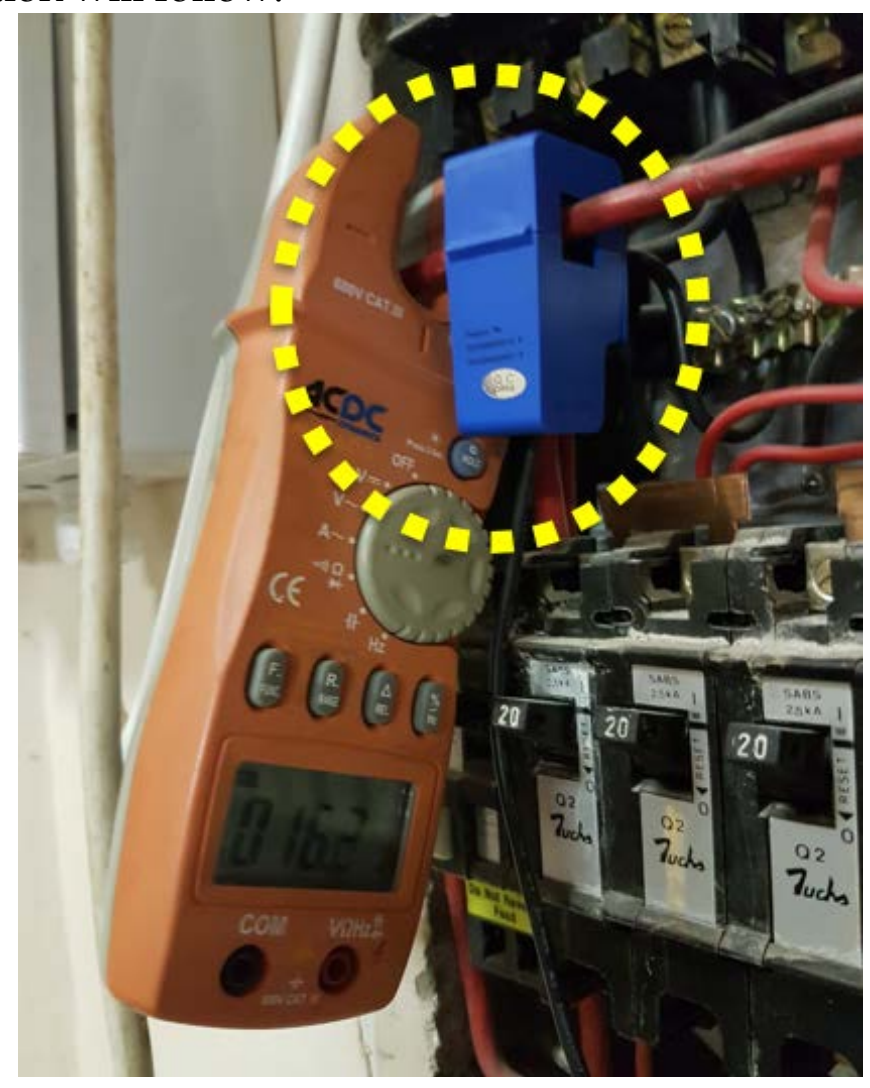

Figure 3-100 A CT coupled in a distribution board of a residential home

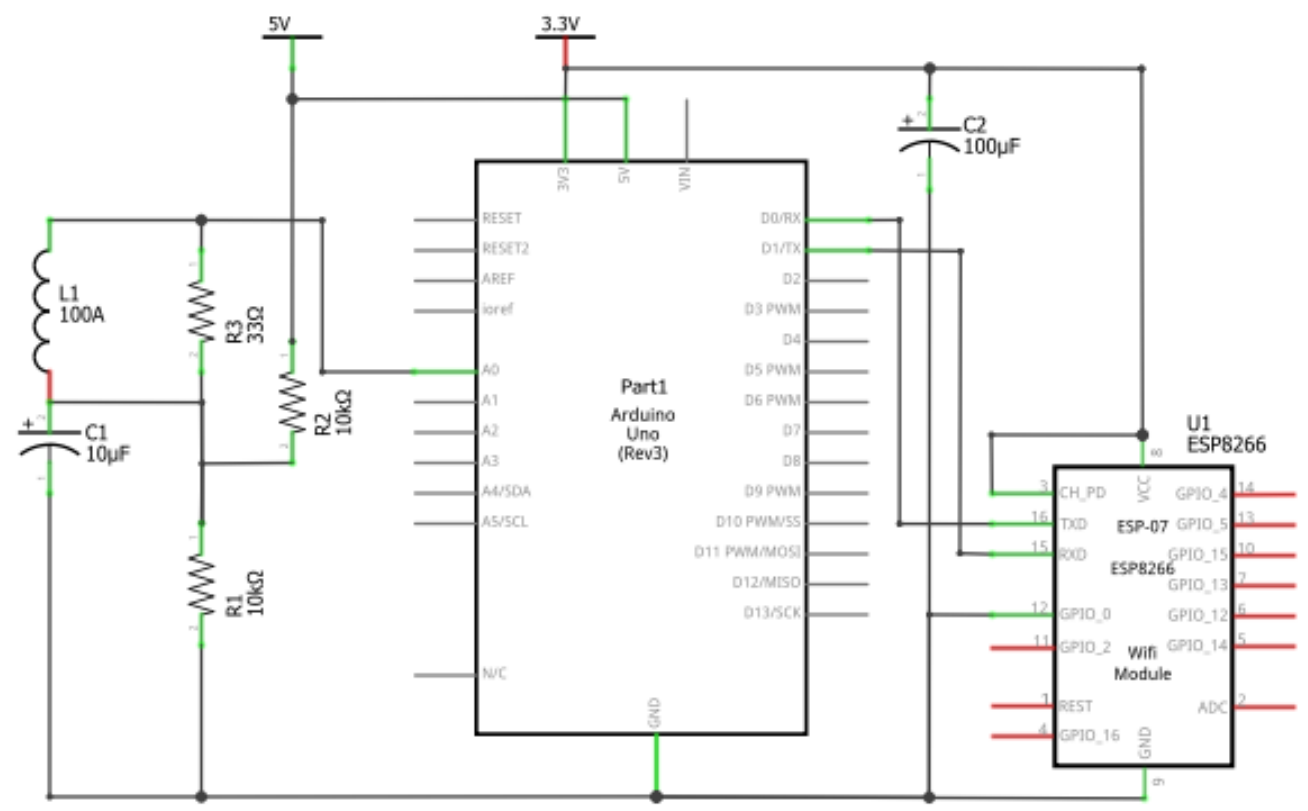

Figure 4- Circuit diagram of noncomplex energy monitor system 
The CT's primary to secondary winding ratio can be calculated from the specified primary and secondary current ratio, as shown in Equation (1). The secondary current must be calculated next in order to determine a suitable burden resistor which is required by the CT sensor. The burden resistor is dependent on the maximum system current. Although this is a 100 A CT sensor, it may be that the maximum possible system current is less than $100 \mathrm{~A}$. In this paper, the burden resistor is calculated for a maximum current of 100 A RMS. Current will flow through the burden resistor that will result in a specific voltage that can be read by the analog to digital converter of the microcontroller. The $100 \mathrm{~A}$ specified rating of the CT is an RMS value and needs to be converted to a maximum value (See Equation (2). The maximum value is needed to complete the calculation of the calibration factor that is discussed later in this section. The two $10 \mathrm{k} \Omega$ resistors (R1 and R2 in Figure 4) form a voltage divider circuit limiting the maximum input voltage to the Arduino to $2.5 \mathrm{~V}$, which is developed across the burden resistor, R3. The burden resistor may then be calculated using Equation (4). The closest E12 value for the calculated burden resistor is then chosen. The voltage generated across the burden resistor by the current flowing in the secondary of the CT sensor is used as an input voltage to the analog to digital converter in the Arduino microprocessor (pin A0 in Figure 4). The last calculation (Equation (5)) is performed to determine a calibration factor. The calibration factor is dependent on the burden resistor and is used in the software to convert the measured voltage from the Arduino microcontroller (less than $2.5 \mathrm{~V}$ ) into a real-time current value (maximum of $100 \mathrm{~A}$ ).$$
I p(\text { mesured })=\sqrt{2}
$$

$$
\begin{aligned}
& n=\frac{I s}{I p} \\
& =\frac{100 A}{50 m A} \\
& =2000 \\
& \text { Is }=\frac{I p}{n} \\
& =\frac{141.4 A}{2000} \\
& =0.0707 \mathrm{~A} \\
& \text { Calibration value }=\frac{n}{R(\operatorname{burden}(E 12))}
\end{aligned}
$$$$
=\sqrt{2} \times 100
$$$$
=141.4 \mathrm{~A}
$$

$$
\begin{aligned}
& R(\text { burden })=\frac{V(\text { sensor })}{I S} \\
& =\frac{2.5 \mathrm{~V}}{0.0707 \mathrm{~A}} \\
& =35.4 \Omega
\end{aligned}
$$

$$
\times \text { Irms }
$$

(5) Where:

$n=$ Transformer ratio

Is $=$ Secondary current

I $p$ = Primary current

$R($ burden $)=$ Burden resistor on secondary of $C$

$V($ sensor $)=$ Maximum voltage across the CT

\section{Processing of sensor data}

In this section, important aspects of the Arduino's microprocessor code is discussed. The entire code cannot be shown due to space constraints. Please note that the line numbers are only used to assist in the description of the required code. 
The WiFi name (line 1) and password (line 2) must be defined, as well as the IP address (line 3 ) of the cloud server. In line 4 the key for the specific channel within the cloud server where the power reading must be uploaded is specified. This key can be obtained by the researcher from his or her account on the ThingSpeak cloud server. Line 5 includes the library for the energy calculations and line 6 the mathematical library. The energy calculations done in the library is to convert the measured voltage across the burden resistor, that was read by the analog to digital converter on the Arduino microcontroller, to a real-time current value.

1. \#define SSID "SSID-WiFiname"

2. \#define PASS "password"

3. \#define IP "184.106.153.149"

4. String msg = "GET / update?key=KEY"

5. \#include "EmonLib.h"

6. \#include <math.h>

The calibration factor (60) that was calculated by using Equation (5), as well as the analog port (0) is stipulated in command line 7. This port is one of four analog input ports that may be used on the Arduino microprocessor to connect to the CT sensor.

7. emon1.current $(0,60)$;

The voltage across the burden resistor of the CT is sampled via an analog to digital converter in the Arduino microcontroller. In line 14, a library function is called where the RMS current is calculated from the voltage that is read from analog port (A0). Ten readings are taken in a second, after which a delay of $1000 \mathrm{~ms}$ is set (line 13). This means that readings are obtained for every odd second (1, 3, 5 etc.) and then averaged according to line 18. The power calculation is done in line 19. This power value is the average power over a ten cycle period and is passed back to the main routine in the code (line 20).

8. void updateReadings ()\{

9. double Total $=0$;

10. int var $=0$;

11. while $(\operatorname{var}<10)$

12. \{

13. delay(1000);

14. Irms $=$ emon1.calcIrms $(1480)$;

15. Total=Irms+Total;

16. var++;

17. $\}$

18. Irms=Tot $/ 10$;

19. Power $=230 *$ Irms;

20. $\}$

\section{WiFi communication and cloud storage}

The average power that was obtained in the previous section must now be communicated to the cloud server, ThingSpeak. This is accomplished by the use of 
the ESP8266 WiFi module. The code for communication and transmission of the data by the ESP8266 module is shown in the following subroutine.

21. void WrightThingSpeak ()\{

22. String $\mathrm{cmd}=$ "AT+CIPSTART $=\backslash " T C P \backslash ", \backslash " '$;

23. $\mathrm{cmd}+=\mathrm{IP}$;

24. $\mathrm{cmd}+=" \backslash ", 80 "$;

25. $\mathrm{cmd}=\mathrm{msg}$;

26. $\mathrm{cmd}+=$ "\&field1=";

27. $\mathrm{cmd}+=$ Power;

28. cmd $+=" \backslash \mathrm{r} \backslash \mathrm{n}$ ";

29. $\}$

In the previous subroutine, AT commands are used to communicate with the ThingSpeak cloud server via the ESP8266 module. The IP address of ThingSpeak (line 23), the port used (line 24), and the position of the calculated power value (line 27 ) is set. In the ThingSpeak cloud server, the data is captured and recorded and can be retrieved by the researcher at any time and anywhere. As an example, the recorded data for 24 April 2017 is displayed in Figure 5.

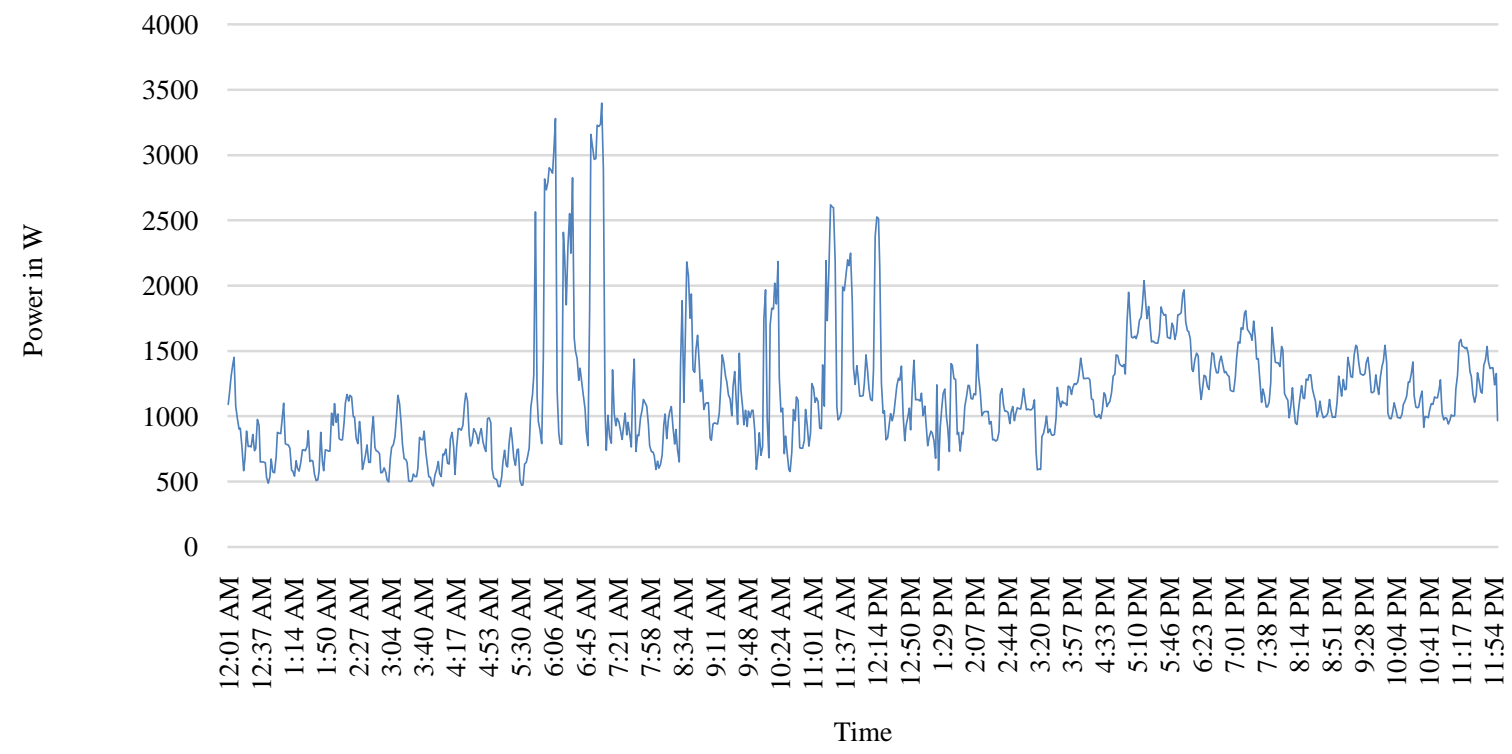

Figure 5- Example of energy data that was collected for 24 April 2017

It is evident that there are many individual peaks as appliances are switched on and off. General higher consumption can be noticed between 06h00 and 07h00 where the household is getting ready for work. In this period they have used hot water to shower, that will trigger the geyser thermostat, and have prepared food using a microwave oven and kettle. Another higher consumption area is evident between $11 \mathrm{~h} 00$ and $12 \mathrm{~h} 00$ that can be contributed to the preparation of lunch, which is the main meal of the family. A third and smaller peak can be noticed between $17 \mathrm{~h} 00$ and 19h30 when various technological devices are being used by the family that has returned from work.

To confirm the validity of the recorded values, the Root-mean-square (RMS) current was also measured (using the multimeter shown in Fig. 4.) and compared to the 
recorded values. As can be seen from Figure 6 the values were very close, and the maximum deviation between the measured and recorded values were $1.6 \%$.

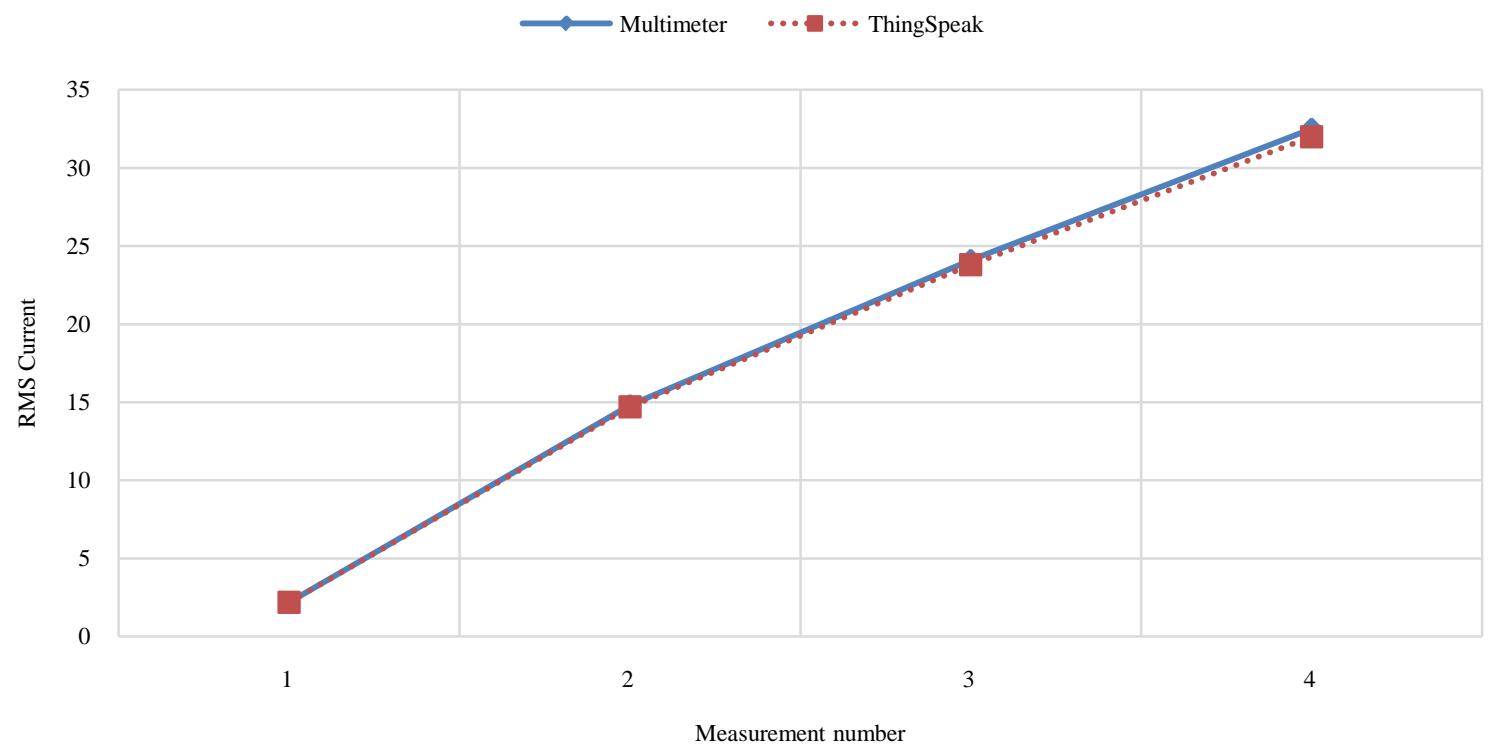

Figure 6- Comparison between multimeter and ThingSpeak readings

\section{Conclusions}

The purpose of this paper was to describe the design and setup of a non-complex cost-effective energy monitoring system that an average residential homeowner may use to identify sources of energy consumption. This system is non-complex in that it only requires a CT sensor, Arduino microprocessor, ESP8266 WiFi module and internet connectivity. These components are furthermore costeffective, as they may be purchased in SA for around \$100. No dedicated PC is required to capture and record the data, and no expensive software is required to use the components. The data may be viewed at any time and anywhere, making it very convenient for the homeowner who may want to keep an eye on his home's energy consumption while away on vacation. This enables the continuous monitoring of heavy intensive electrical energy guzzlers, such as water pumps. These are required to supply adequate water from bore holes in drought-stricken areas or to maintain the circulation of water in swimming pools.

Further research may include the analysis of data over longer periods of time for specific households, the use of Long Term Evolution (LTE) technology to transfer the data to the ThingSpeak cloud server and to monitor more sensors with the system. Identifying the power signature of different sources of energy consumption is also planned.

\section{References}

Abo-Zahhad, M., Ahmed, S. M., Farrag, M., Ahmed, M. F., \& Ali, A. (2015). Design and implementation of building energy monitoring and management system based on wireless sensor networks. Paper presented at the Computer Engineering \& Systems (ICCES), 2015 Tenth International Conference on.

Acharya, C., \& Kuzhalvaimozhi, S. (2015). Irrigation and Internet of Things Platform. 
Aguilar-Acevedo, F., \& Alejo, V. G. (2013). Using open-source platform for trajectory control of DC motors. Paper presented at the Power, Electronics and Computing (ROPEC), 2013 IEEE International Autumn Meeting on.

Bohora, B., Maharjan, S., \& Shrestha, B. R. (2016). IoT Based Smart Home Using Blynk Framework. Zerone Scholar, 1(1), 26-30.

Di Nisio, A., Di Noia, T., Carducci, C. G. C., \& Spadavecchia, M. (2015). Design of a low cost multipurpose wireless sensor network. Paper presented at the Measurements \& Networking (M\&N), 2015 IEEE International Workshop on.

Fan, Y. B., \& Liu, H. (2014). Study on Application Model of Internet of Things for Green Manufacturing. Applied Mechanics and Materials, 484-485, 187-190.

Hertzog, P. E., \& Swart, A. J. (2015). A customizable energy monitoring system for renewable energy systems. Paper presented at the SAUPEC 2015, University of Johannesburg.

Hertzog, P. E., \& Swart, A. J. (2016). Arduino-Enabling engineering students to obtain academic success in a design-based module. Paper presented at the Global Engineering Education Conference (EDUCON), 2016 IEEE.

Jamieson, P. (2010). Arduino for teaching embedded systems. are computer scientists and engineering educators missing the boat? Proc. FECS, 289-294.

Junior, L. A., Neto, O. T., Hernandez, M. F., Martins, P. S., Roger, L. L., \& Guerra, F. A. (2013). A low-cost and simple arduino-based educational robotics kit. Cyber Journals: Multidisciplinary Journals in Science and Technology, Journal of Selected Areas in Robotics and Control (JSRC), December edition, 3(12), 1-7.

Kumar, Y. N., Bindu, P. H., Sneha, A. D., \& Sravani, A. (2013). A Novel Implementation of Phase Control Technique for Speed Control of Induction Motor Using ARDUINO. International Journal of Emerging Technology and Advanced Engineering, 3(4), 469-473.

Mageshvaran, R., \& Jayabarathi, T. (2015). GSO based optimization of steady state load shedding in power systems to mitigate blackout during generation contingencies. Ain Shams Engineering Journal, 6(1), 145-160.

Malagi, M. (2017). Heath monitoring system based on IoT.

Montori, F., Bedogni, L., \& Bononi, L. (2016). On the integration of heterogeneous data sources for the collaborative Internet of Things. Paper presented at the Research and Technologies for Society and Industry Leveraging a better tomorrow (RTSI), 2016 IEEE 2nd International Forum on.

NERSA. (2013). NERSA's decision on Eskom's Revenue Application for the Third Multi-Year Price Determination period 2013/14 to 2017/18. Retrieved from http://www.nersa.org.za/

Peng, C., \& Qian, K. (2014). Development and application of a Zigbee-based building energy monitoring and control system. The Scientific World Journal, 2014.

Swart, A. J., Pienaar, H. C., \& Schoeman, R. M. (2013). Cost-effective energy monitoring of domestic off-grid PV systems. JEPE, Journal of Energy and Power Engineering, 5, 182-188. doi:10.4236/epe.2013.54B035. 


\title{
A Gender Identification of Russian Text Author on Base of Multigenre Data-Driven Approach using Machine Learning Models
}

\author{
Aleksandr Sboev \\ NRC “Kurchatov Institute”, MEPhI National Research Nuclear University \\ Moscow, Russia \\ Ivan Moloshnikov, Dmitry Gudovskikh and Roman Rybka \\ NRC "Kurchatov Institute", \\ Moscow, Russia
}

\begin{abstract}
In this work data-driven approaches to identify the gender of author of Russian text are investigated with the purpose to clarify, to what extent the machine learning models trained on texts of a certain genre could give accurate results on texts of other genre. The set of data corpora includes: one collected by a crowdsourcing platform, essays of Russian students (RusPersonality), Gender Imitation corpus, and the corpora used at Forum for Information Retrieval Evaluation 2017 (FIRE), containing texts from Facebook, Twitter and Reviews. We present the analysis of numerical experiments based on different features(morphological data, vector of character n-gram frequencies, LIWC and others) of input texts along with various machine learning models (neural networks, gradient boosting methods, CNN, LSTM, SVM, Logistic Regression, Random Forest). Results of these experiments are compared with the results of FIRE competition to evaluate effects of multi-genre training. The presented results, obtained on a wide set of data-driven models, establish the accuracy level for the task to identify gender of an author of a Russian text in the multi-genre case. As shown, an average loss in F1 because of training on a set of genre other than the one used to test is about $11.7 \%$.
\end{abstract}

Keywords: data-driving, machine learning, multigenre gender identification.

\section{Introduction}

A few last years the task of author's profiling identification with data driven approach is investigated greatly from different points of view because of its indisputable importance for practical implementations, commercial and governmental. Recently a new accent of this task has emerged: to what extent the 
machine learning models trained on text of some certain genre could give accurate results on texts of other genres. This question was partly addressed at the PAN 2016 (Rangel F., 2016) competition where Twitter was used for training, and different corpora from social media, blogs, essays, and reviews were used for evaluation. A similar competition on Forum for Information Retrieval Evaluation 2017 (Litvinova T., 2017) took place for Russian texts. But the format of the competition was hardly appropriate to clarify this question of solving multi-genre task of profiling, because according to the competition rules only the single best run was taken into account without cross-validations. This is particularly discernible for such languages as Russian, for which the size of existing corpora is currently not so large (600 authors with 1 to 200 tweets per author, compared to 428 authors in English and 250 authors in Spanish with up to 1,000 tweets per author) and, consequently, the result of a single run is more stochastic. As a result, this makes it difficult to evaluate multigenre effects. The purpose of this paper is to evaluate the accuracy of solving multigenre profiling task more correctly with cross-validation, using an extended set of text features along with neural net and machine learning models.

Models, which are effective and largely independent of genre and external dictionaries, have been proposed in our previous work (Sboev A., 2017). The sets of used features included: morpho-syntactic, linguistic features (LIWC), a generalized dictionary approach of low displacement rank (LDR), along with different variants of vector representation of the text. The adaptations of these models and more complex models, based on convolutional neural networks (CNN), long short-term memories (LSTM), gradient boosting methods, are described in Section Materials and Methods, see Subsection Models. The experiments (described in the section Materials and Methods: Experiments) were carried out on the basis of various combinations of these corpora and models with various features. All used corpora and subcorpora are described in Section Materials and Methods: Datasets description. The results are summarized in Section Results and Conclusions.

\section{Material and methods \\ Dataset description}

The Gender Imitation-Crowdsource (CS) corpus was collected using the crowdsourcing platform. Each participant wrote 3 texts on one topic out of: a letter to a friend, a post for a dating site, a complaint letter to the boss or a negative review on the tour operator. The first text had to be written in the natural style (these texts are further referred to as ' $\mathrm{CS}$ a' collection), the second in form to mimic opposite gender ( $\left.C S b^{\prime}\right)$, and the third in form to imitate different style but not the gender ('CS c').

At the preprocessing stage, the texts were manually and automatically checked for borrowing on the Internet. After this stage, the corpus contained 5150 documents of, 1205 unique authors, 615 female and 588 male. Some authors wrote several texts for each collection, in which case we combined these texts into a single document. The average length of combined documents is about 300 words The number of authors 
was balanced by excluding excessive authors, after balancing the resulting dataset contained about 800 authors on average.

We tried combining corpus documents into learning samples in several ways: ' $C S^{\prime}$ - contains parts $\mathrm{A}, \mathrm{B}$ and $\mathrm{C}$ from each author as separate texts.

'CS combined' - in this sample parts A, B and C were combined into one document for each author.

'Cs $\mathrm{ab}^{\prime}$ - in this sample parts A and B were combined into one document for each author.

The corpus 'Cs free theme' was collected according to the same exercise as Gender Imitation Croudsource but on a free topic. This corpus was also split to 'Cs free theme', 'Cs free theme $A^{\prime}$, 'Cs free theme $B^{\prime}$, 'Cs free theme $C^{\prime}$, and 'Cs free theme $\mathrm{AB}^{\prime}$, analogously to ' $\mathrm{CS}$ ', 'CS a', 'CS b', 'CS c', and 'CS ab' respectively.

In addition, we used the Gender Imitation corpus (GI) that was collected under the same rules as CS, but offline, in a fully controllable environment. This corpus also have parts 'GI a', 'GI b', 'GI c', 'GI combined' and 'GI ab' formed in the same way.

RusPersonality(RusPer) (Litvinova T. Z. O., 2016): is a corpus of Russian-language texts labeled with a large amount of metadata on their authors like gender, age, personalities, education level, neuropsychological testing data, etc. The corpus currently contains over 1850 documents, 230 words long in average, from 1145 respondents. This paper uses a part of the corpus, consisting of 1108 texts on two topics: letter to a friend and picture description.

RusProfiling (RusProfiling, 2017): texts collected from different social media platforms as Twitter and Facebook, along with reviews. In this paper, this data set was divided into subsamples, in order to study the influence of the corpus features on the quality of the model and to evaluate the possibility of genre-independent classification:

Reviews - manually collected reviews (641 men, 392 women).

Twitter - twitter messages (998 men, 543 women). 1000 messages were collected for each user, and then merged into one text.

Facebook - messages from walls, combined into one text (136 men, 114 women).

\section{Features}

Group 1. Linguistic Inquiry and Word Count (LIWC)

LIWC - is a set of psychosocial dictionaries (Tausczik, 2010) that describe linguistics categories (the number of words of certain parts of speech, some lexical-thematic groups, the frequency of punctuation marks, etc.) adapted for Russian language (Litvinova, 2017)

Group 2. LDR - Lower dimentional reduction

In this model the document representation is as a vector of dependencies on categories (class-dependent vector). It is calculated based on the matrix of TF-IDF terms and class-dependent term weights. Each document is represented as $F\left(c_{1}\right)$, 
$F\left(c_{2}\right), \ldots, F\left(c_{n}\right) \sim \forall c \in C$, where $n$ - number of categories, $F\left(c_{i}\right)$ contains: the average weight of a document calculated as the sum of weights of its terms divided by the total number of vocabulary terms of the document, the standard deviation of weights of documents, the minimum weight among the term weights of document, the maximum weight found in the document, the sum of weights of the terms of the document divided by the total number of terms of the document, the proportion between the number of vocabulary terms of the document and the total number of terms of the document. The model is described in the work "A Low Dimensionality Representation for Language Variety Identification" (Rangel, 2017).

Group 3 Model Synt

As input to neural network each document is represented as a sequence of words, where each word is encoded by a syntactic pair "word-parent". The word and the parent are encoded by three types of features: unique index of lemma, Word2vec vector for word, (Kutuzov, 2017), binary encoded morphological tag.

\section{Group 4. Model NN}

As input for neural network the morphological characteristics of words are used: noun, verb, nominative case, masculine gender, feminine gender, etc.

Group 5. Symbol model.

In this model symbol-representation of text was used where each symbol convert to a one hot encoding vector. All English and Russian characters are used for encoding.

The features vector (groups 3, 4, 5) for the input document is formed based on the distribution of weights of the pre-trained neural networks described above.

Group 6. The vector of document is formed on the basis of the calculation of the term frequency-inverse document frequency (TF-IDF) of symbols n-grams in range from 3 to 5 letters.

\section{Models}

1) NNSyntMorph. Deep neural network based on morpho-syntactic features. The topology is shown on Fig. 1. Batch size is 32, early stopping after 5 epochs. 


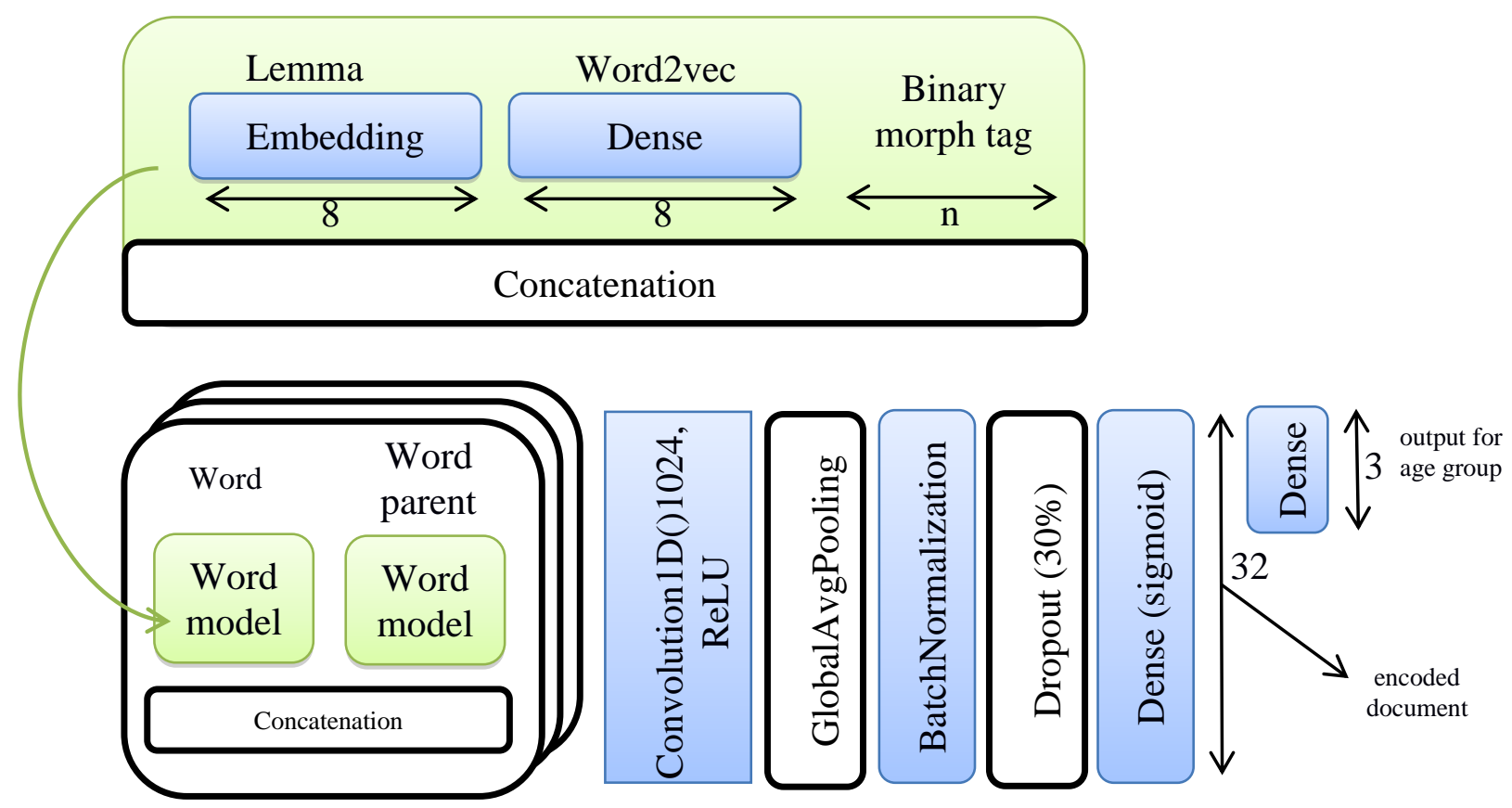

Figure 1 - Topology of morpho-syntactic neural network (NNSyntMorph)

2) Model NN. The topology of the network was taken from the work to determine the gender of the author, it based only on the morphological characteristics of words. (Sboev, 2016). A complicated neural network combining CNN, MLP and LSTM includes:

- 1st, 3rd, 5th CNN layers: Number of convolution kernels to use $=128$, the extension of each filter $=2$, activation function is ReLU

- 2nd, 4th, 6th layers: MaxPooling (pool length = 2)

- 7th layer: Long-Short Term Memory (output dimension =128)

- 8th layer: dropout layer. (Fraction of the input units to drop =0.5)

- 9th layer: fully connected NN layer (Number of hidden neurons $=10$, activation function $=$ softmax)

Learning parameters:

The learning process includes cross-validation with 10 permutation-and-split iterations, $80 \%$ of samples for training, $20 \%$ for testing.

3) Symbols model. This model uses symbol-representation of text where each symbol is converted to a one-hot encoded vector. The topology and parameters of network:

- 1st, 3rd, CNN layers: Number of convolution kernels to use $=8$, the extension of each filter $=2$, activation function is ReLU

- 2nd, 4th, 6th layers: MaxPooling (pool length = 2)

- $5^{\text {th }} \mathrm{CNN}$ layer: Number of convolution kernels to use $=32$, the extension of each filter $=2$, activation function is ReLU

- 7th layer: Global average pooling operation for temporal data.

- 8th layer: dropout layer. (Fraction of the input units to drop $=0.3$ )

- 9th layer: fully connected NN layer (Number of hidden neurons $=3$, activation function $=$ softmax) 
The optimizer in models 1), 2), and 3) is the Adam algorithm (Kingma, 2014) based on gradient descent, with mean squared error as the optimization score function.

4) We used conventional models: RandomForestClassifier, LogisticRegression and Gradient Boosting Classifier (Gradient Boosting). These standard models were used with default hyperparameters of the sklearn library. Support vector machine(SVM) with linear kernel. As hyperparameters the following were used: regularization parameter $\mathrm{C}$ is 1.0, L2-norm used in the penalization and squared hinge-loss function. Each model uses features shown in Table 3.

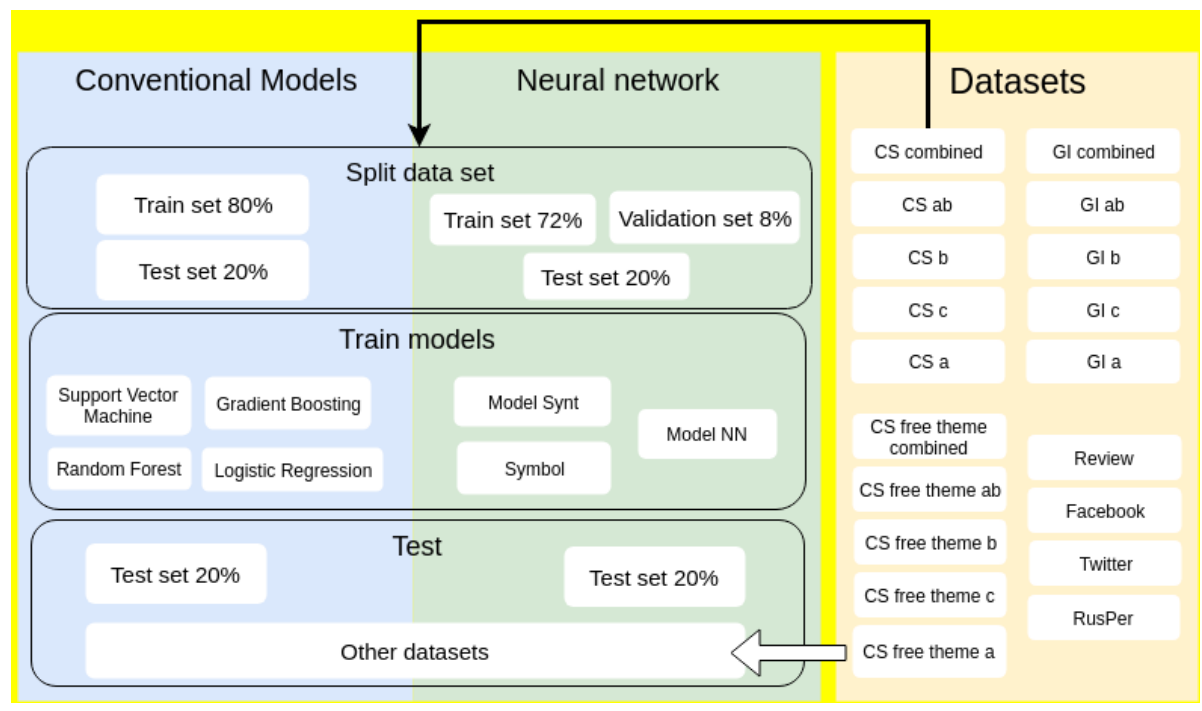

Figure 2. General flow diagram of experiments.

\section{Experiments}

We have performed a set of author identification experiments for identifying author gender by text features, employing various neural nets and machine learning models. The textual training sets are of different genres: Twitter (as at PAN@FIRE 2017, that allows to compare our results with its results); Facebook; different partitions of CS (a, b, c, ab, combined), RusPersonality, and Gender Imitation corpus. In case of conventional models the corpus is split to $20 \%$ for testing set, and $80 \%$ for training, or in case of neural network models $72 \%$ for training, $8 \%$ for validation, and $20 \%$ for testing. To investigate the relation of two genres, say, 1 and 2 , we compare the results of two types of calculations, cross-genre and single-genre. The first type is to train (as well as validate when necessary) on texts of genre 1 with testing on texts of genre 2, and then vice versa: to train on genre 2 with testing on genre 1 . The second type is to use texts of the same genre for training and testing. The general flow diagram of the experiments is shown in Figure 2. Each training set is balanced by excluding excessive examples, and 10-fold cross-validation is performed. 
Table 1 - Cross-genre testing with non-deceptive corpora. Rows indicate training sets (number of examples, average document length), columns - testing sets (number of examples, average document length). F1-score(\%) is presented, with standard deviation in brackets.

\begin{tabular}{|c|c|c|c|c|c|c|c|c|c|}
\hline Corpus & $\begin{array}{c}\text { Reviews } \\
(784,62)\end{array}$ & $\begin{array}{c}\text { RusPer } \\
(1150, \\
173)\end{array}$ & $\begin{array}{c}\text { Cs a } \\
(1664, \\
84)\end{array}$ & $\begin{array}{c}\text { Cs free } \\
\text { theme a } \\
(320,102)\end{array}$ & \begin{tabular}{|c|} 
Facebook \\
$(228$, \\
$1389)$
\end{tabular} & $\begin{array}{c}\text { Twitter } \\
\text { (1086, } \\
\text { 1692) }\end{array}$ & $\begin{array}{l}\text { GI a } \\
(90, \\
150)\end{array}$ & Average & Std \\
\hline $\begin{array}{c}\text { Reviews } \\
(784,62)\end{array}$ & $\begin{array}{l}79 \\
(03)\end{array}$ & $\begin{array}{c}72 \\
(02)\end{array}$ & $\begin{array}{c}63 \\
(02)\end{array}$ & $\begin{array}{c}81 \\
(02)\end{array}$ & $\begin{array}{l}79 \\
(03)\end{array}$ & $\begin{array}{c}71 \\
(01)\end{array}$ & $\begin{array}{c}68 \\
(04)\end{array}$ & 73 & 6 \\
\hline $\begin{array}{c}\text { RusPer } \\
(\mathbf{1 1 5 0 , 1 7 3 )}\end{array}$ & $\begin{array}{c}73 \\
(01)\end{array}$ & $\begin{array}{c}82 \\
(02)\end{array}$ & $\begin{array}{c}71 \\
(01)\end{array}$ & $\begin{array}{c}87 \\
(01)\end{array}$ & $\begin{array}{c}75 \\
(05)\end{array}$ & $\begin{array}{c}72 \\
(01)\end{array}$ & $\begin{array}{c}75 \\
(04)\end{array}$ & 76 & 5 \\
\hline $\begin{array}{c}\text { Cs a } \\
(1664,84)\end{array}$ & $\begin{array}{c}72 \\
(02)\end{array}$ & $\begin{array}{c}76 \\
(03)\end{array}$ & $\begin{array}{c}78 \\
(02)\end{array}$ & $\begin{array}{c}85 \\
(02)\end{array}$ & $\begin{array}{c}69 \\
(11)\end{array}$ & $\begin{array}{c}67 \\
(01)\end{array}$ & $\begin{array}{c}80 \\
(03)\end{array}$ & 75 & 5 \\
\hline $\begin{array}{c}\text { Cs free } \\
\text { theme a } \\
(320,102)\end{array}$ & $\begin{array}{c}71 \\
(01)\end{array}$ & $\begin{array}{l}75 \\
(02)\end{array}$ & $\begin{array}{c}64 \\
(04)\end{array}$ & $\begin{array}{c}82 \\
(06)\end{array}$ & $\begin{array}{l}77 \\
(04)\end{array}$ & $\begin{array}{c}70 \\
(03)\end{array}$ & $\begin{array}{c}69 \\
(06)\end{array}$ & 73 & 5 \\
\hline $\begin{array}{l}\text { Facebook } \\
(228,1389)\end{array}$ & $\begin{array}{c}67 \\
(02)\end{array}$ & $\begin{array}{c}66 \\
(01)\end{array}$ & $\begin{array}{c}51 \\
(03)\end{array}$ & $\begin{array}{c}71 \\
(02)\end{array}$ & $\begin{array}{c}84 \\
(06)\end{array}$ & $\begin{array}{c}66 \\
(01)\end{array}$ & $\begin{array}{c}59 \\
(04)\end{array}$ & 66 & 7 \\
\hline $\begin{array}{c}\text { Twitter } \\
(1086,1692)\end{array}$ & $\begin{array}{c}72 \\
(01) \\
\end{array}$ & $\begin{array}{c}73 \\
(01) \\
\end{array}$ & $\begin{array}{c}62 \\
(02) \\
\end{array}$ & $\begin{array}{c}79 \\
(01) \\
\end{array}$ & $\begin{array}{r}85 \\
(02) \\
\end{array}$ & $\begin{array}{c}79 \\
(02) \\
\end{array}$ & $\begin{array}{c}69 \\
(03) \\
\end{array}$ & 74 & 6 \\
\hline $\begin{array}{c}\text { GI a } \\
(90,150)\end{array}$ & $\begin{array}{l}57 \\
(04)\end{array}$ & $\begin{array}{c}58 \\
(03)\end{array}$ & $\begin{array}{c}56 \\
(02)\end{array}$ & $\begin{array}{c}58 \\
(03)\end{array}$ & $\begin{array}{c}49 \\
(08)\end{array}$ & $\begin{array}{c}51 \\
(02)\end{array}$ & $\begin{array}{c}61 \\
(06)\end{array}$ & 56 & 3 \\
\hline Average & 70 & 72 & 64 & 78 & 74 & 68 & 69 & & \\
\hline Std & 5 & 6 & 6 & 8 & 9 & 6 & 5 & & \\
\hline
\end{tabular}

\section{Results}

Table 1 demonstrates the F1-scores of the experiments mentioned above with corpora without other gender imitation or style distortion (so, for datasets containing A, B, and C partitions, only A subsets were selected). Rows correspond to datasets used for training, and columns correspond to testing datasets; so that on the diagonal (marked grey) are the non-cross-genre results, trained and tested on the same datasets. Each cell of the table shows the F1-score and standard deviation obtained by the model that is the best for that particular pair of training and testing set. Std denotes the standard deviations for average values in columns and rows.

These results show that, in general, in case of evaluating different genre corpora without gender deception the resulting F1 are in range $49 \%-87 \%$, average $70.5 \%$. The result accuracy mostly depends on the size of the training set: the smaller the set is (see the corpora sizes in row and column heads), the lower F1 is. For example see Table 1, lines with GI a and Facebook, which give lower result on every testing dataset. 
Table 2 - Cross-gender testing, including corpora with gender imitation or style distortion. Before slash - F1 (standard deviation in brackets) of training on a dataset stated in the row and testing on a dataset stated in the column, after slash -vice versa, training on a dataset in the column, and testing on a dataset in the row. Names of dataset in columns and rows consists number of examples and average document length.

\begin{tabular}{|c|c|c|c|c|c|c|c|}
\hline Corpus & $\begin{array}{c}\text { Reviews } \\
(784,62)\end{array}$ & $\begin{array}{c}\text { RusPer } \\
(\mathbf{1 1 5 0 , 1 7 3 )}\end{array}$ & $\begin{array}{c}\text { Facebook } \\
(228,1389)\end{array}$ & $\begin{array}{l}\text { Twitter } \\
(1086, \\
1692)\end{array}$ & Average & Std & $\begin{array}{r}\text { Same- } \\
\text { genre } \\
\text { test }\end{array}$ \\
\hline Cs $(4996,82)$ & $\begin{array}{c}58(02) / \\
52(01)\end{array}$ & $\begin{array}{c}65(10) / \\
56(01)\end{array}$ & $\begin{array}{c}57(03) / \\
46(02)\end{array}$ & $\begin{array}{c}57(03) / \\
53(01)\end{array}$ & $\begin{array}{r}59 / \\
52\end{array}$ & $\begin{array}{r}3 / \\
3\end{array}$ & $\begin{array}{c}59 \\
(01)\end{array}$ \\
\hline $\begin{array}{l}\text { Cs free theme } \\
(960,104)\end{array}$ & $\begin{array}{c}55(03) / \\
59(02)\end{array}$ & $\begin{array}{c}63(06) / \\
62(01)\end{array}$ & $\begin{array}{c}61(05) / \\
52(02)\end{array}$ & $\begin{array}{c}58(03) / \\
58(01)\end{array}$ & $\begin{array}{r}59 / \\
58\end{array}$ & $\begin{array}{r}3 / \\
3\end{array}$ & $\begin{array}{c}61 \\
(02)\end{array}$ \\
\hline GI $(250,117)$ & $\begin{array}{c}54(02) / \\
57(03)\end{array}$ & $\begin{array}{c}50(04) / \\
56(03)\end{array}$ & $\begin{array}{c}45(07) / \\
50(02)\end{array}$ & $\begin{array}{c}48(02) / \\
56(02)\end{array}$ & $\begin{array}{r}49 / \\
55\end{array}$ & $\begin{array}{r}3 / \\
2\end{array}$ & $\begin{array}{c}70 \\
(04)\end{array}$ \\
\hline Average & $56 / 56$ & 59 / 58 & $54 / 49$ & $54 / 56$ & & & \\
\hline Std & $2 / 3$ & $6 / 3$ & $6 / 2$ & $4 / 2$ & & & \\
\hline Cs ab $(1544,77)$ & $\begin{array}{c}55(02) / \\
49(02)\end{array}$ & $\begin{array}{c}55(02) / \\
51(01)\end{array}$ & $\begin{array}{c}52(04) / \\
45(01)\end{array}$ & $\begin{array}{c}50(02) / \\
49(02)\end{array}$ & $\begin{array}{r}53 / \\
49\end{array}$ & $\begin{array}{r}2 / \\
2\end{array}$ & $\begin{array}{c}58 \\
(02)\end{array}$ \\
\hline Cs free theme $a b$ & $\begin{array}{c}49(01) / \\
53(01)\end{array}$ & $\begin{array}{c}53(03) / \\
53(01)\end{array}$ & $\begin{array}{c}54(03) / \\
47(01)\end{array}$ & $\begin{array}{c}50(04) / \\
52(01)\end{array}$ & $\begin{array}{r}52 / \\
51\end{array}$ & $\begin{array}{r}2 / \\
2\end{array}$ & $\begin{array}{c}66 \\
(05)\end{array}$ \\
\hline GI $a b$ & $\begin{array}{c}52(02) / \\
54(02)\end{array}$ & $\begin{array}{l}49(01) / \\
52(02)\end{array}$ & $\begin{array}{c}47(04) / \\
48(02)\end{array}$ & $\begin{array}{c}48(02) / \\
54(02)\end{array}$ & $\begin{array}{r}49 / \\
52\end{array}$ & $\begin{array}{r}2 / \\
2\end{array}$ & $\begin{array}{c}62 \\
(11)\end{array}$ \\
\hline Average & 52 / 52 & 52 / 52 & $51 / 47$ & 49 / 52 & & & \\
\hline Std & $2 / 2$ & $2 / 0,7$ & $3 / 1$ & $0,9 / 2$ & & & \\
\hline Cs b $(1666,79)$ & $\begin{array}{c}53(01) / \\
46(03)\end{array}$ & $\begin{array}{c}46(02) / \\
44(01)\end{array}$ & $\begin{array}{c}42(03) / \\
41(03)\end{array}$ & $\begin{array}{c}42(02) / \\
42(01)\end{array}$ & $\begin{array}{r}46 / \\
43\end{array}$ & $\begin{array}{r}4 / \\
2\end{array}$ & $\begin{array}{c}78 \\
(02)\end{array}$ \\
\hline $\begin{array}{c}\text { Cs free theme } b \\
(320,103)\end{array}$ & $\begin{array}{c}53(02) / \\
50(02)\end{array}$ & $\begin{array}{c}47(02) / \\
38(01)\end{array}$ & $\begin{array}{c}43(02) / \\
39(02)\end{array}$ & $\begin{array}{c}48(01) / \\
37(01)\end{array}$ & $\begin{array}{r}48 / \\
41\end{array}$ & $\begin{array}{r}3 / \\
5\end{array}$ & $\begin{array}{c}82 \\
(04)\end{array}$ \\
\hline GI b $(84,100)$ & $\begin{array}{c}50(03) / \\
42(05)\end{array}$ & $\begin{array}{c}45(03) / \\
40(02)\end{array}$ & $\begin{array}{l}42(08) / \\
43(04)\end{array}$ & $\begin{array}{c}47(03) / \\
46(03)\end{array}$ & $\begin{array}{r}46 / \\
43\end{array}$ & $\begin{array}{r}3 / \\
2\end{array}$ & $\begin{array}{c}66 \\
(14)\end{array}$ \\
\hline Average & $52 / 46$ & 46 / 41 & 42 / 41 & 46 / 42 & & & \\
\hline \multirow{2}{*}{$\begin{array}{r}\text { Std } \\
\text { Same-genre test }\end{array}$} & $1 / 3$ & $0,7 / 2$ & $0,4 / 1$ & $2 / 3$ & & & \\
\hline & $79(03)$ & $82(02)$ & $84(06)$ & $79(02)$ & & & \\
\hline
\end{tabular}


Training and testing on different genres cause an average loss in f1-measure is approximately $11.7 \%$. As it follows from Table 1, the best result of testing on Crowdsourcing and Gender Imitation texts is shown by training on RusPersonality texts, which is not surprising, because the methodologies to form these corpora were similar, so these texts were written in a similar stylistic manner.

Table 3 - Cross-genre testing results for "combined" partitions of CS, CS-free-theme, and Gender Imitation datasets. The layout is same as in Table 2.

\begin{tabular}{|c|c|c|c|c|c|c|c|}
\hline Corpus & $\begin{array}{c}\text { Reviews } \\
(784,62)\end{array}$ & $\begin{array}{c}\text { RusPer } \\
(1150,173)\end{array}$ & $\begin{array}{l}\text { Facebook } \\
(228,1389)\end{array}$ & $\begin{array}{c}\text { Twitter } \\
\text { (1086, } \\
\text { 1692) }\end{array}$ & Average & Std & $\begin{array}{c}\text { Same- } \\
\text { genre } \\
\text { test }\end{array}$ \\
\hline Cs combined & $\begin{array}{c}57(01) / \\
54(01)\end{array}$ & $\begin{array}{c}61(01) / \\
57(01)\end{array}$ & $\begin{array}{c}59(02) / \\
51(01)\end{array}$ & $\begin{array}{c}57(03) / \\
54(02)\end{array}$ & $\begin{array}{r}59 / \\
54\end{array}$ & $\begin{array}{r}2 / \\
2\end{array}$ & $\begin{array}{c}60 \\
(05)\end{array}$ \\
\hline $\begin{array}{l}\text { Cs free theme } \\
\text { combined }\end{array}$ & $\begin{array}{c}56(13) / \\
62(03)\end{array}$ & $\begin{array}{c}62(03) / \\
58(02)\end{array}$ & $\begin{array}{c}62(06) / \\
57(01)\end{array}$ & $\begin{array}{c}57(03) / \\
56(02)\end{array}$ & $\begin{array}{r}59 / \\
58\end{array}$ & $\begin{array}{r}3 / \\
2 \\
\end{array}$ & $\begin{array}{c}70 \\
(21)\end{array}$ \\
\hline $\begin{array}{l}\text { Gender } \\
\text { Imitation } \\
\text { combined }\end{array}$ & $\begin{array}{c}51(01) / \\
56(06)\end{array}$ & $\begin{array}{c}50(04) / \\
56(03)\end{array}$ & $\begin{array}{l}49(04) / \\
48(04)\end{array}$ & $\begin{array}{c}49(02) / \\
59(04)\end{array}$ & $\begin{array}{r}50 / \\
55\end{array}$ & $\begin{array}{r}0,8 / \\
3\end{array}$ & $\begin{array}{c}60 \\
(11)\end{array}$ \\
\hline Average & 55 / 57 & $58 / 57$ & 57 / 52 & $54 / 56$ & & & \\
\hline Std & $2 / 3$ & $5 / 0,7$ & $5 / 3$ & $4 / 2$ & & & \\
\hline Cs C $(1666,82)$ & $\begin{array}{c}72(01) / \\
60(03)\end{array}$ & $\begin{array}{c}72(01) / \\
66(00)\end{array}$ & $\begin{array}{c}65(03) / \\
52(03)\end{array}$ & $\begin{array}{c}65(04) / \\
61(01)\end{array}$ & $\begin{array}{r}69 / \\
60\end{array}$ & $\begin{array}{r}4 / \\
4\end{array}$ & $\begin{array}{c}71 \\
(01)\end{array}$ \\
\hline $\begin{array}{c}\text { Cs free theme C } \\
(320,106) \\
\end{array}$ & $\begin{array}{c}68(02) / \\
72(04) \\
\end{array}$ & $\begin{array}{c}73(03) / \\
78(02) \\
\end{array}$ & $\begin{array}{c}67(05) / \\
62(04) \\
\end{array}$ & $\begin{array}{c}67(02) / \\
71(02) \\
\end{array}$ & $\begin{array}{r}69 / \\
71 \\
\end{array}$ & $\begin{array}{r}2 / \\
4 \\
\end{array}$ & $\begin{array}{c}74 \\
(05)\end{array}$ \\
\hline GI C $(76,97)$ & $\begin{array}{c}52(02) / \\
67(06)\end{array}$ & $\begin{array}{c}49(05) / \\
67(04)\end{array}$ & $\begin{array}{c}41(11) / \\
56(02)\end{array}$ & $\begin{array}{c}48(05) / \\
61(05)\end{array}$ & $\begin{array}{l}4 / \\
63\end{array}$ & $\begin{array}{r}3 / \\
4\end{array}$ & $\begin{array}{c}58 \\
(12)\end{array}$ \\
\hline Average & 64 / 66 & 65 / 70 & 58 / 57 & $60 / 64$ & & & \\
\hline Std & $8 / 4$ & $10 / 5$ & $11 / 4$ & $8 / 4$ & & & \\
\hline $\begin{array}{c}\text { Same-genre } \\
\text { test }\end{array}$ & $79(03)$ & $82(02)$ & $84(06)$ & $79(02)$ & & & \\
\hline
\end{tabular}

Tables 2 and 3 present cross-genre testing results for datasets with gender imitation and style distortion. The F1-scores are averaged over all the models used, in contrast to best models' results in Table 1 . In addition, average F1-score and standard deviation over similar partitions $(\mathrm{B}, \mathrm{C}, \mathrm{AB}$, combined, and $\mathrm{ABC}$ contains parts $\mathrm{A}, \mathrm{B}$ and $\mathrm{C}$ of all authors as separate texts) of different datasets are shown. For comparison, the rows and columns "same-genre test" present the same-genre testing results like the diagonal in Table 1: training and testing on the same dataset of the corresponding row or column. 
Among Internet-texts training sets, the best F1 results for testing on GI and CS datasets are shown by training on Reviews (average result is 58\%), then Twitter (56.6\%), and slightly worse on Facebook (51\%). Generally, the presence of texts with gender and style distortions in testing set increases the magnitude of loss in F1 up to $30 \%$.

Table 3 represents ranked levels of efficiency of different input text features with various machine learning models, calculated over all test experiments. Formula 1 demonstrate how to evaluate the rank.

$$
\operatorname{Rank}=\frac{\sum_{j=1}^{n} i \in M}{n}(1)
$$

Table 3 - Rank of models based on results of all experiments.

\begin{tabular}{|l|l|}
\hline Model name & Rank \\
\hline Gradient Boosting (Group 6. Char ngram) & 2,44 \\
\hline SVM(with LIWC and LDR features) & 3,21 \\
Random Forest ( based on LIWC and LDR features) & 3,49 \\
\hline Logistic Regression(with LIWC and LDR features) & 3,62 \\
NNSyntMorph & 4,6 \\
\hline Model NN & 4,64 \\
\hline Symbols model & 5,99 \\
\hline
\end{tabular}

where $\mathrm{i}$ - a place in $\mathrm{j}$-th experiment, place means the position in the F1 rating of the set of models $M$ for $j$-th pair of training and testing datasets, $n$ is the total number of such pairs. In Table 4 our results are compared to that of FIRE best runs, and these results, in general, are close.

Table 4 - Comparison with results of FIRE competition. Names of dataset in columns and rows contains number of examples and average document length. F1-score is presented with standard deviation consist in brackets.

\begin{tabular}{|c|c|c|c|c|c|c|}
\hline Train $\backslash$ Test & $\begin{array}{l}\text { Reviews } \\
(784,62)\end{array}$ & $\begin{array}{l}\text { RusPer } \\
(\mathbf{1 1 5 0 , 1 7 3 )}\end{array}$ & $\begin{array}{l}\text { RusPer } \\
(370,150)\end{array}$ & $\begin{array}{l}\text { Facebook } \\
(228,1389)\end{array}$ & $\begin{array}{l}\text { Twitter } \\
(1086,1692)\end{array}$ & GI combined \\
\hline $\begin{array}{l}\text { Twitter } \\
(1086,1692)\end{array}$ & $\begin{array}{l}72 \\
(01)\end{array}$ & $\begin{array}{l}73 \\
(01)\end{array}$ & - & $\begin{array}{l}85 \\
(02)\end{array}$ & $\begin{array}{l}79 \\
(02)\end{array}$ & $\begin{array}{l}59 \\
(04)\end{array}$ \\
\hline $\begin{array}{l}\text { Twitter FIRE (600, } \\
\text { 1600) }\end{array}$ & 61 & - & 78 & 93 & 68 & 65 \\
\hline
\end{tabular}

\section{Conclusion}

The presented results, obtained on a wide set of data-driven models, establish the accuracy level for author gender identification task on the Russian texts in the case of multi-genre. That is, we trained our models on texts of one genre and tested on texts of other genres. 
The obtained loss in F1 compared to non-multi-genre case about $10 \%$ in average (for texts without gender imitation and style distortion) and depends on the size of training set. The presence of texts with gender deceptions and style distortions in testing set makes the magnitude of loss yet more in F1, up to $30 \%$. The most effective model in our case is the Gradient Boosting model with symbolic n-gram representation of input text (Table 3). In future work we will continue collecting our crowdsourcing corpus, which would enlarge the size of training sets on cross-genre base to create highly effective model appropriate for the wide range of different genres, while at the same time effective for texts with gender deceptions.

\section{Acknowledgements}

This research is supported by the Russian Science Foundation, project No 161810050. This work has been carried out using computing resources of the federal collective usage center Complex for Simulation and Data Processing for Megascience Facilities at NRC “Kurchatov Institute”, http://ckp.nrcki.ru/

\section{References}

Kingma, D. P. (2014). Adam: A method for stochastic optimization. Retrieved from arXiv preprint arXiv:1412.6980

Kutuzov, A. \&. (2017). Building web-interfaces for vector semantic models with the webvectors toolkit. In Proceedings of the Software Demonstrations of the 15th Conference of the European Chapter of the Association for Computational Linguistics, pp. pp. 99-103.

Litvinova T., R. F. (2017). Overview of the RUSProfiling PAN at FIRE Track on Cross-genre Gender Identification in Russian. Notebook Papers of FIRE, pp. 810.

Litvinova T., Z. O. (2016). "Ruspersonality": A Russian corpus for authorship profiling and deception detection. In Intelligence, Social Media and Web (ISMW FRUCT), 2016 International FRUCT Conference on. IEEE, pp. pp. 1-7.

Litvinova, O. S. (2017). Deception detection in russian texts. In Proceedings of the Student Research Workshop at the 15th Conference of the European Chapter of the Association for Computational Linguistics, pp. pp. 43-52.

Rangel F., R. P. (2016). Overview of the 4th author profiling task at PAN 2016: crossgenre evaluations. In Working Notes Papers of the CLEF 2016 Evaluation Labs. CEUR Workshop Proceedings/Balog, Krisztian [edit.]; et al., pp. pp. 750-784.

Rangel, F. F.-S. (2017). A low dimensionality representation for language variety identification. Retrieved from arXiv preprint arXiv:1705.10754

RusProfiling. (2017). RusProfiling Lab 2017 Rusprofiling corpus of russian texts. Retrieved from [online]: http:/ / rusprofilinglab.ru/rusprofiling-at-pan/

Sboev A., M. I. (2017). A comparison of Data Driven models of solving the task of gender identification of author in Russian language texts for cases without and with the gender deception. In Journal of Physics: Conference Series (Vol. 937, No. 1). IOP Publishing., p. p. 012046. 
Sboev, A. L. (2016). Machine learning models of text categorization by author gender using topic-independent features. Procedia Computer Science, 101, pp. pp. 135142.

Tausczik, Y. R. (2010). The psychological meaning of words: LIWC and computerized text analysis methods. Journal of language and social psychology, 29(1), 24-54. 


\title{
A Fuzzy Approach to Prioritisation of Security Requirements in a Constrained Agile Software Development Environment
}

\author{
Nalindren Naicker \\ Durban University of Technology, \\ Durban, South Africa \\ Manoj Maharaj \\ University of KwaZulu-Natal \\ Durban, South Africa
}

\begin{abstract}
Requirements Engineering (RE) is software engineering process that takes place early namely, during the planning phases of software development. The spate of hacking incidents, more especially the ransomware infections, recently has suggested a more robust approach to web application security during RE. This will not only prevent these vulnerabilities but also the necessary rework after a hacking incident. The prioritisation process in RE is instrumental in ensuring if a security requirement gets implemented or is kept on hold indefinitely. A desktop literature review revealed ample scope for fuzzy TOPSIS as a security requirements prioritisation technique. The aim of this research study was to assess the viability of a new and more inclusive technique of more precisely ranking security requirements. A fuzzy automated tool was developed to test this new approach to ranking security requirements. The method will ensure the implementation of the most important security requirements to secure the system. The Design Science Research Methodology guided the development of the automated fuzzy software tool. The automated tool was evaluated in a qualitative study at 17 software development companies. The researcher used structured interviews and document reviews as the primary research instruments. Qualitative data was analyzed deductively using content analysis. It was found that an immediate benefit of the tool is that it prevented biases and autocratic leaders from influencing decision making during security risk analysis. The study concluded that the automated fuzzy tool showed positive results for ranking security requirements in Agile RE. Further research on the usability of the software tool is recommended.
\end{abstract}

Keywords: Agile, Security, Requirements Engineering, Fuzzy TOPSIS. 


\section{Introduction}

In real life decision makers very often are required to make important choices from several alternatives. The selection of an incorrect alternative based on subjective human judgement can have negative consequences. In software development for example the incorrect decision of a project manager on the choice of what features to implement can impact the security of applications. A more precise way at arriving at decisions involving subjective human preferences is required. Multi-criteria decision making (MCDM) problems integrated with fuzzy set theory provides a suitable solution to arrive at a precise judgement based on imprecise information (Lima et al. 2014). This approach is investigated in pursuit of a more rigorous method to prioritise security requirements in a non-prescriptive software development methodology such as Agile Software Development (ASD).

\section{Research Methodology}

A research gap was identified for an automated requirements prioritisation tool to manage the requirements prioritisation process. A desktop literature review into the various requirements prioritisation techniques revealed that a gap existed for the utilization of fuzzy TOPSIS as a prioritisation technique (Achimugu, Selamat, Ibrahim, \& Mahrin, 2014). Literature has highlighted a shortage of automated tools to support secure RE processes (Souag, Mazo, Salinesi, \& Comyn-Wattiau, 2015). Therefore, the development of an automated tool using the fuzzy TOPSIS approach to support secure RE activities in Agile Software Development will contribute to the existing body of knowledge and fill a research gap.

\section{Participants}

Developers from seventeen software development companies were interviewed based on quota sampling to evaluate the automated fuzzy tool. The composition of the companies in terms of their specialisations were as follows: Gaming (5), Medical (3); Transport (1); Finance (2); Education (2) and Non-Specific (4). The tool was constructed from the fuzzy TOPSIS method based on fuzzy set theory.

\section{Procedure}

The interview schedule was constructed through a process of synthesis from the evaluation frameworks in the literature on requirements prioritisation techniques. Questions were carefully crafted to evaluate the fuzzy TOPSIS technique for requirements prioritisation. Recruitment of participants took place at the Company. Before the data collection session, signed consent forms were obtained from participants. The rights of all respondents were respected by treating them equally with dignity and respect. The freedom of choice of the individual was respected, for example they were allowed to discontinue with the interviews at any time, participants were free to express concerns at any time and they were given access to any information that they required. Participants were provided with repeated assurance of anonymity. Interviews in this study were audio recorded. The researcher ensured that the recording was audible for the transcriber and sufficient 
time was allocated for transcribing. Qualitative data was analysed using content analysis. Coding was a way of indexing or categorizing the text in order to establish a framework of thematic ideas about it. A computer program, Nvivo Pro was used for filing and retrieving coded information.

\section{Fuzzy TOPSIS method}

Fuzzy TOPSIS is a method used when a group decision is required for the selection of an alternative from a number of alternatives (possible choices) using multiple decision making criteria to rate alternatives (Martin, 2011). Normally a linguistic rating scale such as very poor, poor, satisfactory, good and very good are used to rate alternatives based on set criteria. The linguistic rating, for example good is regarded as vague and imprecise. It is difficult to distinguish which alternative is better from a choice of two alternatives when both are given a rating of good. The vague rating good can be easily represented as a triangular fuzzy number using fuzzy set theory, for example using a numeric scale of 0-9, good represented as a triangular fuzzy number can be written as $(7,8,9)$. In the fuzzy TOPSIS approach linguistic ratings are replaced by triangular fuzzy numbers provide mathematically precise ways of arriving at decisions involving human judgement (Sodhi, 2012). The final judgement integrates the expert opinion of all decision makers.

In software development, the choice of a security requirement (security feature of the system) for development from the product backlog (store) requires multiple decision makers who rate requirements based on multiple criteria from a collection of requirements (alternatives). Incorrect decision making can result in low customer satisfaction, losses and hence project failure.

The fuzzy TOPSIS algorithm comprises of the following steps, according to (Sodhi, 2012):

\section{Step 1:}

Decision makers must determine the linguistic and fuzzy weighting of evaluation criteria and alternatives.

\section{Step 2:}

(i) Aggregate weights of each criterion as follows:

Let $K=$ number of decision makers

Let $j=1,2, \ldots . n$ representing the criteria

$$
\begin{aligned}
& \widetilde{w}_{j}^{k}=\left(w_{j 1}, w_{j 2}, w_{j 3}\right) \\
& \text { where: } w_{j 1}=\min _{k}\left\{w_{j k 1}\right\}, w_{j 2}=\frac{1}{K} \sum_{k=1}^{k} w_{j k 2}, w_{j 3}=\max _{k}\left\{w_{j k}\right\}
\end{aligned}
$$

(ii) Aggregate weights of each alternative as follows:

Let $k=$ number of decision makers

Let $j=1,2, \ldots . n$ represent the criteria

Let $i=1,2, \ldots$. represent the alternatives 
Aggregate weightings $\tilde{x}_{i j}=\left(l_{i j}, m_{i j}, u_{i j}\right)$ of alternatives $(i)$ with respect to each criterion (j) based on the fuzzy ratings by the $k$ decision maker is expressed by the calculations below:

$$
l_{i j}=\min _{k}\left\{l_{i j}^{k}\right\}, m_{i j}=\frac{1}{K} \sum_{k=1}^{k} m_{i j}^{k}, u_{i j}=\max _{k}\left\{u_{i j}^{k}\right\}
$$

(iii) Aggregate fuzzy decision matrix for the alternatives $(\widetilde{D})$ is expressed below:

$$
\widetilde{D}=\begin{array}{r}
C_{1} \\
A_{1} \\
A_{i} \\
A_{n}
\end{array}\left[\begin{array}{cccc}
\tilde{x}_{11} & \tilde{x}_{12} & C_{j} & \tilde{x}_{1 j} \\
\vdots & \vdots & \vdots & \tilde{x}_{1 m} \\
\tilde{x}_{n 1} & \tilde{x}_{n 2} & \tilde{x}_{n j} & \tilde{x}_{n m}
\end{array}\right]
$$

\section{Step 3:}

The normalized fuzzy decision matrix $\widetilde{(R})$ is written as follows:

Let $\tilde{R}=$ normalized decision matrix

Let $j=1,2, \ldots . n$ represent the criteria

Let $i=1,2, \ldots$. s represent the alternatives

Let $\left(l_{i j}, m_{i j}, u_{i j}\right)=$ aggregated weighting of alternative (i) with respect to criterion ( $j$ )

Let $\tilde{r}_{i j}=$ triangular fuzzy number of alternative (i) with respect to criterion (j) in matrix $r$ with $n$ rows and s columns

The normalized decision matrix is calculated as follows:

$$
\begin{aligned}
& \qquad \widetilde{(R})=\left[\tilde{r}_{i j}\right]_{n x s} \\
& \text { where } \tilde{r}_{i j}=\left(\frac{l_{i j}}{u_{j}^{+}} ; \frac{m_{i j}}{u_{j}^{+}} ; \frac{u_{i j}}{u_{j}^{+}}\right) \text {and } u_{j}^{+}=\max _{\mathrm{i}} u_{i j} \quad \text { (benefit criteria) } \\
& \text { therefore } u_{j}^{+}=\max _{\mathrm{i}}(9,9,9)
\end{aligned}
$$

\section{Step 4:}

The weighted normalized decision matrix, $\widetilde{(V)})$ is computed as follows:

Let $j=1,2, \ldots . n$ represent the criteria

Let $i=1,2, \ldots$. represent the alternatives

The weights of the evaluation criteria, $\left.\widetilde{(w}_{j}\right)$ is multiplied by the elements $\tilde{\mathrm{r}}_{\mathrm{ij}}$ of the normalized fuzzy decision matrix.

$$
\begin{aligned}
& \widetilde{(V})=\left[\tilde{v}_{i j}\right]_{n x s} \\
& \text { where } \tilde{v}_{i j}=\tilde{r}_{i j} \times \widetilde{w}_{j}
\end{aligned}
$$

\section{Step 5:}

The Fuzzy Positive Ideal Solution (FPIS, A \pm ) and the Fuzzy Negative Ideal Solution (FNIS, A=) is calculated as follows:

Let $j=1,2, \ldots . n$ represent the criteria

$\mathrm{A}^{+}=\left\{\tilde{v}_{1}^{+}, \tilde{v}_{j}^{+}, \ldots, \tilde{v}_{n}^{+}\right\}$

$\mathrm{A}^{-}=\left\{\tilde{v}_{1}^{-}, \tilde{v}_{j}^{-}, \ldots, \tilde{v}_{n}^{-}\right\}$

where $\tilde{v}_{j}^{+}=(1,1,1)$ and $\tilde{v}_{j}^{-}=(0,0,0)$

\section{Step 6:}

The distances $d_{j}^{+}$and $d_{j}^{-}$of each alternative from respectively $\tilde{v}_{j}^{+}$and $\tilde{v}_{j}^{-}$are calculated as follows:

Let $j=1,2, \ldots . n$ represent the criteria

Let $i=1,2, \ldots$. s represent the alternatives 


$$
\begin{aligned}
& d_{i}^{+}=\sum_{j=1}^{n} d_{v}\left(\tilde{v}_{i j}, \tilde{v}_{j}^{+}\right) \\
& d_{i}^{-}=\sum_{j=1}^{n} d_{v}\left(\tilde{v}_{i j}, \tilde{v}_{j}^{-}\right)
\end{aligned}
$$

Where $\mathrm{d}$ represents the distance between two triangular fuzzy numbers. For example the distance $\mathrm{d}\left(\tilde{v}_{i j}, \tilde{v}_{j}^{+}\right)$where $\tilde{v}_{i j}=\left(\mathrm{l}_{1}, \mathrm{~m}_{1}, \mathrm{u}_{1}\right)$ and $\tilde{v}_{j}^{+}=\left(\mathrm{l}_{2}, \mathrm{~m}_{2}, \mathrm{u}_{2}\right)$ is expressed as follows:

$$
\mathrm{d}\left(\tilde{v}_{i j}, \tilde{v}_{j}^{+}\right)=\sqrt{\frac{1}{3}\left[\left(l_{1}-l_{2}\right)^{2}+\left(m_{1}-m_{2}\right)^{2}+\left(u_{1}-u_{2}\right)^{2}\right]}
$$

\section{Step 7:}

The closeness coefficient $\left(\mathrm{CC}_{\mathrm{i}}\right)$ is calculated as follows:

$$
\mathrm{CC}_{\mathrm{i}}=\frac{d_{i}^{-}}{d_{i}^{+}+d_{i}^{-}}
$$

\section{Step 8:}

Define the ranking of the alternatives according to the closeness coefficient, $\mathrm{CC}_{\mathrm{i}}$, in decreasing order. The best alternative is closest to the FPIS and farthest to the FNIS.

A worked example using the algorithm is presented in Appendix 1. An automated fuzzy tool using a high level programming language was developed based on the fuzzy TOPSIS algorithm as shown in Figure 1 below.

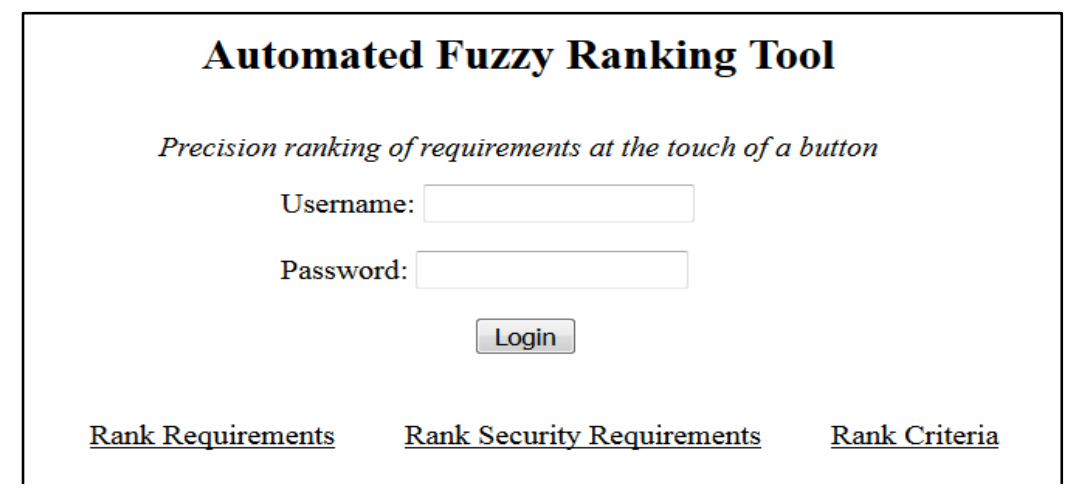

Figure 1: Login Screen of the Automated Fuzzy Tool

\section{Overview of the Automated Fuzzy Tool}

The automated fuzzy tool was developed using the Design Science Research Methodology (DSRM) stages.

\section{Stage 1: Identify problem and motivate}

The search for a new method of requirements prioritisation was driven by the following. Firstly, requirements prioritisation is imperative in Agile Software Development. Secondly, the selection of requirements is based on multiple decision makers and multiple evaluation criteria. After conducting an extensive desktop literature review of requirements prioritisation research, a new approach using the mathematical fuzzy TOPSIS algorithm as a basis is presented. It is aligned specially for use in Agile Software Development and will ensure that the selection of 
requirements meet the satisfaction of project goals and more importantly the customers' expectations.

\section{Stage 2: Define objectives of a solution}

The following objectives were defined:

Investigate current practice of security requirements prioritisation and design an automated fuzzy tool that can promote best practice in Agile Software Development; investigate how the automated fuzzy tool can be merged with conventional practices to prioritise the product/sprint backlogs and construct an automated fuzzy tool artifact for improving Multi-Criteria Decision Making (MCDM) when ranking requirements using the fuzzy TOPSIS method as a basis for the development.

\section{Stage 3: Design \& development}

The design and development of the Automated Fuzzy Software Tool was implemented in an object oriented paradigm using C\#.

\section{Stage 4: Demonstration}

The automated fuzzy tool was demonstrated and tested by software developers using the test website www.fuzzytool.co.za. ${ }^{1}$ The tool was demonstrated using an illustrative example similar to appendix 1 . The results were viewed by stakeholders. The demonstration was validated and verified showing the construction of all intermediate judgement matrices, distance measurement calculations and how final closeness coefficient $(\mathrm{CCi})$ values were computed using illustrated examples. The stakeholders were given a walk-through of the calculations to prove the correctness of the results given by the tool at the time of the demonstration. These calculations shown manually to stakeholders took place programmatically at the backend of the automated fuzzy tool web application.

\section{Analysis of Results}

The results were analysed under stage 5 of the Design Science Research Methodology.

\section{Stage 5: Evaluation}

Software developers occupying various developer roles were interviewed to provide feedback on their evaluation of the automated fuzzy tool. The artifact was evaluated individually by three representatives of intended users of the web application tool. The evaluators represented different Agile Software Development roles namely, customer, business analyst and project manager. The evaluation was conducted at the study sites using requirements from various types of projects. For example, large projects with many requirements, small projects with few requirements, projects with detailed requirements and projects with a mix of detailed and high level requirements. The evaluation framework focused on the following themes as synthesized and referenced from the literature review namely (Hasan, Mahmood,

\footnotetext{
${ }^{1}$ The online web application is currently live.
} 
Alam, Hasan, \& Rahman, 2010; Tuunanen \& Kuo, 2015): ease of use; total time taken; scalability; suitability to high level requirements; suitability to detailed requirements; correctness and scale of measurements.

Each theme is presented below from the data analysis of evaluators' responses.

\section{Ease of use}

This evaluation criterion describes end users experience on how easy it was to use the automated fuzzy tool. The informants indicated that the tool had simple instructions that were fairly easy to follow. Informants indicated that the use of the drop down list boxes and radio buttons ensured that user input was swift and error free. However the following were cited as potential improvements: Increase text field sizes to accommodate longer inputs and outputs; provide better hints to users during input and provide a graphical output for the end user.

\section{Total time taken}

This evaluation theme focused on the time taken to complete the process. In general evaluators indicated that the process was very quick. Time delays experienced were based on how long it took users to make a decision on a requirement.

\section{Scalability}

There were no issues reported on the ability of the tool to handle projects of various sizes.

\section{Suitability to high level requirements}

High level requirements are not specified with a great deal of detail and is elicited in the early stages of a project. The consensus reached was that the automated fuzzy tool is efficient for high level requirements.

\section{Suitability to detailed requirements}

Detailed requirements are specified generally after the analysis of requirements. Generalised requirements become more clearly defined as the project progresses. All participants indicated that the tool is suitable for detailed requirements as the prioritisation was completed just as efficiently as high level requirements.

\section{Correctness}

The correctness of the tool was illustrated to evaluators through a stepwise walkthrough of the manual calculations using their live input. The nature of the illustration was as per the demonstration mentioned in stage 4 above. Calculations were presented at the feedback session after the demonstration. All evaluators were satisfied with the explanations and accepted the results of the tool as accurate.

\section{Scale of measurements}

The automated fuzzy tool is based on fuzzy TOPSIS which presents output on a ratio scale (Karlsson, Wohlin, \& Regnell, 1998). All evaluators agreed that the scale of measurement was able to present the output with the magnitude of the rating and not just output an ordered list of requirements. Evaluators found information on the magnitude extremely useful because if the differences between two requirements were marginal, more practical decisions can be taken during implementation, for 
example, a requirement ranked slightly lower could be implemented immediately if resources for the higher rated requirement were not available for some reason. Magnitude also gives the developers a general idea on the importance of a requirement.

It was suggested that it would be more meaningful to the end users if the magnitude was expressed graphically. This will be most valuable as differences in CCi values of requirements can be easily detected at a glance rather than inspecting the numerical $\mathrm{CCi}$ values. All informants were in agreement that this modification will enhance the presentation of the output as well as make the tool easier to interpret.

\section{Expected Value for developers}

The automated fuzzy tool provides the following benefits to requirements engineers, namely, dcision making is more accurate and precise (ratio data) ensuring that security requirements that are truly valuable gets implemented first. It promotes collective decision making by ensuring that a number of stakeholders must make their input before a final decision is reached. It also prevents biases and autocratic leaders from influencing decision making during security risk analysis. This was a common complaint in the field work. An additional application of the tool is its use in ranking normal requirements in the product backlog and the sprint backlogs.

\section{Conclusion}

The overall results yielded by the automated fuzzy tool were trustworthy and correct. The tool worked efficiently for a mix of high level as well as detailed requirements. The tool can be used at any stage in Agile RE, for example at the initial planning stage when requirements are more high level or prior to the iterations, after requirements analysis when more detailed requirements are specified or during the security risk analysis stage when ranking security requirements. The tool can be used by Agile RE practitioners to ensure that the security requirements prioritisation process is inclusive of all stakeholders and decisions are based on logical principles. Further research on the use of the software tool is envisaged.

\section{References}

Achimugu, P., Selamat, A., Ibrahim, R., \& Mahrin, M. N. (2014). A systematic literature review of software requirements prioritization research. Information and Software Technology, 56(1), 568-585.

Hasan, M. S., Mahmood, A., Alam, M. J., Hasan, S., \& Rahman, F. (2010). An evaluation of software requirement prioritization techniques. International Journal of Computer Science and Information Security (IJCSIS), 8(9).

Karlsson, J., Wohlin, C., \& Regnell, B. (1998). An evaluation of methods for prioritizing software requirements. Information and Software Technology, 39(1415), 939-947. 
Martin, H. A., Zarchi, M. K., Azizollahi, S. (2011). The Application of Fuzzy Topsis Approach to Personnel Selection for PAdir Company, Iran. Journal of Management Research 3(2), 1-13.

Sodhi, B., Prabhakar T.V. . (2012). A Simplified Description of Fuzzy Topsis. Kanpur: Dept. of Computer Science and Engineering.

Souag, A., Mazo, R., Salinesi, C., \& Comyn-Wattiau, I. (2015). Reusable knowledge in security requirements engineering: a systematic mapping study. Requirements Engineering, 1-33.

Tuunanen, T., \& Kuo, I.-T. (2015). The effect of culture on requirements: a valuebased view of prioritization. European Journal of Information Systems, 24(3), 295313.

\section{APPENDIX 1}

\section{Application of Fuzzy TOPSIS to Requirements Engineering}

In this section an illustrated example from a small scale project shows the application of fuzzy TOPSIS to rank security requirements. To promote understanding a step by step description is given. The mathematical translation follows each step. The example is an application on how fuzzy TOPSIS is used to show the preferences by decision makers to security requirements from a list of security requirements.

A security risk analysis has resulted in four security requirements being identified. Due to constraints in budget, only the top two requirements can be accepted into the product backlog. Prioritise these security requirements for an online book store project, consisting of the following elicited security requirements:

- A1 Sales information to be modified only by members of the sales team.

- A2 Modification to any data in the system must be logged.

- A3 Prevent SQL injections on all input fields.

- A4 Allow authorized users to access the system.

Three decision makers, namely the project manager (DM1), the book store client (DM2) and the business analyst (DM3) must rank the requirements based on the following benefit criteria:

- C1 Business value: Valuable to the business.

- C2 Size: Capable of finishing in a sprint.

- C3 Effort: Mental effort is manageable to implement in a sprint.

- C4 Core: The system must have this feature. 


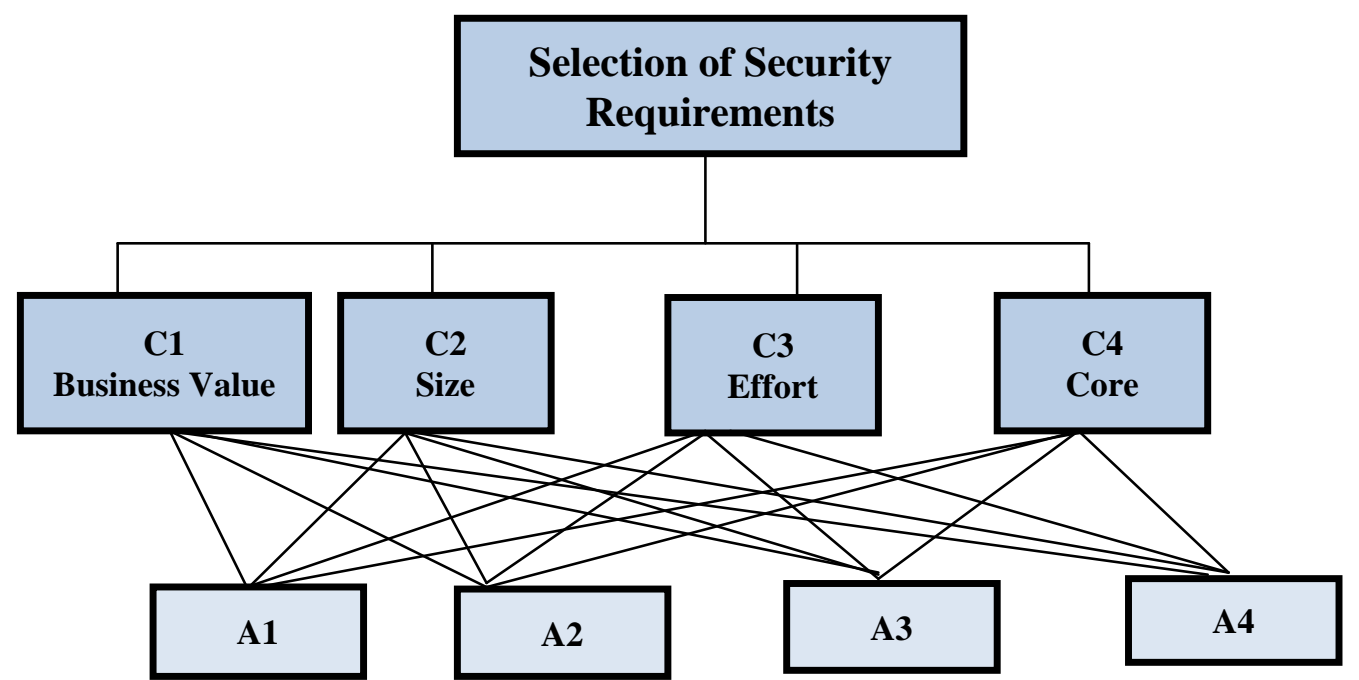

Figure 1: Hierarchical structure of selection process of the Top 2 security requirements

Figure 1 shows the hierarchical structure for the selection of the best two requirements. Each decision maker must rate each requirement on the four decision making criteria. This is a multiple criteria and multiple decision making problem. Fuzzy TOPSIS will be used to solve this multi-criteria, multi-decision making problem. The steps for the solution are given below with explanations.

Step 1: Determine the fuzzy weighting of evaluation criteria and fuzzy weighting of alternatives

(i) Linguistic scales for evaluation criteria must be represented as a triangular fuzzy number

Criteria

$\begin{array}{ll}\text { C1 } & \text { Business value } \\ \text { C2 } & \text { Size } \\ \text { C3 } & \text { Effort } \\ \text { C4 } & \text { Core }\end{array}$

The fuzzy ratings for the criteria expressed as a fuzzy triangular number are illustrated in Table 1. The rating scale is determined by decision makers through consensus. In this example the linguistic rating scale ranges are from Very Low (VL) to Very High (VH) and the fuzzy rating scale ranges from 1 to 9 . Any appropriate rating scale can be chosen.

Table 1: Fuzzy ratings for the criteria by decision makers

\begin{tabular}{|l|c|}
\hline Linguistic term & $\begin{array}{c}\text { Fuzzy triangular } \\
\text { number }\end{array}$ \\
\hline Very Low $(\mathrm{VL})$ & $(1,1,3)$ \\
\hline Low $(\mathrm{L})$ & $(1,3,5)$ \\
\hline Medium $(\mathrm{M})$ & $(3,5,7)$ \\
\hline High $(\mathrm{H})$ & $(5,7,9)$ \\
\hline Very High $(\mathrm{VH})$ & $(7,9,9)$ \\
\hline
\end{tabular}

(ii) Represent linguistic scales for alternatives as a triangular fuzzy number. The alternatives in this instance are the set of requirements from the project backlog. Requirements (Alternatives)

- A1 Sales information to be modified only by members of the sales team 
- A2 Modification to any data in the system must be logged

- A3 Prevent SQL injections on all input fields

- A4 Allow authorized users to access the system

Table 2: Fuzzy ratings for the alternatives by decision makers

\begin{tabular}{|l|c|}
\hline Linguistic term & Fuzzy triangular number \\
\hline Very weak (VW) & $(1,1,3)$ \\
\hline Weak $(\mathrm{W})$ & $(1,3,5)$ \\
\hline Average $(\mathrm{A})$ & $(3,5,7)$ \\
\hline Good (G) & $(5,7,9)$ \\
\hline Very Good (VG) & $(7,9,9)$ \\
\hline
\end{tabular}

Step 2: Expert decision makers must rate the criteria and the alternatives with respect to criteria using appropriate linguistic variables. Construct aggregated fuzzy weights vector for criteria and construct the aggregated fuzzy decision matrix for alternatives.

(i) Decision makers $D M_{1}, D M_{2}$ and $\mathrm{DM}_{3}$ weigh the criteria using the linguistic rating scale for criteria based on their expert knowledge and experience.

Table 3 shows decision makers ratings of criteria, for example criterion $1\left(C_{1}\right)$ were rated as $\mathrm{H}\left(\mathrm{DM}_{1}\right), \mathrm{H}\left(\mathrm{DM}_{2}\right)$ and $\mathrm{L}\left(\mathrm{DM}_{3}\right)$ using linguistic scales.

Table 3: Linguistic Scales for Rating Criteria by Decision Makers

\begin{tabular}{|l|c|c|c|}
\hline \multirow{2}{*}{ Criteria } & \multicolumn{3}{|c|}{ Decision-makers } \\
\cline { 2 - 4 } & DM1 & DM2 & DM3 \\
\hline C1 & H & H & L \\
\hline C2 & H & VH & M \\
\hline C3 & VH & VH & VL \\
\hline C4 & H & H & M \\
\hline
\end{tabular}

(ii) The decision makers $D M_{1}, D M_{2}$ and $D M_{3}$ ratings must be represented as triangular fuzzy numbers in terms of the numeric rating scale for criteria.

Table 4 shows the conversions from a linguistic scale to a triangular fuzzy number. For example the rating for $C_{1}$ by DM1 is $\mathrm{H}(5,7,9)$, DM2 is $\mathrm{H}(5,7,9)$ and DM3 is $\mathrm{L}(1,3,5)$ respectively.

Table 4: Fuzzy weights for criteria

\begin{tabular}{|l|c|c|c|}
\hline Criteria & \multicolumn{3}{|c|}{ Decision-maker } \\
\hline & DM1 & DM2 & DM3 \\
\hline$C_{1}$ & $5,7,9$ & $5,7,9$ & $1,3,5$ \\
\hline$C_{2}$ & $5,7,9$ & $7,9,9$ & $3,5,7$ \\
\hline$C_{3}$ & $7,9,9$ & $7,9,9$ & $1,1,3$ \\
\hline$C_{4}$ & $5,7,9$ & $5,7,9$ & $3,5,7$ \\
\hline
\end{tabular}

Now taking $C_{1}$ as an example, the aggregated rating for $C_{1}$ is calculated as follows (min $(5+5+1), 7+7+3 / 3, \max (9+9+5))$. Similarly the aggregated rating for all other criteria are calculated. Then assemble the fuzzy decision matrix of criteria as shown in the Table 5. 
Table 5: Aggregate Fuzzy Weights for criteria

\begin{tabular}{|l|c|c|c|}
\hline \multicolumn{1}{|c|}{ Criteria } & \multicolumn{3}{c|}{ Weight } \\
\hline C1 & 1 & 5.7 & 9 \\
\hline C2 & 3 & 7 & 9 \\
\hline C3 & 1 & 6.3 & 9 \\
\hline C4 & 3 & 6.3 & 9 \\
\hline
\end{tabular}

Mathematically aggregate weights of each criterion are calculated as follows:

Let $k=$ number of decision makers

Let $j=1,2, \ldots . n$ representing the criteria $\widetilde{w}_{j}^{k}=\left(w_{j 1}, w_{j 2}, w_{j 3}\right)$

where: $w_{j 1}=\min _{k}\left\{w_{j k 1}\right\}, w_{j 2}=\frac{1}{K} \sum_{k=1}^{k} w_{j k 2}, w_{j 3}=\max _{k}\left\{w_{j k 3}\right\}$

(iii) Expert decision makers rating for the alternatives with respect to criteria using the appropriate linguistic variables. Thereafter the aggregated fuzzy decision matrix is constructed.

Table 6 shows that decision makers rating of alternatives in terms of the criteria based on linguistic the rating scale for alternatives.

Table 6: Linguistic assessments for 4 alternatives by decision makers

\begin{tabular}{|l|c|c|c|c|c|c|c|c|c|c|c|c|}
\hline \multirow{3}{*}{ Criteria } & \multicolumn{10}{|c|}{ A1ternatives } \\
\cline { 2 - 16 } & \multicolumn{3}{|c|}{} & \multicolumn{1}{|c|}{ A2 } & \multicolumn{4}{c|}{ A3 } & \multicolumn{3}{c|}{ A4 } \\
\cline { 2 - 15 } & DM1 & DM2 & DM3 & DM1 & DM2 & DM3 & DM1 & DM2 & DM3 & DM1 & DM2 & DM3 \\
\hline C1 & A & VG & W & A & W & A & G & VG & VW & G & A & VG \\
\hline C2 & VG & A & VG & W & A & VG & A & G & W & VG & VG & VW \\
\hline C3 & VW & W & A & G & G & W & A & VG & G & VW & A & VG \\
\hline C4 & VG & G & G & VG & W & G & A & VW & A & VG & G & G \\
\hline
\end{tabular}

Table 7 is populated by representing each entry in Table 6 as triangular fuzzy numbers in terms of the rating scale for alternatives.

Table 7: Aggregated fuzzy decision matrix

\begin{tabular}{|c|c|c|c|c|c|c|c|c|c|c|c|c|}
\hline \multirow{3}{*}{ Criteria } & \multicolumn{12}{|c|}{ Alternatives } \\
\hline & \multicolumn{3}{|c|}{ A1 } & \multicolumn{3}{|c|}{ A2 } & \multicolumn{3}{|c|}{ A3 } & \multicolumn{3}{|c|}{ A4 } \\
\hline & DM1 & DM2 & DM3 & DM1 & DM2 & DM3 & DM1 & DM2 & DM3 & DM1 & DM2 & DM3 \\
\hline $\mathrm{C1}$ & $3,5,7$ & $7,9,9$ & $1,3,5$ & $3,5,7$ & $1,3,5$ & $3,5,7$ & $5,7,9$ & $7,9,9$ & $1,1,3$ & $5,7,9$ & $3,5,7$ & $7,9,9$ \\
\hline C2 & $7,9,9$ & $3,5,7$ & $7,9,9$ & $1,3,5$ & $3,5,7$ & $7,9,9$ & $3,5,7$ & $5,7,9$ & $1,3,5$ & $7,9,9$ & $7,9,9$ & $1,1,3$ \\
\hline $\mathrm{C} 3$ & $1,1,3$ & $1,3,5$ & $3,5,7$ & $5,7,9$ & $5,7,9$ & $1,3,5$ & $3,5,7$ & $7,9,9$ & $5,7,9$ & $1,1,3$ & $3,5,7$ & $7,9,9$ \\
\hline C4 & $7,9,9$ & $5,7,9$ & $5,7,9$ & $7,9,9$ & $1,3,5$ & $5,7,9$ & $3,5,7$ & $1,1,3$ & $3,5,7$ & $7,9,9$ & $5,7,9$ & $5,7,9$ \\
\hline
\end{tabular}

Now the decision maker's fuzzy ratings are aggregated. Table 6 shows that alternative 1 based on criterion 1 was rated as $A\left(D_{1}\right), V G\left(D_{2}\right)$ and $W\left(D_{3}\right)$. The decision makers corresponding fuzzy rating were $\mathrm{A}(3,5,7), \operatorname{VG}(7,9,9)$ and $\mathrm{W}(1,3,5)$ as shown in Table 7 above. Therefore the aggregated rating of the 3 decision makers for alternative 1 using criterion 1 is $(1,5.7,9)$ as shown in Table 8 . In the same way assemble the aggregated fuzzy decision matrix for all other alternatives as shown in the Table 8. 
Aggregate weights of each alternative are calculated as follows:

Let $k=$ number of decision makers

Let $j=1,2, \ldots . n$ represent the criteria

Let $i=1,2, \ldots$. sepresent the alternatives

Aggregate weightings $\tilde{x}_{i j}=\left(l_{i j}, m_{i j}, u_{i j}\right)$ of alternatives $(i)$ with respect to each criterion ( $\left.j\right)$ based on the fuzzy ratings by the $k^{\text {th }}$ decision maker is expressed by the calculations below:

$$
l_{i j}=\min _{k}\left\{l_{i j}^{k}\right\}, m_{i j}=\frac{1}{K} \sum_{k=1}^{k} m_{i j}^{k}, u_{i j}=\max _{k}\left\{u_{i j}^{k}\right\}
$$

The aggregated fuzzy decision matrix is shown in Table 8.

Table 8: Aggregated fuzzy decision matrix

\begin{tabular}{|l|c|c|c|c|c|c|c|c|c|c|c|c|}
\hline \multirow{2}{*}{ Criteria } & \multicolumn{10}{|c|}{ A2 } & \multicolumn{1}{|c|}{ A1ternatives } \\
\cline { 2 - 14 } & \multicolumn{3}{|c|}{ A1 } & \multicolumn{1}{c|}{ A3 } & \multicolumn{3}{c|}{ A4 } \\
\hline C1 & 1 & 5.7 & 9 & 1 & 4.3 & 7 & 1 & 5.7 & 9 & 3 & 7 & 9 \\
\hline C2 & 3 & 7.7 & 9 & 1 & 5.7 & 9 & 1 & 5 & 9 & 1 & 6.3 & 9 \\
\hline C3 & 1 & 3 & 7 & 1 & 5.7 & 9 & 3 & 7 & 9 & 1 & 5 & 9 \\
\hline C4 & 5 & 7.7 & 9 & 1 & 6.3 & 9 & 1 & 3.7 & 7 & 1 & 7.7 & 9 \\
\hline
\end{tabular}

The aggregate fuzzy decision matrix for the alternatives $(\widetilde{D})$ is expressed below:

$$
\widetilde{D}=A_{1} A_{i}\left[\begin{array}{cccc}
C_{1} & C_{2} & C_{j} & C_{m} \\
A_{n} & \tilde{x}_{12} & \tilde{x}_{1 j} & \tilde{x}_{1 m} \\
\vdots & \vdots & \vdots & \vdots \\
\tilde{x}_{n 1} & \tilde{x}_{n 2} & \tilde{x}_{n j} & \tilde{x}_{n m}
\end{array}\right]
$$

\section{Step 3: Normalise the fuzzy decision matrix}

Normalise the fuzzy decision matrix of the alternatives $\widetilde{(D})$. All criteria are benefit criteria. A matrix is normalized to ensure that the value for each entry is in the interval $[0,1]$. It ensures that the values of each criterion are comparable. In fuzzy data the normalization is achieved by dividing each entry in the fuzzy triangular number by max value of the triple for each alternative (Sodhi 2012). For example consider the entry $(1,5.7,9)$ for criterion 1 for alternative1 illustrated in Table 8 . Normalisation is achieved by dividing each value in the triple by 9 . Therefore the normalized entry is $(1 / 9,5.7 / 9,9 / 9)=(0.333,0.633,1)$ as shown in Table 9.

Table 9: Normalised Aggregated fuzzy decision matrix for alternatives

\begin{tabular}{|l|c|c|c|c|c|c|c|c|c|c|c|c|}
\hline & \multicolumn{3}{|c|}{ A1 } & \multicolumn{3}{c|}{ A2 } & \multicolumn{3}{c|}{ A3 } & \multicolumn{3}{c|}{ A4 } \\
\hline C1 & 0.333 & 0.633 & 1 & 0.111 & 0.478 & 0.778 & 0.111 & 0.633 & 1 & 0.333 & 0.778 & 1 \\
\hline C2 & 0.333 & 0.856 & 1 & 0.111 & 0.633 & 1 & 0.111 & 0.556 & 1 & 0.111 & 0.7 & 1 \\
\hline C3 & 0.111 & 0.333 & 0.778 & 0.111 & 0.633 & 1 & 0.333 & 0.778 & 1 & 0.111 & 0.556 & 1 \\
\hline C4 & 0.556 & 0.856 & 1 & 0.111 & 0.7 & 1 & 0.111 & 0.411 & 0.778 & 0.111 & 0.856 & 1 \\
\hline
\end{tabular}

The normalized fuzzy decision matrix $\widetilde{(R})$ is written as follows:

Let $\tilde{R}=$ normalized decision matrix

Let $j=1,2, \ldots . n$ represent the criteria

Let $i=1,2, \ldots$. sepresent the alternatives

Let $\left(l_{i j}, m_{i j}, u_{i j}\right)=$ aggregated weighting of alternative (i) with respect to criterion ( $\left.j\right)$

Let $\tilde{r}_{i j}=$ triangular fuzzy number of alternative (i) with respect to criterion (j) in matrix $r$

with $n$ rows and s columns 
The normalized decision matrix is calculated as follows:

$$
\widetilde{(R)}=\left[\tilde{r}_{i j}\right]_{n x s}
$$

where $\tilde{r}_{i j}=\left(\frac{l_{i j}}{u_{j}^{+}} ; \frac{m_{i j}}{u_{j}^{+}} ; \frac{u_{i j}}{u_{j}^{+}}\right)$and $u_{j}^{+}=\max _{i} u_{i j} \quad$ (benefit criteria)

therefore $u_{j}^{+}=\max _{i}(9,9,9)$

Step 4: Compute the weighted normalised decision matrix

Table 10 shows the aggregated weights for each criteria. This table is used together with Table 11 to calculated the weighted normalized decision matrix , for example, the weighted normalised value for alternative $1\left(\mathrm{~A}_{1}\right)$ is obtained by multiplying the criterion $1\left(C_{1}\right)$ the values for $C_{1}(1,5.7,9)$ from Table 10 by the elements $(0.333,0.633,1.00)$ from the normalized fuzzy decision matrix in Table 11.

Table 10: Aggregate fuzzy weights for criteria

\begin{tabular}{|l|c|c|c|}
\hline & Criteria & \multicolumn{3}{|c|}{ Weight } \\
\hline C1 & 1 & 5.7 & 9 \\
\hline C2 & 3 & 7 & 9 \\
\hline C3 & 1 & 6.3 & 9 \\
\hline C4 & 3 & 6.3 & 9 \\
\hline
\end{tabular}

Table 11: Normalised Aggregate fuzzy decision matrix for alternatives

\begin{tabular}{|l|c|c|c|c|c|c|c|c|c|c|c|c|}
\hline & \multicolumn{3}{|c|}{ A1 } & \multicolumn{3}{c|}{ A2 } & \multicolumn{3}{c|}{ A3 } & \multicolumn{3}{c|}{ A4 } \\
\hline C1 & 0.333 & 0.633 & 1 & 0.111 & 0.478 & 0.778 & 0.111 & 0.633 & 1 & 0.333 & 0.778 & 1 \\
\hline C2 & 0.333 & 0.856 & 1 & 0.111 & 0.633 & 1 & 0.111 & 0.556 & 1 & 0.111 & 0.7 & 1 \\
\hline C3 & 0.111 & 0.333 & 0.778 & 0.111 & 0.633 & 1 & 0.333 & 0.778 & 1 & 0.111 & 0.556 & 1 \\
\hline C4 & 0.556 & 0.856 & 1 & 0.111 & 0.7 & 1 & 0.111 & 0.411 & 0.778 & 0.111 & 0.856 & 1 \\
\hline
\end{tabular}

Table 12 shows the completed weighted normalized fuzzy decision matrix for alternatives.

Table 12: Weighted Normalized Fuzzy Decision Matrix for Alternatives

\begin{tabular}{|l|c|c|c|c|c|c|c|c|c|c|c|c|}
\hline & \multicolumn{3}{|c|}{ A1 } & \multicolumn{3}{c|}{ A2 } & \multicolumn{3}{c|}{ A3 } & \multicolumn{3}{c|}{ A4 } \\
\hline C1 & 0.333 & 3.61 & 9 & 0.111 & 2.723 & 7 & 0.111 & 3.61 & 9 & 0.333 & 4.433 & 9 \\
\hline C2 & 1 & 5.989 & 9 & 0.333 & 4.433 & 9 & 0.333 & 3.889 & 9 & 0.333 & 4.9 & 9 \\
\hline C3 & 0.111 & 2.1 & 7 & 0.111 & 3.99 & 9 & 0.333 & 4.9 & 9 & 0.111 & 3.5 & 9 \\
\hline C4 & 1.667 & 5.39 & 9 & 0.333 & 4.41 & 9 & 0.333 & 2.59 & 7 & 0.333 & 5.39 & 9 \\
\hline
\end{tabular}

The weighted normalized decision matrix, $\widetilde{(V})$ is computed as follows:

Let $j=1,2, \ldots . n$ represent the criteria

Let $i=1,2, \ldots$. s represent the alternatives

The weights of the evaluation criteria, $\left(\widetilde{w}_{j}\right)$ is multiplied by the elements $\tilde{\mathrm{r}}_{\mathrm{ij}}$ of the normalized fuzzy decision matrix.

$$
\begin{aligned}
& \widetilde{(V)}=\left[\tilde{v}_{i j}\right]_{n x s} \\
& \text { Where } \tilde{v}_{i j}=\tilde{r}_{i j} \times \widetilde{w}_{j}
\end{aligned}
$$

Step 5: Fuzzy Positive-Ideal solution (FPIS) values and the Fuzzy Negative-Ideal solution (FNIS) values are determined 
The best performance values for each alternative are referred to as the FPIS and the worst performance values are referred to as FNIS (Vinodh et al. 2010). Table 13 shows the weighted normalized fuzzy decision matrix for alternatives. This table is used to calculate the FPIS and FNIS for alternatives under each criterion.

Table 13: Weighted Normalized Fuzzy Decision Matrix for Alternatives

\begin{tabular}{|l|c|c|c|c|c|c|c|c|c|c|c|c|}
\hline & \multicolumn{3}{|c|}{ A1 } & \multicolumn{3}{c|}{ A2 } & \multicolumn{3}{c|}{ A3 } & \multicolumn{3}{c|}{ A4 } \\
\hline C1 & 0.333 & 3.61 & 9 & 0.111 & 2.723 & 7 & 0.111 & 3.61 & 9 & 0.333 & 4.433 & 9 \\
\hline C2 & 1 & 5.989 & 9 & 0.333 & 4.433 & 9 & 0.333 & 3.889 & 9 & 0.333 & 4.9 & 9 \\
\hline C3 & 0.111 & 2.1 & 7 & 0.111 & 3.99 & 9 & 0.333 & 4.9 & 9 & 0.111 & 3.5 & 9 \\
\hline C4 & 1.667 & 5.39 & 9 & 0.333 & 4.41 & 9 & 0.333 & 2.59 & 7 & 0.333 & 5.39 & 9 \\
\hline
\end{tabular}

Table 14 shows the FNIS and FPIS values. Table 2.16 is used for the calculation of FPIS and FNIS, for example row 1 of Table 2.16 show that the FNIS value is 0.111 and the FPIS value is 9. These values are now written in row 1 of Table 14 under FNIS(A-) and FPIS(A+) respectively.

Table 14: FNIS and FPIS

\begin{tabular}{|c|c|c|c|c|c|}
\hline \multicolumn{3}{|c|}{ FNIS(A-) } & \multicolumn{3}{c|}{ FPIS(A+) } \\
\hline 0.111 & 0.111 & 0.111 & 9.000 & 9.000 & 9.000 \\
\hline 0.333 & 0.333 & 0.333 & 9.000 & 9.000 & 9.000 \\
\hline 0.111 & 0.111 & 0.111 & 9.000 & 9.000 & 9.000 \\
\hline 0.333 & 0.333 & 0.333 & 9.000 & 9.000 & 9.000 \\
\hline
\end{tabular}

The Fuzzy Positive Ideal Solution (FPIS, $\mathrm{A}^{ \pm}$) and the Fuzzy Negative Ideal Solution (FNIS, $\mathrm{A}=$ is calculated as follows:

$$
\begin{aligned}
& \text { Let } j=1,2, \ldots . n \text { represent the criteria } \\
& \mathrm{A}^{+}=\left\{\tilde{v}_{1}^{+}, \tilde{v}_{j}^{+}, \ldots, \tilde{v}_{n}^{+}\right\} \\
& \mathrm{A}^{-}=\left\{\tilde{v}_{1}^{-}, \tilde{v}_{j}^{-}, \ldots, \tilde{v}_{n}^{-}\right\} \\
& \text {where } \tilde{v}_{j}^{+}=(1,1,1) \text { and } \tilde{v}_{j}^{-}=(0,0,0)
\end{aligned}
$$

Step 6: Calculate the distance of each alternative from FPIS and FNIS

Table 15 shows the distance values for each alternative. For example, the distance between alternative 1 criterion $1(0.333,0.361,9.00)$ from Table 13 and FNIS for criterion 1 $(0.111,0.111,0.111)$ from Table 14 is calculated as follows:

$$
\begin{aligned}
\mathrm{d} & =\sqrt{\frac{1}{3}\left[(0.333-0.111)^{2}+(0.361-0.111)^{2}+(9.00-0.111)^{2}\right]} \\
& =5.517
\end{aligned}
$$

Table 15: Distances from FPIS and FNIS for alternatives

\begin{tabular}{|l|c|c|c|c|c|c|c|c|}
\hline & \multicolumn{4}{|c|}{$\mathbf{d}^{-}$} & \multicolumn{4}{c|}{$\mathbf{d}^{+}$} \\
\cline { 2 - 9 } & A1 & A2 & A3 & A4 & A1 & A2 & A3 & A4 \\
\hline C1 & 5.517 & 4.254 & 5.515 & 5.708 & 5.892 & 6.388 & 6.002 & 5.656 \\
\hline C2 & 5.987 & 5.535 & 5.408 & 5.656 & 4.935 & 5.656 & 5.809 & 5.535 \\
\hline C3 & 4.140 & 5.599 & 5.831 & 5.492 & 6.599 & 5.891 & 5.535 & 6.035 \\
\hline C4 & 5.844 & 5.530 & 4.064 & 5.793 & 4.719 & 5.662 & 6.330 & 5.420 \\
\hline
\end{tabular}


The distances $d_{j}^{+}$and $d_{j}^{-}$of each alternative respectively from $\tilde{v}_{j}^{+}$and $\tilde{v}_{j}^{-}$are calculated as follows:

Let $j=1,2, \ldots . n$ represent the criteria

Let $i=1,2, \ldots$. represent the alternatives

$$
\begin{aligned}
& d_{i}^{+}=\sum_{j=1}^{n} d_{v}\left(\tilde{v}_{i j}, \tilde{v}_{j}^{+}\right) \\
& d_{i}^{-}=\sum_{j=1}^{n} d_{v}\left(\tilde{v}_{i j}, \tilde{v}_{j}^{-}\right)
\end{aligned}
$$

Where $\mathrm{d}$ represents the distance between two triangular fuzzy numbers. For example the distance $\mathrm{d}\left(\tilde{v}_{i j}, \tilde{v}_{j}^{+}\right)$where $\tilde{v}_{i j}=\left(\mathrm{l}_{1}, \mathrm{~m}_{1}, \mathrm{u}_{1}\right)$ and $\tilde{v}_{j}^{+}=\left(\mathrm{l}_{2}, \mathrm{~m}_{2}, \mathrm{u}_{2}\right)$ is expressed as follows:

$$
\mathrm{d}\left(\tilde{v}_{i j}, \tilde{v}_{j}^{+}\right)=\sqrt{\frac{1}{3}\left[\left(l_{1}-l_{2}\right)^{2}+\left(m_{1}-m_{2}\right)^{2}+\left(u_{1}-u_{2}\right)^{2}\right]}
$$

\section{Step 7: Obtain the closeness coefficient $\left(\mathrm{CC}_{\mathrm{i}}\right)$}

Table 16 shows the values for the Closeness Coefficients of the four alternatives. The alternative with the highest closeness coefficient represents the best alternative as it is closest to the FPIS and farthest from FNIS.

Table 16: Closeness Coefficients of 4 Alternatives

\begin{tabular}{|l|c|c|c|c|}
\hline & A1 & A2 & A3 & A4 \\
\hline d- & 21.488 & 20.918 & 20.818 & 22.649 \\
\hline d+ & 22.145 & 23.597 & 23.676 & 22.647 \\
\hline CCi & $\mathbf{0 . 4 9 2}$ & $\mathbf{0 . 4 7 0}$ & $\mathbf{0 . 4 6 8}$ & $\mathbf{0 . 5 0 0}$ \\
\hline
\end{tabular}

The Closeness Coefficient values of alternative 1 calculated as 21.488 (d-) / 21.488 (d-) + $22.145(\mathbf{d}+))$ is 0.492 . Similarly the values for the closeness coefficient of other alternatives in Table 2.19 are calculated.

The closeness coefficient $\left(\mathrm{CC}_{\mathrm{i}}\right)$ is calculated as follows:

$$
\mathrm{CC}_{\mathrm{i}}=\frac{d_{i}^{-}}{d_{i}^{+}+d_{i}^{-}}
$$

Step 8: Define the Ranking of Alternatives

Define the ranking of the alternatives according to the closeness coefficient, $C_{i}$, in decreasing order. The best alternative is closest to the FPIS and farthest to the FNIS.

Order:

A4 Allow authorized users to access the system

A1 Sales information to be modified only by members of the sales team

A2 Modification to any data in the system must be logged

A3 Prevent SQL injections on all input fields

The results of fuzzy TOPSIS indicate that only A4 and A1 must be sent to the product backlog. 


\section{APPENDIX 2 \\ INTERVIEW QUESTIONNAIRE: TOOL EVALUATION}

\section{Preamble}

Thank you for volunteering to participate in this research. A new software tool has been developed to address the prioritisation of clients security requirements using a computerized Fuzzy based system as a more effective and efficient way to rank normal requirements and non-functional requirements.

The purpose of this questionnaire is to therefore:

- Elicit the views of requirements engineers on the extent to which the new software tool is able to meet their needs.

\section{INTERVIEW QUESTIONS: Guided questions for stakeholders of the Agile Project} SECTION A: GENERAL

1. Researcher: What is your role in the Agile Software Development project?

Respondent:

2. Researcher: What techniques are presently used to rank client requirements?

Respondent:

3. Researcher: What are the limitations of current techniques?

Respondent:

4. Researcher: Who are the stakeholders involved in the ranking of client requirements presently?

Respondent:

\section{SECTION B: EASE OF USE}

1. Researcher: Describe your experience with the ease of use of the software tool? Respondent:

2. Researcher: What changes would you make to improve the ease of use? Respondent:

3. Researcher: Comment on your satisfaction with the user interface. Does it promote easy Use?

Respondent:

\section{SECTION C: FUNCTIONALITY AND CAPABILITY OF THE SOFTWARE TOOL}

1. Researcher: Discuss your satisfaction with the time taken by the tool to effectively rank requirements?

Respondent:

2. Researcher: Comment on the correctness of the automated fuzzy tool?

Respondent:

3. Researcher: Comment on the scale of measurement used?

Respondent: 
4. Researcher: Comment on the scalability of the tool. How is the performance of the tool as the number of requirements increases?

Respondent:

5. Researcher: Is the tool suitable for high level requirements as well as detailed requirements?

Respondent:

\section{SECTION D: OVERALL}

Researcher: Final comments on the automated fuzzy software tool in comparison to current Methods?

Respondent: 


\title{
Challenges of Big Data from a Philosophical Perspective
}

\section{Sunil Choenni}

Ministry of Justice and Security, WODC

The Hague, The Netherlands

\section{Niels Netten}

Rotterdam University of Applied Sciences, Creating 010

Rotterdam, The Netherlands

\section{Mortaza Bargh}

Rotterdam University of Applied Sciences, Creating 010

Rotterdam, The Netherlands

\section{Rochelle Choenni}

University of Amsterdam

Amsterdam, The Netherlands

\begin{abstract}
Due to the many potential applications of Big Data, the expectations are high. However, there are some fundamental objections on the straightforward use of Big Data outcomes. In this paper, we take a philosophical view on the Big Data approach and discuss these objections. Formally, Big Data induces models from very large data sets, which are nevertheless incomplete. In many cases these data sets might be skewed as well. This gives rise to the question to what extent induced models represent the real world adequately, and therefore are sufficiently grounded to base new policies on. We argue that caution is needed in interpreting these models and well thought through strategies are required for using the models in practice in a responsible way. We discuss two strategies that may be used.
\end{abstract}

Keywords: Big Data, induction, models, incompleteness.

\section{Introduction}

Nowadays, our environment has become more complex as it is equipped with many devices, such as camera's and mobile phones that together generate huge amounts of data. These data are of several types. To exploit this data, traditional database techniques are not advanced enough, giving rise to the concept of Big Data. Big Data 
refers to both structured and unstructured data sets that are so large in volume and complex that traditional systems are not capable of managing, storing and/or processing them within a reasonable time frame. These data sets have proven to encapsulate invaluable and sometimes unexpected information. The data sets have traditionally been used to find some useful insights to enable, for example, cost and time reductions. There are various organizations and companies across a wide range of public and private sectors that are now trying to process raw data in order to derive information useful for specific applications such as public administration, health care, insurance, finance, transportation, logistics and retail (Roger et al., 2012). There are multiple factors that help for effectively retrieving valuable information. Not only does the amount of data available play an important role, the way in which the data is processed has equally important role. Therefore, new intelligent techniques are needed to optimally process the data (Kim et al., 2014).

This need has led to increasing amount of research among data scientists and analysts, regarding questions on how to process and manage the data. However, how to interpret and implement the results in practice is in its childhood. In this paper, we discuss the challenges and pitfalls of implementing the results obtained from data analytics tools.

Results from Big Data are often used on a large scale to make predictions about a wide range of matters (Tien, 2013; Choenni, 2000; Netten et al., 2018). In business settings, for example, predictions can be made about the most optimal business strategy to follow. As the predictions are fully based on the input data, the accuracy of these predictions depends on, among others, the suitability of the data. When for example government institutions use these methods to make predictions about certain future behaviors and potentially impose new regulations based on them, it becomes a necessity to be able to guarantee reasonably accurate results. This entails that the data sets provided to the algorithm are a sufficient reflection of the real world (Choenni et al., 2018).

This gives rise to the question of to what extent Big Data and the models derived from the data are able to represent the real world. More importantly, it gives rise to the question as to whether the use of this approach to gain information on a large scale of complex (social, business, economic, etc.) phenomena is justified. Does this approach provide results that are sufficiently grounded to base new policies on? This paper describes various challenges raised by this Big Data analytics approach. Both theoretical and more practical aspects will be discussed. Firstly, a more thorough explanation will be given on the way in which Big Data models are used. Secondly, the reasoning method behind this approach will be questioned by proposing David Hume's induction problem (Hume, 2003). Then an attempt will be made to provide a solution to this problem through Bayesianism. Lastly, some critical problems to the interpretation and use of the results will be discussed. 


\section{Big Data}

In some sense Big Data can be regarded as a unique collection of the concepts coming from different fields in computer science and the related fields (Netten et al., 2016). The implementation of these concepts gives rise to many novel applications potentially. Two views may be taken on the exploitation of Big Data. In the first view, referred to as the black box, the implementation of the concepts and the relationships between the concepts are not clear and considered as a black box. We exploit these concepts by offering 'big data sets' and some constraints to the black box and observe the results that are provided by the black box. In case that we are not satisfied by the results, we alter some of the constraints and perhaps also the data and offer them again to the black box. We may repeat this process until we are satisfied by the results provided by the black box.

In the second view, referred to as the open box, the implementation of the concepts and their relationships is fully documented and we are able to track exactly how the results are obtained. In case we are not satisfied by the results obtained from Big Data, we may find out which concepts and relationships contribute to this dissatisfaction and adapt them accordingly to achieve satisfactory results. Due to the complexity of contemporary Big Data systems, questions need to be asked about the feasibility of the open box approach.

We take another view on Big Data, referred to as glass-box, which is in between the two (black box and open box) views. In this view, it is not necessary to know precisely all the ins and outs with regard to the implementation of the used concepts and their relationships. However, the crucial concepts of Big Data and possible relationships between the concepts are specified. Furthermore, it should be transparent how Big Data results are obtained. An example of a glass box view is depicted in Figure 1. In this example, we distinguish a set of algorithms for data processing, data analytics, and data visualization, a Hadoop cluster, and some data storage facilities. We see that the algorithms may exploit the Hadoop cluster for their jobs, and this cluster interacts with the data storage facilities. A Hadoop cluster consists of several machines, which are used to process a (complex) job. Via the socalled "map reduce" paradigm, a job is split into a set of smaller jobs and these jobs are distributed among the several machines for processing. Once a machine has completed its job, the result is collected. The collected results are composed to a final result.

Furthermore we see in Figure 1, the output of the data processing algorithms are used as input by the data analytics algorithms, and so on. 


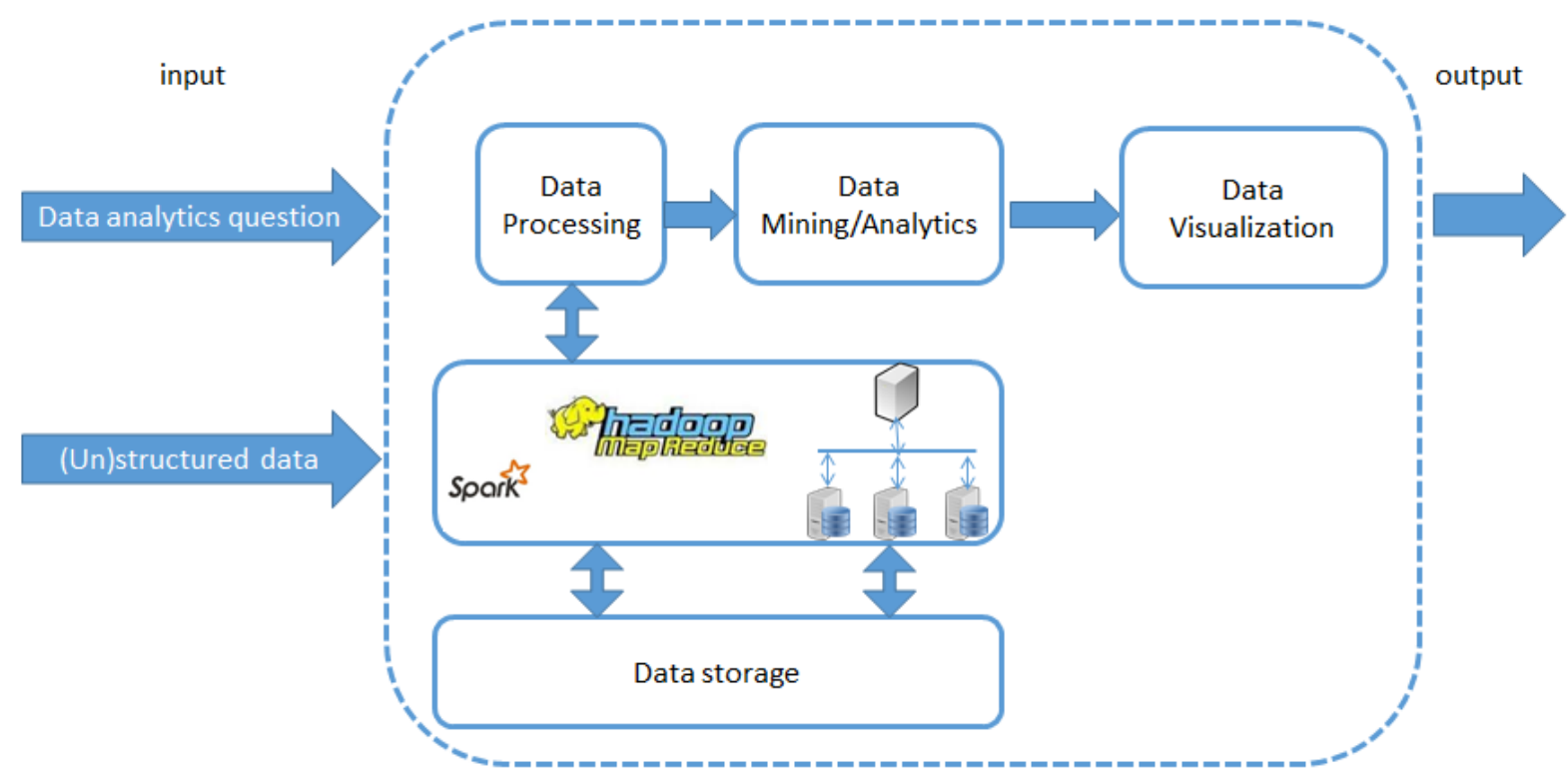

Figure 1 - Illustration of a glass box view

A crucial concept of Big Data is data analytics on which we will focus on in this paper. Data analytics has the goal of learning and drawing conclusions from large amount of integrated data. In order to analyze these large amounts of data, data mining is a key methodology for data analysts. Informally data mining can be regarded as the practice of examining large data sets in order to generate new information. On the other hand, the data set is supposed to represent the real world. If for example a correlation is found between advertisements with luxurious cars and high selling rate, then the result might be a prediction that new commercial campaigns should again involve these cars. This is based on the assumption that if the correlation between luxurious cars and high selling rates has been observed many times already, this correlation will also hold in the future.

\section{Challenges}

Formally, data mining is the induction of a model of the environment from large data sets (Choenni et al., 2005). When such models are generated, they are used to retrieve information from (like for prediction of unforeseen outcomes based some observed events). Inductive reasoning has been the subject of philosophical debate for years. Those models that are based on inductive reasoning pose two critical problems as described below.

Firstly, inductive reasoning assumes that when repeating patterns are observed these patterns will always exist and repeat. For example, when we continuously see that a certain event $X$ leads to a certain event $Y$, we will automatically assume that the next time we observe $X$ it will lead to $Y$ again. Generally, you observe some concrete examples where something is true and from there a universal rule is induced. However, Hume argued that this is in fact a logically incorrect way of reasoning as inductive inferences do not necessarily have to be correct. This will be discussed more thoroughly later on in this paper. 
The second problem stems from the fact that the models based on Big Data cannot fully capture the real world. Models are merely reflections of the real world and therefore they will necessarily miss some (relevant) parts of the real world. As the model might miss crucial information it can be an insufficient and thus skewed representation of the world. If this is the case, results derived from the model may be meaningless in the real world. This means that even when we have a perfectly sound reasoning method to generate the model from, we would still never be sure whether the information derived from it is correct since the model might be incomplete.

Furthermore, models are largely used for two reasons. Either as a way of understanding the environment or as an attempt to predict the environment. These reasons are both contradicting as well as reinforcing. Models that make good predictions do not necessarily have to be insightful(being contradictory), but insightful models can result in better predictions (being reinforcing). Each time the results are retrieved and applied to the real world, the model therefore needs to get updated. Essentially, the model continuously needs feedback to adjust its structure and parameters in order to remain as close of an accurate representation of the world as possible, see Figure 2.

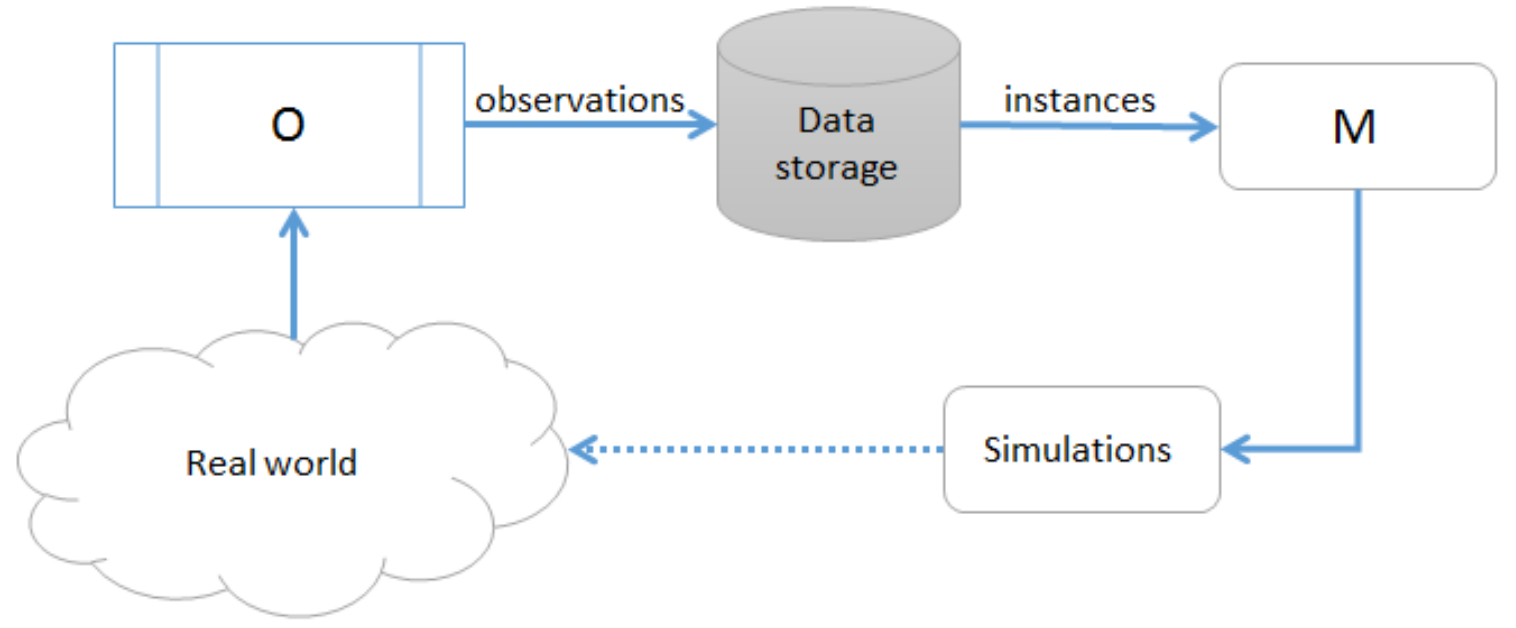

Figure 2 - Relationship between Big Data results and the real world

This new model can then again be used as an up to dated representation of the world to generate information from. Later on, we will explain how this approach in combination with the fact that models are incomplete can pose some ethical problems.

\section{A Philosophical View}

The problem of induction concerns the justification of inductive methods. Hume described them as methods that predict or infer that "instances of which we have had no experience resemble those of which we have had experience" (Hume, 2003). He argued that all human knowledge is derived from ideas and the relationship between ideas and impressions. People can recognize structural patterns systematically in these, which gives rise to concepts such as causality. However, it 
would also be possible to imagine that causality would for example cease to exist tomorrow. Nothing guarantees that patterns that we see today, must necessarily still exist tomorrow. For example, when ice is heated, we expect it to melt. However, it is possible to imagine that from tomorrow on this will no longer be the case. This is what Hume denoted as the induction problem. If nothing guarantees that these relationships will hold, we cannot really gain information from experience. So why do we intuitively reason inductively anyway?

Hume found the answer in habits and associations. When the same sequence of events keeps being observed many times, associations are automatically learned. For example, fire and warmth and ice and cold. Therefore, a certain cause does not have to be necessarily but is just expected by habit. Hume did not find this particularly problematic. If we assume a uniform nature, then it is logical that we would expect certain behaviors based on associations (Glymour, 2015). This does, however, not hold for the luxurious cars and advertisement example proposed earlier. Suppose that the observation has been made many times within the database that a relation between luxurious cars and high selling rates exists. As a result, the prediction is then yielded that a next advertisement will again be most successful if it involves luxurious cars. However, this would not necessarily be logical according to Hume's reasoning about the induction problem. Hume saw a sense of logical reasoning behind induction if we assumed a uniform nature. However, concepts like consumer behavior can be expected to be much less uniform (i.e., more dynamical) than the nature behavior is. It is therefore not completely intuitive to expect all concepts to appear to be uniform.

A well-known answer to Hume's induction problem is Immanual Kant's transcendental argument. Kant found the answer in the transcendental categories of relations. These are synthesized by empirical intuition and create as well as limit all human knowledge by synthetic a priori judgments. These categories form causality under time and space (Kant, 2004). The idea is that we cannot have knowledge of the world in itself, but only of how the world appears to us. However, we know that observations cannot be made without concepts such as causality. Therefore, Kant argued that these concepts precede the experience and can thus escape skepticism. Kant did not prove the existence of these concepts, he merely proved that someone cannot observe anything without adding it to the experience. Again, this argument does not translate well to cases like the luxurious cars and advertisements discussed earlier. In data mining, inductive reasoning is often used to explain abstract concepts like consumer behavior. These are not relations that are experienced.

Many more attempts were made to refute the induction problem that remained unsatisfying. Nowadays, it still seems like there is no solution to the induction problem that can guarantee the correctness of the results. However, in the twentieth century a movement called Bayesianism proposed an insightful alternative. The novel idea was that belief and knowledge do not necessarily have to be either correct or incorrect. Rather, they can come in degrees that conform to certain constraints related to the axioms of probability theory (Grimmett \& Stirzaker, 2001). They 
therefore did not attempt to prove that induction leads to knowledge, but that it can result in a certain degree of knowledge. Probabilistic Bayesianism has now gained a well-established position in philosophy. It is in close correspondence to our intuition. If we see something happen a million times, it is highly likely, but not certain, that it will happen again. The inferences from induction can therefore gain an epistemic status somewhere between the two extremes of right and wrong. This status depends on the quality of the evidence and can be adjusted in the future when new evidence comes to light. This approach to inductive inferences is the only justification to our reasoning with Big Data. Consequently, all results we derive in this manner cannot be perceived as knowledge, but merely as probable conclusions. Whilst this does provide some justification, it is a weakness of the theoretical foundations on which this approach to Big Data is based.

It should be clear that a Big Data approach will not lead to universal truths but at most to some probable conclusions from a philosophical point of view. Furthermore, the probable conclusions are bounded by time and space. Therefore, caution and a good understanding of the probable conclusions are needed in exploiting the results obtained by (Big) Data analytics. To what extent caution should be taken depends on the application at hand. An important parameter that determines this caution is the velocity with which a concept is changing.

\section{Illustrative examples}

As explained in the foregoing, Big Data is collected and by means of data mining a model of the world is generated. This prior model will be used to apply Artificial Intelligence (AI) techniques on, which outputs certain results. The information from these results is then processed in the real world. As the results are processed, the world has changed and therefore the model needs to get updated. The prior model is replaced by a second model on which techniques can be applied to retrieve new information. For smaller simple cases this seems like a reasonable approach. However, when this is applied on a large scale by, for example, government institutions, this approach can pose some ethical problems. This will be illustrated by the following example. Suppose a government institution is in charge of policies regarding law reinforcement. The institution now races for example the challenge of fining as many drivers that commit traffic violations as possible. Therefore, a model of the world is induced from Big Data and some AI techniques are applied to them in order to answer the question of which strategy the policemen should follow.

Now suppose that the data include many correlations between drivers with a certain ethnic profile and the committing of traffic violations. It would seem beneficial, but may be unethical or against the law, to instruct all policemen to keep an extra eye on these drivers. This is where the problem arises. As the policy is now made to watch out for these drivers, the perceived world will be altered based on results that were retrieved from the previous model. Naturally, if many policemen are now focusing on these drivers, the same drivers will again get fined more often. This however does not necessarily have to mean that they do indeed commit traffic violations more often than the drivers with another profile. However, with every iteration of 
this method, the system will keep getting reinforced that it was initially right. With every new model these drivers will get over represented more and therefore the output will remain the same (i.e., the drivers from that certain ethnic profile will be picked up). This would be an ethical problem as policemen are now continuously targeting the same group of people with that certain ethnic profile.

The problem with this approach stems from the fact that the policies that are based on the model will also determine the righteous of the model. Thus, essentially, the model is only reinforcing itself. This illustrates the same problem that Karl Popper already identified. If you are trying to prove the hypothesis that all swans are white, just searching for extra white swans as evidence is not a valid method (Popper, 2005). In this case the hypothesis would be the retrieved result that drivers of the certain ethnicity are committing more violations, which you are then searching evidence for by ordering policemen to target these specific drivers. Therefore, when this method is applied on a large scale, the AI algorithms may actually and eventually influence the real world instead of simply capturing information from it. It could essentially give back that group of drivers accusing them of committing more traffic violations and the outcome will always hold true according to the model. In the context of mortgage provisions, the above discussed issues (may) play a role as well (Berkovec et al., 1994).

In brief, we argue that the real world rules may change. Then, the current rules may not apply in the future, which actually asks for continuous learning. However, even if we want to learn the new rules, the learning is useless if the old rules dictate the scope and space of observations, such that the changes in the real world cannot be observed.

\section{Towards Solutions for Big Data Challenges}

Given the fact that the interpretation of data analysis results is far from trivial, we propose to consider these results as a central body of knowledge. From this knowledge, we can derive a hypothesis for an individual case. Then, we search for other evidence(s) that may support or weaken this hypothesis. As an example, suppose that we feed a Big Data tool with a large amount of data of those who were involved in car accidents. After analyzing the data, the tool produces the following profile as result "young men living in areas with zip code 1234 have a higher than average probability to cause car accidents". Now we have a young man, named Mr. Green, who lives in this area (with zip code 1234). A challenging question is how to apply the profile in the case of Mr. Green.

Despite the fact that the profile is a statistical truth and cannot be projected on a specific individual as fact, we search for a way to exploit the knowledge that is captured in the profile. Therefore, we formulate the following hypothesis for Mr. Green: "Mr. Green will cause car accidents". To evaluate this hypothesis, we may use two strategies as described below. 
In the first strategy, we search for evidences that support the hypothesis, e.g., Mr. Green caused car accidents in the past or an expert agrees with the hypothesis. Note that these evidences should not be based on or derived from the data that are used in the data analysis. Using the same data will shed no new light on the hypothesis, instead it will incorrectly strengthen the hypothesis further. If enough supporting evidence has been collected, the hypothesis can be accepted and the case of Mr. Green should be investigated further. In our example, suppose we examine the drives of Mr. Green to find more evidences that support the hypothesis (that he causes a car accident). Assume that we find that Mr. Green was involved at several car accidents in the past and has filed several insurance claims. However, it was not always clear whether Mr. Green caused the accidents or others who were involved in the accidents. Furthermore, a check on his bank accounts shows that they were practically empty at the time of the accidents. This may be considered as evidence for Mr. Green, who may swindle insurance companies. A disadvantage of the strategy of collecting supporting evidences is that it may strengthen confirmation biases and lead to a self-fulfilling prophecy, i.e., a false hypothesis might become true due to this bias.

In the second strategy, we search for evidence that weakens the hypothesis "Mr. Green will cause car accidents". If we find a set of evidence that gives rise to rejecting the hypothesis, no action should be taken against Mr. Green. Unlike in the previous strategy, the same data set from which the hypothesis is derived may be used to search for evidences that weaken the hypothesis. The data that are used to induce the hypothesis from the profile can be used to infer other profiles that may be in advantage of Mr. Green. For example, if we are able to derive a new profile like: "Men living in zip code 1233 and have not filed a car insurance claim in the past 5 years and do not drive in leased cars are cautious drivers". If Mr. Green satisfies this new profile, then this will weaken the hypothesis that Mr. Green will cause car accidents frequently. Other data sets can also be used to search for evidences to weaken the hypothesis. A disadvantage of this strategy of collecting weakening evidences is that it may lead to a self-denying prophecy, i.e., a true hypothesis might become false due to bias.

Which strategy to use for which application depends on the nature of an application and the impact of possible false positives and false negatives. We note that a false positive refers to an accepted hypothesis while it is false, and a false negative refers to a rejected hypothesis while it is true. The first strategy tends to reduce the false negatives and to increase the false positives, while the reverse is true for the second strategy. The second strategy is applied in contemporary judicial courts. The public prosecutor makes a statement, which is based on police investigations of a suspect. Subsequently, in court the lawyer of the suspect aims to disprove this statement by presenting counter proofs. Such a strategy is chosen to only convict someone if he is indeed guilty, i.e., to avoid false positives. In sensitive applications that have a large impact on someone's life, the second strategy is recommended. The first strategy, on the other hand, focuses on strengthening a hypothesis and avoiding false negatives. 
In some application areas that are related to public security (like searching for terrorists), the first strategy might be useful.

Independent of which strategy is used for an application, it makes sense to have an estimate of the impact of false positives and false negatives, and a procedure to anticipate on them. One of these strategies (or perhaps a mix of both) should be tailored to the application at hand.

\section{Conclusion}

The use of inductive reasoning to retrieve information from Big Data can, to a certain extent, be justified if we take a Bayesianistic position. This, however, means that we have to give up the idea that the results that we obtain are certainly true. Thus, we acknowledge that we might only be dealing with probable information. But if our model is induced and induction is only probable, the results can be wrong. This, combined with the fact that models can never be an exact representation of the real world anyway, results in much room for error. In practice, the model gets continuously updated to compensate for these errors. However, as explained earlier, when government institutions start applying this method on large scale this can pose some challenges. As illustrated, it can result in ethical problems.

All in all, a lot of caution is needed when using this approach. It is important to be aware of the fact that there are many contributing factors that can be the cause of error. It is clear that induction does not always provide correct results. However, as mentioned before, even if it did, it would still not be possible to completely trust the output. After all, then we would still be dealing with a model which in itself is not an exact representation of the world. Therefore, there is too much uncertainty and the information gained is thus not reliable enough to directly base policies on, especially not when being applied to sensitive cases such as with the example of accident causing drivers. It could possibly have negative consequences as wrong interpretations of the results could impact the real world adversely. Nevertheless, as induction is for a large part a justifiable reasoning method, the results are not entirely useless. The results should be interpreted as rough estimates that can serve as guidelines for further research. Based on this research and with human intervention, these results can then indirectly be used to base policies on. We have discussed two preliminary strategies to benefit from Big Data. However, these strategies need to be further elaborated, which is a topic for our future research.

\section{References}

Berkovec, J. A., Canner, G. B., Gabriel, S. A., \& Hannan, T. H. (1994). Race, redlining, and residential mortgage loan performance. The Journal of Real Estate Finance and Economics, 9(3), 263-294.

Choenni, S. (2000). Design and implementation of a genetic-based algorithm for data mining. In VLDB (pp. 33-42), Proceedings of the $26^{\text {th }}$ International Conference on the Very Large Databases, Morgan Kaufmann. 
Choenni, S., Bakker, R., Blok, H. E. \& Laat, de, R. (2005). Supporting technologies for knowledge management. In Knowledge Management and Management Learning (pp. 89-112). Springer, Boston, MA.

Choenni, S., Bargh, M. S, Netten, N. \& Braak, van den, S. (2018). Using data analytics results in practice: Challenges and solution directions. In ICT-Enabled Social Innovation for the European Social Model (IESI-ESM), F. Davide and G. Misuraca (eds), IOS PRESS (to appear).

Glymour, C. (2015): Thinking Things Through: an introduction to philosophical issues and achievements. MIT Press: (pp. 171-179).

Grimmett, G., \& Stirzaker, D. (2001). Probability and random processes. Oxford University Press.

Howson, C. \& Urbach, P. (2006) Scientific reasoning: the Bayesian approach. Open court Publishing: Chapter 1.

Hume, D. (2003): A treatise of human nature. Courier Corporation: section VI.

Kant, I. (2004): Prolegomena to Any Future Metaphysics: That Will Be Able to Come Forward as Science: With Selections from the Critique of Pure Reason. Cambridge university press: (pp. 79-122).

Kim, G., Trimi, S. \& Chung, J. (2014) Big-Data Applications in the Government Sector. Communications of the ACM, 57,3, 78-85.

Netten, N., Braak, van den , S., Choenni, S. \& van Someren, M. (2016). A Big Data Approach to Support Information Distribution in Crisis Response. In Proceedings of the 9th International Conference on Theory and Practice of Electronic Governance (pp. 266-275). ACM.

Netten, N., Bargh, M. S. \& Choenni, S. (2018). Exploiting Data Analytics for Social Services: On Searching for Profiles of Unlawful Use of Social Benefits. In Proceedings of the 11th International Conference on Theory and Practice of Electronic Governance. ACM.

Popper, K. (2005). The logic of scientific discovery. Routledge.

Roger, C.H, Chiang, H. L. \& Storey, V.C. (2012). Business intelligence and analytics: from big data to big impact. MIS quarterly (pp. 1165-1188).

Tien, J.M. (2013). Big data: Unleashing information. Journal of Systems Science and Systems Engineering (pp. 127-151). 


\title{
Reliability of a Directly Cooled PV/T System
}

\author{
Busiso Mtunzi \\ National University of Science and Technology, \\ Bulawayo, Zimbabwe
}

Edson Meyer

Fort Hare Institute of Technology

University of Fort Hare,

Alice, South Africa

\begin{abstract}
The objective of this paper was to quantify and evaluate the effect of water absorption on a directly cooled PV module (PV/T). A design experiment was assembled and used in analysing the performance of the directly water cooled PV module when compared to a naturally cooled PV module. The results revealed that not only the power output of the PV/T dropped, but also the series and shunt resistances of the PV/T changed, and these were found to bring about changes in the generated power. A drop in output power impacted negatively on the performance of the PV/T system. This was found to have an effect on the reliability of the PV systems, hence compounding on the system's ability to deliver the rated power during its life time. The Photovoltaic Module data considered in this paper was for a period of one year.
\end{abstract}

Keywords: Photovoltaic Thermal Module, Shunt Resistance, Reliability, Series Resistance, Performance ratio.

\section{Introduction}

In this paper, the power decline over time, also known as degradation rates for two identical PV modules were analysed. Two modules were analysed, a naturally air cooled module (M1), and a photovoltaic thermal hybrid system (PV/T), (M2). The $\mathrm{PV} / \mathrm{T}$ (M2) module was cooled by water in direct contact with the back of the module.

Generally, a higher degradation rate on photovoltaic modules implies less power production. Understanding photovoltaic module degradation dynamics is important as it helps in determining the PV module's half-life; hence its reliability. Carrying out 
reliability tests on new PV system designs is important as this gives PV modules' behaviour when placed outdoors.

PV modules' manufacturers and users always need to know the product's long term reliability and its performance. The investors always want to know if the project is bankable. A warranty of 20 to 25 years is usually given by most PV module manufacturers and of late, warranties of up to 30 years have been given by some manufacturers (Vazquez \& Rey-Stolle, 2008). However, according to Canadian Solar (2017), by the end of year 25, the actual power output has been found to be no less than $80.7 \%$ of the labeled power output. This could mean that by the end of 30 years, the PV module power output would still be way above $50 \%$ of labelled power output. These warranties are meant for ordinary PV modules. The advent of PV hybrid systems has brought about modifications on the PV modules and the redesigned PV modules (PV/T) may/ may not meet the indicated life span. Introduction of water as a cooling media may impact negatively or positively on the PV modules, hence causing a change on the module's reliability.

In reliability engineering, it is well-known that any equipment or device would typical go through three phases during its field time (Zielnik, 2015) and these are; Infant mortality, Random failure and Wear out failure.

The three phases are shown in figure 1:

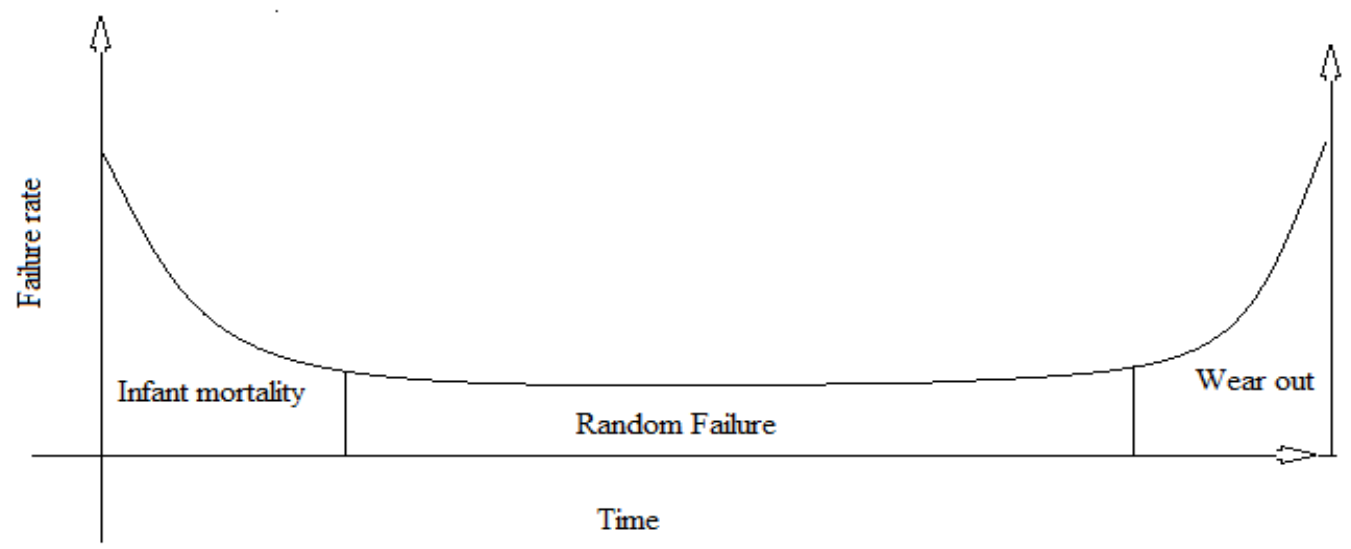

Figure 1- Classic bath tub curve showing how component failure rate can vary with time.

(Zielnik, 2015)

Infant mortality region refers to early life failures of the PV modules, while random failures refer to failures that may be caused by effects such as lighting. The lightning has the same probability of occurring in the system's life, hence a constant level on the graph. The wear out failure in this case could be due to contact oxidation on the module's cells. These three phases could be applied to PV modules and are needed to help the investors and manufacturing companies to understand the module's behaviour. This information is needed to help find the gateway of improving the PV product life or its availability when in the field.

When the radiation falls onto a PV module, part of the absorbed radiation is used by the PV cells to generate electricity while the other part generates heat on the module. 
The photon energy needs to be at least equal or more than the band-gap energy $\Delta \mathrm{E}_{\mathrm{g}}$ of the semiconductor (1.1eV for the crystalline silicon). Photons with energy slightly above $\Delta \mathrm{E}_{\mathrm{g}}$ are partly absorbed by the PV cells and they will generate an electron hole pair per photon. The excess energy will be transferred in the form of heat to the module. It is not all the wavelengths that generate electricity. Some of the wavelengths with energy less than $\Delta \mathrm{E}_{\mathrm{g}}$ will just pass through the PV cells and get absorbed by water, contributing towards the thermal energy. The solar cells have a high absorption coefficient and produce infrared rays. The infrared rays produced by the solar cells get reflected back by the glass causing greenhouse effect in the module hence, heating the module. The flow of heat to the back of the module passes through several thermal resistances causing a heating effect at the back of the module.

In this paper the investigations were carried out for a year.

\section{Design}

This study was designed to examine the performance of two photovoltaic modules, one naturally cooled and the other directly water cooled. A performance ratio analysis was used to check on the effects of directly water cooling of a photovoltaic module. The two modules were placed side by side facing north at an angle of Latitude tilt of $33^{\circ} \mathrm{S}$.

Two PV systems were analysed using their performance ratios and their power output. Both, the performance ratio and power output of the two modules were determined from the field measurements. The field measurements included the I-V characteristics, irradiance in the plane of array, back of module temperatures, inlet and outlet temperatures on module M2 as well as visual inspection. The block diagram showing the experimental set used in the experiment was as shown in figure 2 (Mtunzi, Meyer, Simon \& Malape, 2012).

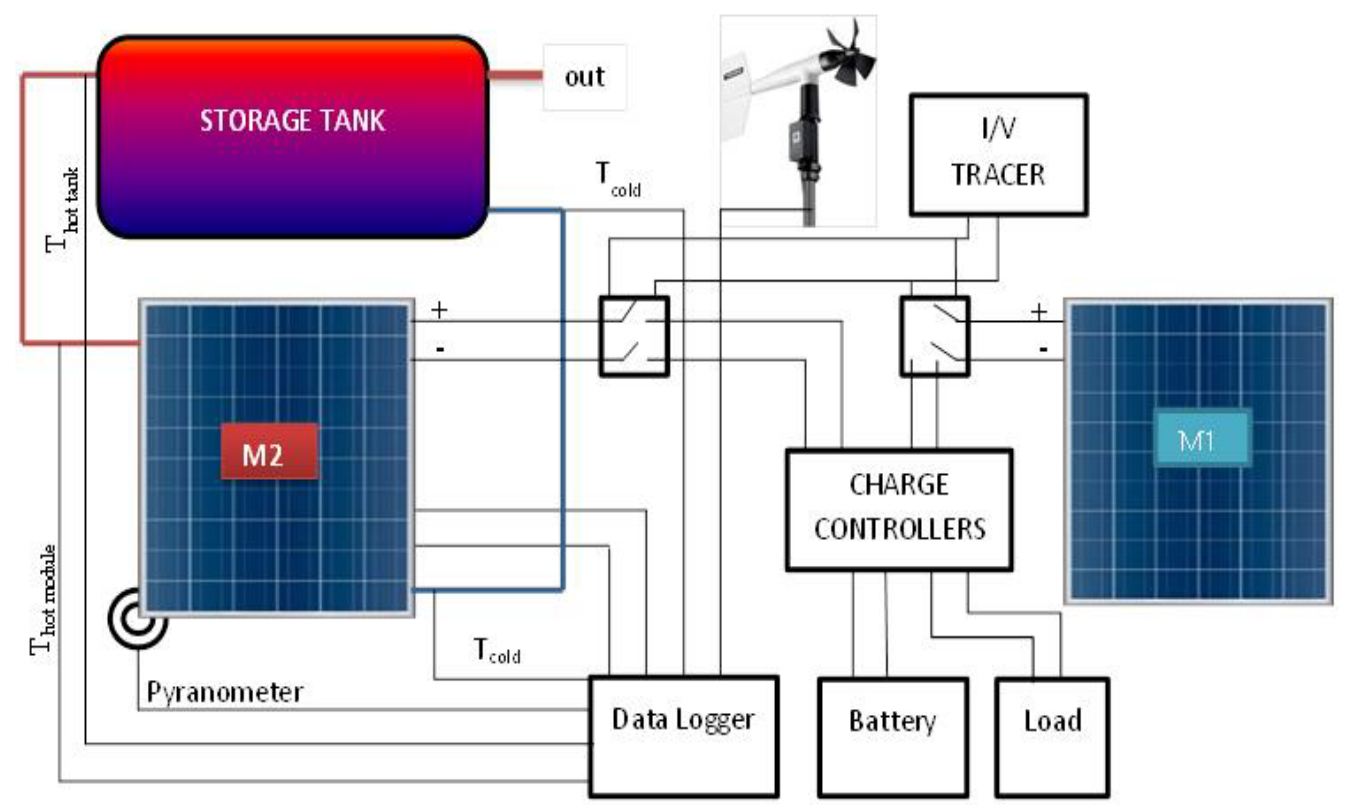

Figure 2- Schematic diagram of the PVT system with data acquisition system (Mtunzi, et al., 2012). 
The respective components were put together to build up the system.

\section{Materials and Procedure}

Two photovoltaic modules used in the study were SW80 Poly/RIA type. They are polycrystalline-si technologies with 36 cells connected in series. Their name plate ratings at standard test conditions (STC) $\left(1000 \mathrm{~W} / \mathrm{m}^{2}, 25^{\circ} \mathrm{C}\right.$ cell temperature and AM1.5 global spectrum) are as cited in Table 1 (SW80, 2009).

Table 1-The SW80 Poly/Ria corresponding STC and NOCT values

\begin{tabular}{|c|c|c|}
\hline $\begin{array}{l}\text { Performance under } \\
\text { standard test conditions (STC) }\end{array}$ & $\begin{array}{c}\text { STC } \\
\text { Rated }\end{array}$ & $\begin{array}{c}\text { Performance at } 800 \mathrm{~W} / \mathrm{m}^{2} \\
\text { NOCT Rated, AM } 1.5 \text { at } 45^{\circ} \mathrm{C}\end{array}$ \\
\hline STC Power Rating $\mathrm{P}_{\mathrm{mp}}(\mathrm{W})$ & 80.00 & 57.30 \\
\hline Open circuit voltage $\mathrm{V}_{\mathrm{oc}}(\mathrm{V})$ & 21.50 & 19.40 \\
\hline Short Circuit currency $\mathrm{I}_{\mathrm{sc}}(\mathrm{A})$ & 4.82 & 3.98 \\
\hline Peak Current $\mathrm{I}_{\max }(\mathrm{A})$ & 4.48 & 3.57 \\
\hline Peak Voltage $V_{\max }(\mathrm{V})$ & 17.90 & 16.10 \\
\hline Efficiency $\eta \%$ & 11.13 & 9.97 \\
\hline Aperture Area $\left(\mathrm{m}^{2}\right)$ & & $1.06 \times 0.68$ \\
\hline
\end{tabular}

A data-taker data logger (DT80) was used to log temperatures at the back of the modules, flow rate of water, wind speed and irradiance. Figure 4 shows the data logger used in the research.

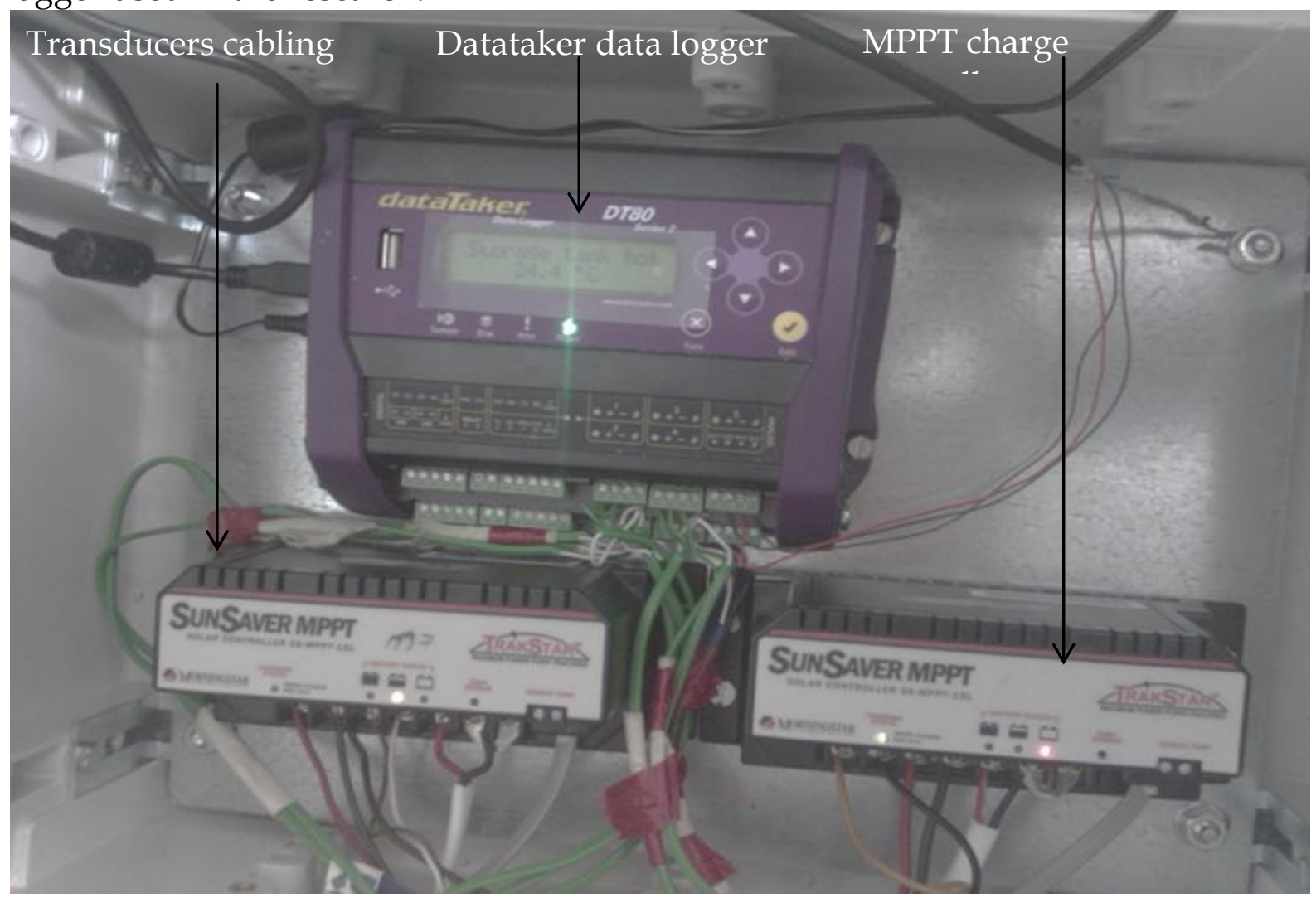

Figure 4 - Datataker datalogger DT80 and Maximum Power Point Tracking (MPPT) charge controllers. 
In the study, type $\mathrm{K}$ thermocouples were used for temperature measurements at the back of the modules and at the inlet and outlet of the box container as well as the inlet point to the storage tank.

The solar irradiance was measured in the plane of the module using a SOZ-03 reference cell (ISO9060), Figure 5 illustrates the crystalline reference cell (SOZ-03 , 2011).

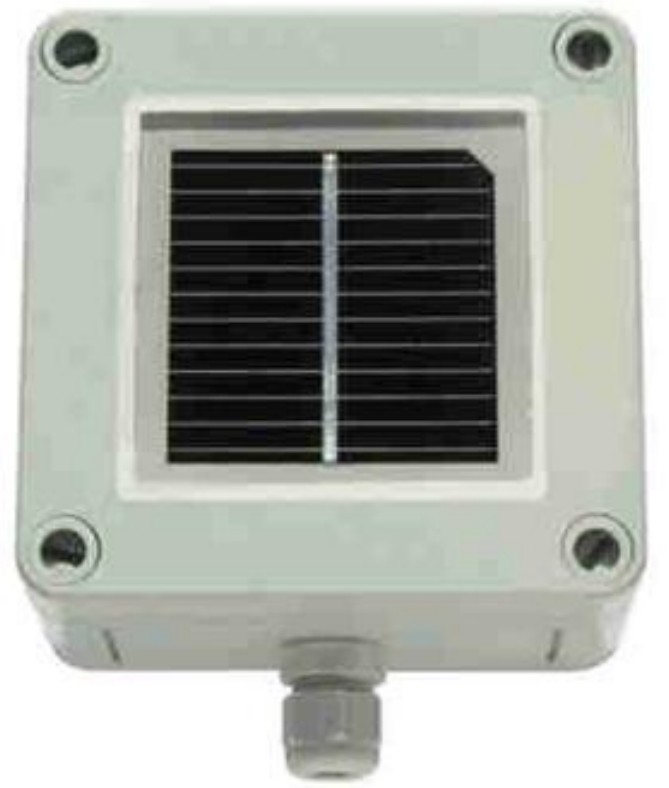

Figure 5- SOZ-03 Pyranometer

The SOZ-03 pyranometer consists of a mono-crystalline silicon cell $\left(50 \times 50 \mathrm{~mm}{ }^{2}\right)$ with special solar glass. The pyranometer is laminated, hence high UV-resistance and long-term stability. The SOZ-03 also consists of an optional integrated signal amplifier (output 0-10 V) and / or temperature sensor (Pt100 or Pt1000). It was used together with a PVPM system. The accuracy of the SOZ-03 is $\pm 5 \%$.

The PVPM is an instrument that was used for peak power measurements and current/voltage curve tracer measurements for the PV modules. The field tests through the use of a PVPM enabled the measurements of the I-V-curves of photovoltaic modules. The device could measure and calculate the peak power Ppk, the Rs and Rp resistances directly at the place of assembly of the PV system. After a measurement the data could be stored automatically in a non-volatile storage in the instrument, PVPM, 2010. The I/V measurements were taken at 10 minutes intervals each day. With I/V characteristics then the efficiency and power output of the module were determined. Figure 6 shows the picture of a PVPM 1000C40 instrument. 


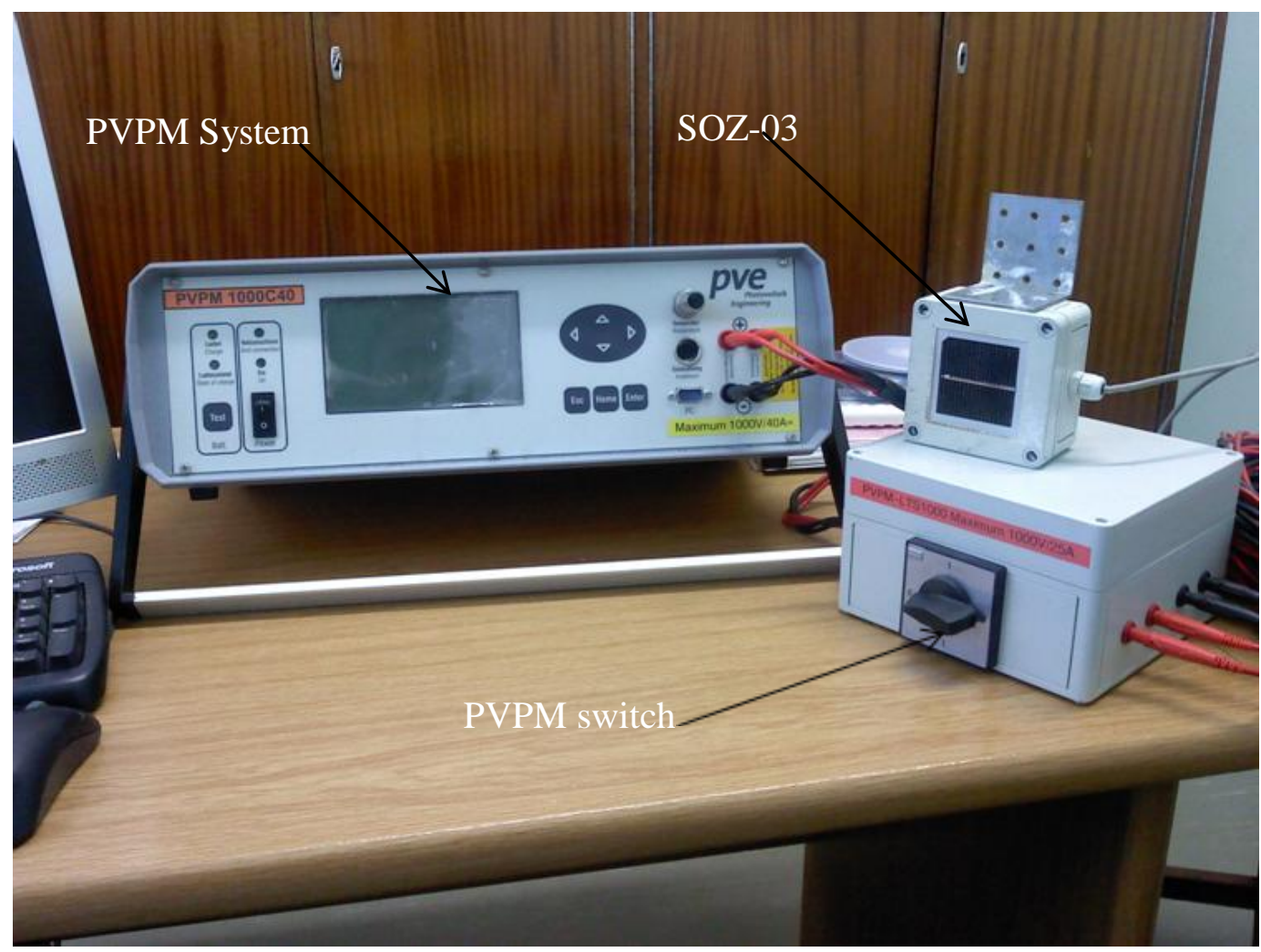

Figure 6 - The PVPM 1000C40 Instrument

The SOZ-03 measured irradiance and the PVPM used these values in converting the daily measured performance values to STC values. The peak power measurements of the PVPM have an accuracy of +/- 5\% (PVPM, 2010).

All instruments were connected as illustrated in the block diagram in figure 2 and measurements were then taken.

\section{Results and discussion}

\section{I-V Characteristics for Modules M1 and M2 (PV/T)}

The PV/T showed a broader distribution of degradation rates than module M1. This was attributed to the presence of water at the back of module M2 (the PV/T). Module M2 was found to be characterised by an initial higher maximum power as compared to moduleM1. For initial measurements taken at solar noon Module M1 was found to have an initial power output of $66.19 \mathrm{~W}$ while M2 had an initially maximum power output of $69.96 \mathrm{~W}$

The current voltage (I-V) measurements taken at the same time were as shown in figure 7. 


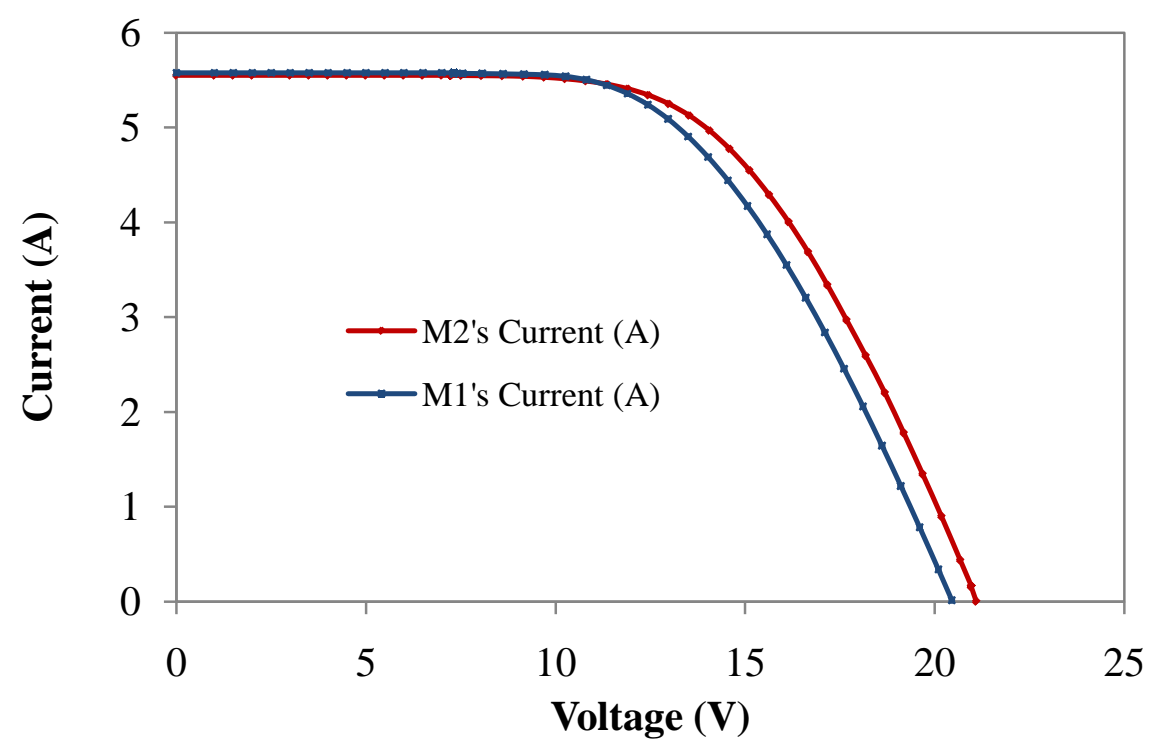

Figure 7- Initial I-V characteristics for modules M2 and M1

As shown on figure 7, the short circuit current generated by the two modules had a small difference, with M1 having a short circuit current of 5.58A and M2 giving $5.55 \mathrm{~A}$. A percentage difference of $0.54 \%$ between the two modules' current was noted. However the open circuit voltage of M2 was found to be $21.10 \mathrm{~V}$ while that of M1was 20.45V. The open circuit voltage for M2 was found to be higher by $3.1 \%$. Module M2 was also found to have a fill factor of $60 \%$ while M1 had a fill factor of $58 \%$ showing a better performance of M2 when compared to M1.

The two modules were continuously monitored every day and on the $7^{\text {th }}$ month from the installation day, the I-V characteristics of the modules were as illustrated in figure 8 .

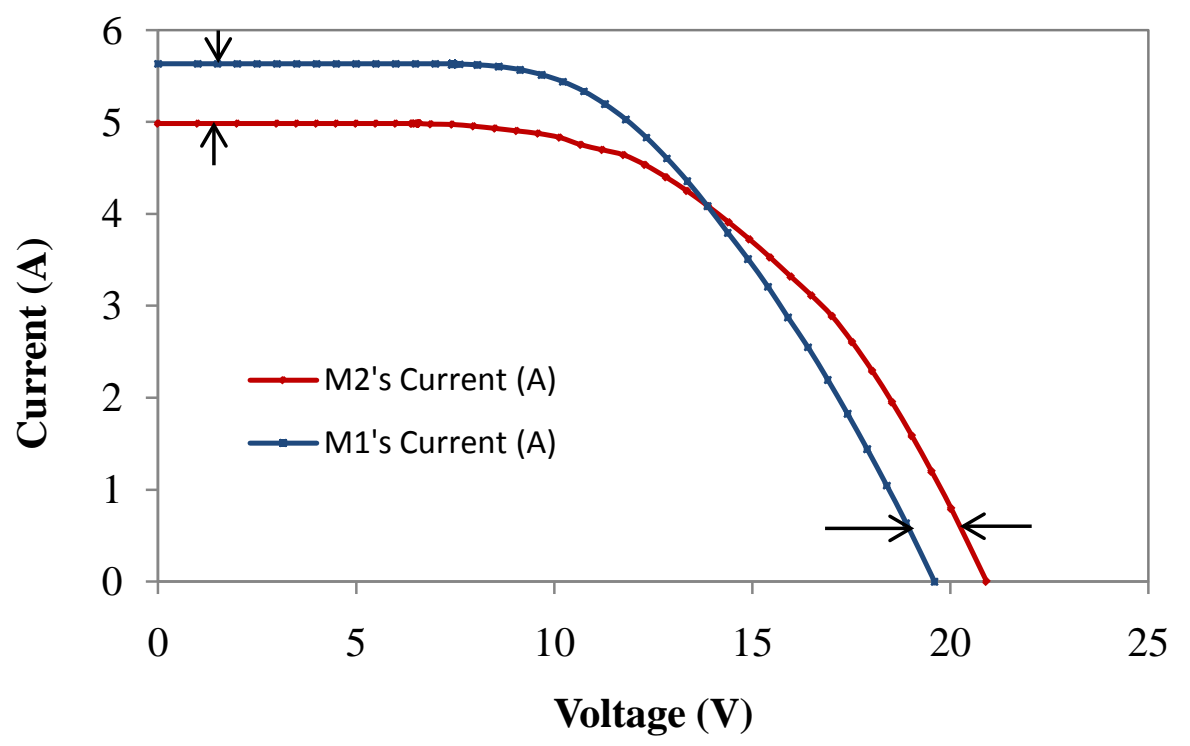

Figure 8- I-V characteristics for modules M2 and M1 taken at $12 \mathrm{~h} 10$

The measurements were also taken around the solar noon and the irradiance was found to be $1012 \mathrm{~W} / \mathrm{m}^{2}$. As shown in figure 8 , module M2 had a lower short circuit 
current $\left(I_{s c}\right)$ of $4.98 \mathrm{~A}$ as compared to M1's current of 5.63A. The fall in current on module M2 was due to degradation of the module. The module's degradation was likely brought about by its water absorption. A percentage drop of $11.55 \%$ in short circuit current by M2 was noted when the two modules were compared. The open circuit voltage of M2 was however found to maintain a higher value as compared to that of M1. Module M2 had an open circuit voltage of $20.9 \mathrm{~V}$ while that of M1 was 19.6V. M1's open circuit voltage was lower than that of M2 by $6.22 \%$ and this difference was attributed to water cooling effect on module M2's back of module when compared to M1. This difference in open circuit voltage was found to have a contributing factor on the modules' fill factors. Module M1's fill factor was found to be $53.94 \%$ while that of M2 was 54.46\%. Lower Fill factors were noted when these values were compared to the initial measurements. These changes were attributed to changes in the series and shunt resistances of the two modules.

As time went on, the performance of module M2 kept on dropping when compared to that of module M1. This continued performance drop contributed negatively towards the reliability of the M2 module. Figure 5 shows the changes that took place on the series and shunt resistances of the two modules. The changes in some of the module M2's electrical characteristics affected its series and shunt resistances, hence the drop in its electrical energy production. The changes in series and shunt resistances of the modules were as illustrated in Figure 9.

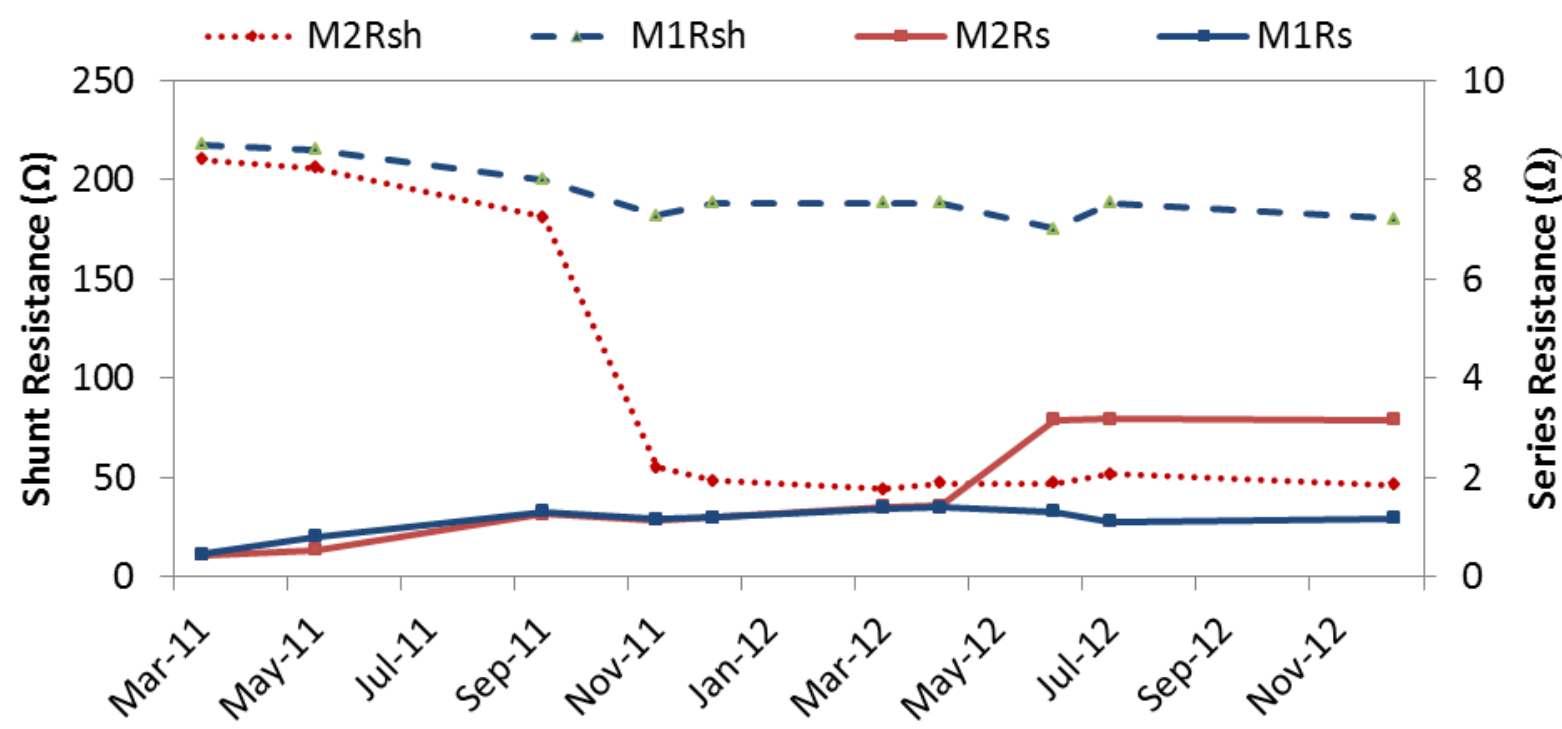

Figure 9- Series and shunt resistance variations of modules (M1) and (M2).

Figure 9 shows a $78 \%$ drop in shunt resistance for M2 as compared to $17 \%$ drop in shunt resistance of module M1. The sudden drop in power output by module M2 is therefore explained by these changes. A major drop in shunt resistance was noted towards the year end. The series resistance increased on both modules and this was assumed to be due to oxidation of the cells, hence the increase in series resistance. This sudden increased in series resistance was suspected to be due to more water absorption by the module as evidenced by the browning of cells shown in figure 10 . 


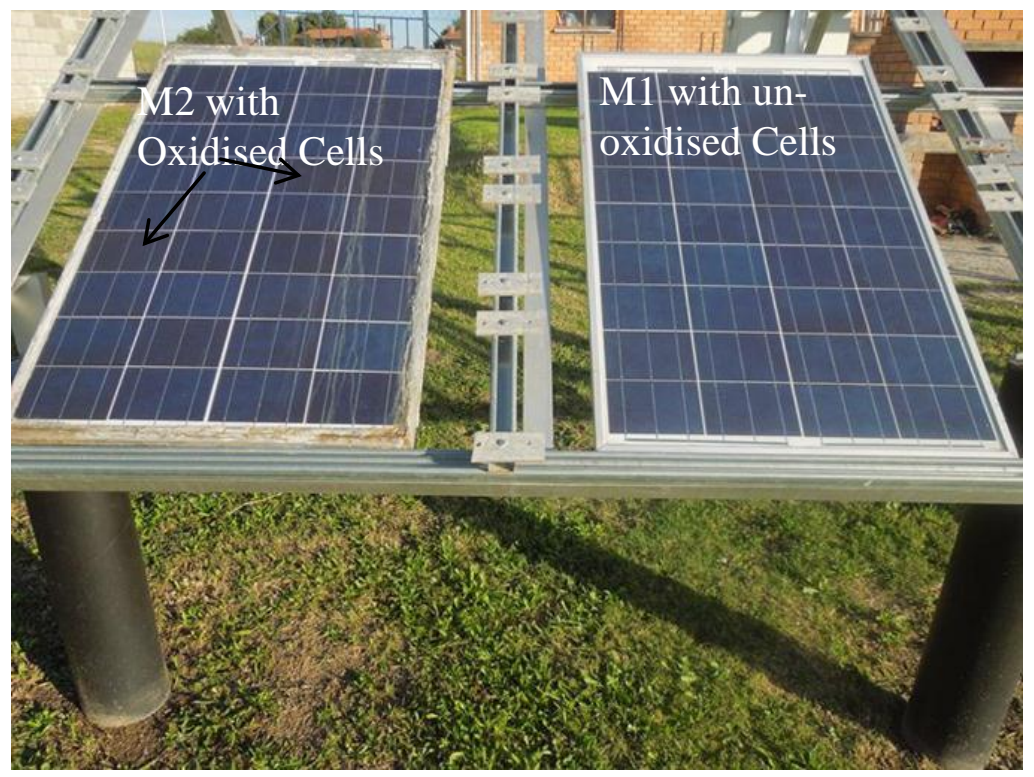

Figure 10- Showing oxidised cells on M2.

Not all cells were oxidised, but just a few on M2 as shown in figure 10. The oxidised cells contributed towards performance reduction in the module M2. The percentage increase in series resistance of the two modules when compared to measurements made in March 2011, were found to be $62 \%$ for M1 and $86 \%$ for M2. This shows the effect of water ingress on modules, hence a need to come up with a water tight system to avoid water ingress.

The I/V curve for module M1 in figure 11 was measured using the PVPM system (Kunz \& Wagner, 2004).

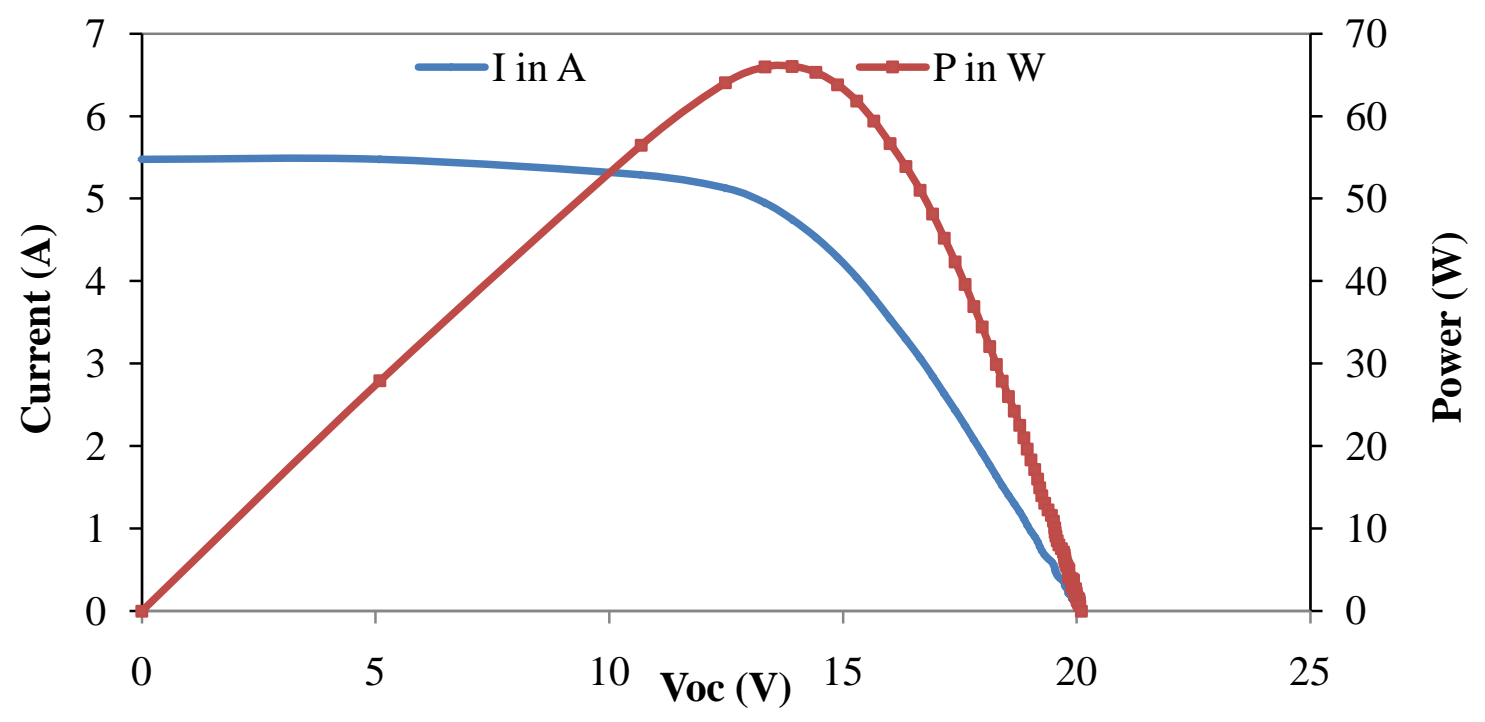

Figure 11- Year-end M1's I/V characteristic taken at 12:30pm

Figure 11 illustrates the measurements carried out towards year end. The corresponding series and shunt resistances of module M1 were found to be $1.18 \Omega$ and $180 \Omega$ respectively and a maximum short circuit current of 5.48A was noted. At the same time measurements were taken across module M2, and a short circuit current of 5.26A was noted as shown in figure 12. 


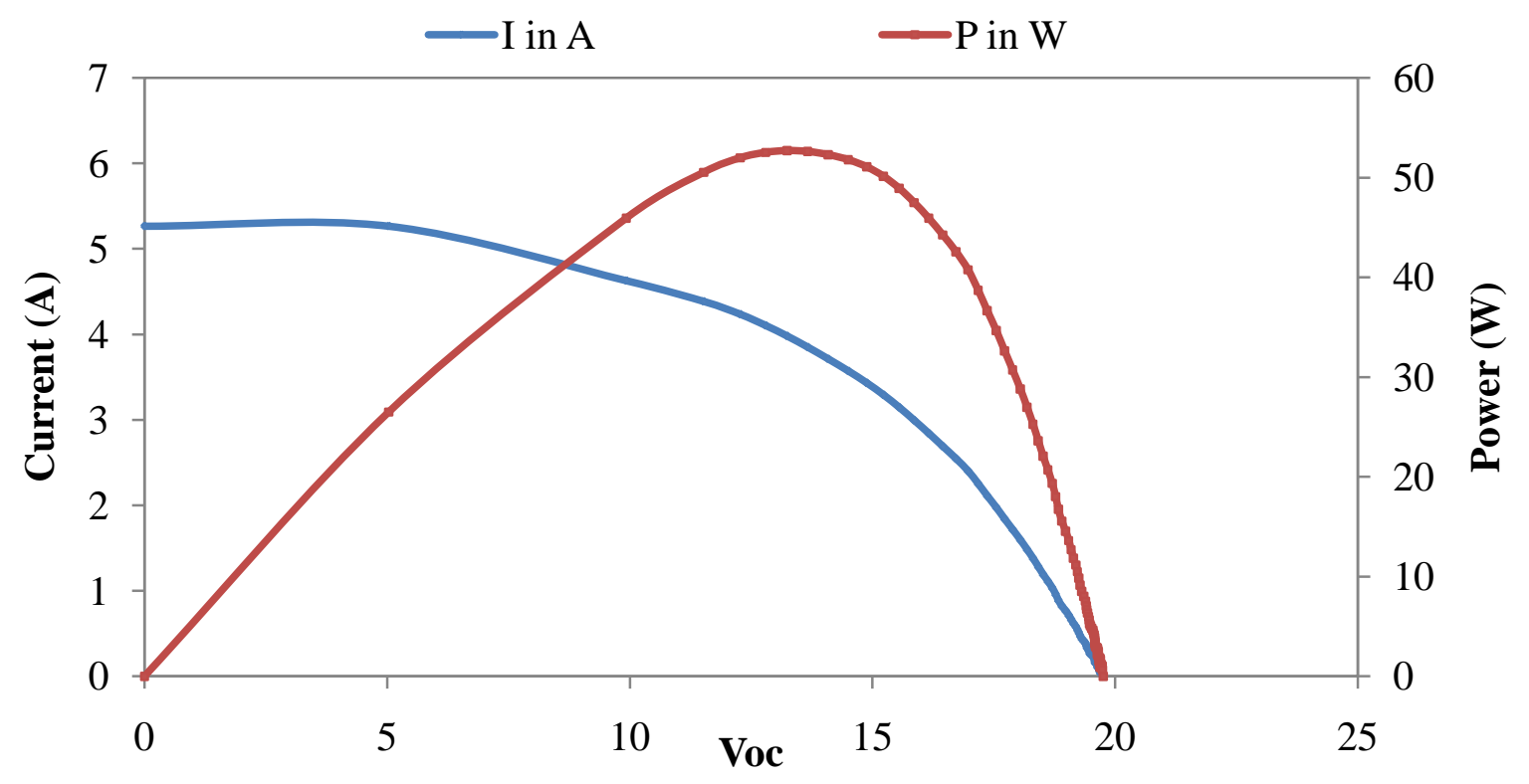

Figure 12- Year end's M2 I/V characteristic.

The series and shunt resistances of M2 were found to be $3.15 \Omega$ and $45.97 \Omega$ respectively. This confirmed the effects of water ingress on the module M2. The series resistance of M2 increased overall by $179 \%$ while its shunt resistance decreased further by $16.42 \%$.

To quantify power losses in the modules, the power degradation rates of the two PV systems were analysed using the performance ratios. The graphs shown in figure 13, shows the normalised graphs of the performance ratios of the two modules.

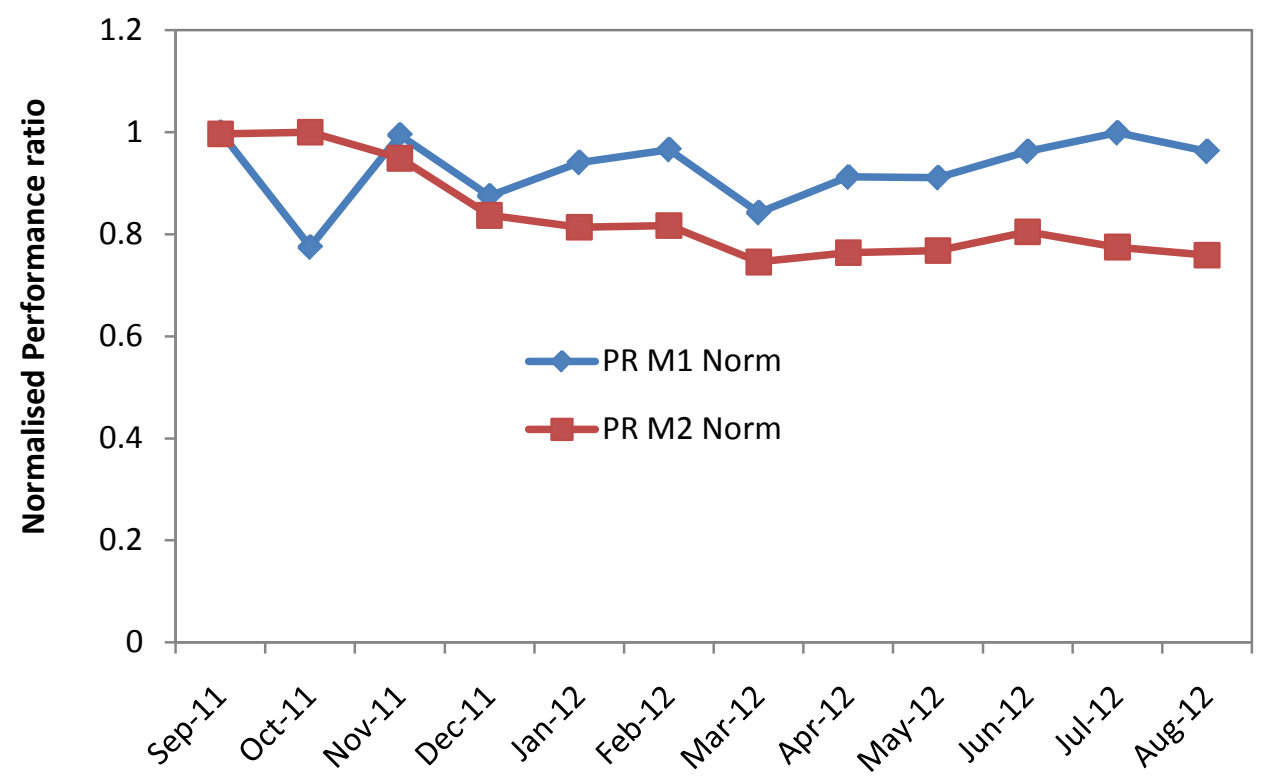

Figure 13- Normalised performance ratio of the two modules.

From figure 13, it can be seen that the performance ratio of the directly water cooled PV module M2 decreased from $100 \%$ to $76 \%$ while that of naturally cooled module decreased to $96 \%$ in a space of a year. The performance response shown in figure 13 
is in agreement with findings made by Kurtz (Kurtz, 2009). With these findings, it can be concluded that, though both electrical and thermal energy could be obtained from a directly water cooled module, methods of preventing water ingress in the directly water cooled module are needed.

\section{Conclusion}

The reliability of the directly cooled module was found to be compromised by water ingress. The electrical performance ratio of the directly cooled PV module dropped by $24 \%$, while that of a naturally cooled PV module dropped by $4 \%$. Water ingress on the directly water cooled module contributed towards increased series resistance and decreased shunt resistance. The overall series resistance of the directly water cooled PV module increased from $0.43 \Omega$ to $3.15 \Omega$, while its shunt resistance decreased from $210 \Omega$ to $45.97 \Omega$. To improve on the directly water cooled module's reliability, ways of preventing water ingress need to be accommodated or investigated.

\section{References}

Canadian Solar. (2017). Limited warranty statement Photovoltaic Module products. Retrieved from https://www.canadiansolar.com/downloads/warranties/v3.2/PV_Module_ Warranty_en-v3.2.pdf

Mtunzi, B., Meyer, E.L. Simon, M. \& Malape, M.A. (2012). Variations in the I/V characteristics of directly water cooled and naturally cooled PV modules.27th European Photovoltaic Solar Energy Conference and Exhibition. (pp 3992 3996). Frankfurt, Germany.

Kunz, G. \& Wagner, A. (2004, June 7-11). Internal series resistance determinated of only one $I-V$ curve under illumination. The $19^{\text {th }}$ European Photovoltaic Solar energy conference, Paris. France. Retrieved from https://www.interempresas.net/FeriaVirtual/Catalogos_y_documentos/803 19/Determinacion-de-la-Rs.pdf.

Kurtz,S. (2009). Reliabilty issues for photovoltaic modules. NREL/PR-520-46481. Retrieved From https://www.nrel.gov/docs/fy10osti/46481.pdf

PVPM. (2010). Operational user's manual. Retrieved from http://website2010.pvengineering.org/fileadmin/user_upload/download/ Operation_Manual/Manual_PVPM.pdf.

SOZ-03. (nd). Technical data. Retrieved from: http://www.nesdatalogger.de/produkte_soz03.htm

SW80, (2014). Sunmodule-SW-80-90-poly-RIA. Retrieved from: http://www.rectifier.co.za/wp-content/uploads/2014/05/Sunmodule-SW80-90-poly-RIA-en.pdf

Vazquez, M. \& Rey-Stolle, P. (2008). Photovoltaic module reliability model based on field degradation studies. Progress in photovoltaics: research and applications, Wiley 
interscience.

Retrieved

from

https://onlinelibrary.wiley.com/doi/abs/10.1002/pip.

Zielnik, A.K. (2015). White paper on Photovoltaic module weather durability and reliability testing. Solar energy competence center. Retrieved from https://www.atlasmts.com/-

/media/ametekatlas/files/applications/photovoltaics/atlas_wp104_atlas_25 plus_rev2_2015-05-12.pdf?la=en 


\title{
Analyzing the Performance of Identical PV Modules in a Semi-Arid Region over a 2-year Period
}

\author{
Arthur James Swart \\ Department of Electrical, Electronic and Computer Engineering, \\ Central University of Technology, \\ Private Bag X20539, Bloemfontein, South Africa, 9300
}

\begin{abstract}
It is assumed that similar PV modules are expected to perform equally well under the same atmospheric conditions, especially with regard to their rated output power. However, this is not always the case due to a number of factors, which include cell degradation overtime and cell manufacturing. It has been reported that PV modules within arrays need to be identical. This is required to have equal currents flow through the branches of an array in order to prevent power mismatches, hot spots and a lower overall output power. The purpose of this paper is to analyze the performance of three identical $10 \mathrm{~W}$ PV modules which were used over a 2-year period in a semi-arid region, in order to identify any anomalies. An experimental research design is employed where three identical $10 \mathrm{~W}$ PV modules were each connected to its own power loads that were to extract more than $90 \%$ of the rated power of the module over a 2-year period. A data logging circuit is included that provides power conditioning between the PV modules and an Arduino UNO board, which serves as the data logger. This logger relays the measured data to LabVIEW where the processing, display and recording is done. Two key anomalies that were identified relate to the abnormal degradation in output power of one of the modules and the abnormal improvement in output power when a newly manufactured module is introduced into the system.
\end{abstract}

Keywords: Arduino, LabVIEW, metrology, LED, degradation.

\section{Introduction}

"Time doesn't change, time reveals." This Arabian proverb well illustrates that the passing of time reveals all. In fact, a well-known English proverb says, "time will tell." Of course, time cannot talk, but instead reveals certain attitudes, outcomes and answers to specific questions. For example, research has shown that photovoltaic (PV) modules tend to degrade by approximately 1.2\% per year (Bartolo \& Yousif, 2017). This required time to prove. In fact, all research requires time to reveal the answers or solutions to research questions or problems. One problem associated 
with PV research is the lack of abundant empirical data regarding the practical application of PV modules in different atmospheric environments. In fact, the International Solar Energy Society has, as its purpose, the encouragement of basic and applied solar research, the promotion of science and technology relating to the application of solar energy and the compilation and dissemination of research relating to all aspects of solar energy (Cox, 2013).

Basic and applied PV research must continue, and be reported on, in an effort to contribute to the scientific body of knowledge which may lead to an improved understanding of PV technologies with the ultimate goal of improving the manufacturing process. This is what the majority of literature has focused on during the past decade with regard to PV research. The rationale has really been limited to two aspects, namely the optimization of Silicon and the discovery of new materials which may be used in the manufacturing process (Sun, 2013). However, this paper is limited only to the application of PV modules in a given atmospheric environment, so as to try to better understand its operation and performance.

Recent publications have focused on the performance of PV modules under uniform shading conditions (Arthur James Swart \& Pierre E. Hertzog, 2016), on the operation of LED's as viable power loads for PV modules (Swart \& Hertzog, 2017) and on the importance of the tilt angle for optimum performance of stationary PV modules (Swart, 2017). These publications fall under the basic and applied research category, which is required for producing explicit knowledge (Edwards, 2001). This knowledge may help to either develop new products or to improve the features of existing products in response to fluctuating markets and industry trends (Bosua \& Venkitachalam, 2013).

The purpose of this paper is to analyze the performance of three identical $10 \mathrm{~W} \mathrm{PV}$ modules in a semi-arid region over a 2-year period, using empirical data, in order to identify any anomalies that may contribute to a better understanding of these modules. The target location is in the middle of South Africa, which is in the Southern Hemisphere. This is a semi-arid region with day-time temperatures well beyond $40^{\circ} \mathrm{C}$ in the summer months. The paper firstly discusses PV cell manufacturing and energy provision. The practical setup and methodology is then presented. Descriptive results follow with succinct conclusions.

\section{PV cell manufacturing and characteristics}

The majority of PV cells are manufactured from wafers of crystallized Silicon or more recently, thin film amorphous Silicon (Holstein, 2015). The basic manufacturing process is shown in Figure 1, where the raw material Silicon is formed into a large ingot. These ingots are usually formed by cooling molten Silicon into a form (usually a cube), after which it is cut into substrates having a thickness of several hundred micrometers (usually called wafers). Three general methods are used to make these ingots; (1) pulling an ingot from a melt (e.g. using the Czochralski process); (2) solidifying a melt in a crucible by directional solidification techniques; or (3) pouring a melt from a crucible into a mold using casting 
techniques (Cortellini et al., 2014). The cutting of ingots is typically performed by a wire cutter and results in a rough surface on the silicon face (Lee \& Yeo, 2017). Metal contacts are added to enable electron flow. A glass substrate and protective film are added to prevent moisture infiltration. An aluminum frame is finally added to provide support that enables installation.

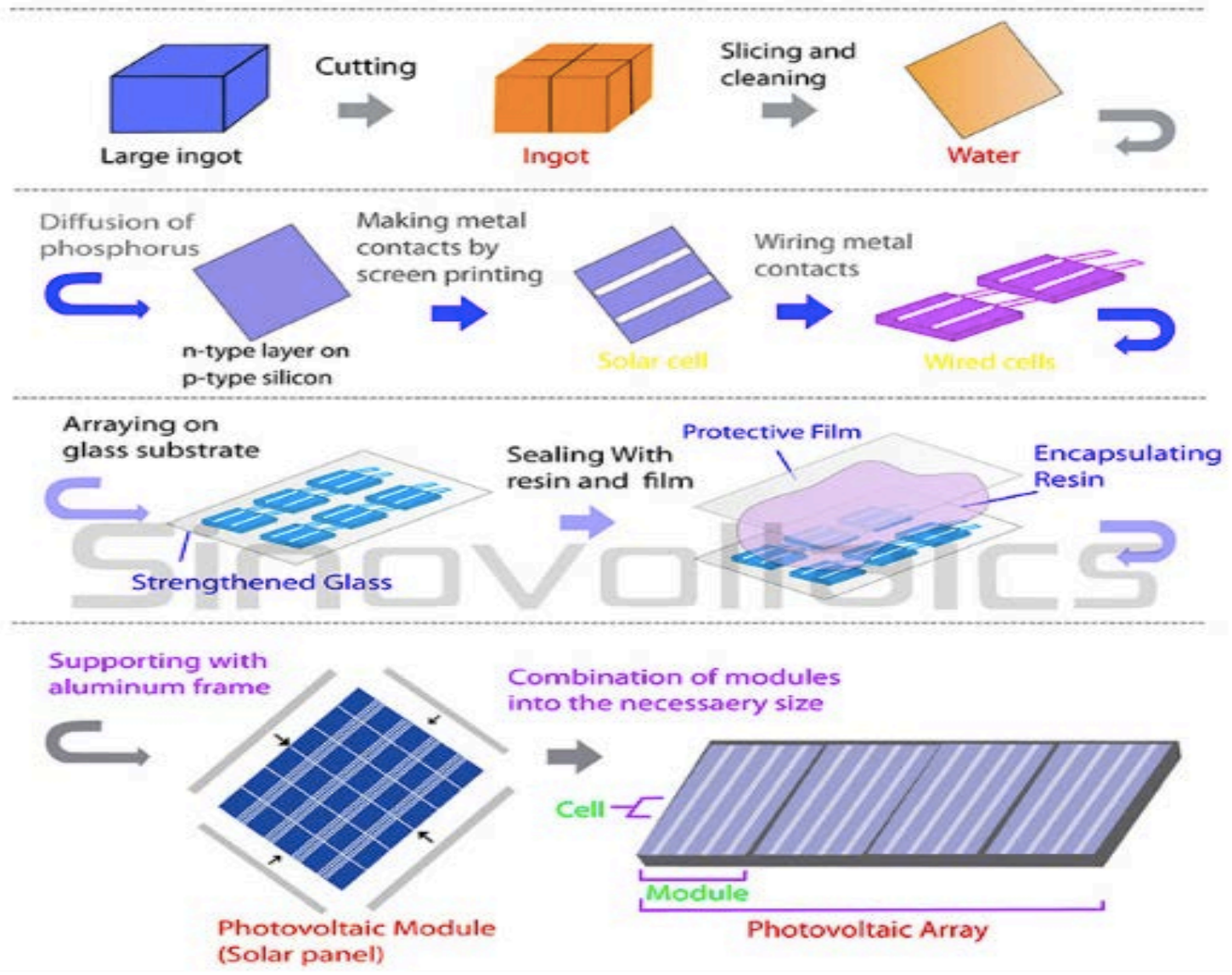

Figure 1 - PV Cell manufacturing process (SINOVoltaics.com, 2017)

Different manufacturing methods are used to achieve different efficiencies, which have an impact on the cost and size of the PV cell (Table 1 presents a concise summary of the major types used today). Mono-crystalline and poly-crystalline PV cells still dominate the market, primarily due to their higher efficiencies. After the initial manufacturing process, single cells are tested for their power output and binned with similar cells (Kim \& Krein, 2013). PV cell manufacturing is not an uniform process, with output cell power generally falling over a given distribution (Kim et al., 2015).

A key requirement for PV system, is that all PV cells, or modules, need to be roughly identical (Lorenzo et al., 2014). Mismatch power losses arise when PV cells or modules with different current-voltage characteristics are connected (Massi Pavan et al., 2014). Differences arise from the unavoidable fabrication spread or from nonuniform irradiance or working temperature within an array (Luque \& Hegedus, 2011). 
This fabrication spread arises in the manufacturing process, which produces PV cells with relatively large tolerances in their power output capability. Industry has been able to reduce these tolerances to around 3\% by using specific techniques (Ancuta \& Cepisca, 2011). However, notable differences still exist in the electrical characteristics of nominally identical PV cells or modules (Massi Pavan et al., 2014).

It is well documented that PV cells degrade from extreme temperatures, UV exposure, and mechanical damage. Long-term UV exposure or cycling temperatures can lead to the internal resistance of the cell increasing due to infiltration of contaminants, such as moisture. Elevated temperatures can also lead to a decrease in shunt resistance, or resistance of the path to ground, when metal ions migrate through the cell. Furthermore, the module's anti-reflective coating (protective film) can deteriorate due to heat, UV exposure, and exposure to contaminants. Degradation resulting from mechanical damage typically results from poorly installed PV cells that are stressed by wind loads or are in a place where objects are contacting them (Bartolo \& Yousif, 2017). All of these factors can lead to a PV cell degrading, resulting in decreased power output.

Table 1 - PV Module parameters (adapted from (Holstein, 2015))

\begin{tabular}{|c|c|c|c|c|}
\hline PV Cell & Type & $\begin{array}{c}\text { R\&D } \\
\text { Efficiency }\end{array}$ & $\begin{array}{l}\text { Commercial } \\
\text { Efficiency }\end{array}$ & $\begin{array}{c}\text { Market } \\
\text { Share }\end{array}$ \\
\hline sc-SI & $\begin{array}{l}\text { Silicon mono-crystalline } \\
\text { wafer }\end{array}$ & $25 \%$ & $13-20 \%$ & $42.2 \%$ \\
\hline mc-SI & $\begin{array}{l}\text { Silicon multi-crystalline } \\
\text { / polycrystalline wafer }\end{array}$ & $20.3 \%$ & $11-15 \%$ & $45.2 \%$ \\
\hline ribbon-SI & $\begin{array}{l}\text { Silicon ribbon multi- } \\
\text { crystalline wafer }\end{array}$ & & $11 \%$ & $2.2 \%$ \\
\hline Heterojunction c-SI & $\begin{array}{l}\text { Crystalline silicon wafer } \\
\text { layered by a-SI }\end{array}$ & $23 \%$ & $18+\%$ & $5.2 \%$ \\
\hline a-SI & $\begin{array}{l}\text { Thin film, amorphous } \\
\text { silicon }\end{array}$ & $10.1 \%$ & $6-9 \%$ & $4.2 \%$ \\
\hline Multi-junction a-SI & $\begin{array}{l}\text { Thin film, a-SI with } \\
\text { alloy materials }\end{array}$ & $12-13 \%$ & & \\
\hline $\mathrm{CdTe}$ & $\begin{array}{l}\text { Thin film, cadmium } \\
\text { telluride }\end{array}$ & $16.5-16.7 \%$ & $10-11 \%$ & \\
\hline CIS & $\begin{array}{l}\text { Thin film, copper } \\
\text { indium disulfide }\end{array}$ & & $8.6-10 \%$ & \\
\hline CIGS & $\begin{array}{l}\text { Thin film, copper } \\
\text { indium gallium } \\
\text { disulfide/diselenide }\end{array}$ & $19.9-20.1 \%$ & $13.1 \%$ & \\
\hline
\end{tabular}


Each individual PV cell has an internal resistance that depends on various parameters, which include cell's physical structure, metal connectors and overall construction. As the PV cells degrade, this internal resistance usually increases, with increased thermal loss being shown (Adcock, 2016). This may further cause less current to flow, impacting on the overall output power of the cell. This effect can be magnified if multiple PV modules, or cells, are wired in series because, similar to shading effects, if one module has a higher degradation rate than other modules in the same string, then it will affect the entire string (Poissant, 2017).

Hot spots generally occur when a large number of series connected PV cells are dissipating power in a shaded cell. This results in the individual PV cells, or modules, being forced to operate at a power level other than their own, which leads to losses in overall output power (Larsen \& Lindquist, 2014). These hot spots are problematic in PV systems that further accelerate cell degradation and lower system performance (Kim \& Krein, 2013). They mainly occur during the day when ambient temperatures are above the STC level when the maximum power current is being drawn.

PV modules convert a certain amount of solar radiation into electrical energy, while the rest is converted into heat energy, leading to a significant rise in a PV module's temperature. This elevated temperature deteriorates the power output and induces structural degradation, resulting in a reduced PV lifespan. It has been shown that PV cells degrade faster in hot climates due to long-term thermal ageing caused by their elevated operating temperatures (Kurtz et al., 2011). Additionally, PV modules lose structural integrity due to delamination that is caused by prolonged operation under elevated temperatures (Saly et al., 2001). By studying the physical mechanisms by which PV cells degrade, design modifications can be implemented to create longer lasting PV modules (Mathews et al., 2014).

Multiple PV cells are generally interconnected to increase current flow and output voltage in a singular PV module. Similarly, multiple PV modules are interconnected to increase the overall output power from a PV array. These types of arrays are often installed on the roofs of residential homes, giving rise to the term PV roofinstallation. These installations have flourished over the past few years (Swart, 2016), as many seek to minimize their dependence on the national gird, by-pass the effects of load shedding and reduce their electrical bills.

\section{Provision of electrical energy}

The national grid has continued to grow globally over the past few decades, with more and more communities being afforded the privilege of having electricity. For example, since 1991, ESKOM (national energy utility in South Africa) has connected over 4.2 million homes to the national grid, largely on a prepaid metering system, and it has provided non-grid access to 38,000 others (Albert et al., 2014). This nongrid access would primarily occur by using renewable energy systems, such as PV systems and wind turbine systems. However, connection to the national grid has 
two major disadvantages, that being load shedding and increased electrical energy costs.

Load shedding is defined as the set of controls, which results in a decrease of load demand in the power system in order to achieve a new equilibrium state (Reddy, 2017). Load shedding occurred regularly between 2013 and 2015 in South Africa, with many parts of the country experiencing no power for up to 2 hours per day. This enabled the system to achieve equilibrium, as specific neighborhoods where deliberately disconnected from the national grid while other remained connected. This created more problems for consumers, especially in terms of small and medium enterprises that were dependent on electrical energy to maintain their cold storage and power their electronic registers. In fact, load-shedding has caused significant damage to national economies of many countries, and even the closure of some industries in Pakistan, resulting in loss of production and jobs (Mirza et al., 2015). A similar detrimental result of load shedding has been observed in South Africa.

Electrical costs have continued to increase worldwide. For example, energy costs have risen steadily for the last 12 years in the United States, and will continue to do so for the next 20-25 years (Deb, 2014). This has also been experienced in South Africa. For example, the National Energy Regulator of South Africa (NERSA) has, since 2008, granted ESKOM an annual average increase of about $22 \%$ per annum in tariffs (Parsons et al., 2015). Recently, NERSA approved an average price increase of approximately 13\% for the year 2015-2016 (Gupta \& Inglesi-Lotz, 2016), with more increases planned for the future.

To negate the effects of load shedding and electrical energy cost increases, small and medium enterprises and residential homeowners need to look to renewable energy sources, including micro-solar $(10-10 \mathrm{~kW})$ and pico-solar $(<10 \mathrm{~W})$ off-gird PV systems. Obtaining a deeper understanding of how PV modules in these systems perform within various atmospheric environments may help to further understand its performance and promote its adoption.

\section{Practical setup}

The practical setup consists of three identical PV systems comprising $10 \mathrm{~W}$ polycrystalline PV modules (short circuit current of $0.78 \mathrm{~A}$ and an open circuit voltage of $20.8 \mathrm{~V}$ ), a data logging interface circuit, an Arduino UNO board and LabVIEW software (see Figure 2). This may be classified as a pico-solar system, as the output power is lower than $10 \mathrm{~W}$ (Cuciureanu et al., 2016). Three different power loads (resistor, LED lamp and solar controller) where connected to the modules for a period of 15 months (starting 17 Feb 2017), in order to validate the use of a regulated LED lamp as a viable power load for such modules (A.J. Swart \& P.E. Hertzog, 2016). The resistor and LED lamps were swapped around on 8 May 2017, in order to validate the performance of the power loads and not the performance of the PV module. The solar controller was consistently connected to PV3 for the entire time period (17 February 2017 to 21 August 2017). On 22 August 2017, all three PV modules were connected to identical LED lamps, in order to validate any significant 
differences between the modules which may have arisen over the previous 18 months (the initial PV modules used in this research are identical and were purchased from one batch and manufacturer in 2014).

Previous research has shown that a solar controller with its battery and associated load outperformed a LED lamp during the summer season for the same research site (A.J. Swart \& P.E. Hertzog, 2016). In fact, the solar controller extracted 3.9\% more energy from the PV module as compared to the LED lamp. A second set of confirmatory results further established the reliability and validity of the electronic measuring process and methodology (Swart \& Hertzog, 2017). The results indicated, that during the winter season, the regulated LED lamps outperformed the solar controller by extracting, on average, $1.5 \%$ more power from its PV module. During this time of the research, all the PV modules were producing more than $90 \%$ of their rated output power, depending on the load condition which was being used.

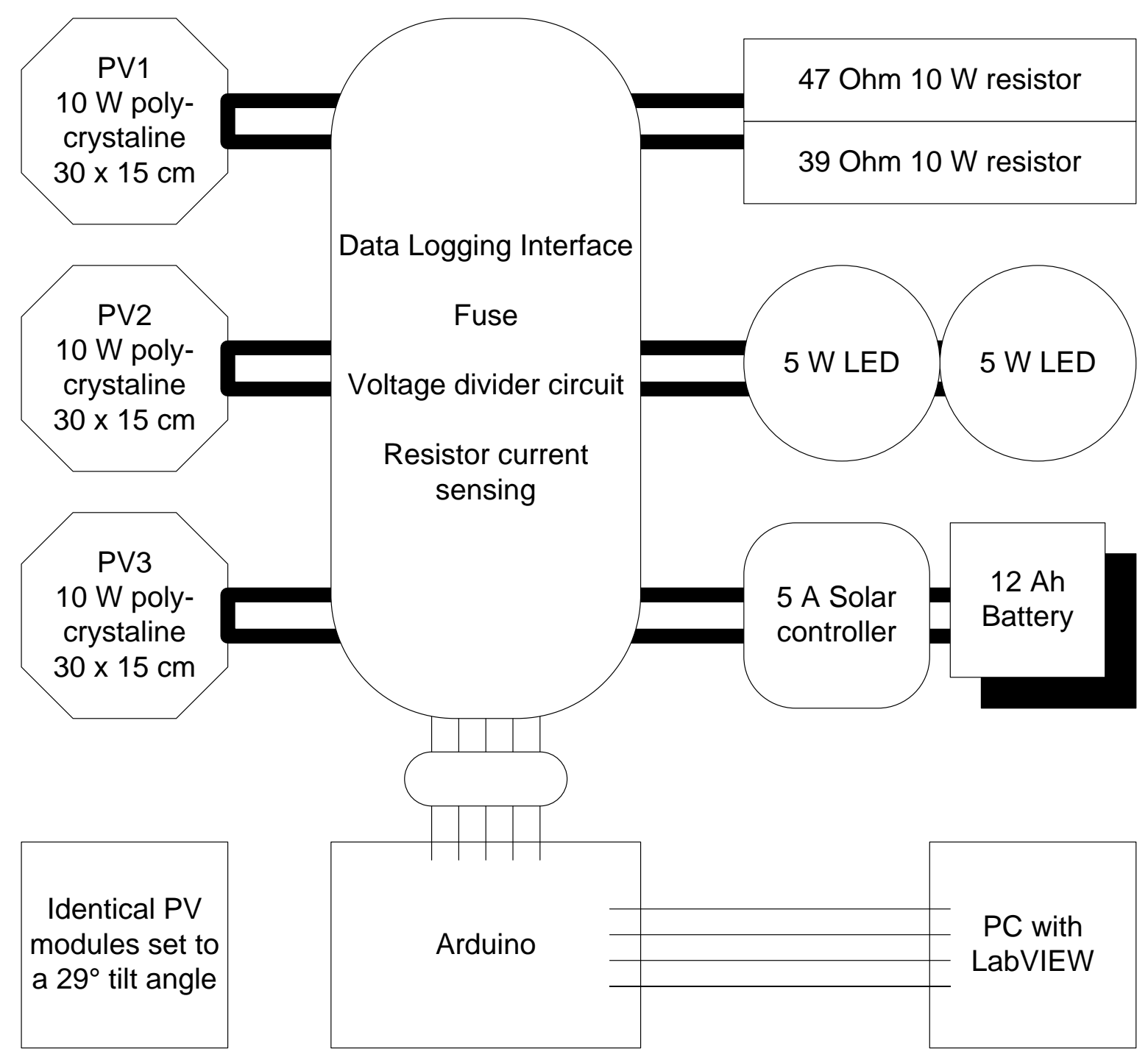

Figure 2 - Block diagram of the practical setup for the time period from 17 February 2017 to 21 August 2017 
Each load was connected in series with a $6 \mathrm{Ohm} 10 \mathrm{~W}$ series resistor for current sensing measurements. A simple voltage divider circuit was used to ensure that the input voltage to the Arduino UNO board would never exceed $5 \mathrm{~V}$. The three PV modules were mounted onto an aluminium frame and set to the same tilt angle of $29^{\circ}$ (also same orientation angle of $0^{\circ} \mathrm{N}$ ), equating to the latitude value of $29^{\circ}$ for the Central University of Technology in the Free State province of South Africa (Hertzog \& Swart, 2015a, 2015b). This area is known as a semi-arid part of South Africa, where around 55\% of its annual rainfall occurs between January and April (Snyman et al., 1987), with very little rainfall occurring between May and August. The output power of these PV modules was recorded and analyzed using LabVIEW software in conjunction with an Arduino UNO board. The reliability and validity of the electronic measurements where obtained by first calibrating the three PV systems (28 October 2015 - 16 February 2016). This process was discussed by Swart and Hertzog (A.J. Swart \& P.E. Hertzog, 2016). The research methodology is presented next.

\section{Research methodology}

An experimental research design was used. The system was initially set up and calibrated over a three-month period (28 October 2015 - 16 February 2016). The three identical PV modules (from the same batch and same manufacturer) were set to the same tilt angle of $29^{\circ}$. Different unique power loads were then connected on 17 February 2017, resulting in only one different variable between the three systems. All other variables were kept constant for the three systems (environmental, orientation, tilt, etc.). However, it is important to note that the PV modules were first installed in October 2015, resulting in a total of two years of experimental (empirical) data.

Voltage readings were obtained by using a standard voltage divider circuit $(147 \mathrm{k} \Omega$ resistor in series with a $100 \mathrm{k} \Omega$ resistor). The obtained values are multiplied by a predetermined factor for calibration and to compensate for any interface losses, being displayed on the front panel of the LabVIEW software that is visible to the user. This value is then filtered by a Butterworth Filter to reduce high frequency components or noise that may originate from the Arduino's analog read circuit or from the data logging interface. The user interface is shown in Figure 3. 


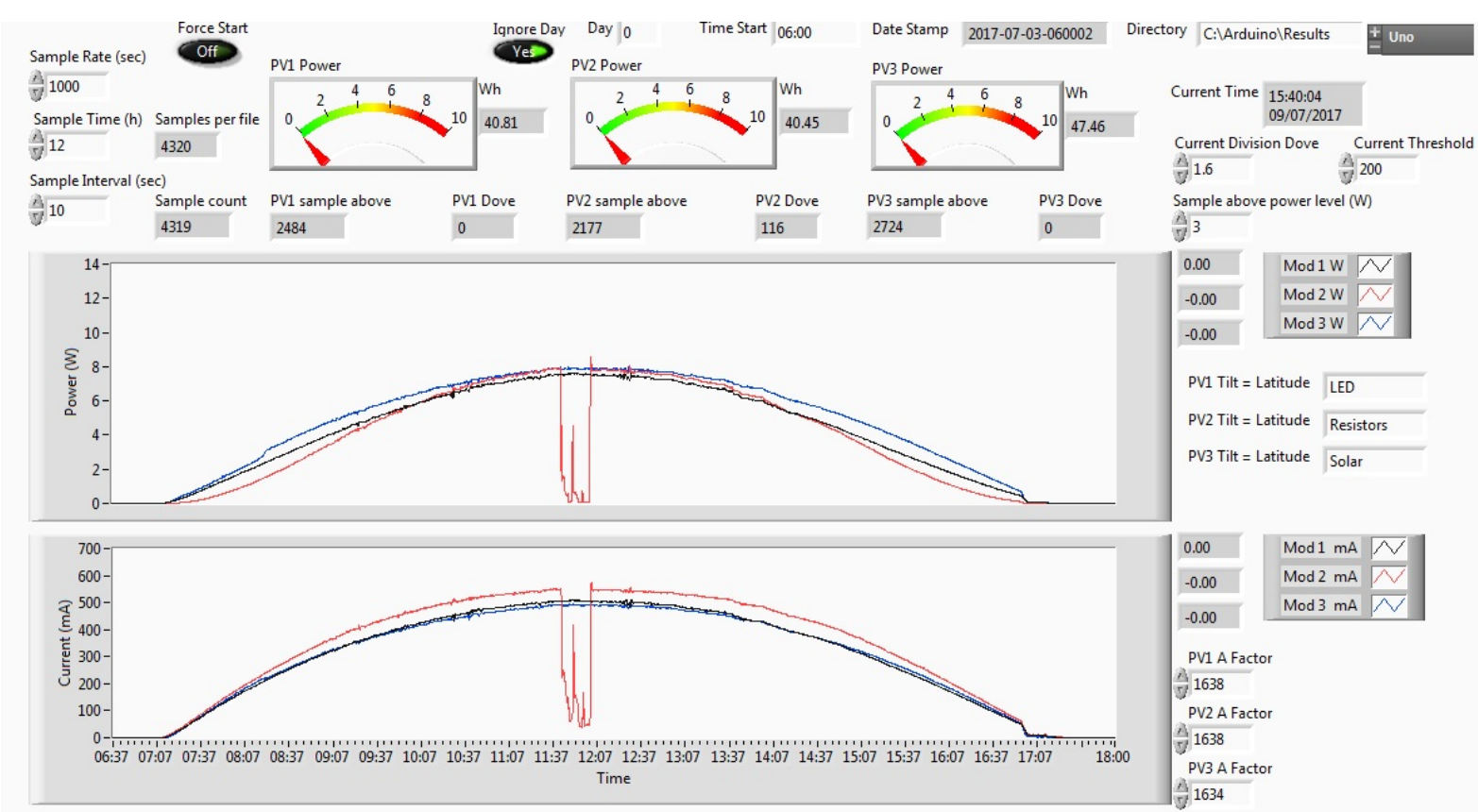

Figure 3 - Screen shot of the LabVIEW interface showing the performance of the three PV modules for 3 July 2017

Current measurements are obtained by measuring the voltage across a low value high power precision resistor (6 Ohm $10 \mathrm{~W} 1 \%$ ). Multiplying the voltage and current readings within LabVIEW yields power readings (in Watts) that are written to a matrix for recording purposes. The instantaneous readings per sample are also shown on the user interface, as shown in Figure 3 (data represents 3 July 2017, where the three power curves of the different load profiles seem to be very similar - far right-hand circle shows which loads were connected to which PV modules). The total amount of power extracted per day for each PV system with its own unique power load is then written to a singular text file for further analysis (Wh value next to the power scale showing the numerical values of $0-10$ ). The results from this text file is then analysed for the 2-year period. It contains the date stamp, power count above $3 \mathrm{~W}$ (number of 10 second intervals per day in which the PV module provides more than $3 \mathrm{~W}$ to its associated power load), maximum $\mathrm{Wh}$ values per day and maximum PV surface temperature per day.

\section{Results and discussions}

The analysis of the recorded data is shown in Figures 4 through 7. Figure 4 highlights the maximum recorded surface temperature (which came from PV3) of the three PV modules for the 2-year period. This suggest that the three modules were exposed to high temperatures, as PV3 regularly reached the $50^{\circ} \mathrm{C}$ mark for the summer season (November through April of 2016 and 2017). This would cause a faster degradation of the PV cells as compared to cooler atmospheric environments. 


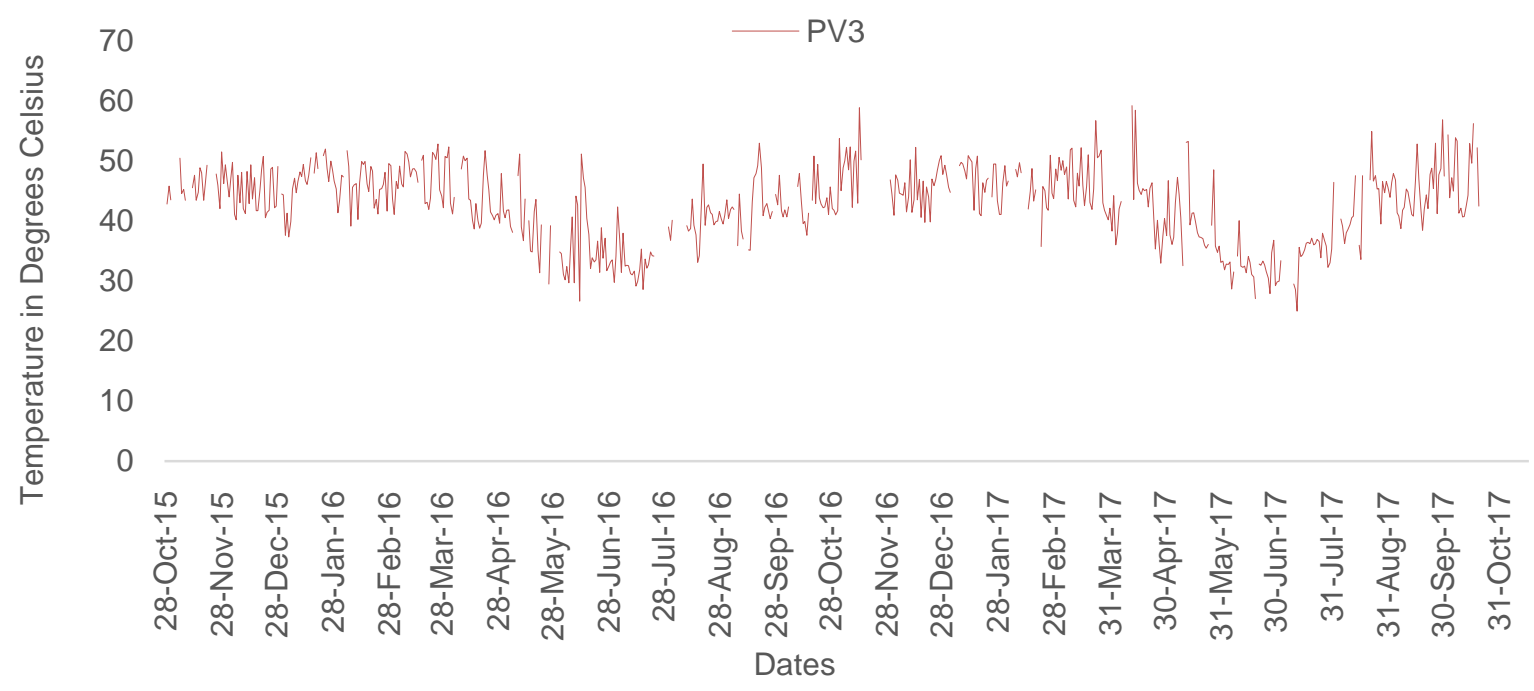

Figure 4 - PV3 surface temperatures for a two-year period

The power difference between the three PV modules is shown in Figure 5, where PV1 is contrasted to PV2 and PV3 is contrasted to PV2. The period from 28 October 2015 to 17 February 2016 was used for setup and calibration purposes, which was detailed by Swart and Hertzog (A.J. Swart \& P.E. Hertzog, 2016). The major portion of Figure 5 (17 February 2016 through 8 May 2017) indicates that PV1 (connected to the load resistor) produced, on average, $6 \mathrm{~W}$ less than PV2 (connected to the LED lamp), while PV3 (connected to the solar controller) produced, on average, $1 \mathrm{~W}$ more than PV2 (connected to the LED lamp). Results remain fairly consistent for this major portion of the figure. PV3 (solar controller) does extract more energy than PV2 (LED lamp) for February through April (summer seasons for 2016 and 2017), but PV2 (LED lamp) extracts more energy than PV3 (solar controller) for May through July of 2016 (winter season). PV1 (load resistor) consistently extracts less energy than PV2, and subsequently than PV3. Its profile would typically fall in between the dotted black line and the solid red line shown in Figure 5.

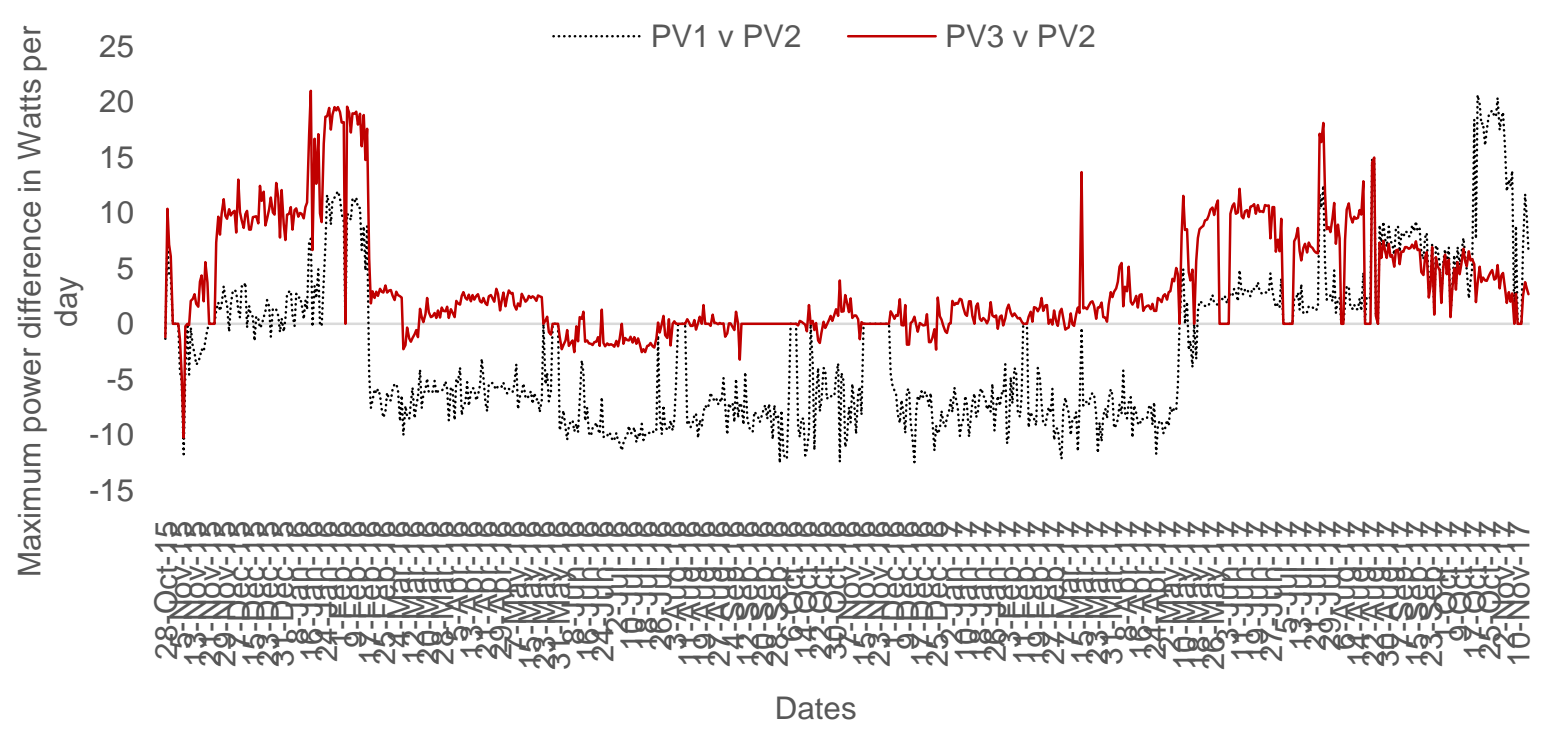

Figure 5 - Power difference (shown in Watts) between PV1 and PV2, and between PV3 and $P V 2$, for a two-year period 
This suggests that a solar controller and LED lamp respond equally well as load resistors for pico-solar systems, with variations between summer (solar 3.9\% more than the LED) and winter seasons (LED 1.5\% more than the solar). This confirms previously published work in this regard (Swart \& Hertzog, 2017). However, this is not observed from 8 May 2017 onwards, as PV1 has now been connected to the LED lamp and PV2 to the resistor load (see Figure 3 for the load profile connections on the right-hand side). PV1 (connected to the LED lamp) now extracts more energy than compared to PV2 (connected to the load resistor) while PV3 (still connected to the solar controller) rises to around $5 \mathrm{~W}$ more than PV2 (load resistor). Then on 22 August 2017, all three PV modules are connected to identical LED lamps. PV1 and PV3 now follow a similar curve.

However, it is expected that PV2 should follow this same curve, as it now has the same load profile. In other words, the two curves shown in Fig. 5 (differences between PV1 and PV2 and between PV3 and PV2) should ideally be around the 0value mark (no perceived differences should exist as they have the same load profile). This does not materialize, indicating that PV2 has significantly reduced in performance as compared to PV1 and PV3. Recall that these three modules had consistently been working since 28 October 2015, which seems to indicate that PV2 has degraded more significantly that PV1 and PV3. These modules would have been exposed to both high temperatures and many dust storms during the 2-year period. Instead of replacing PV2, it was decided to replace PV1 and compare its new performance to that of PV3, as they had followed a parallel power curve since 28 October 2015.

PV1 was replaced with a brand-new PV module that was manufactured in 2017. It had never been used before. Immediately, a major difference occurred, as seen from 17 October 2017 onwards. PV1 (a brand-new PV module manufactured early in 2017) now produced approximately $15 \mathrm{~W}$ more than PV3 (well-used 2-year old PV module that had reached $50^{\circ} \mathrm{C}$ on a regular basis). However, the graph tends to suggest that this major difference is reducing with time. Further empirical results will confirm this.

Figure 6 (a) further validates that the solar controller (connected to PV3 representing the outer circle) generally extracted more electrical energy than the LED lamp (connected to PV1) and the load resistor (connected to PV2), when considering the power level count. The LabVIEW system was designed to count the number of 10 seconds intervals in which the PV modules produced more than $3 \mathrm{~W}$. The median (2228), the average (1953) and the maximum (3199) counts for PV3 are more than that of PV1 and PV2. This indicates that PV3 was the first to reach the $3 \mathrm{~W}$ mark early in the morning, producing $3 \mathrm{~W}$ for a longer period of time than compared to PV1 and PV2.

Figure 6 (b) shows the total Watts produced per day for the three PV modules. PV3 produced the highest median $(53 \mathrm{~W})$ and average value $(48 \mathrm{~W})$ for output power 
over the 2-year period. However, the maximum power produced for one day was achieved by PV1 $(82 \mathrm{~W})$ when it was replaced with a newly manufactured PV module in 2017. This confirms that both consistent use and high temperatures do degrade PV cells, as the newly installed PV module outperformed PV2 and PV3.
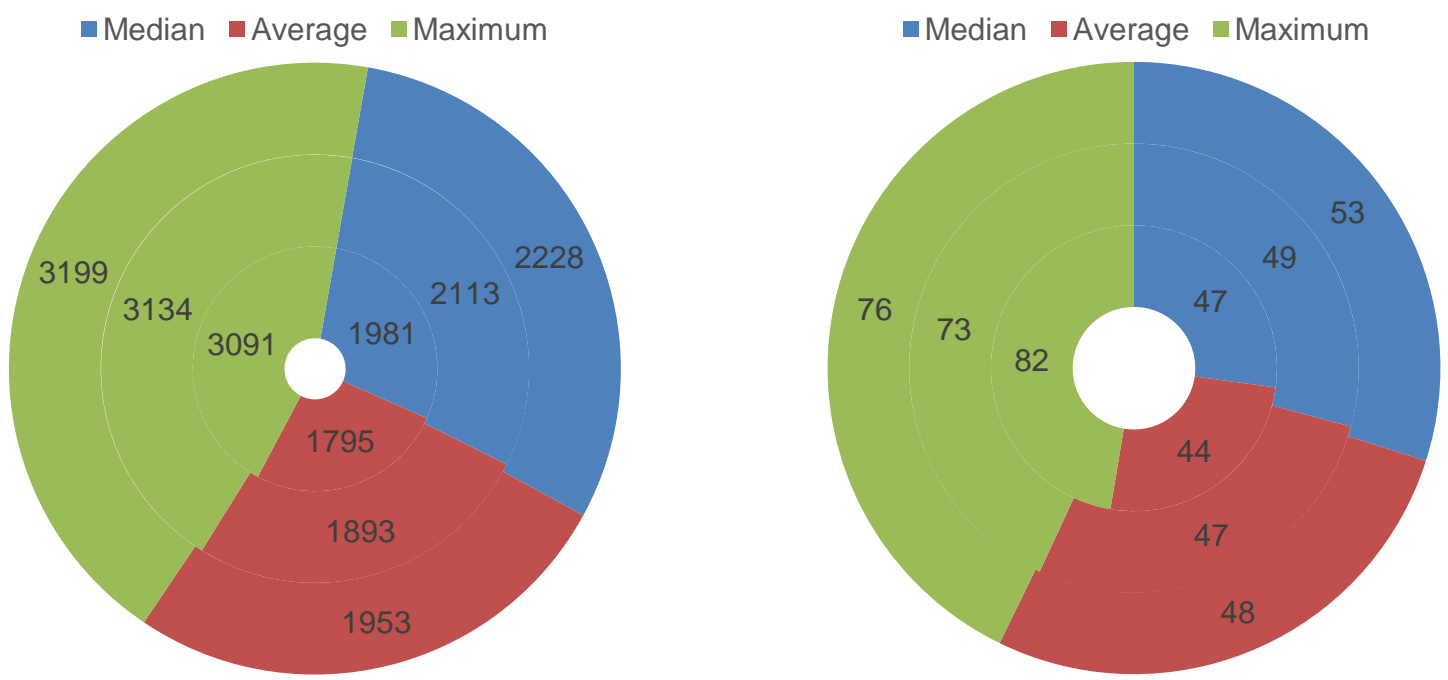

Figure 6 - Power statistics for the 2-year period based on:

(a - Left) power count value above 3 W (PV3 outer circle, PV2 middle circle and PV1 inner circle)

(b-Right) Watts per day (PV3 outer circle, PV2 middle circle and PV1 inner circle)

Figure 7 illustrates the frequency count values (number of days for which each PV module produces a specific amount of Watts per day). All three PV modules produced $10 \mathrm{~W}$ or less for approximately 50 days of the 2-year period. However, PV3 produced more than $70 \mathrm{~W}$ for 204 days within the 2-year period, which is more than PV2 (137 days) and PV1 (85 days). This suggests that PV3 produced more electrical energy for longer periods of time during the day while also producing higher values of electrical energy due to its connection to a solar controller.

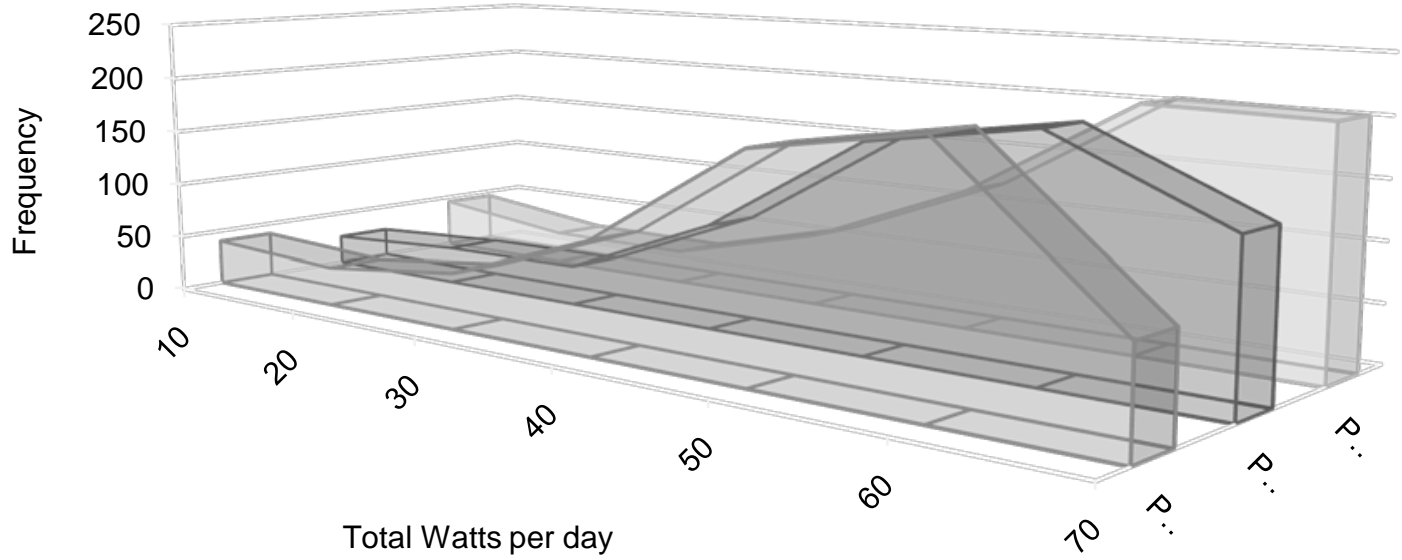

Figure 7 - Frequency of Watts per day generated by the PV modules for the 2-year period 


\section{Conclusion}

Two key anomalies were identified. Firstly, the abnormal degradation in the output power of a well-used PV module. Secondly, the abnormal improvement in the output power of a newly installed PV module.

PV2 shared the same atmospheric conditions as PV1 and PV3 over the 2-year period. It was connected to a load resistor for the last 6 months, which cannot be faulted for the abnormal degradation in output power as PV1 was connected to this same load resistor for the first 18 months. However, PV1 did not exhibit the same abnormal degradation as PV2 did over the last 3 months of the period. PV2 did not follow the same curve as compared to PV1 and PV3, despite having the same load and being exposed to the same atmospheric conditions for the last 3 months of the 2-year period.

PV1 was replaced with a newly manufactured module, after which it produced approximately $15 \mathrm{~W}$ more than PV3 which produced the highest power value over the 2-year period. Two plausible reasons exist for this. Firstly, the new module installed for PV1 may have a higher efficiency, as it was manufactured 3 years after the original module which was used. Secondly, PV3 would have degraded by approximately $2.4 \%$ over the 2-year period, which is in-line with published research (Bartolo \& Yousif, 2017).

Another key observation relates to the similar performance of LED lamps and solar controllers as viable power loads for testing PV modules in pico-solar systems. The LED lamp outperformed the solar controller in the winter season $(1.5 \%$ more output power) while the solar controller outperformed the LED lamp in the summer season (3.9\% more output power). The resistor proved to be a poor power load.

Time has revealed a better understanding of these PV modules in that one PV module degraded faster than other identical PV modules over a 2-year period in a semi-arid region of South Africa. A key recommendation is to initiate an annual maintenance program that may check the output power and performance of individual identical PV modules within a greater array. The contribution of this empirical data for a pico-solar system over a 2-year period may further be used to design and improve simulation software models that are often used as the starting point for determining the viability of a given PV installation within a specific atmospheric environment.

\section{References}

Adcock, G. (2016). Electro-thermal modelling of large PV array degradation for thermography and peak power conditioning monitoring.

Albert, P. J., Werhane, P., \& Rolph, T. (2014). Technology and Capacity Building Global Poverty Alleviation: A Case Book (pp. 13-111): Springer.

Ancuta, F., \& Cepisca, C. (2011). Failure analysis capabilities for PV systems. Recent Res. Energy, Environ. Entrep. Innov, 109-115. 
Bartolo, K., \& Yousif, C. (2017). Assessing PV Module Degradation and the Potential of Using Greenhouse Roofs for Supplemental PV Power Generation in Malta Mediterranean Green Buildings \& Renewable Energy (pp. 223-233): Springer.

Bosua, R., \& Venkitachalam, K. (2013). Aligning strategies and processes in knowledge management: a framework. Journal of Knowledge Management, 17(3), 331-346.

Cortellini, E. A., Reilly, C. J., \& Pujari, V. K. (2014). Crucible body and method of forming same: Google Patents.

Cox, A. (2013). Solar and geothermal. Information Sources in Energy Technology: Butterworths Guides to Information Sources, 263.

Cuciureanu, D., Nituca, C., Chiriac, G., \& Sticea, D. (2016). Analysis of the photovoltaic panels currently in use in different locations. Paper presented at the Electrical and Power Engineering (EPE), 2016 International Conference and Exposition on.

Deb, A. K. (2014). Purchasing Energy in a Dynamic Market. Strategic Planning for Energy and the Environment, 34(1), 51-77.

Edwards, J. (2001). Knowledge life-cycles: What to keep and what to throw away. Proceedings of Knowledge Management in OR Groups Farnborough, UK.

Gupta, R., \& Inglesi-Lotz, R. (2016). Detection of multiple bubbles in South African electricity prices. Energy Sources, Part B: Economics, Planning, and Policy, 11(7), 637-642.

Hertzog, P. E., \& Swart, A. J. (2015a). Determining the optimum tilt angles for PV modules in a semi-arid region of South Africa for the summer season. Paper presented at the SATNAC 2015, Arabella, Hermanus, Western Cape.

Hertzog, P. E., \& Swart, A. J. (2015b). The Use of An Innovative Jig to Stimulate Awareness of Sustainable Technologies Among Freshman Engineering Students. Sustainability, 7, 9100-9117. doi:DOI:10.3390/su7079100

Holstein, B. (2015). Analyzing Photovoltaic Potential Using a Geographic Information System: A Case Study of Prince William County Public Schools.

Kim, K. A., \& Krein, P. T. (2013). Hot spotting and second breakdown effects on reverse IV characteristics for mono-crystalline Si photovoltaics. Paper presented at the Energy Conversion Congress and Exposition (ECCE), 2013 IEEE.

Kim, K. A., Shenoy, P. S., \& Krein, P. T. (2015). Converter rating analysis for photovoltaic differential power processing systems. IEEE Transactions on Power Electronics, 30(4), 1987-1997.

Kurtz, S., Whitfield, K., TamizhMani, G., Koehl, M., Miller, D., Joyce, J., . . . Zgonena, T. (2011). Evaluation of high-temperature exposure of photovoltaic modules. Progress in photovoltaics: Research and applications, 19(8), 954-965.

Larsen, A., \& Lindquist, P. (2014). Forecasting mismatch losses: An empirical study investigating module level inverter-and string inverter systems.

Lee, S. J., \& Yeo, H. S. (2017). Textured silicon substrate and method: Google Patents.

Lorenzo, E., Moretón, R., \& Luque, I. (2014). Dust effects on PV array performance: in-field observations with non-uniform patterns. Progress in photovoltaics: Research and applications, 22(6), 666-670.

Luque, A., \& Hegedus, S. (2011). Handbook of photovoltaic science and engineering: John Wiley \& Sons. 
Massi Pavan, A., Mellit, A., De Pieri, D., \& Lughi, V. (2014). A study on the mismatch effect due to the use of different photovoltaic modules classes in large-scale solar parks. Progress in photovoltaics: Research and applications, 22(3), 332-345.

Mathews, S., Peshek, T., Hu, Y., \& French, R. H. (2014). Photovoltaic Degradation Analysis and Simulated Models. Case Western Reserve University2 May, 2014.

Mirza, I. A., Khalil, M. S., Amer, M., \& Daim, T. U. (2015). Assessment of Wind Potential in Kalar Kahar Region by Comparing On-Site Data with NREL Wind Resource Map of Pakistan Policies and Programs for Sustainable Energy Innovations (pp. 55-81): Springer.

Parsons, R., Krugell, W., \& Keeton, G. (2015). ESKOMs further application for a tariff increase. Rhodes University.

Poissant, A. (2017). Optimal replacement strategy for residential solar panels using monte carlo simulations and nonlinear optimization methods.

Reddy, S. S. (2017). Multi-objective based congestion management using generation rescheduling and load shedding. IEEE Transactions on Power Systems, 32(2), 852-863.

Saly, V., Ruzinsky, M., \& Redi, P. (2001). Indoor study and ageing tests of solar cells and encapsulations of experimental modules. Paper presented at the Electronics Technology: Concurrent Engineering in Electronic Packaging, 2001. 24th International Spring Seminar on.

SINOVoltaics.com. (2017). Solar panel manufacturing process: From cell to module. Retrieved from sinovoltaics.com/learning-center/manufacturing/solarpanel-manufacturing-process-from-cell-to-module/

Snyman, H., Venter, W., Van Rensburg, W., \& Opperman, D. (1987). Ranking of grass species according to visible wilting order and rate of recovery in the Central Orange Free State. Journal of the Grassland Society of Southern Africa, $4(2), 78-81$.

Sun, R. (2013). Photovoltaic properties and size-pH phase stability of iron disulfide from density-functional theory. Massachusetts Institute of Technology.

Swart, A. J. (2016). Correlating the switch-on time of two identical PV modules using a simplified measuring approach. Paper presented at the ECTI-CON, 13th International Conference on Electrical Engineering, Electronics, Computer and Information Technology, Chiang Mai, Thailand. ISBN: 978-1-467-39749-0.

Swart, A. J. (2017). Visualising the effect of different tilt angles on the switch-on time of small PV modules using a simplified measuring approach. Paper presented at the AFRICON 2017, The Avenue, Victoria and Alfred Waterfront, Cape Town, South Africa. ISBN: 978-1-5386-2774-7.

Swart, A. J., \& Hertzog, P. E. (2016). Varying percentages of full uniform shading of a PV module in a controlled environment yields linear power reduction. Journal of Energy in Southern Africa, 27(3), 28-38.

Swart, A. J., \& Hertzog, P. E. (2016). Verifying an economic viable load for experimental purposes relating to small scale PV modules. Paper presented at the SATNAC 2016, Fancourt, George, Western Cape. ISBN: 978-0-620-72418-0.

Swart, A. J., \& Hertzog, P. E. (2017). LED's as viable power loads for experimental purposes relating to PV modules in pico-solar systems. Paper presented at the ICETAS 2017, AMA International University Bahrain, Bahrain. 


\title{
Integrating 5S Principles as a Strategy for Improving Clothing Manufacture
}

\author{
Kemlall Ramdass \\ University Of South Africa \\ Johannesburg, South Africa \\ Mothibeli Pita \\ University Of South Africa \\ Johannesburg, South Africa
}

\begin{abstract}
The supply chain in the clothing is continuously influenced by internal and external competition. The industrialisation of the industry continues to impact all stakeholders throughout the world. Competition is rife in terms of value-add, quality, delivery dates and cost. Many of the manufacturers continue to operate without any kind of strategy to improve current conditions. Market share is being transferred outside the boundaries of the country. The retail industry maintained its impetus in terms of supply to the ultimate customer but the sourcing of finished products moved towards the east primarily due to cost competitiveness. In order to improve the current status, 5S principles may be implemented as a competitive strategy. This simple re-organisation of the workplace would significantly improve ergonomics in its totality, thereby creating an environment that is conducive for productive machine operation. It may be used as a competitive strategy that would cultivate strength in manufacturing capabilities. This paper evaluates current paradigms and implements $5 S$ principles as a means of improvement through a case study in one organisation. Data regarding certain aspects were gathered from 45 clothing manufactures in the Kwa-Zulu Natal area.
\end{abstract}

Keywords: clothing industry, 5S principles, industrial engineering, ergonomics, continuous improvement.

\section{Introduction}

The clothing industry in South Africa is plagued by inefficiencies. Considering it a labour intensive industry, simple organisation of work is problematic as the structures in place do not support ergonomic layouts. Organisation of labour, input, process and output are impacted through a lack of an organized systems. Clutter, dis-organisation and a lack of managerial expertise on industrial engineering 
principles in a workplace reduces labour, material and machine productivity as the current labour efficiency is estimated to be in the region of $50 \%$. Numerous organisations in the clothing and textile sector are besieged by inefficiencies throughout the manufacturing process. In order to improve the competitive position of the industry, it is imperative that simple strategies of re-organisation and reengineering be implemented to, not only improve the efficiency, but to also contribute to the health and safety of workers (Barnes, 2005; Berry et al., 2012).

Other factors that influence the inefficiency in the industry include, but are not limited to the following:

- Inability to change with new trends

- Lack of effective leadership and management

- Poor utilisation of resources

- Inverted pyramid in terms of management

- Poor investment in technology

- Lack of managerial qualities

- Inability to implement skills development

- Poor strategy implementation

- Poor relations with labour

- Working in "silos"

- Poor communication among departments

- Poor team spirit (Esset, 2003; Ramdass, 2009)

The industry is plagued with retrenchments and employment levels over the past 30 years. The majority of the sample (73\%) complained about the decline in employment levels during the 1999-2017 periods. The information elucidates that the clothing industry continues on a downward spiral. However, with assistance from government the industry is stabilising in terms of employment (Bendex, 2010).

\section{Method}

The approach of the study considered a qualitative research methodology through that application of a case study. Although case studies endure criticism, they remain robust as an approach for data collection. The methodology allows exploration and understanding of complex phenomena through in-depth scrutiny. The case study methodology was performed at a number of manufacturing organisations as this provided an overview of the status of the industry. Information was gathered through direct observation and communication with supervisors and workers, focus group interviews with employees regarding their experiences and observations and the review of applicable literature from clothing related journals and applicable text. Descriptive statistics was used to explain certain phenomena (Blumberg, et al.2014).

\section{Literature Review}

Several authors provided critical insight into order winning strategies. Stephenson (2015) formalised four competitive priorities which he categorised as price, quality, dependability and flexibility. These dimensions are totally appropriate for the clothing industry as organisations compete within these dimensions. Further 
examination postulates that price and quality are key order winners within the industry.

Goetsch and Davis (2016) identified five different dimensions namely price; quality; dependability; product flexibility; and volume flexibility. These dimensions are similar to that of Stevenson with the addition of flexibility in terms of products and volume. Through simple manipulation and re-organisation, a clothing manufacturer can produce skirts, blouses, shirts, trousers, underwear, dresses and so forth. The supply chain determines the production of garments. In the clothing industry perspective, a pull supply chain system is used where customer orders drive the production line. Brouwer et al, (2002) added to competitive priorities by proposing plant and equipment, production planning and control, labour, product design and management. These are all appropriate as well maintained machinery and skilled labour, together with planning of production, product design and leadership enhance the efficiency and effectiveness of production management (Gapp et.al (2008).

Hill (2000) postulated that it is imperative for an organisation to critically analyse criteria that would "win orders." His "order-winning" criteria added product variety as an important variable. However, in the clothing industry which is extremely competitive, organisations are encouraged to look at niche markets. This means that they need to focus on what they are "good" at. This may mean that organisations may have variety with a particular product. He also mention performance which considers productivity, efficiency and effectiveness as imperative in a competitive environment.

Val Halen (2005) and Chan (2005) further added to the list of criteria and considered variables such as product design, cost of quality, delivery deadlines and customisation. It is imperative to note that these variables are in-exhaustive as many more variables could be added as one analyses the performance criteria of the clothing industry in particular.

In conclusion of the literature review, what separates the clothing industry in South Africa with the international industry is the resistance to change and the lack of motivation and discipline to embrace improvements within the work environment. As a simple strategy for improvement, 5S principles was chosen to improve the conditions in the working environment.

\section{Results and Discussion}

\section{Introduction}

The South African clothing industry has potential for the improvement of productivity in the region of $30 \%$ through the implementation of industrial engineering methodology. It may be noted that several of the larger organisations have radically reduced in size and product offerings over the last 3 decades. One such manufacturer is South African Clothing Industry who had a staff complement 
of over 2000 employees in the 1980's. Currently there are approximately 300 employees. Most of their operations moved into areas such as Lesotho, Isithebe, Newcastle and Swaziland. One of the primary reasons for this is labour cost which ultimately determines product cost as a primary cost factor. As organisations became smaller and disintegrated, many industrial engineering personnel were lost and organisations deteriorated in terms of the application of simple tools that provide a conducive environment for production.

A bag manufacturer was targeted as a potential for implementation of the $5 \mathrm{~S}$ principles. It was observed that one of the potential barriers for improvement is management. It took several discussions to persuade management about the potential for improvement and the importance of being competitive. It is important that leadership is people orientated with an insight into cultural diversity, financial management by understanding organisational costs, service delivery with quality management imperatives and last but not least, continuous improvement with the $5 S$ principles as the basis for improvement.

The results were broken down into themes aligned to the $5 S$ principles.

\section{Theme 1 - organisation (seiri )}

Cooper (1987) and Dizaji et al (2011) mentioned that if one improves a worker's comfort level, then one improves the company's productivity level. Ergonomics is about the design of workplaces that integrates the rhythmical movements of both hands with the material and the sewing machine. The current workplace is plagued with inefficiency in terms of seating, lighting, workplace layout, disposal equipment, input material and the layout of the workplace. This inefficiency at each workplace reduces human-machine interface by approximately $50 \%$.

The typical inefficiency currently experienced include, but are not limited to the following are:

- Poor operator efficiency

- Lost time in terms of machine and operator utilization

- Higher medical costs due to operator carpul tunnel syndrome

- Material costs - due to lost component parts

- Absenteeism due to operator frustration

- Poor quality and increased defects

- Fatigue, strains, injury

- Operator sabotage of machines

- Machine downtime

- Accidents and errors

- Labour turnover

- Poor productivity levels

- Reduced competitiveness (Jaca, et al. 2014) 
The focus group mentioned that space was a major problem and concern as many operators were not visible due to bundles of panels, patterns, thread and pieces of material around them. It was observed that the ergonomic layout of the workplace in terms of work-in-progress inventory, man-machine interface, stretching out for assembly of fabrics was in disarray. Hand movements were non-rhythmic while materials for construction of garments was unorganised. This led to lost components and incomplete orders. Further, the components had to be re-cut to complete the quantity required from the order placed. Groups complained that orders were lost due to the time delay of re-cutting material for the completion of orders.

In order to address the issue operators were asked to apply the concept of stratification management. This was done through a series of mini-workshops with supervisors and workers. This meant evaluating all components within the workspace and separating them into what was required for immediate production and what needed to be removed. Therefore the importance and quantity of all was evaluated on a scale from 1 being most important to 5 being least important. The inventory of items required relocation to a storage area. Stratification management is involved with keeping only what is necessary for immediate production resulting in optimising the workplace for smooth manipulation of hand movements. Thus reorganisation of the workplace eliminates unnecessary inventory and makes everything visible. This improves visibility of the workplace which can be observed from any location within the layout of the factory. It also improves rhythmic movement of handing the components of the bag (Prabhuswamy, et al 2013); Chan (2005).

\section{Theme 2 - neatness (seiton- housekeeping)}

The next theme that impacted the workplace was neatness. Due to the clutter, dust accumulated on many stagnant components that caused absenteeism and unhygienic working conditions. Staff complained that this was a health hazard which led to a number of employees suffering from allergies and related respiratory tract infections.

The clothing industry is labour intensive and predominantly female breadwinners. It is an industry that is plagued with a high rate of absenteeism. There are numerous factors that contribute to absenteeism. Some of the major factors as mentioned by supervisors and machinists are as follows:

- Poor working conditions

- Unhygienic surroundings

- Demotivation

- People behaviour

A small, medium and large organisation was sampled and provided the following results over a week. 
Table 1 Categorising absenteeism

\begin{tabular}{|l|l|l|l|l|l|l|l|}
\hline & Manufacturer & $\begin{array}{l}\text { No of } \\
\text { emp }\end{array}$ & $\begin{array}{l}\text { No of } \\
\text { abs }\end{array}$ & $\begin{array}{l}\text { Direct } \\
\text { Staff }\end{array}$ & $\begin{array}{l}\text { Indirect } \\
\text { Staff }\end{array}$ & \\
\hline Monday & 1 & 50 & 8 & 16 & 4 & 4 & \\
\hline Tuesday & 1 & 50 & 6 & 12 & 2 & 1 & \\
\hline Wednesday & 1 & 50 & 3 & 6 & 3 & 0 & \\
\hline Thursday & 1 & 50 & 5 & 10 & 5 & 0 & \\
\hline Friday & 1 & 50 & 1 & 2 & 1 & 1 & \\
\hline Average & & & & 9.2 & & & \\
\hline Monday & 2 & 120 & 10 & 8 & 5 & 5 & \\
\hline Tuesday & 2 & 120 & 12 & 10 & 4 & 8 & \\
\hline Wednesday & 2 & 120 & 6 & 5 & 3 & 3 & \\
\hline Thursday & 2 & 120 & 9 & 7.5 & 4 & 5 & \\
\hline Friday & 2 & 120 & 11 & 9 & 5 & 6 & \\
\hline Average & & & & 7.9 & & & \\
\hline Monday & 3 & 530 & 28 & 5.3 & 18 & 10 & \\
\hline Tuesday & 3 & 530 & 35 & 6.6 & 22 & 13 & \\
\hline Wednesday & 3 & 530 & 31 & 5.8 & 15 & 16 & \\
\hline Thursday & 3 & 530 & 42 & 7.9 & 15 & 27 & \\
\hline Friday & 3 & 530 & 25 & 4.7 & 13 & 12 & \\
\hline Average & & & & 6.06 & & & \\
\hline
\end{tabular}

The average level of absenteeism is around $7.72 \%$ and correlates with the study by B\&M Analysts which shows that the rate of absenteeism is approximately $7.2 \%$. The predominantly female breadwinners take major strain in terms of family responsibility. One of the major concerns is that employees believe that they are entitled to their sick leave. The full quota of sick leave is taken during the course of the year and this burdens management in terms of overall productivity. An additional factor is the industrial relationship between employer and employee which seems to be irrepairable. There is low morale, lack of trust, inefficiency, poor quality and increased costs (Falkowski and Kitowski, 2013).

Absenteeism can bring a production line to a standstill, especially if it is a key operation. This would result in line balancing problems where operators are reallocated to machining operations depending on their skills. This becomes the weakest link in terms of the theory of constraints and all focus is on the operation which is detrimental to the manufacturer in terms of quality, cost, delivery and profits.

The financial burden on manufacturers is huge due to absenteeism. Some oganisations started an incentive to motivate people to be at work which burdens then financial status even further. Processes and procedures need to be implemented to address this problematic issue in order to improve productivity and the satisfaction of the supply chain. 
Neatness encompasses the total surrounding of the employee which influences the performance of work. Discussions were held regarding all aspects of the workplace. Workstations where evaluated as to what was supposed to be there (following the principles of (JIT)) and what can be removed to ensure a smooth flow of work in process items. Obstructions to work flow were identified and removed by having a clearly defined layout. Decisions regarding the requirements of machinists was undertaken to stipulate that only was is required should be at the workstation. Such decisions were determined by the usage frequency of items. What was strongly stressed was "a place for everything and everything in its place." In this way all obstructions were minimised for easy flow of production.

Mechanical objects that were infrequently used were stored in machine draws (example screwdrivers for the changing of needles). Jigs and fixtures were added to certain machines to enable ease of operation. Other than storage, attention was focussed on the ergonomic layout such as the chair, height of chair, the machine surface, posture of the operator, the pillows that distorted operator posture while seated and the like.

A system was developed for the identification, labelling, and work process flows that everyone understood, followed and maintained. Special attention was be paid to the tidiness of notices and signs as it is a means of communication. These should be placed neatly at a convenient height so that they can be seen and yet not be an obstruction. What was also mentioned was the importance of using posters that remind operators of a healthy workplace. It was agreed that notices should also be of a proper size, and neatly typed with a clear heading.

\section{Theme 3 - cleanliness (seiso)}

In view of the current experiences expressed and observed by $100 \%$ of the workforce, the workplace was in "shables." Untidiness of the workplace is visible to everyone, yet little is being done to improve the situation. In order to maintain a good image of cleanliness, employees were asked to clean their individual workspaces in the last 10 minutes of the day. A layout of the work environment and work spaces was charted to engage all employees individually in cleaning. No areas were left undefined or unaccounted for. Supervisors were required to make decisions in discarding items that were unnecessary to uncover areas for inspections. Discarding unwanted items made the area clear and uncluttered (Becker, 2001).

Visibility is vital in effectively identifying problems in the workplace. In addition it was suggested that machines be fitted with "robot" lights to indicate the urgency of problems that arise. The maintenance of machines, the workplace and the Daily inspection, lubrication and cleaning of equipment is part of preventive maintenance that would increase efficiency of the machinery. It was important that cleaning be done daily while inspections were scheduled to ensure that this was done religiously. Cleaning was extended beyond the work-area to all areas of the organisation even to areas not usually obvious, such as the washroom. 


\section{Theme 4 - standardisation (seiketsu)}

"Seeing is believing"; thus emphasis must be placed on visual management. All workplaces are identical. Standardised conditions on site must be achieved so that work can be done quickly. The challenge faced by $70 \%$ of the organisations was a lack of standardisation. This is an aspect that is difficult to attain due to disagreements among staff. This should be implemented for every task in the organisation, even for the numbering of technical documents and other papers.

Although time was lost in certain instances, operators were asked to move around and observe their colleagues to create consistency among operators. This enables teams to emerge as to making the work environment as pleasant as possible for all. Standardised visual aids like indicators, labels and markers are easily recognisable and make communication simple and understandable, so that everyone can act quickly.

The following figure provides a layout that is standardised and all workstations are the same dependant on the type of machinery used. This is the process where the above three aspects of the $5 \mathrm{~S}$ principles are put to practice at all workstations.

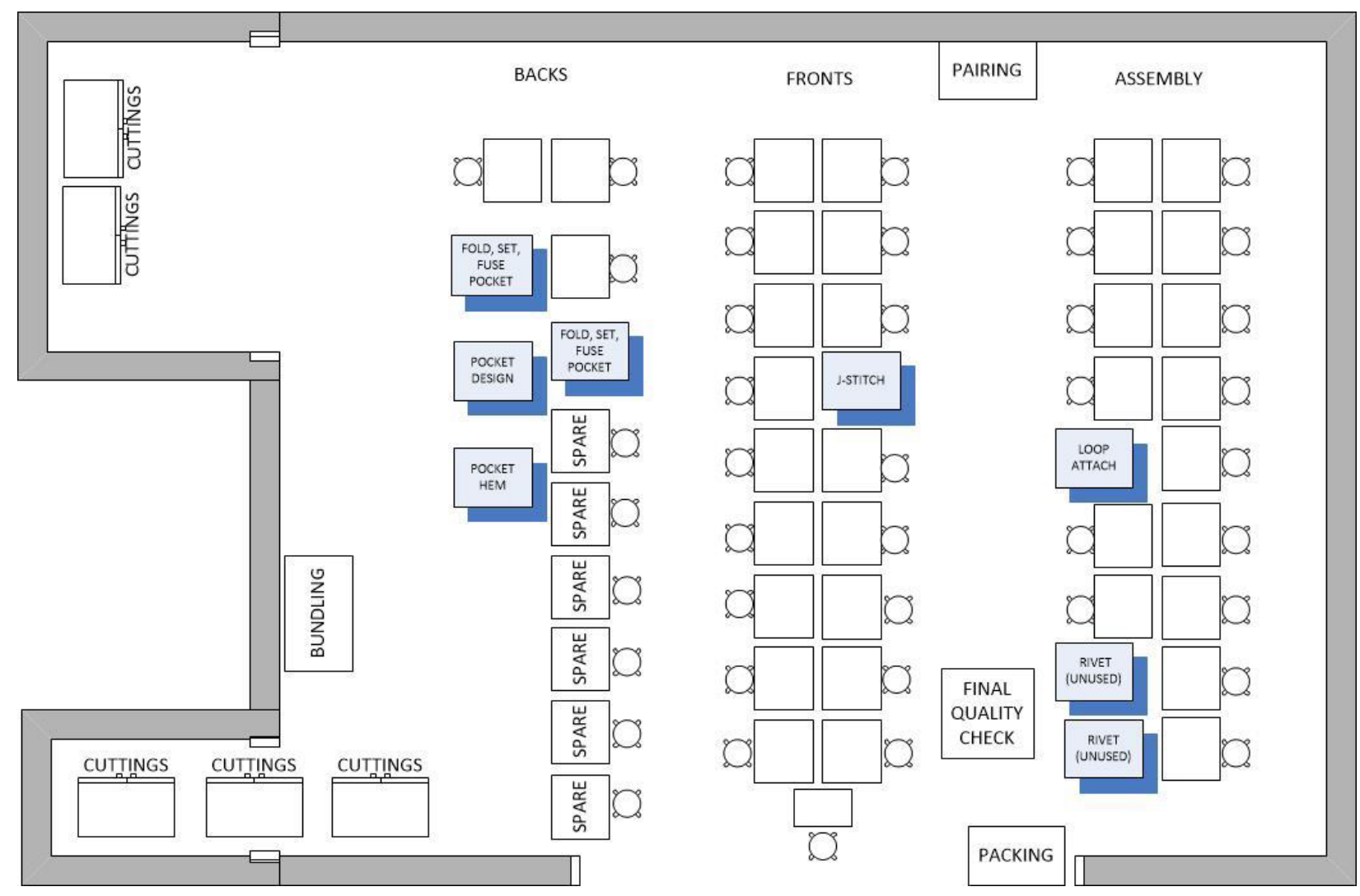

Figure 1. Typical layout of a clothing factory 


\section{Theme 5 - discipline (shitsuke)}

One of the primary concerns by all respondents was the lack of discipline among machinists. Discipline among people of different cultures, age groups and languages are demanding. A decision was taken to engage staff on inculcating habits that promotes "Ubuntu". These can be practised both at home and at work. As the final $S$ in the 5-S, it propagates the 5-S practice. This means conscientiously engaging with the other 4 principles to ensure an orderly and efficient workplace. Continuous engagement of all operators on a regular basis would enable the 4 principles to become a habit. It also means striving for kaizen, which is the Japanese equivalent for continuous improvement.

\section{Conclusion and Recommendations}

Formalising the system of the 5-S principles requires commitment, perseverance and leadership. Management and leadership is required to sustain the system, while teamwork and continuous engagement with staff are vital. It needs to be borne in mind that maintenance is a difficult task, but can be achieve through engagement. The introduction of the $5 S$ principles would enable the organisation to continuously review its strategy towards attaining an environment that is conducive for the optimisation of production. This would definitely serve as a foundation upon which other improvements can be built. The recognition of the application would ensure that integration and practices are well grounded (Whitaker, 2001).

The diligent practise of the 5-S techniques enables immediate impact upon the workplace. This is not only beneficial for the organisation in terms of productivity, but also addresses the workforce environment where transformation takes. The ability to enhance working conditions impacts the following areas:

- worker

- workplace

- machine

- environment

- productivity

- cost

- delivery

The consistent maintenance of the working environment enables the organisation to improve its competitive position in a demanding environment.

\section{Management commitment}

Management together with leadership qualities are imperative for the success of the implementation. To express their commitment, management are required to stand their position "and become the real owners of the organisation." Skills development in legal, business management, human resources and industrial engineering are crucial as a point of departure. It is vital that management take their stand as the leaders of the organisation to enforce discipline. 


\section{Promotional campaign}

In order to achieve acceptance of the principles, a company-wide promotional campaign is recommended. Employees need to understand and take ownership through a team approach. Evaluating individual work areas would eventually encompass the entire organisation. The $5 S$ principles must be in the "face "of all employees and it should become part of the culture of the organisation. As an example, the 5 days of the week could be lined to the 5 principles.

Monday 1st $S$ day - organisation (e.g. throw away things you do not need).

Tuesday 2nd S day - neatness (e.g. name everything and assign locations).

Wednesday 3rd S day - cleanliness (e.g. all- together housecleaning).

Thursday 4th $S$ day - standardisation (visual management and transparency for things).

Friday 5th $S$ day - discipline (e.g. do your own 5-S audit).

\section{Evaluation of 5-S programme}

In order to maintain the effectiveness of the programme, it is important to reward employees accordingly. The encouragement of employees to consistently engage to improve the workplace and their surrounding areas is crucial for the success of $5 S$ principles.

\section{Maintaining the system}

Inspections should be planned from time to time to evaluate the workplace. This should be known by management only. This would prevent last minute efforts to change unacceptable standards. The maintenance of 5S principles is imperative for it to be successful.

In conclusion, it is imperative to engage all employees to buy-in to the system of reorganisation, because it is the workers that would assist in this endeavour and ensure that new changes are maintained.

\section{References}

Becker, J. E. (2001). Implementing 5S: To promote safety \& housekeeping. Professional Safety, 46(8), 29.

Barnes, J. (2005). A strategic assessment of the South African clothing sector. For the National Economic Development and Labour Council (NEDLAC), 29.

Bendix, S.(2010). Industrial relations in South Africa. Juta and Company Ltd.

Berry, C. M., Lelchook, A. M., \& Clark, M. A. (2012). A meta- analysis of the interrelationships between employee lateness, absenteeism, and turnover: Implications for models of withdrawal behavior. Journal of Organizational Behavior, 33(5), 678-699.

Blumberg, B.F., Cooper, D.R., Schindler, P.S. (2014). Business research methods. McGraw-hill education.

Brouwer, W. B. F., Van Exel, N. J. A., Koopmanschap, M. A., \& Rutten, F. F. (2002). Productivity costs before and after absence from work: as important as common?. Health Policy, 61(2), 173-187.

Chan, J. W. (2005). Competitive strategies and manufacturing logistics: an empirical 
study of Hong Kong manufacturers. International Journal of Physical Distribution $\mathcal{E}$ Logistics Management, 35(1), 20-43.

Cooper, C. L. (1987). Coping with stress in organizations: the role of management. Psychosocial Factors at Work and Their Relation to Health, 185-205.

Gapp, R., Fisher, R., \& Kobayashi, K. (2008). Implementing 5S within a Japanese context: an integrated management system. Management Decision, 46(4), 565579.

Goetsch, D. L., \& Davis, S. B. (2014). Quality management for organizational excellence. Upper Saddle River, NJ: pearson.

Dizaji, M. R., Rostamzadeh, R., Sofian, S., \& Rahmani, K. (2011). Relation of 5 S principles and Human Factors Engineering (Ergonomics) in Possibility of TPM Implementation (case study). In International Conference on Sociality and Economics Development, Singapore (pp. 68-73).

Falkowski, P., \& Kitowski, P. (2013). The 5S methodology as a tool for improving organization of production. PhD Interdisciplinary Journal, 4, 127-133.

Jaca, C., Viles, E., Paipa-Galeano, L., Santos, J., \& Mateo, R. (2014). Learning 5S principles from Japanese best practitioners: case studies of five manufacturing companies. International Journal of Production Research, 52(15), 4574-4586.

Prabhuswamy, M. S., Ravikumar, K. P., \& Nagesh, P. (2013). Implementation of kaizen techniques in TPM. IUP Journal of Mechanical Engineering, 6(3), 38.

Ramdass, K. (2016, October). AN EVALUATION OF WASTE AND COSTS AT A TEXTILE FACILITY: A CASE STUDY. In Institute for Industrial Engineering Conference (p. 213).

Stevenson, W. J., \& Sum, C. C. (2015). Operations management. New York: McGraw Hill Education.

Van der Westhuizen, C. (2007) Trade and poverty: A case study of the South African clothing industry. Studies in Economics and Econometrics.1;31(2):109-24.

Van Halen, C., Vezzoli. C., Wimmer, R. (2005). Methodology for product service system innovation: how to develop clean, clever and competitive strategies in companies. Uitgeverij Van Gorcum.

Whitaker, S. C. (2001). The management of sickness absence. Occupational and environmental medicine, 58(6), 420-424. 


\title{
Design and Implementation of an Automatic Electrical Motor Protection
}

\author{
Busiso Mtunzi,Tinashe Shelton Gavhu, Zedekia Madumbu Nyathi, Reginald \\ Gonye and Fidelis Nhenga Mugarsanwa \\ National University of Science and Technology, \\ Bulawayo, Zimbabwe
}

\begin{abstract}
The purpose of this paper was to design and implement an automatic motor protection system prototype. The system designed made use of sensors to measure and provide protection to the motors against temperature, overcurrent, overvoltage and under voltage using an arduino as the controller. Units to monitor temperature, overcurrent, overvoltage and under-voltages were designed and integrated. The temperature and current tolerance of the motor were set at $50^{\circ} \mathrm{C}$ and $0.26 \mathrm{~A}$ respectively. The system could respond accordingly to temperature and current changes by way of stopping the motor when set conditions were exceeded. The data measured was stored on a memory card and could be retrieved at any time to analyse the induction motor behaviour. The system if incorporated on motors, could minimize potential damages to electric motors' windings, hence reducing electric motor maintenance costs for companies.
\end{abstract}

Keywords: Arduino, GSM Module; Induction Motor; Over Voltage; Under Voltage.

\section{Introduction}

This paper looks at the design and implementation of an automatic electrical motor protection and data logging system using a microcontroller as the heart of the system. According to Craig and Multilin (2016), over 1 billion motors are in service in North America alone and these, use over $70 \%$ of the energy used in the manufacturing sector. These motors' failure rate have been found to be as high as $12 \%$ and, $36 \%$ of this failure rate is related to the environmental conditions in which the motor is operating and $33 \%$ is due to electrical related issues. This would apply to all motors in use in general. It has been found that a $10^{\circ} \mathrm{C}$ increase in operating temperature shortens motor life by about half and hence regularly checking of the operating temperature of critical motors would prevent unexpected shutdowns and extend motor life (Bishop, 2013). The most efficient operation and long motor life is only achievable if motors are operated close to the rated voltages and far from the outer limits (Cowern, 2000). 
Monitoring the operating conditions like temperature under which the motors operate and also the current and voltage levels of the motors to make sure they do not operate outside the prescribed ranges will minimise motor failure rate in industry and in turn reduce maintenance costs for companies.

Electromechanical protection method is the mainstream form of technology used to protect electric motors in industry. These electromechanical type of motor protection methods prevent internal thermal damage to the electric motor and their main drawback is that they do not provide any advanced warning that an event is about to occur, and they do not provide any information about why the event occurred (Rockwell Automation [RA], 2016 ).

There is a need for consolidating many motor protection methods into a single electronic device so as to cut on installation costs, component costs, panel size, and maintenance time. Using a microcontroller based motor protection device can also provide communications capabilities to alert for possible motor problems and record on why the motor stopped

\section{Research Methodology}

The motor that was used in designing the motor protection system was a single phase induction motor. Induction motors are currently the most widely used motors in industry, hence their choice in this research activity. The block diagram illustrating the motor protection system was as shown in figure 1 below:

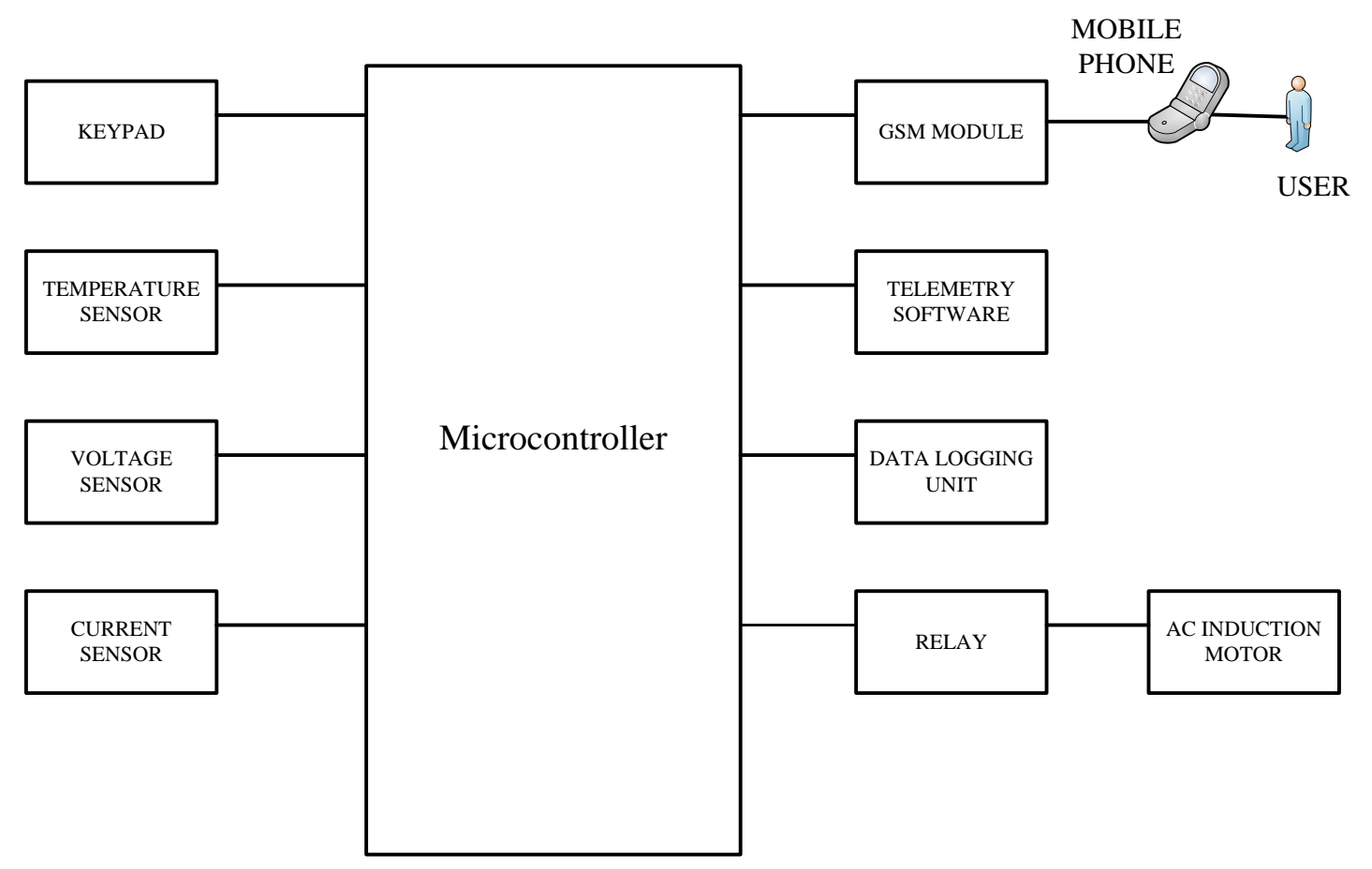

Figure 1 - System Block Diagram 
As can be noted in figure 1, the system consisted of several functional units put together. These included the microcontroller, which provided the brains of the system, sensor units (temperature, voltage and current sensors), and output units which included a data logging unit, the AC induction motor (which responded accordingly if the sensed quantity was exceeded).

\section{Materials and Procedure}

The microcontroller that was used for the system design was the Arduino Mega 2560 shown below in figure 2 .

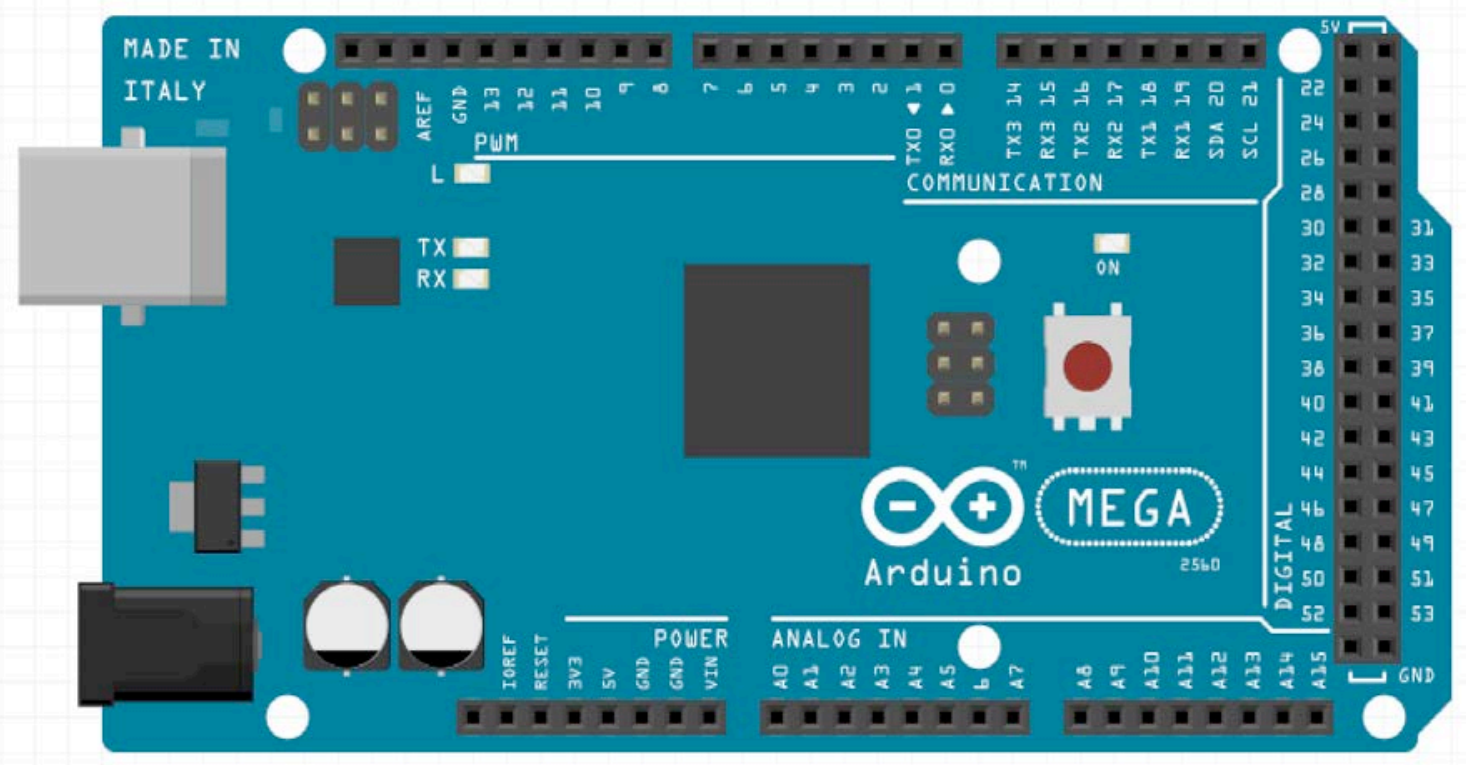

Figure 2 - Arduino Mega 2560

The Mega 2560 is a microcontroller board based on the ATmega2560 which has 54 digital input/output pins with 15 pins which can be used as pulse width modulation (PWM) outputs, 16 analogue inputs, 4 UARTs (hardware serial ports), a $16 \mathrm{MHz}$ crystal oscillator, a USB connection, a power jack, an ICSP header, and a reset button (ARDUINO AG, 2012).

The output unit of this system consisted of a relay which was used to stop and start the motor, a data logging terminal for storing all the measured operating parameters and a GSM module to notify engineers and technicians on the motor sensed quantities.

Motors need to be protected from high ambient temperatures and from high temperature rise due to the motor itself. High temperatures bring about damage to the wiring and insulation. The temperature protection mechanism of the system was set up to measure both the ambient temperature and motor temperature. Manufacturers usually specify the range of temperatures not to be exceeded on motors. They follow the National Electrical Manufacturers Association (NEMA) rates which gives the insulation and temperatures accommodated by electric motors. Class A insulation has a recommended temperature limit of $105^{\circ} \mathrm{C}$, Class $\mathrm{B} 130^{\circ} \mathrm{C}$, Class $\mathrm{F} 155^{\circ} \mathrm{C}$, and Class $\mathrm{H} 180^{\circ} \mathrm{C}$. The motor that was used in this research activity was a class $\mathrm{A}$ motor and had a power rating of 6 Watt. An infrared temperature 
sensor MLX90614 designed for non-contact temperature sensing was used. It has an internal 17-bit analogue to digital converter and a powerful digital signal processor that was used for temperature sensing. The sensor could measure both the ambient and object temperature from a distance.

According to the sensor datasheet, the sensor could measure -40 to $85^{\circ} \mathrm{C}$ for the ambient temperature and -70 to $382.2^{\circ} \mathrm{C}$ for the object temperature. Figure 3 shows the temperature sensor and its connections;
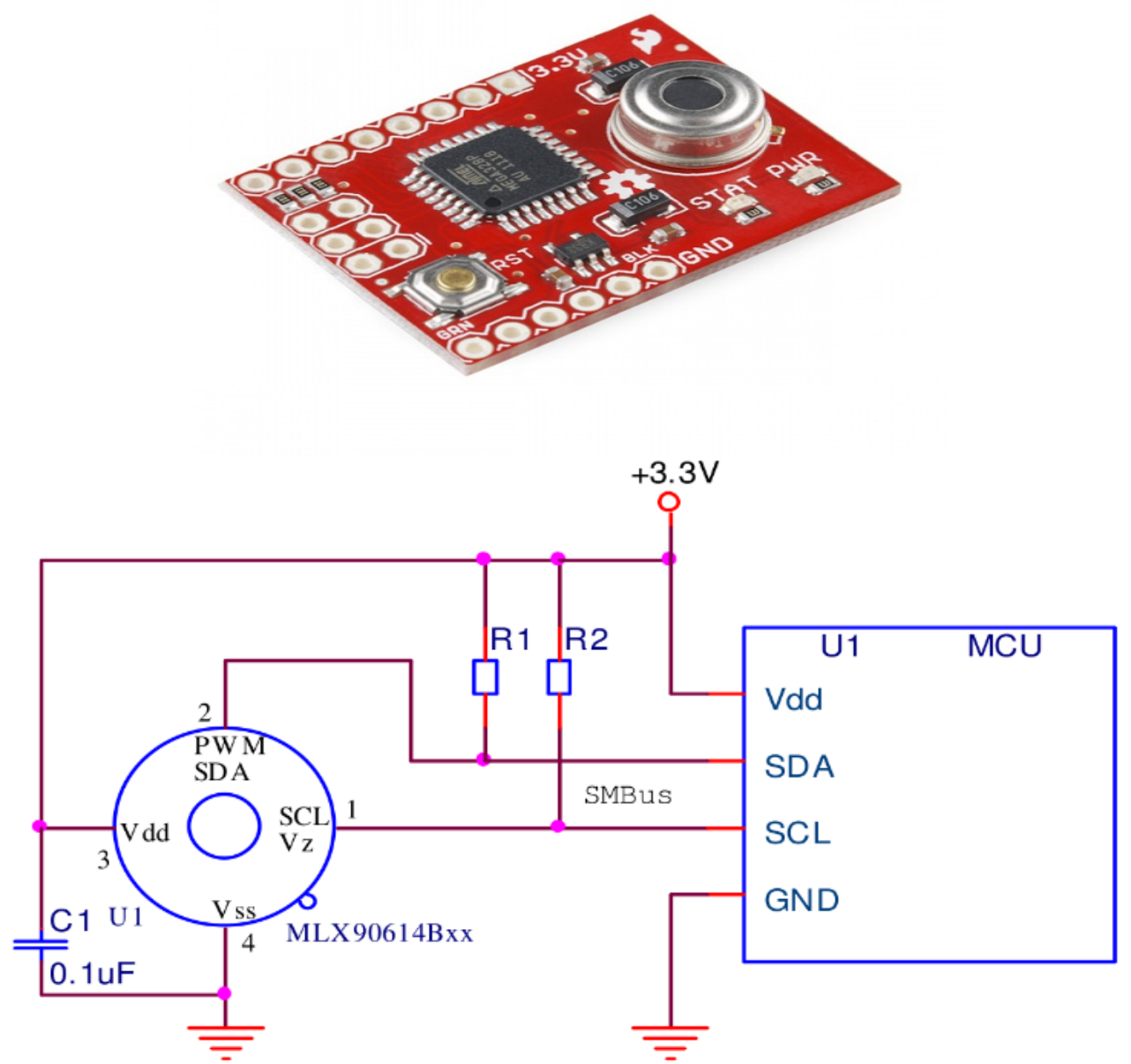

Figure 3 - Infrared Temperature Sensor (type: - MLX90614)

The temperature sensor in figure 3 was calibrated using an ordinary mercury thermometer. A code for the Arduino was developed and used to acquire the temperature readings.

The flowchart illustrating the temperature protection algorithm was as shown in figure 4 in the next page. 


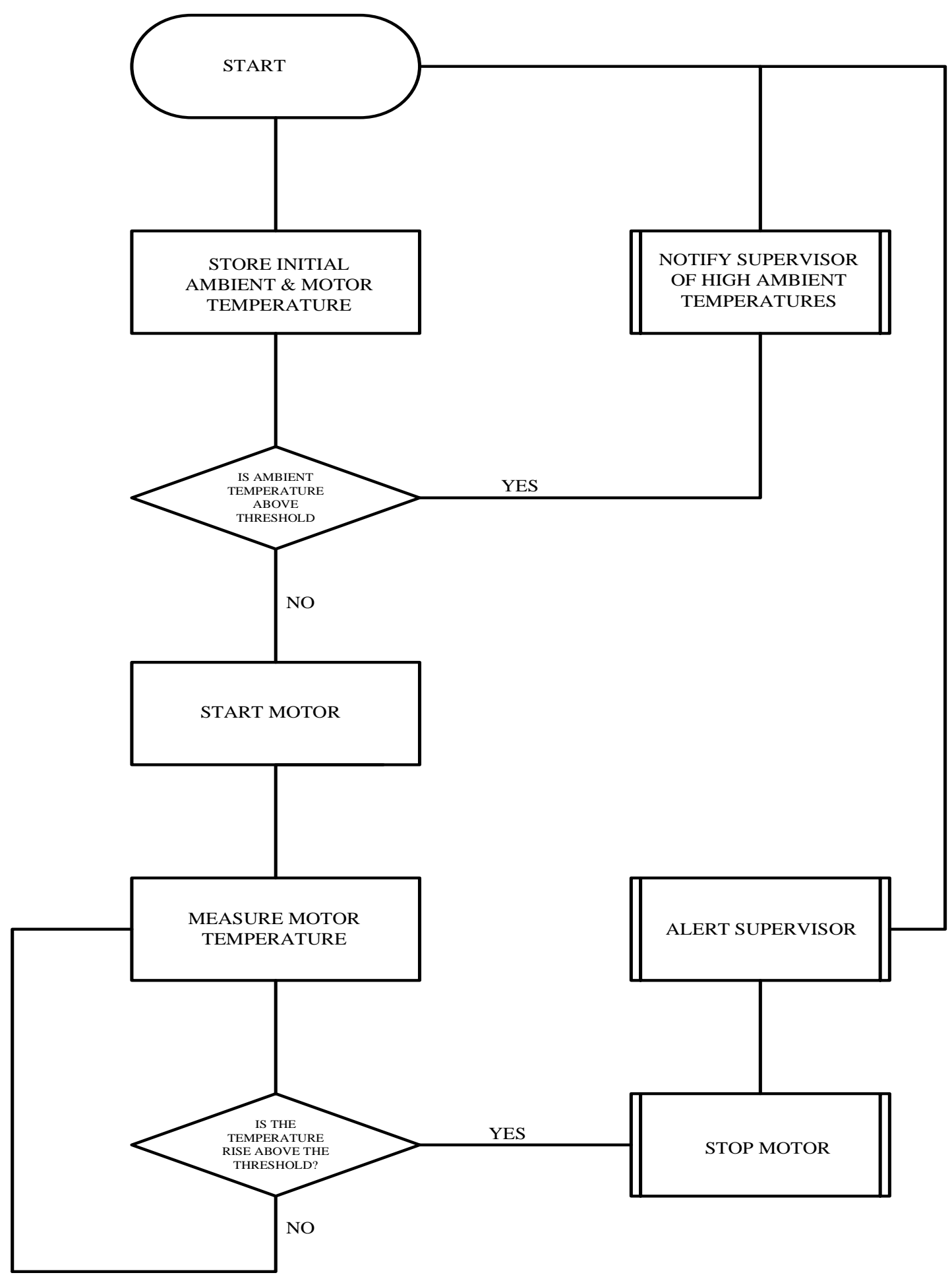

Figure 4 -3 Temperature Protection Flowchart

The flowchart gives the steps that were followed in the temperature protection algorithm of the system.

For overcurrent protection an Allegro ACS712 current sensor was used to measure the current being drawn by the motor. According to National Electrical Manufacturers Association (NEMA), motors with one horsepower output have a service factor of 1.15. A service factor of 1.15 was adopted for the motor which had a rated current of $0.15 \mathrm{~A}$. The motor current protection unit was then designed not to 
allow the motor current to go beyond 1.15 times of the rated current. In this case the current was capped at $0.1725 \mathrm{~A}$. To avoid nuisance tripping of the motor when an overcurrent condition is detected, the current protection system was designed to delay for 5 seconds and if the overcurrent condition persisted then the motor would be disconnected. Figure 5, below shows the Allegro ACS712 (Allegro Microsystem, LLC, 2017).

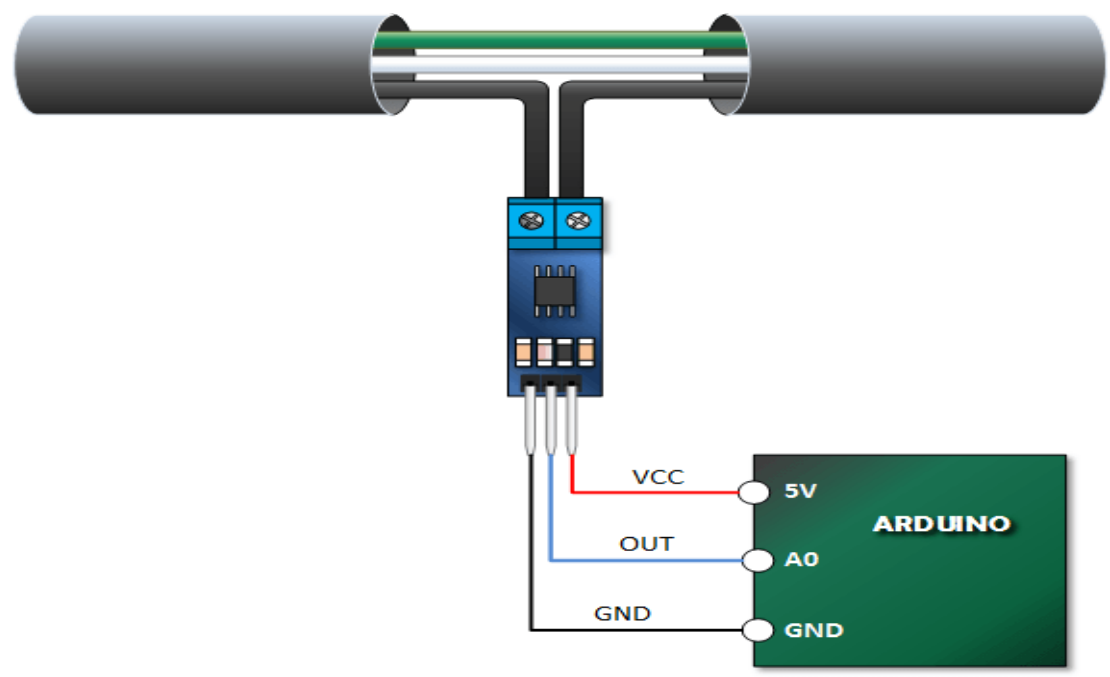

Figure 5 - Allegro Current Sensor (type:- ACS712)

The ACS712 current sensor measures AC current by calculating an RMS current value from the device readings.

For overvoltage and under voltage protection, a voltage sensor was used. Voltage limits were set and whenever these voltage levels where above or below set limits, the motor would be switched off through a relay. The voltage sensor used was a ZMPT101B voltage transformer shown below in figure 6.

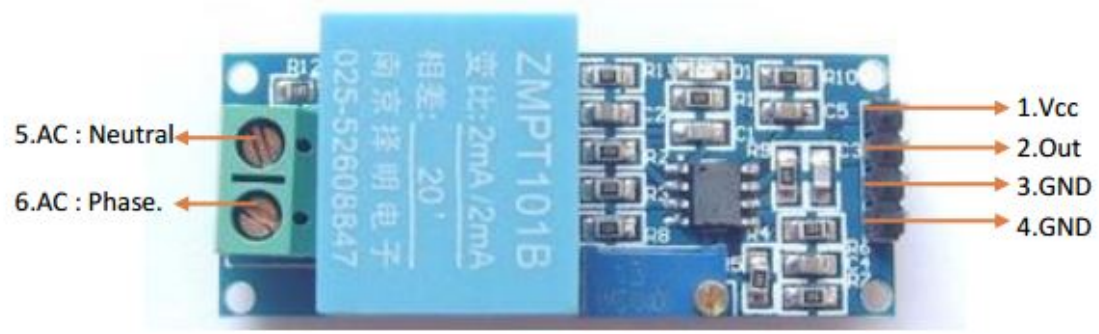

Figure 6 - Voltage Sensor ( type :-ZMPT101B)

The voltage sensor was connected to the Arduino and the voltage levels set and included in the code. Any voltages below or above the set limit would cause the motor to be switched off.

All the measured parameters; voltage, temperature and current, were stored for later analysis in a Micro SD storage card via the Arduino. The real time clock provided a 
timestamp each time data was written to the card. The micro SD card shield was as shown below in figure 7 .

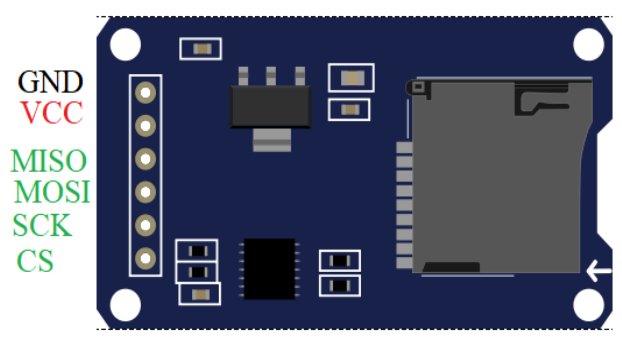

Figure 7 - Micro SD card Shield

The shield communicates with the Arduino via the serial peripheral interface (SPI) which is a synchronous serial data protocol used by microcontrollers for communicating with one or more peripheral devices quickly over short distances. It can also be used for communication between two microcontrollers. With an SPI connection there is always one master device (usually a microcontroller) which controls the peripheral devices. There are three lines common to all the devices:

- MISO (Master in Slave Out) - The Slave line for sending data to the master,

- MOSI (Master Out Slave in) - The Master line for sending data to the peripherals,

- SCK (Serial Clock) - The clock pulses which synchronize data transmission generated by the master

There is also a line specific to each device called the Slave Select (SS) / Chip Select (CS) which can be used by the master to enable and disable specific devices. When the SS/CS pin of a device is low, it communicates with the master and when it is high, it ignores the master. This allows one to have multiple SPI devices sharing the same MISO, MOSI, and CLK lines (ARDUINO AG, 2017).

The real time clock (RTC) used to provide time stamps was as shown below in figure 8.

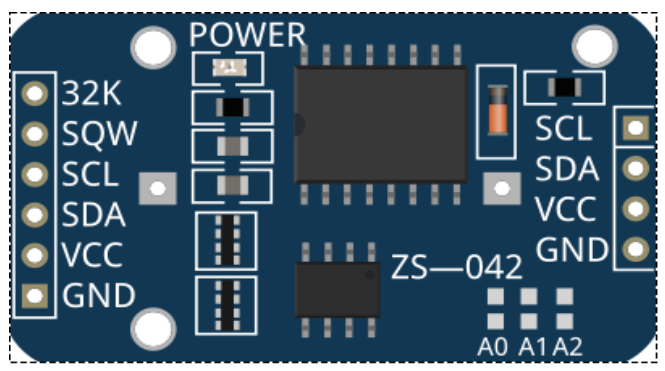

Figure 8 - Real Time Clock 
The real time clock used was a DS1307 RTC which communicates with the microcontroller using $\mathrm{I}^{2} \mathrm{C}$ communication. $\mathrm{I}^{2} \mathrm{C}$ uses two lines to communicate with the slave devices; they are called Serial Clock (SCL) and Serial Data (SDA). The SCL line is the clock signal which synchronizes the data transfer between the devices on the $I^{2} \mathrm{C}$ bus and it is generated by the master device and the SDA line carries the data. Since each device has a unique address, the master specifies the address of the device it wants to communicate with (Nedelkovski, .2015).

The circuit diagram of the data logging unit is shown below in figure 9 .

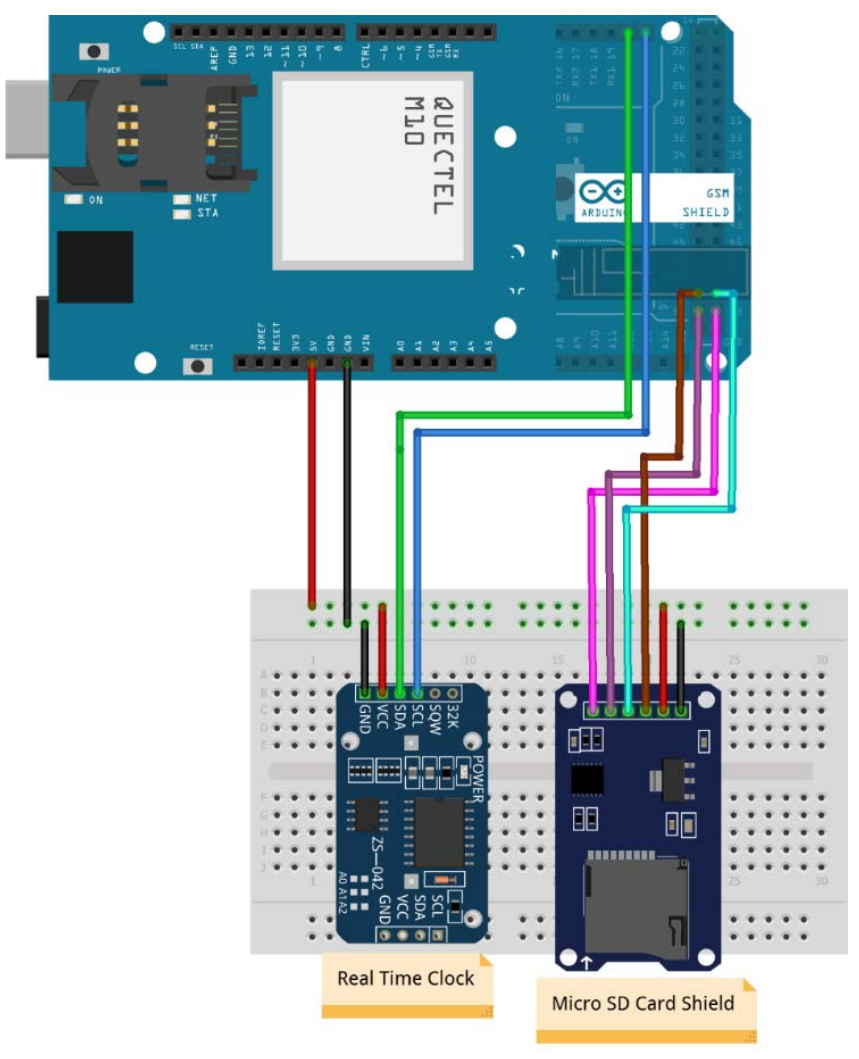

Figure 9 - Data Logging Unit

The data logging and function of the real time clock were verified when all the units were brought together to see if the values for voltage, current and temperature were saved in the memory card with timestamps. A code was then written for the full circuit.

The functional units were consolidated to give the full circuit of the system which was as shown in figure 10. 


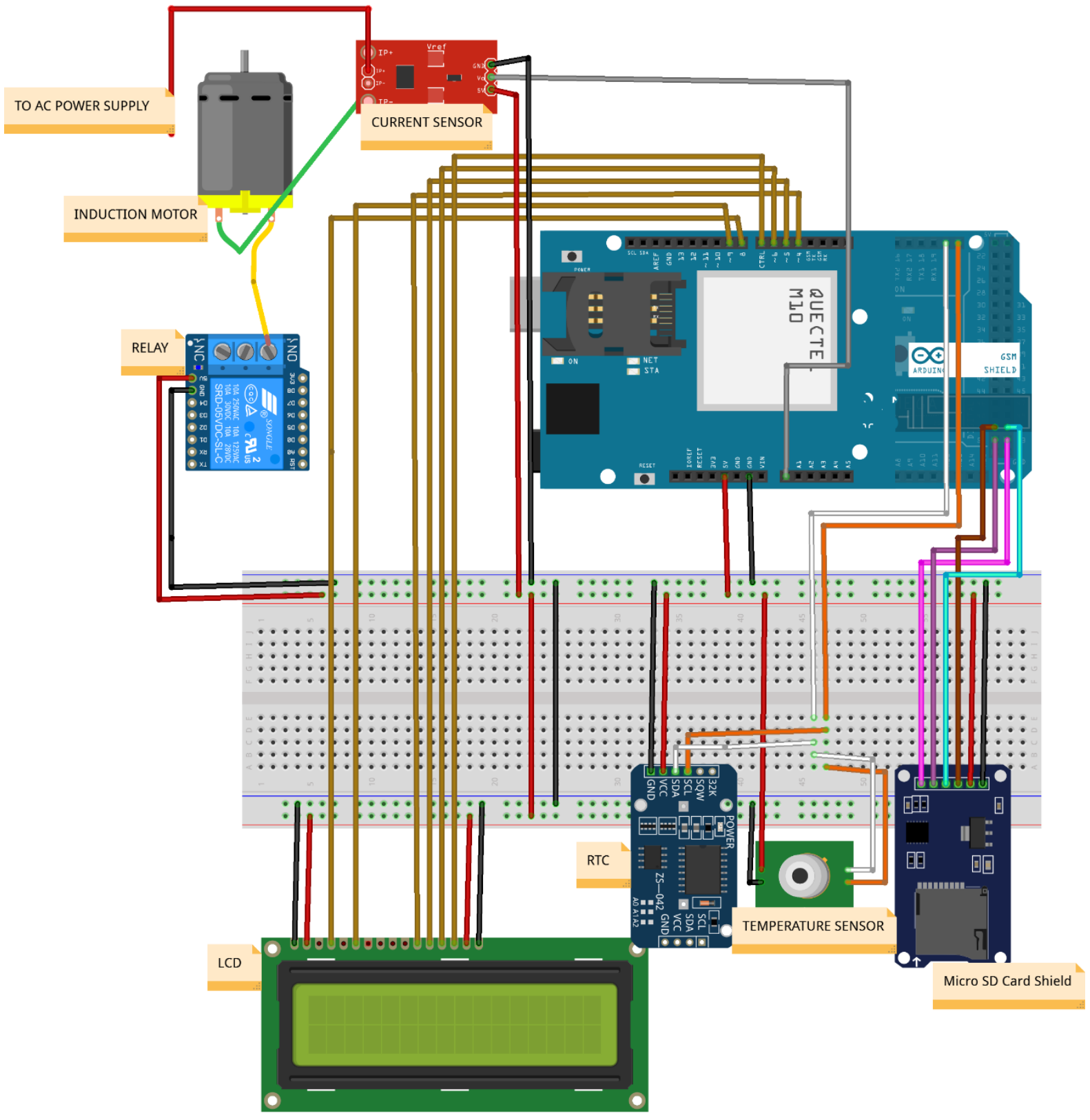

Figure 10 - Full Circuit Diagram

Figure 10 shows a full circuit diagram illustrating the interconnections of the system. The functional units of the system were integrated including the data logging function to see how they work together. The setup was as shown in figure 11. 


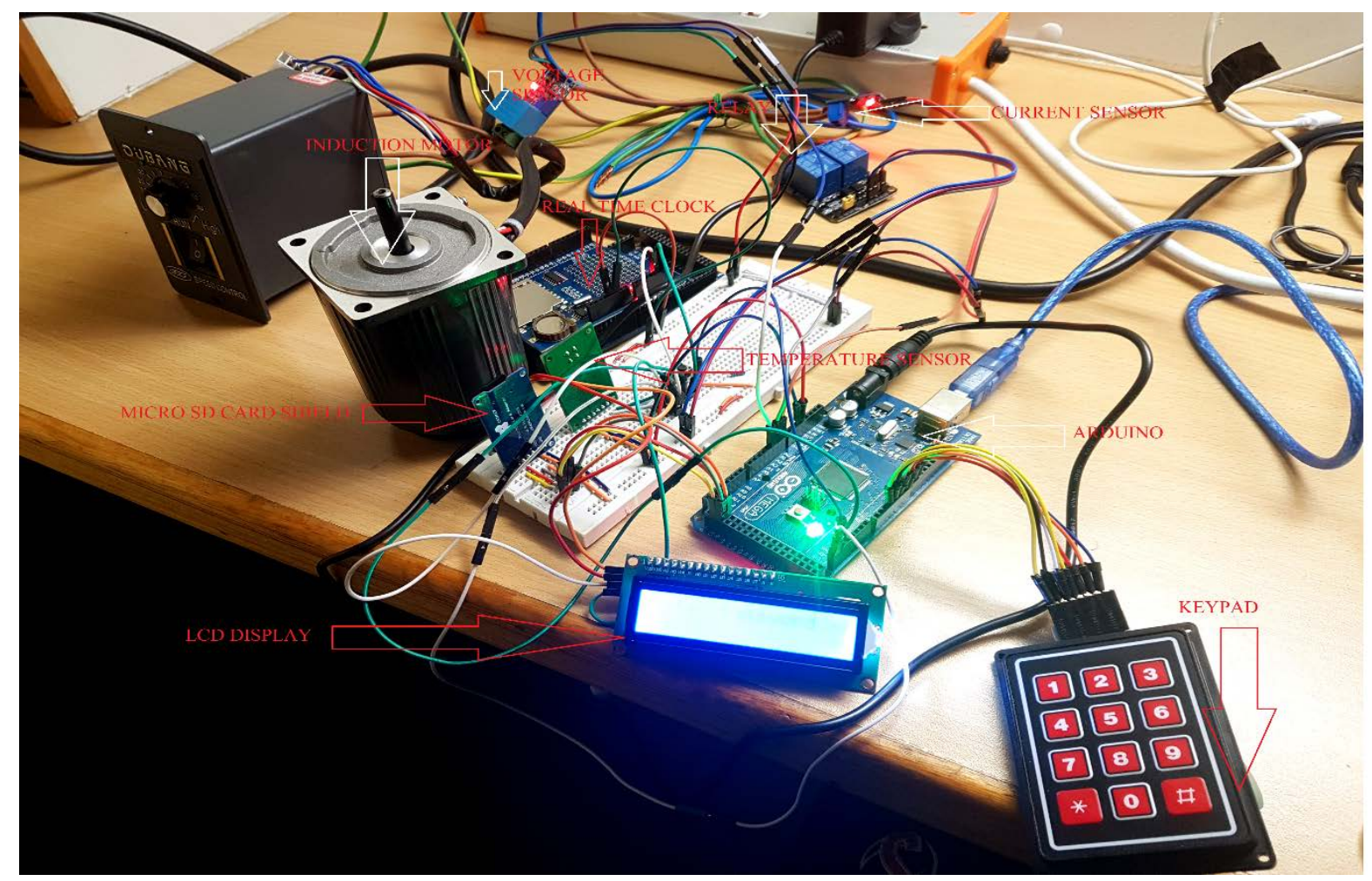

Figure 11 - Fully Integrated System

The system was tested to check on its response on the current protection unit, temperature protection unit and the overall system with all the units integrated. The motor used for the prototype was a single phase 220 Volts, $6 \mathrm{~W}$ induction motor. In the case of overcurrent, overvoltage and under-voltage, when detected, the system was designed to have a delay of up to 5 seconds and after these seconds, it would check if the condition still exists and if so, then the motor would trip. The value of the delay time could be changed to suit different practical conditions.

The Arduino Integrated Development Environment (IDE) serial monitor was also used to view the system prompts and to view the data being acquired by the system as well as its outputs.

\section{Results and discussion}

Some of the current and temperature monitoring results taken were considered. Figure 12 below shows the response of the system on the Serial Monitor due to an overcurrent condition. 


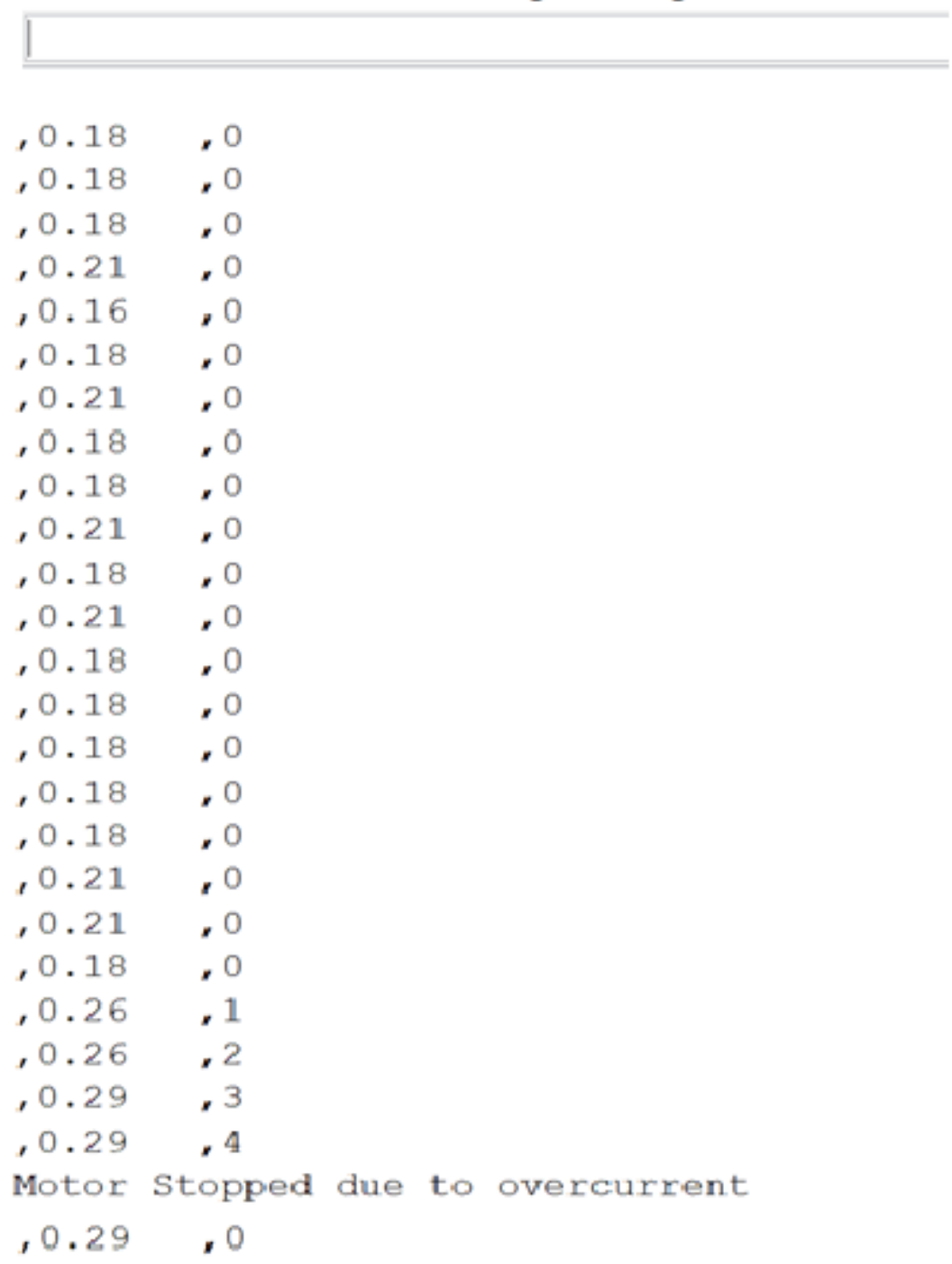

Figure 12 - Overcurrent Condition Shown On Serial Monitor

As shown in figure 12, the serial monitor showed the current level in Amps and the time in seconds from the system. The same was logged on the system. The overcurrent rose from 0.26 A to $0.29 \mathrm{~A}$, and occurred for 4 seconds and thereafter the motor was stopped on the $5^{\text {th }}$ second as per the design.

Figure 13 shows the temperature values and a high motor temperature condition being displayed on the serial monitor. 


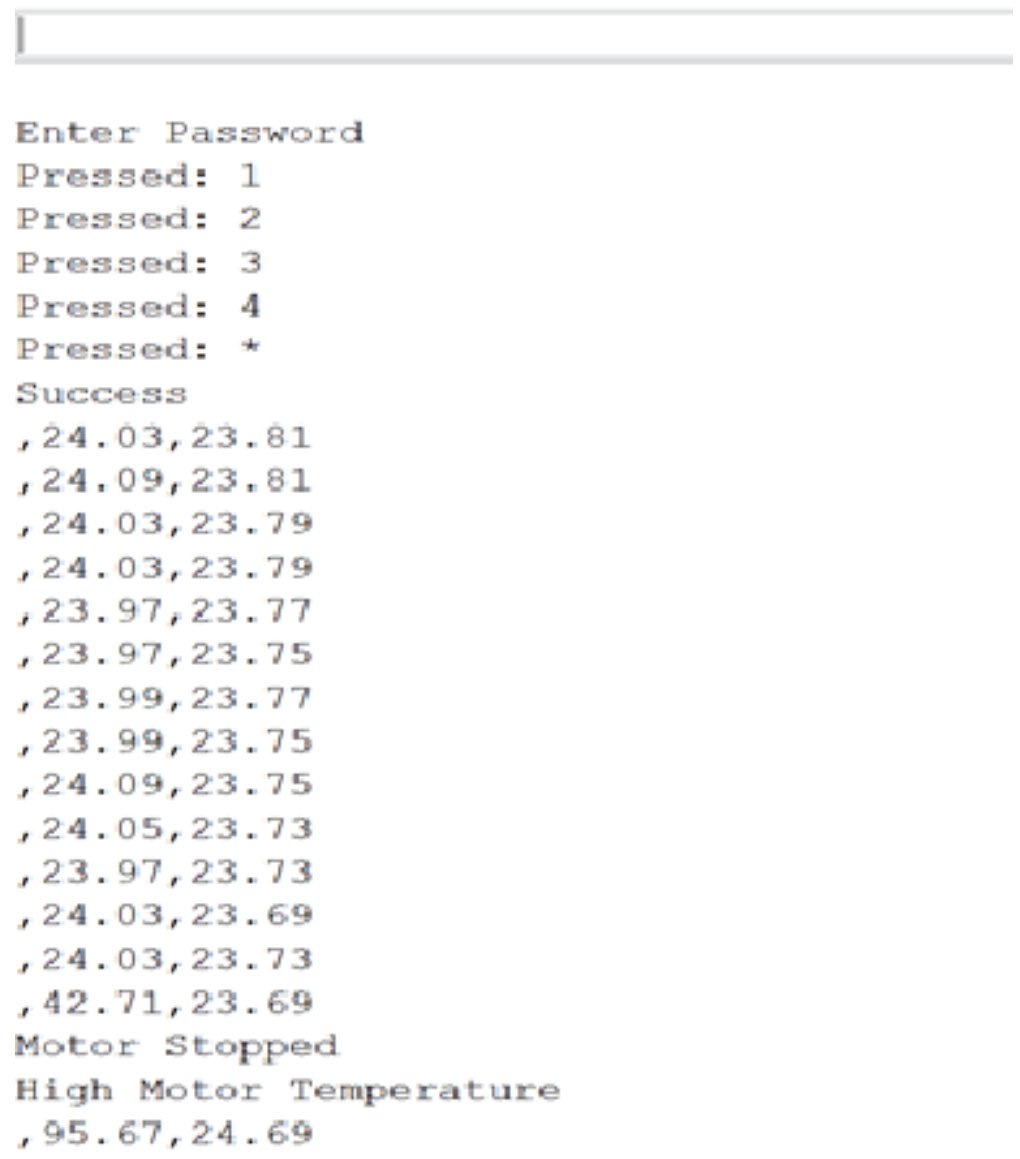

Figure 13 - Temperature Condition Shown On Serial Monitor

Figure 13 shows the motor and ambient temperature on the serial monitor. The left column shows the motor temperature and the right column shows the ambient temperature. The maximum ambient and motor temperatures were set at $50^{\circ} \mathrm{C}$ for this test and as illustrated the motor temperature rose from $24.03^{\circ} \mathrm{C}$ then to $42.71^{\circ} \mathrm{C}$ and the to $95.67^{\circ} \mathrm{C}$, which was a high motor temperature and automatically brought the motor to a stop. This high temperature condition was also displayed on the LCD as shown in figure 14. 


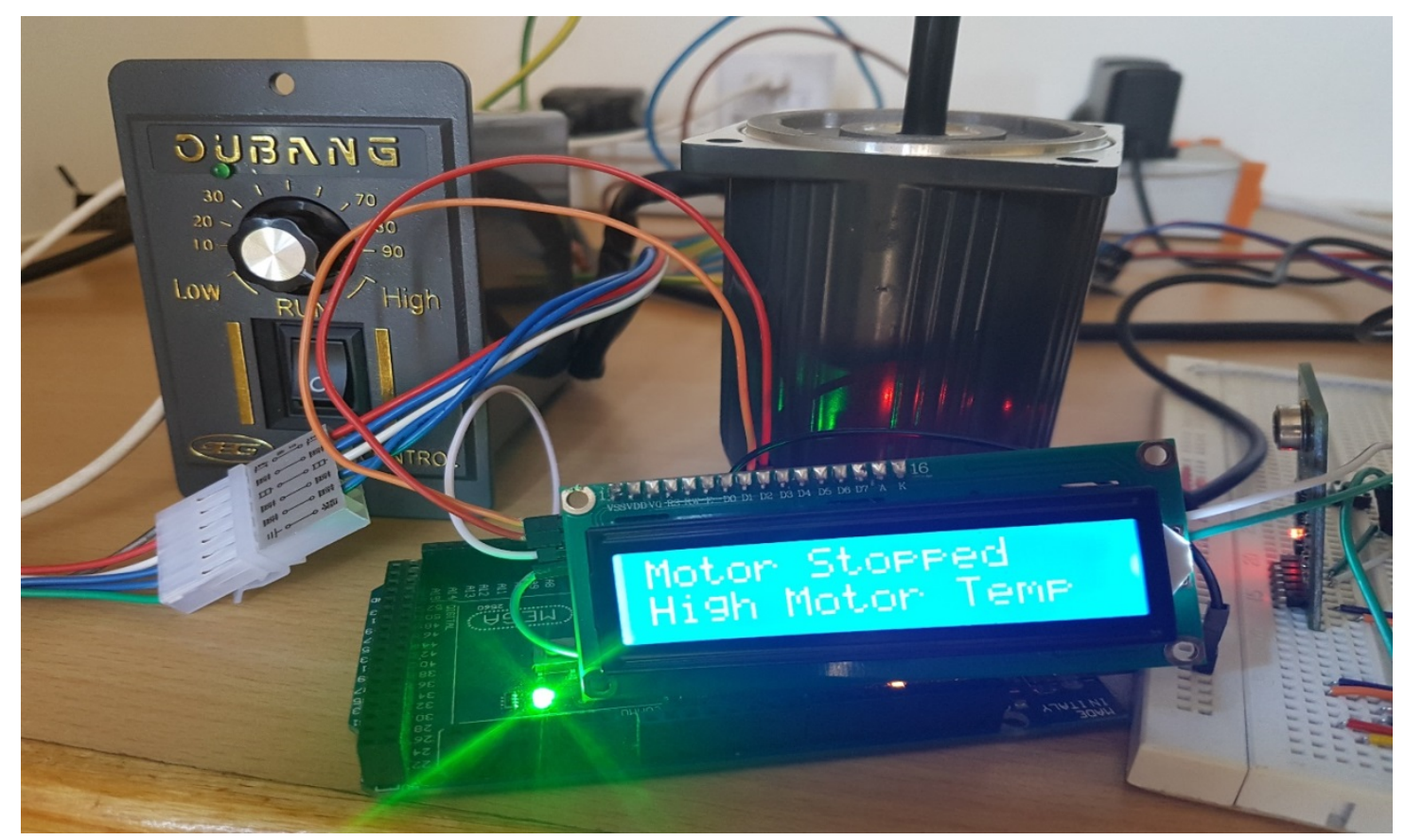

Figure 14 - High Temperature Condition On LCD

Data for both, temperature variations and current variations could also be displayed on the serial monitor. Table 1 below shows results displayed for a High Motor Temperature Condition test in the Integrated System as displayed in the serial monitor;

Table 1 - High Motor Temperature Condition tested in the Integrated System

\begin{tabular}{|c|c|c|c|c|c|}
\hline Time(s) & Voltage & Current & $\begin{array}{c}\text { Motor } \\
\text { Temperature }\end{array}$ & $\begin{array}{c}\text { Ambient } \\
\text { Temperature }\end{array}$ & $\begin{array}{c}\text { Event } \\
\text { Seconds }\end{array}$ \\
\hline 12 & 211.73 & 0.21 & 22.35 & 24.75 & 0 \\
\hline 14 & 213.24 & 0.18 & 22.35 & 24.77 & 0 \\
\hline 15 & 208 & 0.21 & 22.37 & 24.77 & 0 \\
\hline 16 & 209.58 & 0.18 & 22.35 & 24.75 & 0 \\
\hline 17 & 211.22 & 0.18 & 22.41 & 24.77 & 0 \\
\hline 19 & 211 & 0.21 & 22.43 & 24.75 & 0 \\
\hline 20 & 212.95 & 0.21 & 22.47 & 24.77 & 0 \\
\hline 21 & 206.79 & 0.21 & 22.43 & 24.77 & 0 \\
\hline 22 & 210.61 & 0.21 & 22.43 & 24.77 & 0 \\
\hline 24 & 211.29 & 0.21 & 22.19 & 24.81 & 0 \\
\hline 25 & 211.92 & 0.18 & 22.1 & 24.77 & 0 \\
\hline 26 & 208.22 & 0.18 & 22.37 & 24.77 & 0 \\
\hline 27 & 212.29 & 0.18 & 22.51 & 24.75 & 0 \\
\hline 29 & 208.12 & 0.21 & 22.43 & 24.77 & 0 \\
\hline 30 & 209.17 & 0.21 & 22.29 & 24.77 & 0 \\
\hline 31 & 210.03 & 0.18 & 22.43 & 24.77 & 0 \\
\hline 33 & 207.55 & 0.18 & 76.99 & 25.09 & Motor Stopped \\
\hline
\end{tabular}

Table 1, shows currents' flowing through the motor, ambient and motor temperature the motor is exposed to and a high temperature of $76.99^{\circ} \mathrm{C}$ that was detected by the 
system. This high temperature caused the motor to be brought to a stop. The table further shows the temperature, current and voltage levels across the motor.

Graphically, the variations of the motor current when the current increases beyond the set limit is shown in figure 15 .

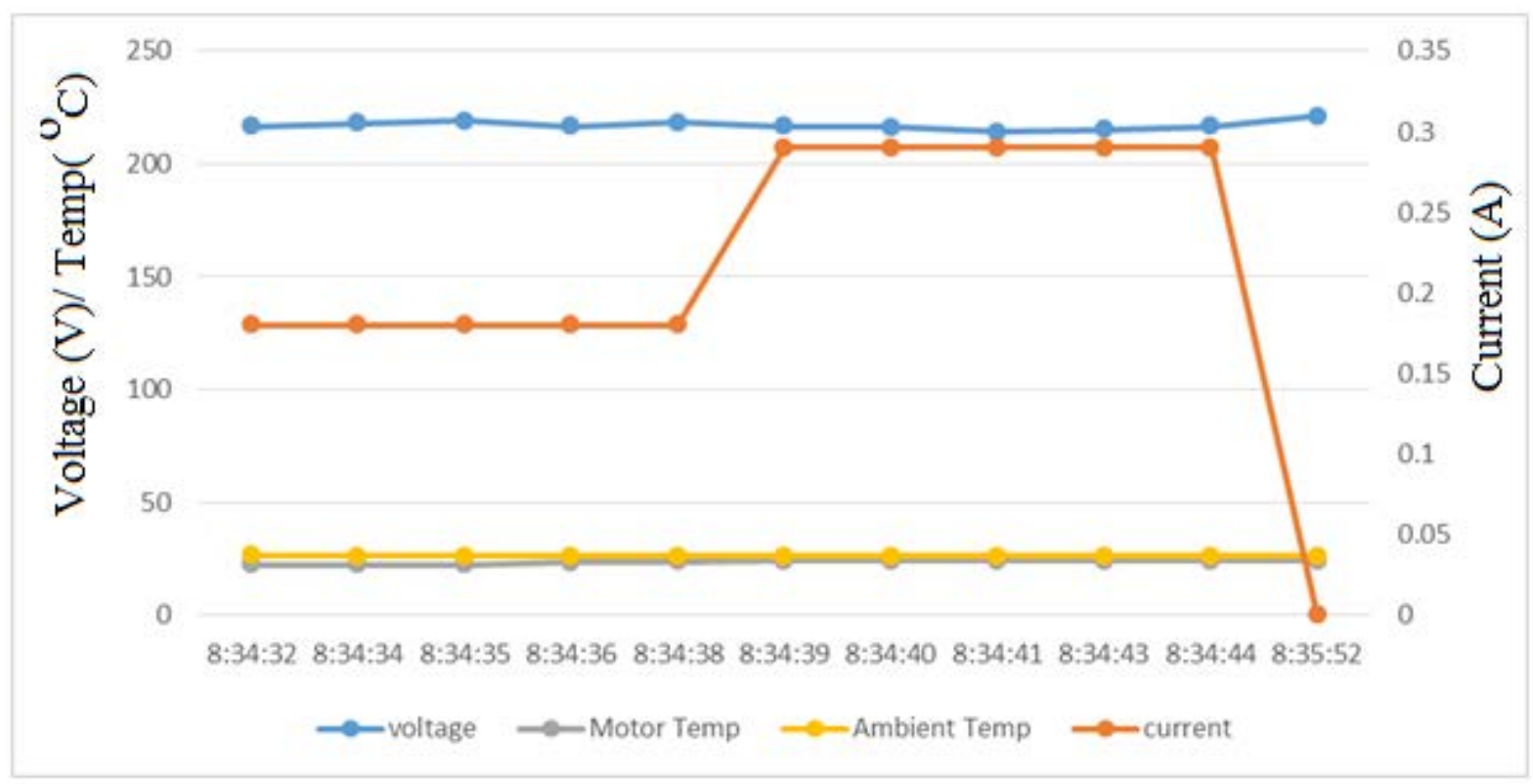

Figure 15 - High motor current condition.

From figure 15 it can be noted that when the current drawn by motor rose to $0.29 \mathrm{~A}$, the motor current dropped to zero, indicating a high current flow through the motor.

The ambient temperature, motor temperature and the input supply voltage remained constant due to the fact that there were no disturbance with regards these parameters on the motor.

For high temperature variations, figure 16 illustrates the response of the system.

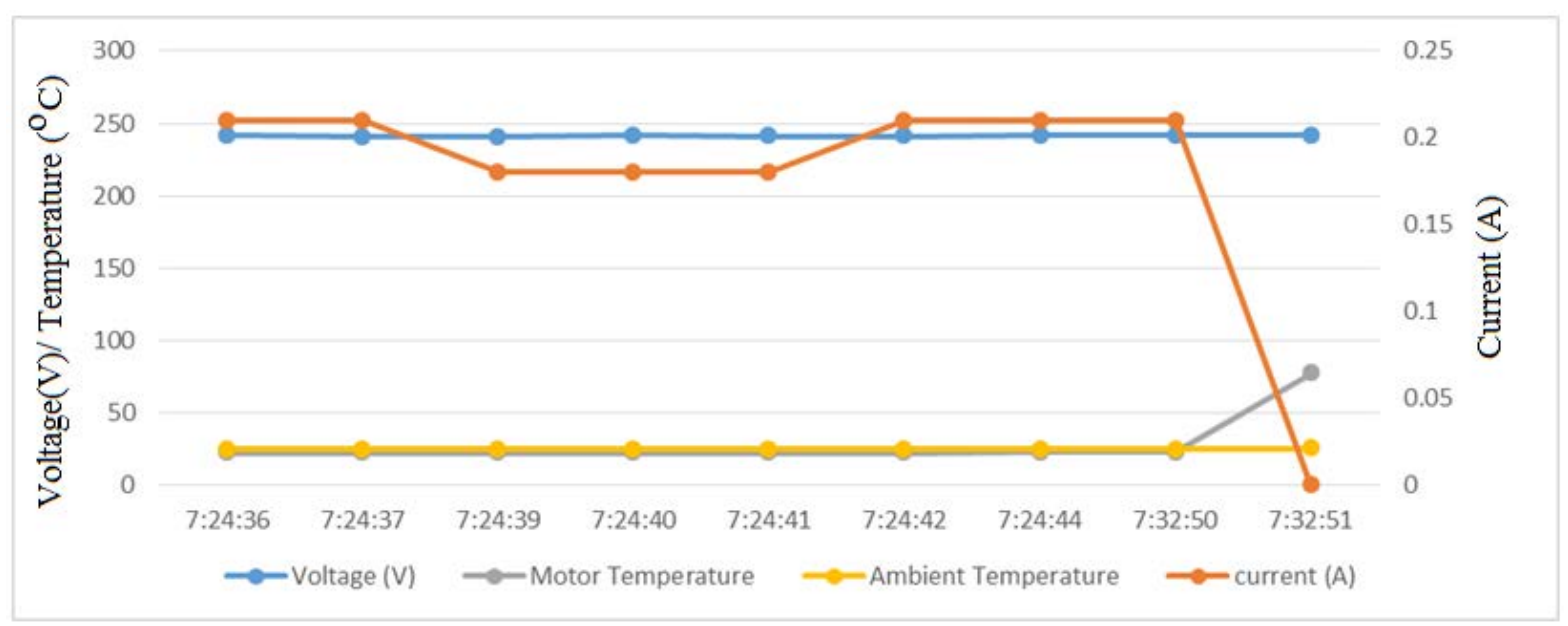

Figure 16 - Current response of motor to temperature changes 
The moment the temperature starts to rise beyond the set value, the current supply to the motor is immediately cut through the use of a relay getting a command from the code stored in the arduino.

\section{Conclusion}

An automatic motor protection system was designed. The system could stop the motor when set conditions were exceeded. It is evident that electromechanical systems can be protected from overcurrent and high temperature levels when in use. Such systems would be able to save lots of capital in terms of purchasing new motors by industries if put into use.

\section{References}

Allegro Microsystems. (2017). ACS712: Fully Integrated, Hall-Effect-Based Linear Current Sensor IC with 2.1 kVRMS Voltage Isolation and a Low-Resistance Current Conductor. Retrieved from: http://www.allegromicro.com/en/Products/Current-Sensor-ICs/Zero-ToFifty-Amp-Integrated-Conductor-Sensor-ICs/ ACS712.aspx

ARDUINO AG. (2012). Arduino MEGA 2560 \& Genuino MEGA 2560. Retrieved from https:/ / www.arduino.cc/en/Main/ArduinoBoardMega2560.

ARDUINO AG. (2017). SPI library. Retrieved from: https://www.arduino.cc/en/reference/SPI.

Bishop T. H. (2013). Increase of motor reliability by monitoring and reducing operating temperature: Retrieved from

http:/ / www.plantengineering.com/single-article/increase-motor-reliabilityby-monitoring-and-reducing operatingtemperature/8fbd6b07e6090125cb576f9e667d8ca4.html

Craig. W and Multilin. GE. (2010). Motor Protection Principles. Retrieved from https:// www.1-3.com/private/ieee/Motor\%20Protection\%20Principles.pdf.

Cowern E. (2000). The Highs and Lows of Motor Voltage. Retrieved from http:/ / ecmweb.com/ design/highs-and-lows-motor-voltage.

Nedelkovski, D. (2015). How I2C Communication Works and How To Use It with Arduino. How to Mechatronics. Retrieved from

http://howtomechatronics.com/tutorials/arduino/how-i2c-communicationworks-and-how-to-use-it-with-arduino/.

Rockwell Automation. (2016).Electromechanical Temperature Controls. Retrieved from https://ab.rockwellautomation.com/Sensors-Switches/TemperatureSensors/Electromechanical. 


\title{
2018 International Conference on Multidisciplinary Research Track 1: STEM (Science, Technology, Engineering \& Mathematics) ISBN: 978-99949-0-384-9
}

\section{Customer Satisfaction: A South African Case}

\author{
Kemlall Ramsaroop Ramdass \\ University Of South Africa, \\ Johannesburg, South Africa
}

\begin{abstract}
Customer satisfaction is one of the most important variables when applying the ISO 9000 quality management system. Customer satisfaction forms the basis of quality improvement initiatives and therefore needs to be measured through its dimensions and specifications. For these reasons it is vital for the organisation to develop a plan of action to address and improve customer satisfaction on a continuous basis. The primary objective of this research was to assimilate characteristics that would improve quality and customer satisfaction on an automotive seat cover production line. "Sewing", the organisation is currently experiencing difficulties with regards to the quality of its products. The current high level of customer complaints as well as internal data related to scrap, rework, and quality issues indicate that product quality is a major contributor to this dissatisfaction. The investigation applied the qualitative research methodology using a case-study as the primary instrument for data collection.
\end{abstract}

Keywords: customer satisfaction, quality, industrial engineering, continuous improvement.

\section{Introduction}

The plant is divided into two operating units; Sewing (the unit) which stitches the complete seat cover and Seat Assemblies (the unit) which trim and assemble the complete seat sets as a JIT (Just-in-Time) supplier for a large automotive manufacturer. Customers are very particular about the quality of the product they receive as this automotive manufacturer is on the upper end of the automotive chain. Thus, Sewing has received numerous complaints from the customer on the quality of their products. The industry demands zero defects and tolerance on product manufacture. In view of the operation being a labour intensive one, it places major strain on all operations and operators. Taking into account that the products manufactured by Sewing are extremely labour intensive, it is a challenging task for perfection in all operations. Sewing has recently received various complaints and corrective action requests from customers regarding the quality of supplied product which clearly impacted negatively on customer satisfaction. 


\section{Method}

The research approach that has been selected for this study is a qualitative research design in conjunction with a case study methodology. The data collection was done through interviews with operators, supervisors, managers and technical personnel through discussions. Applicable literature was reviewed to develop an in-depth understanding into the said phenomenon. The case study methodology enables the researcher to focus on the core issues impacting all operations with a focus on quality. The approach may be considered controversial, but is widely used to gain in-depth explanations regarding the aspects that may impact quality on the product.

\section{Literature Review}

Customer satisfaction is measured through the lens of the customer in relation to the experience gained though the utilisation of a product (Goetsch and Davis, 2014; Kaynak; 2003). According to Foster (2015) the customer's experience is based on several characteristics such performance, conformance, reliability, features, serviceability, durability, aesthetics and perceived quality. In addition, product quality is also measured on customer service characteristics in terms of assurance, empathy, tangibles, reliability and responsiveness. Thus, quality management dimensions play a crucial role in customer satisfaction and require measurements on a continuous basis (Kaynak, 2003; Basu and Wright, 2003).

Goetsch and Davis (2015) defined total quality management as a philosophy that was built on a corporate vision focussed on customer satisfaction through corporate engagement on continuous improvement. To achieve this vision an organisation is required to develop systems and processes that strive to perfect customer requirements. The other stakeholders are; employees, suppliers, business partners, society and owners (shareholders). These business goals are also referred to as Critical Success Factors (CSFs). These factors are aligned towards customer and other stakeholders' satisfaction (Brandt, 2008).

Having identified the critical success factors is not enough to continuously improve the satisfaction of customers (and other stakeholders' satisfaction). Measurable goals are formulated in the form of Key Performance Indicators (KPIs). Juran (2014) postulated these as "measurable goals for control subjects".

Juran identified and categorised the control subjects into the following five quality goals:

- Product performance

- Competitive performance

- Quality improvement

- The cost of poor quality

- The performance of macro processes

These control subjects serve the same purpose as the critical success factors discussed above, which is to identify the important goals in achieving the mission of the organisation. 


\section{Quality dimensions related to the automotive industry}

Curkovic, Vickery \& Droge (2000) alluded to seven competitive characteristics. These were product quality, design quality, conformance to specifications, product durability, and product reliability. The service characteristics were pre-sale customer service, product support and customer responsiveness. This approach emphasised that both product quality and service quality are imperative the for automotive supply chain (Baird, Jia Hu, and Reeve, 2011; Cyrne, 2005).

It would seem obvious that seat covers is defined as the product of Sewing, but the following view point should also be considered. Since none of the design and development rights related to these seat covers belong to Sewing, one can argue that the product (seat covers) belong to the customer and Sewing is merely providing a manufacturing service for the customers product. When defining the product of Sewing as a manufacturing service, the quality dimensions are redefined as in table 1. By redefining the product of Sewing as providing a manufacturing service; reliability, durability and product design becomes relevant as service dimensions (Terziovski, 2006; Talib, Rahman, and Qureshi, 2011).

Table 1. Product definition: Provision of Manufacturing Service

\begin{tabular}{|l|l|l|}
\hline $\begin{array}{l}\text { Quality } \\
\text { dimension }\end{array}$ & Description to & $\begin{array}{l}\text { Relevance tor Sewing } \\
\text { Lear }\end{array}$ \\
\hline $\begin{array}{l}\text { Product (or } \\
\text { service) reliability }\end{array}$ & $\begin{array}{l}\text { Reduction of manufacturing system failure } \\
\text { or malfunction }\end{array}$ & Relevant \\
\hline $\begin{array}{l}\text { Product (or } \\
\text { service) durability }\end{array}$ & $\begin{array}{l}\text { Maximisation of the time to system } \\
\text { replacement (or part of the system) }\end{array}$ & Relevant \\
\hline $\begin{array}{l}\text { Conformance to } \\
\text { specifications }\end{array}$ & $\begin{array}{l}\text { Provision of manufacturing services that } \\
\text { meet established performance standards. }\end{array}$ & Relevant \\
\hline $\begin{array}{l}\text { Service Design } \\
\text { quality }\end{array}$ & $\begin{array}{l}\text { Provision of cover manufacturing services } \\
\text { with superior design quality. }\end{array}$ & Relevant \\
\hline $\begin{array}{l}\text { Pre-sale (or Pre- } \\
\text { launch) customer } \\
\text { service }\end{array}$ & $\begin{array}{l}\text { Customer service during the pre-launch } \\
\text { phase. }\end{array}$ & Relevant \\
\hline Product support & Provide after sale customer service. & Relevant \\
\hline $\begin{array}{l}\text { Responsive to } \\
\text { customer }\end{array}$ & Timely response to needs. & Relevant \\
\hline
\end{tabular}

The SERVQUAL quality dimension specifically for the organisation is listed in the table 2.

Table 2. Service quality dimensions defined for Sewing

\begin{tabular}{|l|l|}
\hline Dimension & Definition as related to Lear Sewing \\
\hline Reliability & $\begin{array}{l}\text { Ability to perform the promised cover manufacturing } \\
\text { service dependably, accurately and on time without system } \\
\text { failures or breakdown in supply. }\end{array}$ \\
\hline Assurance & Employees courteous and knowledgeable ability to inspire \\
\hline
\end{tabular}




\begin{tabular}{|l|l|}
\hline & trust and confidence. \\
\hline Tangibles & Appearance of personnel, physical facilities and equipment. \\
\hline $\begin{array}{l}\text { Conformance to } \\
\text { specifications }\end{array}$ & Manufacture to specifications \\
\hline $\begin{array}{l}\text { Pre-launch customer } \\
\text { service }\end{array}$ & Customer service during the pre-launch phase. \\
\hline Responsiveness & Timeous response to needs and wants including complaints. \\
\hline
\end{tabular}

\section{Continuous Improvement Capability}

According to Bessant \& Francis (1999) Continuous Improvement (CI) can be considered as "dynamic capability". They mention the capability of organisations to harness all the possessed potential to continuously improve all facets. They mention that CI offers mechanisms for rejuvenation in innovation and learning. This relates to "kaizen" and transcends to the "lean" approach (Gryna, 2014). This capability is viewed as a cluster of behavioural routines that can offer a significant competitive potential (Eskildsen and Kristensen, 2007). These behaviour patterns are consume time to institutionalise, and are difficult to transfer. A model developed by Bessant and Francis describe the various levels in the development of CI capability and can be used to position a particular organisation on this journey.

\section{Results and Discussion}

\section{Current level of customer satisfaction}

Managers responded that they were of the opinion that customer satisfaction was at an acceptable level. Managers described the level of customer satisfaction as; "Satisfactory", "Fairly good," "customer is relatively happy", "No major customer concerns, only minor complaints". One manager mentioned that quality and customer satisfaction comes at a price. He mentioned appraisal costs with regards to inspection and quality control mechanisms that were in place. He also referred to failure costs with regards to scrap and rework that was at a high level.

\section{Factors that impact on customer satisfaction}

Managers' response to factors they believed impacted customer satisfaction is listed in table 3 below. The left column lists the factors while the right column indicates the number of managers that responded to the factor.

Table 3. Factors impacting on customer satisfaction - Management

\begin{tabular}{|l|l|}
\hline Factors & $\begin{array}{l}\text { Number of times } \\
\text { mentioned }\end{array}$ \\
\hline Delivery on time & 6 \\
\hline Product quality (conformance) & 6 \\
\hline Cost & 2 \\
\hline Responsiveness to customer complaints & 1 \\
\hline Re-occurring customer complaints & 1 \\
\hline
\end{tabular}




\section{Product quality vs service quality}

Managers were asked to differentiate between product and service quality and provide their opinion as to the importance of these two equally important variables in terms of customer satisfaction. This is summarised in the figure below.
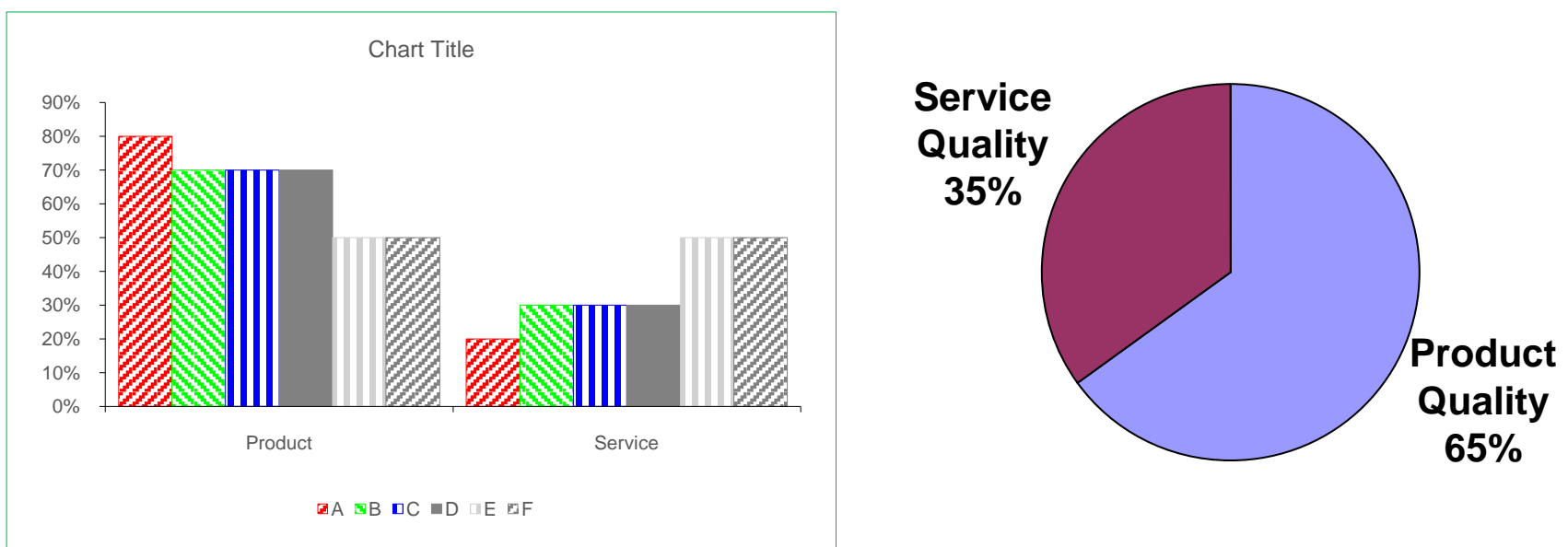

Figure 1. Percentage split between service quality and product quality.

The response was summarised and concluded as a 65/35 split respectively. This seems to be a realistic assumption as product quality dimensions are more diverse and include customer satisfaction as one of the variables. A manager commented that the input into product quality is diverse and difficult to manage if the system is not geared to manage it. Product quality encompasses stringent controls at every stage from raw material evaluation to a completed seat. Service quality requires human interaction and is equally important in ensuring customer satisfaction.

\section{Quality dimensions}

Managers were asked to rate the importance of quality dimensions pertaining to the seat production line. In the rating 1 was considered most important while 5 was the least important. The summary is provided in the next table.

Table 4. Quality dimensions - Management rating

\begin{tabular}{|l|l|l|l|l|l|l|l|}
\hline \multirow{2}{*}{ Dimension } & \multicolumn{4}{|l|}{ Managers } & \multirow{3}{*}{ Total } \\
& response & \\
\cline { 2 - 6 } & 1 & 2 & 3 & 4 & 5 & 6 & \\
\hline Conformance to specifications & 3 & 1 & 1 & 2 & 1 & 2 & 10 \\
\hline Reliability & 2 & 4 & 2 & 1 & 2 & 1 & 12 \\
\hline Responsiveness & 1 & 2 & 3 & 4 & 3 & 3 & 16 \\
\hline Pre-launch customer service & 4 & 6 & 4 & 3 & 4 & 4 & 25 \\
\hline Assurance & 6 & 3 & 5 & 5 & 5 & 6 & 30 \\
\hline Tangibles & 5 & 4 & 6 & 6 & 6 & 5 & 32 \\
\hline
\end{tabular}


As deduced from the table management consider conformance, reliability and responsiveness (in this order) as the most important quality variables while tangibles, assurance pre-launch customer service were of lesser importance.

\section{Product Quality}

Product quality is determined by customers. Managers were tasked to evaluate the current level of product quality and to identify opportunities for improvement. The majority of managers mentioned that there was room for improvement, although product quality was at an acceptable level. Managers detailed a list of improvement initiatives that could be implemented.

- Extraction and analysis of data regarding defects

- Quality cycle approach on defects

- Team approach on the resolution of problems

- Scrap area to be relocated to a more visible area on the production line..

- Evaluation of supplier quality

- Evaluation of operator skills

- Continuous operator training and multi-skilling

- Evaluation of absenteeism

- Discipline on production lines to be improved.

- Root cause analysis to eliminate quality problems

- Stabilisation of machines and operators

- Maintenance schedule for all machines (Gregory, 2008).

\section{Service Quality}

Managers mentioned that responsiveness and reaction time required improvement. In addition a quality satisfaction survey was to be implemented to receive comments from customers. All information required screening and documentation to prevent re-occurrence (Gayriel, 2006; Green, 2006).

\section{Continuous Improvement}

Managers were asked to respond regarding their perception of the current continuous improvement strategy in all areas relating to product and service quality improvement. Managers complained about available resources, skills and training of operators, discipline in the department and the amount of waste in the organisation. They re-iterated that they were currently touching the surface and much more needs to be done on product and service quality.

\section{Employee survey}

The total staff compliment of Sewing at the time of the survey was 33 employees. Of the 33 employees, 24 responded to the survey. This equates to a response rate of $73 \%$. The results of the individual surveys was tabled and captured in an excel spreadsheet. The average response to each of the statements (both the agreement rating and importance rating) was calculated. These average response values were then plotted on a scatter chart to give a graphical presentation of the results (Figure 2). The agreement responses, which represent the perceived values, were plotted on the vertical axis and the importance values which represent the expected values 
were plotted on the horizontal axis. The diagonal line indicates the area on the scatter gram where the perceived value is equal to the expected value. Should the responses to a statement fall on this line, it would indicate that for the relevant statement, the perceived level of deployment for the approach equals the level that is expected for that approach. The distance between where a statement is plotted on the scatter chart and the diagonal line, indicates the difference between the perception and expectation ratings. If the statement is plotted above the diagonal line, it is an indication that the perception related to that statement is higher than the expectation. When plotted below the diagonal line it is an indication that the perception regarding the statement is below the expectation.

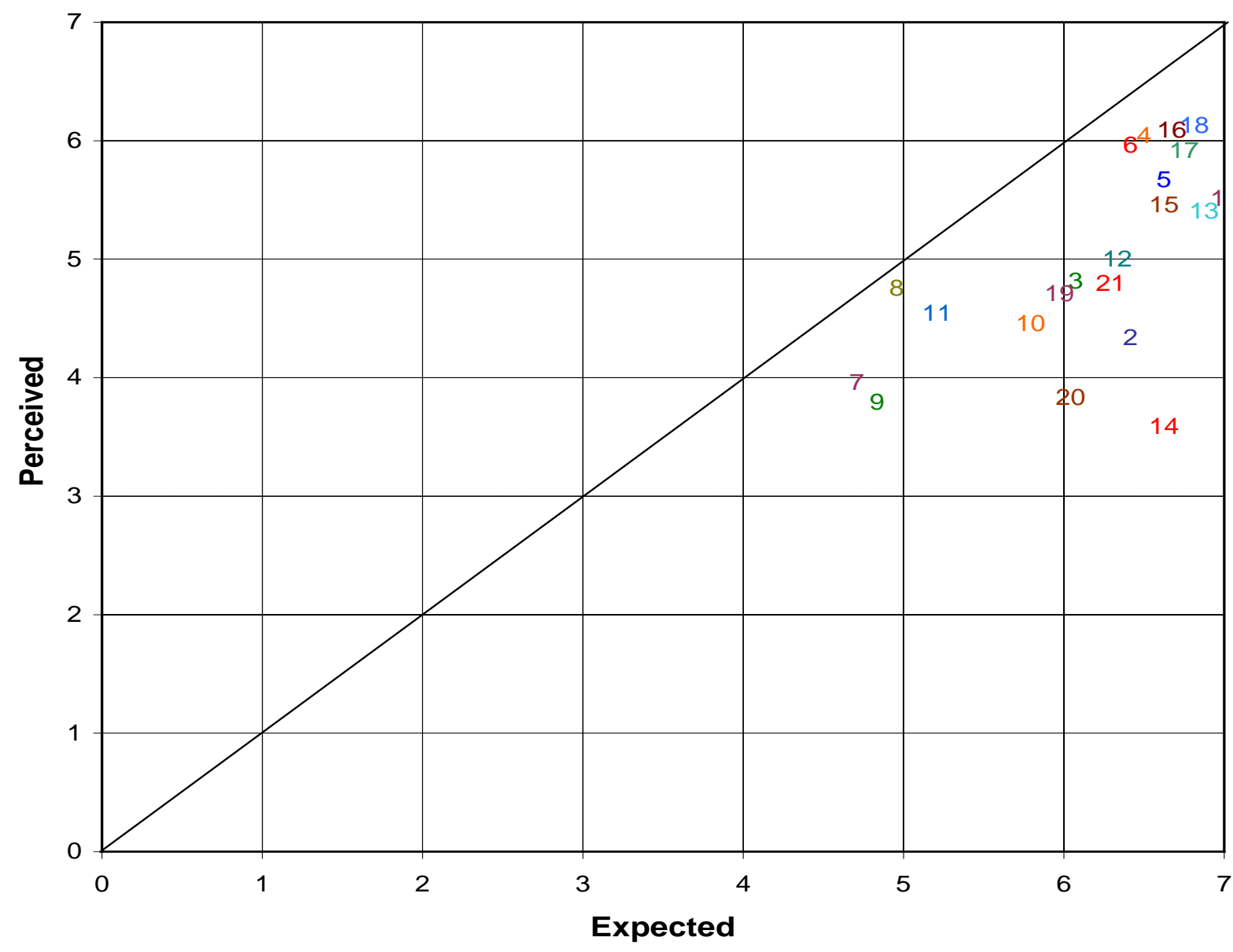

Figure 2. Scatter Chart of Employee Survey Results

The response to statement number eight, which stated that Sewing's physical facility is very appealing, is the closest to the diagonal line. This means that the level of agreement to this statement (perception) as it relates to Sewing is very close to the importance (or expectation) for this statement. The actual average importance rating for this statement is 4.95, which indicate that the importance of this statement as it relates to customer satisfaction was not very high. The average agreement rating to this statement is 4.75 , which indicated a slight but not high agreement to the statement. This is fairly close to the expectation value. On the other extreme, statement number 14, this stated that the reject rate of Sewing covers at the customer is exceptionally low, is the furthest away from the diagonal line. The expectation 
rating for this statement is 6.63, which indicates that Sewing employees believe that this statement is of very high importance to customer satisfaction, thus the expectation regarding the statement is very high. The actual perception rating for this statement is 3.58, which indicate a slight disagreement to the statement. The difference between this perceived value of 3.58 and the expected value of 6.63 indicates the gap that exists between the expectation and actual perception.

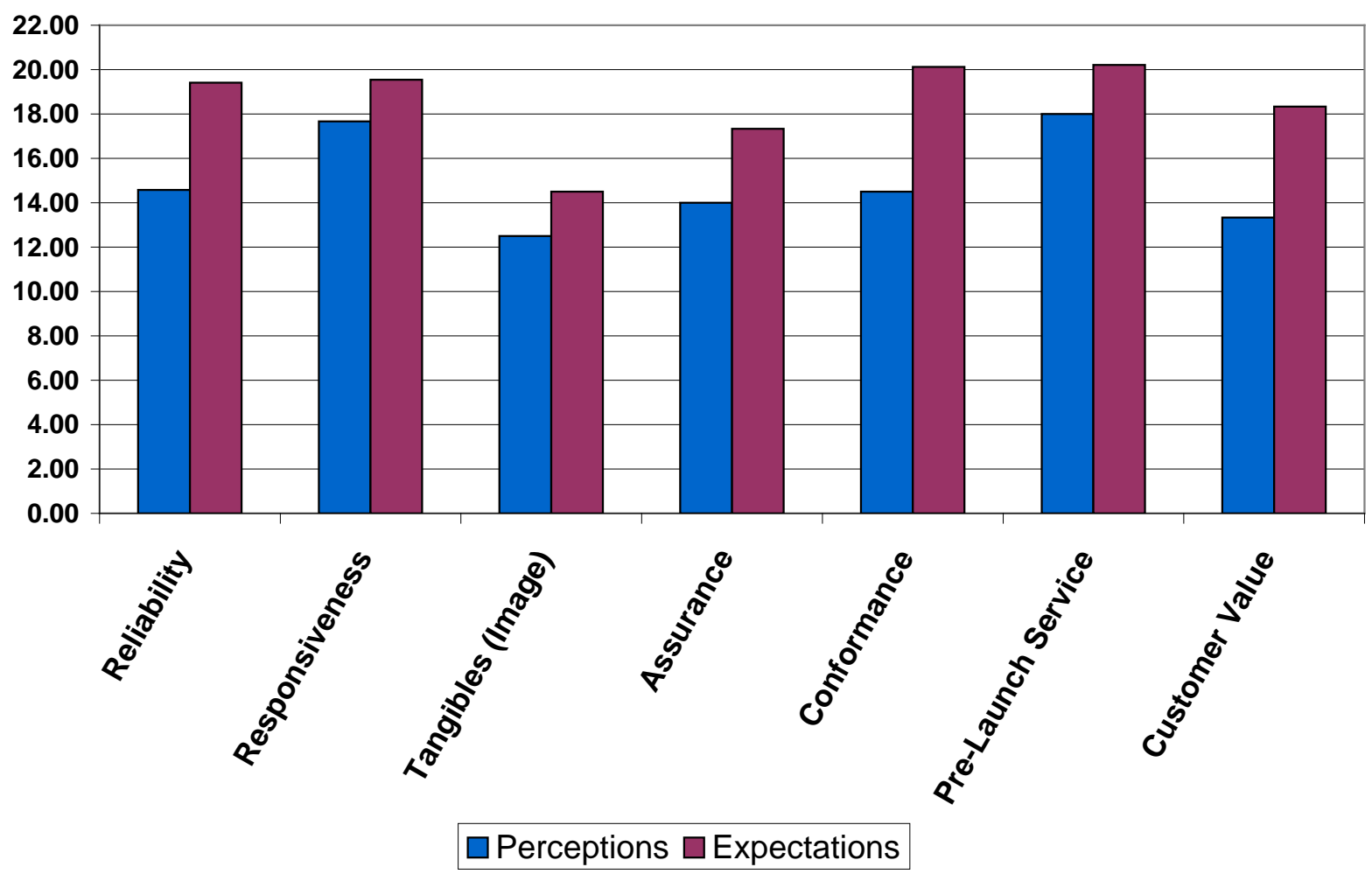

Figure 3. Perception/Expectation Histogram per Quality dimension

The importance or expectation rating indicated by the maroon coloured bars in the figure 3 indicate that Sewing employees' consider conformance, pre-launch customer service, responsiveness and reliability almost equally important with regards to customer satisfaction. The quality dimensions assurance and tangibles are rated as the least important quality dimensions.

For these quality dimension ratings the perception rating was again subtracted from the perception rating to illustrate the gap between the employees' perception and expected ratings for the relevant quality dimension. This is illustrated in figure 4 . 


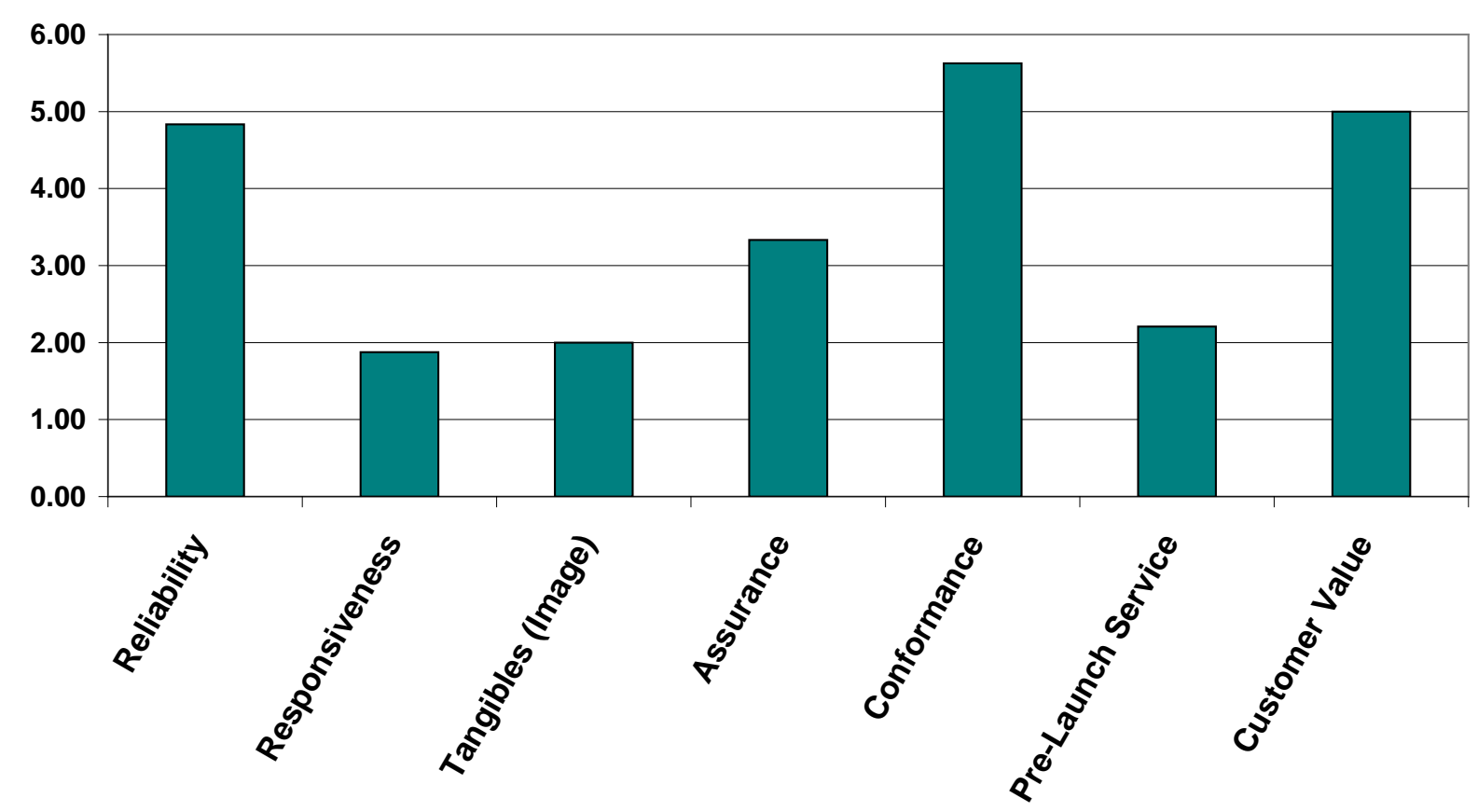

Figure 4. Gap Analysis Histogram per Quality dimension

This graph indicates that the employees of Sewing identify conformance, reliability, and customer value (in this order) as the areas where the biggest gaps exist between expectation and perception. The three quality dimensions; responsiveness, tangibles and pre-launch customer service are the quality dimensions perceived to be the closest to their respective expectations. This indicates that the biggest impact on customer satisfaction can be made by focusing on product conformance, reliability and customer value.

\section{Focus groups}

\section{Supervisor focus group}

The main issues that were raised during the supervisor focus group discussion as per the main focus points given in paragraph 5.4.1 are summarized below:

The first point for discussion was related to the feedback supervisors receive from the quality department regarding the quality of production. According to the supervisors their main source of feedback is directly from the final inspectors, as they always have up to date information available and they can enquire about the data at any time during their shift. Final inspectors would also alert the supervisors to major or re-occurring quality problems. Other feedback that they receive from quality is a weekly overview of the defects per operator, which is used to identify operators that need to be counselled or disciplined. Operators who exceed a certain amount of defects in a week are issued with a disciplinary warning. The overall perception of the supervisors is that the feedback they receive from the quality department is effective and that it does give a clear indication of the quality problems on the lines. 
The next point of discussion was the action that is taken on reported quality data and whether supervisors believe that these actions are sufficient. Supervisors admit that they themselves do not do enough to address the issues recorded by the inspectors. When machine breakdowns or supply problems occur they need to focus their attention on these problems, and once these are resolved they need to switch their focus to achieving the target, as these problems leave them with less time to do so. Supervisors claim that they do however address the major quality problems, as and when they are highlighted by the inspectors. Supervisors are also of the opinion that some inspectors do not always bring major or re-occurring problems as detected at final inspection to their attention. Quality problems are not only pointed out by inspectors, but also by the operators themselves. According to the supervisors a large amount of quality problems is related to machine settings which require theirs or a mechanics immediate attention, as most of the operators do not have the skills or knowledge to adjust and correct machine settings.

Supervisors were asked to give their opinions on the support they receive from their managers and other departments in solving quality problems. Supervisors indicated that their major frustrations in this regard are the lack of support from their managers and some of the maintenance staff. Supervisors expect their managers to assist them with major quality problems and expect the managers to involve other departments to assist with problems when required. They believe that their managers are not always interested in listening to their explanation or concerns regarding problems and believe that managers are more interested to know what disciplinary action was taken with regards to quality problems. For this reason supervisors would often contact other departments directly for assistance (the engineering department was mentioned as an example).

Supervisors also indicated that other departments such as cutting, logistics and maintenance do not share their sense of urgency regarding time, and a lack of timely service from these department often impact on the time available to achieve target. Supervisors also believe that there are employees in the company (with special problem solving skills) who could offer more assistance with regard to their quality related problems. The supervisors high-lighted a recent event where a large number of front backrest covers was rejected. In this instance they expected to see more support and involvement from other departments, including the continuous improvement department, in a team effort to solve the quality problems.

Another topic during the supervisor function group discussion was the problems and obstacles supervisors face with regards to addressing quality problems. In this regard supervisors mentioned machine problems. Some of the machines on the lines are fairly old and constantly cause quality problems. Incorrect machine settings, even on new machines, are another obstacle that the supervisors face. Even supervisors struggle to correct settings on some machines and have to rely on mechanics to adjust settings. Another frustration mentioned here was that some mechanics do not give feedback to the operator or to them as to what they have done to correct the problem. The efforts of one of the mechanics who usually explain to 
operators what he is doing while setting the machine was however appreciated by supervisors. Supervisors believe that if other mechanics share this approach their operators would become more knowledgeable regarding machine settings and rely less on mechanics over time. The combination of absenteeism and a lack of multi skilled operators pose another problem, as supervisors find it difficult to get suitable replacement for key operations. These situations impact negatively on quality and the ability of the line to reach target. Another problem mentioned was the supply of mixed parts from logistics (two product variants supplied in the same bundle). The cause of mixed parts is usually disputed between production and logistics. However the problem is mostly only picked up in the sewing line while parts are being sewed together, and as there is no proof that suggests otherwise, it is assumed that parts were mixed on the line and rejects get recorded against the operators.

The supervisors were asked about their opinions regarding the level of selfinspection done by operators. The supervisors believe that their operators do put in the effort to inspect their own work as properly as time would allow. They pointed out that due to the uneven spread of work load on the line; some operators don't have the same amount of available time to do proper self-inspection as others. When unforeseen delays occur, these operators are put under even more pressure and the thoroughness of their self inspection could suffer as a result.

The last point of the discussion focused on the effectiveness of our current corrective action system. Supervisors are of the opinion that not all required parties are involved in the corrective action procedure and that this procedure does not resemble a team effort. When a corrective action is issued to production they believe it is often only handled as merely a paper exercise without real investigation into causes and solutions. The impression supervisors get when instructed to carry out activities related to corrective actions is that managers are only interested to get the paper work done and that there seam to be little focus on the actual problem, other than demanding disciplinary action.

\section{Focus Group - Quality staff}

The focus group conducted with the staff of the quality department focused on some of the same issues that have been addressed with inspectors and supervisors. The purpose of this is to look at similarities and difference in opinions from different view-points. The quality personnel were asked to give their opinion as to the effectiveness of the current method of data recording and analysis. Quality personnel commented that they believe the current system is generally effective and that it does give a good indication of what the quality situation is, but that errors do occur. Incidents where inspectors have to be asked to correct mistakes on forms and clear up issues on their recording sheets often occur. Incomplete information on data recording forms, such as the date, name of inspector, production line or shift causes major frustration when data is captured from these sheets. Quality staff is also of the opinion that inspectors sometimes neglect to record all the defect data, which leads to data never being captured or reported. The statement was also made that inspectors sometimes record defects under the wrong defect category. For example 
an inspector might record a defect as caused by bad cutting, when the defect was in fact caused by misalignment or other operator related issue. The reason for this could be lack of experience or lack of proper investigation into the problem causing the defect.

Quality staff was asked to comment on the noticeable response to the reported data and whether they believe that this response is sufficient. The general consensus reached by the group is that the response to data is very limited. The reporting of information every morning has become a routine exercise with no follow-up action. It was also noted that the reported data is captured twice onto separate spreadsheet, for different purposes. Firstly the previous day's data get recorded from the inspector's record sheets onto a spreadsheet to report the daily scrap and rework figures by line (not operator or defect type specific). Later in the week the same record sheets are used again for capturing the weekly defect figures which then include the defects per operator and defect type. A lot of effort is put into generating these two reports. The data that is recorded for the daily scrap report is captured by a quality technician, who works overtime every morning in order to capture data before the daily morning meeting. The data capturing for the weekly report is usually done during the weekend (also on overtime) in order to have it complete by the following Monday. The weekly report with defect per operator is then solely used by the managers to identify the operators who have reached their pre-set reject limit and who as a result, will forfeit their production bonuses. Other than for the purpose described above, no further action seems to be taken on the recorded data. As this data is not broken down into specific variants, it is also not possible to use this data to statistically prove the effect on some change. This problem was observed by the author, when asked to investigate what effect a recent change on a specific variant of a component had on the reported defects.

The last discussion point dealt with the effectiveness in which customer complaints are being handled. The quality team are of the opinion that response to customer complaints is generally very good and that quick action is usually taken to address these complaints. Further comments were that they believe that their problem containment actions, problem awareness and problem solving methods are effective and that problems are usually resolved quickly. However the concern is that the effects of these actions are usually short lived. It seems like once the problem is brought under everyone's attention quality alerts have been posted where required and awareness training was done, the problem seem to be resolved for a while, but as the focus shift away again to other issues the discipline to uphold the actions that were put in place seem to fade. Thus the re-occurrence of customer complaints is mostly attributed to the lack of self-discipline. This lack of self-discipline is not only applicable to the operators and inspectors, but to all involved in keeping the proposed actions and procedures in place including management. 


\section{Operator and Inspector Interviews}

\section{Final Inspector Interviews}

The response received from the final Inspectors are summarised below:

Two of the six inspectors interviewed indicated that the current list of defects are sufficient for the defects they encounter. The other four inspectors all named or described defects they are not able to classify under any of the current defect categories. The unlisted defects described by the inspectors are listed below:

- $\quad$ Defects related to piping (a design seam on some variants)

- Defects related to the isofix label (An isofix label indicate the positioning of a Child seat bracket, which get sewn into the cover.)

- Foreign objects (When particles get stuck between material layers or seams)

- Wrong identification label sewn onto cover.

- Mixed panels (For example when a panel from a sport cover is sewn into a standard cover)

It was also observed that some of the defects that is currently listed on the current defect listing are not used at all. These are defects that were relevant to previous projects, but due to the nature of the current components have become irrelevant.

The second question is related to the effectiveness and user-friendly layout of the recording sheet. The recording sheet is split into a top and bottom section (reworks and scrap). The top section is used for recording of defects that can be reworked and is split into two horizontal halves. On the left side inspectors tally the defects next to the specific defect that is listed on the form and on the right side they record the operator number with the defect code. The bottom section is a mirror image of the top section, but here the defects, resulting in a cover being scrapped, are recorded in the same way as for reworks.

The general response of all inspectors was that this recording form was effective and easy to use. It was however noted that the inspectors had to write very small in order to fit the information in the narrow lines provided. This is because the defect listing of 40 items appear twice on the sheet (scrap and rework sections) which result in eighty lines that are spaced very narrowly on an A4 sheet. As one of the inspectors explained how they write the operator number of the defect code on one side and then tick of the defect on the left side, she realised that she neglected to complete the left side for the previous recorded defect. Another issue that is related to the first interview question, was that inspectors indicated that they sometimes have to tipex or scratch out a description on the defect listing that is not used to write a description for a defect that is not listed in order to the record that defect. This presents a problem to the accuracy of the reported data, because this is not done consistently by the various inspectors, and when quality technicians record the defects into the system for further reporting they use the defect code (and not the description) to identify the defect type. 
The third question relates to the inspectors opinion of the level of self inspection done by the operators. The author received mixed responses to this question. Two of the inspectors interviewed are of the opinion that operators on their lines are careless and that they generally neglect to do self inspection. One inspector responded that she believed operators generally do put in an effort to do self inspection due to the fact that their clock numbers get recorded and that they could loose their production bonus or even receive a disciplinary warning for high rejects. The other three inspectors also indicated that they believe that here is a noticeable degree of effort from operators to do self inspection, but not yet sufficient.

The fourth question addresses the re-occurrence of defects. One of the inspectors replied that she noticed a random occurrence of various types of defects and could not single out major re-occurring defects. The other inspectors all indicated that there are a number of defects that they can single out as occurring more often than others. These defects as high-lighted by some of the inspectors, are given in below:

- Needle holes (usually associated with a bad rework)

- Seam width not to specification

- Backtacks not in order

- Loose hanging threads

- Skew seams

- Edge trimming problems

The purpose of the last question was to determine if inspectors believed that sufficient action is being taken on the data they collect. Two of the inspectors responded that they believe sufficient action is being taken on the reported problems. The observed response or action is supervisors addressing the quality problems with the individual operators. The other four inspectors were of the opinion that problems are not addressed sufficiently. A statement made by one of the inspectors was that they only see action taken on the lines when issues are raised by the customer. The perception of another inspector was that, no action will be taken, even if she recorded a relatively high number of defect incidents.

\section{Operator Interviews}

The response to the operator interviews is summarised below:

All operators interviewed claim that they know the specifications for their operations. Ninety percent of operators referred to their start-up check sheets with hand written specifications, when asked about the availability of these specifications. According to most of these operators these specifications originated from the work instruction. It was observed that hand written information gets copied weekly from one start-up form to the next.

Three of the operators indicated that they receive feedback regarding the quality of their production lines. The other 17 operators indicated that they do not receive feedback regarding performance of the group line) on a regular basis. Individual 
problems are generally dealt with each operator separately. Only major quality problems are discussed in a group setup.

Eight out of the twenty operators indicated that there are times when they have to use their machines even when they are not functioning properly, and could impact on the quality of their work. This occurs when they are waiting for a mechanic to change settings, or when a mechanic is waiting for an ordered part. The other sixty percent of operators indicated that they will not work on a machine unless it is functioning properly. Machine problems thus affects their ability to reach target but not their ability to produce quality work.

All operators interviewed indicated that they do proper self inspection. Only two out of the 20 operators indicated that time pressure could sometimes affect their ability to do proper self inspection. The remaining operators indicated that they have enough time to do self-inspection. The table below summarises defect listing of seat covers at Sewing.

Table 6. Defect Listing for W204 Seat Covers

\begin{tabular}{|l|l|l|l|}
\hline 1 & $\begin{array}{l}\text { THREAD TENSION / LOOSE } \\
\text { SEAM }\end{array}$ & 21 & DIRTY, MARKED, STAINED \\
\hline 2 & DAMAGED & 22 & CUTTING (SIZE / SHAPE / NIPS) \\
\hline 3 & $\begin{array}{l}\text { LISTING (LENGTH, TWISTED, } \\
\text { NARROW) }\end{array}$ & 23 & COLOUR SHADING \\
\hline 4 & BACKTACK N.O.K. & 24 & NO CLOCK NUMBER \\
\hline 5 & SEWN IN PLEATS & 25 & REINFORCEMENT TAPE \\
\hline 6 & DROPPED STITCHES & 26 & $\begin{array}{l}\text { COTTON } \\
\text { PROBLEMS(BREAK,TWIST,STUCK)) }\end{array}$ \\
\hline 7 & NOTCH ALIGNMENT N.O.K. & 27 & SEAM BURST \\
\hline 8 & STITCH LENGTH N.O.K. & 28 & CREASES \\
\hline 9 & NEEDLE HOLES & 29 & SEAM PARAMETER \\
\hline 10 & SEAM WIDTH & 30 & MISSING PANELS \\
\hline 11 & $\begin{array}{l}\text { TOP STITCH N.O.K. - ALIGNMENT } \\
\text { TO CENTRE }\end{array}$ & 31 & LOOSE LAMINATION \\
\hline 12 & STITCH TENSION & 32 & MISSING PROFILES \\
\hline 13 & AIRBAG LABEL & 33 & EDGE TRIMMING \\
\hline 14 & AIRBAG SEAM / JOINING & 34 & PROFILE WRONG WAY ROUND \\
\hline 15 & MIXED BATCH NUMBERS & 35 & SEAM MISSING \\
\hline 16 & JOINING ALIGNMENT & 36 & $\begin{array}{l}\text { HOLES MISSING/HOLES BOTH } \\
\text { SIDES }\end{array}$ \\
\hline 17 & LOOSE HANGING THREADS & 37 & PNA \\
\hline 18 & OVERLAPPING N.O.K. & 38 & BAD REWORKS \\
\hline 19 & LEATHER DEFECTS & 39 & CLOSED SEAM \\
\hline 20 & FABRIC DEFECT & 40 & SKEW SEAM \\
\hline
\end{tabular}

The main objective or goal identified for this project is the improvement of quality and customer satisfaction. The purpose of the conclusion is to present a framework 
for an improvement plan and its deployment, which can be used to address these factors and causes.

\section{Conclusion}

\section{Improvement Plan for Sewing}

The main elements of the proposed plan for Sewing is based on the PDCA approach discussed in the following sections.

The analysis of the set of causes related to quality and customer satisfaction within Sewing has been done during the empirical study discussed. The next step of the process would be to establish the goals of the improvement plan. These goals should address those critical success factors highlighted during the empirical study. Critical success factors was discussed as part of the literature review. These critical success factors should be established and agreed upon by management. However based on the preceding empirical study, critical success factors for Sewing should for example include, amongst others, at least the following three critical success factors:

- Conformance: Continually improve on product quality (improve on conformance and reduce defects)

- Reliability: Improve the reliability of the manufacturing system

- Customer value: Improve the effectiveness of cost reduction initiatives and aim to continuously add value to the customer.

Out of the seven quality dimensions studied during the employee survey, the above 3 dimensions demonstrated the biggest gap between the level of expectation and the current perception. In order to address the critical success factors, measurable goals or key performance indicators should be established. Measurable goals are required to be established by the management team under direction of the CI department and should be based on a thorough analysis of the current state within the organisation.

The next step is the establishment of the plan which entail the development of improvement projects that would lead to the goals. Each of these improvement projects would have its own measurable goals which when put together will lead to achieving the organisational goals established above. A measurement system should be put in place in order for the plan to be measured against the set goals. This plan should follow the continuous PDCA cycle as illustrated in figure 4 to continuously improve on the effectiveness of the plan. The outline of what is discussed here and the rest of the deployment process is summarised in the following section. 


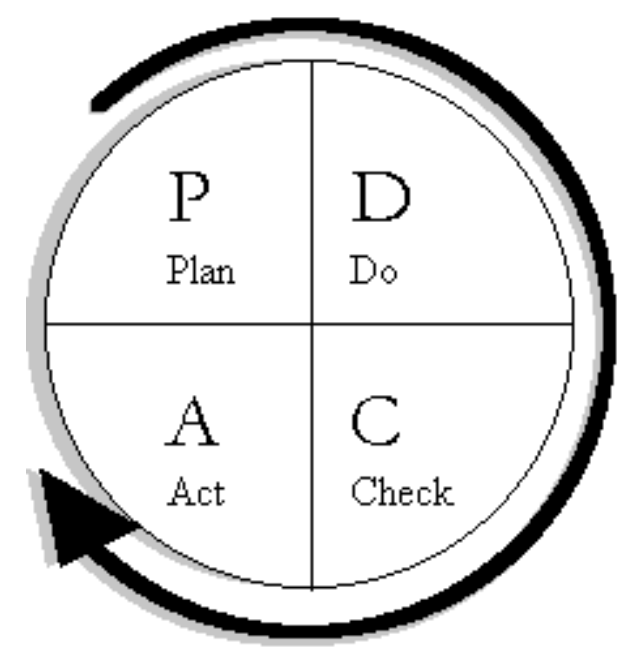

Figure 4. The PDCA Deming Wheel (Plan - Do - Check - Act)

\section{Deployment of the plan and the PDCA-cycle}

Policy deployment (also referred to as Hoshin Kanri) is the process whereby the goals of the organisation are put into action through an action plan. This is also described as the process of deploying the goals and initiatives of corporate top-level management down to the lower levels of the corporate hierarchy. Dahlgaard \& Dahlgaard (2002) stated that the policy deployment process is about a cause-effect relationship and proposed that the policy deployment process be implemented in a rotational PDCA cycle. The process they proposed is as follows:

Plan

1. Analyse the set of causes

2. Establish the goals

3. Establish a plan (the hypothesis), which is expected to lead to the goals.

4. Establish a measurement system Do

5. Plan for the implementation and measurements system

6. Implement the plan (a test of the hypothesis about cause and effect relationship) Check

7. Compare goals and results

8. Identify gaps (positive as well as negative gaps)

9. Analyse the cause for significant positive as well as negative gaps Action

10. For Good results (positive gaps as well as expected results): standardize the causes that had a good effect

11. For Bad result (negative gaps): improve the plan and rotate the PDCA cycle again

Another process for policy deployment proposed by Nanda (2003) is developed around the FAIR model and also involves the PDCA approach for project execution. 
FAIR is an acronym which stands for: focus, alignment, integration, and responsiveness.

\section{Continuous improvement \& policy deployment}

Sewing currently has access to various resources related to Lean manufacturing and Six Sigma through the CI department. However based on the negative feedback received from management and staff interviews as well as the feedback received from the employee survey, the evolution of CI within Sewing currently would be rated between level 1 and 2 when using the model developed by Bessant \& Francis. This negative perception of continuous improvement within Sewing can be partly attributed to the seemingly isolated way in which the CI department seems to function. Statements used to describe level $1 \& 2$ according to the model by Bessant \& Francis that resembles the current CI situation within Sewing for example, are as follow:

- CI happens as a result of the learning curve (level 1)

- effects are associated with a particular new process but then fades out again (level1)

- Formal attempts to create and sustain CI (Level 2)

- Structured and systematic CI (Level2)

- However effects are often short lived and localised (Level 1)

- Training started in basic CI tools, e.g. Kaizen Team work (Level 2)

- Dominant mode of problem-solving is by specialists (Level 1)

In order to move Sewing to higher levels in the evolution of $\mathrm{CI}$, improvement projects that are aligned with the overall goals and objectives of the organisation must be identified and conducted, there should be an increased focus on employee training, the levels of experimentation must be increased (for example incorporating design of experiments) and everyone must get actively involved.

\section{Other Issues raised by Sewing employees}

Some of the main concerns that were raised during interviews with management and staff are summarised below.

- Problems associated with gathering, feedback, analysis and reliability of quality data.

- The lack of a team approach in addressing quality issues and continuous improvement initiatives.

- Perceptions that some staff members have of management as to the lack of interest and seriousness that they show in solving quality problems.

- Negative perception of staff and management as to the effectiveness of the current continuous improvement and waste (including cost) reduction strategies.

- Short lived solutions to quality problems resulting in re-occurrence of the same problems.

- Absenteeism and the lack of multi skilled operators to alleviate the problem.

- The overall reliability of the manufacturing system and equipment. 


\section{Recommendations}

One of the first steps in addressing the improvement of quality at Sewing should include a review of the methods used to collect, analyse and report quality data. The introduction of a computerised data collection system involving scanning of covers at inspection stations is proposed. This method is already in use at various other automotive sewing plants, and speed up the collection, analyses and reporting of quality data, with less risk of errors. This method also allows for more detailed analysis of quality data due to the detailed product information that is recorded via scanning. Another major benefit of this system would be that quality technicians would need to spend less time on data capturing activities and can focus more of their time on addressing quality issues on the production floor.

It is recommended that the improvement plan discussed should follow the projectby-project approach where project teams address the measurable goals of the plan. To further the achievement of this goal and to emphasise the team approach. Sewing should adopt the matrix organisation structure to launch continuous quality improvement and cost \& waste reduction projects. This structure should give the continuous improvement managers and appointed project leaders an equal level authority over their project team members as the functional managers. This team approach will also address the negative perceptions as to the effectiveness of continuous improvement strategies and would improve confidence in management's earnestness to address and resolve quality issues. The team approach should also be emphasised on the production floor through the effective use of the quality circle (or green area) approach, where feedback is given and supervisors and operators can discuss problems and issues on a daily bases. This approach should also aim to address the following points listed by Deming; create a constancy of purpose, adopt a new philosophy, break down barriers between departments, put everybody to work and institute education and self-improvement.

The current situation regarding machine down time, the employee survey, as well as comments by staff during interviews, all indicate that Sewing does not resemble an organisation with a proper preventive maintenance system. The limitation of the current preventative system was also high-lighted during a recent internal audit. Although a computerised system is in place, there seems to be limited knowledge amongst maintenance personal regarding the full functionality of the system. A proposal is that a proper investigation should be done to improve the current preventative maintenance ability at Sewing. At another sewing plant in Rosslyn, maintenance activities have effectively been incorporated with the company's Information System (XPPS - System) which allows for better down time tracking, maintenance scheduling and problem identification. The proposed investigation should include a benchmarking exercise with this Sewing Plant in Rosslyn.

As recommendations for further research, it is recommended that separate detailed studies be conducted for each of the other two quality dimensions that showed the largest negative gaps in this study, namely Reliability and Customer Value. A study to improve the reliability of the organisation should focus on improving 
preventative maintenance and improve the effectiveness of the maintenance team at Sewing. The proposed study to improve Customer Value should specifically focus on improving the cost and waste reduction initiatives at Sewing to increase the overall value that Sewing can provide to their customer. The Kaizen team-worker recently adopted by the Corporation can play a major part in addressing the overall objective of increasing customer value.

\section{References}

Basu, R. \& Wright, N. (2003). Quality Beyond Six Sigma. Butterworth-Heinemann.

Baird, K., Jia Hu, K., \& Reeve, R. (2011). The relationships between organizational culture, total quality management practices and operational performance. International Journal of Operations \& Production Management, 31(7), 789-814.

Bessant, J. \& Francis, D. (1991). Developing strategic continuous improvement capability, International Journal of Operations \& Production Management, vol 19, no. 11, pp $1106-1119$

Brandt, D. (2008). Stricken with Lean. Industrial Engineer, vol. 40 no. 3/4, pp 52-53

Burke, R. (2007). introduction to project management - one small step for the project manager. Burke Publishing, 2007.

Curkovic, S., Vickery, S. \& Droge, C. (2000). An empirical analysis of the competitive dimensions of quality performance in the automotive supply industry. International Journal of Operations Management, vol. 20, no. 3, pp 386-403

Cyrne, R.O. (2005). Motivating employees to higher levels of performance: Lear Corporation. Unpublished master's thesis. Business School Netherlands

Dahlgaard, J.J. \& Dahlgaard S.M.P (2002). From defect reduction to reduction of waste and customer/stakeholder satisfaction (understanding the new TQM metrology). Total Quality Management, vol. 13, no. 8, pp 1069 -1085

Davison, L. \& Al Shagana, K. (2007). The Link between Six Sigma and Quality culture - An Emperical Study. Total Quality Management, vol. 18, no. 3, pp 249265

Eskildsen, J. \& Kristensen K. (2007). Customer Satisfaction - The Role of Transparency. Total Quality Management, vol 18 no. 1-2, pp 39 - 47

Ferguson, D. (2007). Lean and six sigma: The same or different? Management Services, vol. 51, no.3, pp 12-13

Foster S.T. (2015). Managing Quality. An Interactive Approach (2nd ed.). New Jersey: Pearson Prentice Hall

Gavriel, M. (2006). Quality of Design and Quality of Conformance: Contingency and Synergistic Approaches. Total Quality Management, vol. 17, no. 2, pp 205 - 219

Green, F.B. (2006). Six-Sigma and the revival of TQM. Total Quality Management, vol. 17 , no. 10 , pp $1281-1286$

Gregory, A. (2008). A lean marriage. Works Management, vol. 61, no. 6, pp 18-21

Gryna F.M. (2014). Quality Planning and Analysis. From Product Development through Use (6 $6^{\text {th }}$ ed.). New York: McGraw-Hill/Irwin 
Herman, J. (2007). Six Sigma is a key Implement in the quality toolbox. Automotive News, vol. 81, no. 6267, pp 24

Juran, J.M. (2015). Juran on the Leadership for Quality - an Executive Handbook. London, U.K.: The Free Press, Collier Macmillan.

Kamensky, J. (2008). Is Lean Six Sigma “Cool?" Ask Employees of FT. Wayne, IN, American Society for Public Administration, PA Times, April 2008, pp 9

Katz, J. (2007) Lean Takes the driver seat at Lear "Total Lean behaviour" has helped auto supplier stay competitive in a struggling market. Industry Week.com, December 2007,

Kaynak, H. (2003). The relationship between total quality management practices and their effects on firm performance. Journal of operations management, 21(4), 405435.

Kaye, M.M. \& Dyason, M.D. (1999). Achieving a competitive focus through self assessment. Total Quality Management, vol 10, no.3, pp 373-390

Kettinger, W.J. \& Lee, C.C. (2005). Zones of Tolerance: Alternative Scales for Masuring Information Systems Service Quality. MIS Quarterly, vol. 29, no. 4, pp 607-623

Khanna, V.K., Vrat, P., Shankar, R. \& Sahay, B.S. (2006). Usage of Quality Tools in the Indian Automobile Sector. Journal of Management Research, vol. 6 no. 3, pp 157-169

Levin, D.Z. (2000). Organizational Learning and the Transfer of Knowledge: An Investigation of Quality Improvement. Organization Science, vol. 11, no. 6, pp 630-647

Lewis, T. (2007). Continental CEO: Suppliers must cut costs or get out. Automotive News, vol. 81, no. 6265, pp 22-22

McManus, K. (2007). The trouble with teams. Industrial Engineer, vol. 39, no. 10, pp 20

Mikytuck, H. (2006). Driving Incremental Value Through Lean Six Sigma. Industry Week.com, September 2006. Retrieved from the World Wide Web 20 April, 2008: http://www.industryweek.com/PrintArticle.aspx?ArticleID=12585

Mohamed, Z. (2002). Beyond TQM implementation: The new paradigm of TQM sustainability. Total Quality Management. Vol. 13, no. 8, pp 1161-1172

Nanda, V. (2003). A process for the deployment of corporate quality objectives. TQM $\mathcal{E}$ Business Excellence. Vol 14, no. 9, pp 1015-1021

Niefer H. (2005) DaimlerChrysler in South Africa. Automotive Industries, May 2005.

Parasuraman, A., Zeithhaml, V. A. \& Berry, L. (1991). Refinement and Reassessment of the SERVQUAL Scale. Journal of Retailing, vol. 67, no. 4, pp 420-450

Parasuraman, A., Zeithhaml, V. A. \& Berry, L. (1988). Communication and Control Process in the Delivery of Service Quality. Journal of Marketing, vol. 52, April 1988, pp 35-48

Philip Crosby Associates (2004), Quality management guru's consultancy: "customer satisfaction = failure". Winter Park, Fla. And Boston, Mass., January 22, 2004. Retrieved from the World Wide Web 15 April, 2008: http://www.capabilitygroup.com/images/PCA_TCG_2004.pdf. 
Rossiter, R (2006a).Second-Quarter CEO Update. Retrieved from the World Wide Web 4 September 2007: http://learnet.lear.com/ceo/archive/073106

Rossiter, R (2006b).Fourth-Quarter CEO Update. Retrieved from the World Wide Web 4 September 2007: http://learnet.lear.com/ceo/archive/01272006

Vasilash G.S. (2002) No secrets - Marginal - automobile industry customer satisfaction. Automotive Design \& Production, Oct 2002, vol. 114, no. 10, pp 8,

Witzenburg, G. (2004) Mending fences: OEMs and suppliers find that building better relationships leads to building better product. Automotive Industries, Feb 2004.

Wood, G.R. \& Munshi, K.F. (1991) Hoshin Kanri: a system approach to breakthrough improvement. Total Quality Management, vol. 2, no. 3, pp 213-226

Sachdev, S.B. \& Verma, H.V. (2002). Customer Expectations and Service Quality Dimensions Consistency. Journal of Management Research, vol. 2, no. 1, pp 4452.

Sachdev, S.B. \& Verma, H.V. (2004). Relative Importance of Service Quality Dimensions: A Multisectoral Study. Journal of Service Research, vol. 4, no. 1, pp 94-116

Söderquist, K. \& Motwani, J. (1999). Quality Issues in Lean Production implementation: a case study of a French automotive supplier. Total Quality Management, vol. 10, no.8, pp 1107-1122

Stevenson, W.J. (2007). Operations Management (9 $9^{\text {th }}$ ed.). New York: McGrawHill/Irwin

Suarez, S. (1992). THREE EXPERTS ON QUALITY MANAGEMENT. TQL Publication No. 92 - 02, July 1992: Retrieved from the World Wide Web 4 March 2008: http:// deming.eng.clemson.edu/pub/psci/files/3expert.txt

Terziovski, M. (2006). Quality management practices and their relationship with customer satisfaction and productivity improvement. Management Research News, 29(7), 414-424.

Talib, F., Rahman, Z., \& Qureshi, M. N. (2011). A study of total quality management and supply chain management practices. International Journal of Productivity and Performance Management, 60(3), 268-288. 


\title{
Poor Road User Practices: Colombo Urban Roads a Case Study
}

\author{
K. S. Weerasekera \\ Department of Civil Engineering, \\ The Open University of Sri Lanka, \\ Nugegoda, Sri Lanka
}

\begin{abstract}
A road user behaviour study was conducted in a busy suburban area outside SouthEastern city limits of Colombo, the capital of Sri Lanka. The study area consisted of heterogeneous vehicle composition; typically combined with high proportion of motorcycles and three-wheelers which are poor in adhering to road rules that could commonly see in most of the South Asian developing countries. Although heavy investments are utilized in road improvement projects in these countries, the outcomes gained often seems to be unsatisfactory. The intent of this study was to find out the reasons for this unsatisfactory outcomes of the investments spent on road improvement projects and look into possible measures to overcome these unsatisfactory outcomes.
\end{abstract}

The study was conducted in two parts; (i) road user behaviour study, and (ii) field consultation study. Through the road user behaviour study the undesirable practices of all road users such as; passenger vehicles, motorcycles, three-wheelers, buses, trucks, pedestrians and excessive noise were directly identified. It was seen from the consultation study results the varied expectations of different categories of road stake holders' were different from category to category and biased towards their advantage. Finally through this study it was observed that the maximum benefits expected from investing on road improvement projects are not fully realized when poor road user practices are being adopted by the stake holders'.

Keywords: poor road practices, Colombo urban roads, road indiscipline.

\section{Introduction}

Poor vehicle composition; especially with high proportion of motor-cycles and threewheelers combined with other motorized vehicles may result in dual problem of poor traffic flows and high rate of road risk on urban roads (Weerasekera, 2008; Bandara, 2011). This was evident from a study conducted around relatively busy suburban areas of Nawala and Nugegoda (i.e. outside South-Eastern city limits of 
Colombo, Sri Lanka). It was observed that inefficient use of the road system and poor road user behaviour is a major contributor to traffic problems, even where there is no shortfall in road capacity. Hence the heavy investments spent on road improvements are not fully harnessed unless road user behaviour practices are improved (Edirisinghe; 2011, Jayatilake et al., 2017; Dharmakeerthi \& Sathyaprasad; 2017). This study intends to highlight the importance of improving the road user behaviour to maximize the benefits obtained from road improvement projects and increase road efficiency.

The area indicated in Figure 1 was selected as the area of study which is the SouthEastern city limits of Colombo. During the study number of road users who are accustomed to the area for the last 25 years, consisting of drivers, residents, businessmen, daily office travelers and pedestrians of the area were consulted. Consultations consisted of question and answer sessions posing different types of questions regarding road user behaviour patterns of multitude of road users who are familiar with the study area.

\section{Methodology}

\section{Road user behaviour \& field consultations}

Due to the increase of road users over the time and improvement of vehicle engine efficiencies the problem of accidents is becoming more severe in highway engineering. Hence the responsibility of traffic engineers to carry out systematic studies of traffic accidents and to find out the main causes for accidents and finally to come-up with preventive or remedial measures is important. Level of safety of a road system is influenced by the behaviour practices of all road users, not only driver behaviour alone. The other types of road users in addition to standard passenger car \& SUV drivers include; motor-cycle riders, three-wheel operators, bus and truck drivers and pedestrians. Colombo traffic composition is different from most of the developed countries due to its extremely heterogeneous vehicle fleet and very high proportion of motor-cycles and three-wheelers. Hence all the road users and their all behaviour practices have to be studied carefully to improve road safety and road efficiency under the existing extremely complicated situation. This has been observed by Roberts and Simmonds (1995) and Howorth, (1995) in their work too.

In addition to consultations, an observational study was performed to observe and determine road user behaviour operations. Twelve observation locations were selected as indicated in Figure 1 which is distributed along the road network under study. The observations carried-out were initially focused to study the behaviours reflecting violations of road rules and other dangerous road behaviour practices. The road user characteristics which carefully observed were; lane discipline, roadside parking \& friction, turning movements, failure to giveaway, irregular lane swapping, bus driver arrogant behaviour and not properly stopping at bus-stops, road rage by large vehicles and the pedestrian movements. Each study location (see Figure 1) was under observation for one-hour duration during busy daytime. 


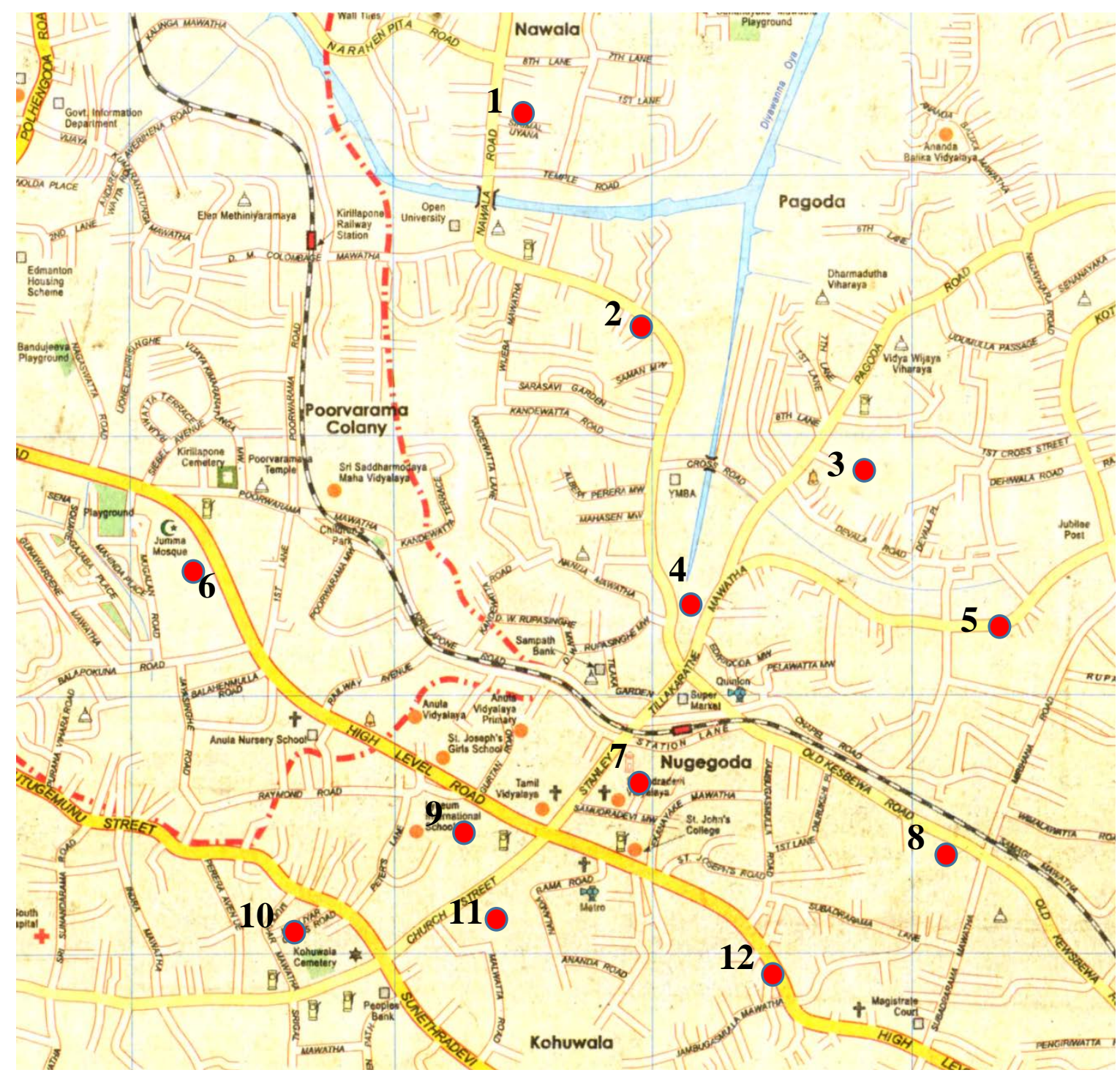

Figure 1 - Road network of the study area

\section{Road user behaviour study results}

Different types of road-user behaviour practices for different categories of road-users were identified and are categorized as indicated in Table 1 below.

Table 1 - Observed behaviour types of different road-user types

\begin{tabular}{|l|l|}
\hline Road-user type & \multicolumn{1}{c|}{ Observed behaviour type } \\
\hline 1. All vehicles & $\begin{array}{l}\text { - Reluctance to giveaway at signalised junctions and } \\
\text { small roundabouts } \\
\end{array}$ \\
& $\begin{array}{l}\text { - Reluctance to giveaway at prioritized junctions } \\
\text { - Unauthorized roadside parking }\end{array}$ \\
\hline
\end{tabular}




\begin{tabular}{|l|l|}
\hline & - Unnecessary lane changes and overtaking \\
\hline 2. Motor-cycles & $\begin{array}{l}\text { - Pillion passengers not wearing helmets } \\
\text { - Squeeze through other piled vehicles } \\
\text { - Keeping too close to other vehicles } \\
\text { - Riding on sidewalks } \\
\text { - Sometimes excessive speeding } \\
\text { - Cutting across lanes dangerously } \\
\text { - Riding while talking through mobile phones } \\
\text { - Overtaking from left }\end{array}$ \\
\hline - Not sticking to lanes \\
\hline - Sudden lane changes \\
- Excessive noise \\
- Three-wheelers on wrong side \\
- Cutting across lanes dangerously \\
- Overtaking from left
\end{tabular}


It could be seen that similar results have been obtained by Haworth (1995) in Dhaka, Bangladesh which has somewhat similar traffic conditions to Colombo.

\section{Field consultation study results}

The results obtained from consultation studies are summarized in Figure 2 below.

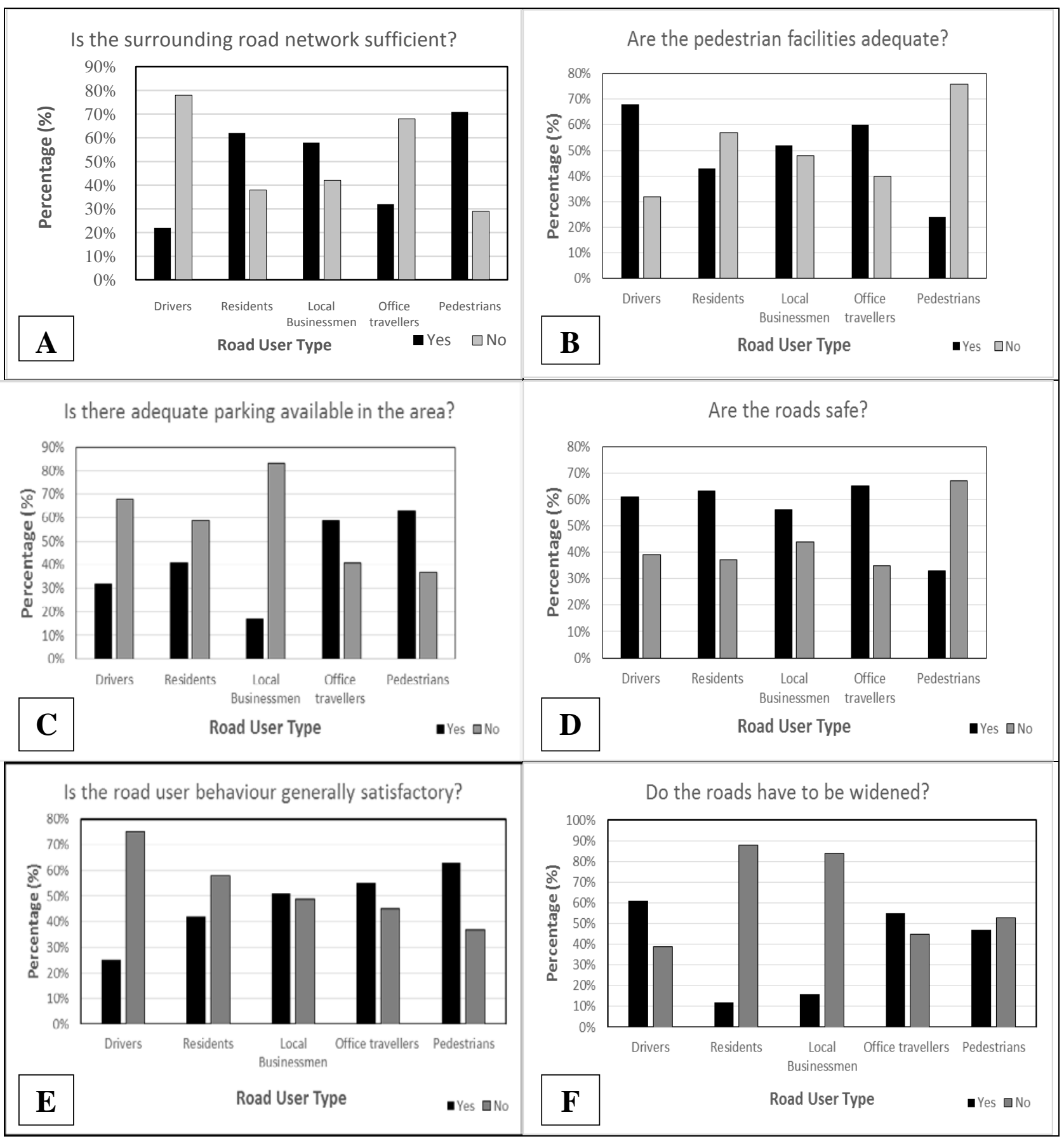

Figure 2 - Consultation study results

\section{Discussion}

It is clear from Figure 2A that drivers are the most unhappy about the inadequacy of surrounding road network and closely followed by office travellers. On contrary it is 
observed that pedestrians are happy about the surrounding road network. It also observe that residents and local businessmen too are generally satisfied with the adequacy of the surrounding road network.

From Figure $2 \mathrm{~B}$ it is seen that pedestrians are generally worried about the inadequate pedestrian facilities available. Generally residents too are not much satisfied about the adequacy of pedestrian facilities that are available currently. On contrary drivers are least worried about the availability of pedestrian facilities.

Figure 2C clearly indicates the unhappiness of local businessmen and drivers about the inadequacy of parking facilities. It's also clear even the local residents expect a higher level of parking facility in the area. It also could be noted that pedestrians and office travellers are not bothered about parking facilities available in the surrounding areas.

From Figure 2D it is indicated that except pedestrians all the other categories generally satisfied about the safety level of these urban roads. Majority of the pedestrians do conclude that the roads are not safe.

Figure 2E indicates that mainly drivers and residents complain about the unsatisfactory level of road user behaviour, but local businessmen and office travellers are not much concerned about the road user behaviour. The pedestrians have an opposite view that they think the road user behaviour is satisfactory from their point of view.

From Figure 2F it is interesting to note that greater majority of residents and local businessmen are not in favour of widening the roads fearing that their land will get affected. But on contrary all the other categories majority do agree in widening the roads.

It could be seen from the consultation study results that the expectations of different categories of stake holders' were different from category to category probably biased towards their advantage. For instance many drivers and through travellers preferred road widening to be carried-out, but residents and road side business community were against road widening since they were going to be affected. It also could see that pedestrians were unaware of the road capacity situation, but they were much worried about the lack of pedestrian facility in the area. When it comes to adequacy of parking in the area mainly business community and drivers were not happy about the inadequacy in parking availability although travellers and pedestrians were least bothered about parking facility in the area. It can be seen that generally all the categories of road users were concerned about the road safety; especially pedestrians and drivers had a high priority concern for improving road safety. Most of the drivers were not satisfied with the behaviour of the road users in general. In this regard pedestrians were the least bothered. 


\section{Conclusions}

Road safety depends when road user behaviour is combined with vehicle and road characteristics. Hence road user behaviour of vehicle drivers is due to; lack of understanding of road signs and some carriageway markings, lack of knowledge of priority rules, right of way concept is not properly understood and practiced, reversing to main roads obstructing the main flow is commonly observed, roadside parking causing side friction, and road raging by bus and heavy vehicle drivers. It could be clearly seen that efficiency of a road much depends on smooth traffic flow along the roads with; (i) least side friction along mid-blocks, and (ii) orderly maximum throughputs at intersections. The main reasons that increase side-friction and flow in mid-blocks is due to; unnecessary lane changing on roads, roadside parking and slowing down due to business activities along the sides of main roads, vehicles cutting across lanes and parking along opposite side of the road and buses overtaking and competing with each other.

The road regulations and conditions that the road users have to adhere are; condition of vehicle (road worthiness), adhere to speed limits in urban areas, adhere to give-way and priority regulations, adopt correct overtaking practices, buses to stick to assigned bus stops, adhere to post accident practices, adhere to correct parking practices, stick to lane discipline and strictly adhere to obligations at pedestrian road crossings.

\section{References}

Bandara, J. M. S. J. (2011). Need for an integrated highway management system: Developments in Sri Lankan context. Proceedings of the Joint Int. Symposium on Social Management Systems (SMSS-2011), (pp. 700-703) Colombo, Sri Lanka.

Dharmakeerthi, U. G. S. \& Sathyaprasad, I. M. S. (2017). Development of trip attraction rates and parking standards for supermarkets in Kandy area. Proceedings of the 5th International Symposium on Advances of Civil and Environmental Engineering Practices for Sustainable Development (ACEPS-2017) March 2017 (pp. 198-205) University of Ruhuna, Galle, Sri Lanka.

Edirisinghe, J. E. (2011). Driver behaviour at pedestrian crossings. Proceedings of the Joint International Symposium on Social Management Systems (SMSS-2011), (pp. 696-700) Colombo, Sri Lanka.

Howorth, N. (1995). Road user behaviour and safety in a developing country. Proceedings of the 7th World Conference on Transport Research (WCTR), July 1995, (pp. 302-306), Sydney, Australia.

Jayatilake, P. A. S. R., Premachandra, B. M. N. M. and Wickramasinghe, W. M. V. S. K. (2017). Variation of the start-up time of pedestrians with their familiarity to the signal phase. Proceedings of the 5th International Symposium on Advances of Civil and Environmental Engineering Practices for Sustainable Development (ACEPS-2017) March 2017 (pp. 206-212) University of Ruhuna, Galle, Sri Lanka.

Roberts, M. \& Simmonds, D. C. (1995). A strategic modelling approach for urban transport policy development. Proceedings of the 7th World Conference on Transport Research (WCTR), July 1995, (pp. 285-289), Sydney, Australia.

Weerasekera, K. S. (2008). Towards Better Roads. P \& P Associate Press, Rajagiriya, Sri Lanka. 


\title{
From Sustainable Engineering Education to Knowledge Transfer: A Preview
}

\author{
Nadiir Bheekhun \\ Aerospace and Communication Technology Research Group, Department of \\ Engineering and Technology, Faculty of Information Sciences and Engineering, \\ Management \& Science University \\ 40100 Shah Alam, Selangor Darul Ehsan, Malaysia
}

\section{Abd Rahim Abu Talib}

Aerodynamics, Heat Transfer and Propulsion Research Group, Department of Aerospace Engineering, Faculty of Engineering, Universiti Putra Malaysia 43400 Serdang, Selangor Darul Ehsan, Malaysia

\begin{abstract}
Science, as discovery, reminds us to praise the Creator for the divine, implausible, magnificent beauty and intricacy of His Creations. Engineering, as invention, helps us to unfold the creations further by developing products using the existing materials and the forces of nature to serve humanity. To do so without compromising the ability of future generations to meet their own needs, sustainable engineering concepts are required. Hence, the engineering education needs to anticipate and adapt itself to the socio-technological ever-evolving challenges in order to contribute towards the sustainability of the globe in an effective manner. A sustainable engineering curriculum would be one that is comprised of a good blend of engineering, scientific, technological knowledge, plus managerial, innovation, economic, communication, and more importantly ethical and moral knowledge and skills. This paper highlights the evolution of engineering education and emphasizes on the significance of ethics and moral values, managerial skills, emotional awareness, training and development, technopreneurship and knowledge transfer in engineering education.
\end{abstract}

Keywords: engineering ethics, emotional intelligence, knowledge society, technopreneurship, technology transfer.

\section{Introduction}

To seek knowledge is elemental. Applying and imparting the acquired knowledge with proper principles is what would contribute towards the transformation of the society in a constructive and positive manner. The process thereby knowledge is 
being nurtured can be termed as education. Sustainability, on the other hand, can be broadly defined as the ability of doing something in a constant and renewable manner. The mechanism to achieve sustainability is called as sustainable development (herein referred to as SD). According to the United Nations' Our Common Future, also known as the Brundtland Report (Brundtland, 1987), sustainable development is a type of development that meets the needs of the present without compromising the ability of future generations to meet their own needs. Literature indicates that SD is a multifaceted, multidisciplinary and intertemporal complex concept which encompasses three dimensions namely, social, economic and environmental (Griggs et al., 2013; Holden et al., 2017; Lehtonen, 2004; Lélé, 1991; Omer, 2008). Sustainable Engineering Education (herein referred to as SEE) is therefore an infusion of the SD concept into engineering education to support the global visionary knowledge society. The latter has been defined in numerous forms: it is a society about creating, sharing and using knowledge to bring prosperity and a sense of well-being to its people (Al-Hawamdeh, 2002); it is a society in which the knowledge sector represents the most significant share of the economy (Rohrbach, 2007); it is a society fortified by a up-to-date information as well as wellbuilt physical infrastructure allowing participation in the different socio-economic and political activities (Lor et al., 2007), inter alia.

"Engineering is that profession in which knowledge of the mathematical and natural sciences gained by study, experience, and practice is applied with judgment to develop ways to utilize, economically, the materials and forces of nature for the benefit of mankind". - Engineers

\section{Council for Professional Development (1961/1979)}

Engineers are therefore intellects that apply interpersonal judgment to analyze, design, construct and operate the products and services. The know-how of creative problem-solving under restrictions, the insights of extent to compromise, the wisdom to develop intuitive communication, the aptitude to identify risky or failure scenarios and act responsively - these are the features required to make a reliable engineer, combining not only math, natural sciences but also social sciences, creativity with ingenuity and the universal characteristics, moral values and ethics. Engineering has an intrinsic relationship with the society unlike science which is not bound by the immediate needs of society and is driven by curiosity or inherent dedication to solving nature's elaborate complexities and/or seeking answers to natural phenomena. Considering the ever-growing challenges worldwide, engineering-oriented solutions are critically demanded. Traditional engineering education has to be properly tailored with respect to technological advancement without compromising any standards or professionalism. As a consequence, the branches of engineering have expanded into scores of different engineering expertise compared to other subjects. Fields ranging from agricultural to aeronautical, electrical to telecommunications, civil to naval architecture, chemical to biochemical and many more have been established in the academia. Furthermore, the areas of specialization are narrowed to specific applications at degree level by means of minor technical modules. It is undeniably, that numerous technological-driven institutions are already emphasizing on engineering sustainable development in 
terms of economic and environmental contributions, but does our actual engineering curriculum instill satisfactorily essence of ethics and moral values and their significance in the society? Does the pedagogical approach inform engineering apprentices of the essentials matters in sustainability of mankind and its development? Are our graduates ready to identify and manage their own emotions in this world filled with odds? Does engineering education provide ample opportunities for training and development for interpersonal skills? Do we, the Higher Education Institutions (HEI) which is considered as the beacons of hope, amity, and ideas are promoting the spirit of technopreneurship? Are academicians transferring knowledge effectively to the society? This preview intends to highlight these philosophical viewpoints as an impulse towards SEE.

\section{Transformation in Engineering Education}

\section{"The only constant in life is change."- Heraclitus}

A timeline of the future global visions for engineering education and trends in industry is shown in Figure 1. It can be noticed that eras of engineering education and trends in industry have been evolved from 1900 up to now and are still being developed. The eras of engineering education have been changed from practiceoriented into engineering science based content and we are now moving towards integrative and innovative era. Froyd (Froyd et al., 2012) shared five major shifts during the past century: (1) a shift from hands-on and practical emphasis to engineering science and analytical emphasis (2) a shift to outcome-based education and accreditation (3) a shift to emphasizing engineering design (4) a shift to applying education, learning and social-behavioral sciences research (5) a shift to integrating information, computational and communication technology in education.

\section{Taking the Long View}

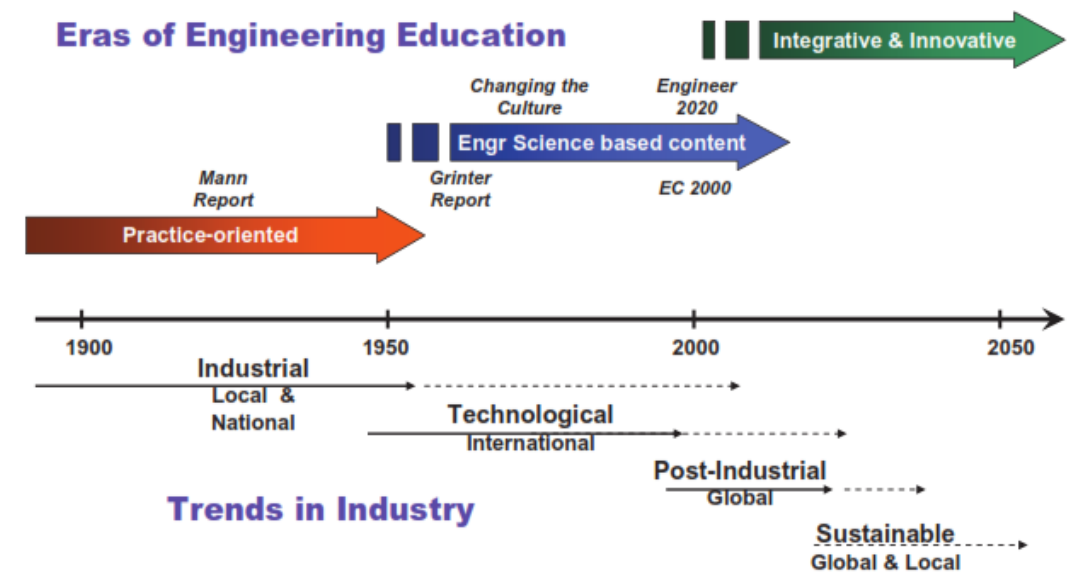

Figure 1: Long view of engineering education (Sunthonkanokpong, 2011) 
While the first two shifts have been realized already but continue to have implications up to now, the last three shifts are still being implementing. The first shift is about mathematical modeling and theory-based pedagogy. The second shift concerns internationalization of engineering curricula and engineering practices that are regulated by the Washington Accord. It gives international recognition to the degrees of engineers for member countries enhancing the employment opportunities of their citizens across the world. The accord requires that member nations set up suitable accreditation standards which would ensure a minimum quality of attainment for their engineering graduates. This improves the overall standard of training across all engineering schools to match the international standards set. It also works as quality framework that responds to difficulties that graduates face after their studies. It is worthwhile to mention that engineering technology degrees are now being run by institutions in many countries and graduates are rather referred to technologists rather than engineers as the students learn more through hands-on training as opposed to theory. The Sydney Accord is the mutual recognition agreement (MRA) which pertains to four-year engineering technology programs accredited by its signatories in their jurisdictions since 2001. The incorporation of design courses is usually achieved by a cumulative project during the first year of the curricula. The fourth shift can be noticed that it is directly related to work-integrated learning (WIL) which is a way of equipping graduates with attributes that make them work-ready by going through a compulsory workplace learning form (WPL). Qualities such as work diligence, qualitative efficiency, quantitative efficiency and teamwork are simultaneously being fostered in the engineering interns. WPL focuses on developing competencies. Lastly, the fifth shift is about the impact of information, communication and computation technologies (ICCT) on engineering education. Examples are content delivery methods, personal response systems, intelligent tutors, simulation software, remote laboratories amongst others (Froyd et al., 2012).

\section{Gaps in Engineering Education}

Although during the past 100 years, numerous challenges in engineering education have been tackled by the global community of educators and researchers, either enthused by the development of new technologies or the need for a restructuring the engineering curricula based on suggestions for more effective approaches to teaching and learning, some gaps still exist and hence SEE cannot be fully realized. Quite some time ago, in 1997, Nichols and Weldon (Nichols, 1997) argued that engineering education process and professional societies pay insignificant attention to the relationship between engineering and the society. They proposed a Venn diagram (Figure 2) which shows the engineering relationship with societal needs, scientific knowledge and creativity with different regions intersected. Region A solely describes the domain where capabilities of solving scientific problems lie. Region B shows the innovative capacity in engineering domain. Together, intersection of region $\mathrm{A}$ and $\mathrm{B}$ creates region $\mathrm{C}$, where engineering design are a result of real world problem solving from the merging of engineering science and creativity. Region D illustrates the domain where region A, B and societal needs 
meet. This region exemplifies the ideal role of engineering and the individual engineer.

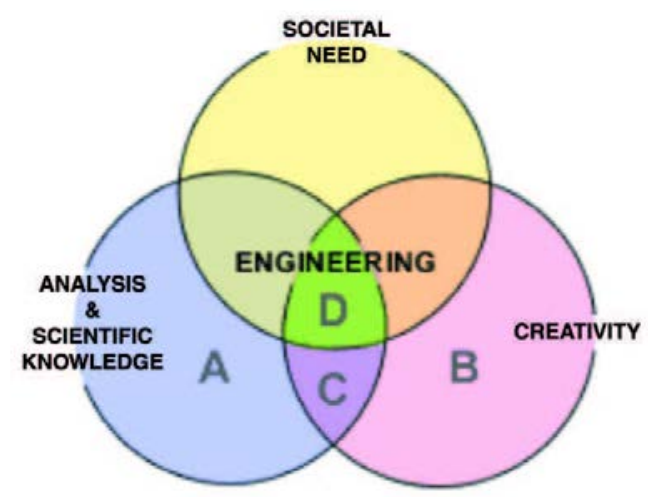

Figure 2: Venn Diagram of the relationship of engineering to societal needs, scientific knowledge and creativity (Nichols, 1997)

Engineering education has to be therefore in harmony with the society in order to ensure its sustainability. Socio-behavioural development of young professionals is the key for a better and safer modern future world. Apart from the scientific understanding of engineering science, the engineer has to be able to appreciate and prioritise the society's needs, propose optimum solutions, subjected to the available resources and time. It is hence essential to reform the way that an engineer thinks, decides, and deals with members of his society. This is dictated by the ethics and moral values and shall be reminded and inculcated in our engineers' mind. Key qualities are integrity, discipline, dedication, responsibility, transparency and commitment. If not, negative emotion such as ego will be developed. In their paper entitled as "Linking Ego and Moral Development: The Value Consistency Thesis", Levine et al. (Levine et al., 1992) addressed the relationship between ego and moral development, as represented by the theories of Erikson and Kohlberg. Engineering ethics which is the study of the moral issues and decision confronting individuals and organizations involved in engineering (Catalano, 2010), should be embedded into the curriculum. As a result, it will increase the ability of engineers to confront the urgent moral questions raised by technological activity, boost the ability to identify, formulate and solve engineering ethical problems, and improve the ability to communicate effectively with personnel of other related disciplines. The programmes should initiate awareness on moral autonomy, defined as the ability to think critically and independently about moral issues and to apply this moral thinking to situations that arise in the course of professional engineering practice. Proper, intensive, and practical- oriented training and practice of ethical decisionmaking approach is imperative. There is an urgent need to have qualified, wellinformed engineers who will voluntarily, effectively, and wholeheartedly implement SD principles in its entirety. A revolution in ethics is needed to improve the chance of success for SD in engineering education. Decision makers that include engineers, the community, and society needs to redefine, address, and prioritize the real issue facing this generation and future generations. The difference between public interest, self-interest, organizational interests, society's interest, and future 
generations' interest should be clearly and explicitly dealt with in relevant courses. Al-Rawahy (Al-Rawahy, 2013) has stressed that this is an important area where young engineers need to be exposed to and rehearsed on. Conflict of interest is and will continue to be a sticky problem for decision makers who are not fully aware of the distinction between these paradigms. Engineering code of ethics require engineers to consciously place the public interest above all other interests. It is primordial to incorporate practical examples and incidents that will practically teach students of the reality that is practiced by working engineers. Besides engineering ethics, it is thought that spirituality also contributes to the development of the engineer's personality philosophically, sociologically, and psychologically.

Contemporary themes addressing the essentials issues in sustainability of mankind and its development need to be made conscious to engineering students. These may include industrial and economic development, environmental impacts, globalization, population growth and its general impact on resource use, availability, and environment, the social-cultural-political-ethical-and moral impacts of development, global crises and problems that confront mankind and the wider environment, and their relevant impacts on society and future generations. This will further enable them to see beyond technical issues to the societal implications of technology, adapt to the rapid fluctuations of the consumer market and new technologies, solve interdisciplinary challenges, and combine a depth of knowledge of a subspecialty with the breadth of understanding required for real-world engineering (Al-Rawahy, 2013; Albu et al., 2004; Crawley et al., 2007; Mills et al., 2003).

Even though it is asserted by universities that an engineer's role in a company is one of leadership and implicit management, some engineering education curricula still lack the management skills component. Emotional Intelligence, coined as EQ, is a concept that was developed by Salovey and Meyer (Salovey et al., 1990) and later expanded by Goleman (Goleman, 1996). He identified that IQ is less important for success in life and work than EQ - a set of skills comprising of five characteristics that are not directly related to academic ability, namely self-awareness, selfregulation, motivation, empathy, and social skills. He thought that these qualities can be taught to engineering students as preparation for their professional working life. Moreover, Goleman (Goleman, 1996) stated that emotional intelligence abilities were about four times more important than IQ in determining professional success and prestige, even for those with a scientific background. He mentioned that engineering education has ignored this range of skills in the past, skills that incorporate communication and collaborative abilities, teamwork, selling an idea, accepting criticism and feedback, learning to adapt, and leadership. The professionals involved in international business will need to be equipped with new and expanded competences and skills from an intercultural perspective. Technical skills alone are no longer sufficient in this brave new world of globalisation and digitalisation; intercultural awareness and EQ skills are prime components that will facilitate the adaptation of future engineering graduates. The engineer's requirements continue to expand and evolve beyond the basic technical engineering 
knowledge and therefore universities need to recognise that the skills base now needed in the knowledge era has extended to greater emotional awareness. EQ tests should be performed with students. A person who owns high EQ test has the ability to self-assess, knows the strengths and weaknesses of themselves and others, has empathy and understanding of others' feelings. He/she will always know how to keep calm and optimistic in life even in the most difficult challenges. Regular mentoring activities should be conducted to further strengthen the relationship between lecturers and students, thereby recognizing the fact that we are first and foremost, all human beings, irrespective of our qualifications and achievements in life. Interpersonal skills, such as team building, communication skills, conflict management, persuasion skills, affirmation skills, adaptability and negotiation skills may be addressed. The activities may also assist mentees in discovering their interests and talents, how they learn best, possibilities of a career path, and how to make decisions and start planning. In short, the curricula should have attributes of human touch as well.

Training and development programmes are often considered as a pivotal human resource aspect to achieve sustainability in this dynamical business environment. Imparting codified knowledge and technical skills-knowledge would fulfil the requirements of academia-industry-government while maintaining the standard of the organisation internationally. Upgrading engineering students through add-ons training, such as soft skills, business standards, and manufacturing operations amongst others can accelerate their performance, mould their thinking and ensure they are sufficiently energised to meet the objectives, hence mission and vision of the organisation they will be working for. The establishment can stay competitive in today's ever-changing marketplace whilst ensuring its future engineers are being up-to-date with the current state-of-art management and technologies. They should gain the intellectual skills needed for lifelong learning. Upon graduation, the engineers should have vision, innovation, creativity and the spirit of technopreneurship. The importance of technological entrepreneurship needs to be addressed to engineering students by the faculty through approaches such as:

- Developing of modules in technopreneurship;

- introducing of new postgraduate courses on entrepreneurial engineering;

- Organising workshops and certified trainings in conjunction with consultancy firms;

- Forming a research group exploring on technological entrepreneurship;

- Implementing of an entrepreneurial centre for enhancement of entrepreneurship for graduated engineers;

- Promoting of alumni-industry collaborations.

\section{Knowledge Transfer}

Knowledge transfer (KT) is the process in which ideas are being shared and/or commercialise from the academic environment to the outside realm (industry/community) to generate a knowledgeable and high income nation. In other words, it is a process of knowledge creation and application, knowledge 
mobilization and exchange, information search and transformation as well as the learning process at and outside the workplace (Awang et al., 2009). The importance of technology transfer and its relevance in the formation of a knowledge-based economy has nowadays been recognised to altered extent by nations. Universities and research councils already have Technology Transfer Offices (TTO) in order to provide platforms for transfer of technologies being developed within institutions to industries. It is the role of these offices to collect invention disclosures from engineers and technologies, to assess the disclosures for merit, to propose the category of intellectual property protection that is most relevant, to ensure the proper filings or registrations are carried out, to market the inventions to potential commercial partners through licensing efforts, to monitor the patent filings and license agreements and screen revenue generation and distribution from licensed technologies/services amongst others. However, many innovations that are being generated at research organisations do not find their way to the market for various reasons including as outlined below:

- Not market-focused: solutions do not meet the society's demands;

- Lack of Technology Transfer managers: well-versed personnel to facilitate technology transfer, in terms of invention disclosure assessment, IPRs, marketing technologies, negotiation of deals, basics of license agreements and spinout company formation are scarce, which causes delay in transferring the knowledge;

- Missing of direct indicators of impact: Many governments and organisations across the world have been utilising metrics that are not true indicators of success in measuring the impact of technology transfer. Such a gap can be seen in many countries where the numbers have only led to the disappointment of impact seekers. Greater effort needs to be deployed in measuring the true impact of technology transfer and to determine its spillover effects to an economy.

Considering the above second point, it should be understood that formation of Technology Transfer Managers (TTM) is necessary to accelerate the rate of transferring technology. Do the actual TTM really focus on commercialising the outcomes of university research? Do they satisfy their key responsibilities which are:

- To work with academic researchers thereby commercialising new university technologies;

- To identifying commercial strategies for transferring technologies to industry, including supporting proof-of-concept activities and marketing to potential end-users;

- To negotiate appropriate licensing contracts, in conjunction with legal experts;

- To manage the creation of spin-out companies and supporting a culture of entrepreneurship within the university and local innovation ecosystem;

- To ensure technology transfer activities support the generation of research impact by university researchers. 
In addition, successful technology transfer managers should also demonstrate proven competence in:

- Grasping technical concepts quickly, and translating these into commercially viable proposals;

- Working effectively with key stakeholders, assertively influencing them and where appropriate showing tenacity and persistence in business development situations;

- Deploying excellent interpersonal and communication skills, both verbally and in writing;

- Successfully negotiating and licensing commercial contracts with industry;

- Working successfully within a close-knit team;

- Managing a large portfolio of complex projects to a successful commercial conclusion.

Most technology transfer platforms are lacking trained and accredited individuals with the skills mentioned above to deliver on knowledge and technology transfer requirements. The lack of expertise is crippling the transfer of knowledge and innovations from research organisations to the industry. It has further resulted in reduced commercialisation rate in terms of the number of innovations that are protected and the number of innovations transferred to industry or another entity for further development and commercialisation. The development of skill set relating to technology transfer and commercialisation should become a pressing need to developing countries. Governments should push their national agenda towards innovation and commercialisation which can spur talent development in the industry.

While implementing the New Economic Model for Malaysia which is a blend of high income, inclusiveness and sustainability for a better quality of life, a Knowledge Transfer Programme (KTP) has been initiated in such a way that:

- It recognises a broad range of activities to support mutually beneficial collaborations between universities, industries and communities (government agencies/ non-government organizations/ public sectors);

- It provides the platform for the exchange of tangible and intangible intellectual property, expertise, learning and skills between academia, industry and the community;

- The forms of interactions may include joint research, consultancy, education, training, graduate development, conferences, sharing of physical facilities and student placement.

$\mathrm{KTP}^{\prime} \mathrm{s}$ objective is to recognise and promote the transfer of knowledge via the exchange of creative and innovative ideas, research findings, experiences and skills between IPTA, research organizations, industries, government agencies and the wider community. 


\section{Conclusion}

Engineering turns knowledge into technology which is vital in addressing global challenges such as sustainability, climate change, poverty and the other humanityoriented goals. Yet, as technology advances, the engineering education needs to ensure conformity with the economic, environmental and social domains in order to realise sustainable development of the society, or rather knowledge-society. The first two aspects are undoubtedly being encouraged sufficiently by academic institutions but relatively less attention is given to the contribution of engineering to the society. Propositions such as an engineering ethics dosage with a touch on spirituality is required in the curriculum, contemporary topics to educate the engineers about sustainability of the society has to be addressed, the emotional intelligence concept has to be integrated during formation to nurture selfawareness, self-regulation, motivation, empathy and social skills, and finally training and development programmes including technopreneurship workshops should be conducted to address and confront the new set of realities whilst simultaneously ensuring revised standards and norms are being met, as innovation and creativity are crucial to the global competitive arena. Overall, an engineer should (1) be technically adept, broadly knowledgeable and a lifelong learner (2) be ethical and sincere (3) exhibit a technopreneurial spirit, innovative, and understand world markets (4) be pro-active and possess emotional intelligence (5) know how to translate technological innovation into commercially-viable products and services thereby contributing towards the betterment of mankind. Knowledge transfer programmes should be initiated and steadily encouraged to improve the quality of life whilst ensuring high income, inclusiveness and sustainability.

\section{Acknowledgements}

The authors would like to thank the Ministry of Higher Education of Malaysia for their financial support through the Fundamental Research Grant Scheme FRGS/TK05/UPM/02/7 No. 5524896.

\section{References}

Al-Hawamdeh, S., Hart, T.L. (2002). Information and Knowledge Society. Singapore: McGraw-Hill.

Al-Rawahy, K. H. (2013). Engineering education and sustainable development: The missing link. Procedia-Social and Behavioral Sciences, 102, 392-401.

Albu, M. M., Holbert, K. E., Heydt, G. T., Grigorescu, S. D., \& Trusca, V. (2004). Embedding remote experimentation in power engineering education. IEEE Transactions on Power Systems, 19(1), 139-143.

Awang, A. H., Hussain, M. Y., \& Malek, J. A. (2009). Promoting knowledge transfer in science and technology: A case study of Technology Park Malaysia (TPM). Croatian Economic Survey(11), 95-113.

Brundtland, G. H. (1987). Our common future-Call for action. Environmental Conservation, 14(4), 291-294.

Catalano, G. D. (2010). Tragedy in the gulf: A call for a new engineering ethic. Synthesis Lectures on Engineers, Technology, and Society, 5(1), 1-93. 
Crawley, E., Malmqvist, J., Ostlund, S., \& Brodeur, D. (2007). Rethinking engineering education. The CDIO Approach, 302, 60-62.

Froyd, J. E., Wankat, P. C., \& Smith, K. A. (2012). Five major shifts in 100 years of engineering education. Proceedings of the IEEE, 100(Special Centennial Issue), 1344-1360.

Goleman, D. (1996). Emotional Intelligence. Why It Can Matter More than IQ. Learning, 24(6), 49-50.

Griggs, D., Stafford-Smith, M., Gaffney, O., Rockström, J., Öhman, M. C., Shyamsundar, P., . . . Noble, I. (2013). Policy: Sustainable development goals for people and planet. Nature, 495(7441), 305-307.

Holden, E., Linnerud, K., \& Banister, D. (2017). The imperatives of sustainable development. Sustainable Development, 25(3), 213-226.

Lehtonen, M. (2004). The environmental-social interface of sustainable development: capabilities, social capital, institutions. Ecological economics, 49(2), 199-214.

Lélé, S. M. (1991). Sustainable development: a critical review. World development, 19(6), 607-621.

Levine, C., Jakubowski, L., \& Cote, J. (1992). Linking ego and moral development: The value consistency thesis. Human Development, 35(5), 286-301.

Lor, P. J., \& Britz, J. J. (2007). Is a knowledge society possible without freedom of access to information? Journal of information science, 33(4), 387-397.

Mills, J. E., \& Treagust, D. F. (2003). Engineering education-Is problem-based or project-based learning the answer. Australasian journal of engineering education, 3(2), 2-16.

Nichols, S. P. (1997). Professional responsibility: The role of the engineer in society. Science and Engineering Ethics, 3(3), 327-337.

Omer, A. M. (2008). Energy, environment and sustainable development. Renewable and Sustainable Energy Reviews, 12(9), 2265-2300.

Rohrbach, D. (2007). The development of knowledge societies in 19 OECD countries between 1970 and 2002. Social Science Information, 46(4), 655-689.

Salovey, P., \& Mayer, J. D. (1990). Emotional Intelligence. Imagination, Cognition and Personality, 9(3), 185-211. doi: 10.2190/DUGG-P24E-52WK-6CDG

Sunthonkanokpong, W. (2011). Future global visions of engineering education. Procedia Engineering, 8, 160-164. 


\title{
Five Lessons Learned Using Water-Scrum-Fall in South Africa
}

\author{
Laurie Butgereit \\ Nelson Mandela Metropolitan University \\ Port Elizabeth, South Africa
}

\begin{abstract}
The term Water-Scrum-Fall was coined by West et al. in 2011 to describe a hybrid methodology which is a by-product of attempting to introduce Scrum into an organisation which usually operates with a Waterfall Methodology. Water-ScrumFall typically appears in an organisation which had been using the Waterfall Methodology and in which a development team attempts to migrate to Scrum (or some other Agile Methodology). In such a hybrid, the first few phases (such as feasibility studies, funding exercises, requirements gathering, etc) are executed using a Waterfall Methodology. Then the development and possiby testing phases are executed using Scrum and are broken up into small sprints. The actual deployment, however, is then executed using again the Waterfall Methodology. This paper looks at a specific project in a South African listed company in which the development team implemented Scrum for the development phase and a large portion of the testing phase. This hybrid methodology was not necessary successful. This paper describes five of the lessons learned using Water-Scrum-Fall.
\end{abstract}

Keywords: waterfall, scrum, water-scrum-fall.

\section{Introduction}

In 1970, Dr Winston Royce developed the Waterfall Methodology (or Model) for software development. The Waterfall Methodology is a non-iterative methodology where a software project moved in its entirety from one step or phase to the next step or phase (Charvat, 2003).

In 2001, however, a number of people met in a ski resort in Utah, United States, and clarified certain values and principles which they held with respect to software development. These values and principles became known as the Agile Manifesto (Agile Alliance, 2017; Fowler \& Highsmith, 2001). A number of new software development methodologies were created using this Agile Manifesto including XP, Lean Development and Scrum. 
Attempting to transition an organisation from a Waterfall Methodology to an Agile Methodology is not without its challenges (Krasteva \& Ilieva, 2008; Sumrell, 2007; Sureshchandra \& Shrinivasavadhani, 2008). In many large organisations which have been typically doing Waterfall Methodologies, it is the development team who decides to implement an Agile Methodology. As will be more fully described in the Section appropriately entitled Water-Scrum-Fall, this hybrid methodology has been named Water-Scrum-Fall by West (West, Gilpin, Grant, \& Anderson, 2011).

This paper specifically looks at some of the lessons learned in using Water-ScrumFall in a corporate environment in South Africa. The background to Waterfall Methodology, Agile Methodologies, and the hybrid Water-Scrum-Fall is provided in the next four sections. A description of the research environment is in the subsequent section. The specific lessons learned are then itemised followed by concluding remarks.

\section{Overview of Waterfall and Agile}

The Waterfall Model is a non-iterative methodology favouring a number of discrete phases or steps such as a requirements gathering phase, followed by a design phase, an implementation phase, a testing phase, a deployment phase, etc. The project moves through these steps in one unit. In other word, one phase must be completely finished before the project can migrate to the next phase. These distinct phases make it easy for project managers because there is well defined documentation which accompanies the project as it moves from phase to phase (Charvat, 2003). In contrast, however, the Waterfall Methodology is often difficult for the end user or customer because the first time that the end user or customer actually views the product is during the last phase.

The Agile Manifesto clarified that the signatories valued (Agile Alliance, 2017; Fowler \& Highsmith, 2001): Individuals and interactions over processes and tools; Working software over comprehensive documentation; Customer collaboration over contract negotiation; Responding to change over following a plan. The Agile Manifesto also describes twelve guiding principles which emphasized frequent face-toface meetings between business and development groups along with continuous software delivery.

\section{Comparing Waterfall and Agile}

The Waterfall Methodology is process oriented. There are well defined processes (or phases) which must be followed. On the other hand, Agile Methodologies are more people oriented.

The Waterfall Methodology is a command and control methodology were project managers assign tasks to various people. In contrast, Agile Methodologies are more collaborative when team members co-operate and collaborate to complete the required tasks. In addition, Agile team members themselves select which tasks out of a backlog of tasks they wish to work on. 
The Waterfall Methodology depends on formal sign-off documents as a project moves from phase to phase. Agile Methodologies, however, depend more on informal meetings and face-to-face communication.

And, finally, the Waterfall Methodology is guided by tasks and processes whereas Agile Methodologies are guided by customer requirements (Nerur, Mahapatra, \& Mangalaraj, 2005).

\section{Adaption of Agile Methodologies in South Africa}

The adoption of various Agile Methodologies has grown world-wide. In his masters' dissertation, South African Master's student Vanker has researched the adoption of Agile methodologies inside South Africa in his Master's dissertation The Adoption of Agile Software Development Methodologies by Organisations in South Africa. Vanker collected 85 responses from 25 software development companies in South Africa. Of those 85 responses, 71 (or 83.5\%) used Scrum as their Agile methodology (Vanker, 2015).

\section{Scrum}

Scrum is one of the many Agile Methodologies. Jeff Sutherland was one of the original signatories to the Agile Manifesto and is often attributed to be the founder or author of Scrum. Sutherland himself, however, attributes many of the ideas basic to Scrum as coming from a 1986 article by Takeuchi and Nonaka in the Harvard Business Review entitled The New New Product Development Game (Sutherland, 2012; Takeuchi \& Nonaka, 1998).

Regardless whether Sutherland or Takeuchi and Nonaka were the author/s of the Scrum Methodology, Scrum teams are self-organising teams which collaborate to do the planning, design, development, and testing of a software project in an iterative manner (Sutherland, 2012; Sutherland \& Schwaber, 2013). Scrum defines a number of artifacts, a number of roles and a number of meetings or events.

The artifacts include the Product Backlog, the Sprint Backlog, and the Product Increment. The roles include the Product Owner, the Scrum Master, and the Team Members. The meetings or events include Sprint Planning, Daily Standup, Sprint Review, Sprint Retrospective and on-going Backlog Grooming.

\section{Water-Scrum-Fall}

In 2011, Dave West coined the term Water-Scrum-Fall. Water-Scrum-Fall describes a situation where a programming team (and possibly the testing team) are "doing Scrum" while the rest of the organisation is still doing Waterfall Methodology as can be seen in Figure 1 (West et al., 2011).

West uses the term water to describe the upfront work done by the organisation in doing feasibility studies, funding exercises, and requirements gathering. West uses the term scrum to describe the middle process where developers use the Scrum methodology to do actual development (and possible testing). And finally, West 
uses the term fall to describe the final deployment step where the organisation's existing release policy is still implemented.

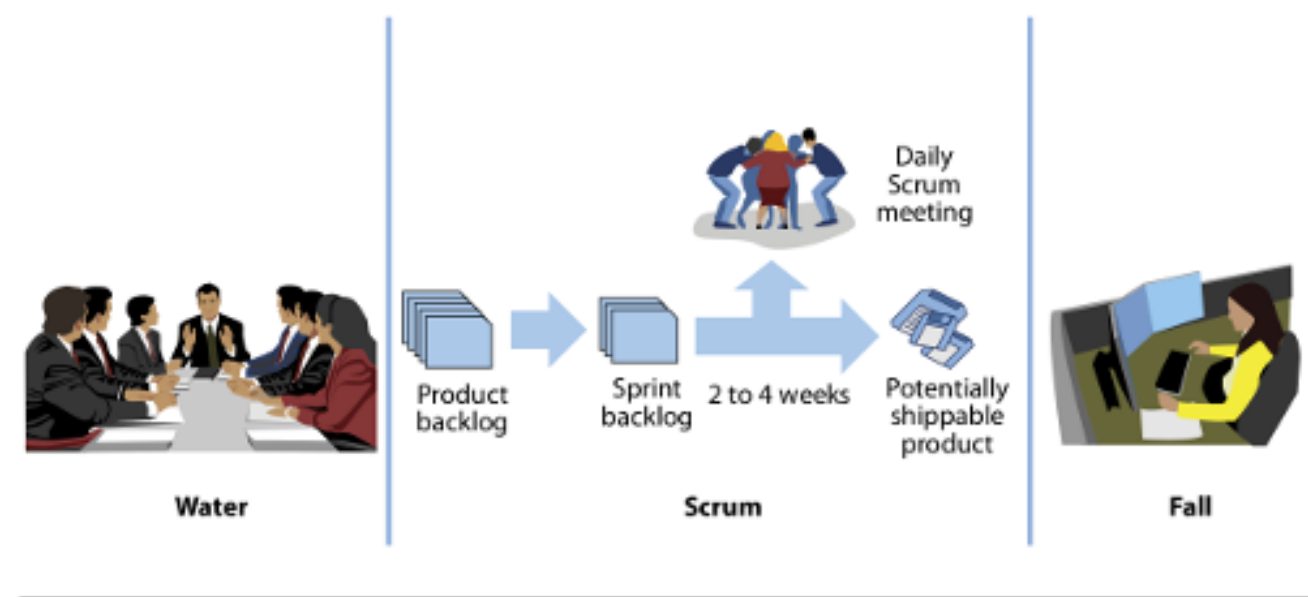

Figure 1: Water-Scrum-Fall as described by West (2011)

\section{Research Environment}

The company under research is a corporate listed on the Johannesburg Stock Exchange. The company is one of the largest vendors of secure tokens which can be redeemed for electronic goods such as airtime, data, wifi connectivity, electricity, and gambling facilities. In addition the company supplies other electronic facilities such as bill payments, traffic fine payments, event ticketing, bus ticketing, and lotto.

The company offers a switch which connects to the various cell phone providers, bill payment aggregators, ticketing agents, etc, available in South Africa. Prior to this research, the company offered a Windows Operating System (OS) based device which provides a user friend graphical user interface (GUI) interface to the switch. The Windows based device was placed at merchant stores and shops and would enable the merchant to on-sell the various products and facilities offered by the company to the end customer. The company did not deal with the end consumer of the product directly. All transactions when through a middle merchant.

The lessons learned itemised in this paper are specifically from a project to rewrite this Windows OS based GUI into an Android based app which could be deployed on much less expensive devices with embedded thermal printers.

Over the period of time which these lessons learned are obtained, the team varied from three (a product owner, a project manager/scrum master, one development team member) to eight (a product owner, a business analyst, a GUI specialist, a tester, a project manager/scrum master, and three development team members).

\section{Lessons Learned}

The project described in this paper entailed rewriting a Windows OS based application into an Android app. During this rewrite exercise, a number of improvements were made to the original flow of information and screens. In otherwords, it was not intended that the Android app would be an exact copy of the 
Windows OS application. Certain operations would be streamlined, some new functionality would be added, and some obsolete functionality would not be incorporated in the Android app.

This section itemises five of lessons learned while using Water-Scrum-Fall in the project.

\section{Lesson \#1 - A Waterfall Project Manager and an Agile Scrum Master are two completely different types of roles}

A project manager who works in a Waterfall methodology is accustomed to a command-and-control structure where the project manager assigns work to team members and the team members follow instructions. In contrast, however, in a Scrum environment, the team members' daily stand-up generates a list of impediments which are hindering their progress and the team members expect the Scrum Master to clear the impediments. This is, in effect, the team members giving instructions to the Scrum Master. In the case where a previous project manager is now filling the role of a Scrum Master, reversing the flow of instructions from project manager to team member and then from team member to Scrum Master can be difficult for a person to get used to.

During the course of the project under discussion in this paper, there were times when the team members looked to the Scrum Master and said things like "The lack of good test data is impeding my progress" or "The corporate firewall is blocking the Android devices from connecting to the server", the new Scrum Master did not understand those sentences to be instructions to him to solve the impediments. He understood the sentences to merely status updates and he expected the team members to solve their own problems. This change in attitude took a while to achieve.

This is a common problem as organisations migrate to scrum. For example, in some of their online training materials, Berteig Consulting argues that the number one common myth about Scrum is that a Project Manager is the same as a Scrum Master (Berteig, 2015).

\section{Lesson \#2 - A Business Analyst is not a proxy for a Product Owner}

Over the course of this project, there were periods of time when the Product Owner attended the various Scrum meetings (especially the daily stand-ups) and there were times when the Product Owner did not attend those meetings. When the Product Owner did not attend the meetings, the Business Analyst attended as a proxy for the Product Owner. When this happened, the Business Analyst did not feel she could make decisions on behalf of the Product Owner. This slowed down development. Questions which the development team had about things such as screen flows or colour schemes could not be resolved quickly.

In researching this phenomenon after it happened, it was found that this appears to be a common issue. Various Scrum training organisations such as Manifesto (Bates, 
2018) and Roman Pichler (Sumrell, 2007) (both based in the UK) maintain that a Business Analyst must not attend as a proxy for the Product Owner.

\section{Lesson \#3 - Formal Specifications are not Tasks or User Stories}

Formal specifications as written up by a Business analyst often consist of ten to twenty tasks or user stories. In the beginning of the project under research, it was assumed that one specification correlated to one task. The specification (masquerading as a task) was placed in the backlog as written. It was quickly learned that the specification was, in fact, multiple user tasks or stories. As the project advanced, the development team learned to break up any formal specification presented by the Business Analyst into smaller tasks or user stories.

\section{Lesson \#4 - Continuous Delivery can not happen using Water-Scrum-Fall}

The first principle of the Agile Manifesto is "Our highest priority is to satisfy the customer through early and continuous delivery of valuable software". In a Waterfall environment or a Water-Scrum-Fall hybrid environment, the last step of software delivery or software deployment is controlled by a gate keeper who collects sign-off documents from testers and product owners before deploying software.

This not only extends the period of time between deliveries, it is often complicated in a Water-Scrum-Fall environment because one specification may be broken up into multiple tasks or user stories.

\section{Lesson \#5 - MVPs can not be Updated and Deployed Quickly using Water-Scrum- Fall}

A number of Agile Methodologies including Scrum and Lean Development support the concept of a Minimum Viable Product (MVP). An MVP is the smallest version of the product which can provide some value for the end user or customer. Through the employment of continuous integration and continuous delivery techniques, an Agile team improves on the MVP and supplies new versions of the MVP to the end user or customer weekly or bi-weekly. In a Water-Scrum-Fall environment, however, the actual deployment of the software is typically still under control of a Waterfall Methodology and it is not possible for the Agile team to timeously deploy new software to the end user.

In the case of this project, often three months passed between new versions of the MVP.

\section{Conclusion}

As organisations try to move from a Waterfall Methodology to an Agile Methodology, there is often a hybrid state where parts of the project is still managed using a Waterfall methodology and parts of the project is executed using an Agile Methodology. West named this hybrid methodology Water-Scrum-Fall.

This paper looked at a specific project to rewrite a Windows OS based application as an Android app. The development team adopted a Scrum Methodology as defined 
by Sutherland. However, the other steps of the project such as requirements gathering, testing, and implementation/deployment remained under a Waterfall methodology.

Five important lessons were learned during this project. Three of the lessons dealt with the issue of there not being a one-to-one mapping between the roles in the two different methodologies and the documents in the two different methodologies: 1) project leaders are not the equivalent of scrum masters 2) business analysts are not proxies for product owners and 3) specifications are not the equivalent of user stores or tasks. Two of the lessons dealt specifically with the difficulty (or even impossibility) of trying to do continuous delivery in a Waterfall environment.

Large organisations often find smaller organisations strongly competing with them in the marketplace. The new smaller organisations were often created Agile at their inceptions and the larger organisations struggle to compete. A new small Agile organiation can get an MVP of a new product to market very quickly while the larger organisaation is still struggling with writing user requirements documents.

If such large organisations wish to attempt to embrace Agility successfully, these five lessons learned and itemised in this paper need to be avoided.

\section{References}

Agile Alliance. (2017). Agile alliance website. Retrieved March 17, 2018, 2018, from https://www.agilealliance.org/

Bates, S. (2018). Scrum in practice: the role of the business analyst. Retrieved March 17, 2018, March 13, 2018, from https://manifesto.co.uk/scrum-in-practice-therole-of-the-business-analyst/

Berteig, M. (2015). Scrum myth 01 - the ScrumMaster is a project manager. Retrieved March 17, 2018, 2018, from https://www.youtube.com/watch?v=a7mTMgUEjUQ

Charvat, J. (2003). Project management methodologies: selecting, implementing, and supporting methodologies and processes for projects. New Jersey: John Wiley.

Fowler, M., \& Highsmith, J. (2001). The agile manifesto. Software Development, 9(8), 28-35. Retrieved from http://andrey.hristov.com/fhtstuttgart/The_Agile_Manifesto_SDMagazine.pdf

Krasteva, I., \& Ilieva, S. (2008). Adopting an agile methodology: Why it did not work. Paper presented at the Proceedings of the 2008 International Workshop on Scrutinizing Agile Practices Or Shoot-Out at the Agile Corral, pp. 33-36.

Nerur, S., Mahapatra, R., \& Mangalaraj, G. (2005). Challenges of migrating to agile methodologies. Communications of the ACM, 48(5), 72-78.

Sumrell, M. (2007). From waterfall to agile-how does a QA team transition. Paper presented at the Proceedings of AGILE, pp. 291-295.

Sureshchandra, K., \& Shrinivasavadhani, J. (2008). Moving from waterfall to agile. Paper presented at the Agile, 2008. AGILE'08. Conference, pp. 97-101. Retrieved from http:/ / ieeexplore.ieee.org/abstract/document/4599456/ 
Sutherland, J. (2012). The scrum papers: nuts, bolts, and origins of an agile framework. Cambridge, Massachusetts: Scrum, Inc.

Sutherland, J., \& Schwaber, K. (2013). The scrum guide: the definitive guide to scrum: the rules of the game

Takeuchi, H., \& Nonaka, I. (1998). The new new product development game. Japanese Business: Part 1, Classics Part 2, Japanese Management Vol.2: Part 1, Manufacturing and Production Part 2, Automotive Industry Vol.3: Part 1, Banking and Finance Part 2, Corporate Strategy and Inter-Organizational Relationships Vol.4: P(TRUNCATED), 64(1), 321. Retrieved from https://hbr.org/1986/01/the-new-new-product-development-game

Vanker, C. (2015). The adoption of agile software development methodologies by organisations in south africa. Unpublished University of KwaZulu-Natal,

West, D., Gilpin, M., Grant, T., \& Anderson, A. (2011). Water-scrum-fall is the reality of agile for most organizations today. Forrester Research, 26 


\section{Track 2: Arts, Humanities \& Social Sciences}

Sociology

Psychology

Political Science

Religion \& Philosophy

Language \& Literature

Law, Justice \& Human Rights

History

Art, Music \& Design

Communication \& Media 


\title{
Herding Behaviour and Investment Decisions of Individuals with Fraudulent Microfinance Institutions in Ghana
}

\author{
Anselm Komla Abotsi, (Ph.D.) \\ Department of Economics Education, \\ University of Education, Winneba \\ P. O. Box 25, Winneba-Ghana \\ Theophilus Edward Richardson (Ph.D.) \\ Department of Economics Education, \\ University of Education, Winneba \\ P. O. Box 25, Winneba-Ghana
}

\begin{abstract}
Access to credit and financial services of sections of the societies in low-income developing countries has led to the proliferation of genuine and fraudulent microfinance institutions in recent times and this has been the subject of intense interest by researchers. This study explores the sociological and psychological factors that influence investment decisions of individuals with microfinance institutions to maximize their economic wellbeing in spite of the high incidence of atrophy of such institutions and fraud. This is an exploratory research which adopted a descriptiveanalytic approach in the analysis. In all 165 respondents were purposively selected from five administrative areas in the Brong Ahafo Region in Ghana. The study found that out of the total respondents of 165 who ever engaged in business with microfinance institutions, 119 of them have ever been swindled by microfinance institution. The respondents exhibited herding behaviour in their investment decisions with these fraudulent microfinance institutions. These fraudulent microfinance institutions used interest rate as a conduit to deceive and lure people to save with them. The respondents used bounded rationality in their investment decisions which resulted in the bad investment with these fraudulent microfinance institutions.
\end{abstract}

Keywords: Herding behaviour, bounded rationality, investment decision, fraudulent microfinance. 


\section{Introduction}

Access to credit and financial services of sections of the societies (usually the poor) in low-income developing countries has been the subject of intense interest and research by both the academia and international financial institutions (IFIs) since the early 1990s. Globally, microfinancing of microenterprises has emerged as an effective strategy for poverty reduction espercially in empowering women, assisting vulnerable groups, and improving standards of living (Goldberg, 2005; Imai, Arun and Annim, 2010; Mahjabeen, 2008). Widespread market failures and institutional failures that diminished the ability of the self-employed, individual and family (micro) enterprises to access credit from formal banking institutions turned attention to the informal institutions which have served the needs of the poor and the segmentation of financial markets in low-income developing countries. There was a shift in focus to harnessing the potential of informal financial institutions to serve as a vehicle for the mobilization and channelling of financial resources to the poor through the activities of informal savings systems. Referred to as the 'rural microfinance revolution', informal savings systems were expected to promote greater poverty reduction, improve the standard of living, and empowerment of women.

The introduction of the financial sector adjustment programme (FINSAP) in 1988 as part of neoliberal policy reforms in Ghana introduced in April 1983 created a liberalized monetary system which has since fostered the proliferation of microfinance institutions. While the interest in microfinance institutions have spawned substantial literature that have focused extensively on their scope and coverage, sustainability, greater poverty reduction and greater empowerment of the poor and marginal groups, especially women, there has also been pervasive failures of such institutions and instances of fraud and the loss of deposits of customers (the cases of Pyram and R4 scams in 1995 in Ghana being classic cases). These developments by no means appear to have diminished investor interest in these institutions; on the contrary, it seems to be on the upward. Substantial studies exist on the connections between investor behaviour and crises in financial markets in Europe and the Gulf states (Balcilar, Demirer and Hammoudeh, 2013; Corredor, Ferrer and Santamaria, 2015; Galariotis, Krokida and Spyrou, 2016). Yet hardly any studies have been done on the behaviour of investors in financial markets in Ghana, and this is a very important gap in the literature. Ghana is one country which has implemented one of the most far-reaching neoliberal economic policy reforms encompassing total liberalisation of the financial sector since the early 1980s.

This paper is primarily concerned with the economic behaviour of investors in financial markets in Ghana, with particular reference to microfinance institutions. Specifically, it examines the motivations that influence the decisions of individuals and households to whether or not invest in microfinance institutions in spite of the high incidence of atrophy of such institutions and fraud, with a special focus on sociological and psychological factors. We argue that neoclassical economics has severe limitations in explaining economic behaviour in financial markets, in particular its axiom of rational, atomistic, utility-maximising individuals and the neglect of collectivities and collective action problems, and that sociological and 
psychological factors are inherently economic and rational in character and are important variables in explaining the behaviour of agents in financial markets.

In this paper, we adopt a descriptive-analytic approach to study the interactions between the institutions' behaviour, the influence of society, and the preferences of individuals and households to maximize their economic wellbeing in the long term. This approach is appropriate because it enables us to explain the dynamics and effects of the sociological and psychological factors, in addition to the purely economic motives, on the decisions to invest in microfinance institutions and the benefits and costs of the decisions. The major contribution of the paper is that while recognizing economic rationality as an important explanatory factor, we emphasize the limits of the fundamental axioms of neoclassical economics in explaining the economic behavior of actors in financial markets; instead, we emphasize the relative importance and explanatory power of the concepts of 'bounded rationality' and herd behaviour.

\section{Theoretical Discussion and Literature review}

Three main theoretical paradigms in contemporary financial economics provide the analytical framework for explaining the evolution and developments in financial market systems and the impact of policy reforms. In the main, market conditions can be linked to the stage and nature of institutional development. These paradigms are presented as complementary to each other but differentially focused on government failure/policy-based or structural/institutional and non-economic psychological and sociological explanations.

\section{Government Failure or Policy-based Explanation: The Financial Repression Thesis}

The starting point of the theoretical discussions on financial systems (and especially microfinance institutions) is the analysis of government failure. The financial repression hypothesis (Fry, 1982, 1988; McKinnon, 1973; Shaw, 1973) attributes underdeveloped and inefficient financial systems to government policy failure as a consequence of excessive government intervention. Other analysts perceive repressive policies as the prime cause of fragmentation of financial markets in developing countries (Roe, 1991). Financial repression may take various forms: the central bank may impose credit ceiling or rationing on the commercial banks, or insist that the banks lend to certain priority sectors; or nominal interest rates may be kept artificially low, so that in the context of high inflation the real interest is negative, and the acquisition of interest-bearing financial assets is discouraged (Thirlwall, 1999). In particular, ceilings on deposit and loan rates tend to raise the demand for investible funds and depress the supply. Unmet demand for funds compels financial intermediaries to ration credit by means other than the interest rate, while an informal market develops at uncontrolled rates. The result is the emergence of a fragmented financial or credit markets in which favoured borrowers obtain funds at subsidized, often highly negative real interest rates in the formal markets, while others are forced to seek credit in inefficient, expensive informal markets (Aryeetey, Hettige, Nissanke and Steel, 1997). Thus financial repression leads to misallocation of savings and credit. Another major criticism of government- 
backed loan programs is that the default rates are very high and that most of the beneficiaries appeared to be the wealthy borrowers.

According to this perspective, financial liberalization would lead to financial deepening; improved efficiency in financial resource allocation to the most productive sectors; and increased flow of funds between segments, including improved access to formal finance of previously marginalized savers and borrowers.

\section{Market Failure and Institutional Failure Argument and Microfinance}

Conventional economic theory postulates that economic transactions in conditions of perfectly competitive markets (characterized by perfect competition and perfect information dissemination) are characterised by efficient allocation of resources and socially optimal outcomes, what is widely referred to as the efficient market hypothesis $(\mathrm{EMH})$. However, in practice, markets do not work well all the times: sometimes, markets do fail, and market failure occurs when a competitive market fails to allocate scarce societal resources optimally to achieve the maximum possible social welfare. By the same token, credit market failures lead to inefficient allocation of credit among the members of the society which is particularly pernicious to the poor and underprivileged. In particular, rural credit markets in developing countries are characterized by pervasive market imperfections, resulting from institutional failures which lead to the unavailability of credit to the rural poor. Hoff and Stiglitz (1990) put forward an explanation for persistent market and institutional failures based on imperfect information on creditworthiness and differences in the costs of screening, monitoring and enforcing contract across lenders. Institutional failures, which are legendary, are thought to result from the difficulties in enforcing and monitoring repayments, scarcity of suitable collateral and under-developed property rights, the underdevelopment of complementary institutions such as insurance markets, and a high degree of covariant risks among borrowers resulting from insecure sources and volatility of income (Besley, 1994; Rao, 2012). In the circumstances, banks are unlikely to satisfy the credit demands of the self-employed and family (micro) enterprises due to high transactions costs, relative to commercial bank spreads between the interbank rate and lending rate, and smallholder farmers may have extremely limited access to formal sector credit in a liberalized financial market in which credit is allocated purely on commercial criteria; and whereas banks are unwilling to extend secured lines of credit to micro enterprises, banks in Ghana have been historically oriented to large firms, reflecting the penchant for shorttermism.

Many economic decisions are however based on expected future economic conditions. Ignorance and uncertainty about the future mean that many decisions may turn out to be incorrect. Thus in the presence of the classic problems of information asymmetry and costly contracting, which are most widespread in lowincome developing countries, market failures are conceived to result from adverse selection and moral hazard which undermine the operation of financial markets. Regarding enforcement problems in credit markets, the borrowers' commitment to repay in the future may not be credible. This brings up the centrality of acceptable collateral to reduce uncertainty by shifting the risk from lender to borrower. The 
structural collateral is compounded by weaknesses inherent in the infrastructure that supports the financial system. For example, formal financial sector's ability to offset the risk of default may be limited by the absence of a well-functioning insurance market and of markets for the sale of confiscated collateral (Binswanger and Rosenzweig, 1986), coupled with the social difficulties of seizing the assets owned by the poor, which may be more costly to sell than their worth on second-hand markets (Roth, 2000).

\section{Herding Behaviour}

Despite the influential impact of the Keynesian revolution on economic thinking, and in particular, the importance of psychological and sociological factors that influence the financial behaviour of investors, and the inherent tendency of the financial market towards instability and collapse, ideas about the behaviour of individuals, households, and society and of financial markets continue to be dominated by neoclassical economic theory. Neoclassical economics rests on a number of theoretical assumptions in hypothesizing about the behaviour of economic agents. First, a fundamental axiom of neoclassical economics is that perfect and efficient markets are populated by individuals, not households, nor societies, nor communities. Second, individuals are rational, choosing, and maximizing in the pursuit of their preferences. Third, these preferences are purely private in character. From this narrow and restrictive perspective, the theory asserts that there are rational, independent, atomistic, and self-interested individuals who have at their disposal and use all relevant information efficiently, and endlessly seek to systematically maximize their unique private preferences.

A fundamental flaw of neoclassical economics, however, is that in practice it turns its methodology on its head, suggesting the recognition that some aspects of life are inherently collective or social rather than purely private, and individuals' and societies' preferences may and do often coincide (see Buchanan, 1978). The central problem for Keynesian economic theorizing and behavioral economics is the explanation of the central role of psychology, emotions, and sociological influences on the decisions of individuals that originate from the wider network of social ties in which the individual is embedded which formed the basis of much economic analysis. Thus rather than rational, atomistic, utility-maximizing individuals, actors tend to behave as collectivities, such as herds, with each individual motivated by the desire to minimize his/her exposure to risks or losses but to maximize his/her welfare function, which is usually similar to the society's overall welfare function. Though such behaviour may be inherently rational, the individual's behaviour is likely to be constrained by incomplete information and the limits imposed by human cognitive power or human and social conventions. The building blocks of the idea of 'bounded rationality' are thus derived from the recognition of a world in which market failure, information asymmetry, cognitive sightedness, institutions such as social conventions and norms, and human exuberance most often, if not always, influence economic decisions (Simon, 1978). From this perspective, a number of economic and non-economic models, which emphasise the important role of sociological, psychological and emotional factors, have been developed to explain herd behaviour in financial markets (Baddeley, 2010). 
The conceptualization and definition of herding can be traced to the original influences of Keynesian economic thinking which focused on the significance of socio-psychological factors in explaining the economic behaviour of individuals; specifically, Keynes emphasized the motivations of individuals to imitate and follow the crowd in a world of uncertainty (Keynes, 1930). Herding has thus been defined as the phenomenon of individuals deciding to follow others and imitating group behaviours rather than deciding independently and atomistically on the basis of their own, private information. Keynes conceptualized herding as a response to uncertainty and individuals' perception of their own ignorance: people may follow the crowd because they think that the rest of the crowd is better informed. Such a behaviour has the potential to not only generate economic instability, but it can also create speculative episodes in financial markets.

A very important insight provided by Keynes relates to the ways in which sociopsychological influences result in imitative learning, reputation building and/or 'beauty contests' or profit-making. In this view, (Baddeley, 2010) in a seminal paper has highlighted the futility of attempts by neoclassical economists to explain herding using rational expectations theory by retaining rationality 'assumptions but in a weakened form', i.e. by deploying 'a range of different statistical hypotheses' which incorporate sociological influences while psychology and emotional factors are accorded very little role (Baddeley, 2010). On the contrary, the paper emphasizes an eclectic approach as essential to understanding how and why herding and social influence evolve in economic and financial context. Bringing together ideas from the social and behavioural sciences including economics, sociology, psychology, evolutionary biology and neuroscience, the most powerful explanation of herding and social influence emphasise the dual roles played by reason and emotion. As Baddeley (2010) has perceptively observed, emotions and socio-psychological traits exert a major moderating influence on herding and imitation in economic and financial decision-making.

This paper, therefore, integrates insights from economics and other social sciences to provide an understanding of how reason and emotion interact to generate herding in individuals' investment decisions in microfinance institutions in Ghana.

\section{Microfinance}

Microfinance is a catch-all term. It refers broadly to the provision of financial products targeted at low-income groups. The aim of such small-scale financial services may have to do with the desire to help the poor get out of poverty trap by developing new income generating activities or strengthening the existing incomegenerating activities. Microfinance institutions (MFI) to some extent solve the problems of collateral security and information asymmetry by lending to groups rather than individual so that there is joint liability for borrowers. Under the structure of financial services industry in Ghana, microfinance belongs to both deposit-taking institutions and non-deposit taking institution. Therefore in place of collateral security, the forced savings of the group of borrowers or individuals is substituted by some of these institutions. Similarly smaller but frequent repayment 
instalments are found to be more suitable for borrowers having daily or weekly earnings. The local presence of these institutions also enables them to be in constant touch with the borrowers thereby reducing the transaction costs of borrowing (Rao, 2012). Customers of microfinance institution may borrow from the institutions for a variety of purposes ranging from household emergencies, paying of school fees, to investment of their businesses. Customers are also allowed to retain or save money for future use or for unexpected costs.

As of 2016, there were five hundred and sixty-four (564) MFIs in Ghana, which are made up of seventy-one (71) money lending companies, eleven (11) Financial NGOs and four hundred and eighty-two (482) microfinance companies (Bank of Ghana, 2016). In terms of growth, the microfinance sector recorded a marginal growth of 1.9 per cent in total assets in 2016 compared with growth of 30.3 per cent in 2015 . The MFls controlled 1.3 per cent of industry's total assets in 2016, down from 1.7 per cent in 2015 (Bank of Ghana, 2016). In 2016, Bank of Ghana suspended the granting of new licence to MFIs to give greater supervisory attention to the existing licensed institutions perhaps due to the increasing numbers of fraudulent microfinance institutions in Ghana. Some microfinance institutions in Ghana are fraudulently extorting monies from their clients for which reason the Bank of Ghana had to place a moratorium on some of these institutions (Bank of Ghana, 2016). Since the role of microfinance in poverty reduction cannot be overstated, there is the need to explore the sociological and psychological factors that influence the decisions of individuals to whether or not invest in microfinance institutions in spite of the high incidence of atrophy of such institutions and fraud.

\section{Methodology}

This was an exploratory study conducted in five (5) municipalities/ district in the Brong Ahafo Region in Ghana. The five (5) municipalities/ districts include Sunyani East Municipal, Techiman South Municipal, Asunafo North District, Dormaa Central Municipal and Nkoranza South Municipal. The population for the study includes all persons who have ever been engaged in business with microfinance institutions in these administrative areas. The economy of these administrative areas is made up of the agriculture, industry, commerce and the service sectors with agriculture being the predominant economic activity. There are several commercial financial institutions including banks, credit unions and savings and loans institutions among others in these administrative areas.

\section{Research Design and Data}

This is an exploratory research where a quantitative approach was adopted through the use of survey responses from the populace of the five administrative areas who have ever engaged the services of microfinance institutions in these areas. In all 165 respondents were purposively given the opportunity to respond to the survey questions with 33 from each administrative area because they have the best knowledge needed to meet the objectives of this study. The instrument used in the data collection was the questionnaire and the statistical software used in the data analysis was StataCorp 2013. 


\section{Results and discussion \\ Demography characteristics}

Literature has shown that the decisions/choices of individual investors have been influenced by their social settings (Barber and Odean, 2013). Demographic characteristics, such as age, education level, investment experience among others have been found to affect the investor's choice of financial services and their perceived risk from financial service (Falk and Matlulich, 1976; Mitchell and Greatorex, 1993). Relationship between demographic characteristics and herding bias has also been found in literature (Lin, 2011). The cross tabulation of age and education of respondents is presented in Table 1.

Table 1: Cross Tabulation of Age and Education of Respondents

\begin{tabular}{lllllll}
\hline \multicolumn{7}{c}{ Level of Formal Education } \\
\hline $\begin{array}{l}\text { Age of } \\
\text { Respondents }\end{array}$ & $\begin{array}{l}\text { No } \\
\text { Education }\end{array}$ & Primary & Secondary & University & $\begin{array}{l}\text { Other } \\
\text { tertiary }\end{array}$ & Total \\
\hline $21-30$ & $5(41.67)$ & $3(11.54)$ & $37(48.68)$ & $19(67.86)$ & $6(26.09)$ & $70(42.42)$ \\
$31-40$ & $6(50)$ & $11(42.31)$ & $17(22.37)$ & $6(21.43)$ & $7(30.43)$ & $47(28.48)$ \\
$41-50$ & $0(0)$ & $11(42.31)$ & $11(14.47)$ & $2(7.14)$ & $2(8.7)$ & $26(15.76)$ \\
$51-60$ & $1(8.33)$ & $0(0)$ & $9(11.84)$ & $1(3.57)$ & $5(21.74)$ & $16(9.7)$ \\
61 and above & $0(0)$ & $1(3.85)$ & $2(2.63)$ & $0(0)$ & $3(13.04)$ & $6(3.64)$ \\
\hline Total & $\mathbf{1 2 ( 1 0 0 )}$ & $\mathbf{2 6 ( 1 0 0 )}$ & $\mathbf{7 6 ( 1 0 0 )}$ & $\mathbf{2 8 ( 1 0 0 )}$ & $\mathbf{2 3 ( 1 0 0 )}$ & $\mathbf{1 6 5 ( 1 0 0 )}$ \\
\hline
\end{tabular}

Survey data (Figures in parenthesis represent percentages)

The results in Table 1 shows that most of the respondents are between the ages of 21 and 30 years (70) and 31 and 40 years (47) of age; most of whom have attained secondary level of education ( $48.67 \%$ and $22.37 \%$ respectively out of a total of 76$)$. A chi-square test of goodness-of-fit was performed to determine whether the education of respondents was equally distributed across age groups. The education of respondents was not equally distributed across the age groups, $\chi^{2}(16, N=165)=$ 47.82, $\mathrm{p}<.05$. This finding is consistent with Lin (2011) who found that young investors are more prone to herding behaviour than older ones. The respondents between 21 and 30 years who have attained university level of education are 19 which represent $67.86 \%$ out of a total of 28 .

Generally, empirical financial literature has revealed that women exhibit a higher degree of risk aversion and have a lower propensity to undertake risky projects (Croson and Gneezy, 2009; Dohmen, Falk, Huffman, Sunde, Schupp and Wagner, 2005; Fellner and Maciejovsky, 2007; Lusardi and Mitchell, 2008). The results in Table 2 indicate that 88 of the respondents are males and 77 are females. Literature elsewhere has analyzed the impact of marital status on financial choices. Lupton and Smith (2003) and Waite and Gallagher (2000) concluded that single individuals are more risk averse than the married. The cross tabulation of sex and marital status results show that majority of the males (45 representing $51.14 \%$ ) and females (40 representing 51.95\%) respondents are married. This suggests that the married are more prone to herding behaviour. Lin (2011) found that females are more involved in herding behaviour than males. 
Table 2: Cross Tabulation of Sex and Marital Status of Respondents

\begin{tabular}{llll}
\hline \multicolumn{5}{c}{ Sex } & & \\
\hline Marital Status & Male & Female & Total \\
\hline single & $40(45.45)$ & $28(36.36)$ & $68(41.21)$ \\
married & $45(51.14)$ & $40(51.95)$ & $85(51.52)$ \\
divorced & $2(2.27)$ & $5(6.49)$ & $7(4.24)$ \\
widowed & $1(1.14)$ & $4(5.19)$ & $5(3.03)$ \\
\hline Total & $\mathbf{8 8 ( 1 0 0 )}$ & $\mathbf{7 7 ~ ( 1 0 0 )}$ & $\mathbf{1 6 5 ( 1 0 0 )}$ \\
\hline \multicolumn{4}{r}{ Survey data (Figures in parenthesis represent percentages) }
\end{tabular}

A chi-square test of goodness-of-fit was performed to determine whether there was sex difference in the marital status of the respondents. There is no significant sex difference in the marital status of the respondents, $\chi^{2}(16, \mathrm{~N}=165)=4.79, \mathrm{p}=0.188$.

\section{Business characteristics}

\section{Real Sector of Economy and Business Engagement}

The study sought to find out the real sector of the economy where the respondents were engaged in a type of business. The result of the analysis is presented in Table 3. It is not surprising that majority of the respondents (95 representing almost 58\%) are engaged in a business in the primary sector (agriculture) since agriculture predominate the economic activities while $66(40 \%)$ are engaged in a business in the tertiary sector (services). Persons who are interested in agriculture sometimes face credit constraints as banks find it less profitable or more risky to lend to businesses in agricultural production.

Table 3: Sector of Economy and Business Engagement

\begin{tabular}{lcc}
\hline Real Sector of Economy & Number of respondents & Frequency \\
\hline Primary Sector & 95 & 57.58 \\
Secondary Sector & 4 & 2.42 \\
Tertiary Sector & 66 & 40.00 \\
\hline Total & $\mathbf{1 6 5}$ & $\mathbf{1 0 0}$ \\
\hline Survey data (Figures in parenthesis represent percentages)
\end{tabular}

The high risk perception of the agricultural sector and lack of adequate risk management tools are the central reasons why banks have been reluctant in lending to the sector. Therefore farmers would have to look elsewhere for capital injection into their businesses. Though literature has shown that microfinance play an important role in increasing agricultural production (Sulemana and Adjei, 2015), identifying the genuine microfinance institutions has always been a challenge to these farmers.

\section{Savings and income}

According to Keynes, (1936) there are eight kinds of goals which would motivate individuals to abstain from spending out of their incomes. These goals include precaution to build up a reserve against unstable and unforeseen conditions in the future, calculation to enjoy interest, improvement to increase the standard of living, enterprise to secure a certain amount of money for investment among others (see also Canova, Rattazzi and Webley, 2005). Table 4 shows that 58 (35\%) of the 
respondents (165) save below 11\% of their monthly income with 107 (65\%) of them saving more than $11 \%$ of their monthly income.

Table 4: Cross Tabulation of level of monthly income and percentage of monthly income saved

\begin{tabular}{llllll}
\hline \multicolumn{7}{c}{ percentage of monthly income saved } \\
\hline $\begin{array}{l}\text { level of monthly } \\
\text { below }\end{array}$ & $\mathbf{1 1} \%$ & $\mathbf{1 1} \%-\mathbf{2 0} \%$ & $\mathbf{2 1} \% \mathbf{- 3 0} \%$ & more than $\mathbf{3 0} \%$ & Total \\
\hline less than 100 & $4(6.9)$ & $2(5.56)$ & $3(9.68)$ & $1(2.5)$ & $10(6.06)$ \\
$100-500$ & $26(44.83)$ & $15(41.67)$ & $9(29.03)$ & $16(40)$ & $66(40)$ \\
$501-1000$ & $19(32.76)$ & $11(30.56)$ & $15(48.39)$ & $10(25)$ & $55(33.33)$ \\
$1001-1500$ & $4(6.9)$ & $8(22.22)$ & $3(9.68)$ & $7(17.5)$ & $22(13.33)$ \\
1500 and above & $5(8.62)$ & $0(0)$ & $1(3.23)$ & $6(15)$ & $12(7.27)$ \\
\hline Total & $\mathbf{5 8 ( 1 0 0 )}$ & $\mathbf{3 6}(\mathbf{1 0 0})$ & $\mathbf{3 1}(\mathbf{1 0 0})$ & $\mathbf{4 0 ~ ( 1 0 0 )}$ & $\mathbf{1 6 5 ( 1 0 0 )}$ \\
\hline Survey data (Figures in parenthesis represent percentages) & &
\end{tabular}

The analysis shows that out of the 58 respondents who saved below $11 \%$ of their monthly income, $26(44.83 \%)$ earn between 100 and 500 Ghana cedis in a month (Table 4) while 19 (32.76\%) earn between 501 and 1000 Ghana cedis. While 40 of the respondents save more than $30 \%$ of their monthly income, 36 of them save between $11 \%$ and $20 \%$ and 31 save between $21 \%$ and $30 \%$. A chi-square test of independence was performed to examine the relation between level of monthly income and percentage of monthly income saved by the respondents. The relation between these variables was not significant, $\chi^{2}(12, \mathrm{~N}=165)=17.43, \mathrm{p}=0.134$. Though the motive for the savings cannot be predicted, the most probable reasons could be the calculation to enjoy interest, improvement to increase the standard of living, enterprise to secure a certain amount of money for investment.

\section{Business with microfinance institution}

\section{Sources of Information and background check of microfinance institution}

The sources of information have attracted much interest due to their direct influence on investment decisions and market efficiency. As indicated earlier, neoclassical economic theory conceives that there are rational, independent, atomistic, and selfinterested individuals who use all relevant information they have at their disposal efficiently, and endlessly seek to systematically maximize their unique private preferences. The concept of bounded rationality suggests that individuals can only process a limited set of information accessible on the market (Khan, Tan and Chong, 2017). The sources of information on the existence of the microfinance institution in the research areas were found to include friends, radio and newspapers among others. Table 5 shows that 73 of the respondents knew of the existence of the microfinance institutions through friends and 57 through radio advertisements. This finding is consistent with Arnswald (2001) who finds that fund managers search for information from colleagues and other market participants to endorse their own investment decisions besides using fundamental information. In fact, Ivkovic and Weisbenner (2007) postulate word-of-mouth communication as an important information source affecting financial decisions. Some investors would want to reduce search costs and because they lack expertise prefer to use word-of-mouth communication as the main information channel (Khan, Tan and Chong, 2017). 
Table 5: Level of Formal Education and mediums of information on the existence of the microfinance institution

\begin{tabular}{llllll}
\hline \multicolumn{7}{c}{$\begin{array}{l}\text { Mediums of Information on The Existence of The } \\
\text { Microfinance Institution }\end{array}$} & & & \\
Level of Formal & & Newspaper & Radio & Others & Total \\
\hline No Education & $4(5.48)$ & $0(0)$ & $5(8.77)$ & $3(11.11)$ & $12(7.27)$ \\
Primary & $8(10.96)$ & $2(25)$ & $8(14.04)$ & $8(29.63)$ & $26(15.76)$ \\
Secondary & $37(50.68)$ & $3(37.5)$ & $26(45.61)$ & $10(37.04)$ & $76(46.06)$ \\
University & $18(24.66)$ & $2(25)$ & $5(8.77)$ & $3(11.11)$ & $28(16.97)$ \\
Other Tertiary & $6(8.22)$ & $1(12.5)$ & $13(22.81)$ & $3(11.11)$ & $23(13.94)$ \\
\hline Total & $\mathbf{7 3 ( 1 0 0 )}$ & $\mathbf{8 ( 1 0 0 )}$ & $\mathbf{5 7 ( 1 0 0 )}$ & $\mathbf{2 7 ( 1 0 0 )}$ & $\mathbf{1 6 5 ( \mathbf { 1 0 0 } )}$ \\
\hline
\end{tabular}

Survey data (Figures in parenthesis represent percentages)

The analysis in Table 5 show that out of 73 respondents who knew of the existence of the microfinance institutions through friends, 37 (50.68\%) have obtained secondary level of education and those who have obtained university education are 18 $(24.66 \%)$. Similarly $26(45.61 \%)$ out of 57 respondents who knew of the existence of the microfinance institutions through radio advertisement, have obtained secondary level of education. With these levels of education, it is expected that the respondents would have sought for further information on the microfinance institution from the Bank of Ghana before engaging in any business activity with them. More emphasis was placed on friends and this confirms the contention of Menkhoff, Schmidt and Brozynski (2006) that German fund managers place more emphasis on the decisions of colleagues and other market participants to obtain information. A chi-square test of independence was performed to examine the relation between level of formal education of the respondents and mediums of information on the existence of the microfinance institution. The relation between these variables was not significant, $X^{2}$ $(12, \mathrm{~N}=165)=18.22, \mathrm{p}=0.109$.

Table 6: Level of Formal Education and Enquiring Whether the microfinance Have a Valid operation Licence

\begin{tabular}{llll}
\hline \multicolumn{4}{c}{$\begin{array}{l}\text { Enquire Whether MF Have Valid } \\
\text { Licence To Operate }\end{array}$} \\
\hline Level of Formal Education & Yes & No & Total \\
\hline No Education & $7(9.46)$ & $5(5.49)$ & $12(7.27)$ \\
Primary & $8(10.81)$ & $18(19.78)$ & $26(15.76)$ \\
Secondary & $32(43.24)$ & $44(48.35)$ & $76(46.06)$ \\
University & $13(17.57)$ & $15(16.48)$ & $28(16.97)$ \\
Other Tertiary & $14(18.92)$ & $9(9.89)$ & $23(13.94)$ \\
\hline Total & $\mathbf{7 4 ( 1 0 0 )}$ & $\mathbf{9 1}(\mathbf{1 0 0 )}$ & $\mathbf{1 6 5 ( 1 0 0 )}$ \\
\hline
\end{tabular}

Survey data (Figures in parenthesis represent percentages)

Out of the 165 respondents, 91of them never enquired whether the microfinance institution they are engaged in business have a valid license from the Bank of Ghana to operate while 74 actually did enquire (Table 6). The cross tabulation shows that out of the 91 respondents who never enquired whether the microfinance institution they are engaged in business has a valid license, 44 (48.35\%) have obtained secondary level of education while $24(26.37 \%)$ have obtained university and other 
tertiary level of education. Unfortunately, even with the level of education, rather than rational, atomistic, utility-maximizing individuals, the respondents behaved as collectivities, such as herds, with each individual motivated by the desire to minimize his/her exposure to risks or losses without ascertaining the genuineness or otherwise of the microfinance institution. Though such behaviour may be inherently rational, the individual's behaviour is likely to be constrained by incomplete information and the limits imposed by human cognitive power or human and social conventions. According to Khan, Tan and Chong (2017) several studies have found that investors like to seek information from informal communication such as unsystematic and word-of-mouth communications among others (Shiller and Pound,1989) but studies elsewhere also indicate that obtaining information from trustworthy sources contributes more toward active trading than information acquired from less reliable sources (Epstein and Schneider, 2008). A chi-square test of independence was performed to examine the relation between level of formal education of the respondents and whether they enquired if the microfinance institution they are engaged in business have a valid license from the Bank of Ghana. The relation between these variables was not significant, $\chi^{2}(4, N=165)=5.61, p=$ 0.230 .

\section{Kind of Business Engaged with the Microfinance Institution}

The kind of business the respondents engaged with the microfinance institution included either borrowing, saving or both. Table 7 shows that 135 (82\%) out of the 165 respondents only saved with the microfinance institution and 26 saved and borrowed. One of the eight goals according to Keynes (1936) which would motivate individuals to abstain from spending out of their incomes is calculation to enjoy interest. Also among the three motives identified in literature for the desires to hold cash (McKinnon, 1973); the speculative motive is influenced by the level of interest rate and therefore an increase in interest rates will make saving more attractive and should encourage saving. Mwega, Ngola and Mwangi (1990) found that one of the main determinants of savings is interest rate. Since McKinnon (1973) and Shaw (1973) argue that low interest rate discourages savings mobilization, it therefore suggest that a higher interest rate encourages savings. For this reason, interest rate has become an instrument used by fraudulent microfinance institutions to deceive and lure people to save with them so they can swindle them.

Table 7: Cross Tabulation of Promised interest rate and kind of business engaged in with the microfinance institution

\begin{tabular}{|c|c|c|c|c|}
\hline \multirow[b]{2}{*}{$\begin{array}{l}\text { Promised interest } \\
\text { rate }\end{array}$} & \multicolumn{3}{|c|}{$\begin{array}{l}\text { kind of business engaged in with the microfinance } \\
\text { institution }\end{array}$} & \multirow[b]{2}{*}{ Total } \\
\hline & Borrowing & Saving & Borrowing and Saving & \\
\hline $1 \%-20 \%$ & $1(25)$ & $22(16.3)$ & $13(50)$ & $36(21.82)$ \\
\hline $21 \%-40 \%$ & $3(75)$ & 17 (12.59) & $9(34.62)$ & $29(17.58)$ \\
\hline $41 \%-60 \%$ & $0(0)$ & $72(53.33)$ & $4(15.38)$ & $76(46.06)$ \\
\hline above $60 \%$ & $0(0)$ & $24(17.78)$ & $0(0)$ & $24(14.55)$ \\
\hline Total & $4(100)$ & $135(100)$ & $26(100)$ & $165(100)$ \\
\hline
\end{tabular}


Table 7 shows that out of the 135 respondents who only saved with the microfinance institution, $96(71.11 \%)$ of them save because of the promised of interest rate of over 41 percent deposit interest rate. A chi-square test was performed to find out if there is difference between the promised interest rate and kind of business the customers are engaged in with the microfinance institution. There is significant difference between these variables, $\chi^{2}(62, \mathrm{~N}=165)=140.85, \mathrm{p}=0.000$. Though the basic component of assessment of capital investments is risk assessment, this seems to have been ignored by the respondents in their quest to get higher returns on their investment. Deposit interest rate in Ghana averaged 17.71 percent from 1980 until 2015, reaching an all-time high of 35.76 percent in 1997 and a record low of 8.89 percent in 2006. In 2015, deposit interest rate in Ghana increased to 13.30 percent from 12.90 percent in 2014 (World Bank, 2016). Therefore, a promise of deposit interest rate of over 41 percent by any microfinance institution should be an indication of a very high risk business venture. In light of this high level of deposit interest rate, it is the expectation that customers will do further background investigation on the credibility of the activities of those microfinance institutions with the central bank (Bank of Ghana) before engaging in any business with them. Failure to do so was likely to leave the customers with only some limited information to process in relation to the decision to invest with those institutions, a behaviour referred to as bounded rationality (Simon, 1978).

As a result of this bounded rationality, the customers take bad investment decisions to invest with these fraudulent microfinance institutions. The results in Table 8 shows that out of the total respondents of 165 who ever engaged in business with microfinance institutions, 119 of them have ever been swindled by the microfinance institution. A chi-square test was performed to find out if there is difference between the promised interest rate and whether the respondents were ever swindled by the microfinance institution. There is low significant difference between these variables, $\chi^{2}(31, \mathrm{~N}=165)=41.94, \mathrm{p}=0.091$.

Table 8: Cross Tabulation of promised interest rate and whether the respondent was ever swindled by this microfinance institution ever been swindled by the microfinance institution

\begin{tabular}{llll}
\hline $\begin{array}{l}\text { Promised interest } \\
\text { rate }\end{array}$ & Yes & No & Total \\
\hline $1 \%-20 \%$ & $20(16.81)$ & $16(34.78)$ & $36(21.82)$ \\
$21 \%-40 \%$ & $19(15.97)$ & $10(21.74)$ & $29(17.58)$ \\
$41 \%-60 \%$ & $60(50.42)$ & $16(34.78)$ & $76(46.06)$ \\
above $60 \%$ & $20(16.81)$ & $4(8.7)$ & $24(14.55)$ \\
\hline Total & $\mathbf{1 1 9 ( 1 0 0 )}$ & $\mathbf{4 6}(\mathbf{1 0 0 )}$ & $\mathbf{1 6 5 ( 1 0 0 )}$ \\
\hline Survey
\end{tabular}

Survey data (Figures in parenthesis represent percentages)

The analysis in Table 8 shows that 60 (50.42\%) of the respondents who have ever been swindled were promised an interest rate of between $41 \%$ and $60 \%$ and 20 $(16.81 \%)$ were promised an interest rate of above $60 \%$. The microfinance institutions capitalised on the bounded rationality behaviour of their customers due to information asymmetry to swindle them. 
A cross tabulation analysis of the source of motivation to do business with the microfinance institution and whether the respondent was ever swindled by this microfinance institution was done. Source of motivation to do business with the microfinance institution included income generation, valid licence by the microfinance to operate from Bank of Ghana, easy access to loan, expansion of business, save for contingencies, children's education and friends advice which is consistent with the eight goals postulated by Keynes (1936) to motivate individuals to abstain from spending out of their incomes. Table 9 shows that 88 (73.95\%) out of the respondents who have ever been swindled by the microfinance institutions saved with the intention of generating income. A chi-square test was performed to find out if there is difference between the Source of motivation and whether the respondents were ever swindled by this microfinance institution. There is significant difference between these variables, $\chi^{2}(6, N=165) 17.33, p=0.008$.

Table 9: Source of motivation and ever been swindled by the microfinance institution

\begin{tabular}{|c|c|c|c|}
\hline \multirow[b]{2}{*}{ Source of motivation } & \multicolumn{3}{|c|}{$\begin{array}{l}\text { ever been swindled by the microfinance } \\
\text { institution }\end{array}$} \\
\hline & Yes & No & Total \\
\hline income generation & $88(73.95)$ & $22(47.83)$ & $110(66.67)$ \\
\hline valid licence from BoG & $3(2.52)$ & $0(0)$ & $3(1.82)$ \\
\hline easy access to loan & $16(13.45)$ & $11(23.91)$ & $27(16.36)$ \\
\hline expansion of business & $6(5.04)$ & $5(10.87)$ & $11(6.67)$ \\
\hline save for contingencies & $2(1.68)$ & $5(10.87$ & $7(4.24)$ \\
\hline children's education & $3(2.52)$ & $1(2.17)$ & $4(2.42)$ \\
\hline friends advice & $1(0.84)$ & $2(4.35)$ & $3(1.82)$ \\
\hline Total & $119(100.00)$ & $46(100.00)$ & $165(100.00)$ \\
\hline
\end{tabular}

Survey data (Figures in parenthesis represent percentages)

\section{Conclusion}

The phenomenon of individuals deciding to follow others and imitating group behaviours rather than deciding independently and atomistically on the basis of their own, private information - referred to as herding- has an influence on their financial decisions. This paper adopted a descriptive-analytic approach to study the interactions between the institutions' behaviour, the influence of society, and the preferences of individuals and households to maximize their economic wellbeing. The study found that out of the total respondents of 165 who ever engaged in business with microfinance institutions, 119 of them have ever been swindled by the microfinance institution. The respondents exhibited herding behaviour in their investment decisions with these fraudulent microfinance institutions since most of the respondents $(78.78 \%)$ knew of the existence of these microfinance institutions through friends and radio advertisements. These fraudulent microfinance institutions used interest rate as a conduit to deceive and lure people to save with them so they can swindle them. Out of the 165 respondents, 91of them never enquired whether the microfinance institution they are engaged in business have a valid license from the Bank of Ghana to operate in the country. The respondents used bounded rationality in their investment decisions by failing to do any background check on these institutions and relied on the little information from 
friends to process in relation to the decision to invest with these institutions. The respondents, therefore, took bad investment decisions to invest with these fraudulent microfinance institutions as a result of this bounded rationality.

\section{References}

Arnswald, T. (2001). Investment behaviour of German equity fund managers: an exploratory analysis of survey data (Discussion Paper No. 08/01). Frankfurt.

Aryeetey, E., Hettige, H., Nissanke, M. and Steel, W. (1997). Financial Market Fragmentation and Refroms in Sub-Saharan Africa (Technical Paper series No. 356). Washington, DC.

Baddeley, M. (2010). Herding, social influence and economic decision-making: sociopsychological and neuroscientific analyses. Philosophical Transactions of the Royal Society B, 365(1538), 281-290.

Balcilar, M., Demirer, R. and Hammoudeh, S. (2013). Investor Herds and RegimeSwitching: Evidence from Gulf Arab Stock Markets. Journal of International Financial Markets, Institutions and Money, 23(1), 295-321.

Bank of Ghana. (2016). Annual Report. Retrieved December 31, 2017, from https://www.bog.gov.gh/privatecontent/Publications/Annual_Reports/Annu al Report 2016 final 24th June.pdf

Barber, B.M. and Odean, T. (2013). The behaviour of individual investors. In Handbook of the Economics of Finance. Elsevier, B.V. Retrieved from https:/ / faculty.haas.berkeley.edu/\%0Aodean/Papers current versions/behavior of individual investors.pdf

Besley, T. (1994). How do market failures justify interventions in rural credit markets? World Bank Research Observer, 9(1), 27-47.

Binswanger, H. P. and Rosenzweig, M. R. (1986). Behavioural and Material Determinants of Production Relations in Agricultur. The Journal of Development Studies, 22, 503-539.

Buchanan, J. M. (1978). From Private Preference to Public Philosophy: The Development of Public Choice in Institute of Economic Affairs. In The Economics of Politics. London, UK: Institute of Economic Afffairs.

Canova, L., Rattazzi, A. M. and Webley, P. (2005). The hierarchical structure of saving motives. Journal of Economic Psychology, 26, 21-34.

Corredor, P., Ferrer, E. and Santamaria, R. (2015). The Impact of Investor Sentiment on Stock Returns in Emerging Markets: The Case of Central European Markets. Eastern European Economics, 53(4), 328-355.

Croson, R. and Gneezy, U. (2009). Gender Differences in Preferences. Journal of Economic Literature, 47, 448-474.

Dohmen, T., Falk, A., Huffman, D., Sunde, U., Schupp, J. and Wagner, G. G. (2005). Individual Risk Attitudes: New Evidence from a Large, Representative, ExperimentallyValidated Survey (DIW Discussion Papers No. 511). DIW Discussion Papers.

Epstein, L.G. and Schneider, M. (2008). Ambiguity, information quality, and asset pricing. Journal of Finance, 63(1), 197-228.

Falk, H. and Matlulich, S. (1976). The effect of personal characteristics on attitude toward risk. The Journal of Risk and Insurance, 43(2), 215-241. 
Fellner, G. and Maciejovsky, B. (2007). Risk Attitude and Market Behaviour: Evidence from Experimental Asset Markets. Journal of Economic Psychology, 28, 338-350.

Fry, M. J. (1982). Models of Financially Repressed Developing Economies". World Development, 10(9), 731-750.

Fry, M. J. (1988). Money, Interest and Banking in Economic Development. (Baltimore: John Hopkins University Press.

Galariotis, E. C., Krokida, S-I and Spyrou, S. I. (2016). Bond Market Investor Herding: Evidence from the European Financial Crisis. International Review of Financial Analysis, 48, 367-75.

Goldberg, N. (2005). Measuring the Impact of Microfinance: Taking Stock of What We Know. Grameen Foundation USA Publication Series (Washington, DC: Grameen Foundation).

Imai, K. S., Arun, T. and Annim, S. K. (2010). Microfinance and household poverty reduction: New evidence from India. World Development, 38(12), 1760-1774.

Ivkovic, Z. and Weisbenner, S. (2007). Information diffusion effects in individual investors' common stock purchases: covet thy neighbors' investment choices. Review of Financial Studies, 20(4), 1327-1357.

Keynes, J. M. (1936). The general theory of employment, interest and money. London: Macmillan.

Khan, M.T.I., Tan, S. and Chong, L. (2017). Information sources and investing decisions - a path modeling approach. Managerial Finance, 43(8), 928-947.

Lin, H. W. (2011). Elucidating rational investment decisions and behavioral biases: evidence from the Taiwanese stock market. African Journal of Business Management, 5(5), 1630-1641.

Lupton, J. P. and Smith, J. P. (2003). Marriage, Assets and Savings. In GROSSBARDSHECHT \& S. Societies (Eds.), Marriage and the Economy: Theory and Evidence from Advanced Industrial (pp. 129-152). Cambridge: Cambridge University Press.

Lusardi, A. and Mitchell, O. S. (2008). Planning and Financial Literacy: How Do Women Fare? American Economic Review, 98, 413-417.

Mahjabeen, R. (2008). Microfinancing in Bangladesh: Impact on households, consumption and welfare. Journal of Policy Modeling, 30, 1083-1092.

McKinnon, R. I. (1973). Money and Capital Market in Economic Development. Washington, DC: The Brookings Institution.

Menkhoff, L., Schmidt, U. and Brozynski, T. (2006). The impact of experience on risk taking overconfidence, and herding of fund managers: complementary survey evidence. European Economic Review, 50(7), 1753-1766.

Mitchell, V.W. and Greatorex, M. (1993). Risk perception and reduction in the purchase of consumer services. The Service Industry Journal, 13(4), 179-200.

Mwega, F., Ngola, S. and Mwangi, N. (1990). Real Interest Rates and the Mobilization of Private Saving in Africa: A casev study of Kenya (Research Paper No. 2).

Rao, D. S. (2012). Credit Market Failures and Microfinance. International Journal of Research in Management E Technology, 2(3), 294-297.

Roe, A. R. (1991). Financial Systems and Development in Africa. a conference report 
of an EDI Policy Seminar held in Nairobi, 29 January-1 February 1990.

Roth, A. E. (2000). Game Theory as a Tool for Market Design. In and S. T. Fioravante Patrone, Ignacio García-Jurado (Ed.), Game Practice: Contributions from Applied Game Theory (pp. 7-18). Dordrecht: The Netherlands: Kluwer.

Shaw, E. (1973). Financial Deepening in Economic Development. Oxford: Oxford University Press.

Shiller, R.J. and Pound, J. (1989). Survey evidence on diffusion of interest and information among investors. Journal of Economic Behavior E Organization, 12(1), 47-66.

Simon, H. A. (1978). Rationality as Process and Product of thought. American Economic Review, 68, 1-16.

Sulemana, A. and Adjei, S. A. (2015). Microfinance Impact On Agricultural Production In Developing Countries - A Study Of The Pru District In Ghana. International Journal of Academic Research and Reflection, 3(3), 26-44.

Thirlwall, A. P. (1999). Growth and Development: with Special Reference to Developing Economies (6th ed.). London: Macmillan publishers.

Waite, L. and Gallagher, M. (2000). The Case for Marriage: Why Married People are Happier Healthier, and Better Off Financially. New York: Doubleday.

World Bank. (2016). World Development Indicators. Retrieved December 31, 2017, from http:/ / databank.worldbank.org/data/reports.aspx?source=worlddevelopment-indicators\# 


\title{
Martha Nussbaum's Theory of Emotion and Death Issues
}

\author{
Vasil Gluchman \\ University of Prešov, \\ Prešov, Slovakia \\ Marta Gluchmanová \\ Technical University in Košice, \\ Prešov, Slovakia
}

\begin{abstract}
The aim of the article is exploring Martha Nussbaum's theory of emotions and their meaning in human life. Authors pay attention also to one of the most frequent emotions in Nussbaum's writings, to the fear of death. She explores the fear of death especially in her research of Hellenistic philosophy and ethics. Following this research, Nussbaum argues that the fear of death is understandable in case of premature death of young people. According to her, death is an interruption of human activities, for this reason, it is bad. Authors confirm that despite Nussbaum's little focus on the meaning and role of positive emotions in human development and human flourishing, her theory of emotions as one of the core capabilities is a very useful tool how to improve human life and to reach good life and well-being of human beings. It also concerns her view that philosophy can be like medical treatment very useful diminishing the fear of death.
\end{abstract}

Keywords: Martha C. Nussbaum, emotions, human development, human flourishing, fear of death.

\section{Introduction}

Human development is one of the noble goals of almost whole human history. A turn of the $20^{\text {th }}$ and $21^{\text {st }}$ centuries offers to us, on the one hand, the new ideas how to go on with human development. One of the significant contemporary theories is Martha Craven Nussbaum's theory of human development based on the capabilities approach (Nussbaum, 2002; 2003; 2011; 2016). Nussbaum, following Amartya Sen, defines capability as the opportunity to achieve plans and goals of a human being has reason to value in its human development. The aim of the article is to analyze the meaning of emotions in human development. 


\section{Capabilities approach and theory of emotions}

Nussbaum's capabilities approach includes mental states which mean being able to have connections to things and people outside ourselves and in their means of human development; to like very much those who love and take care for us, to mourn at their absence; generally, to love, to mourn, to experience longing, gratefulness, and justified anger.

What concerns emotions in Nussbaum's theory, it possible to see them as a significant contributor to the method we think and learn about human development and also life's deepest secrecies, especially our personal vulnerability, our reliance on others, and about ways we can help out the flourishing of ourselves and other people (Plumb, 2014). According to Nussbaum, the emotions are, indeed, a form of knowledge through which we think about things that we take care about but that are beyond our control. With Stoic philosophy as her guide, Nussbaum opens a much broader view of the emotions and of their contribution to moral thinking as one of the most significant factors of human development (Nussbaum, 2016).

Nussbaum states her theory of the emotions as neo-Stoic. We have already laid the groundwork to realize what this might mean. The Stoic opinion of the emotions is that they are a form of knowledge, that is, a way of reasoning about things in our human development. Different from other forms of knowledge, the things that are the object of the emotions are things that concern to us, that we take care about or feel connected to. Given the changes of life and the ultimate finitude of objects of concern, however, they are also features of our lives that are eventually out of our control. If something happens somehow to either improve or jeopardize an object of concern, our emotions are how we think about it (Plumb, 2014). Nussbaum claims, emotions are eudemonistic, that is, they refer intrinsically to a human being's sense of human flourishing and human development. Nussbaum wants to maintain that our eudaimonia, or flourishing happiness as a form of human development, depends upon our formulating a cluster of aims and goods to which we join value, which in turn are constituent elements of a conception of what it means for us to live good life, to reach well-being and human flourishing. For compassion to then occur, we must make the eudaemonistic decision that the other's well-being is involved in our personal conception of well-being, good life and human flourishing (Gallagher, 2009).

In Nussbaum's estimation, the reality of the emotions is open-ended, and everemerging than many would hope. Still, as a form of rational thought, emotions are far from being unreasonable or hopelessly not capable of contributing to the good life, well-being, and human development. The emotions take part in the real world, where real objects of concern flourish or feel pain. The evaluations of the emotions are subject to being more or less right or wrong, and the perceptions and beliefs that support them, and the judgments that they sustain are still cooperative to both epistemic and, particularly, the phronetic reasoning of our human development involving human flourishing. The advantage of Nussbaum's neo-Stoic theory of 
emotions, it that, at the same time as it considers the complexity of the emotions, it retains a keen sense of their capacity to support to moral reasoning and human flourishing and development of the human beings (Plumb, 2014). Nussbaum's reason for exploring emotions also in animals is to point out how emotions are "elements of our common animality with considerable adaptive significance: so their biological basis is likely to be common to all" (Nussbaum, 2003, 141).

According to Gallagher, an encounter with Nussbaum's rich and nuanced exploration provides a possibility to reflect upon one's own emotionality as a part of our human nature, on the things one takes cares about in life, and about one's own sense of neediness and vulnerability in face of the finitude of things of the world. It provides one a chance to reason about the various alternatives one might use to deny or rage against one's own sense of vulnerability and, in doing so, be a negative force in the lives of other people as well as how to make them better (Gallagher, 2009). The two cardinal ways that Nussbaum sees this occurring are, first, through overcoming the emotion of disgust, because it is a barrier of our human development as well as due to a fact that an emotion divides us from others insofar as it pushes the other away as different and subordinate to us; and, second, by cultivating the mental capacity of imagination, which Nussbaum believes will make possible us to understand and feel the pain of those who undergo tragic events who are not in our recent empirical orbit, not just humans, but also animals (Gallagher, 2009).

The identification of emotion as part of moral thinking and human development holds the potential to promote reflection of marginalized groups through the tying of fairness to equality: since compassion is conceptualized as a pain that is felt for others where there is a breach of capabilities and where there is inequality. Moreover, the encouragement of emotional recognition with each and every other, by taking the other into one's concept of ' $\mathrm{I}$ ', offers a way of negotiating the controversial elements of individual and collective-focussed approaches to organizing difference in our effort how to overcome our limits in human development and human flourishing.

Nussbaum's idea of empathy is one of the brightest: empathy is "an imaginative reconstruction of another person's experience without any particular evaluation of that experience" (Nussbaum, 2002, 302). According to her, the evaluation that a person is in anxiety, and a desire to do something about it, is a judgment of compassion rather than empathy. It is significant to note that she is not claiming that this is all there is to compassion, but that compassion, when it overcomes negative emotions such as disgust, envy, and shame, entails evaluation. She argues that sympathy is also close to the term compassion, though lacking its strength (DeaneDrummond, 2017).

According to Celia Deane-Drummond, for Nussbaum, compassion has three elements that require a reasoned decision. The first decision of compassion is one of measurement of size-it means what has happened to the human being or other creature is a serious event. The second decision is one of nondesert: the being did not 
deserve this to happen to it. The third decision is eudaemonistic, meaning this being is important in terms of my goals (Deane-Drummond, 2017). Nussbaum also names wonder the ability to reason the worth of the other even apart from our own sense of flourishing and human development (Nussbaum, 2002). For Nussbaum, empathy requires recognition of the otherness, rather than simply feeling as if it were its pain, which is an emotional infection. Thus, empathy means both an awareness of another's pain and yet knowing that it is not mine (Nussbaum, 2002). Empathy requires sure imagining of what the other being (not only human being) is feeling and is a prelude to compassion where those feelings are connected with bad feelings. Empathy, however, can result in a lack of compassion, as when enemies read the intentions of their rivals or enemies and manipulate them for their own purposes. Nussbaum also reasons that compassion may be without empathy, however, empathy is a good 'pilot for compassion (Nussbaum, 2002).

According to Deane-Drummond, Nussbaum's analysis is extremely illuminating in clarifying the relationships between empathy and compassion, as well in her examination of the Greek ancient literature in order to define more specifically what compassion means for human development and human flourishing. However, her idea that empathy is merely a pilot for compassion seems a little bit weak. So while theoretically, in accordance with her definitions, it might be possible to show compassion without empathy, compassion is more significant and fuller as a virtue if it is inclusive of empathy rather than excluding it of human nature and human development. Modifying her definition, any account of compassion needs therefore to involve in a primary meaning a positive affirmation of the evaluation of compassion toward the other informed by love for the other as a part of human flourishing and human development, rather than, in the way that she sets up her argument, an answer to large negative events that have happened to another that were underserved. Such answers can be included, but her idea of ordering toward "wonder" needs modifying to one of effective compassion guided by love (DeaneDrummond, 2017).

Nussbaum's explaining compassion that focuses on the specific cognitive evaluations required in compassionate deeds fails to consider adequately the priority of relations between love and compassion in human flourishing and human development, specifically, that part of compassion that is actively involved, rather than just in answer to another's distress. While empathy exactly speaking means the ability to feel what the other feels, and therefore is potentially either passive in the ethical and moral sphere, compassion is more positive and aside from some uncommon exceptions requires the capacity of empathy for its using in human development and individual flourishing (Deane-Drummond, 2017).

\section{A fear of death in Nussbaum's theory of emotions}

In Theory of Desire Nussbaum very intensively focuses her research on the fear of death, especially in context with Hellenistic philosophy and ethics, in Epicurus and Lucretius. She expressed that we see in society people who are ignorant of life and 
death issues on the first look. Their response can be that they do not have fear of death and they are full of optimism. However, Nussbaum states that the fear of death is deeply rooted in their hearts (Nussbaum, 2009).

Following research on Hellenistic ethics, she affirms the diagnostic argument confirming deeply rooted the fear of death. According to Nussbaum, the diagnostic argument has four parts:

"1. A description of a pattern of behavior that seems to lack adequate explanation. It will be argued that the most powerful explanation of these symptoms is fear of death.

2. A description of a subjective condition which, although not consciously felt as fear, lacks, as described, adequate explanation. Again, it will be argued that the fear of death is the best explanation of these inner symptoms.

3. A description of occasions of confession or acknowledgment: situations in which the patient, dropping her habitual defenses, will grant that fear is in fact what she feels.

4. In the background, a normative picture of the healthy unconstrained person, a person whose life is not burdened by fear and who lacks, in consequence, the bad symptoms associated with it" (Nussbaum, 2009, 196).

Nussbaum mentioned Epicurus' and Lucretius' rejecting the fear of death as counterproductive for human life and very useful to religious beliefs and religious authorities. She follows their reasoning and affirms that death frustrates human projects and desires. The fear of death is not only the fear that makes human projects empty but also they are diminished (Nussbaum, 2009).

The fear of death can be, according to Nussbaum, rational for a person who has valuable activities, it means who is able to love, work, choose, enjoy the beauty. "Death will be most terrible when it is, in conventional human terms, premature; for then the value of many preparatory activities - activities involving training oneself so as to be able to act in some valued way in future - will be completely lost, in that they will never lead on to the fruition that gives them their entire point. To devote a large part of one's life to merely preparatory activities is characteristic of youth. But the elderly, too, have valuable lives; and their activities, too, are interrupted by death. It would perhaps be irrational for an elderly person to devote the whole of his or her time to activities whose value is entirely preparatory and instrumental; but even the activities that are constitutional of a good life can, as we have said, be interrupted. And even if there should be a person for whom death arrives just as all current projects are, for the moment, complete and at a standstill - if such a thing ever happens for a person who loves living - still, the bare project to form new projects is itself interrupted; and it seems that this project is itself a valuable one in a human life..." (Nussbaum, 2009, 209-210).

Nussbaum presents the view that philosophy is able to answer the fundamental human life questions including questions of life and death. Philosophy, according to 
her, can be like therapeutic medical treatment helping people to understand their lives and also the fear of death in contexts with human activities and their life plans.

\section{Conclusion}

However, some of the authors argue that Nussbaum' pays a little interest in positive emotions and her capabilities approach list reflects a Benthamite opinion of positive emotions that are out-dated in light of current psychological research. Positive emotions not only make people feel good; they also broaden and build, and allow human development and also human flourishing. They hope that the research on the positive values of positivity will move Nussbaum to the acceptance of positivity as a valued capability (Jayawickreme \& Pawelski, 2013). Really, we think that despite a critique of Marta Nussbaum's little focus on the meaning and role of positive emotions in human development and human flourishing, her theory of emotions as one of the core capabilities is a very useful tool how to improve human life and to reach good life and well-being of human beings. It concerns also her approach to the fear of death as well as the usefulness of philosophy like medicine for the mental health of people.

\section{Acknowledgement}

The paper is a part of project VEGA 1/0521/17 Critique of theoretical and methodological issues in contemporary bioethical debates on death and dying.

\section{References}

Deane-Drummond, C. (2017). Empathy and the Evolution of Compassion: From Deep History to Infused Virtue. Zygon, 52, 258-278.

Gallagher, P. (2009). The Grounding of Forgiveness: Martha Nussbaum on Compassion and Mercy. American Journal of Economics and Sociology, 68, 231252.

Jayawickreme, E. \& Pawelski, J. O. (2013). Positivity and the capabilities approach. Philosophical Psychology, 26, 383-400.

Nussbaum, M. C. (2002). Education for Citizenship in an Era of Global Connection. Studies in Philosophy and Education, 21, 289-303.

Nussbaum, M. C. (2003). Upheavals of Thought: The Intelligence of Emotions. Cambridge, MA: Cambridge University Press.

Nussbaum, M. C. (2009). The Therapy of Desire: Theory and Practice in Hellenistic Ethics, $3^{\text {rd }}$ ed. Princeton, NJ \& Oxford: Princeton University Press.

Nussbaum, M. C. (2011). Creating Capabilities: The Human Development Approach. Cambridge, MA \& London: The Belknap Press of Harvard University Press.

Nussbaum, M. C. (2016). Anger and Forgiveness: Resentment, Generosity, Justice. New York: Oxford University Press.

Plumb, D. (2014). Emotions and human concern: Adult education and the philosophical thought of Martha Nussbaum. Studies in the Education of Adults, $46,145-162$. 


\title{
Radio Listening Clubs in Malawi and How they Contribute to Expanding Ordinary People's Capabilities
}

\author{
Peter Mhagama \\ University of Malawi, \\ Blantyre, Malawi
}

\begin{abstract}
This article examines how radio listening clubs (RLCs) established by community radio stations in Malawi help to expand ordinary people's capabilities. The RLCs provide a platform for ordinary people to assemble to listen to a designated radio programme, discuss the content for its merit and then decide how best to use the information to improve their livelihoods. The programmes are produced in such a way that they raise pertinent issues faced in the community and offer listeners suggested solutions to overcome those problems. The findings discussed in the paper are based on data collected through focus group discussion with members of RLCs of Nkhotakota community radio, semi-structured interviews with its deputy station manager and other key informants, and participant observation of what goes on in the radio station. The article argues that the RLC has potential to expand the capabilities of ordinary people because the information they receive, share and discuss empowers them to make informed decisions and to be knowledgeable. By providing a forum for the discussion of issues affecting ordinary people, this article concludes that RLCs can help to raise people's awareness to existing problems and influencing them to do something about their situation. In this way, RLCs can help expand ordinary people's capabilities to achieve a life that they value.
\end{abstract}

Keywords: capabilities, community radio, human development, ordinary people, radio listening clubs.

\section{Introduction}

The paper examines how radio listening clubs (RLCs) in Malawi contribute to expanding capabilities of ordinary people in rural areas. A listening club is a 'small listening and discussion group that meets regularly to receive a special radio programme, which the members then discuss' (Rogers et al., 1977 cited in Manyozo, 2012: 29). Members discuss the content of the programme for its merit and then decide how best to use the information in their daily activities. The discussion is 
conducted in such a manner that it enables sharing of knowledge and raising issues concerning community problems and finding solutions to those problems (Mchakulu, 2007). Using a case study of Nkhotakota community radio station and its RLCs in Malawi, the article demonstrates how this arrangement can enhance the lives of rural people because the information they receive, share and discuss can enable them to make informed decisions and to be knowledgeable, hence expand their capabilities. The article gives an overview of RLCs, the motivation behind their establishment, the data collection method used in the research and finally discusses how RLCs can expand the capabilities of ordinary people.

\section{Origins of RLCs}

The Development Broadcasting Unit (DBU) (2000) defines a Radio Listening Club (RLC) as "a community based group organized by community members themselves and uses radio programmes to facilitate development discourse within their own community". The RLCs that this article examines were those that were established by Nkhotakota community radio station and some NGOs working in the community. The concept of RLCs in Malawi dates back to 1966 when they were called farmers' forum listening groups (Mackie, 1971). The origins of radio forums more generally can be traced further back to Canada during the Second World War in 1940 (Lewis and Booth, 1989) where they were called farm forums. The radio forums of Canada were "organized community-based radio structures to facilitate local people's participation in ongoing or impeding rural development projects" (Flor, 1995 cited in Manyozo, 2012: 30). From Canada radio forums spread to India in 1949 and then Africa particularly in Ghana between 1964 and 1965 where they aimed at educating adults and stimulating village self-help efforts (Berrigan, 1979: 22). Since then RLCs spread to other countries such as Malawi in 1966, Tanzania in 1967 and Senegal in 1969. These countries adopted their own models of implementation depending on their needs. For example, in Tanzania, between 1977 and 1978 Audio Cassette Listening Forums (ACLF), using audio cassette recorders, provided a development programme that enabled women to recognize the importance of their role (Berrigan, 1979: 48).

In Malawi, since independence in 1964, the Malawi Broadcasting Corporation (MBC) Radio $^{1}$, a public broadcaster, acted as a communication tool in agricultural and rural development (Mackie, 1971). It was meant to compensate for the many logistical, financial, staffing and transport constraints in the Ministry of Natural Resources' Extension Service Department with regards to "teaching Malawi's farmers better agricultural methods" (Mackie, 1971: 106). The Extension Service model is a "system of 'extending' new technology from the research plots of agricultural universities out to farmers through district extension agents" (White, 2009: 13). It was "an integral part of the modernization paradigm which hoped to raise productivity in

\footnotetext{
${ }^{1}$ After independence, Malawi was a single party state. During the one party regime (1964-1994) there was only one radio station in the country, the MBC. The MBC Act (1964) vested all broadcasting powers in this station, implying that there was no provision for the establishment of other radio stations, let alone television (Manjawira and Mitunda, 2011).
} 
developing countries by rapid transfer of technology from the First to the Third worlds" (White, 2009: 13). It is reported that the introduction of the farmers' forum listening group project in July 1966 in Malawi, was cost-effective and proved to be an effective rural development communication strategy in the sense that it increased farmers' knowledge and also provided a link between farmers and agricultural service providers (Mackie, 1971: 108).

In recent years, in Malawi, the Development Broadcasting Unit (DBU) of the MBC reintroduced RLCs under the Ndizathu Zomwe (Our Own) Project. The DBU is "a new structure within MBC, established in 1999 to liaise with the station's Programmes Department to effectively engage in development programming through participatory communication activities, to promote national dialogue and development issues" (Sisya, 2003 cited in Manyozo, 2005: 4). The project (Ndizathu Zomwe) was jointly implemented by the $\mathrm{MBC}$ and the Radio for Development $(\mathrm{RfD})^{2}$ of the United Kingdom (UK), with support from the British Government's Department for International Development (DFID) ${ }^{3}$. The idea behind the RLCs was to engage members in "participatory production of programmes based on development discourses among the network of radio listening clubs" (Manyozo, 2012: 144). The DBU initially established fifteen RLCs across the country, which later increased to more than thirty. Although this number is too small for the whole country, it was reported that the DBU has "promoted a sense of ownership of the national airwaves as it enables communities to determine programme content through their chosen discourse, as evidently, the day and time of broadcast were decided by the communities themselves" (Chirwa et al., 2000, cited in Manyozo, 2005: 6).

Therefore, when the first community radio station in Malawi, Dzimwe, was established in 1998, it adopted this format because it is a perceived success story. Other community radio stations which were established later, for example, Nkhotakota community radio established in 2003, also adopted the same initiative of setting up RLCs but went beyond participatory programme production to include other activities. The aim was "to facilitate people's participation at all levels of development efforts to identify and implement appropriate and sustainable policies, programmes, and technologies to reduce poverty and improve people's livelihoods" (Chirwa, 2005: 34). This article examines in more detail the activities which take place in the RLCs and demonstrate how they have the potential to expand people's capabilities.

\section{Participatory Communication and the Capabilities Approach}

\footnotetext{
${ }^{2}$ RfD now named Media For Development (MFD) was established as a private production company in the mid 1990s in the UK. Its primary communication tool was radio broadcasting of educational programmes produced and researched in collaboration with African broadcasters (MFD, n.d.).

${ }^{3}$ DFID was established in 1997 to lead the UK's work to end extreme poverty by creating jobs, unlocking the potential of girls and women and helping to save lives when humanitarian emergencies hit in 28 countries across Africa, Asia and the Middle East (www.gov.uk).
} 
Since the RLCs were established to ensure that its members are involved in participatory radio programme production 'based on development discourses' (Manyozo, 2012), I employed the participatory communication theory to help understand how the clubs contribute to development but that kind of development which is related to building capabilities. Therefore, I also analyse the capabilities approach to demonstrate how RLCs help to expand capabilities. According to Carpentier (2011) in 1975, the Dag Hammarskjöld Foundation released a report entitled 'What Now? Another Development'. Servaes (1999: 78-9) notes that the phrase 'Another development' in the title indicates "the ambition to develop another type of development grounded in the focus on the people's basic needs (such as eradication of poverty), self-reliance, ecological sensitivity, sustainability and participation". The report was released after scholars and development agents blamed the modernization paradigm which dominated intellectual thinking around 1945-1965 (Servaes, 1995: 40) for lack of participation of the beneficiary communities. This lack of participation was blamed for the failure of most development projects (Melkote, 1991). The Dag Hammarskjold Report views the former approaches to development (the modernization paradigm) as "reductionist and top-down, and more supportive of transnational capital than development and poverty reduction" (Carpentier, 2011: 50). In the modernization paradigm development was viewed as modernization and focused primarily on economic growth only Forster-Carter, 1985; Lerner, 1958). Instead the Dag Hammarskjold report emphasizes another development characterized by a diversity of approaches, and that development should "focus on the needs of the lower echelons" of the social system (Potter et al, 2008 cited in Carpentier, 2011: 50). This pointed to the idea that development that focuses on human development and capabilities is more empowering and meaningful and accords individuals more personal freedom than any other notion of development. This is where the issue of grassroots participation came in, and participation is defined as a process of empowering people to participate in identifying development problems or designing development programmes (Melkote, 1991).

Thus by the late 1980s the term participation was mainstreamed in large-scale development programmes and by early 1990s, many international agencies like SIDA and USAID had mainstreamed participation and empowerment in the development projects that they were implementing worldwide (Tandon, 2008: 289).

Here emphasis shifted from the broader levels of participation in developing societies to participation in setting of development projects, where it is aimed at empowering people, capturing the indigenous knowledge and ensuring the sustainability and efficiency of the interventions (Hickey \& Mohan, 2004 cited in Carpentier, 2011: 51).

Thus participation requires a higher level of people's involvement in the development projects. "Here individuals are active in development programmes and processes, they contribute ideas, take initiatives, articulate their needs and problems and assert their autonomy" (Ascroft and Masilela, 1989 in Melkote, 1991: 237). 


\section{Development Redefined}

The inclusion of the term 'participation' in development discourse shifts the emphasis from an understanding of development in the modernization paradigm as economic growth to that of development as expanding and facilitating human freedom and capabilities (Sen, 1992; Nussbaum, 2011). This is what Sen (1992) describes as the 'capabilities approach'. According to Alkire and Deneulin (2009: 31) "the key idea of the capability approach is that social arrangements should aim to expand people's capabilities - their freedom to promote or achieve what they value doing or being". Although social arrangements can vary from community to community and also depending on the stage of development each society is at, in this paper the RLCs are considered as the social arrangements which can help to expand people's capabilities. The RLCs are located in rural areas where essential basic resources are non-existent or limited. To ensure that these people live the lives they desire, it requires a number of social arrangements, one of which is the RLCs to try and help expand their capabilities. Extending the definition of the capabilities approach, Nussbaum (2011: 20) argues that "by capabilities, what is meant is "not just abilities residing inside a person but also the freedoms or opportunities created by a combination of personal abilities and the political, social, and economic environment". This is not the responsibility of government alone but also various development agents and agencies including the media playing a role. This paper will, therefore, argue and demonstrate how RLCs can not only enhance ordinary people's capabilities but also create opportunities for people to improve their social, cultural and economic conditions. In the capabilities approach, development is "a process of expanding the real freedoms that people enjoy" (Sen, 1999: 3). The freedoms might be the outcome of improved well-being such as literacy, hygiene, food availability and having smaller families as a result of information addressing these issues (Stevenson, 1988: 13). This definition implies that when ordinary people are given resources such as information and an enabling environment to exercise their creative potential and natural talents they can achieve things which can bring them happiness. Breaking the chains of ill health, lack of food, poverty, lack of access to information and other social ills which perpetually keep people in bondage can ultimately bring them freedom, which is development, according to Sen (1999). The purpose of development is "to create an enabling environment for people to enjoy long, healthy, and creative lives" (Nussbaum, 2011: 1). This is the meaning of development that is adopted in this paper which aims to investigate how RLCs help to expand people's capabilities.

\section{Methods}

\section{Participants}

Data presented in this paper are drawn from a research project conducted in Malawi. The data were collected through semi-structured interviews with the deputy station manager of Nkhotakota community radio station and some key informants drawn 
from some NGOs working with the radio station in the communities. I also conducted focus group discussions (FGDs) with the listeners of the radio station in their RLCs. These FGDs were conducted in six different RLC in Nkhotakota. Participant observation was another technique used to collect data. This involved observing what goes on in the radio station in terms of programme production, presentation, and daily operations or management. As part of participant observation, I had an opportunity to interact with and interview some selected radio practitioners such as the programme manager and production manager of the station. All the observations were recorded in a diary while all the interviews and FGDs were recorded and transcribed, and used as a resource for this study. The data were analyzed using qualitative thematic analysis.

\section{Results and discussion}

\section{Nkhotakota community radio station and its radio listening clubs}

Nkhotakota Community Radio station started broadcasting in 2003 with financial assistance from UNESCO. It is located in Nkhotakota district and targets a population of 303, 659 (the population of the district) which is mostly rural. The majority of the people (73 \%) in the district are involved in subsistence farming and $32.1 \%$ of the population lives in poverty (National Statistics office (NSO), 2012). The illiteracy rate in the district is $15.1 \%$ (NSO, 2012: 23). Nkhotakota community radio station has 32 RLCs that is, about ten clubs in each of the six Traditional Authorities 4 (TA's or Senior Chiefs) in the district (interview with deputy station manager, 31 October 2012). While some of these clubs were established by the radio station, others were established by NGOs such as Farmers Voice Radio (FVR) 5 , Total Land Care (TLC) and Sasakawa Global 2000. The RLCs are composed of the chairperson, secretary, treasurer, and members. The chairperson, treasurer and secretary are elected positions by the members. The chairperson is the leader and facilitator of the club meetings. Each club is supposed to have twenty-five members but due to large numbers of people in the villages willing to join, the total number of members exceeds twenty-five in some instances. Membership in these clubs is open to anyone willing to take part. Members of the clubs are trained by the radio practitioners (interview with deputy station manager, 31 October 2012).

\footnotetext{
4 There are 28 districts in Malawi. Administratively, the districts are subdivided into Traditional Authorities (TA's) presided over by senior chiefs. The TA's are composed of villages and these are the smallest administrative units presided over by village headmen/women (National Statistical Office (NSO) 2011, 13).

${ }^{5}$ In Malawi, FVR is a consortium made up of the Ministry of Agriculture, Irrigation and Water Development (MoAIWD); Bunda College of Agriculture, a former constituent college of the University of Malawi; Centre for Alternatives for Victimized Women and Children (CAVWOC); Creative Centre for Community Mobilization (CRECCOM); Farm Radio Malawi; Malawi Broadcasting Corporation (MBC); and all community radio stations in Malawi with funding from the Bill and Melinda Gates Foundation of the USA. The pilot phase which run from July1999 to December 2012 was implemented by the American Institute for Research (AIR) in partnership with, in the case of Malawi, the organizations mentioned above (http://www.creccom.org/project.php?project=44).
} 
Nkhotakota community radio station established its RLCs to achieve three major aims. First, as support networks to ensure that there is a listening audience. Members of the RLCs give feedback to the station on its programming and daily operations. This is also a way of ensuring audience feedback and it also functions as a form of audience research. Secondly, the clubs aimed to generate income for the radio station. The station sells station branded plain paper to RLCs and individuals at a profit. This paper is used for writing letters to the station. In an effort to make their clubs visible the clubs compete among themselves by buying many sheets of paper and writing many letters, and in the process make more money for the station (interview with deputy station manager, 31 October 2012). Finally, some programmes were established with the aim of imparting specific messages targeting specific groups of people in the community. For example, these could be programmes aimed at spreading messages about HIV/AIDS, safe motherhood, programmes targeting fishermen, and so on. The programmes can be sponsored programmes by NGOs or produced by the community radio station itself. Therefore, the third reason was to ensure that programmes produced to achieve specific development purposes have an impact on their target audiences. The impact expected could be change in behaviour or adoption of a new farming technique. This implies that some specific programmes have their own listening clubs whereby members use the information listened to directly in their activities. Club members discuss what they listen to in groups and share knowledge to enhance understanding. The interview excerpt below explains the aim of establishing RLCs:

The aim was to improve listenership because we realized that when they listen to the radio in groups, they are able to discuss what they listen to. Again the level of understanding is different so in groups they can share the knowledge which each person gets from the radio. The other reason is that we wanted to make sure that these programmes that have been produced for different reasons really bring an impact on the listeners. Some clubs were specifically created for specific programmes so that the people are really able to grasp what they listen to in these programmes. We want the people to make use of what they listen to on these programmes. Some listening clubs were created mainly to enhance health in our areas; others were created to enhance agriculture activities in the district; some were created to enhance HIV and AIDS awareness while others were created to mitigate the impact of climate change. That's the whole essence of establishing the radio listening clubs (interview with deputy station manager, 31 October 2012).

As Megwa (2007: 53) argues, "community support and participation are critical to the existence and survival of community-based organizations including community radio stations". Therefore, establishment of RLCs as support networks can be viewed as a strategic move. It would be almost impossible to broadcast at community level where there is no community support since community radio stations are ostensibly established for them. 


\section{Activities done in RLCs and how they contribute to building capabilities}

As Banda (2007, 132) has rightly observed, the concept of RLC is 'misleading' because it assumes that club members merely listen to the radio programmes. Yet there are many activities that take place apart from listening to the radio programmes. Listening clubs are one way of engaging people in development discourses and sharing of ideas. This has some properties of the public sphere although this happens in a mediated and managed context. Among the activities that are done in the RLCs include listening to designated radio programmes, discussing the content of the programme for its merit and deciding how to use the information in their activities. The content of the programme is also used to conduct drama performances and poetry recitals carrying messages on modern methods of farming, HIV/ AIDS, family planning, safe motherhood and other social issues affecting the people. Members of the clubs support one another in the their farming activities and also materially and they also conduct income generating activities to support their activities. To ensure sustainability of the clubs, some NGOs such as FVR also introduced activities such as the village bank and cooperatives to help farmers generate income for themselves and for the club as a whole. Club members access the money through loans or interest on shares to meet their economic needs (interview with FVR Projects Officer, 14 March 2013).

\section{Access to development information and sharing of ideas in the clubs}

The opportunity given to club members to discuss programme content with the aim of enhancing collective understanding "enables literates and illiterates to leap the illiteracy barrier" (Rogers et al., 1977 cited in Manyozo, 2012: 30). Due to high illiteracy levels among the listeners, some people find it hard to understand some of the information or concepts broadcast on the radio station. However, as Held (2006: 237) argues, "through sharing information and pooling knowledge, public deliberation can transform individuals' understanding and enhance their grasp of complex problems". This is demonstrated through some radio programmes in which members listen to development information. For example, in the agricultural programmes, farmers are advised to plant maize, which is a staple food crop in Malawi, at a spacing of 15 centimetres apart using the 1-1 maize (sasakawa) planting system to maximize production. This is contrary to the traditional practice of planting 3 maize seeds per station at a spacing of 30 centimetres. The ridges have to be 25 centimetres apart. Within their clubs, those who are literate demonstrate to the others how to achieve the prescribed measurements using sticks. The participants explained that:

Individually you can hear on the radio that to grow maize using the sasakawa type of farming, you have to make your ridges at a spacing of 25centimeters apart but without knowing what it really means. However, in our club we do have some members who can demonstrate using measuring instruments such as sticks. Sometimes we invite our agricultural advisors to explain to us as a group. Therefore, when we go to our farms we know exactly what to do with the measurements. On the other hand, non-club members have problems with such measurements (FGD 3, 3 November 2012) 
The manner in which members of RLCs discuss issues in groups can result in shared interpretation, shared meaning and eventually collective or individual action because the knowledge acquired from the clubs is directly applied to their activities. This point is illustrated by the farmers' response that:

In our meetings, we also make sure that every member knows how to do these things on their own with the help of those members who have understood them better, for example, this practice of growing maize at 15 centimetres apart (FGD 3, 3 November 2012).

This is a way of overcoming a problem arising from a lack of literacy. It is consistent with Chapman et al's. (2003) argument that RLCs "are being employed as an extension tool for their ability to reach many illiterate people, providing them with development information in the language they can understand" (cited in Manyozo, 2012: 154). Through the sharing of information, club members become their own and each others' development agents. This form of access to crucial information means that "community members have a platform for all manner of individual and collective self-expression" (Howley, 2010: 16). Therefore, RLCs also play an interventionist role by providing a platform where ordinary people can access development information and solve development conundrums for individual and community advancement. In all this, the community radio station is at the centre of the clubs' activities, both as a source of and as a tool for the dissemination of that information.

\section{RLCs as a platform for building ordinary people's capabilities}

It has been argued that, "while the community radio movement is worldwide, its resonance is most felt in places where the world's most vulnerable people eke out precarious livelihoods in a bid to escape poverty" (da Costa, 2012: 138). As reported earlier, the RLCs discussed in this article are located in rural areas where resources are limited, as such ordinary people join clubs with the aim of benefiting from the opportunities that are found there some of which can help alleviate their poverty. For example, it was reported that:

We assist each other in the groups. A person cannot stand alone. For instance, if one falls sick, the club members help that person to cultivate in his gardena thing that cannot happen when you are not in a club (FGD 4, 4 November 2012).

Despite the problems that individual members may face, members of RLCs share common problems and they realize that if they want to advance they have to uplift one another in times of problems to make sure that they are progressing together by assisting one another. According to the UNDP (1994: 13) "the purpose of development is to create an environment in which all people can expand their capabilities, and opportunities can be enlarged for both present and future generations". Here we note that RLCs can help to create such an environment. This is the main reason why ordinary people join RLCs, the hope of being assisted by 
others in the face of problems. To illustrate this point one farmer confessed how he was specifically assisted by his club members:

Being in a club is very important. For instance, I did not buy fertilizers this year because I didn't have money, but my fellow club members helped me with some fertilizers and now at least I have maize. If I were not in the club, I couldn't have harvested the maize that I have now since I didn't have the fertilizers (FGD 4, 4 November 2012).

Alkire (2010: 25) argues that people are "not only the beneficiaries of development; they are also agents, whose vision, ingenuity and strength are vital to advancing their own and others' well-being". The RLC, therefore, can be an effective means of mobilizing people to participate in self-help initiatives. It can also enable people to play the role of development agents for each other.

The difference between those who joined RLCs and those people who are not members of any club is that the benefits discussed above are not available to nonmembers. One participant explained that:

Most of the times when, something is beginning not all people join instantly. After they have appreciated the benefits from members who joined in the first place, then the rest join the grouping. Likewise here, they are currently inspired by what we gain from the club, hence prospects of joining us. For instance, when we experiment what we have heard on the radio it is observed that we produce more yields than anyone else. So they usually ask what our secret is. Then we tell them that although you listen to the radio on your own, it is totally different with us, because we do schedule a time to discuss them in detail (FGD 3, 3 November 2012).

It can be argued that participating in RLCs can help community members achieve collective capabilities. Collective capabilities are defined as "the newly generated functioning bundles a person obtains by virtue of his/her engagement in a collectivity that help her/him achieve the life he/she has reason to value" (Ibrahim, 2006: 398). The collectivity here is the RLC. Ibrahim further gives two characteristics of collective capabilities. First, collective capabilities 'are only present through a process of collective action", and secondly, that collective capabilities are "the new choices that the individual alone would neither have nor be able to achieve unless he/she joins a collectivity, such as a self-help group" (Ibrahim, 2006: 398). In the case of RLCs, collective capabilities can be achieved through group listening to the radio, discussing the message and adopting the best practices contained in the message; for example, modern farming practices. This can help to expand people's capabilities when they act jointly or individually to implement the decisions made. It was reported that as a result of the benefits realized by early adopters, the late adopters are persuaded to join clubs with an aim of realizing the same benefits; for example, one farmer testified that:

I was one of the hard nuts to crack. I did not know that planting one maize seed per station is good and I could scorn those people who used to follow this method. Now when the advisor came to us together with Mr Nkhata [chairman of the club], they explained the importance of planting one maize 
seed per station and they asked me to make manure and plant my maize seeds as advised. I found that it worked very well then I completely changed my mindset and I have finally adopted the use of manure and planting one seed per station (FGD 4, 4 November 2012).

It is common knowledge that people resist change sometimes. However, as Megwa (2007: 54) argues, "when an individual, for example, is trained to perform certain functions at a community radio station or learns from listening to community radio programmes, it is generally accepted that this knowledge and skill will be diffused to other members of the community". Therefore, participation in RLCs can help through interpersonal influence to persuade those people who resist change to see the importance of adopting new innovations and act in concert with the others for individual and collective capabilities.

\section{Access to material resources}

Banda (2007: 143) argues that "the knowledge that the clubs were organized structures, complete with rules and decision-making processes, presented an attractive forum for several development partners and policy-making elites to work with the clubs". The participants in this study reported that participating in RLCs results in easy access to loans, farm inputs and other material resources: "We are encouraged because in our groups it is now easy to access loans" (FGD 3, 3 November 2012). The RLCs act as a platform to help farmers find organizations which assist them with free seeds, fertilizers, and to find markets for their produce. Farmers reported that:

We have other organizations in this village that help us. We have NASFAM [National Smallholder Farmers' Association of Malawi] which assists us with groundnut seeds. NASFAM assists us because we are united and we do our activities as one (FGD 4, 4 November 2012).

When people are already organized in clubs, NGOs and other development agents find it easier to mobilize them for a particular development project in the community. Community radio stations link RLCs to different organizations which come to assist members in various ways. These opportunities are not available to community members who are not in clubs.

Furthermore, when the farm inputs are not sufficient for everyone, club members share the few resources that are there to ensure that everyone benefits equally. When a member of a club fails to repay a loan, club members mobilize themselves to bail out their colleague.

Sometimes organizations come and tell us that there is a chance of getting fertilizer on loan but there is need for a subscription fee. So what we do in our clubs is to contribute money as a club to pay for this subscription fee. When we find that the fertilizer is not enough then we just share among ourselves the little that we manage to buy. The other advantage of being in groups is that it is easier for organizations to reach us in our groups than when we are not in groups (FGD 4, 4 November 2012). 
Therefore, RLCs can be used as collateral when accessing loans. As Putman (2002) argues, "a group whose members manifest trustworthiness and put trust in one another will be able to accomplish much more than a group that is comparable but lacks trust" (cited in Damasio, 2011: 40). Farmers' participation in RLCs can also help them to develop trust in one another through how their activities are coordinated and this can improve interpersonal relationships.

\section{RLCs as a forum for women empowerment}

Lennie and Tacchi (2013: 65) argue that "while the participation of women is a fundamental principle for development, it is often difficult to carry through because of gender inequalities in many societies". Although, the whole of the rural population can be considered as marginalized, women are the most affected as they face marginalization in the family and village setting because of their considered low status or subordinate position in a male dominate society. According to Held (2006: 89), it is the subordination of women that "has created fundamental 'hindrances to human improvements'". The situation is especially aggravated by their lack of access to information that could help them mitigate or manage the problems that they face (Sterling et al., 2009: 145). Therefore, improving women's status "is essential if we are to move the world towards a better life for individuals" (Mongella, 1995: 121).

I also found that women's participation in RLCs increases the opportunity for them to access and discuss crucial information necessary to help improve their livelihoods. In almost all the RLCs I visited, there were more women than men. The reasons for this are varied; some have to do with gender roles, others have to do with traditional practices and economic reasons. For example, members of some RLCs reported that men are usually busy with work and sometimes their work demands going away from home frequently. On the other hand, women remain at home most of the times so they have time to participate in club activities where radio listening is one of the activities. For example, it was reported in one FGD that:

Being a lakeshore area, boys and men normally prefer going to the lake to fish unlike being in groups because they make money from fishing. This explains why we have more female members in our groups because women and girls are at home when men have gone fishing (FGD 6, 6 November 2012).

The participants further explained that while the men are away fishing or doing other jobs, it is mostly women who are involved in farming, hence their increased interest in joining farmers clubs. The women claimed that they are always determined to succeed in implementing what they have learnt from the programmes. Women see themselves as being responsible for the upbringing of children and so if they do not work hard in the garden then there will be no food for the children. Furthermore, because of their considered low status in society, women are said to welcome development news more positively than men so that they can improve their livelihoods through the benefits that may come through joining clubs (FGD 6, 6 November 2012). 
Another reason for the gender imbalance in RLCs is that sometimes ownership of the radio set is problematic. For example, an FVR projects manager explained that:

Some men think that they own the radio set so when they are going away they take it with them so that they can listen to it when doing their daily duties. Sometimes men hide batteries so they can listen to their favourite programmes at their own time when they come back. This leaves women with no opportunity to listen to the radio hence they opt to join radio listening clubs and listen to the radio in their clubs (interview, 16 March 2013).

According to a Farm Radio International (2011: 15) report, 75 percent of males and 56 percent of females in Malawi own radio sets. Although these figures do not indicate listening habits between men and women, it can be inferred that more men than women have access to a radio set. Therefore, in their quest for information more women join RLCs so that they can listen to a radio there. The RLCs serve to increase women's access to radio and to information necessary to improve their livelihoods.

However, unlike Manyozo (2005) whose study of participation in RLCs in Malawi found that there was male dominance in the dialogues that characterize RLCs despite women being in the majority, I found that when making contributions, both men and women expressed their views equally. I observed this when conducting FGDs in the clubs. It can be suggested, therefore, that over the years the RLCs have empowered women in the research area to participate without fear in issues that affect them. By participating in RLCs, more women can gain confidence to come in the open and engage in activities that improve not only their lives but that of the whole family.

To illustrate that RLCs can offer a potential solution to the lack of access to information, the participants claimed that:

Here along the lakeshore area, women were not engaging in any economic activities, only men did. These days, because of the programmes which are broadcast on the radio, women have become as active as men economically. They are now doing business just like their male counterparts (FGD 1, 2 November 2012).

This claim can be read as meaning that discussion of programme content in the RLCs has encouraged women to think about gender roles and to do something about it to reverse the situation. The claim that more and more women are now involved in small scale businesses is a sign of building capabilities as result of access to information through community radio but discussed in the RLC. The people feel that they are informed and so their awareness has been raised, and because of this, they are motivated to change. The women further claimed that they are now closing the gaps in the gender divide by contributing equally to the running of their families instead of just depending on men. One woman explained that:

As women we were just depending on our husbands for everything.

Currently, many women are farming on their own thereby benefiting their homes as well (FGD 3, 3 November 2012).

Women's ability to contribute to the family income or food basket is indicative of 'women's agency' (Sen, 1999). As a result, women's "contribution to the prosperity 
of the family is then more visible, and she also has more voice, because of being less dependent on others" (Sen, 1999: 192). The RLCs, therefore, can provide a forum for increasing women's capacity to participate or engage in economic activities and to access information which contributes to their self-development and that of the family. Such a forum can enable women to discuss issues affecting them, make informed decisions, and to speak out their mind and to have a voice just as their male counterparts. In this way, RLCs can be said to play a role in empowering marginalized groups such as women and to help expand their capabilities.

Furthermore, young women claimed that the discussions which take place in RLCs have also helped them to gain confidence which enables them to among other things, negotiate use of a condom during sex, as illustrated in this response:

We as girls are equipped with skills necessary to curb harassment. We can be bold and firm on the use of condoms and in addition, if one violates our rights, we know where to go and report the human rights violations (FGD 6, 6 November 2012).

Consequently, this has the potential of reducing women's vulnerability to abuse because the information they receive from the community radio and the discussion they hold in the RLCs can raise their awareness about such issues. This information can help empower them to defend their rights by reporting abuse cases and human rights violations. The spread of HIV/AIDS can further be reduced among the youth in the process. Therefore, instead of suffering in silence, RLCs provide a forum where the youths' personal problems such as sexual harassment and abuse are shared and discussed, solutions suggested, and the necessary course of action taken by the affected person. This is particularly significant for people living in rural areas where there are no or very few places or organizations where abuse cases can be reported. There are also no places where people can receive counseling on the problems affecting them. Therefore, by listening to specific programmes on the community radio together and discussing problems in groups, the youth can enlighten one another about how to mitigate those problems.

\section{Facilitating self-representation in development dialogues}

Fraser and Estrada (2001: 20) argue that "in poor communities, local authorities and politicians can easily take advantage of citizens, either individually or as a group, in part because the marginalized and oppressed have no way to complain". Confirming this argument, a study by Chirwa et al. (2000) found that "RLCs sometimes face outright hostility from service providers such as district assemblies, civil society and NGOs, who usually do not appreciate listening to audio-tapes that articulate problems of a particular community or the idea of driving to communities for dialogues" (cited in Manyozo, 2012: 154). However, I found that all service providers mentioned by Chirwa et al. (2000) avail themselves to the RLCs except politicians especially MPs who shun such meetings. This leaves the electorate angry and frustrated as demonstrated in the excerpt below: 
We have been asking for an opportunity to meet our MP but to no avail. Meanwhile we have just stopped because we think they regard us as unimportant people (FGD 5, 5 November 2012).

The participants reported that other office bearers at district level are readily accessible to answer people's questions: “Officials from the district hospital do come and partly the officials from the council. They explain and give the subjects a chance to speak out their views" (FGD 6, 6 November 2012). According to Tettey (2011: 245) "This live exchange provides a unique opportunity for ordinary citizens to directly confront or engage with public officials in ways that the normal ordering of power relations would otherwise not allow". It can allow ordinary people to make repeated calls for effective delivery of social services and implementation of projects. The reason why politicians shun such meetings is not clear. However, community radio stations in Malawi are not allowed to broadcast any political and religious news to avoid promoting 'hate speech'. Section 9.6 of the license conditions under 'Live Broadcasts' in Sub-section 9.6.3 it is stated that, "the authority shall restrict the Licensee from live broadcasts if the Licensee broadcasts hate speech whether live or recorded" (GoM, 2008). However, this cannot be the reason why politicians fail to avail themselves for such interfaces because responding to people's development needs is not tantamount to promoting hate speech. Consequently, the topics of discussion in the clubs fall short of empowering ordinary citizens politically. Discussion of governance or anything political is frowned upon as such topics of discussion are limited to service provision such as construction of school blocks, health centres, poverty alleviation, health and agriculture news (Mchakulu, 2007; Manyozo, 2012).

Another challenge that RLCs face in enhancing capabilities emanates from their relationship with community radio stations. Since radio programmes produced and recorded in the RLCs are edited by radio practitioners before they are broadcast, the power to decide what content to broadcast remains with the radio producers. For example, Members of Mpamantha farmers Club complained that some of the programmes which they produced and recorded some time back before this research had not been aired on the radio. In this case, the radio producers act as gatekeepers of the programmes that are recorded in the RLCs. Although this could be a source of conflict between members of RLCs and radio producers, such tension is diffused by the radio producers who use their power to provide any convincing explanation reassuring the members that all is well. As Carpentier and Dahlgren's (2011: 8) argue, "the presence of a participatory culture cannot be conflated with participation itself and its logics of equal(ised) power relations". This arrangement can create an illusion of participation which can make ordinary people feel as though they are involved in a democratic process when in reality they are not (Dahlgren, 1995).

\section{Conclusion}

In conclusion, this chapter has discussed the concept of RLCs, and how it is helping to expand ordinary people's capabilities. The discussion has demonstrated how RLCs can create and expand people's capabilities to achieve more than what 
individuals can actually achieve if they acted alone. The RLCs contribute to raising people's awareness to existing problems and influencing them to do something about their situation through discussing the content of a radio programme and finding solutions to their problems. The people's claims about the positive changes that have taken place in their lives and community which they attribute to the activities done in the RLCs are evidence of increased agency. By providing a forum for the discussion of issues affecting ordinary people and allowing them to engage in different economic activities for their benefit, RLCs can be said to help expand ordinary people's capabilities. They contribute to alleviate poverty "which robs people of the freedom to satisfy hunger, or to achieve sufficient nutrition, or to obtain remedies for treatable illnesses, or the opportunity to be adequately clothed or sheltered, or to enjoy clean water or sanitary facilities" (Sen, 1999: 4). Attainment of these things can enable people enjoy the life they value.

\section{References}

Alkire, S. (2010). Human development: Definitions, critiques, and related concepts. Background paper for the 2010 Human Development Report. OPHI Working Paper No. 36. Oxford: OPHI.

Alkire, S. \& Deneulin, S. (2009). The human development and capability approach. In Deneulin, S \& Shahani, L. (Eds.) An introduction to the human development and capability approach: Freedom and agency (pp. 22-48). London: Earthscan,.

Banda, F. (2007). Radio listening clubs in Malawi and Zambia: Towards a participatory model of broadcasting. Communicare, 26(1), 130 -148.

Berrigan, F. J. (1979). Community communications: The role of community media in development. Paris: UNESCO.

Carpentier, N. (2011). Media and participation: A site of ideological-democratic struggle. Bristol: Intellect.

Carpentier, N. \& Dahlgren, P. (2011). Introduction: Interrogating audiences Theoretical horizons of participation. Communication Management Quarterly, 21, 7-12.

Chirwa, W.C., Kayanula, E. \& Lijenda, B. (2000). The Development Broadcasting Unit (DBU), Malawi Broadcasting Corporation: A tracer study of Kanthu N'khama radio program. Blantyre: DBU and MBC, Unpublished.

Chirwa, W.C. (2005). Community broadcasting in Malawi: Which way forward. A country assessment paper prepared for AMARC. Chancellor College: University of Malawi.

da Costa, P. (2012). The growing pains of community radio in Africa: Emerging lessons towards sustainability. Nordicom Review, Special Issue 33, 135- 148.

Damasio, M. J. (2011). Social capital: Between interaction and participation. Communication Management Quarterly, 21, 37-60.

Dahgren, P. (1995). Television and the public sphere: Citizenship, democracy and media. London: Sage.

Development Broadcasting Unit (DBU), (2000). The development broadcasting unit. Retrieved from http://www.mbcradios.com/aboutmbc/dbu.htm. 
Farm Radio International. (2011). Participatory radio campaigns and food security: How radio can help farmers make informed decisions. Retrieved from http://www.farmradio.org/pubs/ farmradioprcreport2011.

Foster-Carter, A. (1985). The sociology of development. Ormskirk: Causeway.

Fraser, C. \& Estrada, S. R. (2001). Community radio handbook. Paris: UNESCO.

GoM, (2008). Malawi Communications Regulatory Authority community sound broadcasting license. The Malawi Government Gazette. XLV(21).

Held, D. (2006). Models of democracy (3rd ed.). Cambridge: Polity Press

Howley, K. (ed.). (2010). Understanding community media. Thousand Oaks: Sage.

Ibrahim, S. S. (2006). From individual to collective capabilities: The capability approach as a conceptual framework for self-help. Journal of Human Development, 7(3), 397-416.

Lennie, J. \& Tacchi, J. (2013). Evaluating communication for development: A framework for social change. Oxon: Routledge.

Lerner, D. (1958). The passing of the traditional society: Modernizing the Middle East. New York: Free Press.

Lewis, P. M. \& Booth, J. (1989). The invisible medium: Public, commercial and community Radio. London: MacMillan.

Mackie, W.E. (1971). Radio broadcasting in Malawi: A search for identity and service. PhD diss., University of Missouri at Columbia.

Manyozo, L. (2005). The practice of participation in broadcasting for development in postindependent Malawi. Journal of Social Development in Africa, 20(1), 77 - 105.

Manyozo, L. (2012). People's radio: Communicating change across Africa. Penang: Southbound.

Mchakulu, J.E.T. (2007). Youth participation in radio listening clubs in Malawi. Journal of Southern African Studies, 33(2), 251-265.

Megwa, E. R. (2007). Community radio stations as community technology centers: An evaluation of the development impact of technological hybridization on stakeholder communities in South Africa. Journal of Radio Studies, 74(1), 49-66.

Melkote, S.R. (1991). Communication for development in the Third World: Theory and practice for empowerment (1 $1^{\text {st }}$ ed.). London: Sage.

Mongella, G. (1995). Moving beyond rhetoric. In Women: Looking beyond 2000. New York: United Nations Publications.

National Statistical Office, (2012). Integrated household survey 2010-2011: Household socio-economic characteristics report. Zomba-Malawi.

Nussbaum, M. C. (2011). Creating capabilities: The human development approach. Cambridge: The Belknap Press.

Sen, A.K. (1992). Inequality re-examined. Oxford: Clarendon Press.

Sen, A. (1999). Development as freedom. Oxford: Oxford University Press.

Servaes, J. (1995). Development communication-for whom and for what? Communicatio, 21(1), 39-49.

Servaes, J. (1999). Communication for development: One world, multiple cultures. Cresskill, NJ: Hampton Press. 
Sterling, S. R., O'Brien, J. \& Bennett, J. K. (2009). Advancement through interactive radio. Inf Syst Front, 11, 145-154.

Stevenson, R. L. (1988). Communication, development, and the third world. New York: Longman.

United Nations Development Programme (UNDP). (1994). Human development Report 1994: New dimensions of human security. New York: Oxford University Press.

Tandon, R. (2008). Participation, citizenship and democracy: Reflections on 25 years' of PRIA. Community Development Journal, 43(3), 284-296.

Tettey, W. J. (2011). Talk radio and politics in Ghana: Exploring civic and (un)civil discourse in Public Sphere. In Gunner, L., Liganga, D. \& Moyo, D. (eds.). Radio in Africa: Publics, cultures, communities. Johannesburg: Wits University Press, 19-35.

White, R. A. (2009). Grassroots, participatory communication in Africa: 10 major lines of research. Communication Research Trends, 28(1), 3-27. 


\title{
The Pass-Through of Global Food Price Inflation to Domestic Prices Inflation: Empirical Evidence from South Asia
}

\author{
Selliah Sivarajasingham \\ University of Peradeniya, \\ Peradeniya, Sri Lanka \\ Navaratnam Balamurali \\ The Open University of Sri Lanka \\ Nawala, Sri Lanka
}

\begin{abstract}
This study investigates and assesses how the international food price surge affects domestic inflation process in South Asia. Global food price indices (GFPI) are collected from FAOSTAT website. Data for domestic prices (CPI and CFPI) are collected from CEIC website. Exchange rates for each country are collected from IMF website. The empirical statistical results are derived by using a battery of parametric and non-parametric econometric techniques using monthly data of price series for the study period, 2005M1 to 2017M12. The co-integration analysis results confirm that the global food prices and domestic prices are co-integrated. Granger-causality test reveals the unidirectional causal relationship running from global food prices to domestic prices over the study period for Pakistan and Sri Lanka. However, In the case of India, Bangladesh and Nepal, samples do not show the evidence of causal relationship in the short run. However, in the long run, GFPI Granger cause local prices in all countries. Therefore, Governments from South Asia need to develop a safety net program for the poor and a longer term poverty reduction strategy. Policy attention needs to shift towards efforts to increase food production. The results of this study have shown various policy implications for monetary policy, food and agricultural policy and trade policy for South Asia.
\end{abstract}

Key words: food prices, Pass- through, co-integration, South Asia.

\section{Introduction}

Higher food prices erode the purchasing power of households. The soaring food prices may have tremendous impact on the real incomes of poor households in developing countries, South Asia in particular. It fuels inflation risks and hits the 
most vulnerable. The people who spend a large share of their income on food will be mostly affected. The food price surge during recent years has aroused intense concern worldwide about the impact on the poor in developing and emerging countries. International food prices are still above their average levels. The risk of higher food prices was highlighted in a recent World Bank publication (Global Development Finance, 2007). Rapidly rising food prices pose important macroeconomic policy challenges for decision makers in emerging and developing countries.

South Asia is the vulnerable region to food price inflation. According to the International Food Policy Research Institute (IFRI), South Asia and SSA are the regions with highest Global Hunger Index (GHI) scores and the highest poverty rates (IFRI, 2008, 2013). Sri Lanka's rank based on 2013 GHI scores (GHI=15) comes under serious category. Food prices have largest weight around more than 40 percent in the average household's consumption basket in South Asia. In globalized world, domestic food prices closely move with international food prices. These price movements lead to changes in local producer and consumer price. It influences headline inflation, local food price inflation and inflation expectations either directly or through second round effects.

Considering these transmission channels, several studies have empirically examined the relationship between global food prices and domestic inflation: Shawarby and Selim (2012), Jalil and Zea (2011), Van Duyne (1982), and El- Sakka et al. (2005). While a vast literature has examined the transmission effects of global food prices to domestic, no in-depth quantitative study investigated the pass-through effects of international food price inflation on domestic consumer price inflation for South Asia. This study attempts to fill this gap in the literature by examining pass-through and spillover effects of international food prices to the domestic inflation in South Asia. This study attempts to answer the following questions: i) to what extent world food prices pass-through domestic prices in South Asia? ii) is there long run relationship between global food price and domestic prices in each of country in South Asia?. iii) Have domestic food price inflation spillover into headline inflation?, The extent of transmission is a fundamental and important issue because it is the domestic prices that affect the welfare of poor consumers; hence it has important implications for economic welfare.

The main objective of this study is to investigate in-depth the pass through of international food prices to consumer prices in the domestic inflation dynamics in South Asia during the period of 2005M1 to 2017M12. In particular, this investigation will show the extent and speed of transmission of international food price changes to the domestic prices in South Asia. This study will uncover a robust empirical relationship between GFPI and domestic prices. Currency movements, domestic physical infrastructure, market failure and government policies to stabilize the prices may determine the size of the impact of the pass through. However, this study focuses only how pass-through occurs. 
The structure of the paper is as follows. Section 2 describes data and methodology of the study. Section 3 discusses the empirical results obtained from various econometric techniques applied in this study and the final section concludes, and gives some recommendations from the results obtained.

This study contributes to the literature and seeks to provide updated estimates of global food price pass-through effects using econometric methodologies. Consequently, this study provides a more comprehensive analysis of pass-through effects in South Asia by focusing on the short run and long runs. In order to conduct monetary policy and food policy successfully, a thorough understanding of price transmission mechanism is required. The understanding of the dynamic passthrough relationship between global food prices and domestic prices has important benefits for analyzing and forecasting inflation and implication for monetary, food, trade and agricultural policies.

\section{Data and Methods}

This study area covers some selected South Asian countries, namely, Bangladesh, Nepal, India, Pakistan, and Sri Lanka. The data related for Afghanistan, Bhutan, and Maldives are not available for all sample period. The sample period of the study is 2005M1 to 2017M12. Time series (monthly) data are used for this study. Global food price indices (GFPI) are collected from FAOSTAT website. Data for domestic prices (CPI and CFPI) are collected from CEIC website. Exchange rates for each country are collected from IMF website.

There are eight countries namely, Afghanistan, Bangladesh, Bhutan, Maldives, Nepal, India, Pakistan, and Sri Lanka form South Asia but this paper analysed the data from the selected five countries except Afghanistan, Bhutan, and Maldives out of the above mentioned eight countries because of the unavailability of adequate data.

This study adopts a simple model based on the Law of One Price (LOP) to express the relationship between global food prices and domestic prices and test the transmission effect.

The empirical statistical results are derived by using descriptive statistics, confidence ellipse, non-parametric regression (Kernel), Granger causality test, co-integration and error correction technique. To estimate the transmission effects of global food price changes on domestic prices, the models were formulated for each domestic price based on LOP. Further, Johansen co-integration analysis is adopted to examine the long run equilibrium relationships and Error correction model is used to capture short run dynamics around a long term equilibrium relationship between time series and to estimate feedback speed. Granger causality test is employed to identify the direction of causal relationship between the variables. 


\section{Results \& Discussion}

The time series plot in Figure-1shows that international food prices, Consumer Price index, domestic food price index move upward closely. Local food price has been higher and increasing trend compared to global food price dynamics. Global food price series are more volatile than domestic price series. However, local food prices in South Asia follow global food price movements.

Global food price inflation has asymmetric effects on domestic food prices and overall price behaviour. Pass-through of food price hikes is significantly higher than that of food price falls in South Asia. Domestic price trends of food items have broadly mirrored global trends. However, while domestic prices rise in tandem with global food prices they do not tend to decline to the same extent that global food prices do. This may be owing to local market inefficiencies, domestic monopolies, and limited global trade integration.

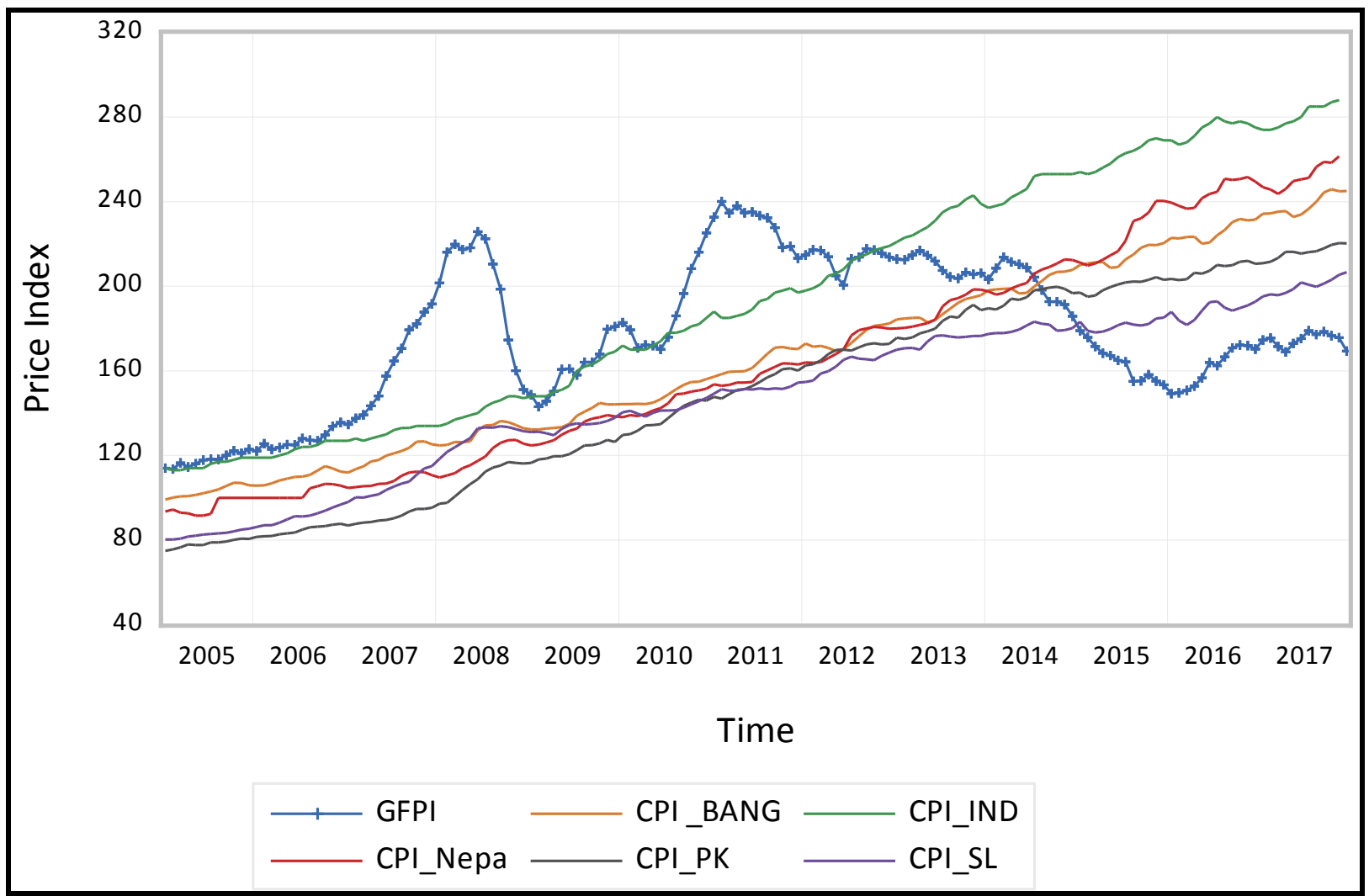

Figure 1- CPI and CFPI, GFPI Dynamics in selected countries, 2005M1-2017M12

The overall shape of the confidence ellipse indicates that world food price, CPI are and positively correlated. Therefore, international food prices have been claimed that it has been their main driving factor. The volatility of global food price inflation is also positively related to domestic inflation. we can observe the underlying dynamic inter relationship between global food price and domestic prices from the confidence ellipse graphs in Figure 2, -Figure 6 . The overall shape of the confidence ellipse indicates that CFPI, GPFI, CPI are positively correlated. The Lowess linear fit indicates that the dynamic relationships are nonlinear and positive. 


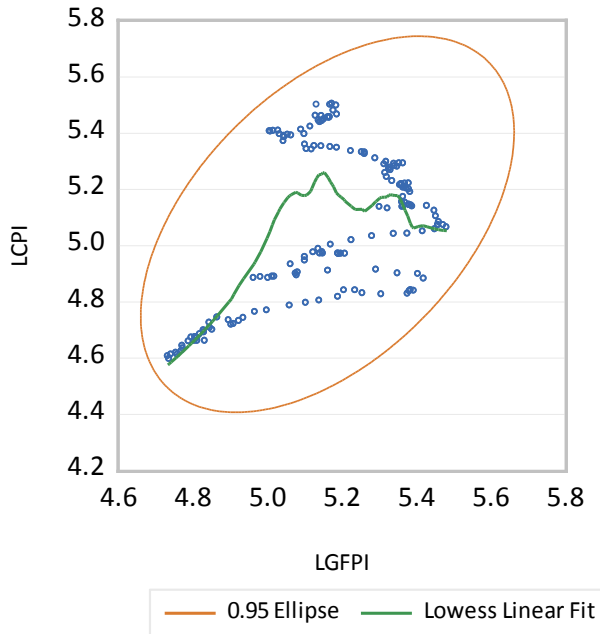

Figure 2 - Bangladesh

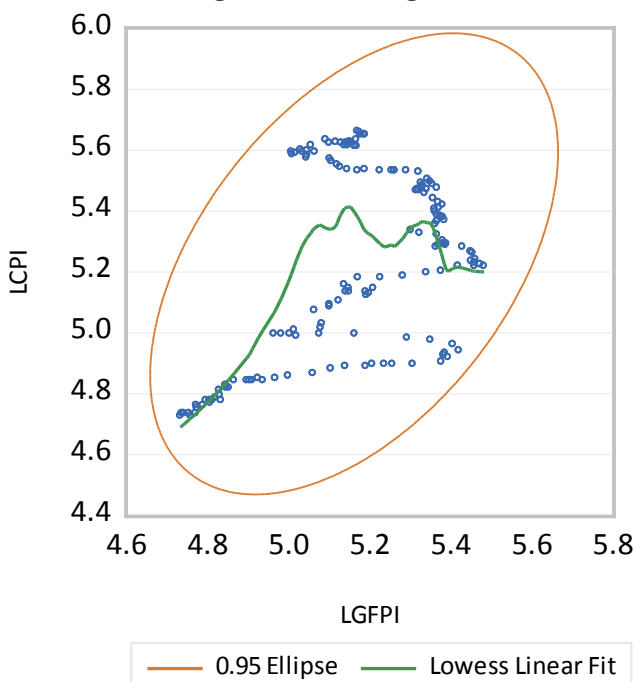

Figure 4 - India

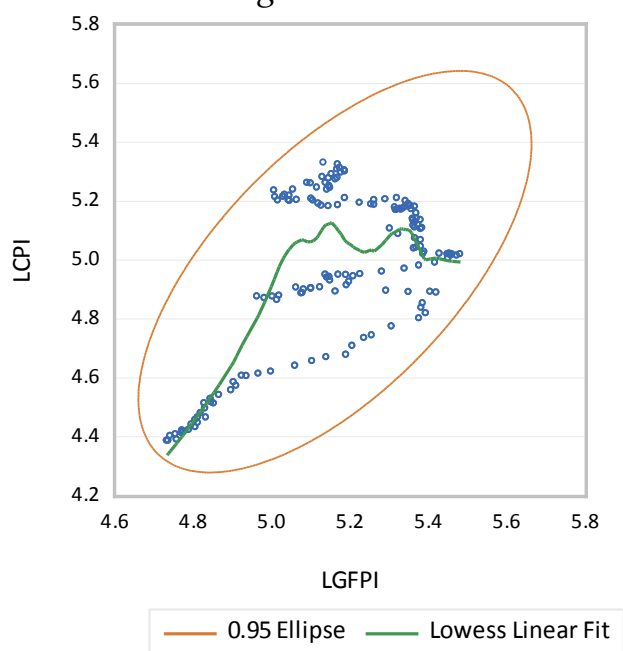

Figure 6 - Sri Lanka
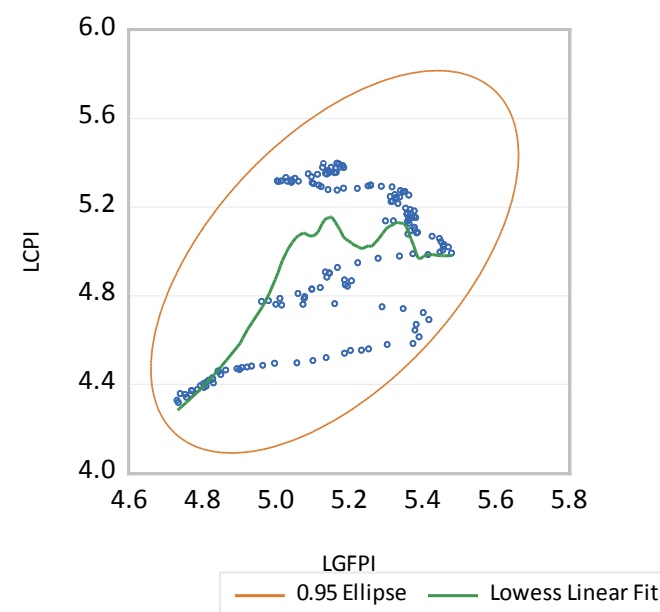

Figure 3 - Pakistan

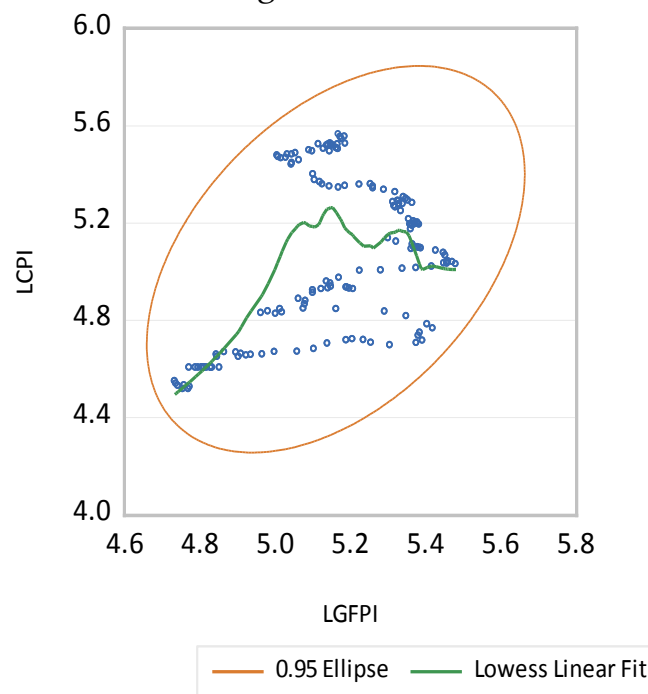

Figure 5 - Nepal

\section{Unit Root Tests of Variables}

The ADF unit root test indicated that all these price series are non-stationary series and integrated order one, I(1). These series in first difference are stationary, I(0). All " $\mathrm{P}$ " values of the ADF test statistic for each variable of every country in this study 
are less than 0.05 . The first difference series are strongly mean reverting and $\mathrm{I}(0)$. Therefore, all the series in this study are I(1) in level form.

Table 1- Unit Root Test Results for Variables.

\begin{tabular}{|c|c|c|c|c|c|c|c|c|c|}
\hline \multirow[t]{3}{*}{ Countries } & \multicolumn{4}{|c|}{$\begin{array}{c}\text { Level } \\
\text { Intercept with trend }\end{array}$} & \multicolumn{4}{|c|}{$\begin{array}{c}\text { First Difference } \\
\text { Intercept with trend }\end{array}$} & $\begin{array}{l}\text { Order } \\
\text { of } \\
\text { integrat } \\
\text { ion }\end{array}$ \\
\hline & \multicolumn{4}{|c|}{$\begin{array}{c}\text { ADF } \\
\text { t- test statistics }\end{array}$} & \multicolumn{4}{|c|}{$\begin{array}{c}\text { ADF } \\
\text { t- test statistics }\end{array}$} & \\
\hline & LCFPI & LCPI & LER & LGFPI & LCFPI & LCPI & LER & LGFPI & \\
\hline Sri Lanka & $\begin{array}{l}-1.69 \\
(0.75) \\
\end{array}$ & $\begin{array}{l}-1.48 \\
(0.83) \\
\end{array}$ & $\begin{array}{l}-2.57 \\
(0.29)\end{array}$ & $\begin{array}{l}-2.11 \\
(0.53) \\
\end{array}$ & $\begin{array}{l}-7.62^{*} \\
(0.00) \\
\end{array}$ & $\begin{array}{l}-6.98^{*} \\
(0.00 \\
\end{array}$ & $\begin{array}{l}-4.60^{*} \\
(0.00)\end{array}$ & $\begin{array}{l}-4.59^{*} \\
(0.00)\end{array}$ & $\mathrm{I}(1)$ \\
\hline India & $\begin{array}{l}-1.75 \\
(0.72) \\
\end{array}$ & $\begin{array}{l}-0.76 \\
(0.96) \\
\end{array}$ & $\begin{array}{l}-2.34 \\
(0.40)\end{array}$ & $\begin{array}{l}-2.11 \\
(0.53) \\
\end{array}$ & $\begin{array}{l}-6.87^{*} \\
(0.00)\end{array}$ & $\begin{array}{l}-6.01^{*} \\
(0.00) \\
\end{array}$ & $\begin{array}{l}-6.59^{*} \\
(0.00)\end{array}$ & $\begin{array}{l}-4.59^{*} \\
(0.00)\end{array}$ & $\mathrm{I}(1)$ \\
\hline Bangladesh & $\begin{array}{l}-3.13 \\
(0.10)\end{array}$ & $\begin{array}{l}-3.33 \\
(0.06) \\
\end{array}$ & $\begin{array}{l}-2.00 \\
(0.59)\end{array}$ & $\begin{array}{l}-2.11 \\
(0.53) \\
\end{array}$ & $\begin{array}{l}-7.21^{*} \\
(0.00)\end{array}$ & $\begin{array}{l}-7.29^{*} \\
(0.00) \\
\end{array}$ & $\begin{array}{l}-6.58^{*} \\
(0.00)\end{array}$ & $\begin{array}{l}-4.59^{*} \\
(0.00)\end{array}$ & $\mathrm{I}(1)$ \\
\hline Pakistan & $\begin{array}{l}-0.21 \\
(0.99) \\
\end{array}$ & $\begin{array}{l}-0.06 \\
(0.99) \\
\end{array}$ & $\begin{array}{l}-1.73 \\
(0.73)\end{array}$ & $\begin{array}{l}-2.11 \\
(0.53) \\
\end{array}$ & $\begin{array}{l}-6.56^{*} \\
(0.00)\end{array}$ & $\begin{array}{l}-5.03^{*} \\
(0.00) \\
\end{array}$ & $\begin{array}{l}-4.66^{*} \\
(0.00)\end{array}$ & $\begin{array}{l}-4.59^{*} \\
(0.00)\end{array}$ & $\mathrm{I}(1)$ \\
\hline Nepal & $\begin{array}{l}-3.04 \\
(0.12)\end{array}$ & $\begin{array}{l}-2.56 \\
(0.29)\end{array}$ & $\begin{array}{l}-2.39 \\
(0.38)\end{array}$ & $\begin{array}{l}-2.11 \\
(0.53) \\
\end{array}$ & $\begin{array}{l}-6.89^{*} \\
(0.00)\end{array}$ & $\begin{array}{l}-6.66^{*} \\
(0.00) \\
\end{array}$ & $\begin{array}{l}-6.91^{*} \\
(0.00)\end{array}$ & $\begin{array}{l}-4.59^{*} \\
(0.00)\end{array}$ & $\mathrm{I}(1)$ \\
\hline
\end{tabular}

Note: * indicates significance at $5 \%$ level. $\mathrm{P}$ value is in parenthesis. Unit root test regression model is selected with intercept and trend.

\section{Co-integration analysis}

Cointegration analysis is performed using the Johansen method to estimate long run equilibrium relationship between global food price and domestic prices in South Asia. Two models are estimated separately for two domestic prices, namely, CPI and CFPI. Unit root test results are given in Table 1 indicate that all series are in the same order of integration, I(1).

\section{Determining the Optimal Lag}

Before performing the test for the existence of co-integration, it is vital to choose an appropriate lag order of the model specification. The Johansen method is known to be sensitive to the lag length (Banerjee et al., 1993). Therefore, first, the optimal lag is estimated. The appropriate lag length for the co-integration test is selected based on VAR lag order selection criteria. The optimal lag for each domestic price of all countries in level series are reported in Table .2

Table 2 - VAR Lag Order Selection Criteria

\begin{tabular}{|l|l|l|l|l|l|l|l|l|l|l|}
\hline & \multicolumn{3}{l|}{ Sri Lanka } & \multicolumn{2}{l|}{ India } & \multicolumn{2}{l|}{ Bangladesh } & \multicolumn{2}{l|}{ Pakistan } & \multicolumn{2}{l|}{ Nepal } \\
\hline Model & LCPI & LCFPI & LCPI & LCFPI & LCPI & LCFPI & LCPI & LCFPI & LCPI & LCFPI \\
\hline SC & -17.32 & -16.11 & -16.30 & -15.31 & -17.31 & -16.53 & -16.37 & -16.02 & -15.62 & -15.14 \\
\hline Lag & 2 & 2 & 2 & 2 & 2 & 2 & 2 & 2 & 2 & 2 \\
\hline
\end{tabular}

Then, co-integration tests are carried out for two domestic price models. Domestic prices are deliberately chosen as the dependent variable (normalized to one) to 
examine the global food price transmission to the domestic prices in South Asia. The Johansen test procedure for co-integration among the variables is used by looking at a rank of the long run impact matrix (referred to as the $\Pi$ matrix in many expositions) via its eigenvalues. Using Johansen's $(1988,1991)$ maximum eigenvalue and trace tests, a sequential procedure is used to decide the number of co-integrating relations. Two sets of models employed to test co-integration using Johansen's method are:

1. Model 1 LCPI $=\mathrm{f}($ LGFPI, LER $)$

2. Model LCFPI $=\mathrm{f}($ LGFPI, LER)

The results of the Johansen co-integration test for each domestic price model are presented in Table 3.

Table 3 - Rank Test of Cointegration for Global Food Price Transmission.

\begin{tabular}{|c|c|c|c|c|c|c|c|}
\hline & & No o & integ & Trace Stat & No of $\mathrm{Cc}$ & ation (r) & \\
\hline Country & Model & $\mathrm{H}_{\mathrm{o}}$ & $\mathrm{H}_{1}$ & & $\mathrm{H}_{\mathrm{o}}$ & $\mathrm{H}_{1}$ & \\
\hline Sri Lanka & $\begin{array}{l}\text { LCPI } \\
\text { LCFPI }\end{array}$ & $\begin{array}{l}r=0 \\
r \leq 1 \\
r=0 \\
r \leq 1\end{array}$ & $\begin{array}{l}r \geq 1 \\
r \geq 2 \\
r \geq 1 \\
r \geq 2\end{array}$ & $\begin{array}{c}32.41(29.79)^{*} \\
8.79(15.49) \\
45.35(35.19)^{*} \\
13.42(20.26)\end{array}$ & $\begin{array}{l}r=0 \\
r=1 \\
r=0 \\
r=1\end{array}$ & $\begin{array}{l}r=1 \\
r=2 \\
r=1 \\
r=2\end{array}$ & $\begin{array}{c}23.62(21.13))^{*} \\
7.19(14.26) \\
31.92(22.29)^{*} \\
7.95(15.89)\end{array}$ \\
\hline India & $\begin{array}{l}\text { LCPI } \\
\text { LCFPI }\end{array}$ & $\begin{array}{l}r=0 \\
r \leq 1 \\
r \leq 2 \\
r=0 \\
r \leq 1 \\
r \leq 2\end{array}$ & $\begin{array}{l}r \geq 1 \\
r \geq 2 \\
r \geq 3 \\
r \geq 1 \\
r \geq 2 \\
r \geq 3\end{array}$ & $\begin{array}{c}53.98(35.19)^{*} \\
20.8(20.26) \\
7.90(9.16) \\
43.71(35.19) * \\
21.27(20.26) \\
9.01(9.16)\end{array}$ & $\begin{array}{l}r=0 \\
r=1 \\
r=2 \\
r=0 \\
r=1 \\
r=2\end{array}$ & $\begin{array}{l}r=1 \\
r=2 \\
r=3 \\
r=1 \\
r=2 \\
r=3\end{array}$ & $\begin{array}{c}33.69(22.29){ }^{*} \\
12.37(15.89) \\
7.90(9.16) \\
20.79(17.79)^{*} \\
9.15(11.22) \\
9.01(9.16)\end{array}$ \\
\hline Bangladesh & $\begin{array}{r}\text { LCPI } \\
\text { LCFPI }\end{array}$ & $\begin{array}{l}r=0 \\
r \leq 1 \\
r=0 \\
r \leq 1\end{array}$ & $\begin{array}{l}r \geq 1 \\
r \geq 2 \\
r \geq 1 \\
r \geq 2\end{array}$ & $\begin{array}{c}40.04(42.91) \\
11.72(25.87) \\
29.79(24.27)^{*} \\
6.35(12.32)\end{array}$ & $\begin{array}{l}\mathrm{r}=0 \\
\mathrm{r}=1 \\
\mathrm{r}=0 \\
\mathrm{r}=1\end{array}$ & $\begin{array}{l}r=1 \\
r=2 \\
r=1 \\
r=2\end{array}$ & $\begin{array}{c}28.31(25.82)^{*} \\
6.85(19.38) \\
23.44(17.79)^{*} \\
6.34(11.22)\end{array}$ \\
\hline Pakistan & $\begin{array}{l}\text { LCPI } \\
\text { LCFPI }\end{array}$ & $\begin{array}{l}r=0 \\
r \leq 1 \\
r=0 \\
r \leq 1\end{array}$ & $\begin{array}{l}r \geq 1 \\
r \geq 2 \\
r \geq 1 \\
r \geq 2\end{array}$ & $\begin{array}{c}59.10(35.19) \\
17.43(20.26) \\
52.28(35.19)^{*} \\
16.20(20.26)\end{array}$ & $\begin{array}{l}r=0 \\
r=1 \\
r=0 \\
r=1\end{array}$ & $\begin{array}{l}r=1 \\
r=2 \\
r=1 \\
r=2\end{array}$ & $\begin{array}{c}41.66(22.29)^{*} \\
13.37(15.89) \\
36.08(22.29) \\
12.45(15.89)\end{array}$ \\
\hline Nepal & $\begin{array}{l}\text { LCPI } \\
\text { LCFPI }\end{array}$ & $\begin{array}{l}r=0 \\
r \leq 1 \\
r=0 \\
r \leq 1 \\
r \leq 2\end{array}$ & $\begin{array}{l}r \geq 1 \\
r \geq 2 \\
r \geq 1 \\
r \geq 2 \\
r \geq 3\end{array}$ & $\begin{array}{c}27.73(24.27)^{*} \\
8.72(12.32) \\
40.11(35.19)^{*} \\
21.52(20.26) \\
8.77(9.16)\end{array}$ & $\begin{array}{l}\mathrm{r}=0 \\
\mathrm{r}=1 \\
\mathrm{r}=0 \\
\mathrm{r}=1 \\
\mathrm{r}=2\end{array}$ & $\begin{array}{l}\mathrm{r}=1 \\
\mathrm{r}=2 \\
\mathrm{r}=1 \\
\mathrm{r}=2 \\
\mathrm{r}=3\end{array}$ & $\begin{array}{c}19.00(17.79)^{*} \\
8.61(11.22) \\
18.58(22.29) \\
12.75(15.89) \\
8.77(9.16)\end{array}$ \\
\hline
\end{tabular}

Note: $r=$ number of cointegration,relationships * denotes Ho is rejected at $5 \%$ level.

$5 \%$ critical value in parenthesis. 
The Rank test (Trace and Maximum eigenvalue tests) shows that the null hypothesis of no co-integration, $\mathrm{H}_{0}: \mathrm{r}=0$ is rejected at the 5 percent level in favour of at least one co-integrating relationship. For Nepal, only trace statistics of LCFPI indicates two co-integrating relationships. Max-eigenvalue indicates no co-integrating relationship.

The co-integrating vector and speed of adjustment parameters are estimated for characterizing the extent of global food price transmissions and the disequilibrium behavior of domestic prices respectively. The results indicated that GFPI and domestic prices obey long run equilibrium relationships. This implies that GFPI and domestic prices never diverge too far from each other, they are in co-movement.

\section{Long Run Global Food Price Transmission Elasticities}

The long run parameter estimates from the co-integration analysis are reported in Table .4, for each model of CPI and CFPI.

Table 4 - Global Food Price Transmission Elasticities in Long Run, 2005M1-2017M12

\begin{tabular}{|c|c|c|c|c|c|c|c|}
\hline \multirow{2}{*}{\multicolumn{2}{|c|}{ Countries }} & \multicolumn{3}{|c|}{$\begin{array}{c}\text { Model 1: } \\
\text { LCPI =f ( LGFPI,LER) }\end{array}$} & \multicolumn{3}{|c|}{$\begin{array}{c}\text { Model 2: } \\
\text { LCFPI =f (LGFPI, LER) }\end{array}$} \\
\hline & & \multirow{2}{*}{$\begin{array}{c}\begin{array}{c}\text { Coefficien } \\
\mathbf{t}\end{array} \\
0.600^{*}\end{array}$} & \multirow{2}{*}{$\begin{array}{c}\text { SE } \\
0.062\end{array}$} & \multirow{2}{*}{$\begin{array}{l}\text { t-value } \\
9.677\end{array}$} & \multirow{2}{*}{$\begin{array}{c}\text { Coefficient } \\
0.597^{*}\end{array}$} & \multirow{2}{*}{$\begin{array}{c}\text { SE } \\
0.136\end{array}$} & \multirow{2}{*}{$\begin{array}{r}\text { t- value } \\
4.389\end{array}$} \\
\hline Sri Lanka & LGFPI & & & & & & \\
\hline & LER & $1.424^{*}$ & 0.102 & 13.921 & $1.839^{*}$ & 0.216 & 8.513 \\
\hline \multirow{2}{*}{ India } & LGFPI & $0.730^{*}$ & 0.321 & 2.274 & $0.908^{*}$ & 0.172 & 5.279 \\
\hline & LER & $1.096^{*}$ & 0.384 & 2.843 & $1.410^{*}$ & 0.204 & 6.911 \\
\hline \multirow[t]{2}{*}{ Bangladesh } & LGFPI & $0.074^{*}$ & 0.013 & 5.547 & 1.331 & 1.843 & 0.722 \\
\hline & LER & 0.100 & 0.071 & 1.412 & 0.02 & 2.213 & 0.009 \\
\hline \multirow[t]{2}{*}{ Pakistan } & LGFPI & $0.482^{*}$ & 0.132 & 3.651 & $0.543^{*}$ & 0.132 & 4.133 \\
\hline & LER & $1.055^{*}$ & 0.128 & 8.242 & $1.220^{*}$ & 0.128 & 9.531 \\
\hline \multirow[t]{2}{*}{ Nepal } & LGFPI & 0.494 & 0.991 & 0.498 & $1.622^{*}$ & 0.488 & 3.323 \\
\hline & LER & 0.876 & 1.153 & 0.759 & $1.277^{*}$ & 0.580 & 2.201 \\
\hline
\end{tabular}

Note: * indicates significant at 5\% level, others are not significant. Critical values for 5\% level is 1.645 .

Table 4 shows that the coefficient for GFPI in each CFPI and CPI model is statistically different from zero at the 5 percent level, except LCFPI for Bangladesh, and LCPI for Nepal. This implies that GFPI is influencing domestic price positively and significantly. Therefore, one can conclude that GFPI co-moves with (cointegrated) domestic food prices (CFPI), overall consumer price (CPI) indicating that a long run relationship exists between GFPI and CFPI and CPI. The estimated significant coefficient of GFPI implies that GFPI transmits positively and significantly to domestic food and overall prices. 
The estimated transmission elasticity indicates that when GFPI increases by one percent, average CPI will increase by $0.60 \%$ for Sri Lanka, $0.73 \%$ for India, $0.48 \%$ for Pakistan, $0.07 \%$ for Bangladesh, domestic average consumer food price (CFPI) increases by $0.73 \%$ for Sri Lanka, $0.90 \%$ for India, $0.54 \%$ for Pakistan and $1.62 \%$ for Nepal. All transmission elasticities are positive and significantly different from zero.

Error correction model results show that, global food price inflation has positive and statistically significant response on headline inflation and domestic food inflation in the short run. The coefficient of error correction term is statistically significant for each model (CPI, CFPI) for each country and has expected negative sign in the case of headline, food inflation equations. This indicates that global food price has long run causal relationship with headline inflation, domestic food inflation in the long run. All of the coefficients of error correction term are statistically significant at the 5 percent level and have correct signs and absolute values of them are less than one. This implies the stability of the corresponding model and each response variable (domestic prices) moves towards the long run equilibrium path. The estimate of adjustment coefficient for DLCPI, DLCFPI models shows that the disequilibrium is corrected in every month significantly.

Table 5 - Estimates of adjustment coefficient

\begin{tabular}{|l|c|c|}
\hline Country & LCPI & LCFPI \\
\hline Sri Lanka & $-0.049^{*}(-4.347)$ & $-0.043^{*}(-5.613)$ \\
\hline India & $-0.090^{*}(-5.887)$ & $-0.024^{*}(-2.223)$ \\
\hline Pakistan & $-0.029^{*}(-5.59)$ & $-0.060^{*}(-5.752)$ \\
\hline Bangladesh & $-0.256^{*}(-5.07)$ & $-0.002^{*}(-4.260)$ \\
\hline Nepal & $-0.003^{*}(-4.276)$ & $-0.025^{*}(-1.878)$ \\
\hline
\end{tabular}

Granger causality test shows that global food inflation Granger cause headline inflation, domestic food inflation in Pakistan and Sri Lanka. In other countries, there is no evidence that GFPI Granger cause local prices in the short run.

Table 6 - Granger causality Test results

\begin{tabular}{|l|l|c|c|c|}
\hline Countries & Null Hypothesis & Obs & F-Statistics & P value \\
\hline Sri Lanka & DLGFPI $\stackrel{\text { No }}{\longrightarrow}$ DLCFPI & 153 & $6.177^{*}$ & 0.003 \\
& DLGFPI $\stackrel{\text { No }}{\longrightarrow}$ DLCPI & & $9.620^{*}$ & 0.0001 \\
\hline Pakistan & DLGFPI $\stackrel{\text { No }}{\longrightarrow}$ DLCFPI & 153 & $4.963^{*}$ & 0.008 \\
& DLGFPI $\stackrel{\text { No }}{\longrightarrow}$ DLCPI & & $4.126^{*}$ & 0.018 \\
\hline India & DLGFPI $\stackrel{\text { No }}{\longrightarrow}$ DLCFPI & 153 & 3.123 & 0.079 \\
\hline
\end{tabular}




\begin{tabular}{|l|l|l|l|l|}
\hline & DLGFPI $\stackrel{\text { No }}{\longrightarrow}$ DLCPI & & 2.222 & 0.966 \\
\hline Nepal & DLGFPI $\stackrel{\text { No }}{\longrightarrow}$ DLCFPI & 153 & 0.408 & 0.666 \\
& DLGFPI $\stackrel{\text { No }}{\longrightarrow}$ DLCPI & & 0.236 & 0.789 \\
\hline Bangladesh & DLGFPI $\stackrel{\text { No }}{\longrightarrow}$ DLCFPI & 153 & 2.382 & 0.095 \\
& DLGFPI $\stackrel{\text { No }}{\longrightarrow}$ DLCPI & & 1.947 & 0.146 \\
& & & \\
\hline
\end{tabular}

\section{Conclusions and policy recommendation}

We find evidence of a statistically and economically significant international food price pass-through to domestic inflation dynamics in South Asia in the long run. The results of this study show domestic headline inflation, food inflation have been accompanied by the spikes in international food prices. Local consumer food price increases in higher rate than overall CPI prices due to global food price changes. The co-integration test results confirm that global food price inflation, local headline inflation and domestic food inflation are co-integrated in South Asian countries. It seems that the global food prices, both inflation and volatilities, affect the national food markets all countries in South Asia. Therefore, every government must develop a safety net program for the poor. Safety net interventions need to be made consistent with a longer term poverty reduction strategy and fiscal sustainability. Policy attention needs to shift toward efforts to increase food production and lower the vulnerability of the poor.

\section{References}

Banerjee, A., Galbraith J., \& Hendry, D. (1993). Cointegration, Error Correction and the Econometric Analysis of Non-stationary Data. Oxford: Oxford University Press.

Duyne, V. (1982). Food Prices, Expectations, and Inflation. American Journal of Agricultural Economics, 64(3), 419-43

El-Sakka, M. \& Ghali, K. (2005). The Sources of Inflation in Egypt: A Multivariate Co-integration Analysis. Review of Middle East Economics and Finance 3(3): 257269.

Jalil, M., \& Zea, E. T. (2011). Pass-through of international food prices to domestic inflation during and after the great recession: evidence from a set of Latin American economies. Desarrollo y Sociedad Journal, 67, 135-179.

Shawarby, S.A. \& Selim, H. (2012). Are International Food Price Spikes the Source of Egypt's High Inflation?, Policy Research Working Paper (6177). 


\title{
Inflation rate and Poverty: Does Poor Become Poorer with Inflation?
}

\author{
Mahua Paul \\ Assistant Professor at Institute for Studies in Industrial Development \\ Vasant Kunj, New Delhi \\ Pooja Sharma \\ Phd Scholar at Energy Studies Program, SIS, JNU \\ Assistant Professor Department of Economics, \\ Daulat Ram College, University of Delhi, New Delhi,
}

\begin{abstract}
This paper examines the inflation rates for the period 2005-06 and 2011-12 and concludes that inflation in 2005-06 hurts the poor more since the rise in food prices was substantial in this period and food constitutes a substantial proportion of their total expenditure._The paper observes that there was a substantial rise in Inflation which was experienced in the food grains like egg, fish, and meat, while the growth of inflation rate was observed minimal in case of beverages, footwear, and edible oil. Further, the impact of inflation on poor varied not only across both rural and urban areas but also the impact was different for different time periods. The paper also depicts shifts in the pattern of inflation rate across the expenditure classes between 2005-06 and 2011-12 and establishes inverse association between inflation rate and expenditure in the year 2005-06 for both rural and urban areas while the relationship was completely distinct in case of 2011-12 for both rural and urban areas. The study clearly reveals that bottom thirty percent of the population in rural areas observes same inflation rate as their urban counterparts for the year 2005-06. Finally, the paper concludes that the impact of inflation is not only commodity specific but also decile class specific. Further, the impact of inflation is observed to be different for rural and urban areas. This establishes the role of policy and government intervention through the public distribution scheme favoring the poor section with the aim of minimizing the gap of the impact of inflation experienced by poor and rich.
\end{abstract}

Keywords: Inflation rates, Total Expenditure, Decile classes, Public Distribution Scheme. 


\section{Introduction}

With the population of more than 1.2 billion, India's integration into the global economy has been accompanied by economic growth. This has launched the country amongst the global player (World Bank, 2018). Consequently, India has emerged as the world's third-largest economy in purchasing power parity. The growth rate of $7.6 \%$ in 2015 constitutes a substantial consumer market in the world (Hindustan Times March 07, 2017). Moreover, agricultural revolution has led to a drastic transformation of the Indian economy from a chronic dependence on grain imports into an agricultural economy, a prominent net exporter of food. It is evident that India is growing at a rapid rate but this growth needs to be well complemented by the status of poverty in the country. A higher economic growth is generally transformed to rise in aggregate demand in which case inability of developing nations to meet the rising demand results in inflationary tendencies in the economy.

The consumer prices increased to 4.28 percent in March 2018. Inflation rate had reached an all-time high of 12.17 percent in November of 2013 and the lowest of 1.54 percent in June 2017. The Asian Development Bank Report of 2011 stated that the global food prices increased by more than $30 \%$ in the first two months of 2011. A $10 \%$ increase in domestic food prices in developing Asia created around 64.4 million poor people, implying that a $30 \%$ increase in global food prices would increase the percentage of poor by $5.7 \%$. 'According to United Nation's Millennium Development Goals (MDG) programme, almost 21.9\% people out of 1.2 billion of Indians lived below the poverty line of \$ 125 in 2011-2012 (Mehra, 2016)'.

Observing above statistics it is plausible that there exists a relationship between inflation and poverty. The paper attempts to explore the impact of inflation on poor, whether inflation makes poor people poorer. In order to examine this relationship, it is important to define inflation and poverty in the Indian context. Inflation is a rise in the average price level of commodities in commodity basket which is captured by consumer price index and producer price index, considered as economic indicators. These indicators capture the price fluctuations for goods and services. Though the indicators serve the same purpose, the mix of a basket of goods and services along with the prices for the specific goods and services.

Since inflation depicts rise in the average price of consumption basket, it affects the budget of the individual households of the economy, thus affecting the livelihood of the poor section of the society. The genesis of this relationship between inflation and poverty lies in the theory that the wages are sticky and takes time to move while prices fluctuate at a greater speed. The purchasing power of poor section of society can be conceptualized by defining 'Poverty'.

Poverty is defined in two different perspectives one from income method and other from a consumption point of view. Poverty is still a very significant issue and concern in India, being a developing country. In order to understand the definition of poverty which in its simplest form can be defined as lack of sufficient income needed to obtain basic minimum necessities of life. Poverty has a very wide scope of unfolding and representing economic, social and political issues and concerns. The 
term poverty reflects, low-income levels, hunger, lack of assets, poor health, insecurity, social exclusion, political powerlessness.

There are two fundamental approaches to comprehend the concept of poverty. The first approach pertains to absolute poverty while the second method is the notion of relative poverty. The notion of absolute poverty pertains to the consumption expenditure considering a specified minimum standard. According to this method, all individuals whose consumption expenditure is below a specific standard are classified as poor. While the relative concept of poverty refers to a state when the level of income or consumption expenditure of a family or an individual falls below a predetermined level. The distribution of income of different fractile groups is estimated and a comparison is also carried out between the standard of living of people in both bottom and the top layer of the population in order to evaluate a relative standard of poverty. Therefore, a relative definition of poverty is more applicable for developed nations in contrast to the absolute notion of poverty which is more prevalent in case of developing nations.

The estimation of poverty is carried out by Planning Commission, the nodal agency of Government of India which estimates, the incidence of poverty at the national level and state level separately for urban and rural areas. The methodology was recommended by Expert Group chaired by Prof. D.T. Lakdawala, Dr. Tendulkar, and Dr. C. Rangarajan etc. According to the definition suggested by the expert group, the incidence of poverty is measured by poverty ratio. Poverty ratio is defined as a number of poor to the total population expressed as a percentage. This is also referred as headcount ratio. The poverty line which is quantified as per capita consumption expenditure over a month along with class distribution is used to define poverty ratio as prescribed in the survey of National Sample Survey Office (NSSO).

Inflation increases poverty, the problem of poverty is aggravated when the prices of commodities increase. Inflation is therefore considered as 'cruelest tax 'on the poor. Cardoso (1992) argued that inflation increases poverty in two ways: Inflation tax reduces disposable real income. Another reason is that when nominal wages increase less than the price of goods consumed by wage earners then automatically the worker's real wages decline. The author evidently showed that the main effect of inflation on poverty was through real wages. Increased level of inflation resulted in increased poverty. It is, therefore, crucial to examine the trends in the pattern of inflation.

The pattern of inflation in the three different time periods has been analyzed. The analysis assumes selected commodity specific inflation rates for all expenditure group as prescribed by Economic survey and thus evaluates inflation rates for each expenditure group. Having computed inflation rate for expenditure group, inflation rate expenditure function is estimated. Another crucial assumption made by the paper is fitting the Lorenz curve to the distribution of expenditure. The third assumption is that the study computes the class-specific inflation rates from the estimated inflation expenditure function. 
In order to comprehend the linkage between inflation and poverty, it is crucial to acknowledge the inherent stickiness of wages. When the country faces inflationary tendencies, the general price level of commodities increases, making the basic or essential commodities unaffordable by a poor section of society mainly because the wages do not increases or catches up with the rising prices due to the stickiness of wages which lowers their purchasing power, consequently making the poorer section even poorer.

Talukdar (2012) studied the effect of inflation on poverty in developing countries. The author analysed the effect of inflation on poverty for 115 developing countries over the period from 1981 to 2008. The author deduced that inflation is generally positively correlated with poverty while income, educational attainment, and quality of governance show a negative correlation with poverty.

Cardoso (1992) highlights the regressive nature of inflation tax and the extent of impact on those individuals below the poverty line. The paper argues that inflation affects poverty mainly through the impact on real wages. The authors evidently prove that the wages increase at a much lesser rate than the prices during the times when inflation was rising in Latin America. Finally, the study highlights that the stabilization programs implemented through incomes policy have not helped poor in Latin America, implying that inflation has affected poor drastically.

The decline in real wages during inflation was observed in Latin America during the last two decades while examining the period 1960-1997, observed that poverty maxima coincided with inflation maxima (Braumann, 2004). The author concluded that standard of living of poor were most hurt by macroeconomic policies that are inflationary. Ravallion (1998) examined food prices between 1959-1994 and evaluated its impact on poverty. The study concluded a strong positive correlation between higher prices and poverty. Later in 2002, Datta and Ravallion depicted that India's poor were adversely affected mainly by affecting real wage of unskilled workers.

The present paper analyses the pattern of inflation rates for 2005-06 and 2011-12 and examine whether 2011-12 inflation hurt the poor more. It involves the following steps, Firstly, it evaluates inflation rates for expenditure group and estimates inflation rate expenditure function; Secondly, it fits Lorenz curve to the expenditure distribution and estimates decile class specific per capita expenditure. Thirdly, decile class specific inflation rates from the estimated inflation expenditure distribution (function) are computed.

The study is organized into the following sections. Section I deals with theoretical background elaborating the model estimation and procedures. Section II analyzes the All India data for NSS round, it also displays the detailed account of equations of Lorenz curve estimation. Finally, Section III discusses results and discussions, Section IV concludes the findings. 


\section{Theoretical Background: Model and Estimation Procedures}

There exist five-step estimation procedures where the relationship between inflation rate and total expenditure is established. The method will eventually help in estimating Lorenz curve.

\section{Computation of Price Index}

The method of Laspeyres price index is adopted to generate inflation rate. The laspeyres price index for the time period $t$ is given by

$$
\mathrm{I}_{\mathrm{t}}=\frac{\sum \mathrm{p}_{\mathrm{it}} \mathrm{q}_{\mathrm{it}}}{\sum \mathrm{p}_{\mathrm{i} 0} \mathrm{q}_{\mathrm{i} 0}}
$$

Where $q_{\text {it }}$ be the quantity of ith item priced at $p_{\text {it }}$ consumed by the individual at a given level of expenditure $e_{t}$ in the year $t . p_{i 0}$ and $q_{i 0}$ are the price and the quantity associated with the base year 0 .

2. Computation of Inflation rate

Inflation rate $\prod_{\mathrm{t}}$ can be calculated by the following formula

$$
\prod_{\mathrm{t}}=\mathrm{I}_{\mathrm{t}}-\mathrm{I}_{\mathrm{t}-1} / \mathrm{I}_{\mathrm{t}-1}
$$

The above formula indicates the rate of increase in the value of base year commodity bundle of an individual when prices used in the valuation are changed from the period $t-1$ to $t$.

\section{Engel Curve}

The engel curve can be expressed as a function of total expenditure in the base year 0 represented as $\mathrm{e}_{0}$. As a result, the relationship between inflation rate and total expenditure in the base year can be expressed as

$$
\prod_{\mathrm{t}}=\mathrm{f}\left(\mathrm{e}_{0}\right)
$$

The above equation represents a relationship between the rate of change in consumer price index of a consumer from one time period $\mathrm{t}-1$ to another time period $t$, represented by $\prod_{t}$ and the total expenditure of the consumer for the base year 0 , represented by $\mathrm{e}_{0}$ 


\section{Estimation Procedure: Model Specification.}

In order to test the relationship between inflation and total expenditure a quadratic model specification is adopted as described below:

$$
\prod_{\mathrm{t}}=\mathrm{d}_{0}+\mathrm{d}_{1} \mathrm{e}_{0}+\mathrm{d}_{2} \mathrm{e}_{0}^{2}
$$

The decile class specific inflation rate can be estimated by the above equation. The explanatory variable is per capita expenditure levels of decile classes in the base year. With the help of Mean per capita expenditure (e) and Lorenz curve of expenditure distribution in the base year will estimate expenditure levels of decile classes. Lorenz curve can be estimated as described in the following section Kakwani (1981).

\section{Estimating expenditure level for decile classes}

Lorenz curve can be specified as follows:

$$
\mathrm{L}(\theta)=\theta-\mathrm{A} \theta^{\alpha}(1-\theta)^{\beta}
$$

Where $L(\theta)$ is the share of the poorest $\theta$ proportion of the population in the total expenditure and $A, \alpha$ and $\beta$ are the parameters. The expenditure level for the decile class or in other words poorest $\theta$ persons denoted by $e_{\theta}$ is given by

$$
\mathrm{e}_{\theta}=\mathrm{e} \mathrm{L}(\theta) / \theta
$$

\section{Analysis of All India data}

The methodology mentioned in the above section has been applied to all India rural consumer expenditure data retrieved from $62^{\text {nd }}$ and $68^{\text {th }}$ NSS round and commodity specific inflation rates obtained from Office of Economic Adviser, Ministry of Commerce and Industry, Government of India (Table 1).

Table 1: Commodity - specific Inflation rates

\begin{tabular}{|l|l|l|l|l|}
\hline \multirow{2}{*}{ NSS Item } & \multicolumn{4}{|l|}{ Inflation Rate (\%) } \\
\cline { 2 - 5 } & $2005-06$ & $2006-07$ & $2009-10$ & $2011-12$ \\
\hline Cereal & 6.0 & 16.7 & 61.2 & 76.2 \\
\hline Pulses \& Pulse Products & 7.8 & 30.2 & 56.4 & 65.3 \\
\hline Milk \& Milk Products & 0.8 & 8.5 & 45.3 & 90.7 \\
\hline Edible Oil & -5.9 & 2.5 & 14.4 & 35.7 \\
\hline
\end{tabular}




\begin{tabular}{|l|l|l|l|l|} 
Egg, Fish \& Meat & 6.3 & 12.8 & 51.5 & 114.3 \\
\hline Vegetables & 13.7 & 14.3 & 61.8 & 79.3 \\
\hline Fruits (Fresh) & 3.3 & 9.7 & 36.2 & 86.4 \\
\hline Fruits (Dry) & 3.3 & 9.7 & 61.8 & 79.3 \\
\hline Sugar & 8.8 & 7.4 & 61.9 & 67.7 \\
\hline Salt & 4.4 & 22.7 & 70.2 & 76.2 \\
\hline Spices & -5.5 & 36.7 & 82.7 & 137.5 \\
\hline Beverages, etc. & 2.5 & 3.0 & 18.2 & 41.3 \\
\hline Fuel \& Light & 13.6 & 20.9 & 32.1 & 69.0 \\
\hline Clothing & 0.6 & 7.1 & 30.8 & 70.5 \\
\hline Footwear & 7.8 & 14.5 & 34.7 & 43.8 \\
\hline
\end{tabular}

Notes:

The prices of essential commodities registered a high rate of inflation in 2011-12. The spices and food items like egg, fish, and meat have exhibited a very high growth rate from 2005-06 to 2011-12. The products like milk and milk products, fresh fruits along with vegetables and dry fruits have reasonably shown an excessive rise in growth rates. While on the other hand, food commodities like cereal, pulses and pulse products, sugar and salt have shown an average growth rate in the inflation rate. It is observed that inflation rate of fuel and lighting, as well as clothing, has also observed an average rise in inflation rates. The commodities like beverages, footwear, and edible oil have also shown a rise in inflation rate but at a substantially less rate as compared to other commodities.

Further, it is observed that inflation rate of vegetables, fuel, and light (energy) was maximum in 2005-06 while egg, fish, meat, and spices were the main drivers of inflation for the year 2011-12. This clearly reveals that for the year 2005-06, the pattern of inflation rate across decile classes is expected to be similar or in other words, the relationship between inflation and decile class is expected to be same. This deduction is subjected to the fact that the inflation in 2005-06 is driven mainly by the essential food items like vegetables and necessary commodities like fuel and light which are essential commodities for consumption both for rural and urban areas.

However, in case of the year 2011-12, since the inflation is mainly driven by food items like egg, fish, meat, and spices which are consumed comparatively more by the people belonging to higher decile group, it is expected that rural and urban areas will witness a different relationship between inflation rate and total expenditure. 
Table 2: Inflation Expenditure Equations (2005-06 and 2011-12)

\begin{tabular}{|l|l|l|l|}
\hline Rural & $\mathrm{r}=7.35$ & $-0.00536 \theta$ & $\begin{array}{l}+2.66 \mathrm{E}-06 \theta^{2} \\
\mathrm{R}^{2}=0.99\end{array}$ \\
\hline $2005-06$ & $\mathrm{r}=10.38$ & $-0.000995 \theta$ & $\begin{array}{l}-1.12 \mathrm{E}-06 \theta^{2} \\
\mathrm{R}^{2}=0.95\end{array}$ \\
\hline Urban & \multicolumn{3}{|l|}{} \\
\hline $2005-06$ & $\mathrm{r}=6.74$ & $-0.0024 \theta$ & $\begin{array}{l}+7.87 \mathrm{e}-07 \theta^{2} \\
\mathrm{R}^{2}=0.98\end{array}$ \\
\hline $2011-12$ & $\mathrm{r}=10.65$ & $-0.0037 \theta$ & $\begin{array}{l}-1.77 \mathrm{E}-07 \theta^{2} \\
\mathrm{R}^{2}=0.97\end{array}$ \\
\hline
\end{tabular}

The equation of Lorenz Curve: $L(\theta)=\theta-A \theta^{\alpha}(1-\theta)^{\beta}$

Parameters of Lorenz curve were estimated from NSS data on Consumption Expenditure by regressing

$$
\operatorname{Iog}(\theta-L(\theta)) \text { on } \log \theta \text { and } \log (1-\theta),
$$

Where $L(\theta)$ is the share of the poorest $p$ pro-portion of the population in the total expenditure and $A, \alpha$ and $B$ are parameters.

Estimated Lorenz function is given in the table below.

Table 3: Parameters of Lorenz curves (2004-05)

\begin{tabular}{|l|l|l|l|}
\hline Rural & & & \\
\hline $\log (\theta-\mathrm{L}(\theta))$ & 0.51 & $2.10 \log (\theta)$ & $\begin{array}{l}0.71 \log (1-\theta) \\
\mathrm{R}^{2}=0.98\end{array}$ \\
\hline Urban & & & \\
\hline $\log (\theta-\mathrm{L}(\theta))=$ & -0.21 & $1.21 \log (\theta)$ & $\begin{array}{l}0.38 \log (1-\theta) \\
\mathrm{R}^{2}=0.99\end{array}$ \\
\hline
\end{tabular}

The fit is good, we have estimated expenditure level of the rural and urban decile classes using an equation -

$$
\mathrm{e}_{\theta}=\mathrm{e} \mathrm{L}(\theta) / \theta
$$

The above Table2 represents the inflation expenditure function for the period 200506 and 2011-12. The results of estimated inflation - expenditure function indicate that inflation has equally affected adversely rural and urban areas in both the years 2005-06 and 2011-12. In 2005-06 the inflation affected rural areas more than the urban areas while in 2011-12 the adverse effect of inflation was more in the year 2005-06. High values of coefficient of determination $\mathrm{R}^{2}$ reveals that the regression 
equation of inflation expenditure is a good fit both for rural and urban areas and also that the quadratic equation is able to explain a substantial proportion of inflation rate over the previous round $\left(62^{\text {nd }}\right)$ NSS expenditure groups.

The parameters of Lorenz function curve were estimated for 2005-06 and 2011-12 NSS rounds( $62^{\text {nd }}$ and $\left.68^{\text {th }}\right)$ by regressing $\log (\theta-L(\theta))$ on $\log (\theta)$ and $\log (1-\theta)$ as depicted in Table 3 above. The estimated Lorenz curve is well depicted in Table 3. The results reveal that the estimated Lorenz function or equation is a good fit indicated by the high coefficient of determination.

Table 4: Decile Class Specific Expenditure Levels (2004-05)

\begin{tabular}{|l|l|l|}
\hline \multirow{2}{*}{ Decile classes } & \multicolumn{2}{|c|}{ Per capita expenditure (Rs per month) } \\
\cline { 2 - 3 } & Rural & Urban \\
\hline I & 49.04 & 55.6 \\
\hline II & 84.73 & 100.34 \\
\hline III & 109.94 & 144.4 \\
\hline IV & 128.8 & 191.95 \\
\hline V & 146.35 & 246.13 \\
\hline VI & 168.16 & 310.35 \\
\hline VII & 203.34 & 390.08 \\
\hline VIII & 260.6 & 492.9 \\
\hline IX & 357 & 638.5 \\
\hline X & 558.78 & 1052.36 \\
\hline
\end{tabular}

Using estimated inflation expenditure function and mean expenditure level of the decile classes, decile class specific inflation rates are computed

Table 5: Inflation rates for decile classes for 2005-06 and 2011-12

\begin{tabular}{|c|c|c|c|c|}
\hline \multirow[t]{2}{*}{ Decile classes } & \multicolumn{2}{|l|}{ Rural } & \multicolumn{2}{|l|}{ Urban } \\
\hline & 2005-06 & 2011-12 & 2005-06 & 2011-12 \\
\hline I & 6.63 & 10.44 & 6.26 & 10.63 \\
\hline II & 6.54 & 10.47 & 6.21 & 10.62 \\
\hline III & 6.48 & 10.5 & 6.17 & 10.6 \\
\hline IV & 6.43 & 10.52 & 6.11 & 10.58 \\
\hline $\mathrm{V}$ & 6.38 & 10.53 & 6.05 & 10.56 \\
\hline VI & 6.33 & 10.56 & 5.98 & 10.54 \\
\hline VII & 6.24 & 10.59 & 5.9 & 10.51 \\
\hline VIII & 6.10 & 10.65 & 5.78 & 10.47 \\
\hline IX & 5.85 & 10.74 & 5.62 & 10.42 \\
\hline$X$ & 5.34 & 10.94 & 5.16 & 10.27 \\
\hline
\end{tabular}


Inflation rates varied significantly across classes in both the periods. There has been a shift in the pattern of inflation across decile classes; inflation rates tend to be positively related to total expenditure in 2005-06 and negatively related in 2011-12. The empirical results also show that in contrast to 2005-06, both rural and urban classes experience a higher order of inflation. Rural-Urban differences, as well as inter-class differences,, are less in magnitude.

Figure 1: Inflation rates by Decile classes 2005-06

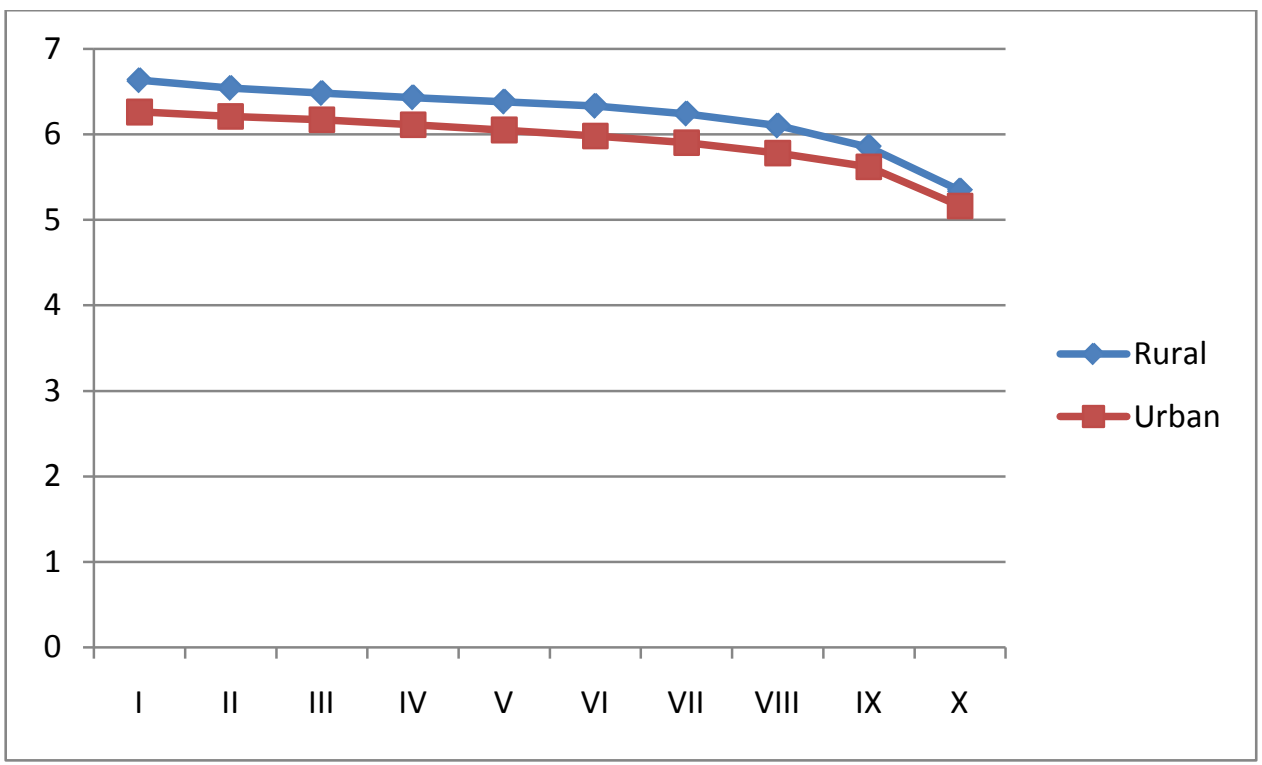

Source: Author's Calculation

Figure 2: Inflation rates by Decile classes 2011-12

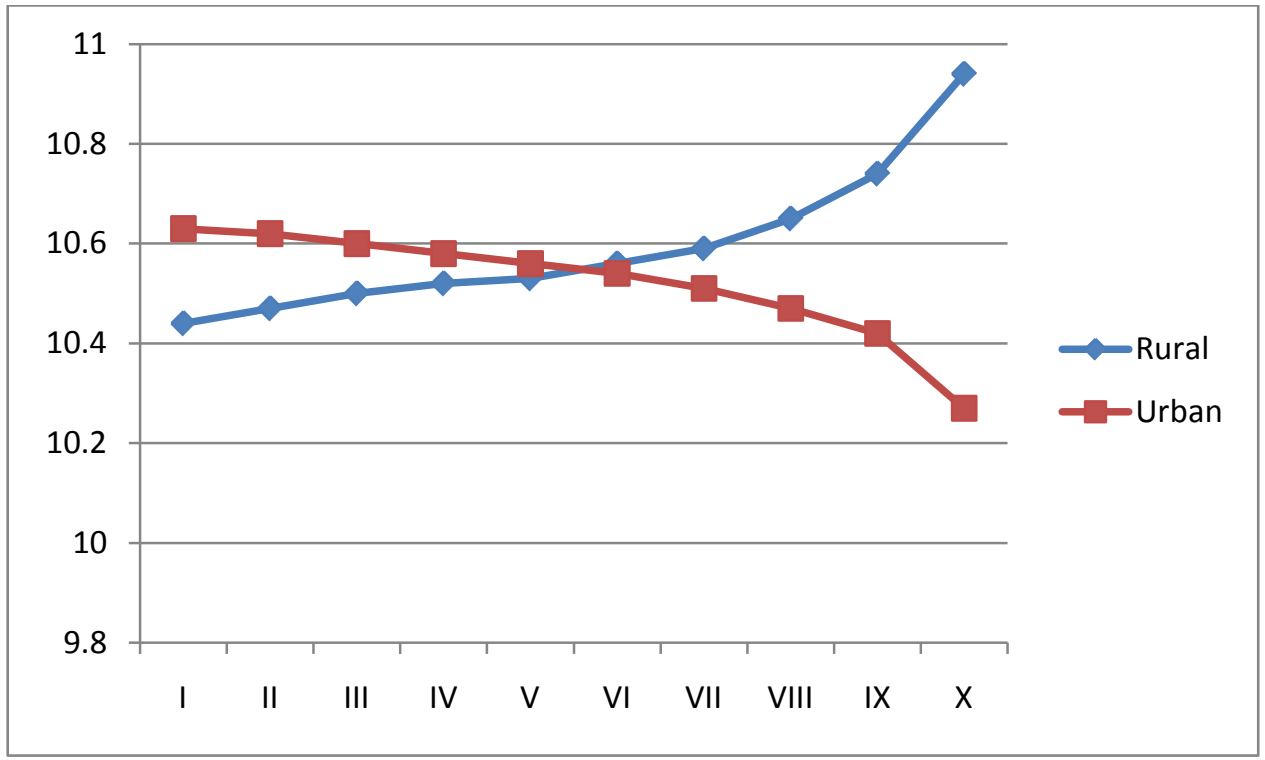

Source: Author's Calculation 


\section{Results and Discussion}

The estimated inflation - expenditure function and the mean expenditure levels of decile classes as shown in Table 4 are further used to compute the decile class specific inflation rates given in Table 5. The variability of inflation rates across classes is evidently reflected in both the selected periods. The above figures, figure 1 and figure 2 reveal that inflation rate tends to be inversely related to total expenditure in 2005-06 for both rural and urban areas. This is attributed to the fact that inflation is driven by the essential commodities like vegetables, fuel, and light which are equally consumed both in rural as well as urban areas. This reflects the rationale that with the rise of inflation the total expenditure also declines across the decile classes, suggesting an inverse relationship or in other words, a poor section of population experience higher inflation as compared to higher section and this difference is higher for rural areas as compared to urban areas. In 2005-06, rural areas get affected by the inflation rate more than the urban areas. This pattern of inflation rate is justified rationally since food grain account for larger share of in the budget of rural consumers. Another unique feature observed is that rural-urban differences are similar in magnitude compared to inter-class differences.

However, in 2011-12, the inflation rate is negatively related to total expenditure in urban areas while positively related to rural areas. This indicates that the pattern of inflation is distinct in both rural and urban areas in the year 2011-12. Inflation rates are negatively related to total expenditure indicating that poor population in urban areas experience high inflation as compared to people belonging to upper decile class group. However, in rural areas, there exists a positive relation between inflation rate and total expenditure. This reflects that in rural areas higher decile population experiences high inflation. This result is attributed to the fact that the inflation rate in the year 2011-12 is mainly driven by food items like egg, fish, meat, and spices which are mainly consumed by the upper decile class of the population. Therefore it is plausible to observe that for the bottom 60 percent of population inflation rate for urban areas is more than their rural counterparts since in urban areas this section of the population must be consuming above mentioned food items more than their rural counterparts.

However, inflation rate experienced by the upper $70 \%$ of the population in rural areas is much more than experienced by their urban counterparts. As the rise in the price of given food items made the rural upper decile class suffer much more than their urban counterparts considering that upper $70 \%$ of the population is consuming the above mentioned food items.

It is further observed that bottom 30 percent rural population experienced an inflation rate of 10.5 percent while bottom 30 percent urban population experienced an inflation rate of 10.6 percent in the year 2011-12. This clearly reflects the phenomenal success of Public distribution system (PDS) which made sure the impact of inflation being equal for both rural and urban areas for bottom $30 \%$ of the population. However, bottom 30 percent of the rural population experienced $6.5 \%$ of Inflation rate as compared to the bottom 30 percent of the urban population that experienced 6.2 percent of inflation rate which reflects that rise in food grain prices 
has affected poor people of rural areas slightly more than the bottom poor people of urban areas.

The inference from the above analysis clearly entails that if the rise in nominal expenditure of bottom classes can neutralize the effect of inflation on their real standard of living. But this conclusion is uncertain as the data on expenditure distribution in the year 2005-06 is not available. But it is expected that nominal expenditure of poor would have increased sufficiently to neutralize the effect of inflation since the wages of casual labor are not indexed.

\section{Conclusion}

The study examines the impact of inflation rate in both rural and urban areas selecting two distinct periods 2005-06 and 2011-12. By analyzing the commodityspecific inflation rates from the period 2005-06 to 2011-12 for both rural and urban areas, it is observed that the spices and food items like egg, fish, and meat have exhibited a very high growth rate from 2005-06 to 2011-12. The products like milk and milk products, fresh fruits along with vegetables and dry fruits have reasonably shown an excessive rise in growth rates. The commodities like beverages, footwear, and edible oil have also shown a rise in inflation rate but at a substantially less rate as compared to other commodities. Analysing the inflation rate across commodities it is evident that inflation rate for essential commodities is quite high for the year 2005-06 while the commodities consumed by upper decile class of population depicted a substantial rise in the inflation rate in 2011-12.

The inflation expenditure function for the period 2005-06 and 2011-12 is estimated indicating that inflation has equally and adversely affected rural and urban areas in both the years. In 2005-06, the inflation affected rural areas more than the urban areas while in 2011- 12, the adverse effect of inflation was more in the year 2005-06. A substantially high value of the coefficient of determination signifies the estimated Lorenz curve.

The paper establishes an inverse association between inflation rate and expenditure in the year 2005-06 both for rural and urban areas while the relationship was completely distinct for rural and urban areas for the year 2011-12. The study clearly reveals that bottom thirty percent of the population in rural areas observes same inflation rate as their urban counterparts for the year 2005-06. Finally, the paper concludes that the impact of inflation is not only commodity specific but also decile class specific. The results also reveal that the bottom $30 \%$ of the population experience almost similar impact of inflation in 2005-06 but in 2011-12, the impact of inflation on $30 \%$ of the population was adverse for rural areas as compared to urban areas. An efficient Public distribution scheme would be effective enough to reduce the gap between the rural and urban areas when it comes to assessing the impact of inflation rate on poor. The paper, therefore, recommends policies to reduce the impact of inflation on the poor. The paper opens further scope of analyzing the nominal expenditure data of poor people who actually get affected by inflation and 
examine if the rise in nominal expenditure offsets the inflation rate resulting in a neutral effect on real expenditure.

\section{References}

Kakwani, N. (1981). Welfare Measures: An International Comparison, Journal of Development Economics, 8 (1): 21-45.

Braumann, B. (2004). Tu Felix Austria: Evidence for a de-celerator in financial reform. International Economics and Economic Policy, 1(1): 53-72.

Radhakrishna, R. (2006). Consumption and Nutritional Status in India: Emerging Trends and Perspectives, (IGIDR, November).

Cardoso, E., \& Urani, A. (1995). Inflation and unemployment as determinants of inequality in Brazil: the 1980s. In Rudiger Dornbusch and Sebastian Edwards, eds. Reform, Recovery, and Growth: Latin America and the Middle East (151-176). University of Chicago Press.

Radhakrishna, R. \& Ravi, C. (1992). Effects of Growth, Relative Price and Preferences on Food and Nutrition: (Centre for Economic and Social Studies, Hyderabad), Indian Economic Review, New Series, Special Number in memory of Sukhamoy Chakravarty, 27: 303-323.

Datt, G., \& Ravallion, M. (1998). Why have some Indian states done better than others at reducing rural poverty? Economica, 65(257): 17-38.

Radhakrishna, R. \& Rav, C. (1992). Does Inflation Hurt the Poor More? Economic and Political Weekly, 27(4): 157-59.

GOI (Various Years). Wholesale Price Index Data: Office of Economic Adviser, Ministry of Industry

Agrawal, Amol (2008). Dissecting the Food Consumption Pattern of Households in India: IDBI Guilt Report, July, 23.

Mehra, P. (2 April 2016). 8\% GDP growth helped reduce poverty: UN report. The Hindu, Retrieved 16 August 2017.

Samanta, G. P. \& Mitra, Sharmistha (1998). Recent Divergence between Wholesale and Consumer Prices in India: A Statistical Exploration, RBI Occassional Papers, Vol.19, No4.

Ravi, C. (2000), Complete Demand Systems, Welfare and Nutrition: An application to Indian Consumption Data, Centre for Economic and Social Studies, Hyderabad.

Radhakrishna, R., Hanumantha Rao K., Ravi, C. \& Sambi Reddy, B. (2004) Chronic Poverty and Malnutrition in India, Working Paper 11, Chronic Poverty Research Centre, Indian Institute of Public Administration, New Delhi.

Rao, C.H.H. (2000). Declining Demand for Foodgrains in Rural India: Causes and Implications, Economic and Political Weekly, 35(4):201-206 •

Talukdar, S. R. (2012). The effect of inflation on poverty in developing countries: A panel data analysis (Doctoral dissertation). 
Track 3:

Business,

Management \&

Accounting

Economics

Business Models for Economic Growth

Management \& Leadership

Forensic \& Financial Accounting

Knowledge Economy

Marketing \& Digital Marketing

Actuarial Science

Human Resource Management

Transport \& Logistics

Travel \& Tourism 


\title{
Assessment of Final Year Accountancy Students' Knowledge of the Elements of Financial Statements
}

\author{
Stephen Chimtengo, Eric Chimpesa and Rabiya Hanif \\ University of Malawi \\ Blantyre, Malawi
}

\begin{abstract}
The aim of the study was to assess the knowledge of final year accountancy students on the elements of financial statements as contained in the Conceptual Framework for Financial Reporting. The study was exploratory in nature. It involved final year students on the accountancy programmes at colleges and universities in Malawi. The students were required to list the elements of financial statements. The results indicate that the majority of the students were not aware of the elements despite the fact that the financial reporting syllabi for the programmes incorporate the Conceptual Framework for Financial Reporting. It is recommended that financial reporting lecturers at the colleges and universities should adopt the principles based approach to teaching International Financial Reporting Standards. The accountancy students should also be encouraged to consider the Conceptual Framework for Financial Reporting as a foundation for their accounting studies and so endeavour to know its tenets.
\end{abstract}

Keywords: Conceptual Framework, Elements, Financial reporting, Students, Knowledge.

\section{Introduction}

The Conceptual Framework for Financial Reporting plays an important role in corporate financial reporting. It documents the agreed upon concepts which underlie International Financial Reporting Standards (IFRS) (Coetzee and Schmulian, 2011). In this case the International Accounting Standards Board (IASB), a body responsible for setting IFRS fosters consistency in financial reporting. The expectation is that the IFRS as a single set of reporting standards, will be adopted and applied worldwide. Coetzee and Schmulian (2011) note that the Conceptual Framework for Financial Reporting might also enable preparers of financial statements to account for economic events for which there are no reporting standards. The Conceptual Framework will then form a basis for the formulation of relevant accounting policies to inform the accounting treatment of the identified economic events. 
Given the inherent importance of the Conceptual Framework for Financial Reporting in corporate financial reporting, it is imperative that preparers of financial statements and accountancy students should have knowledge of the Conceptual Framework. The curricula of the Accountancy degree programme of various colleges and universities in Malawi have incorporated the International Financial Reporting Standards (IFRS) (Chimtengo et al., 2017). Generally, students are taught the Conceptual Framework for Financial Reporting in year three of their studies. The Conceptual Framework is intended to serve as an introduction to the detailed study of IFRS. Therefore, accountancy students are expected to be familiar with all the concepts in the Conceptual Framework for Financial Reporting.

The scope of the Conceptual Framework for Financial Reporting covers the objective of financial reporting; the qualitative characteristics of useful financial information; the reporting entity; the definition, recognition and measurement of the elements of the financial statements and the concepts of capital and capital maintenance. However, this study focused on the elements of the financial statements. Specifically the study was aimed at assessing the knowledge of final year accountancy students on the elements of financial statements.

\section{Literature Review}

\section{The Conceptual Framework for Financial Reporting}

Miller and Reading (1986) as cited in Solomon and Solomon (2005) defined a conceptual framework as a collection of broad rules, guidelines, accepted truths, and other basic ideas about the field. Solomon and Solomon (2005) noted that conceptual frameworks are intended to perform any function from simply helping to define a discipline to developing a fully comprehensive, analytical framework for the discipline. The concept of the conceptual framework applies to all disciplines and corporate financial reporting is no exception.

In the arena of financial reporting, the IASB developed the Conceptual Framework for Financial Reporting. The Conceptual Framework for Financial Reporting is very important in corporate financial reporting. The framework sets out the concepts that underlie the preparation and presentation of financial statements. The primary purpose of the Conceptual Framework is to assist the IASB by identifying concepts that it will use consistently when developing and revising IFRSs (IASB, 2013). The Conceptual Framework might be regarded as a foundation or a constitution for financial reporting standards (Gore and Zimmerman, 2007; Coetzee and Schmulian, 2011).

Use of the Conceptual Framework for Financial Reporting in Pedagogue and Pedagogues Approaches

Apart from aiding the IASB in the development of IFRSs, the Conceptual Framework can also be used for pedagogic purposes (Fawcett, 1997). Solomon and Solomon (2005) observe that the broader conceptual framework can be used as an introduction to a discipline, or subjects within a discipline. In the case of corporate 
financial reporting, the Conceptual Framework serves as an introduction to IFRS. Therefore it makes sense for students to be taught the Conceptual Framework before they cover the IFRSs. A special feature of the Conceptual Framework is that it is not updated frequently (Janse van Rensburg, Coetzee and Schmulian, 2014; Barth, 2008) unlike IFRS. As a result, the Conceptual Framework provides a good foundation for IFRS lessons.

The Conceptual Framework for Financial Reporting and IFRS are interlinked. The implication of this linkage is that the pedagogical approach adopted when teaching students IFRSs has a bearing on how students understand the Conceptual Framework. Coetzee and Schmulian (2011) underscore the need to attach great importance to the teaching of the Conceptual Framework for Financial Reporting. This calls for the application of an appropriate pedagogical approach. It follows that a good pedagogical approach should be employed to ensure that a good foundation is laid for financial reporting through the Conceptual Framework for Financial Reporting.

There are two approaches to teaching IFRS which may be applied: the traditional and the principles-based approaches (Coetzee and Schmulian, 2011; Persons, 2014). Under the traditional approach, the emphasis is placed on imparting knowledge of the concepts to the students (Coetzee and Schmulian, 2011). In essence the approach address the "what" question. Coetzee and Schmulian (2011) further observe that the role of the students is just to absorb the knowledge being imparted. The students are not taught how to make informed judgements using the knowledge they have acquired. In essence, the traditional approach does not afford students an opportunity to apply judgment. Consequently, this approach has been criticised as not being appropriate and is contrary to the current developments in IFRS education (Barth, 2008; Coetzee and Schmulian, 2011). Persons (2014) further bemoans that the traditional method does not explain the underlying principles used by the IASB or the Financial Accounting Standards Board (FASB) to promote IFRSs or U.S. GAAP respectively.

Unlike the traditional approach, the principles-based approach goes beyond the imparting of knowledge. The approach focuses on the "why" question by using the IASB Conceptual Framework (IASB, 2010) in this way therefore, the students are no longer spectators. They take an active role for their learning as they work through the relevant frameworks (Coetzee and Schmulian, 2011). Patro and Gupta (2012) note that the principles-based pedagogical approach ensures that students develop their ability to apply judgment. The use of the Principles-based approach to teaching IFRS has been greatly recommended (Persons (2014; Patro and Gupta, 2012) to overcome the shortfalls of the traditional approach.

\section{Methodology}

The study was exploratory in nature. The study involved two tertiary institutions offering accountancy programmes. For the sake of this study, the institutions have been named A and B. Institution A is a public university while Institution B is a public college. All respondents were final year students on the accountancy degree 
programmes offered by the two institutions. Ninety-seven students participated in the study. The distribution of students per institution has been shown in Table 1. The students were asked to list the elements of financial statements as contained in the Conceptual Framework for Financial Reporting.

Table 1- Distribution of Students

\begin{tabular}{|c|l|c|}
\hline Institution & $\begin{array}{c}\text { Number of } \\
\text { Respondents }\end{array}$ \\
\hline 1 & A & 67 \\
\hline 2 & B & 30 \\
\hline & Total & 97 \\
\hline
\end{tabular}

\section{Findings}

\section{Items Listed by the Students}

The students were requested to list the elements of the financial statements. The items mentioned by the students have been presented in Table 2 . The results indicate that the students from institution A mentioned 22 items while the students from institution B mentioned 17 items. Overall, the students from the two institutions mentioned a total of 15 common items. Seven items were unique to institution A while respondents from Institution B only mentioned 2 unique items. The items mentioned included the elements of financial statements, description of accounting standards, specific examples of assets and liabilities, qualitative characteristics of useful accounting information and the components of the financial statements.

Table 2- Number of Items Mentioned by the Students

\begin{tabular}{|r|l|c|c|}
\hline No & Item & Institution A & Institution B \\
\hline 1 & Assets & $\checkmark$ & $\checkmark$ \\
\hline 2 & Liabilities & $\checkmark$ & $\checkmark$ \\
\hline 3 & Equity & $\checkmark$ & $\checkmark$ \\
\hline 4 & Income & $\checkmark$ & $\checkmark$ \\
\hline 5 & Expenses & $\checkmark$ & $\checkmark$ \\
\hline 6 & Profit & $\checkmark$ & $\checkmark$ \\
\hline 7 & Capital & $\checkmark$ & \\
\hline 8 & Bank / Cash & $\checkmark$ & $\checkmark$ \\
\hline 9 & Revenue & $\checkmark$ & $\checkmark$ \\
\hline 10 & Investments & $\checkmark$ & \\
\hline 11 & Gain & $\checkmark$ & \\
\hline 12 & Losses & $\checkmark$ & \\
\hline 13 & Cost of sales & & \\
\hline 14 & Tax & & $\checkmark$ \\
\hline 15 & Income statement & $\checkmark$ & \\
\hline
\end{tabular}




\begin{tabular}{|r|l|c|c|}
\hline 16 & Statement of comprehensive income & $\checkmark$ & $\checkmark$ \\
\hline 17 & Statement of financial position & $\checkmark$ & $\checkmark$ \\
\hline 18 & Statement of cash flows & $\checkmark$ & $\checkmark$ \\
\hline 19 & Statement of changes in equity & $\checkmark$ & $\checkmark$ \\
\hline 20 & Notes to the financial statements & $\checkmark$ & $\checkmark$ \\
\hline 21 & Statement of Financial Performance & & $\checkmark$ \\
\hline 22 & Sales & $\checkmark$ & \\
\hline 23 & Clarity & $\checkmark$ & \\
\hline 24 & Comparability & $\boldsymbol{2}$ & \\
\hline & Total number of items mentioned & $\mathbf{2 2}$ & $\mathbf{1 7}$ \\
\hline
\end{tabular}

The aim of the study was to assess the final year accountancy students' knowledge of the elements of financial statements as contained in the Conceptual Framework for Financial Reporting. To this end, the items of interest were assets, liabilities, equity, income and expenses as these are the elements of the financial statements. The number of times that the students mentioned these elements is shown in Table 3.

Table 3- Frequencies of the Elements of Financial Statements Mentioned by the Students

\begin{tabular}{|l|l|c|c|c|c|c|}
\hline \multirow{2}{*}{ No } & \multirow{2}{*}{ Element } & \multicolumn{2}{|c|}{ Institution A } & \multicolumn{2}{c|}{ Institution B } & \multirow{2}{*}{ Overall \% } \\
\cline { 3 - 6 } & Frequency & \% & Frequency & \% & \\
\hline 1 & Assets & 48 & 72 & 14 & 47 & 64 \\
\hline 2 & Liabilities & 48 & 72 & 14 & 47 & 64 \\
\hline 3 & Equity & 40 & 60 & 13 & 43 & 55 \\
\hline 4 & Income & 28 & 42 & 4 & 13 & 33 \\
\hline 5 & Expenses & 39 & 58 & 11 & 37 & 52 \\
\hline
\end{tabular}

The results indicate that 72 percent of the respondents from institution A managed to mention assets and liabilities as elements while sixty percent mentioned equity as an element. Forty two percent and 58 percent of the respondents mentioned income and expenses respectively. On a different note, 47 percent from Institution B indicated assets and liabilities as the elements of the financial statements. Forty three percent of the respondents listed equity as one of the elements. Finally, 13 percent and 37 percent of the students from Institution B indicated income and expenses as forming part of the elements of the financial statements. In terms of the overall picture, almost half of the respondents from the two institutions managed to list assets, liabilities, equity and expenses as elements of the financial statements. It is only 33 percent of the respondents from the two institutions who indicated income as one of the elements.

\section{Students Performance}

The students' performance in terms of how they listed the elements of the financial statements as set has been shown in Figure 1. 


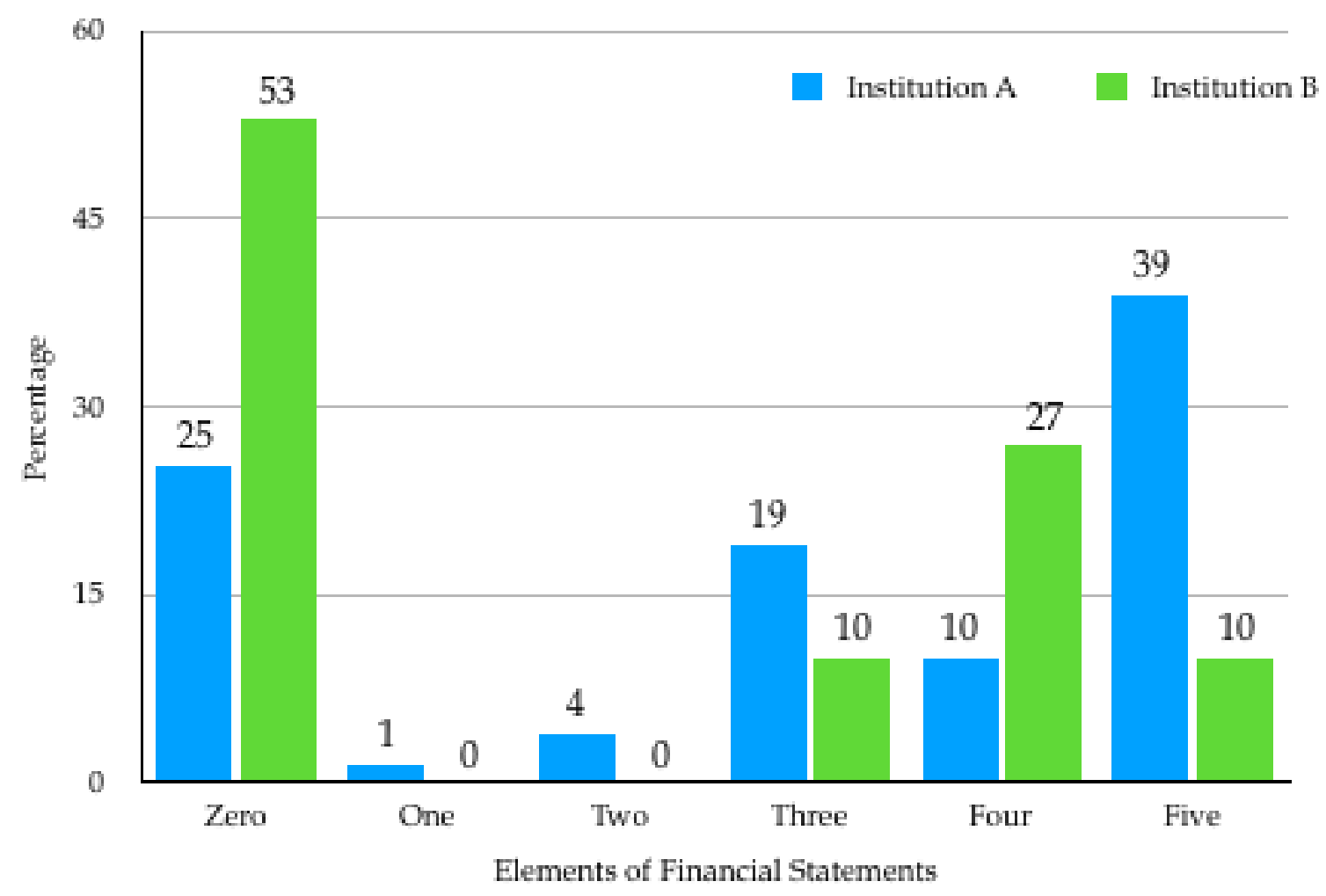

Figure 1 - Students' Performance on the Conceptual Framework

The figure shows that it is only 39 percent of the respondents from Institution A and 10 percent from Institution B who managed to list all the five elements of the financial statements. Twenty seven percent of the respondents from Institution B and 10 percent from Institution A listed four elements. Nineteen percent and 10 percent of the respondents from Institutions $A$ and $B$ mentioned three elements out of the required five elements. The results also indicate that the majority of the respondents from Institution B did not list even a single element while 25 percent of the students from the public university failed to list a single element.

\section{Discussion}

There are five elements of financial statements as per the Conceptual Framework for Financial Reporting. The elements are assets, liabilities, equity, income and expenses. The students were expected to list the five items. This task should have been a simple one to the students. However, the majority of the students listed other items that are not elements of financial statements. The students listed description of accounting standards, specific examples of assets and liabilities, qualitative characteristics of useful accounting information and the components of the financial statements as elements of the financial statements.

The results indicate that the majority of the students are not familiar with the elements of the financial statements. This lack of knowledge on the elements is reflected in the smaller number of the students who listed the five elements. It is only 49 percent of the students who listed the correct number of the elements of financial statements. The results also show that Institution A had the highest number 
of students who managed to mention five of the elements of the financial statements while Institution B had the highest number of students who mentioned 4 elements. Generally, students from Institution A performed better than their counterparts from Institution B. The better performance of students from Institution A could be due to the fact that this institution is well established as it has been offering academic accountancy programmes for over 40 years. Institution B has just started offering academic accountancy programmes. Other than this observation, the Financial Reporting module or its equivalent module on the Accountancy programme for the two institutions has a topic on the Conceptual Framework for Financial Reporting as proved by Chimtengo et al., (2017) in their study of the IFRS content of the Accountancy Degree Syllabi of colleges and universities in Malawi. Chimtengo et al, (2017) further noted that these modules are generally offered in year three of the accountancy programmes at the universities and colleges. In fact, the students on the accountancy programme study the Conceptual Framework before they cover the actual IFRSs. The spirit behind this approach was that the students should appreciate the tenets of the Conceptual Framework before they start covering the IFRS. This approach is line with Barth (2008) who indicated that students would be able to apply judgment when accounting for economic events once they have grasped the foundational concepts contained within a Conceptual Framework.

These findings agree with the finding of a study of Akhter (2013). Akhter conducted a study on students' awareness of International Financial Reporting Standards (IFRS) in India in 2013. He noted that it was only a small number of students who were familiar with the contents of the IFRSs. Patro and Gupta (2012) also observed the lack of awareness of IFRS among Indian management students. Other studies that have been conducted on the awareness of IFRS and related areas (Baba, 2013; Kulkarni and Hyderabad, 2014; Dhankar and Gupta, 2014; Moser, 2014; Balsari and Varan, 2014; Chimtengo, 2017) also show that the level of awareness is generally low among practicing accountants and other IFRS stakeholders.

One of the possible reasons for the students' poor knowledge of the elements of financial statements could be that the students had learnt the Conceptual Framework in the third year of their studies and so they could not explicitly remember the concepts in the Framework in the final year of their studies. As such they now needed to focus on the actual IFRS. This reasoning is flawed because the Conceptual Framework is at the centre of IFRS and as accountants-in-training, the students should be aware of the same at all times.

The other possible reason could be the pedagogy employed when teaching the Conceptual Framework. It could be possible that the lecturers at the two institutions were adopting the traditional approach to teaching the Conceptual Framework. The traditional approach does not address the relationship between the Conceptual Framework and IFRS as hinted by Barth (2008) and Coetzee and Schmulian (2011). Janse van Rensburg et al., (2014) assessed the ability of financial reporting students at a South African University to comprehend the IASB Conceptual Framework using the Cloze method. The results of their study indicated that the majority of the students were at Independent or Instructional level and that fewer students were at 
the Frustration level. It can be construed that the students' ability to comprehend the Conceptual Framework coupled with the right pedagogical approach might enable the students to have a positive attitude towards the Conceptual Framework.

Beyond the first accounting degree, most of the students register with professional accountancy bodies with the aim of becoming qualified accountants. The students would be required to make use of the elements of financial statements and the other concepts within Conceptual Framework of Financial Reporting generally in the course of undertaking these exams. Accounting students are expected to be conversant with IFRS, as these will form a foundation of their working life. Wong and Wong (2013) noted that knowledge of IFRS makes students understand better the financial information and the implications of any changes in the standards. It is evident that the Conceptual Framework for Financial Reporting should be a companion for accounting students. This companionship should even exist beyond the successful completion of professional examinations.

\section{Conclusion and Recommendations}

The objective of the study was to assess the knowledge of final year accountancy students on the elements of financial statements. The findings indicate the majority of the students are not familiar with the elements of financial statements that are just an aspect of the broader Conceptual Framework for Financial Reporting (2010). It is recommended that financial reporting lecturers at the two institutions should ensure that they are applying the Principles- based approach to teaching IFRS as advocated by Coetzee and Schmulian (2011) and Barth (2008). The accountancy students should be encouraged to consider the Conceptual Framework for Financial Reporting (2010) as a foundation for their accounting studies.

This study has focused on year four accountancy students at only two institutions offering accountancy degree programmes in Malawi. Future studies can consider students from a larger number of accountancy colleges and universities in Malawi. Again, the study concentrated on the elements of financial statements. Studies that

can assess the knowledge of the students on all aspects of the Conceptual Framework for Financial Reporting would be valuable.

\section{References}

Akhter, A. (2013). Awareness of international financial reporting standards (IFRS): A study of Post- Graduate Students of Commerce and Management in Kashmir. Business and Management, 14,16-24.

Baba, B. (2013). Assessing Nigeria's journey towards IFRS adoption. Information Management and Business Review, 5, 505-513.

Balsari, C. \&Varan, S. (2014). IFRS implementation and studies in Turkey. Accounting and Management Information Systems, 13, 373-399.

Barth, M. (2008). Global financial reporting: Implications for U.S. academics. The Accounting Review, 83, 1159-1179. 
Coetzee, S. \& Schmulian, A. (2011). The Conceptual Framework for Financial Reporting and Pedagogical Approach Thereto. Summer.

Chimtengo, S. (2017). Accountants' Awareness of Updates of International Financial Reporting Standards (IFRS) in Malawi. Management Research, 9 ,70-85

Chimtengo, S., Hanif., R., Banda, F., Mkandawire, K. and Chidule, C. (2017), Analysis of International Financial Reporting Standards Content of Accounting Degree Curricula in Malawi, International Business and Management, 14, 25-32. doi: http://dx.doi.org/10.3968/9244

Dhankar, R. \& Gupta, A. (2014). Transition to International Financial Reporting Standards (IFRS) or IND as in India. Finance and Management, 6, 609-614.

Fawcett, J. (1997). A Framework for Analysis and Evaluation of Conceptual Models of Nursing, in Perspective on Nursing Theory, Nicoll, L (eds.), Lippincott, Philadelphia, USA, pp. 487-495

Gore, R and Zimmerman, D. (2007), Building the Foundations of Financial Reporting : The Conceptual Framework. The CPA Journal.

Hong, Y.(2008). Do Principles-based Accounting Standards Matter? Evidence from the adoption of IFRS in China, 3321207. Thesis, Drexel University, Drexel University, United States -Pennsylvania.

International Accounting Standards Board (IASB) (2010). The Conceptual Framework for Financial Reporting. IFRS Foundation, London, UK.

Janse van Rensburg, Coetzee and Schmulian, 2014. South African financial reporting students' reading comprehension of the IASB Conceptual Framework. Accounting Education, 32, 1-15.

Kulkarni, P. \& Hyderabad, R. (2014). Implementation of IFRS in India: A Study of Awareness among Practicing Chartered Accountants. Management Thought and Research, IV, 3-14.

Larson,R. \& Street, D. (2011). IFRS Teaching Resources: Available and Rapidly Growing. Accounting Education, 20, 317-338.

Moser,R, (2014). IFRS and convergence in China and the USA. Technology Management in China, 9, 56 - 66.

Patro, A. \& Gupta, V. (2012). Adoption of International Financial Reporting Standards (IFRS) in Accounting Curruclula in India- An Empirical Study. Procedia, 2,227-236. doi: 10.1016/S2212-5671(12) 00083-4

Persons, O.(2014). A principles-based approach to teaching International Financial Reporting Standards (IFRS). Instructional Pedagogies, 13, 1-13.

Wong, H. and Wong, K. (2013). An Empirical Study - Adoption of International Financial Reporting Standards (IFRS) in Hong Kong Education. Management Research, 5, 98-107.

Solomon, A and Solomon, J. (2005). A Conceptual Framework of Conceptual Frameworks: Positioning Corporate Financial Reporting and Corporate Environment Reporting on a Continuum. Working Paper Series 04/ 05. 


\section{Track 4: Education}

Early childhood education

Primary \& secondary education

Curriculum design \& development

eLearning, distance \& blended learning

Massive Open Online Courses (MOOCs)

Higher Education

Special Education, Learning Difficulties and Learner Diversity

Adult and lifelong learning

Education for sustainable development

Education for interdisciplinary thinking

Emerging trends in Education 


\title{
Epistemic Injustice: Barrier to Articulation Management between Higher Education Institutions in South Africa
}

\author{
Darren Brendan Lortan and Savathrie Maistry \\ Durban University of Technology \\ Durban \\ South Africa
}

\begin{abstract}
The call for free education and decolonization of higher education curricula in recent years by university students in South Africa has increased awareness of epistemological issues and the notion of epistemic justice in higher education. In national policies education is presented as a critical means for addressing inequality, poverty and unemployment and the need for integration of the various strands of the post-school education and training system is highlighted. Systemic articulation between institutions of learning and work is viewed as expanding access to education and training opportunities.
\end{abstract}

A national articulation baseline survey involving all public universities and technical vocational education and training (TVET) colleges was undertaken in November 2016 in order to explore the existence and nature of articulation initiatives, and identify enablers and barriers to articulation between higher education institutions in South Africa. The report was completed in October 2017. This paper focuses on findings in the report related to articulation management between TVET colleges and universities. Although not explicitly stated, the findings revealed the prevalence of what we refer to as epistemic injustice, indicated by the extent to which individual and collective attitudes in academia perpetuate discrimination that impacts negatively on students' progression from one institution to another higher learning institution. The need for epistemic justice to be included in the articulation lexicon on a national level is highlighted. One of the recommendations made in the report is that the South African Qualifications Authority should raise the level of awareness of epistemic injustice at institutional and individual levels.

Keywords: epistemic injustice, articulation, individual and collective attitudes, discrimination, epistemic justice. 


\section{Introduction}

National policies such as the White Paper on the Transformation of Higher Education (1997) and the recently developed White Paper for Post School Education and Training (PSET) (2013) conceive of education as a critical means to addressing inequality, poverty and unemployment in the democratic context of South Africa. Centuries of oppressive and divisive colonial and apartheid history left South Africa with a legacy of discrimination in all its national systems, including higher education, at the time of transition to democracy in 1994.

Democratic South Africa inherited a racially segregated, unequal education and training system in which different types of learning did not enjoy parity of esteem and qualifications were not necessarily linked to learning pathways (Department of Higher Education and Training [DHET], 2017). In recent years, South African students' campaign for epistemological relevance at the higher education level has steadily increased. A consequence of students' call for free education and decolonization of higher education curricula is an increased awareness of epistemological issues and the notion of epistemic justice in higher education.

A mandate of the South African Qualifications Authority (SAQA), established post 1994, is to oversee the implementation and further development of the National Qualifications Framework (NQF) which is the means chosen to integrate the education and training system and to enable lifelong learning (Republic of South Africa [RSA], 2008). The NQF is a comprehensive system developed for the classification, registration, publication and articulation of quality-assured national qualifications and part-qualifications. It comprises three co-ordinated qualifications sub-frameworks: General and Further Education and Training, Higher Education and Trades and Occupations, which have the objective of education and skills development (RSA, 2008). Each level of qualification or course of knowledge has to be allotted a certain number of credits at a certain specific level, for enabling these credits to be transferred across the system. The NQF levels serve as guidelines for curriculum development and indicate where curriculum is positioned in the NQF. Level descriptors can be used as a benchmark of higher education to understand curriculum levels of any course being offered at the different institutions of learning. This NQF framework has the potential to influence articulation collaboration and articulation of programmes in any field (RSA, 2008).

The whole framework of credits and qualifications is managed by SAQA which conducts research to support this work and expands its capacity through long-term research partnerships with public higher education institutions (HEIs). While much has been achieved regarding systemic redress, access, progression, quality and transparency in the context of the NQF, the major current focus, clearly expressed in the White Paper for Post-School Education and Training (PSET) is on articulation (Department of Higher Education and Training, 2013). This current focus culminated in the promulgation of the Articulation Policy of the Post School Education and Training System (DHET, 2017). 
SAQA's current research project in partnership with the Durban University of Technology (DUT) which commenced in 2016, explores the enablers and barriers to systemic articulation. The study is entitled: Developing an understanding of the enablers of student transitioning between Technical Vocational Education and Training (TVET) Colleges and Higher Education Institutions (HEIs). It also explores successful articulation models that address identified barriers. In this research partnership, articulation is understood as the enabling of mobility within and between the various learning programmes and institutions (including colleges, universities and workplaces) that comprise the post-school education and training system (SAQADUT Research Proposal, 2016:1). Articulation is intended to broaden access to higher education.

This research initiative necessitated a basic understanding of the prevailing issues related to articulation in the post school sector in South Africa. Consequently, a national articulation baseline survey (NABS) was undertaken in November 2016, prior to the commencement of the larger research project, involving all public higher education institutions (HEIs) and technical vocational education and training (TVET) colleges. The baseline survey aimed to explore the existence and nature of articulation initiatives and identify enablers and barriers to articulation between higher education institutions in South Africa. The survey report was finalized in October, 2017.

This paper focuses on the findings in the survey report related to the management of articulation between TVET colleges and HEIs. The survey revealed the extent to which individual and collective attitudes in academia perpetuate discriminatory practices that impact negatively on students' progression from one institution to another institution of higher learning. As researchers involved in the baseline study, we view this discriminatory individual and collective or institutional attitude in articulation management as epistemic injustice.

This paper intends to create an awareness of the existence of epistemic injustice in articulation management in the PSET sector. In the first section of this paper we review literature on the concepts of articulation including the management of articulation and epistemic injustice. Next, we cover relevant aspects of the national articulation baseline survey, its findings and discussion based on the findings. In conclusion, we propose recommendations to address epistemic injustice in the management of articulation between TVET colleges and universites. We highlight the importance of relational agency theory as a guideline to building collaborative relationships between TVET colleges and universities in order to promote successful systemic articulation; and the role of SAQA as critical for the implementation and realisation of the suggested recommendations.

\section{Literature Review: Key Concepts of Articulation and Epistemic Injustice}

Articulation remains a challenge in the PSET system in South Africa. The focus on articulation is a relatively recent issue in the South African higher education agenda. 
As a consequence, there is a scarcity of literature on articulation for the South African context. The key challenge for education access in South Africa is to design a flexible post school education and training system that enables people to find articulation pathways that meet their needs.

Accordingly, the PSET White Paper (2013: vii-viii) is seen "as a vision for an integrated system of post-school education and training, with all institutions playing their role as parts of a coherent but differentiated whole....The system envisaged must provide paths for articulation between various qualifications, and there should be no dead-ends for students; there should always be a way for someone to improve their qualifications without undue repetition". The PSET system is understood as comprising all education and training provision for those who have completed school, those who did not complete their schooling, and those who never attended school. It consists of the following institutions, which fall under the purview of the Department of Higher Education and Training (DHET):

- 26 public universities

- 50 public TVET colleges

- Public adult learning centres (soon to be absorbed into the new community colleges)

- Private post school institutions

- Sector Education and Training Authorities (SETAs) and the National Skills Fund (NSF)

- Regulatory bodies responsible for qualifications and quality assurance in the post school system, SAQA and the Quality Councils (PSET White Paper, 2013: xi).

The PSET White Paper further points out that in terms of management and articulation there are conflicting and uneven quality assurance mechanisms and articulation possibilities with the mix of qualifications in TVET colleges. They are complex to administer and difficult for learners and parents to understand and often poorly quality assured (PSET, 2013: 14). It is clear that successful articulation in an envisioned integrated system of post-school education and training can only take place by design and not default. Research on understanding articulation and issues related to enabling successful articulation is an important element of the design process.

Articulation is defined and understood in various ways. However, the common factor in most of the definitions is that articulation enables mobility within and between the various learning programmes and institutions (universities, colleges and workplaces) that comprise the post-school education and training system (Makhafola, 2005: 14; N'gethe et al.,2008: xvii; SAQA-DUT research proposal, 2016).

Articulation may be engendered through credit accumulation and transfer (CAT) and Recognition of Prior Learning (RPL). Credit accumulation and transfer means "an arrangement whereby the diverse features of both credit accumulation and credit transfer are combined to facilitate lifelong learning and access to the workplace" (SAQA, 2013: 6). Recognition of prior learning refers to the principles 
and processes by which prior knowledge and skills of a person are made visible, mediated and assessed for the purposes of alternative access and admission, recognition and certification or further learning and development (SAQA, 2013:9).

Lotz-Sisitka (2015) presents a broader understanding of articulation whereby articulation is viewed as 'systemic articulation' or a 'joined up' system incorporating qualifications and various other elements aligned to and supportive of, learning pathways. Articulation could also be seen more specifically, in terms of the structuring of qualifications to allow progression, with or without intra-or interinstitutional agreements for credit accumulation and transfer. Articulation could also refer to the pathways followed by individuals as they progress, and are supported in their learning and work. Aspects such as the quality of qualifications and learning, and career development advice or initiatives are part of articulation. The concept of 'transitions' becomes important, when a learning pathway involves transitions from training to work, from work to training, from school or College to Higher Education (Lotz-Sisitka, 2015).

The recent national policy on articulation states that a well articulated system is one in which there are linkages between its different parts; there should be no silos, no dead ends (DHET, 2017). In support of students' progression, it highlights this critical point-if a student completes a course at one institution and has gained the relevant knowledge and skills at the necessary levels, this must be recognized by other institutions if the knowledge gained is sufficient to allow epistemological access to programme(s) that the student wants to enter. Learners must be supported in their individual learning and work pathways (DHET, 2017: 1).

The policy also highlights that education and training has to address personal, national socio-economic and community needs. By doing so, injustices such as poverty and unemployment can be addressed. It points out that in the current situation in the post-school education and training system, a number of articulation initiatives exist but more work is needed regarding articulation in order to build a truly integrated and articulated post-school system. Even though the NQF has enabling features to promote articulation, barriers to access, articulation and credit transfer still exist (DHET, 2017:1). The following issues are identified in the policy as barriers to articulation:

a. Academic qualifications are considered more valuable and credible than comparable vocational or occupational qualifications;

b. Some qualifications on the NQF are seen to be "dead end" in nature, and do not lead to further learning;

c. There are general perceptions that the purpose and nature of technical and vocational education and training is to offer learning programmes which focus on a relatively narrow band of employment -related or job- specific skills and competencies, when in fact the qualifications / part -qualifications could have wider relevance;

d. There has been inadequate building of coherence between the NQF sub - 
frameworks;

e. There has been an absence of robust articulation arrangements or alignment work between the different programmes and institutional types; and

$\mathrm{f}$. There is a general lack of institutional flexibility to support learners as they 'stop in and stop out' of their studies, where research has shown that this kind of 'staggered pathway' is the norm at all NQF levels (Department of Higher Education and Training, 2017:2).

While not stated explicitly-and this may be attributed to the concept of epistemic injustice being an unfamiliar concept in government lexicon- it is possible to discern hints of epistemic injustice in all of the issues mentioned above but specifically so in the first and third issues identified in the policy.

The management of articulation is an important factor in enabling successful articulation between and among institutions of learning, and between institutions of learning and the workplace. Van Der Waldt and Du Toit (1999) correctly point out that in a country such as South Africa where much needs to be done to reduce poverty, inequality and unemployment with limited resources, it is of cardinal importance for public institutions to manage existing resources more effectively to achieve greater need satisfaction. They add that the public expects the best possible management processes and services from public institutions.

Management may be formally defined as the process whereby human, financial, physical and information resources are employed in order to reach the goals of an organisation (Cronje et al., 2003:122). The four fundamental management tasks or functions are: planning, organising, leading, controlling and staffing (Cronje et al., 2003; Kerzner, 2013). Van Der Waldt and Du Toit (1999) refer to these functions as general or basic and claim that a distinction exists between general management and function-specific management. "Whereas function-specific management refers to aspects such as financial and personnel management, general management refers to the tasks that have to be performed at all management levels... and can best be defined by the types of functions that are undertaken" (Van Der Waldt and Du Toit, 1999: 12). We contend that the management of articulation includes both general management and function-specific management, given the challenges currently experienced in management is pervasive from the highest level of institutional management to the level of individual academics and administrative staff.

The larger SAQA-DUT study is based on the assumption that institutions of higher learning are open social systems and therefore systems theory and the systems approach to management are important for this investigation as it emphasises the interdependent interaction and relationship between parts and the whole (Van Der Waldt and Du Toit, 1999: 64). A system is described as a collection of parts which form some whole and the whole cannot be divided into independent parts; the effects of the behaviour of the parts on the whole depend on the behaviour of other parts (Cole, 2004: 74; Theron and Maphunye, 2005: 26). In the systems approach to management any person or a particular institution may be seen as a system and this 
approach looks at the interconnections between the different parts of the institution to determine how well it is functioning. A systems theory perspective examines a broader picture, one that includes the environment within which the organisation exists and how changes in the environment affect the organisation. Therefore, a systems approach to the management of articulation highlights the interdependency of each system, that is, TVET Colleges and universities and individuals within these institutions. Successful management is essential to enable and contribute to the big picture of access to education and work for the majority in South Africa.

\section{Epistemic Injustice}

In the relationship between TVET colleges and universities on articulation and articulation management, we agree with Catala's (2015) description of epistemic injustice as injustices pertaining to the recognition and transmission of knowledge where a prior situation of social injustice exists, one of unequal power relations between different social groups, including inequalities in epistemic power. Those epistemic inequalities grant different levels of credibility and intelligibility to individuals based on their membership in different social groups, thereby creating epistemic injustice (Catala, 2015). The questioning of not only credibility but the value of qualifications from TVET colleges by some universities blatantly undermines the process of successful articulation. We believe that epistemic injustice presents a formidable barrier to articulation management that needs to be acknowledged as such and addressed from a number of perspectives.

Prior to 1994, the apartheid system had institutionalised racial segregation in all spheres, thereby creating one of the world's most unequal societies. A segregated educational system where inferior education was given to the majority to prepare them for an inferior place in society was one of the foundations on which white 'superiority' was built. Epistemic injustice is only beginning to feature in higher education discourse in South Africa due in part to higher education currently being challenged by students who are demanding relevance of knowledge and the 'decolonization and Africanization' of curricula.

Miranda Fricker's seminal work 'Epistemic Injustice: Power and the Ethics of Knowing' (Fricker, 2007) focuses on two types of injustice, namely testimonial injustice and hermeneutical injustice. In simple terms testimonial injustice describes situations where a speaker's words are perceived as less credible than they ought to be, due to prejudice held by the listener. The prejudice leads to the speaker being fully, or partially, excluded from participating in the production and transmission of knowledge. The speaker suffers a 'credibility deficit' and their words are perceived as less credible than they ought to be. It should be understood that attributing of a credibility deficit does not by itself constitute testimonial injustice. It is the attribution of the deficit together with a prejudice, held by the listener that categorises the injustice as testimonial. Testimonial injustice may often result in damaging consequences for articulation and also the limitation of career progression. Epistemic injustice may also be structural in nature, for example 
members of certain groups may simply not be asked their opinions as they are regarded as invalid (Graham et al., 2017). Hermeneutical injustice describes the inability of individuals to effectively communicate their experiences or knowledge claims, due to a lacuna in the collective or societies' understanding of said experience or knowledge. The existence of this lacuna is due to the relative powerlessness of the social group, to which the individual belongs, and its inability to fully articulate its experience of being disadvantaged. This is a structural injustice in that the listener is not at fault for their inability to fully understand the speaker (Graham et al., 2017).

Fricker's work places emphasis on the individual's experience of epistemic injustice with the goal of identifying and eliminating some of the structural prejudices prevalent in our society. She advances the position that by recognising our own individual prejudices we may contribute to the amelioration of epistemic injustice by our now enlightened individual actions. Contrary to this, Anderson (2012), advocates that some epistemic injustices are best addressed at the societal, as opposed to the individual level, thereby implying the requirement for structural or policy change. This is not to say that structural changes should be implemented at the expense of efforts to promote individual value changes, but rather that the structural change should also motivate an individual response, for without individual support the structural changes will ultimately lack effectiveness (Graham et al., 2017).

We contend that epistemic injustice as revealed in the baseline study, has to be addressed at the individual, collective, institutional and structural levels as presented by both Fricker (2007) and Anderson (2012) respectively.

\section{The Research Problem}

Articulation (within and across) the institutional and qualification landscape is a national imperative that has featured regularly in Higher Education legislation. Although the goal of strengthening relationships between education and training institutions and between these institutions and workplaces is clearly laid out in the White Paper on Post-School Education and Training (MHET, 2013), widespread implementation of legislative imperatives by institutions and workplaces is still needed.

In view of the problems related to students' access and mobility from one institution of learning to another and from institutions of learning to the workplace and vice versa, articulation has become an important investigative issue for SAQA. SAQA's Partnership Research has revealed and sought to address gaps in the understanding of articulation and learning pathways.

The main focusof the SAQA-DUT research partnership is on investigating relationships that enable articulation within and across Technical and Vocational Education and Training (TVET) Colleges and Universities in general, and between TVET Colleges and Universities of Technology (UoTs) in particular.The main focus 
of the research is to understand how to build collaborative/cooperative relationships between TVET Colleges and UoTs which will enable, enhance and promote successful articulation and integrated College-University learning pathways. The research attempts to answer the following broad question: How can collaborative relationships between TVET Colleges and UoTs be developed, which will enable, enhance, and promote student transitioning between TVET Colleges and HEIs?(SAQA-DUT Research Proposal, 2016)

\section{The National Baseline Study}

The national baseline survey is part of the larger SAQA-DUT partnership research project which extends from April 2016 to March 2020. The focus of this study is to determine the enablers and challenges to articulation between TVET colleges and universities and between these institutions and the workplace. The national baseline survey focused on the articulation relationship between all 26 public universities and all 50 TVET colleges, a total of 76 institutions. The 26 public universities include 9 Universities of Technology (UoTs). The rationale for the survey was to obtain 'a big picture' understanding of articulation and articulation issues in the post school education and training sector. The findings of the baseline survey are intended to supplement the SAQA-DUT partnership research project.

\section{Objectives of Baseline Study}

The objectives of the baseline study were to:

i) Understand how articulation is conceptualised in the institution;

ii) Obtain descriptions of existing articulation arrangements associated with the institution;

iii) Obtain descriptions of the management of these articulation arrangements, and challenges;

iv) Capture the extent and nature of the successes and enablers of articulation experienced

This paper focuses on the third objective, specifically in relation to challenges experienced by the institutions regarding the management of articulation.

\section{Design and Methodology}

The design of the baseline survey was exploratory in nature. This is the first time that a national baseline survey was conducted in South Africa to obtain an overall understanding of the enablers and barriers to articulation within the post school education and training sector. Specifically, this is the first survey to explore the management of articulation between TVET colleges and universities. In order to achieve the objectives of the baseline study, the questions were open ended and required descriptive responses. Therefore, the survey methodology was qualitative in nature. Qualitative research is concerned with exploring a phenomenon from the 'interior' and it takes the perspective and accounts of the research participants as starting points. In general, qualitative research is concerned with exploring and answering the 'what', 'why' and 'how' questions (Ritchie et al., 2013:3). 


\section{Participants}

An open ended questionnaire based on achieving the above objectives was jointly constructed by SAQA-DUT research team. The survey was conducted between August and December 2016. Given the time frame available, it was decided to focus on public entities only: all 50 public TVET Colleges and all 26 public HEIs (which included 9 UoTs) were included in the survey. In total the sample consisted of 76 institutions.

\section{Procedure}

For the HEIs, the survey instrument was emailed together with a formal invitation to participate, from SAQA's Chief Executive Officer, to the relevant Deputy Vice Chancellors or Registrars. For the TVET Colleges, the survey questionnaires and invitation letters were emailed to the College Principals. It was necessary to ensure that the highest academic office at each institution dealt with the survey, or delegated it to an appropriate person, recognised to be responsible for articulation arrangements at the respective institutions, in order to obtain the detailed information needed. Up to five follow-ups were made in each instance to enable the researchers to obtain the levels of clarity and detail needed. Forty-nine (98\%) of the Colleges and twenty-five (98\%) of the HEIs completed the surveys.

\section{Data Analysis}

The results of the survey were analysed using a variety of coherent mechanisms which comprised thematic analysis and the analysis of key words following a grounded theory approach. Braun and Clarke (2006:76) define thematic analysis as a method for identifying, analysing and reporting patterns within data. Given that Grounded Theory (Charmaz, 2006; De Vos, 2002) is developed and provisionally verified through systematic data collection and analysis, the Grounded Theory approach is useful for aligning theory in the South African context, regarding articulation, lifelong learning and integrated learning pathways, based on data from the proposed study.

\section{Findings}

\section{Articulation Management Challenges}

Responses to the main challenges experienced in the management of articulation are categorised as: student support and student issues; work placements; resources; curriculum issues between Colleges and HEIs; institutional systems and structures; and the relationships between Colleges and HEIs. Table 1 shows the reported incidence of these categories of challenges. 
Table 1: Articulation management challenges as reported by TVET Colleges and HEIs

\begin{tabular}{|l|c|}
\hline Challenges & $\begin{array}{l}\text { Number of institutions reporting this } \\
\text { challenge }\end{array}$ \\
\hline Student support and student issues & 6 Colleges, 2 HEIs \\
\hline Work placement issues & 8 Colleges, 1 HEI \\
\hline Resources & 4 Colleges (No HEIs) \\
\hline $\begin{array}{l}\text { Curriculum issues between Colleges and } \\
\text { HEIs }\end{array}$ & 8 Colleges, 2 HEIs \\
\hline Institutional systems and structures & 7 Colleges, 1 HEI \\
\hline $\begin{array}{l}\text { TVET College relationships with HEIs; } \\
\text { HEIs lack of understanding/ attitudes } \\
\text { towards 'epistemic } \\
\text { (knowledge-related) injustices' }\end{array}$ & 10 Colleges, 2 HEIs \\
\hline
\end{tabular}

It should be noted that the 'Articulation management challenges' is biased in terms of the College voice; the voices of the universities are relatively silent on the matter. This finding is not surprising given the limited overall articulation approach on the part of the universities: the sense on the part of the universities of what the challenges are would be commensurately limited. From our analysis of the findings on management challenges, we perceive what is considered to be epistemic injustice to be located in the following challenges: curriculum issues between Colleges and HEIs; institutional systems and structures, and the relationships between Colleges and HEIs.

\section{Curriculum-related challenges}

Eight TVET Colleges and two HEIs mentioned curriculum-related issues as being challenging. Firstly, universities accreditation requirements may dictate that exemptions be granted for courses only if there is significant overlap in the content of the course being exempted and the course already passed. The response to these requirements is an examination of the content of the two courses to determine the extent of overlap.

Secondly, it remains a challenge to articulate into Higher Education based on the National Certificate Vocational (NCV) qualifications of TVET Colleges. This may be because of the unfamiliarity of the HEI practitioner with the content of the courses offered at the Colleges, or simply a manifestation of the lack of parity of esteem between the two, where a mismatch is assumed merely because the course is offered elsewhere. Responses that reflects HEIs lack of familiarity with TVET Colleges programmes are as follows: "HEIs not understanding TVET colleges subjects offering and qualifications"; "the non-understanding of what the programme is about and why it's implemented at a TVET College" and "there are still instances in which the qualifications from TVET Colleges are not recognised by Institutions of Higher Learning". There are cases where mismatch of curriculum content occurs, but this is ordinarily established by first examining the content. 
Some Colleges maintained that the universities are not aware of the rules, regarding the articulation of the NCV into Higher Education. One College response highlights this lack of knowledge by HEI personnel: "Our NCV students are made aware of the Level 4 articulation gazette at induction- unfortunately qualifying students still experience problems at some HE institutions when they try to register. The registration personnel on the ground at HE institutions don't appear to be aware of the gazette and the Level 4 NCV students who meet the requirements of the gazette must be considered for registration". A College noted that the same HEI would use different approaches for College students with similar NCV results. Another College claimed that an HEI resisted the admission of students with NCV Level 4 and other TVET qualifications-"HEIs resist and close their doors to consider NCV level 4 and N6 students" and "there was also an impression that the criteria was more strict for College students compared to their Matric counterparts though some of college students already had Matric".

This College suggested that the Department of Higher Education and Training (DHET) should develop guidelines for the recognition of TVET qualifications for entry into HEIs.

\section{Institutional systems and structure-related challenges}

Institutional systems and structures were identified as challenges by seven TVET Colleges and one HEI. These challenges related to a lack of guiding policies and procedures; management information systems (MIS); administration systems; access or placement tests and recognition of prior learning (RPL) procedures within faculties in HEIs. The considerable physical distances between institutions were also seen as being challenging for articulation management.

It was reported that good working relationships between Colleges and HEIs were often dependent on specific individuals in the institutions involved, and that these relationships became a challenge when these 'champions' left the institutions. The following TVET response indicate the lack of institutional systems and structure as a challenge: "not all HEI have developed documentation that assists the articulation process".

\section{TVET College - HEI relationship-related challenges}

The challenge of TVET-HEI relationships for the management of articulation was mentioned by ten TVET Colleges and two HEIs. Colleges identified the autonomy of HEIs as they had the power to change entrance requirements. In addition, the correct departments and contact personnel in HEIs were not necessarily easy to identify. Colleges experienced difficulty in arranging appointments with the Deans in HEIs"Setting up appointments with HEI, getting an appointment with relevant Deans takes a very long time". The HEIs were reported as generally not regarding the TVET Colleges as being equal partners in the PSET system, and the role-players in partnerships often had different agendas-"We find it extremely difficult to engage universities to collaborate with us as we are not regarded as an equal partner" and "there is discrimination in that universities as they take students without practical experience over those that come from a TVET college with practical experience and exposure to the workplace" were responses indicative of prejudicial treatment by HEIs towards TVET Colleges. 
There was generally poor understanding of 'articulation', and a fear of commitment by both TVET Colleges and HEIs to the processes involved. The Gazetted agreements appeared to allow for easy articulation but the HEIs ultimately have a say in admission. Common understanding was lacking regarding implementing the policies for access to qualifications offered by HEIs.

The Colleges struggled to get recognition by HEIs. Front desk officials in most HEIs are not knowledgeable about the qualifications offered at TVET Colleges. The Colleges refer to Government Gazette rules and SAQA 'bands' in order to articulate the TVET offerings correctly, especially for students from NCV programmes wanting to continue their studies at universities, but the NCV qualifications are not well known or acknowledged by the universities. In addition, the quality of NCV Level 4 passes was deemed to be not good enough for universities.

\section{Discussion}

Unhealthy relationships, specifically those that are discriminatory in nature could result in what we refer to as 'epistemic injustice'. The injustices are in relation to the lack of recognition and respect for different kinds of knowledge, especially when it involves the HEIs' lack of understanding of, or indifference to, TVET College qualifications and standards.

In the comparative study of twelve African countries, including South Africa, on differentiation and articulation conducted by N'gethe et al., (2008), the findings reveal that the problem of articulation is a regional one. They point out that:

articulation in Africa seems to be in its infancy and write that " the mobility of staff and students between university and non-university tertiary institutions suffers from a lack of cooperation and absence of dialogue between the two institutional groups. In fact, an unhealthy rivalry often characterises their relationship....graduates seeking 'academic progression' into the university system often encounter a void in which there are no clearly defined articulation pathways to follow, the level to which they are admitted being dependent on the course they wish to pursue and the regulations of the particular university to which they are applying (N'gethe et al., 2008:xviii).

The report stated that the internal structures of institutions tended to inhibit articulation amongst and between institutional types. Consequently, they tend to view articulation-even amongst themselves-as a managerial nuisance that is best avoided (N'gethe et al., 2008: xviii).

The epistemic injustice described in this survey is primarily testimonial in nature. For students to readily obtain epistemological access to programmes as indicated in the recently developed articulation policy, specifically from TVET colleges to universities requires the removal of epistemic injustice. Epistemic justice is a set of principles and practices that collectively constitute the amelioration of recognized 
epistemic injustices. This may be at the individual or structural level. Epistemic justice also includes the process of identifying epistemic injustice and determining the basis upon which to engender epistemic justice (Maistry and Lortan, 2017).

Relationship-building within and between the TVET Colleges, HEIs and workplaces, requires deliberate effort. Edwards' (2010) concept of 'relational agency' is useful for understanding and strengthening relationships. According to Edwards' relational agency theory, the underlying foundation for different systems to work together well are best described in the nature of their interaction and relationship with each other. Relationship building is therefore one of the core elements which is vital for different systems to progress and function effectively (Edwards, 2010:1).

Four ideas are central to relational agency. The first idea is that relational expertise involves additional knowledge and skills over and above specialised core expertise. Second, relational expertise involves understanding and engaging with the motives of others. It allows the expertise offered by others to be surfaced and used. Third, relational expertise is useful vertically (in authority hierarchies), but it is also relevant for horizontal collaboration across practices at similar levels in authority hierarchies. Lastly, relational expertise respects history, but is focussed on the common knowledge created through shared understanding of the different motives of those collaborating, and going forward together (Edwards, 2010). The theory of relational agency can serve as a critical framework that guides the development of constructive, cooperative relationships between the institutions to seek solutions to epistemic injustice as a barrier to successful articulation between the institutions.

\section{Recommendations}

As a first step towards addressing the issue of epistemic injustice, we propose that epistemic justice be included in the articulation lexicon and discourse at a national level. We view the role of SAQA to be significant to this proposal and accordingly recommend that the South African Qualifications Authority not only raise the level of awareness of epistemic injustice at both institutional and individual levels but also seeks appropriate solutions to this barrier.

As a starting point, we suggest the organization of participatory workshops between academia from universities and TVET colleges to address the issue of epistemic injustice at the individual and institutional levels. In addition, publications by SAQA on epistemic injustice as a barrier to systemic articulation management could serve the purpose of raising awareness on the national level.

\section{Conclusion}

This study reveals that epistemic injustice in both testimonial and hermeneutical forms present itself in the articulation pathways between TVET Colleges and HEIs in South Africa. The notion of justice is inextricably intertwined with ameliorating injustices. If epistemic justice is to be understood as a deliberate response to recognised epistemic injustice with the purpose of ameliorating said injustices, then 
a critical component of epistemic justice is the recognition of said epistemic injustice (Maistry and Lortan, 2017).

Therefore, it becomes necessary for TVET Colleges and HEIs to be consciously and jointly aware that epistemic injustice is a critical barrier to effective articulation between TVET Colleges and HEIs. If epistemic injustice is not addressed between the different levels of higher education, then very little progress will be made towards addressing issues of inequality, poverty and unemployment and the legacies of apartheid of inferior and superior knowledge in South Africa.

Epistemic justice directs HEIs to rethink fundamental questions about knowledge: of what should be known; for what purpose; how to know it and what should be the criteria to legitimize knowledge (Restrepo 2014). Epistemic justice demands a shift in attitude by academia in HEIs and to acknowledge and value the knowledge of TVET College students transitioning to HEIs. SAQA has a critical role in creating an awareness of epistemic injustice within the post school sector and making recommendations to the national government on how issues of epistemic injustice could be addressed.

\section{References}

Anderson, E. (2012). Epistemic justice as a virtue of social institutions. Social epistemology, 26(2), 163-173.

Braun, V., \& Clarke, V. (2006). Using thematic analysis in psychology. Qualitative research in psychology, 3(2), 77-101.

Catala, A. (2015). Democracy, Trust, and Epistemic Justice. The Monist. 98 (4), 424440.

Charmaz, K. (2006). Constructing Grounded Theory. Qualitative Research in Organizations and Management: An International Journal, 2(2), 84-103.

Cole, G. A. (2004). Management Theory and practice (6th ed.). London: Thompson.

Cronje, G., Gawie, G. S., \& Motlatla, M. D. (2003). Introduction to Business Management (6th ed.). Oxford: Oxford University Press.

Davids, I., Theron, F. and Maphunye, K.J. (2005). Participatory Development in South Africa: a development management perspective (1 ${ }^{\text {st }}$ ed.). Pretoria: Van Schaik Publishers.

De Vos, A. S., Delport, C. S. L., Fouché, C. B., \& Strydom, H. (2011). Research at grass roots: A primer for the social science and human professions.

Edwards, A. (2010). Being an expert professional practitioner: The relational turn in expertise (Vol. 3). Springer science \& business media.

Fricker, M. (2009). Epistemic Injustice: Power and the Ethics of Knowing. Oxford: Oxford University Press.

Graham, B., Lortan, D., Maistry, S., \& Walker, M. (2017). Using epistemic justice as a framework for underpinning articulation between technical and vocational education and training colleges and higher education engineering programmes. In Collier-Reed, B. (Ed.) Proceedings of the 4th Biennial Conference 
of the South African Society for Engineering Education (SASEE) Conference Proceedings Cape Town Lodge, Buitengracht Street, Cape Town , South Africa, 14-15 June 2017 (pp. 94-102). Cape Town, South Africa:SASEE.

Kerzner, H. R. (2013). Project Management: A systems approach to planning, scheduling and controlling. New Jersey: John Wiley and Sons.

Lotz-Sisitka, H. (2015). Close-out Report for the SAQA-Rhodes University Research Partnership for Learning Pathways research.

Makhafola, M. (2009). Student Access and Articulation. Mthatha: Walter Sisulu University.

Maistry, S. M., \& Lortan, D. B. (2017). Lessons from the global South: knowledge democracy and epistemic justice in higher education institutions in South Africa. Journal for New Generation Sciences, 15(1), 123-139.

N'gethe, N., Subotzky, G. and Afeti, G. 2008. Differentiation and Articulation in Tertiary Education Systems: A Study of Twelve African Countries. Washington: The World Bank.

National Qualificaitons Framework (NQF), Act no 67 of 2008. Pretoria: Government Printer.

Republic of South Africa. Department of Education. (1997). Education white paper 3: A programme for the transformation of higher education. White Paper (General Notice 1196, 24 July 1997). Pretoria: Department of Education.

Republic of South Africa. Department of Higher Education and Training. (2013). White Paper for Post-School Education and Training: Building an expanded, effective and integrated post-school system. White Paper (Government Gazette 37229, Notice 11, 20 November 2013). Pretoria: Department of Higher Education and Training.

Republic of South Africa. Department of Higher Education and Training. (2017). The Articulation Policy for the Post School Education and Training System of South Africa. Policy (Government Gazette 40545, Notice 619, 13 January 2017). Pretoria: Department of Higher Education and Training.

South African Qualifications Authority. (2013) . Policy and Criteria for the Registration of Qualifications and Part-Qualifications on the National Qualifications Framework. Waterkloof: SAQA.

South African Qualifications Authority- Durban University of Technology. (2016). Developing an understanding of the enablers of student transitioning between Technical Vocational Education and Training (TVET) Colleges and Higher Education Institutions (HEIs). Research Proposal.

Ritchie, J., Lewis, J., Nicholls, C. M., \& Ormston, R. (Eds.). (2013). Qualitative research practice: A guide for social science students and researchers (2 ${ }^{\text {nd }}$ Ed.). Los Angeles: Sage.

Van der Waldt, G. \& Du Toit, D. F. P. (1999). Managing for excellence in the public sector. Cape Town: Juta and Company Ltd. 


\title{
Community Based Participatory Research: Exploring the Value of Indigenous Knowledge for Early Childhood Development
}

\author{
Kanya Padayachee and Savathrie Maistry \\ Durban University of Technology \\ Durban, South Africa
}

Darren Lortan

Durban University of Technology

Durban, South Africa

\begin{abstract}
This paper presents the preliminary findings on a current engaged research that aims to develop through Community Based Participatory Research a framework for Integral Education underpinned by indigenous knowledge in Early Childhood Development in South Africa. Indigenous Knowledge is a growing field of inquiry globally and at all levels, particularly for those interested in education innovation. In Early Childhood Development, the inclusion of local resources and positive cultural values and practices has the potential to contribute to significant goals, such as psychosocial and cognitive development towards building strong foundations for lifelong learning. The first stage of a larger study on Integral Education focuses on building values for Early Childhood Development through Indigenous Knowledge. This part of the exploratory phase - the study is still in progress - establishes a significant trend in favour of the inclusion of Indigenous Knowledge in the Early Childhood Development programme.
\end{abstract}

Keywords: Community Based Participatory Research, Early Childhood Development, Integral Education, Indigenous Knowledge.

\section{Introduction}

The purpose of this paper is to present the preliminary findings of part of a research that aims to determine the extent to which Integral Education promoting indigenous knowledge values is practised in early childhood development. Generally, mainstream education tends to focus on the acquisition of knowledge, the development of cognitive skills and individual achievement. In contrast, an Integral Education approach encourages the holistic development of a child by including the emotional, moral, interpersonal, physical, spiritual and cultural developmental 
aspects. Thus, it provides for the inclusion of indigenous knowledge values that promote individual and collective development and within this development locates the individual within the collective.

Both the South African National Curriculum Framework for children from birth to four and the Government of the Republic of South Africa's draft policy on Early Childhood Development make reference, inter alia, to two critical areas that guide this study: the potential for timely interventions in the early years of a child's life and the historical inequities that continue - on a range of levels - to impact negatively the majority of children in South Africa which play themselves out in the cycle of poverty and deprivation and the attendant social ills which children confront, daily. To this end, the application of Integral Education offers an opportunity to concretely ameliorate past failings by including all human dimensions in the learning process, with a focus on the collective. This approach resonates with the philosophical underpinnings of the African Indigenous value system, Ubuntu, which emphasizes the human development of the individual through the well-being of others in the community. Thus, in cultivating social responsibility and civic mindedness, Integral Education and Ubuntu are critical components to be included in an Early Childhood Development programme.

\section{Research problem}

The assumption is made in the study that there is a significant gap in the current Early Childhood Development curriculum in South Africa as it does not apply an Integral Education approach that also promotes Indigenous Knowledge values. This education model, it is argued, can significantly improve the life chances of children, especially the most vulnerable, by developing the mind, body and spirit and creating the enabling environment to make responsible life choices Therefore, the study will combine the African philosophy of Ubuntu in Indigenous Knowledge to develop, implement and evaluate an Integral Education programme for Early Childhood Development that inculcates sustainable social responsibility and civic mindedness in young children.

\section{Literature Review}

The Draft Early Childhood Development Policy (2015) affirms that the government has a responsibility to create the conditions for the realisation of the right of every child to develop optimally (Department of Social Development, 2015: 19). The policy states further that the social returns on Early Childhood Development's public investments in universal comprehensive Early Childhood Development services yield lifetime development returns for the child, his or her family and society. Notably, Early Childhood Development has the potential to contribute significantly to the reduction of key development challenges facing South Africa, particularly poverty and inequality. Still further, that Early Childhood Development investments bring about higher levels of positive self-regulation which lead to significantly less crime and greater public safety," reduced public violences" and greater social cohesion and civic participation (Ibid: 22-23). Thus the policy gives a clear indication of the causal link between adequate and quality provisioning for this phase of 
children's development and the long term benefits to the fabric of society - "positive self-regulation", "less crime", "greater public safety", "greater social cohesion" and "civic participation" (ibid: 22-23).

This study is suggesting that such a turnaround strategy for South African society as that implied in the policy cannot be accomplished by upscaling material provisions and resources and not clearly identifying a behaviour and attitude change programme to support child development. Consequently, it proposes that such changes require the deliberate embedding of a set of core values into the Early Childhood Development programme that can lead to the holistic development of children. According to Wilson (2008:1) early childhood education should address the moral development of the child, especially the caring and compassionate aspects of morality. These cannot be relied on to be fostered only at home and in the communities because the behaviours and attitudes of many adults in South African society, are not exemplars of positive role modeling.

This problem of poor role models for children is further compounded in South Africa by the added socio-political realities of the high prevalence of family fragmentation (Callinicos, 2005:1) and child headed households (Department of Social Development, 2008:18). Thus, for many children the option of cultivating a set of values to guide their moral development may not exist. Pendlebury and Enslin (2007:238) also argue for a values education to mitigate the deleterious effects of apartheid. They maintain that post-apartheid South Africa is replete with widespread corruption at all levels of the public service and heinous crimes persist. Consequently, they argue that values education should be the starting point to overcome these ills.

According to Hawkes (2010:237), values education can positively influence the expansion of universal values, which have such a powerful effect on the culture of the school and on the development of the child and the fostering of a civil, caring and compassionate society. Studies have shown that early childhood interventions in a holistic and integrated manner generally result in pro-social behaviour and effects long term societal behavioural changes (Viviers, 2009: 5). Thus, values cultivated and promoted from the early years of a child's development are most likely to grow strong and sustainable roots to last into adulthood (Kaya, 2013).

Values education, however, falls outside the ambit of the "mainstream," "conventional," or "traditional" forms of education, which tend to focus on the acquisition of knowledge, development of cognitive skills and individual achievement. In contrast, "alternative," "holistic," and "transformative education" include many aspects of an individual (e.g., emotional, moral, interpersonal, and spiritual) and culture (ecological, global, etc.) and may be better suited to incorporate values. According to Esbjörn-Hargens (2011: 2-4) who argues for an approach that honours the strengths while recognising the limits of both mainstream and alternative educational approaches, Integral Education is best suited to accommodate the holistic development of the child and, for this study, especially, 
the devalued and marginalised socio-cultural and spiritual aspects of indigenous knowledge. This view derives largely from Aurobindean philosophy on education which states that true education is integral as it includes all the aspects of the human being: physical, vital, mental, psychic, and spiritual. It is an education designed to foster the evolution of the individual, the nation, and humanity (Vengopal and Kumari, 2010: 59).

Biersteker (2012:2) contends that despite the growing international recognition that good quality Early Childhood Development programming should be sensitive to and build on the local and indigenous knowledge and practices, most local models and curriculum guidelines still privilege Western models. In addition, she notes that while local and global knowledge could exist side by side, there is a conspicuous absence in local curricula and programmes of local knowledge. Thus, the values and principles on which Western Early Childhood Development goals and interventions are based may be inappropriate to local conditions, clash with local values and result in misguided and unsuccessful implementation.

In promoting relevant and culturally appropriate values in young children, the study proposes that indigenous knowledge consistent with the African philosophy and principles of Ubuntu should be used. These values which emphasize that the individual realizes her/his humanness through the well-being of others and the community as a whole (Kaya and Padayachee, 2013) resonate with the overarching ones of social responsibility and civic mindedness. According to Bonn (2007: 2), Ubuntu encompasses compassion, tolerance, care, charity, understanding, empathy, equality, hospitality, honesty, trust, conformity, solidarity, mutual responsibility, taking care of everyone in one's community, respect, dignity and a concern for others' welfare. These moral values safeguard both the conservation of the group/community as a whole and promote the harmonious existence of the individual within the community. It would appear, therefore, that the essential values to cultivate social responsibility and civic mindedness - goals and targets identified in the draft Early Childhood Development Policy - resonate with those of Ubuntu and, to improve impact and sustainability, should be included in an Early Childhood Development values programme.

The dearth of literature on moral regeneration suggests a relatively unchartered field. The urgency, however, to intensify efforts to instill moral and ethical values at all levels, especially in the earliest years, is valid. According to Richardson (2003:5), the moral assault on the majority of South Africans demonstrates a society urgently in need of targeted and concrete efforts to find and sustain shared values for our society. It is in the early childhood period that children develop their basic values, attitudes, skills, behaviours and habits, which may be long lasting. As early childhood education is about laying a sound intellectual, psychological, emotional, social and physical foundation for development and lifelong learning, it has an enormous potential in fostering values, attitudes, skills and behaviours that support sustainable development (Samuelsson and Kaga, 2008:12). 
Thus, the aim of this study is to develop an integral early childhood development programme based on indigenous knowledge and the philosophy of Ubuntu and its associated values for social responsibility and civic mindedness and to assess the impact of implementation to give concrete effect to the ideals of the draft Early Childhood Development policy.

\section{Research Question}

The aim of the research is to determine the extent to which Integral Education underpinned by Indigenous Knowledge Values in Early Childhood Development could contribute to the development of social responsibility and civic mindedness in children aged 3-4 years. It asks the following question: To what extent could Integral Education based on Indigenous Knowledge Values be applied to the Early Childhood Development programme for the age cohort of $3-4$ to develop social responsibility and civic mindedness?

\section{Research Objectives}

The study comprises four phases: exploratory, developmental, intervention and evaluation phases. This paper presents the preliminary findings of the exploratory phase. The four phases and their objectives are presented to provide a comprehensive picture of the study.

The specific objectives in each phase are the following:

\section{Exploratory Phase:}

1. Investigate the nature and extent to which the current Early Childhood Development curriculum and practice could benefit from integral education which incorporates Indigenous Knowledge values and principles of Ubuntu for social responsibility and civic mindedness. Learnings from two international Integral Education organisations are considered to guide the study

2. Identify the theoretical and philosophical considerations that should guide the development of an integral education Early Childhood Development programme based on Indigenous Knowledge for social responsibility and civic mindedness

\section{Developmental Phase:}

3. Develop an integral education early childhood development programme based on the indigenous knowledge values of Ubuntu for social responsibility and civic mindedness

4. Develop a measuring instrument to evaluate the programme

\section{Intervention Phase:}

5. Implement the programme in the selected sample sites over a period of $8-10$ months

\section{Evaluation Phase:}

6. Evaluate the programme by applying the measuring instrument prior to and after implementation

\section{Method}

\section{Participants}

Through stratified, purposive and nonprobability sampling, key informants and target groups were identified in the KwaZulu Natal province in South Africa. The 
study also attempts to learn from Integral Education programmes in Early Childhood Development implemented in Delhi and in California. The sample groups are as follows:

Group 1: ECD staff, comprising Early Childhood Development Supervisors and Practitioners - total of 41 participants

Group 2: Parents of children, community members and elders, constituted of 2 urban groups, 2 rural groups and 2 semi-rural groups - total of 75 participants

Group 3: Key informants, comprising Early Childhood Development national representative bodies, Early Childhood Development research associations, provincial government Department of Social Development, 3 academics involved in Indigenous Knowledge Systems, 2 international Integral Education institutes and 2 Integral Education preschool principals in Delhi.

This paper presents the preliminary findings of data collected from sample group 1, that is, Early Childhood Development staff, comprising supervisors and practitioners - a total of 41 individuals.

\section{Design}

The research design is exploratory in nature as this is the first study based on integral education. The study uses a community based participatory research approach and consists of four phases: exploratory, developmental, intervention and evaluation with qualitative and quantitative research methodologies (Creswell, 2015:14-15). In this mixed methods research, the researcher gathers both qualitative and quantitative data, integrates the two and then draws interpretations based on the combined strengths of both sets of data to better understand the research question (ibid: 38 ).

The exploratory phase uses qualitative research methods of focus group and individual interviews as well as content analysis of practitioners' journals and participant observations of practitioners. The central focus of the exploratory phase is to probe the existing Early Childhood Development programme and activities to determine the extent to which Integral Education underpinned by Indigenous Knowledge values in Early Childhood Development could contribute to the development of social responsibility and civic mindedness in children aged 3-4 years.

During the developmental phase, data from the focus group interviews, individual interviews and participant observations will be integrated and collated to:

i) inform the development of an integral education indigenous knowledge values programme in early childhood development

ii) promote social responsibility and civic mindedness in children within the age cohort of $3-4$ years

iii) develop a measuring instrument to evaluate children's behaviours and attitudes prior to and after the implementation of the programme. 
The programme will be first piloted in three ( 1 urban; 1 rural; 1 semi-rural) of the six sample sites which are 2 urban; 2 rural and 2 semi-rural, reviewed and revised where relevant, and then implemented in all six sites during the intervention phase. Thereafter, it will be evaluated to assess the outcomes.

\section{Materials}

Data was collected through focus groups interviews with staff from six Early Childhood Development sites in KwaZulu Natal in South Africa on the three areas of the research: Integral Education; Indigenous Knowledge and Early Childhood Development. An interview schedule for this sample group included the following questions:

1. What is your understanding of indigenous knowledge?

2. Does the learning/teaching content of the current Early Childhood Development programme include indigenous knowledge material and values?

3. Do you think children can benefit now from Indigenous Knowledge stories/rhymes/songs, etc.?

4. What methods will be best suited for gathering indigenous stories, rhymes, folk tales/lores, games, etc. to promote indigenous knowledge values?

\section{Procedure}

The study is located in 6 public Department of Social Development registered and funded Early Childhood Development sites, situated in urban, rural and semi-rural areas in Umbumbulu, south of Durban, KwaZulu-Natal, South Africa. It is assumed that the urban/rural/semi-rural sample dynamic could influence the findings as a result of such factors as, inter alia: lifestyle influences, value systems, cultural practices, quality of preschools, human and material resources, parental and community structures. It involves appropriately qualified Early Childhood Development practitioners teaching 3 - 4 year-old children, and site supervisors (managers of sites).

\section{Results}

The focus group data will be discussed in the following categories based on the interview guide $(\mathrm{n}=41)$ :

- Conceptualization of Indigenous Knowledge

-Extent of Indigenous Knowledge content in the current Early Childhood Development Programme

- Benefits of Indigenous Knowledge content to Early Childhood Development

- Methods for promoting Indigenous Knowledge Values for Early Childhood Development 


\section{Descriptive statistics}

Table 1 - Summary of the categories and themes in ranking order:

\begin{tabular}{|l|l|l|l|l|}
\hline Category & $\begin{array}{l}\text { Conceptualization } \\
\text { of IK }\end{array}$ & $\begin{array}{l}\text { Extent of IK } \\
\text { content in the } \\
\text { ECD Programme }\end{array}$ & $\begin{array}{l}\text { Benefits of IK } \\
\text { content to ECD }\end{array}$ & $\begin{array}{l}\text { Methods for } \\
\text { promoting IK } \\
\text { Values for ECD }\end{array}$ \\
\hline Theme 1 & $\begin{array}{l}\text { IK has valuable } \\
\text { moral and ethical } \\
\text { teachings }\end{array}$ & $\begin{array}{l}\text { No IK content } \\
\text { because of the } \\
\text { clash with } \\
\text { modernity }\end{array}$ & $\begin{array}{l}\text { IK can offer } \\
\text { solutions to } \\
\text { modern } \\
\text { challenges }\end{array}$ & $\begin{array}{l}\text { Wisdom of } \\
\text { community elders }\end{array}$ \\
\hline Theme 2 & $\begin{array}{l}\text { IK is associated } \\
\text { with inter- } \\
\text { generational } \\
\text { values and }\end{array}$ & $\begin{array}{l}\text { Opportunity to } \\
\text { include IK } \\
\text { content should be } \\
\text { created }\end{array}$ & $\begin{array}{l}\text { IK is associated } \\
\text { with cultural } \\
\text { wellbeing }\end{array}$ & $\begin{array}{l}\text { Appropriate texts } \\
\text { and books, etc }\end{array}$ \\
\hline Theme 3 & $\begin{array}{l}\text { IK promotes } \\
\text { cultural roots and } \\
\text { identity }\end{array}$ & $\begin{array}{l}\text { No IK content, } \\
\text { therefore, lacking } \\
\text { cultural relevance }\end{array}$ & $\begin{array}{l}\text { IK can promote } \\
\text { sound values and } \\
\text { ethics }\end{array}$ & \\
\hline
\end{tabular}

Total number of participants was 41 .

\section{Findings and Discussion}

\section{Conceptualization of Indigenous Knowledge}

In this category, three overarching themes emerged.

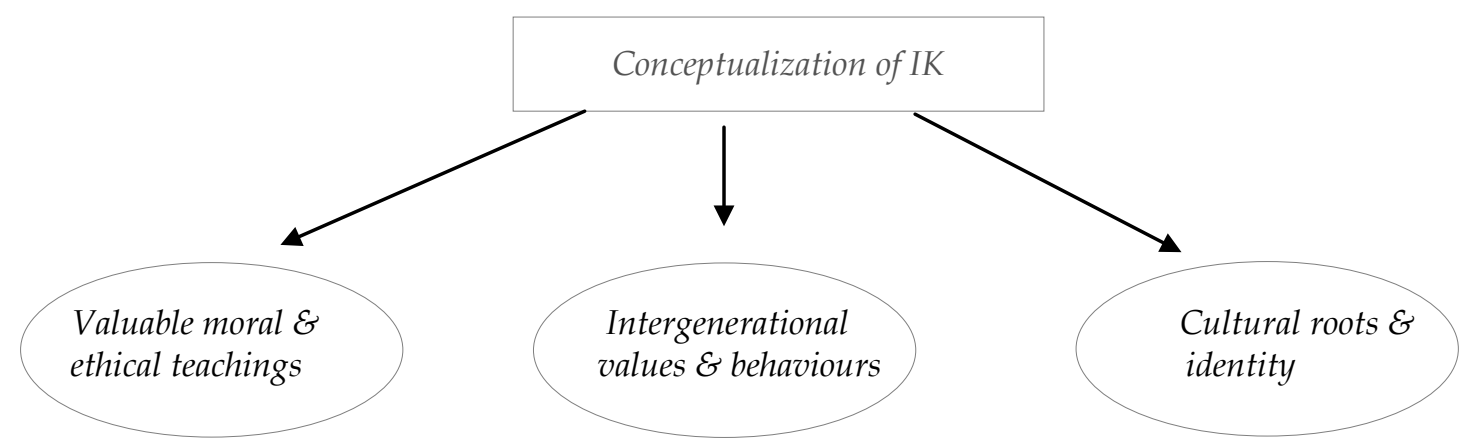

Figure 1: Schematic representation of Category 1: Conceptualisation of Indigenous Knowledge

\section{Indigenous Knowledge has valuable moral and ethical teachings}

The majority of participants identified a clearly defined connection between Indigenous Knowledge and moral and ethical attitudes and behaviours. In the application of indigenous knowledge, honesty and integrity were never compromised and people lived by a strong, collective moral code. Even communally narrated stories could raise a conscience "unembeza" and always necessitated accepting the good and rejecting the bad. Some even spoke about the Indigenous Knowledge guidelines that kept people on a righteous path as opposed to the new South African democratic constitution which they felt was being violated and, as 
such, failed in its main objective of promoting social responsibility. Conversely, Indigenous Knowledge, they maintained, could succeed where modern laws failed or were ineffectual. Phrases and comments about the moral aspect of Indigenous Knowledge included, "awareness of our surroundings", "the value of respect, honesty and love", "good teachings that prevented many current problems, like, teenage pregnancies and the high rate of violence" (the latter two social ills ascribed to the lack of local/community knowledge and western influences), and "groomed us".

Indigenous Knowledge is associated with intergenerational values and behaviours

The second theme was that which related Indigenous Knowledge to their ancestors and forebears and drew a clear link between inheriting a positive value system and the imperative to pass this knowledge on to future generations. Words and phrases included, "respect for nature", "natural and organic remedies", "good foundation", "unbroken chain", "respect for every human being" and "spiritual growth". Some of the older respondents spoke about "still living by the rules of Indigenous Knowledge because they taught us so much of the good". Many recalled the indigenous games their parents taught them and the resultant wholesome lifestyles, which they feared would be lost to the current generation.

\section{Indigenous Knowledge promotes cultural roots and identity}

The third theme was the connection between Indigenous Knowledge and its link with cultural roots and a sense of identity. Most spoke about the growing disintegration of community and the collective and the resultant individualism that now characterises their lives. The often repeated, "It takes a village to raise a child" African phrase featured in this discussion, with reference to the loss of cultural roots. The belief that a people's history is tied to local and indigenous knowledge was also expressed and reiterated in, "If only we could go back to indigenous knowledge, maybe these new generations growing up will have concrete foundations".

\section{Extent of Indigenous Knowledge content in the current ECD Programme}

Three main themes were identified in this second category.

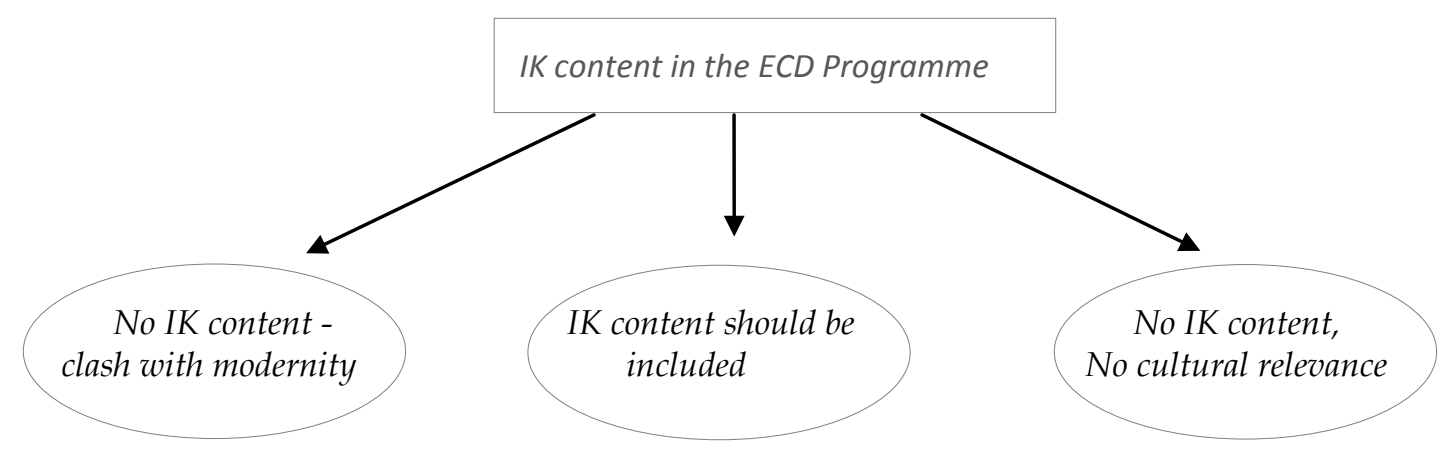

Figure 2: Schematic representation of category 2:

Indigenous Knowledge content in the ECD programme

No Indigenous Knowledge content because of the clash with modernity 
This dominant theme in the second category was that there may not be a place for Indigenous Knowledge in the modern classroom, mainly because of the possible misfit. These doubts were expressed as not "involving those things in the past", because, for instance, "Indigenous Knowledge games are no longer relevant, technology has taken over" and "children are moving away from the old stuff (injula)". Some expressed the view that parents, who had adopted a western culture, were generally against teaching children the roots of Ubuntu.

\section{Opportunity to include Indigenous Knowledge content should be created}

The second theme identified two possibilities, one where opportunities in the existing programme could be used to include Indigenous Knowledge and the second where there was a need for programme developers to create opportunities for Indigenous Knowledge content. In both instances, however, the need for the inclusion of Indigenous Knowledge was clear. For instance, some referred to a topic like, "My Family" which could include family history and identity. Others, said that there was no local, indigenous content, only "Western adopted teachings" , and reinforced this claim by calling for the inclusion of Indigenous Knowledge in Early Childhood Development, "Ligotshwa lisemanzi" meaning "if you want to teach a child, teach them at an early age". Many more voiced the need for Indigenous Knowledge in the curriculum but said, "there is so much we have to do to put it in, especially finding people who have this knowledge".

\section{No Indigenous Knowledge content, therefore, lacking cultural relevance}

This theme in the second category highlighted the absence of Indigenous Knowledge content in the Early Childhood Development programme and the resultant cultural irrelevance to, in this sample, African children. The participants indicated that the teaching/learning content still favoured Western/Eurocentric pedagogy and materials and, as such, excluded the children's own cultural world. They expressed fear of the possibility of the "wiping out" of a whole culture, if "the current older generation doesn't pass the knowledge on". Many spoke about "lost games" ("amagende") or ("amatshe") which "used to sharpen our minds, sight (hand-eye coordination) and motor skills".

\section{Benefits of Indigenous Knowledge content to ECD}

In this third category, the three themes are as follows.

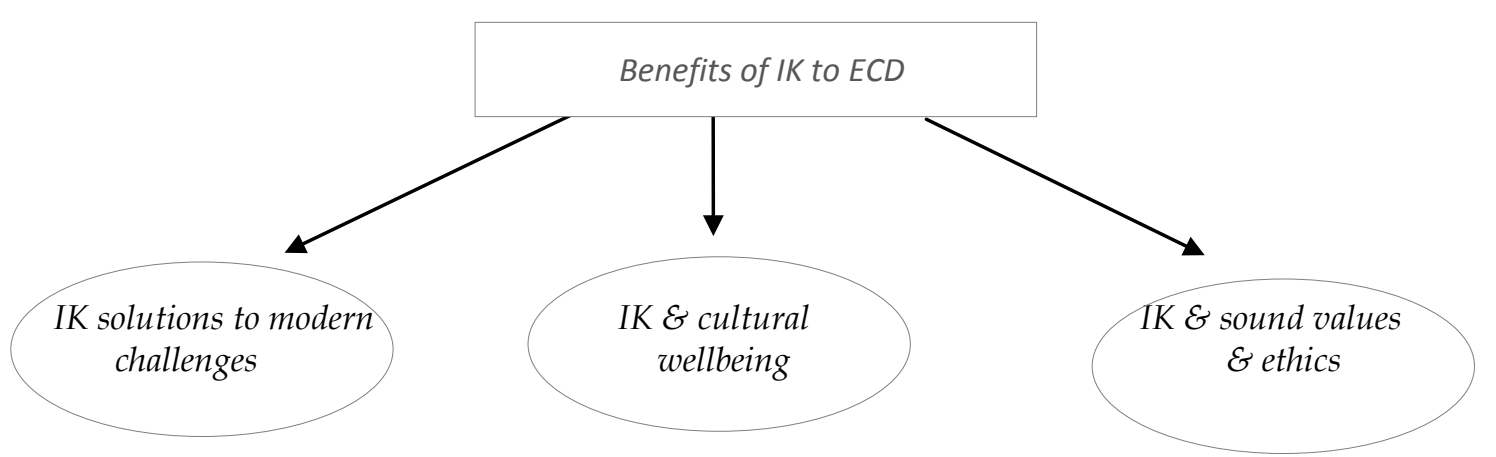

Figure 3: Schematic representation of category 3: Benefits of Indigenous Knowledge to ECD 


\section{Indigenous Knowledge can offer solutions to modern challenges}

The dominant theme in this category was the potential value of Indigenous Knowledge to solve modern challenges. Participants here based this mainly on the indolence of young children and the associated problems. The issue of technology replacing wholesome and natural lifestyle choices featured strongly. They also spoke about the lack of values in children and that a return to the African philosophy and way of life, of Ubuntu, could possibly bring about changes in behaviour and attitude. Here the urban-rural binary was raised in light of the township influences on children as compared to the more natural conditions in which rural children are raised.

\section{Indigenous Knowledge is associated with cultural wellbeing}

The next dominant theme was that of the association between Indigenous Knowledge and cultural wellness. Most indicated that children needed to have a strong sense of their culture, which they said would contribute to firm foundations in life, especially for the growing child. Indigenous Knowledge stories are important "to give them a sense of history and where they come from and, as human beings, you should know where you come from as a whole". Many expressed the view that unless children were affirmed in their own culture, there was limited scope for them to learn about and appreciate the different cultures, especially in a multi-cultural country like South Africa.

\section{Indigenous Knowledge can promote sound values and ethics}

Given the current moral and ethical deficit in South Africa, this dominant theme resonated with most of the practitioners who made the link between Indigenous Knowledge and a sound value system which was needed to ground the children in the early years. Many held the view that telling stories which promote values and ethics from their own culture with their identity was important because children adapt more naturally to what is familiar to them and their worlds. In this regard, they referred to stories like "Unogwaja no chakijana" and also books like "injula" which have values-based stories, rhymes and songs of local knowledge.

\section{Indigenous Knowledge collection methods}

This category identified two themes.

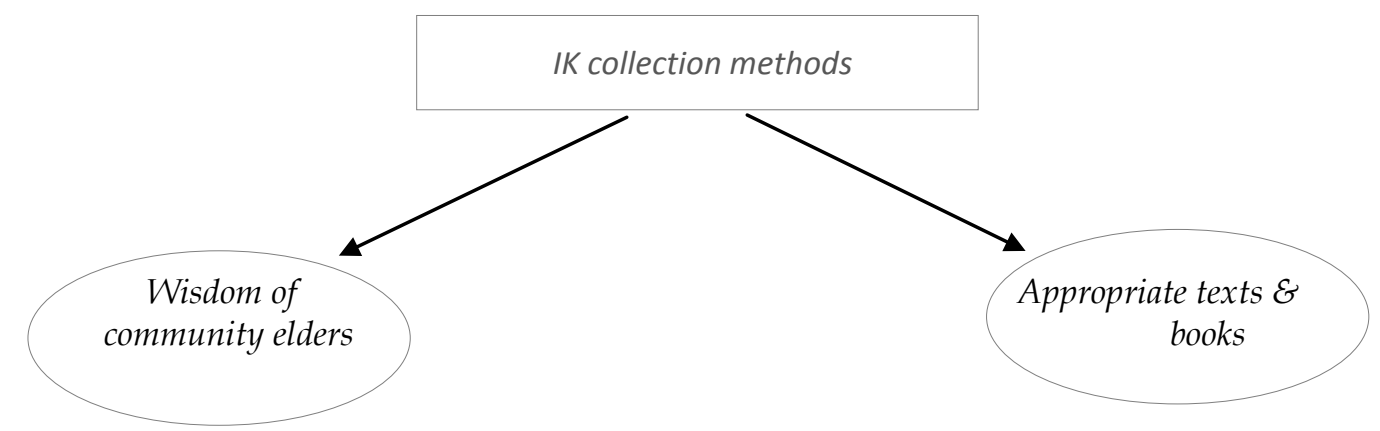

Figure 4: Schematic representation of category 4: Indigenous Knowledge collection methods 


\section{Wisdom of community elders}

In light of the oral nature of African Indigenous Knowledge, and its subsequent devaluing and marginalisation (colonialization and white minority rule in South Africa), Indigenous Knowledge holders are the community elders and the written word is scarce. Consequently, this category identified two themes. The dominant one, was the true knowledge holders, the community elders. Participants showed much reverence when referring to the elders as the main repositories of Indigenous Knowledge. They offered suggestions like "bringing elders to school to teach children their values and benefit from their rich and varied experience. This could also help children "learn more about their history, know what happened in specific locations and keep this knowledge to pass to their children" thus, keeping their culture alive.

\section{Appropriate texts and books, etc.}

In this lesser theme, participants - mainly older - referred to books from their youth, which were introduced to them by their parents. Many were unsure of where to source the books but spoke about these books being expensive in earlier years ("70s '80s), but have since lost their currency and are sold cheaply. They suggested books such as, "Injula", "Inqolobane yesizwe (the roots of the nation)" "Umasihambisane" and "Sikleza ogwansile".

All respondents displayed a clear understanding of the conceptualisation of indigenous knowledge and most were able to recall many of the cultural artefacts songs, poems, stories, proverbs, rhymes, etc. - from their own childhoods and the positive and affirming values they derived from these, which have stayed with them. Significantly, this reinforces one of the primary claims made in the study that behaviour and attitude interventions are crucial and most sustainable at earlier levels, but critical at the Early Childhood Development level for optimal and lifelong learning opportunities. In response to the question on the inclusion of Indigenous Knowledge content in the current Early Childhood Development programme, there was unanimous agreement that there was none, but opportunities to include do exist.

Many were uncertain and unsure about the current relevance of Indigenous Knowledge material, which they believed may be incongruous in the modern classroom. This notion stems from the fallacious argument that indigenous knowledge is primitive and backward and therefore irrelevant and, further, contradicts the holistic and integrated nature of a people's culture. In the wake of this dominant trend, however, all of them agreed that there was opportunity to include Indigenous Knowledge content and they were enthusiastic about the prospect of participating in this study towards the development and implementation of an Early Childhood Development programme using an integral education approach with Indigenous Knowledge values and, in so doing, promote cultural relevance. 
The affirmation of Indigenous Knowledge content was echoed in the third category in their responses to the benefits of the inclusion of Indigenous Knowledge in the Early Childhood Development phase. They all confirmed that these advantages would include the promotion of sound values, morals and ethics, which had the potential to resolve many modern-day problems such as disrespect, irresponsibility, teenage pregnancies, obesity, etc. It could also restore the cultural wellbeing of communities that have been subjected to ongoing western acculturation and devaluing of African ways of being.

Finally, in acknowledging that indigenous knowledge holders are mainly community elders, although some stories, songs and rhymes, etc. have been documented, they looked forward to this study's community focus group sessions that could generate rich material.

This study plans to develop an Integral Education Early Childhood Development programme underpinned by Indigenous Knowledge to redress the indicated shortcomings of the existing Early Childhood Development curriculum. According to Partho (2007), an integral and unitive education addresses the head, the heart, the body and the spirit, an education of the whole person (Partho, 2007: 19). This model, it would seem, could contribute effectively to cultivating sustainable social responsibility and civic mindedness in young children.

\section{Conclusion}

The above preliminary research findings make a strong case for the inclusion of relevant and culturally appropriate indigenous/local knowledge in the Early Childhood Development curriculum and they support the assumption made in this study that there is a significant gap in this curriculum as it does not apply an Integral Education approach, for the holistic development of the child by addressing the head, the heart, the body and the spirit, nor does it include Indigenous Knowledge, for social responsibility through spiritual and moral values and for the preservation of a cultural identity. Such an approach, it is argued, makes allowance for the development and evolution of the self within the collective and, as such, would be more suitable to the purpose of promoting a socially responsible society. Finally, these findings confirm the value placed on Indigenous Knowledge by communities, the sense of loss experienced as a result of the destruction of a well-grounded and innate system and the expectation that its inclusion in the Early Childhood Development programme could auger well for the moral and ethical development of the child from the earliest years. 


\section{References}

Aurobindo, Sri. (1997). The Complete Works of Sri Aurobindo (Vol. 25). Pondicherry, India: Sri Aurobindo Ashram.

Bonn, M. (2007). Children's understanding of 'Ubuntu'. Retrieved August 20, 2015, from

http:/ / www.informaworld.com/openurl?genre=article\&id=doi:10.1080/0300 4430701269291.

Callinicos, L. (2005). Migrant Labour in South Africa Overcoming Apartheid Building Democracy. Retrieved November 24, 2015, from http:/ / overcomingapartheid.msu.edu/multimedia.php?id=65-259-5-2007

Creswell, J. (2015). A concise introduction to mixed methods research. California: SAGE.

Esbjörn-Hargens, S. (2011). Integral Teacher, Integral Students, Integral Classroom: Applying Integral Theory to Education. Retrieved October 24, 2015, from http:/ / nextstepintegral.org/wp-content/uploads/2011/04/IntegralEducation-Esbjorn-Hargens.pdf

Gwele, M., \& Biersteker, L. (2012). Local and Indigenous Knowledge, Practices and Skills in Support of Early Childhood Development: Learnings and Implications (pp. 1-38, Rep.). ELRU.

Hawkes, N. (2010). Values Education and the National Curriculum in England (International Research Handbook on Values Education \& Student Wellbeing) (Lovat, Terry, Toomey, Ron, Clement, Neville, Ed.). The Netherlands: Springer.

Kaya, H., \& Padayachee, K. (2013). Building a Culture of Nonviolence in Early Childhood Development through Indigenous Knowledge Systems. Retrieved November 25, 2015, from http:/ / www.ukzn.ac.za/news/2013/09/17/indigenous-knowledge-systemsand-non-violence-culture

Partho. (2007). Integral Education: A Foundation for the Future. Pondicherry, India: Sri Aurobindo Society.

Pendlebury, S., \& Enslin, P. (2007). What Kinds of People are We?: Values Education After Apartheid (Values Education and Lifelong Learning) (Aspin, D.N. and Chapman, J.D., Ed.). The Netherlands: Springer.

Richardson, N. (2003). Not Another Moral Summit: Problems and Possibilities for Moral Regeneration. Retrieved February 25, 2015, from http:/ / scriptura.journals.ac.za/pub/article/view/893/855

Samuelsson, I., \& Kaga, Y. (2008). The contribution of early childhood education to a sustainable society. Retrieved February 26, 2015, from http://unesdoc.unesco.org/images/0015/001593/159355E.pdf

South Africa, Department of Social Development. (2015). Draft Early Childhood Development Policy (No. 204)(Government Gazzette Number 38558).

South Africa, Department of Education. (1997). Education White Paper 3 (Government Gazette Number 18207). 
South Africa, Department of Social Development. (2008). On a Situational Analysis of Child-Headed-Households in South Africa. Pretoria, Gauteng: UNISA.

South Africa, Department of Social Development. (2012). The South African National Curriculum Framework for children from Birth to Four. Pretoria.

Vengopal, K., \& Kumari, P. (2010). Auroville School and Holistic Education. Encounter Education for Meaning and Social Justice, Volume 23(Number 3), 59-63.

Viviers, A. (2009). Early Child Development: A Strategy to Invest in. In Unicef Country Representative presentation at the Committee for Economic Development and NBI conference on Business Engagement in education policy. UNICEF.

Wilson, R. (2008). Fostering Goodness \& Caring: Promoting Moral Development of Young Children. Retrieved November 20, 2015, from http:/ / www.earlychildhoodnews.com /article_view.aspx?ArticleID=565 


\title{
ResearchGate: Investigating Altmetric and Bibliometric Relationships for Environmental Science Researchers at Unisa
}

\author{
Leslie S Adriaanse \\ Department of Information and Knowledge Management \\ University of Johannesburg \\ Johannesburg, South Africa \\ Chris Rensleigh \\ Department of Information and Knowledge Management \\ University of Johannesburg \\ Johannesburg, South Africa
}

\begin{abstract}
This paper aims to establish the relationships between the altmetrics derived from the academic social networking tool ResearchGate and bibliometrics derived from the citation resources Web of Science, Scopus and Google Scholar to establish which academic social networking tools gives the most advantage. The investigation forms part of a longitudinal study investigating the e-visibility of the environmental science researchers at the University of South Africa during December 2014 and December 2016. The bibliometric indicators from Web of Science, Scopus and Google Scholar, and altmetric indicator data from ResearchGate were collected in six (6) month intervals, and analysed using SPSS to determine the Pearson's rank correlations. The results indicated a positive correlation between the bibliometric indicators derived from Web of Science, Scopus and Google Scholar, and the altmetric indicators derived from the academic social networking tool ResearchGate. The results show that ResearchGate altmetric indicators influence the bibliometric indicators positively. ResearchGate therefore can be recommended for academic social networking as a strategy to influence the Environmental Science researcher's citations positively at Unisa.
\end{abstract}

Keywords: bibliometrics, altmetrics, ResearchGate, citation resources, altmetrics bibliometric correlations 


\section{Introduction}

Bibliometric indicators were traditionally used during research evaluations and research performance measurement to ascertain the research impact of researchers (Hoffman, Lutz, \& Meckel, 2014). With the advent of Web 2.0 Technology, social networking tools with enhanced functionalities permeated academia and the research processes of researchers. The new generation academic social networking tools allow for the discovery, communicating, disseminating and sharing of research during the research workflow and has ushered in a new dimension to measuring scholarly research activity i.e. usage statistics, downloading statistics, and sharing of research (Kim \& Abbas, 2010).

The introduction of the alternative measuring indicators i.e. altmetrics, made it possible to measure the attention and usage the scholarly research on social networking tools was receiving online. It is suggested that altmetrics be an alternative metric indicator to bibliometrics, trying to fill the gap in traditional citations metrics by producing more inclusive and timely metrics which are pertinent to the researcher and the research output (Kim \& Abbas, 2010; Konkiel, 2013). The aim of altmetrics was to capture previously invisible impacts i.e. the impact of research output on a larger audience - society as a whole. Recent studies found, the emergence of relationships between bibliometrics, as performance indicators for the measurement of research performance, and altmetrics, as possible indicators for research impact on social networking tools (Costas, Zahedi, \& Wouters, 2014; Khodiyar, Rowlett, \& Lawrence, 2014; Li, Thelwall, \& Giustini, 2012; Naude \& van Biljon, 2017; Schlögl, Gorraiz, Gumpenberger, \& Jack, 2014).

This paper aims at investigating and reporting the relationships between the traditional research impact represented by bibliometrics, derived from citation resources Web of Science, Scopus and Google Scholar and altmetrics, derived from the social networking tool ResearchGate. Alternative metrics being alternative to bibliometrics represents the societal impact of researchers. The research for this paper focus on Phase 1 of an e-visibility study, which forms part of a larger PhD longitudinal comparative study spanning a two-year period from December 2014 to December 2016. The e-visibility study aims at developing an e-visibility strategy for the researchers at the School of Environmental Sciences (SES) at University of South Africa (Unisa), and comprises of five (5) phases combining bibliometric and altmetric data collection over six (6) month intervals, and two e-visibility surveys determining the e-visibility status of the researchers over the two year period. On commencement of the study, very little research were available on the correlation of bibliometrics and altmetrics for the environmental sciences in South Africa.

The main contribution of this paper is to establish the correlation between the bibliometrics and altmetrics of the SES researchers within a South African context. The premise is that bibliometrics derived from citation resources correlate with the altmetrics derived from social networking tools. 


\section{Research metrics}

The introduction of altmetrics as a possible alternative measurement indicator of research impact necessitates the investigation of the possible influence of altmetric indicators from social networking tools on bibliometric indicator in a research context. Following an overview of the nature of each indicator and their role within research impact.

\section{Bibliometrics}

The number of citations a researcher receives suggests how influential the research is deemed within the subject discipline (Konkiel, 2013), and suggests the rate of citations as an indicator of the researcher's performance. Bibliometrics describes the counting and analysing of published scholarly research and describes the techniques measuring scholarly impact of research (Bornmann, 2014a, 2014b; Roemer \& Borchardt, 2012). It is generally accepted that a citation indicates the utilization of research and gives acknowledgement of the researcher's contribution to the body of knowledge within the specific subject discipline implying research credibility and quality. However, the growing realization that the existing research metrics, comprising of bibliometrics, is seen to be inadequate and exclusive in addressing the full spectrum of research evaluation and do not cater for the attention the research output receives on the Web and on social networking tools (Chen, Tang, Wang, \& Hsiang, 2015; Roemer \& Borchardt, 2012).

\section{Altmetrics}

The emergence of the Web 2.0 technologies, introduced change to the new generation research communities and how they experienced information (Haustein, Peters, Bar-Ilan, Priem, Shema, \& Terliesner, 2014; Yeong \& Abdullah, 2012). Incorporation of social networking tools in the research workflow, have affected how researchers conduct, discuss and disseminate research, significantly becoming a "nutrient rich space for scholars" (Priem, Groth, \& Taraborelli, 2012; Priem, Piwowar, \& Hemminger, 2012). Social networking tools gathers and records the social usage data and scholarly activity of research outputs on the Web according to altmetric type (e.g. reads, sharing, links, counts, views, downloads, bookmarks, saves, annotates, discussing, recommended scholarly items) (Adie \& Roe, 2013; Robinson-García, Torres-Salinas, Zahedi, \& Costas, 2014; Thelwall, Haustein, Larivière, \& Sugimoto, 2013). This trail of social activity and impact gives insight into broader view of the research consumption of research output on the Web i.e. the digital footprint of the research (Kortelainen \& Katvala, 2012; Priem, Groth, \& Taraborelli, 2012). This translates to the societal impact of research. Altmetrics can be described as an attempt to measure the influence and scholarly interaction on the Web (social platforms) and to quantify the social networking resources interactions (Galligan \& Dyas-Correia, 2013; Hassan \& Gillani, 2016; Haustein \& Siebenlist, 2011).

Research impact makes use of traditional bibliometric indicators in the measurement of research impact while societal impact makes use of alternative metrics to measure the societal impact of the research (Bornmann, 2014a). Yeong and Abdullah (2012) suggest that altmetrics is the answer to a new generation of scientists and 
researchers seeking measurement of the more complete and inclusive research impact of their research as researcher.

\section{Relationships between Bibliometrics and Altmetrics}

Various studies have been conducted on establishing the relationships between altmetrics by calculating correlations between altmetrics and bibliometrics (Costas, Zahedi, \& Wouters, 2014; Khodiyar, Rowlett, \& Lawrence, 2014; Li, Thelwall, \& Giustini, 2012; Naude \& van Biljon, 2017; Schlögl, Gorraiz, Gumpenberger, Jack, \& Kraker, 2013; Torres-Salinas, Robinson-Garcia, \& Jimenez-Contreras, 2016).

Prior research on altmetrics bibliometric indicator relationships regarding ResearchGate reported strong positive correlations for altmetric indicators for ResearchGate and citation resources: Scopus, Web of Science and Google Scholar. These include:

- Scopus, Pearson's r=0.98 (significance not listed) (Shrivastava \& Mahajan, 2015);

- Web of Science, Spearman r=0.974 - 0.976 (<0.000 significance) (Onyancha, 2015); and

- Google Scholar, Spearman r=0.956 (<0.05 significance) (Ortega, 2015) and Spearman $\mathrm{r}=0.87$ (significance not listed) (Orduña-malea, Martín-martín, \& Delgado-lópez-cózar, 2016).

\section{Methods}

\section{Participants}

The sample population includes the 62 researchers in the School of Environmental Sciences (SES) at Unisa.

\section{Design}

The aim of this paper is to investigate the relationships with the purpose of establishing correlations between bibliometrics and altmetrics for the School of Environmental Sciences (SES) researchers from University of South Africa.

\section{Materials}

The following combination of altmetric indicator data from ResearchGate and bibliometric indicator data from Web of Science, Scopus and Google Scholar, would help establish the altmetric bibliometric indicator relationships between ResearchGate and Web of Science, Scopus and Google Scholar.:

- Name of author; number of publications;

- Number of citations of researchers of citation resources (Web of Science, Scopus and Google Scholar);

- Number of views (December 2014 to July 2015), downloads and reads from ResearchGate;

- Number of downloads (December 2014 to July 2015), from ResearchGate;

- Number of reads (December 2015 to December 2016), from ResearchGate. 


\section{Procedure}

The collection of data, which included sourcing altmetric indicator data derived from the academic social networking tool ResearchGate, and bibliometric indicator data derived from the citation resources: Web of Science, Scopus and Google Scholar, were extracted, recorded and collected manually during December 2014 and December 2016. The data collected from ResearchGate proved to be a limitation as ResearchGate changed the method of harvesting the altmetric indicators from views and downloads in between July 2015 and December 2015, to reflect reads motivating that reads incorporate both views and downloads to be expressed as reads for a more accurate reflection of the altmetric indicator (Nicholas, Clark, \& Herman, 2016). The data was analysed using SPSS software for statistical analysis, including the Kolmogorov-Smirnov (Lillefors significance correction) normality tests for each value. The Pearson rank correlation was used to calculate the correlations between altmetrics and bibliometrics that is suitable for non-normal distributions typically used in large social network data testing.

\section{Results}

The following results were obtained from the statistical analysis of bibliometrics from Web of Science, Scopus and Google Scholar, and altmetrics from the social networking tool ResearchGate relating to the 62 SES researchers for the two year period from December 2014 to December 2016.

\section{Distribution of bibliometrics and altmetrics}

The distribution of the altmetric and bibliometric indicators during December 2014 to December 2016 for the SES researchers are summarised in Figure 1 and 2.

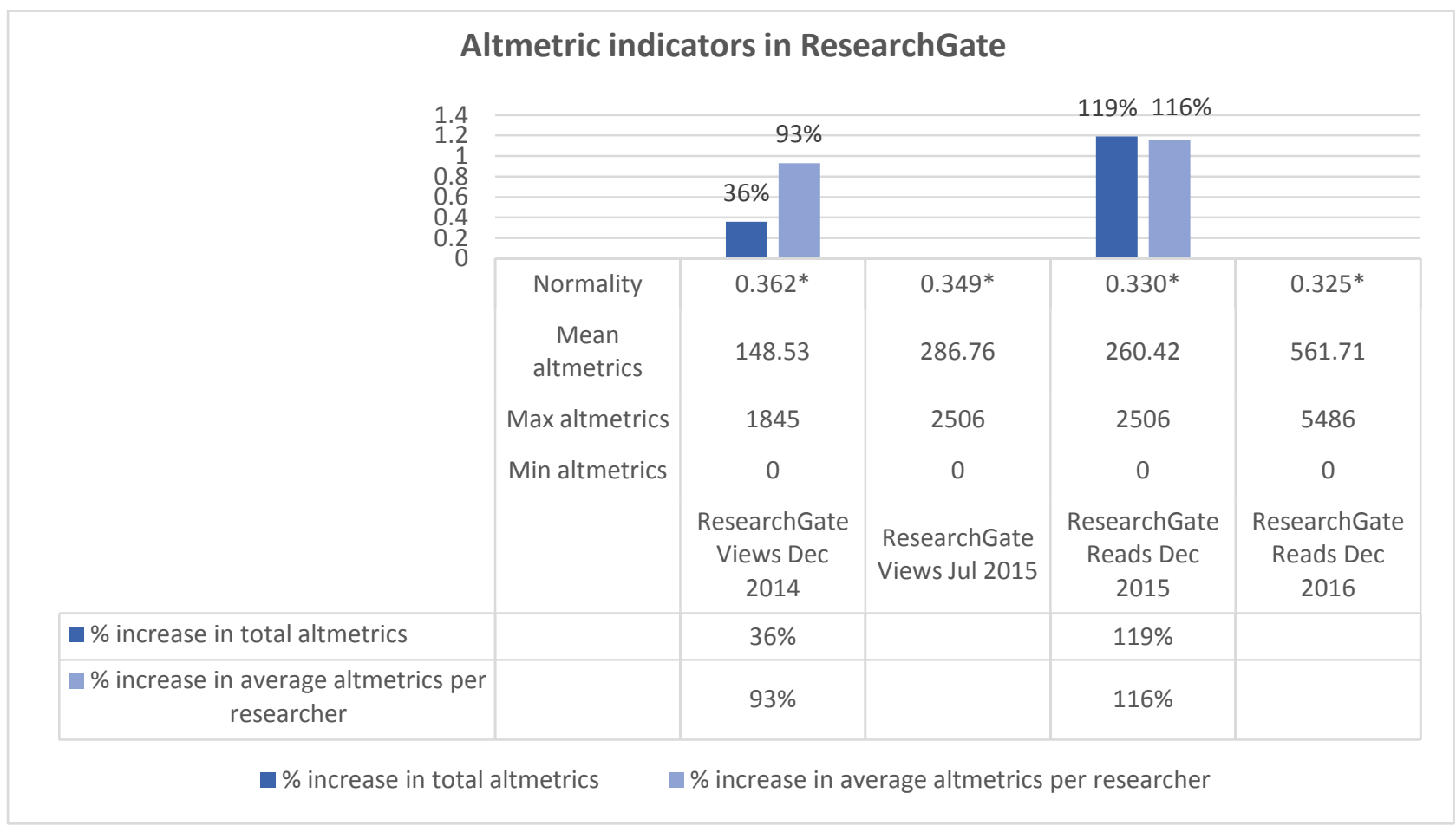

*(Lillefors Sig. correction)

Figure 1 - Distribution of altmetric indicators in ResearchGate 
In Figure 1, the altmetric distribution results indicated an increase in the total reads of $119 \%$ with an increase in average reads per researcher of $116 \%$ for the period December 2015 to December 2016. The results for the period December 2014 to July 2015 for ResearchGate, indicated an increase in total views of 36\% and an increase in the average views on ResearchGate of $93 \%$.

The results for bibliometric distributions indicate the largest increase in total citations (138\%) and average citations (170\%) in Google Scholar, followed by $58 \%$ for total citations and $99 \%$ for average citations in Scopus; and $25 \%$ for total citations and $69 \%$ for average citations in Web of Science - see Figure 2.

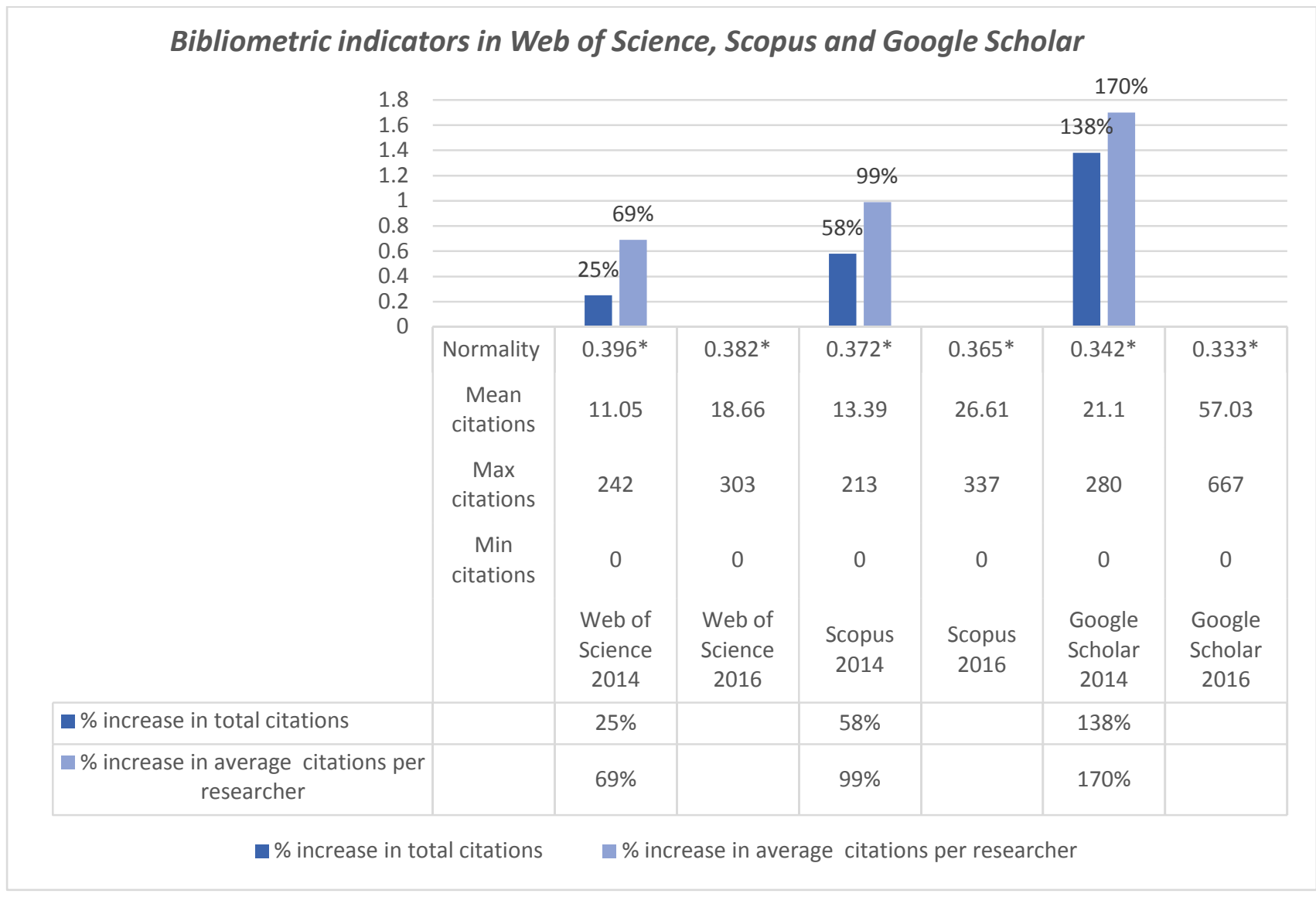

*(Lillefors Sig. correction)

Figure 2 - Distribution of bibliometric indicators in Web of Science, Scopus and Google Scholar

The results indicate an increase in total citations and an increase in average citations for SES researchers across the three citation resources.

\section{Correlations between altmetrics and bibliometrics}

The relationships between bibliometric and altmetrics indicators are demonstrated by the Pearson's rank correlation coefficient between bibliometrics from Web of Science, Scopus, and Google Scholar, and altmetrics in ResearchGate. Table 1 shows the correlation matrix for the correlation analysis of citations from Web of Science, Scopus and Google Scholar; and views and downloads from ResearchGate (December 2014 to July 2015) and reads from ResearchGate (December 2015 to 
December 2016).

Table 1 - Correlation analysis of the rank values for the bibliometrics and ResearchGate as altmetric indicators

\begin{tabular}{|c|c|c|c|c|c|c|}
\hline Pearson correlation & WOS 2014 & WOS 2016 & $\begin{array}{c}\text { Scopus } \\
\mathbf{2 0 1 4}\end{array}$ & $\begin{array}{c}\text { Scopus } \\
\mathbf{2 0 1 6}\end{array}$ & $\begin{array}{c}\text { Google } \\
\text { Scholar } \\
\mathbf{2 0 1 4}\end{array}$ & $\begin{array}{c}\text { Google } \\
\text { Scholar } \\
2016\end{array}$ \\
\hline $\begin{array}{c}\text { ResearchGate Views } \\
\text { Dec 2014 }\end{array}$ & $0.726^{*}$ & $0.783^{*}$ & $0.819^{*}$ & $0.855^{*}$ & $0.919^{*}$ & $0.847^{*}$ \\
\hline $\begin{array}{c}\text { ResearchGate Views } \\
\text { Jul 2015 }\end{array}$ & $0.681^{*}$ & $0.733^{*}$ & $0.774^{*}$ & $0.820^{*}$ & $0.855^{*}$ & $0.840^{*}$ \\
\hline $\begin{array}{c}\text { ResearchGate } \\
\text { Downloads Jul 2015 }\end{array}$ & $0.688^{*}$ & $0.653^{*}$ & $0.635^{*}$ & $0.619^{*}$ & $0.449^{*}$ & $0.614^{*}$ \\
\hline $\begin{array}{c}\text { ResearchGate Reads } \\
\text { Jul 2015 }\end{array}$ & $0.599^{*}$ & $0.644^{*}$ & $0.664^{*}$ & $0.731^{*}$ & $0.802^{*}$ & $0.741^{*}$ \\
\hline $\begin{array}{c}\text { ResearchGate Reads } \\
\text { Dec 2016 }\end{array}$ & $0.549^{*}$ & $0.612^{*}$ & $0.633^{*}$ & $0.696^{*}$ & $0.766^{*}$ & $0.702^{*}$ \\
\hline
\end{tabular}

*(Sig. (2-tailed) $<0.000)$

Correlation matrix legend

\begin{tabular}{|l|l|l|l|l|l|l|l|l|l|l|}
\hline 0 & 0.1 & 0.2 & 0.3 & 0.4 & 0.5 & 0.6 & 0.7 & 0.8 & 0.9 & 1 \\
\hline
\end{tabular}

\section{Discussion of bibliometric correlations for ResearchGate}

The results following include the Pearson's rank correlation for ResearchGate (views and downloads within the period December 2014 to July 2015). The strongest correlation was observed in Google Scholar and views from ResearchGate $(r=0.919$, $<0.000$ significance) in December 2014. The weakest correlation was observed in ResearchGate downloads for July 2015 and Google Scholar December 2014 with Pearson $r=0.449$ (<0.000 significance). The results for ResearchGate and Web of Science, Scopus and Google Scholar found that ResearchGate has an overall positive correlation between Web of Science, Scopus and Google Scholar for the period December 2014 and December 2016. The Pearson's ranks correlations vary from $\mathrm{r}=0.549$ to $\mathrm{r}=0.919$ with only one moderate correlation Pearson $\mathrm{r}=0.449$ for ResearchGate downloads for July 2015 and Google Scholar December 2014.

The importance of calculating the altmetrics bibliometric correlations lies within the ability to identify the significant relationships between altmetrics and bibliometrics relationships between the views and reads from ResearchGate and the citations from Web of Science, Scopus and Google Scholar during the period December 2014 and December 2016. The established correlations between altmetrics and bibliometrics points towards an assumption that altmetrics and bibliometrics are related and can exert influence on each other (Thelwall, Haustein, Larivière, \& Sugimoto, 2013). Previous studies on correlations between bibliometrics and altmetrics indicators have support the findings of this study and have also recorded strong positive correlations (Pearson and Spearman) for Web of Science and ResearchGate views and downloads (Onyancha, 2015); for Scopus and ResearchGate (Shrivastava \& Mahajan, 2015), for Google Scholar and ResearchGate (Orduña-malea et al., 2016; Ortega, 2015). 


\section{Conclusion}

The altmetric bibliometric correlation results, relating to ResearchGate altmetrics and bibliometrics from Web of Science, Scopus and Google Scholar, found an overall positive correlation varying from $\mathrm{r}=0.549$ to $\mathrm{r}=0.919$ with only one moderate correlation Pearson $r=0.449$ for ResearchGate downloads for July 2015 and Google Scholar December 2014.

The strong correlation between the altmetrics derived from ResearchGate and the bibliometrics derived from Web of Science, Scopus and Google Scholar indicate that there was a positive correlation between Web of Science, Scopus and Google Scholar, and ResearchGate. The results provide evidence that altmetrics indicators from ResearchGate associate with the bibliometric indicators and exert influence positively on citations. This positive influence of ResearchGate as academic social networking tool has a positive societal impact on the Environmental Science research output at Unisa. This study therefore recommends the creation and maintenance of a research e-profile on ResearchGate for the positive influence of citation counts on citation resources of researchers.

\section{References}

Adie, E., \& Roe, W. (2013). Altmetric: enriching scholarly content with article-level discussion and metrics. Learned Publishing, 26(1), 11-17. http:/ / doi.org/10.1087/20130103

Bornmann, L. (2014a). Do altmetrics point to the broader impact of research? An overview of benefits and disadvantages of altmetrics. Journal of Informetrics, 8(4), 895-903.

Bornmann, L. (2014b). Validity of altmetrics data for measuring societal impact: A study using data from Altmetric and F1000Prime. Journal of Informetrics, 8(4), 935-950. http:/ / doi.org/10.1016/j.joi.2014.09.007

Bornmann, L., \& Daniel, H.-D. (2008). What do citation counts measure? A review of studies on citing behaviour. Journal of Documentation, 64(1), 45-80. http:/ / doi.org/DOI 10.1108/00220410810844150

Chen, K., Tang, M., Wang, C., \& Hsiang, J. (2015). Exploring alternative metrics of scholarly performance in the social sciences and humanities in Taiwan. Scientometrics, 102, 97-112. http:/ / doi.org/10.1007/s11192-014-1420-6

Costas, R., Zahedi, Z., \& Wouters, P. (2014). Do "altmetrics" correlate with citations? Extensive comparison of altmetric indicators with citations from a multidisciplinary perspective. Journal of the Association for Information Science and Technology, 66(10), 2003-2019. Digital Libraries. http:// doi.org/10.1002/asi.23309

Galligan, F., \& Dyas-Correia, S. (2013). Altmetrics: Rethinking the Way We Measure. Serials Review, 39(1), 56-61.

Hassan, S.-U., \& Gillani, U. A. (2016). Altmetrics of “altmetrics” using Google Scholar, Twitter, Mendeley, Facebook, Google-plus, CiteULike, Blogs and Wiki. ArXiv, 1603.07992, 1-19.

Haustein, S., Peters, I., Bar-Ilan, J., Priem, J., Shema, H., \& Terliesner, J. (2014). 
Coverage and adoption of altmetrics sources in the bibliometric community. Scientometrics, 101(2), 1145-1163. http:// doi.org/10.1007/s11192-013-1221-3

Haustein, S., \& Siebenlist, T. (2011). Applying social bookmarking data to evaluate journal usage. Journal of Informetrics, 5(3), 457-446.

http:/ / doi.org/10.1016/j.joi.2011.04.002

Hoffman, C. P., Lutz, C., \& Meckel, M. (2014). Impact Factor 2.0: Applying Social Network analysis to Scientific Impact Assessment. In 47th Hawaii Interantional Conference on Systems Science (pp. 1576-1585). IEEE Computer Society. http:/ / doi.org/10.1109/HICSS.2014.202

Hood, W. W., \& Wilson, C. S. (2001). The Literature of Bibliometrics, Scientometrics, and Informetrics. Scientometrics, 52(2), 291-314.

Khodiyar, V. K., Rowlett, K. A., \& Lawrence, R. N. (2014). Altmetrics as a means of assessing scholarly output. Learned Publishing, 27(5), S25-S32. http:/ / doi.org/10.1087/20140505

Kim, Y.-M., \& Abbas, J. (2010). Adoption of Library 2.0 Functionalities by Academic Libraries and Users: A Knowledge Management Perspective. The Journal of Academic Librarianship, 36(3), 211-218.

http:/ / doi.org/10.1016/j.acalib.2010.03.003

Konkiel, S. (2013). Altmetrics: A 21st Century Solution to Determining Research Quality. Online Searcher, 37(4), 1-5.

Kortelainen, T., \& Katvala, M. (2012). “Everything is plentiful-Except attention”. Attention data of scientific journals on social web tools. Journal of Informetrics, 6(4), 661-668.

Li, X., Thelwall, M., \& Giustini, D. (2012). Validating online reference managers for scholarly impact measurement. Scientometrics, 91(2), 461-471. http:/ / doi.org/10.1007/s11192-011-0580-x

Naudé, F. (2017). Comparing downloads, mendeley readership and google scholar citations as indicators of article performance. Electronic Journal of Information Systems in Developing Countries, 78(1). http://doi.org/10.1002/j.16814835.2017.tb00572.x

Naude, F., \& van Biljon, J. (2017). Scholarly Impact: a Bibliometric and Altmetric study of the Journal of Community Informatics. The Journal of Community Informatics, 13(1).

Nicholas, D., Clark, D., \& Herman, E. (2016). ResearchGate: Reputation uncovered. Learned Publishing, 29(3). http:/ / doi.org/10.1002/leap.1035

Onyancha, O. B. (2015). Social media and research: an assessment of the coverage of South African universities in ResearchGate, Web of Science and the Webometrics Ranking of World Universities. South African Journal of Libraries and Information Science, 81(1), 8-20. http:/ / doi.org/10.7553/81-1-1540

Orduña-malea, E., Martín-martín, A., \& Delgado-lópez-cózar, E. (2016). The next bibliometrics: Almetrics (author level metrics) and the multilevel faces of author impact. El Profesional de La Informacion, 25(3), 485-496.

Ortega, J. L. (2015). Relationship between altmetric and bibliometric indicators across academic social sites: The case of CSIC's members. Journal of Informetrics, 9, 39- 
49. http:// doi.org/10.1016/j.joi.2014.11.004

Priem, J., Groth, P., \& Taraborelli, D. (2012). The altmetrics collection. PloS One, 7(11), e48753. http:/ / doi.org/10.1371/journal.pone.0048753

Priem, J., Piwowar, H. A., \& Hemminger, B. M. (2012). Altmetrics in the wild: Using social media to explore scholarly impact. arXiv Preprint, 1203.4745, 1-15.

Robinson-García, N., Torres-Salinas, D., Zahedi, Z., \& Costas, R. (2014). New Data, New Possibilities: Exploring the Insides of Altmetric.Com. Nuevos Datos, Nuevas Posibilidades: Revelando El Interior de Altmetric.com., 23(4), 359-366.

Roemer, R. C., \& Borchardt, R. (2012). From bibliometrics to altmetrics. College $\mathcal{E}$ Research Libraries News, (November), 596-600.

Schlögl, C., Gorraiz, J., Gumpenberger, C., \& Jack, K. (2014). Comparison of downloads, citations and readership data for two information systems journals. Scientometrics.

Schlögl, C., Gorraiz, J., Gumpenberger, C., Jack, K., \& Kraker, P. (2013). Download vs. citation vs. readership data: The case of an information systems journal. In Proceedings of ISSI 2013 - 14th International Society of Scientometrics and Informetrics Conference (Vol. 1, pp. 626-634).

Shrivastava, R., \& Mahajan, P. (2015). Relationship amongst ResearchGate altmetric indicators and Scopus bibliometric indicators. New Library World, 116(9/10), 564-577. http:/ / doi.org/10.1108/NLW-03-2015-0017

Thelwall, M., Haustein, S., Larivière, V., \& Sugimoto, C. R. (2013). Do altmetrics work? Twitter and ten other social web services. PloS One, 8(5), e64841. http:/ / doi.org/10.1371/journal.pone.0064841

Torres-Salinas, D., Robinson-Garcia, N., \& Jimenez-Contreras, E. (2016). Can we use altmetrics at the institutional level? A case study analysing the coverage by research areas of four Spanish universities. In Proceedings of the 21st International Conference on Science and Technology Indicators, 14-16 September 2016 (pp. 1-8). Valencia: STI Conference 2016.

Yeong, C. H., \& Abdullah, B. J. J. (2012). Altmetrics: the right step forward. Biomedical Imaging and Intervention Journal, 8(3), 1-2. http:/ / doi.org/10.2349/biij.8.3.e15. 


\title{
mooKIT - A MOOC Platform for Developing Countries
}

\section{Prabhakar T.V.}

Indian Institute of Technology Kanpur (IITK),

Kanpur, India

Balaji Venkatraman

Commonwealth of Learning (COL),

Vancouver, Canada

Revathy K.T.

Indian Institute of Technology Kanpur (IITK),

Kanpur, India

\begin{abstract}
IIT Kanpur (IITK) and Commonwealth of Learning (COL) have been working on Massive Open Online Courses (MOOCs) since 2012. During this collaboration we developed a MOOC Management system and delivered close to 30 courses to about 200000 users in over 100 countries. Our understanding of what features a MOOC Management System should have and how to engage the students in a course has also evolved. In this paper we describe our experiences and trace how some of our software features have evolved. One of the core features is the development and integration of an advanced analytics module. We also discuss how to reduce dropouts from an open online course, a common concern for all MOOCs.
\end{abstract}

Keywords: MOOCs, Analytics, Developing Countries.

\section{Introduction}

IIT Kanpur ran its first MOOC in late 2012, a course on Architecting Software for the Cloud (arch4cloud, 2012). This was run on a customized Sakai platform(Sakai, 2018), a well-known open source learning management system. The course was intended for advanced software developers in the industry and had close to one thousand registrations, a sizable number for such a specialized course. Sakai was adequate for the course but had several issues from our perspective. For one, it required advanced Java Skills to manage daily operations, large amount of computing power and was designed with a classroom -based delivery in mind. We realised that there are no MOOC management systems available in the 'market' for the kind of contexts most 
academic institutions in the developing countries are placed in, and decided to develop one, ground up. Commonwealth of Learning joined IITK as a collaborator in 2013 in creating the requirements and specifications and in running MOOCs. Subsequently, we developed mooKIT and ran 20 courses (m4d, 2018; agMOOCs, 2018; Outreach, 2018). We also adopted mooKIT for running flipped classes at IIT Kanpur (Flipclasses, 2018).

During 2013, one of us proposed that a MOOC should be viewed as an open conference event, that too, an event with serious involvement of media. In other words, a MOOC is primarily a media-rich conference event (Prabhakar, 2013). Based on this, we offered two MOOCs during 2013-15. The external reviewers concluded that oth of them met the then-popular definitions of MOOCs while fulfilling learner expectations (Porter, 2014; Perris, 2015). Based on extensive analysis of literature on research and practice in MOOCs, we proposed a series of recommendations on how MOOCs may be adapted in the context of developing countries to meet the increasing demands of Higher Education with quality assurance and in skills development (Patru and Balaji, 2016). Our understanding of what a MOOC management system should look like, what should be its principle features and what are the limitations from a student perspective evolved over the last 4 years. These are primarily perspectives from a developing country, but many of them are applicable for a global audience as well. We also developed some insights on how to keep the students engaged and increase retention rates. In the first part of the paper we talk about platform experiences and in the second part we discuss best practices.

\section{Challenges and responses}

From our deployment in 2014, we collected data on the access to courses by learners in diverse situations, and made a number of changes to address some of the challenges identified.

Bandwidth: One of the first things we noticed is that a large number of students have a very poor or unstable bandwidth. We needed to come up with application level features that would help the students in such scenarios.

1. We built a bandwidth indicator of the Internet connection quite like the signal strength bars on a cell phone. This gives a visual indication to the student: if the connection was going bad, the videos would not be able to stream, thus allowing the learner to explore other content delivery options(see below).

2. Content Delivery Options:

Audio streaming: The bandwidth required to stream audio is much less ( For example, one can stream standard quality audio at $64 \mathrm{kbps}$ whereas we need at least $400 \mathrm{kbps}$ for 360p (640x360) video. Many MOOC lectures are what we call 'talking head videos' - the Instructor is speaking and there are a set of slides. mooKIT has a feature where the slides and the audio are stored separately on the server. When the connection bandwidth drops below acceptable video streaming levels, the user can 
opt to listen to the talk and stream the audio only. The system will download the slides and play the audio in a synchronised manner giving an almost videostreamed lecture experience at much less bandwidth.

Semi offline: We discovered that sometimes streaming options are not good enough to stream even the audio. Further, some of the lectures are not in the slides +audio format. mooKIT has an option where the videos can be loaded on an SD Card, which can then be loaded on the users mobile phone. The mooKIT app will stream the videos from the card. For information that is changing, like the Forum posts, Announcements etc. an Internet connection is needed. Since these are text-only content, they work with much lower bandwidth requirements. The analytics information, like the number of views by a user are collected by the app and sent to the Instructor, thus validating the participation of the student in the course. The app does opportunistic syncing - that the analytics go the server whenever a connection becomes available. The SD card can be replicated locally and distributed to any number of users.

Fully offline: mooKIT based courses can be distributed on an SD Card to be pursued without any Internet connection. Typical scenarios are archival for self-reference after doing a course, or for distribution of Open Educational Resources, which are structured as a course.

Social network connect: Forums are the counterparts for classroom discussion. The students not only get to clear their doubts but also learn from questions asked by other students. Even more important than that, students often answer the questions by others, thereby gaining in confidence and engagement, building social connects and improving the overall enthusiasm in the course. However, we noticed that there is a small speed bump for this. To participate in the forum, the student has to log into the course. And normally she does it when the new lecture is released. And may not login till the next significant event, like another lecture release or some activity in the Forum post she is following.This may reduce the opportunity to participate in the Forum on a more continuous basis. We realised that many students however are active on Social Networking platforms like Facebook and Twitter. We built a feature in mooKIT where a post in the Forums can appear automatically as a post in a Facebook page corresponding to the course. And any responses to that post (on the Facebook page) will be reflected in the Forum posts in the platform. Thus, a student can follow and participate in the discussions without having to login into the course platform. A sample from a course on Integrated Pest Management is shown below [Figure 1]: 


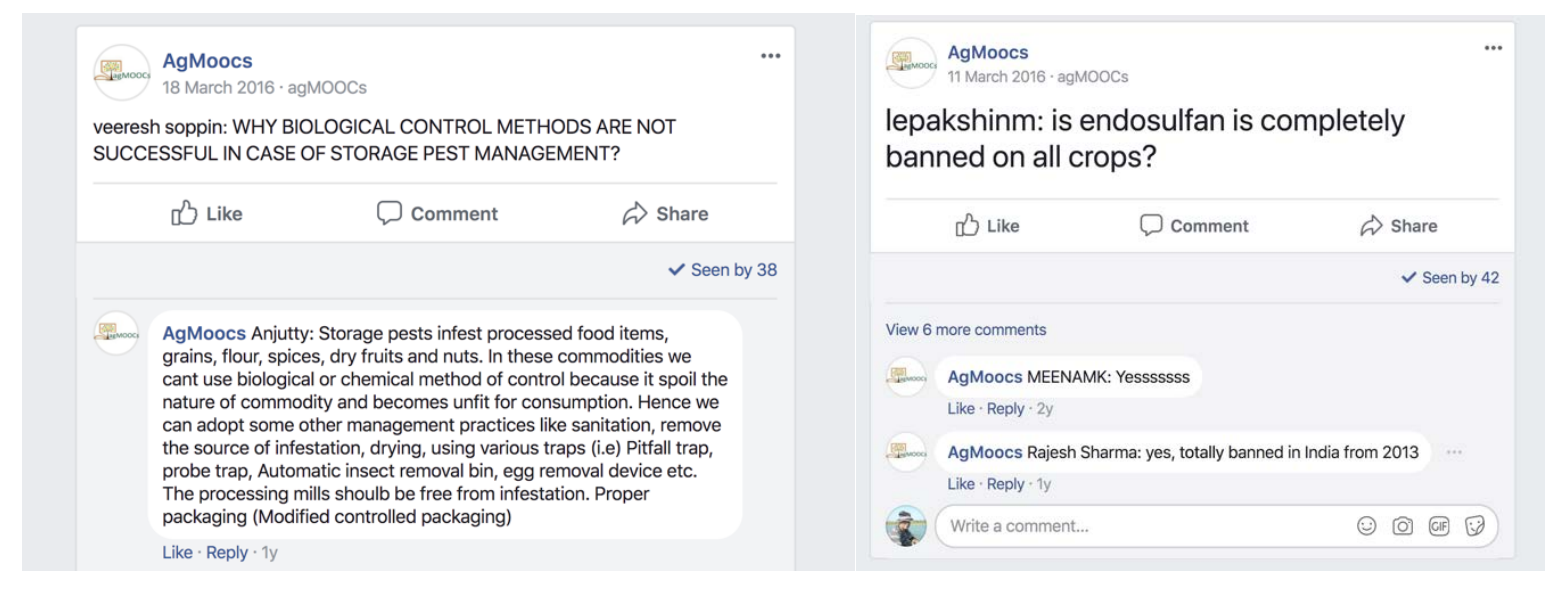

Figure 1: Integration of social media posts in the Forum

Analytics: mooKIT has an easy and intuitive module dedicated to monitor the student participation. The analysis is classified into 3 categories based on the phases involved during the course.

a. Registration: The data collected during the registration results in various insights which the Instructor can use for planning the course. It can be used to check the profile of the registered students. One gets an insight on day-to-day registrations, worldwide distribution of the audience and more.

b. Interactions: After registration, students go through the content, participate in discussions and share thoughts etc. An aggregate analysis of the activities of students during interaction gives an understanding about their behavior like how frequently they login, interact in forums and much more.

c. Evaluation: Students get certified on the basis of their performance in the assessments. An analysis on the performance would help in figuring out how well the content was received by the students, difficulty level of the questions and to identify dropouts etc.

For each of these phases a set of graphs are presented for easy understanding of the data. To collect the data, mooKIT tracks each and every click made by the user.The data set consists of the user ID, the click time, type of request, URL of the resource, IP address from which the request was made, etc.

Registration: When students register, some personal information is collected for Identity Management. Typically, apart from the information like name and email, details such as the age group that the student belongs to, their qualifications, organizational affiliation, location etc are collected.

Daily registrations: Here we display the number of users registered on each day

[Figure 2 ] and on a cumulative basis [Figure 3 ]. This may help in planning the publicity for the course. 


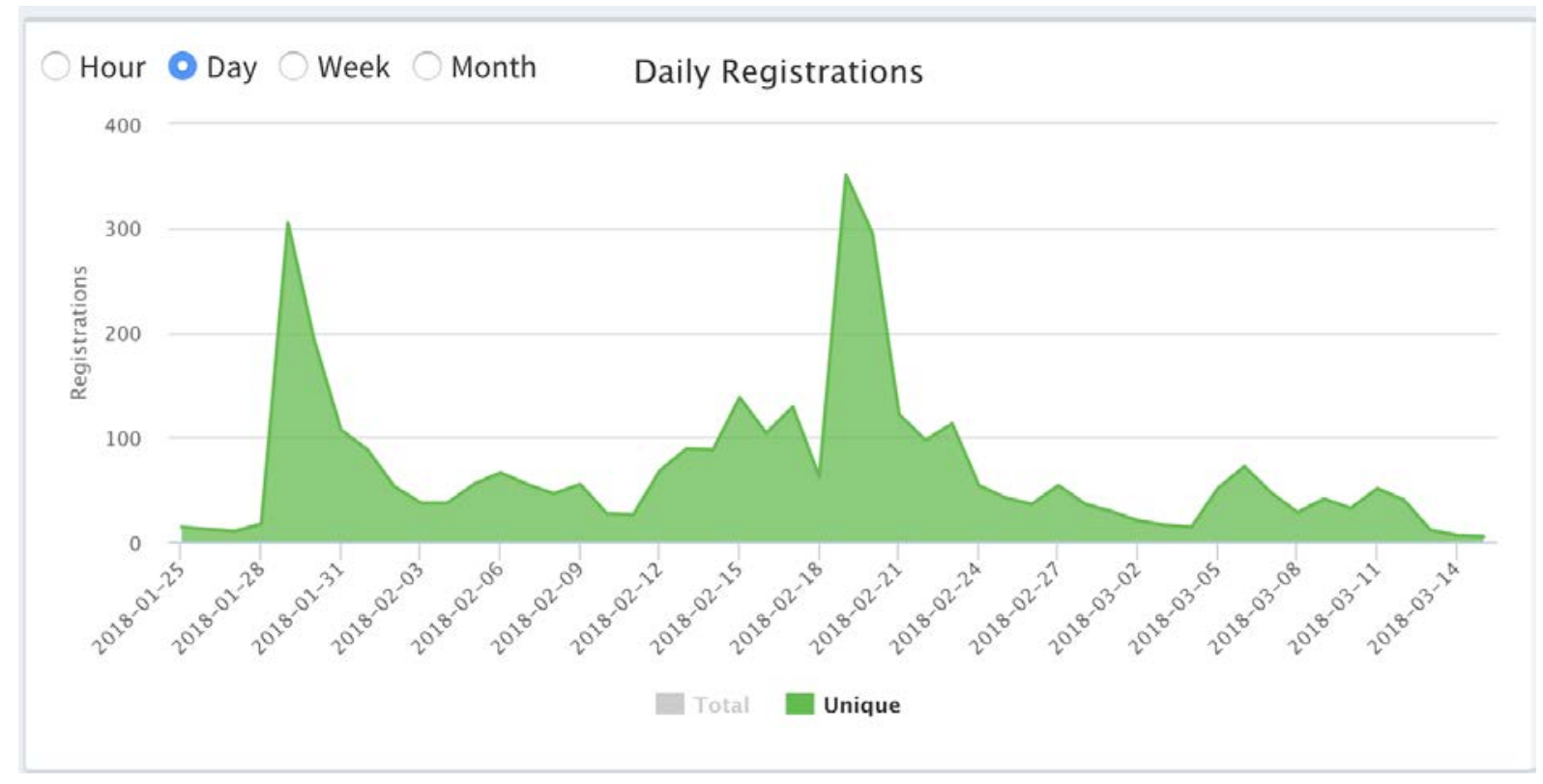

Figure 2: Daily registrations of users

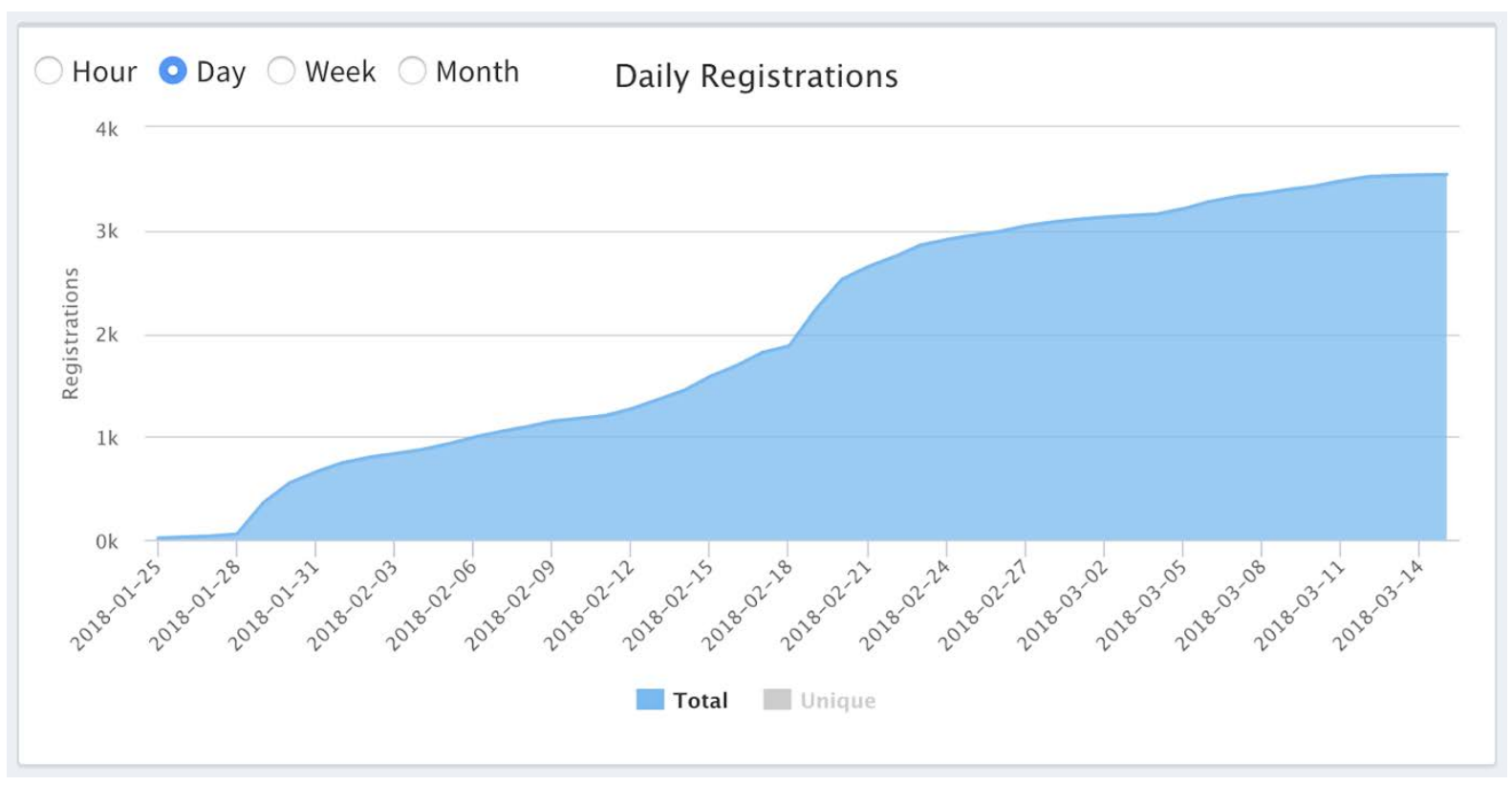

Figure 3: Cumulative registrations

Geographical Reach: We keep note of the location from which users are coming to the course. This data can be very useful to the Instructor and the program managers. The display below [Figure 4] shows a sample distribution of a course in agriculture. 


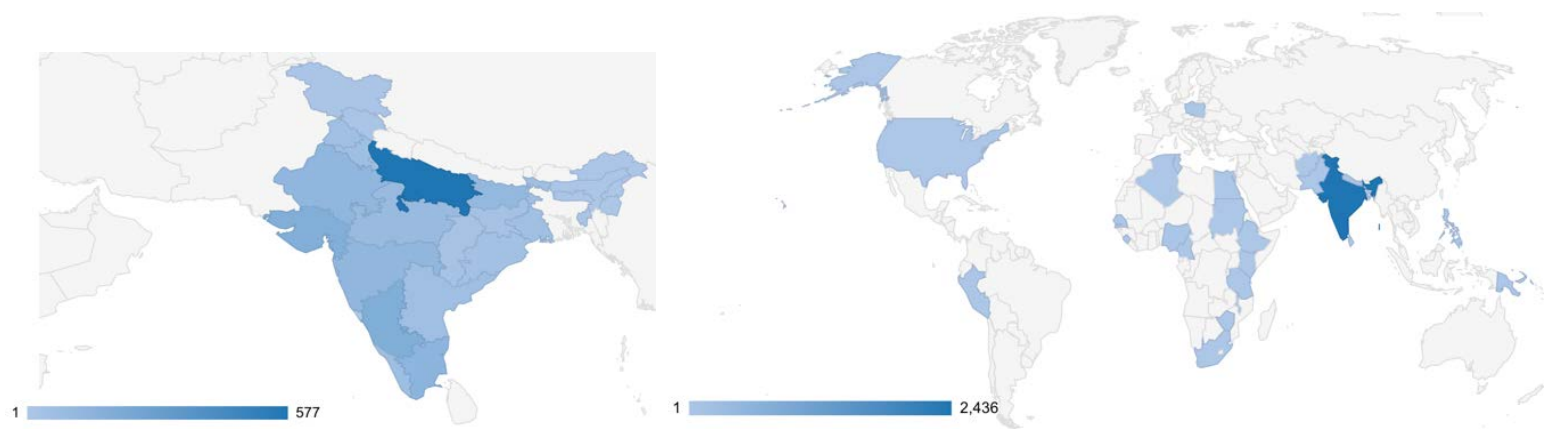

Figure 4: Geographic reach

Other information: In Figure 5 one can see the other information like the gender, age group, qualification and affiliation depicted as pie charts. Such information is valuable also to orient the content and is particularly useful in mentoring processes.

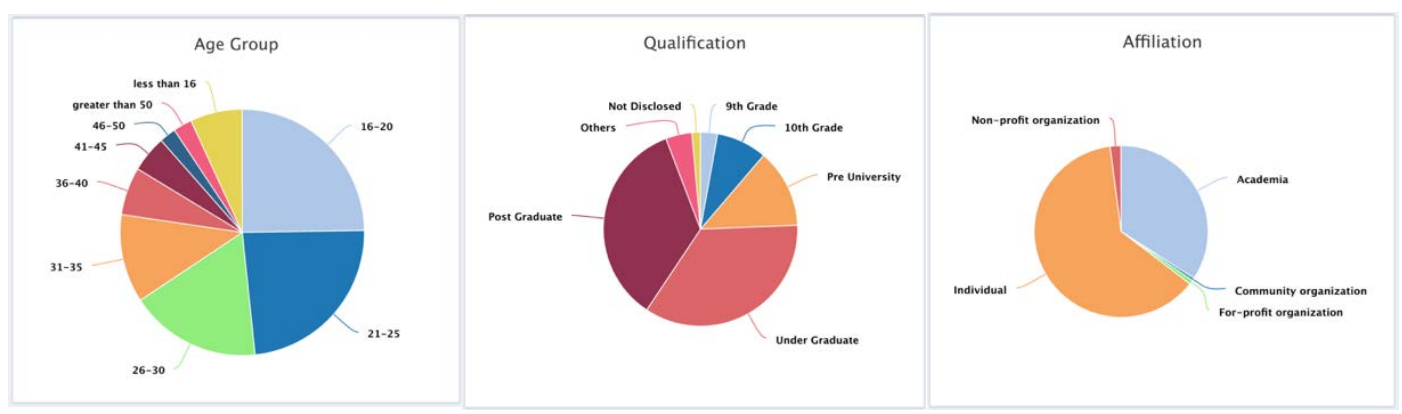

Figure 5: Gender, age group, qualifications data

Interactions: When student's login into the platform, they watch a video, participate in a discussion or simply browse etc. These activities are shown as charts in the Interactions subgroup of mooKIT analytics.

Daily engagement summary: As a course administrator or an instructor, one will be interested in knowing "How many students are active on each day?", "Did they view a lecture or participate in a discussion?" and so on. Also one can correlate the activity of students with a "Deadline for an assessment", an "Announcement which is sent", the "Lecture release schedule", the "Day/Time when the instructors are active in discussions" etc. 


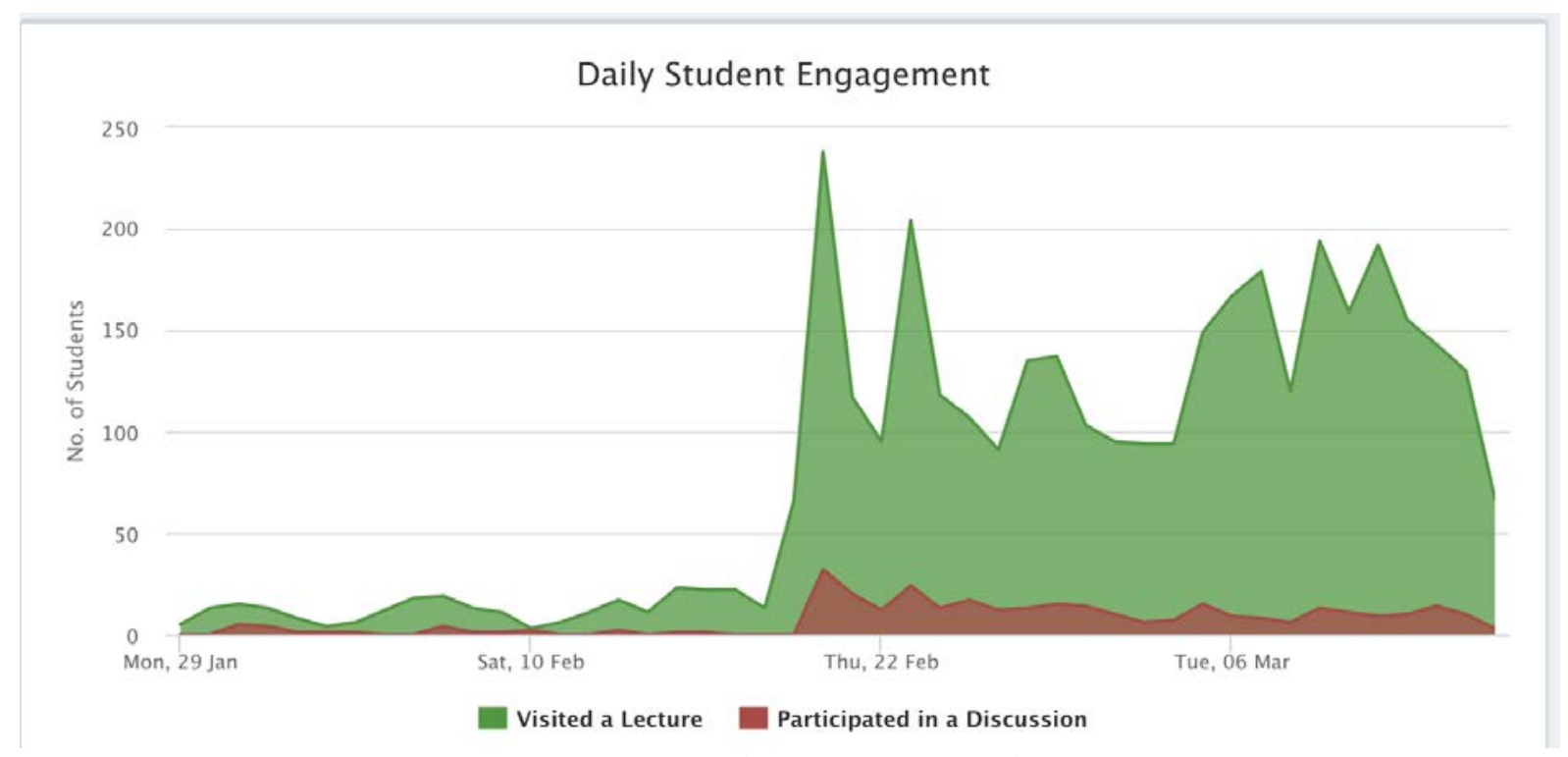

Figure 6: Daily interactions chart

Learner's activity: The Instructor will be able to see every individual students engagement with the course. We track how many times a student has visited a page, how many videos she has viewed completely and so on. A sample of this information is given below.

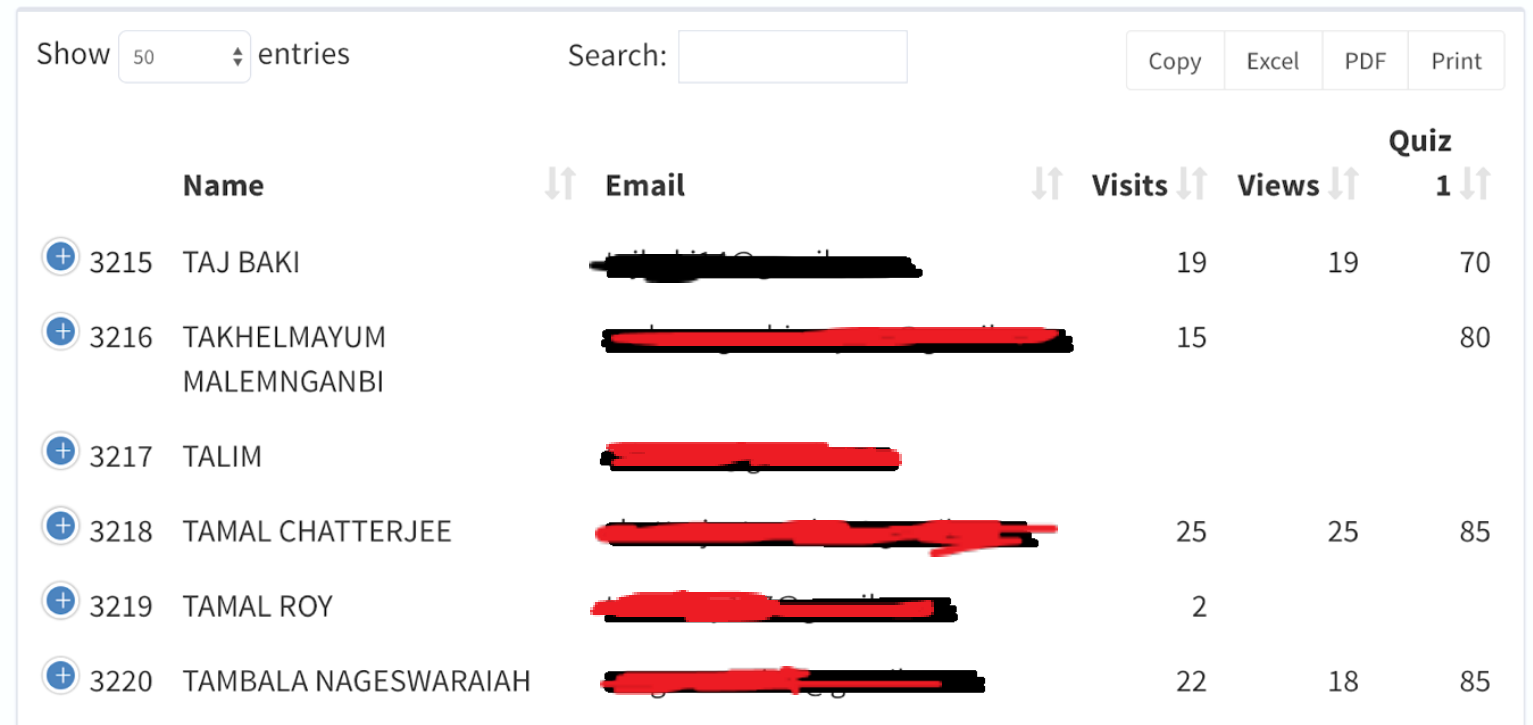

\section{Figure 7: Individual statistics}

Forum analysis: We track the interactions that happen in Forums. That is who is answering whose questions, who are the most active people etc. This can be represented as a graph with nodes standing for the person and a line between nodes ' $a$ ' and ' $b$ ' when ' $a$ ' answers a question posted by ' $b$ '. A sample of this graph is shown below. One may be able visualise inteteresting properties like who is the most active person in a given period, if people forming small groups are forming etc. 


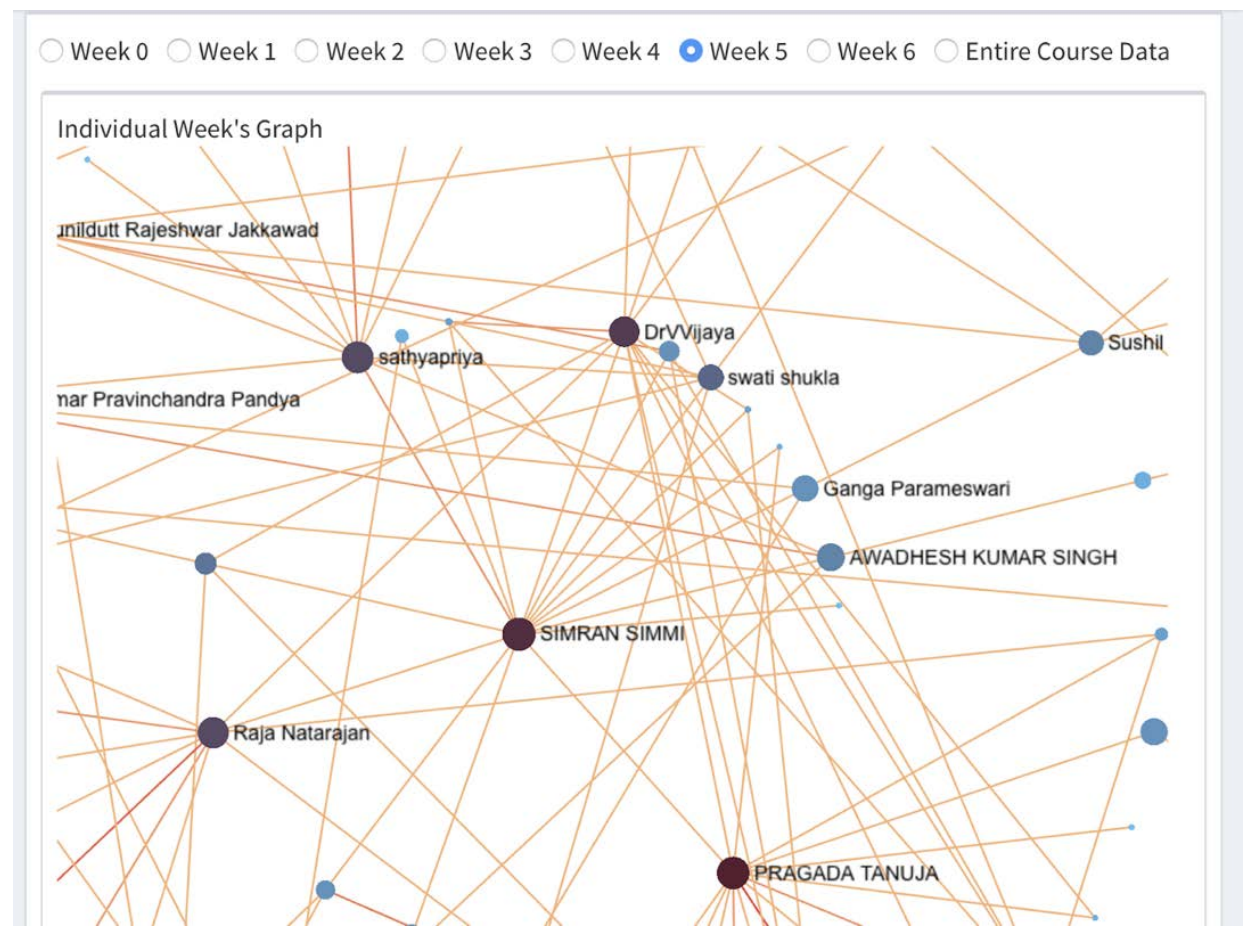

Figure 8: Interaction network

Individual progress report: We provide each individual student a dashboard to keep track of her progress. This gives information about how many videos have been watched, how many are pending etc. A screen capture given below gives an example.

\section{Progress Details}

Week 0

Estimated Time(Videos) : 12m 12s

Week 1

Estimated Time(Videos) : 1h 9m 41s

Lectures

\begin{tabular}{|c|c|c|c|}
\hline & Title & Progress & Duration \\
\hline$>$ & What is Extension & $24 \%$ & $10: 51$ \\
\hline$\checkmark$ & What is NOT Extension & $100 \%$ & $11: 14$ \\
\hline O & Definition and scope of extension & $0 \%$ & $12: 07$ \\
\hline$>$ & Philosophy and Functions of Extension Education & $26 \%$ & $16: 18$ \\
\hline$\bigcirc$ & Principles and Objectives of Extension Education & $0 \%$ & $19: 11$ \\
\hline
\end{tabular}

Figure 9: Individual students dashboard 
Architecture and Implementation : Mookit is built on open source technologies. The major driving principle is that it should be easy to scale. The state of art architecture tries to fully use the client side computing power to achieve this. The diagram below (Figure 10) illustrates the various components and services of mookit. The student interface written in Angularjs runs in the front end, in clients machines(mobiles, laptops). The server only needs to extract and send the data a JSON objects to the client, greatly increasing the scalability of the server.

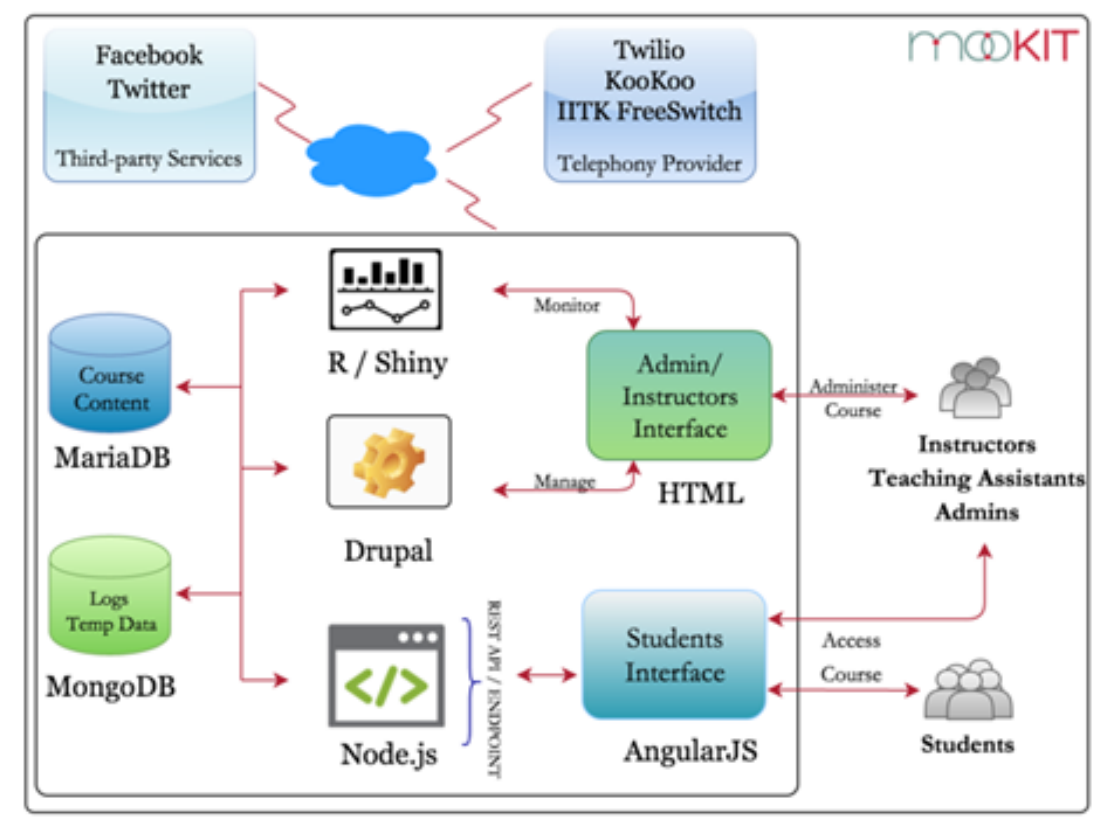

Figure 10: mooKIT architecture

The analytics module runs as a service and communicates with all the courses running on mooKIT. Periodically the data for each course is fetched, cleaned, and stored in the local storage after partial processing. MongoDB is used for local storage for faster reads and writes. Whenever an Instructor interacts with the Analytics module, the data is fetched from local storage, processed and displayed. The architecture is shown below. 


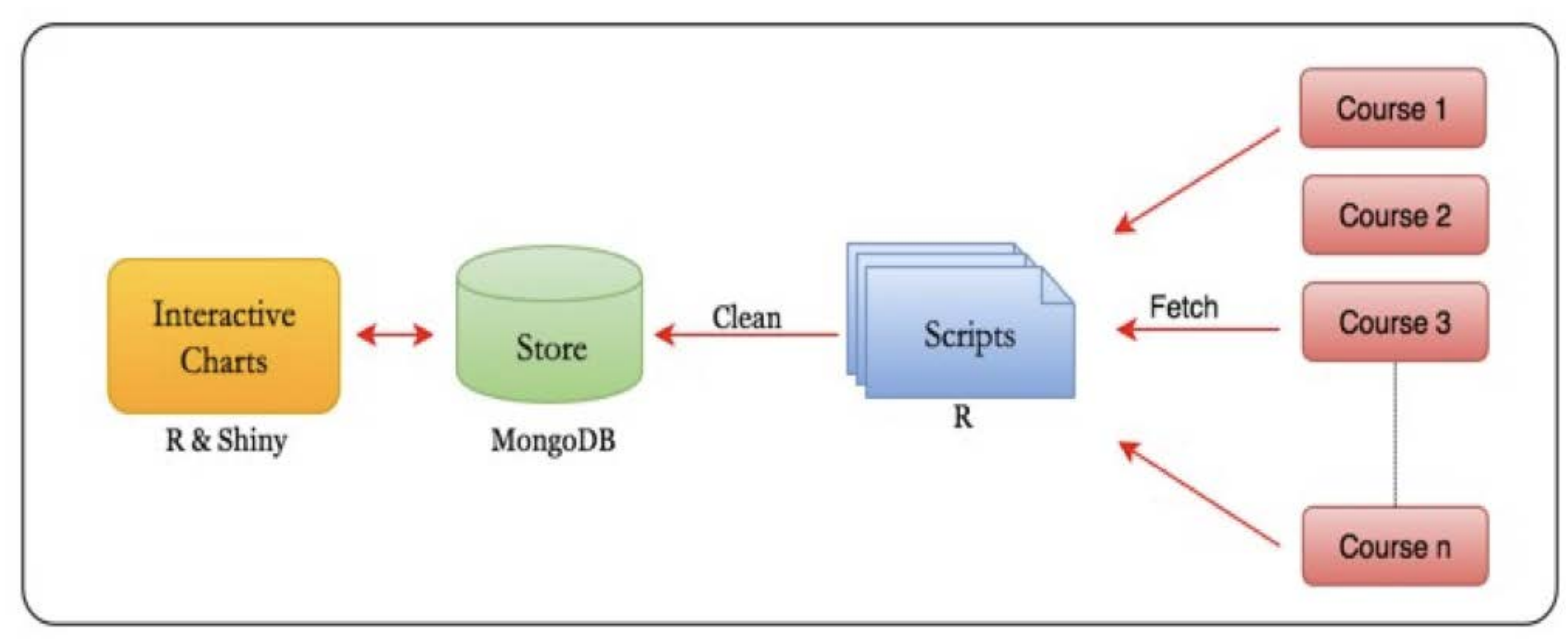

Figure 11: Analytics Processing

\section{Discussion: what are the good practices that emerge from these iterations?}

We ran about twenty courses on the mooKIT platform varying from Mobiles for Development to Physics of Semiconductors. We are also running a series of courses on agriculture(agMOOCs, 2018).

One of the most discussed aspect of MOOCs is the poor completion rates (Hone and El-Said, 2016). It has been observed that only about $10 \%$ of the registered students complete course.

In this section we will present the best some of the practices we found will help in increasing the engagement and retention of students in online courses.

Instructor should participate: Typically, in an online course, the questions posed by the students get tacked by the Teaching Assistants. However, we observed that if the Instructors answers the questions herself, the student engagement increases. The Instructor could also participate in the forums by posing additional questions and giving some titbits in the Forums. This increases the enthusiasm of the students and helps in retention.

Release lectures more than once in a week: Normally, the lectures are released once a week. We noticed that there is a peak in the student engagement with the platform as soon as the lectures are released. The students quickly watch the lectures for the week and do not visit the platform till the next release - which is a week away. Sometimes, a week's absence is too long and might result in losing some students. On the other hand, if the lectures are released 2-3 times a week, the student has a reason to come to the platform more often and engage on a more continuous basis.

Students want to participate: In a normal classroom course, assignments are not always welcome by the student. The fewer the homework tasks and assignments the better. However, in online courses, the students seem to welcome and even ask for 
problems to work on. There could be multiple reasons for this, but such assignments seem to increase the stickiness of the student to the course and help in increasing the completion numbers.

Mid-course incentives: In one of the courses, we noticed that the student engagement was dropping and decided to offer some mobile phones as gifts to the top performing students. This seem to galvanise the students: no further drop offs were noted and some of the students who stopped visiting the course came back and completed the course. The gift itself was very small - a feature phone costing US\$30, but the prestige of winning a prize is what seem to have mattered.

\section{Conclusions}

Massive Open Online Courses (MOOCs) are a powerful tool especially for developing countries where there is a serious need to skill a large number of people in a short time. To help institutions achieve this goal, a number of design issues must be addressed in delivering and managing MOOCs. There are particularly challenging problems in delivering a MOOC when there are bandwidth challenges. The mooKIT has been designed to address these challenges in multiple ways. In this paper we presented how mooKIT solves some of these challenges. Student retention has been flagged as a major problem in MOOCs. We also discussed some techniques the mooKIT course management team has evolved to increase student retention. These have helped in pushing completion rates to as high as $25 \%$ in many of the courses.

\section{References}

agMOOCs (2018). Agriculture MOOCs(2018) https://www.agmoocs.in/

arch4cloud (2012). Architecting for the Cloud https://cse.iitk.ac.in/users/a4c/Arch4Cloud_Brochure.pdf

Flipclasses (2018). IIT Kanpur Flippedclasses Portal https:// onlinecourses.iitk.ac.in/ M4D (2018). MOOCs for Development https:/ / www.mooc4dev.org/

Hone, K S and El-Said, G (2016). Exploring the factors affecting MOOC retention: A survey study. Computers E Education Volume 98, 157-168

Outreach (2018). IIT Kanpur Outreach Portal http://outreach.iitk.ac.in/

Patru , M and Balaji, V (2016) Making Sense of MOOCs: A guide for policy makers in developing countries Paris: UNESCO and Vancouver: COL http://hdl.handle.net/11599/2356

Perris, K (2015) Massive Open Online Course (MOOC) on MOOCs: an evaluation, Vancouver: COL http://hdl.handle.net/11599/568

Porter, D (2014) MOOC on Mobiles for Development Report, Vancouver: COL http://hdl.handle.net/11599/563

Prabhakar, T V (2013) Massive Open Online Courses for Developing Countries (MOOC4D) Video Series, Vancouver: COL http://hdl.handle.net/11599/740

Sakai (2018). Sakai Learning Management System https://www.sakaiproject.org/ 


\title{
A New Approach to Teacher Assessment: Joys and Sorrows of its Introduction. (Case study)
}

\author{
Zdenka Gadusova and Eva Svarbova \\ Constantine the Philosopher University, \\ Nitra, Slovakia \\ Timea Sipkai \\ Lower Secondary School, Pionierov 1 \\ Roznava, Slovakia
}

\begin{abstract}
One of the widely discussed issues in the current world is the quality of teacher's professional competences, which is closely related to the possibilities and ways of their evaluation. The identification and evaluation of teacher's key professional competences is of particular interest for the team of researchers at Constantine the Philosopher University in Nitra (Slovakia) within the research project APVV-14-0446 Assessment of teacher's competences, the output of which should be a new concept of the assessment of teacher's professional competences and the design of a relevant set of assessment tools. The intention of the research team is to encourage teachers' evaluators to use more reflective approach to this task in order to ensure deeper feedback for teachers on their performance.
\end{abstract}

The project started in 2015 and is still in progress. So far the team has managed to agree on 10 professional competences of teacher, which they consider to be the key ones for the high quality performance of teachers. Then, a new stratified approach to their assessment was developed, and for each of the competences, a set of assessment instruments: Assessment Sheets for evaluators, Self-Assessment Sheets for the evaluated teachers and instructions for Post-Observation Interview of the evaluator with the teacher, was designed. The instruments are currently being piloted in schools.

The paper deals with partial results of the piloting of the three tools designed to assess the teacher's competence: can develop pupil's personality and their competences. The piloted tools will be introduced in more details and the issues connected with their acceptance and use by school staff and managers will be discussed.

Keywords: teacher, competence, assessment, evaluator, piloting. 


\section{Introduction}

Teacher competences are understood today as the intersection of theory, practice, and critical reflection of one's own and others' practices, which are mutually intertwined and influenced. The extent and complexity of the competences that are needed in the $21^{\text {st }}$ century is so great that no one is likely to acquire all of them, and even the ones teachers acquire will not be of the same quality (Slavík, 1999; ETUCE, 2008; Schleicher, 2012). It is therefore anticipated that teachers will need to have supported, in particular, the development of their key professional values and mutual relationships: reflective practice, autonomous learning, and engagement in research and innovation, and in collaboration with colleagues and parents engagement in the development of the whole school. We understand the development of competences and their acquisition as lifelong professional education (Blömeke, 2011; Moreno-Murcia et al., 2015). In our research we understand it complexly, not only as the current school practice, but also in the context of undergraduate teacher training and consequently followed by continuous lifelong learning and increasing the professional qualification of the teacher (Jakubovská et al., 2016; Magová et al., 2016; Boboňová et al., 2017; Lomnický et al., 2017; Stranovská et al., 2017, Záhorec et al., 2017). It is necessary to distinguish teaching competences from the competences of the teacher. Teaching competences are focused on the role of the teacher in the classroom; they are directly linked to the "craft" of teaching with professional knowledge and skills mobilized for classroom action (Hagger \& McIntyre, 2006). Teacher competences imply a wider, systematic view of the teacher's professionalism (Pavlov, 2013). In the view of the increasing demands placed on teachers and their work, on the increasing complexity of their tasks, teachers need access to effective personal and professional support throughout their careers - from initial education to induction phase and continuous professional development. It is necessary to support a reflexive approach that would lead teachers to continuous assessment of their work individually and collectively, and to ensure that teachers receive regular feedback on their performance. Based on this, they should update their development and expansion of their competences throughout their careers (Malderez \& Bodoczky, 2009; Kleickmann et al., 2013; Kuhn et al., 2016). However, it is necessary to create a suitable environment and atmosphere, in which the teacher will feel that their work is valued and appreciated, and the school managers are interested in teacher's professional advancement, and thus, the quality of teaching will become the matter of course. Improving the working conditions and the work of the teacher itself will have a positive impact not only on the retention of novice teachers in schools but also on students who are trained to become teachers. To achieve this, a properly and efficiently tailored evaluation of the teacher's professional competences is to a large extent must and it should also be taken into account in teacher's career and professional growth.

\section{Materials and methods}

The identification, development and evaluation of the teacher's competences is the subject of a current research of the team of scientists at the Constantine the Philosopher University in Nitra (Slovakia), within the research project APVV-14- 
0446 Assessment of the teacher's competences, resulting in the design of a new concept in the approach to teacher's professional competences assessment. The concept is based on the identification of ten competences, reflecting the three dominant areas of education and learning: pupil, educational process, and teacher. The strategic aim of the research project is to develop such a system of teacher evaluation which will shift traditional view from the evaluator's activity to the activity of the evaluated.

The identified set of ten teacher's professional competences has become the basis for a stratified approach to assessing the teacher's competences based on the tracking of their demonstration during the lesson, as well as their impact on pupils and their learning outcomes. The selection of ten key professional competences of the teacher was done considering the competence profile of a pedagogical employee, which has been developed - in the line with the European trends and published documents, by Kasáčová and Kosová (2006), and has become a supporting structure for the design of professional standards for different categories of teaching staff in Slovakia (Pavlov, 2013).

Among the identified ten key professional competences of a teacher, the following competences were included:

- can identify the developmental and individual characteristics of the pupil,

- can identify the psychological and social factors of pupil learning,

- can develop the personality of the pupil and their competences,

- can create a positive climate in the class,

- has mastered and can control the content and didactics of the subjects taught,

- can plan and design teaching,

- can select and implement organizational forms and teaching methods,

- can use the material resources and aids in the teaching process,

- can evaluate the progress and results of teaching and learning process,

- can plan and carry out their own professional development.

In order to evaluate each of the stated teacher's competences, for each of them the research team has developed an Assessment Sheet for the evaluator (internal school manager or external member of school inspection) and a relevant Self-Assessment Sheet for the observed and evaluated teacher together with a set of recommended questions and cues for the Post-Observation Interview of the evaluator with the evaluated teacher.

The concept of the quality assessment of Slovak teachers work, designed by the research team of the research project, is thus conceived on three basic elements. The evaluation made by the school head teacher, or their deputy/school representative or external evaluator (using the Assessment Sheet) is a natural starting point. However, the research team considers it necessary to look also for such tools (SelfAssessment Sheet) that would strengthen the self-reflective aspect of the teacher assessment in the context of their work and impact of their teaching on the pupil. Therefore, the second part of the research team's strategy is the focus on the area of autonomous self-evaluation of the teacher, where the main focus goes with being 
aware of own strengths and weaknesses and, in particular, the possibility of subsequent corrections and improvements.

However, the assessment of the teacher by the evaluator as well as the selfassessment of the teacher may, in addition to a great degree of objectivity, include some subjective aspects and opinions of the two actors in the process. To eliminate subjectivity in the assessment, the research team therefore has suggested solving it by developing and using another instrument - the Post-Observation Interview. The aim of the last element of the triadic construction of the proposed concept is to objectify the evaluation process and its outputs. The Post-Observation Interview is designed as a penetration of the views and attitudes of both actors in the assessment process, focusing on various aspects of the selected key competence demonstration. The expected or envisaged relative inconsistency of the assessment findings and conclusions should be directed towards clarifying the positions, and assessment criteria, should lead to the critical thinking of both subjects, pursuing the common goal of improving the educational process as the means of developing the personality of the pupil.

In the paper we deal with the partial results of piloting of one triple of tools that were developed to assess the teacher's competence can develop the personality of the pupil and their competences. We introduce these tools in more detail and, given their current piloting, we also point out how these tools have been accepted by the teaching staff, how useful and helpful they find them and what they see their pros and cons are. On the basis of pilot findings, the revision of the first draft of the instruments will be done.

For piloting the above mentioned evaluation tools assessing the teacher's competence can develop the personality of the pupil and their competences, the research objectives were set as follows:

- Finding out how the evaluators assess the educational performance of teachers related to the competence during the lesson.

- Finding out how teachers assess their own educational impact of the competence on pupils during the lesson.

- Finding out in what way and to what extent these two assessments - in this case, with regard to the teacher's competence to develop the personality of the pupil and their competences, match and differ.

The Assessment Sheet for the evaluator, as well as the Self-assessment Sheet for the teacher, contains 6 items to which the evaluator makes a written record during the lesson observation and the evaluated teacher does the same after the lesson is over. In the end of the Assessment Sheet, the evaluator states their conclusions and recommendations and the evaluated teacher expresses their consent or disagreement.

The sheets contain closed and open items. Closed items provide the possibility to mark one answer on the scale from 1 to 4 , with $1=$ yes, 2 = rather yes, 3 = rather no 
and $4=$ no, the fifth option N/A is applied in case of impossibility to judge if the phenomenon did not appear on the lesson. There are four closed items in both types of the sheets. In the open items, both the teacher and the evaluator write their notes themselves, recording their remarks, findings, attitudes, and opinions. Since it is not possible to pre-define the answers in this case, these items have the advantage of getting subjective opinions. In both types of the sheets, the respondents involved express their minds about pupils' personality development factors; they should state which personality factors have been developed and how the teacher has developed them during each phase of the lesson, and also what has been their impact on pupils. The evaluated teacher expresses about the issue fixed in the item from their point of view, and the evaluator/observer gives their views on the teacher's performance during the lesson. In the last item of the Assessment Sheet (designed to assess teacher's competence to develop pupil's personality and their competences), both respondents participating in the evaluation express their opinion about the overall effort of the teacher aimed at developing pupil's personality and competences during the lesson.

After comparing the two evaluation records, a semi-structured interview of the evaluator with the evaluated one should follow, for which the content frame of the questions was prepared in advance, but the questions can be tailored to what actually happened and took place in the lesson and how the conversation is being unfolded.

\section{Participants}

The presented partial results are from piloting carried out at three lower and one upper secondary school with a sample of ten evaluated teachers and their assessors, out of which three were deputy head teachers and two chairs of the subject committee. Evaluated teachers had a different length of their teaching at school, ranging from 3 to 26 years (one was a novice teacher, three of them have been working at school for 6-10 years, four for 16-20 years, and the other two for over 20 years). Among the evaluated teachers, there was one German language teacher, one Chemistry teacher, one Slovak language teacher, and seven English language teachers. Piloting the sheets was conducted from November 2017 to January 2018.

\section{Results and Discussion}

Since the Assessment Sheet as well as the Self-assessment Sheet includes both closed and open items, the analysis of the collected data from closed items was done using graphs and subsequent interpretation of the results, the statements from the open items were transformed into the key domains and recorded in tables, followed by their comparison and drawing the conclusions. Similarly were elaborated the recordings of the Post-observation interviews.

In the first item of the Assessment Sheet and Self-Assessment Sheet, there are two types of questions, one closed and the other open. The first one was a yes/no question, 
where both the respondents had to indicate whether during the lesson the teacher developed the personality of the pupil and their competences. The answers are shown graphically in Figure 1, where the response of the evaluated teacher in the Self-Assessment Sheet with the response of the evaluator who was observing the teacher during their lesson and recording their findings in the Assessment Sheet are compared. The Figure 1 shows the data recorded for all the ten teachers (T1-T10) involved in piloting as recorded in Assessment (brownish column in the figure) and Self-Assessment (bluish column in the figure) Sheets. The overall finding is that the ratings of teachers and evaluators who were observing the teachers were, in general, positive, since both respondents used only two numbers from the evaluation scale 1 (yes) and 2 (rather yes), and they do not differ significantly in their evaluation, even though it can be seen that teachers are in their self-assessments more selfcritical - only two teachers expressed categorical yes in their Self-Assessment Sheets compared to 6 evaluators, out of which 4 evaluated the teacher better than the teachers themselves. Conformity in evaluation was recorded with six pairs of evaluators.

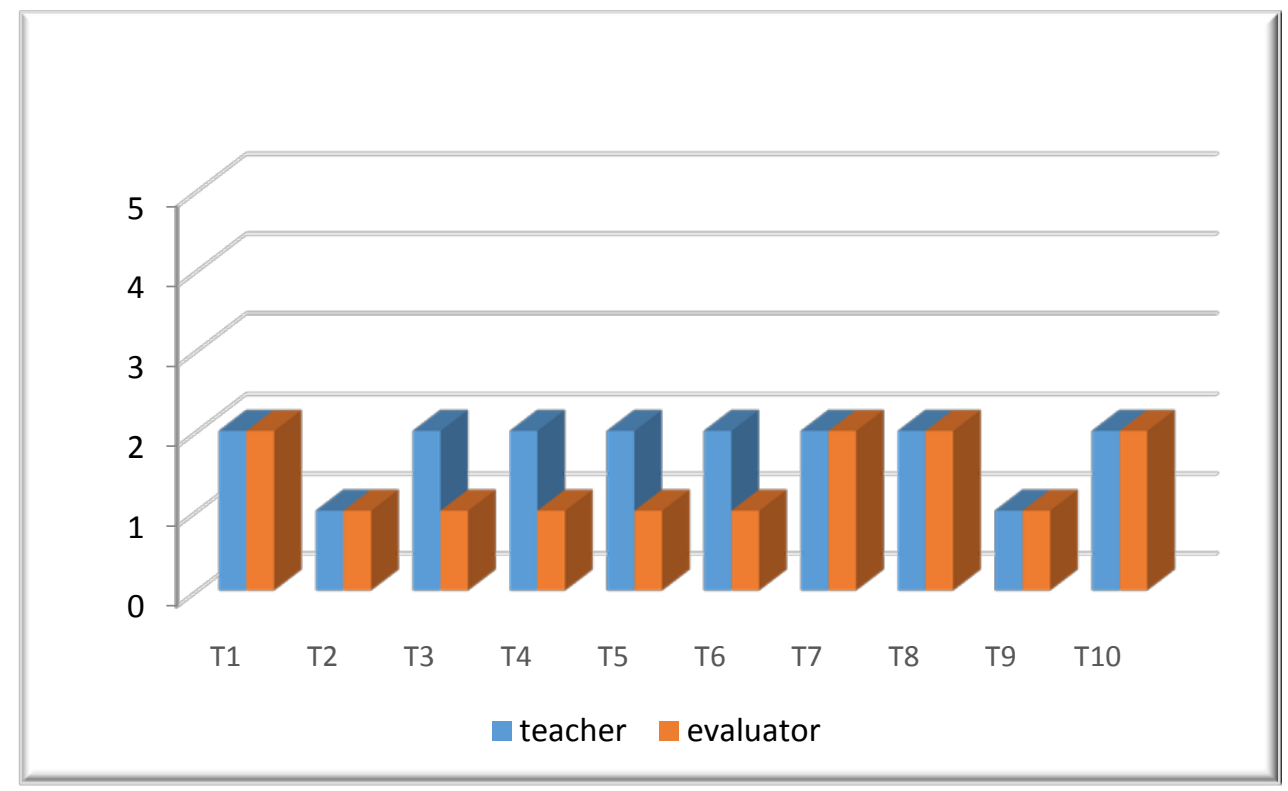

Figure 1 - Recorded opinions about developing pupil's personality and their competences

The answers to the second question of the first item are presented in Table 1, in which we compare teachers' answers with the answers of the evaluators observing their lessons. The question was how the teacher realized the development of the pupil's personality and their competences in the observed lesson. If we compare the key words from the Self-Assessment Sheet of the evaluated teacher with those recorded in the Assessment Sheet of the evaluator, we will find that they are identical in a number of cases. Teachers, however, stated more ways of this competence demonstration than observing evaluators did, but some keywords appeared several times in both sheets. The detailed breakdown of responses is given in Table 1. 
Table 1 - Evidence of pupil's personality and competences development in the observed lesson

\begin{tabular}{|c|c|c|}
\hline $\begin{array}{l}\text { Number } \\
\text { of the } \\
\text { teacher } \\
\text { and their } \\
\text { evaluator }\end{array}$ & Teacher & Evaluator \\
\hline T1 & $\begin{array}{l}\text { individual work } \\
\text { communication }\end{array}$ & pupil's assessment \\
\hline $\mathrm{T} 2$ & self-assessment & self-assessment \\
\hline T3 & $\begin{array}{c}\text { perception/attention } \\
\text { imagination } \\
\text { fantasy } \\
\text { creativity }\end{array}$ & $\begin{array}{l}\text { imagination } \\
\text { fantasy } \\
\text { creativity }\end{array}$ \\
\hline T4 & $\begin{array}{l}\text { own opinion } \\
\text { self confidence }\end{array}$ & own opinion \\
\hline T5 & individual approach & own opinion and attitude \\
\hline T6 & $\begin{array}{l}\text { team work } \\
\text { own opinion }\end{array}$ & $\begin{array}{l}\text { showing interest } \\
\text { own opinion } \\
\text { activity } \\
\text { co-operation }\end{array}$ \\
\hline $\mathrm{T} 7$ & $\begin{array}{c}\text { team work } \\
\text { co-operation } \\
\text { communication } \\
\text { own will }\end{array}$ & group-work \\
\hline T8 & - & reading literacy \\
\hline T9 & $\begin{array}{c}\text { expressing emotions, attitudes, } \\
\text { opinions } \\
\text { co-operation } \\
\text { memory, thinking } \\
\text { creativity }\end{array}$ & $\begin{array}{l}\text { own opinions and attitudes } \\
\text { co-operation }\end{array}$ \\
\hline T10 & $\begin{array}{l}\text { work with a text } \\
\text { critical thinking } \\
\text { fantasy }\end{array}$ & 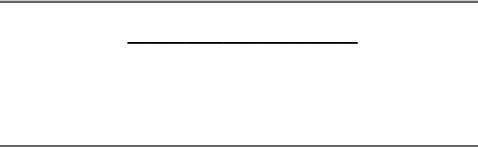 \\
\hline
\end{tabular}

It is clear from the table that the evaluated teachers attach greater importance in the development of a pupil's personality and competences to the fact, whether pupils are able to express their own attitudes and opinions, and how they communicate and think, whether they are creative and have imagination and fantasy, which can be very well demonstrated both in their teamwork and individual work. In the assessments of evaluators, this view is narrower - focusing more on demonstrated pupils' cooperation and expressing their own opinions and views. For both types of respondents (the evaluated teachers and their evaluators), however, it can be seen that their ability to capture the ways and demonstrations of the pupil's personality 
and competence development is rather limited, they often either cannot see or cannot name them.

Item 2 focuses on 4 groups of personality development factors - cognitive, affective, social, and conative. The task of the respondents was to evaluate how the teacher was developing each of these factors in the different phases of the lesson (what methods, forms and strategies they used to do that) and what its impact on pupil(s) was.

The first group of the factors (cognitive ones) is related to the development of memory, perception, thinking, imagination, fantasy and pupils' creativity. Though they were listed in the evaluation sheets, the evaluators had obviously significant problems how to grasp and characterize these factors in pupils. As it can be seen from Table 2, teachers, in the same way as their evaluators, focused more on the forms of teacher's work (individual, group, frontal) with the pupils than on the methods and strategies used in the teacher's work with the pupils, not to mention the identification of the lesson phase when they were used. Even more powerful helplessness is visible in recordings of the impact of methods, forms and strategies on the pupil. Due to the fact that the vast majority of the evaluations were conducted for language lessons, the work with text is very frequent, which is acceptable but less acceptable is what teachers and they evaluators said about the impact this work has on pupils (responsiveness, ability to listen, reading comprehension, answering questions, vocabulary repetition), with the exception of T10, who could see this impact influenced the development of creativity, fantasy and critical thinking of pupils. In the case of the evaluators, unfortunately, most of the statements missed the target.

Table 2 - Cognitive factors of personality development (CFPD)

\begin{tabular}{|c|c|c|c|c|}
\hline \multirow{2}{*}{$\begin{array}{l}\text { Number of the } \\
\text { teacher and } \\
\text { their evaluator }\end{array}$} & \multirow{2}{*}{\multicolumn{2}{|c|}{$\begin{array}{l}\text { CFPD - methods, forms and } \\
\text { strategies used in different } \\
\text { phases of the lesson }\end{array}$}} & \multicolumn{2}{|c|}{ Impact on pupil(s) } \\
\hline & & & teacher & evaluator \\
\hline T1 & individual work & $\begin{array}{c}\text { individual work } \\
\text { thinking } \\
\text { imagination }\end{array}$ & $\begin{array}{l}\text { expressing } \\
\text { opinion } \\
\text { expression of } \\
\text { creativity }\end{array}$ & $\begin{array}{c}\text { expressing } \\
\text { opinion, ideas }\end{array}$ \\
\hline T2 & $\begin{array}{c}\text { motivational } \\
\text { discussion } \\
\text { individual work }\end{array}$ & $\begin{array}{c}\text { motivational } \\
\text { discussion } \\
\text { individual form }\end{array}$ & & \\
\hline T3 & $\begin{array}{c}\text { individual work } \\
\text { group work }\end{array}$ & individual work & - & $\begin{array}{l}\text { development of } \\
\text { imagination, } \\
\text { creativity }\end{array}$ \\
\hline T4 & $\begin{array}{l}\text { expressing the } \\
\text { idea }\end{array}$ & $\begin{array}{c}\text { asking } \\
\text { questions }\end{array}$ & $\begin{array}{l}\text { development of } \\
\text { imagination }\end{array}$ & \\
\hline
\end{tabular}




\begin{tabular}{|c|c|c|c|c|}
\hline T5 & $\begin{array}{c}\text { frontal } \\
\text { reviewing } \\
\text { work with text } \\
\text { creating } \\
\text { dialogues } \\
\end{array}$ & $\begin{array}{l}\text { frontal } \\
\text { reviewing } \\
\text { text creation }\end{array}$ & $\begin{array}{l}\text { creativity in } \\
\text { creating } \\
\text { dialogues } \\
\text { responsiveness }\end{array}$ & $\begin{array}{l}\text { joy of } \\
\text { evaluation } \\
\text { they were } \\
\text { proud of } \\
\text { themselves }\end{array}$ \\
\hline T6 & $\begin{array}{l}\text { asking } \\
\text { questions }\end{array}$ & $\begin{array}{l}\text { asking } \\
\text { questions }\end{array}$ & $\begin{array}{c}\text { logical } \\
\text { explanation }\end{array}$ & pupils' answers \\
\hline T7 & role playing & role playing & & $\begin{array}{l}\text { role in role } \\
\text { playing }\end{array}$ \\
\hline T8 & work with text & work with text & $\begin{array}{l}\text { ability to listen } \\
\text { reading } \\
\text { comprehension } \\
\text { answering } \\
\text { questions }\end{array}$ & $\begin{array}{c}\text { listening } \\
\text { reading } \\
\text { comprehension }\end{array}$ \\
\hline T9 & $\begin{array}{l}\text { listening } \\
\text { reading } \\
\text { description }\end{array}$ & $\begin{array}{l}\text { reading } \\
\text { writing }\end{array}$ & $\begin{array}{l}\text { pupils were } \\
\text { listening } \\
\text { vocabulary } \\
\text { repetition }\end{array}$ & $\begin{array}{c}\text { asking } \\
\text { questions } \\
\text { pupils' answers }\end{array}$ \\
\hline T10 & work with text & $\begin{array}{l}\text { work with text } \\
\text { work with ppt } \\
\text { presentation }\end{array}$ & $\begin{array}{l}\text { development of } \\
\text { creativity, } \\
\text { fantasy, and } \\
\text { critical thinking }\end{array}$ & $\begin{array}{l}\text { listening } \\
\text { sentence } \\
\text { completion }\end{array}$ \\
\hline
\end{tabular}

Likewise, teachers and their evaluators were expected to evaluate the affective factors (emotions, attitudes and beliefs) of pupils' personality development. As can be seen from Table 3, both groups of respondents had a lot of problems with this task, perhaps even greater than with the group of cognitive factors. The methods and strategies used in the various phases of the lesson at teacher's work with pupils that relate to the affective factors evaluated by teachers and their evaluators were difficult for them to identify, and if identified, then not always relevant and appropriate. Similarly, the impact on the pupils was described, with the exception of T7 and T10. In some cases (T1), the impact is judged to be controversial when the evaluated teacher stated a positive impact on the pupil in terms of emotions, attitudes and beliefs, and, on the contrary, the evaluator stated a negative perception, according to which not every pupil worked in the lesson, what can be connected with negative feelings, even with disinterest on the part of the pupils. 
Table 3 - Affective factors of personality development (AFPD)

\begin{tabular}{|c|c|c|c|c|}
\hline \multirow{2}{*}{$\begin{array}{l}\text { Number of the } \\
\text { teacher and } \\
\text { their evaluator }\end{array}$} & \multirow{2}{*}{\multicolumn{2}{|c|}{$\begin{array}{l}\text { AFPD - methods, forms and } \\
\text { strategies used in different } \\
\text { phases of the lesson }\end{array}$}} & \multicolumn{2}{|c|}{ Impact on pupil(s) } \\
\hline & & & teacher & evaluator \\
\hline T1 & $\begin{array}{c}\text { reading } \\
\text { descriptions }\end{array}$ & $\begin{array}{c}\text { picture } \\
\text { descriptions }\end{array}$ & $\begin{array}{l}\text { expressing } \\
\text { emotions, } \\
\text { attitudes and } \\
\text { beliefs }\end{array}$ & $\begin{array}{l}\text { not every pupil } \\
\text { worked }\end{array}$ \\
\hline T2 & $\begin{array}{c}\text { dialogue } \\
\text { individual form }\end{array}$ & $\begin{array}{c}\text { dialogic } \\
\text { methods } \\
\text { individual form }\end{array}$ & & \\
\hline T3 & $\begin{array}{l}\text { expressing } \\
\text { opinion }\end{array}$ & group work & & $\begin{array}{l}\text { expressing } \\
\text { opinion }\end{array}$ \\
\hline T4 & brainstorming & group work & $\begin{array}{l}\text { expressing a } \\
\text { sense of work }\end{array}$ & $\begin{array}{l}\text { expression of } \\
\text { opinion in the } \\
\text { group }\end{array}$ \\
\hline T5 & role playing & dialogue & $\begin{array}{l}\text { personage } \\
\text { empathy }\end{array}$ & $\begin{array}{l}\text { personage } \\
\text { empathy }\end{array}$ \\
\hline T6 & $\begin{array}{l}\text { activation of } \\
\text { less active } \\
\text { pupils }\end{array}$ & eliciting interest & $\begin{array}{l}\text { written } \\
\text { expression of } \\
\text { opinion } \\
\text { oral activation }\end{array}$ & $\begin{array}{l}\text { pupils became } \\
\text { active }\end{array}$ \\
\hline T7 & $\begin{array}{l}\text { motivational } \\
\text { video } \\
\text { drama activity, } \\
\text { solving the } \\
\text { problem } \\
\text { group work }\end{array}$ & $\begin{array}{l}\text { group work } \\
\text { motivational } \\
\text { video }\end{array}$ & $\begin{array}{l}\text { empathy } \\
\text { development } \\
\text { expression of } \\
\text { interest }\end{array}$ & $\begin{array}{c}\text { empathy } \\
\text { development }\end{array}$ \\
\hline $\mathrm{T} 8$ & $\begin{array}{l}\text { work with } \\
\text { video } \\
\text { work with text }\end{array}$ & $\begin{array}{l}\text { work with } \\
\text { video } \\
\text { work with text }\end{array}$ & $\begin{array}{l}\text { looking for } \\
\text { answers } \\
\text { ordering } \\
\text { phrases }\end{array}$ & $\begin{array}{c}\text { answering } \\
\text { questions }\end{array}$ \\
\hline T9 & $\begin{array}{l}\text { emotions, } \\
\text { attitudes }\end{array}$ & 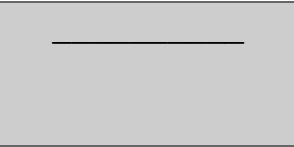 & $\begin{array}{c}\text { expressing } \\
\text { emotions and } \\
\text { attitudes }\end{array}$ & $\begin{array}{l}\text { expressing own } \\
\text { attitudes }\end{array}$ \\
\hline T10 & $\begin{array}{l}\text { work with text } \\
\text { listening }\end{array}$ & $\begin{array}{l}\text { work with text } \\
\text { listening }\end{array}$ & $\begin{array}{l}\text { expressing own } \\
\text { opinions and } \\
\text { attitudes }\end{array}$ & $\begin{array}{l}\text { development of } \\
\text { critical thinking, } \\
\text { expressing own } \\
\text { attitudes }\end{array}$ \\
\hline
\end{tabular}

The third group of factors was the social factors of pupils' personality development (co-operation, empathy, acceptance, communication, coherence - pupils' cohesion, work with tensions in the classroom), to which the respondents should also have stated the methods, forms and strategies used during the lesson and their impact on pupil(s). The teachers and their evaluators succeeded the best with this group of factors - in most cases, they managed to grasp what they were expected to record. Although in self-assessment records the teachers, as well as the evaluators in their 
evaluations of teachers, focused more on the forms of work (pair, group and team work) than on the methods and strategies used in the teacher's work with pupils, in identifying the impact of the methods, forms and strategies used during the lesson, they were much more successful. Both groups of respondents were able to identify relevant aspects of personality development (such as strengthening tolerance and self-criticism, expressing own opinions, cooperating with classmates, assertive responses, accepting refusals, etc.). However, even with social factors controversial statements were recorded as well - T1 or T5.

Table 4 - Social factors of personality development (SFPD)

\begin{tabular}{|c|c|c|c|c|}
\hline \multirow{2}{*}{$\begin{array}{l}\text { Number of the } \\
\text { teacher and } \\
\text { their evaluator }\end{array}$} & \multicolumn{2}{|c|}{$\begin{array}{l}\text { SFPD - methods, forms and } \\
\text { strategies used in different } \\
\text { phases of the lesson }\end{array}$} & \multicolumn{2}{|c|}{ Impact on pupil(s) } \\
\hline & & & & \\
\hline T1 & $\begin{array}{l}\text { communication } \\
\text { mutual } \\
\text { acceptance }\end{array}$ & $\begin{array}{c}\text { erratic } \\
\text { communication }\end{array}$ & $\begin{array}{l}\text { fear to perform } \\
\text { for the class }\end{array}$ & $\begin{array}{l}\text { fear to perform } \\
\text { for the class }\end{array}$ \\
\hline $\mathrm{T} 2$ & $\begin{array}{l}\text { verbal methods } \\
\text { - discussion }\end{array}$ & $\begin{array}{l}\text { dialogic } \\
\text { methods }\end{array}$ & $\begin{array}{l}\text { strengthening } \\
\text { tolerance and } \\
\text { self-criticism }\end{array}$ & $\begin{array}{l}\text { strengthening } \\
\text { tolerance and } \\
\text { self-criticism }\end{array}$ \\
\hline T3 & $\begin{array}{l}\text { pair work } \\
\text { defense of } \\
\text { opinion }\end{array}$ & $\begin{array}{c}\text { defense of } \\
\text { opinion } \\
\text { communication }\end{array}$ & & $\begin{array}{c}\text { development of } \\
\text { empathy, } \\
\text { communication } \\
\text { and acceptance }\end{array}$ \\
\hline T4 & group work & acceptance & $\begin{array}{l}\text { expressing own } \\
\text { opinion } \\
\text { peer co- } \\
\text { operation }\end{array}$ & $\begin{array}{c}\text { expressing own } \\
\text { opinion }\end{array}$ \\
\hline T5 & $\begin{array}{l}\text { creation of } \\
\text { dialogues } \\
\text { co-operation }\end{array}$ & $\begin{array}{c}\text { creation of } \\
\text { dialogues } \\
\text { co-operation }\end{array}$ & $\begin{array}{l}\text { pupils liked to } \\
\text { work in pairs }\end{array}$ & $\begin{array}{c}\text { fear to some } \\
\text { pupils to } \\
\text { perform for the } \\
\text { class }\end{array}$ \\
\hline T6 & $\begin{array}{l}\text { communication } \\
\text { team work }\end{array}$ & $\begin{array}{l}\text { expressing own } \\
\text { opinion }\end{array}$ & $\begin{array}{l}\text { pupils co- } \\
\text { operated }\end{array}$ & $\begin{array}{c}\text { pupils } \\
\text { communicated, } \\
\text { collaborated, } \\
\text { expressed ideas }\end{array}$ \\
\hline T7 & $\begin{array}{l}\text { pair work } \\
\text { creation of } \\
\text { dialogues } \\
\text { acceptance and } \\
\text { rejection of the } \\
\text { proposal }\end{array}$ & $\begin{array}{l}\text { creation of } \\
\text { dialogues } \\
\text { group work }\end{array}$ & $\begin{array}{c}\text { pupils } \\
\text { communicated } \\
\text { assertively } \\
\text { responded } \\
\text { accepted } \\
\text { rejection }\end{array}$ & $\begin{array}{l}\text { communication } \\
\text { co-operation }\end{array}$ \\
\hline T8 & $\begin{array}{l}\text { pair work } \\
\text { role-playing }\end{array}$ & $\begin{array}{l}\text { pair work } \\
\text { role-playing }\end{array}$ & $\begin{array}{l}\text { they were } \\
\text { actively } \\
\text { listening } \\
\text { they felt } \\
\text { themselves in } \\
\text { the roles }\end{array}$ & $\begin{array}{c}\text { actively } \\
\text { collaborated } \\
\text { and } \\
\text { communicated }\end{array}$ \\
\hline
\end{tabular}




\begin{tabular}{|c|c|c|c|c|}
\hline & & & $\begin{array}{l}\text { they practiced } \\
\text { empathy }\end{array}$ & \\
\hline T9 & $\begin{array}{l}\text { co-operation } \\
\text { acceptance } \\
\text { communication } \\
\text { pair work }\end{array}$ & $\begin{array}{l}\text { communication } \\
\text { co-operation } \\
\text { discussion }\end{array}$ & $\begin{array}{c}\text { pupils } \\
\text { collaborated in } \\
\text { creating } \\
\text { interviews, } \\
\text { accepted } \\
\text { opinions and } \\
\text { attitudes }\end{array}$ & $\begin{array}{l}\text { tolerated } \\
\text { opinions }\end{array}$ \\
\hline T10 & group work & group work & $\begin{array}{c}\text { pupils } \\
\text { collaborated } \\
\text { and } \\
\text { communicated }\end{array}$ & $\begin{array}{l}\text { pupils } \\
\text { presented their } \\
\text { views, } \\
\text { actively } \\
\text { listened, } \\
\text { felt in the roles, } \\
\text { expressed } \\
\text { opinions, } \\
\text { discussed their } \\
\text { views in groups } \\
\text { they practiced } \\
\text { empathy }\end{array}$ \\
\hline
\end{tabular}

The last part of the second item in the Assessment and Self-Assessment Sheets was devoted to the conative factors (motivation and values), to which a similar entry was required as in the previous three groups of factors. Since the respondents could see also here that motivation and values are particularly relevant to conative factors, both terms were abundant in the evaluation records. Unfortunately, their narrower specification was lacking, it occurred only as an exception - T1 and T6 (motivation of pupils by means of grades and assessment). In the case of T10 (motivational interview, motivational video), however, the specification is not connected that much with the personality of the pupil, but it is the stimulation of the pupil's specific work. Also with this group of factors, both groups of respondents were much more successful in identifying the impacts of the used methods, forms and strategies on the pupil, especially in terms of values (such as T8 - pupils have acquired values such as friendship, loyalty, honor, or T6 - pupils have respected the rules of joint work), but also motivation (such as T1 - "they showed an effort to cooperate" - the teacher, "some pupils were satisfied and motivated, others were disappointed" - the evaluator). However, as seen from the latter example, the teacher, when assessing conative factors, focused more on individual work of pupils and their performances for the class, the impact of which was evaluated as a manifestation of the cooperation efforts. But the question is the cooperation with whom they had in mind - cooperation of pupils with each other or with the teacher. On the other hand, the evaluator focused on an adequate and fair assessment of pupils that had mostly positive, but in rare cases also negative, impact on pupils. In case of T1, we can see how the statements in this item can reasonably correlate (evaluator's opinions - the strategies used and their impact on the pupil), but at the same time partly contradict (self-evaluation of the teacher - used work forms compared to their impact on the 
pupil(s)) and not even match the content in the records (individual work - the teacher, assessment of pupils - the evaluator).

Table 5 - Conative factors of personality development (CFPD)

\begin{tabular}{|c|c|c|c|c|}
\hline \multirow{2}{*}{$\begin{array}{l}\text { Number of the } \\
\text { teacher and } \\
\text { their evaluator }\end{array}$} & \multirow{2}{*}{\multicolumn{2}{|c|}{$\begin{array}{l}\text { CFPD - methods, forms and } \\
\text { strategies used in different } \\
\text { phases of the lesson }\end{array}$}} & \multicolumn{2}{|c|}{ Impact on pupil(s) } \\
\hline & & & teacher & evaluator \\
\hline T1 & $\begin{array}{l}\text { individual work } \\
\text { performing for } \\
\text { the class }\end{array}$ & $\begin{array}{l}\text { adequate } \\
\text { assessment of } \\
\text { pupils }\end{array}$ & $\begin{array}{l}\text { pupils have } \\
\text { shown an effort } \\
\text { to co-operate }\end{array}$ & $\begin{array}{l}\text { some pupils } \\
\text { were satisfied } \\
\text { and motivated, } \\
\text { others were } \\
\text { disappointed }\end{array}$ \\
\hline T2 & $\begin{array}{l}\text { dialogue } \\
\text { method of } \\
\text { interview }\end{array}$ & $\begin{array}{l}\text { dialogue } \\
\text { method of } \\
\text { interview }\end{array}$ & & \\
\hline T3 & & $\begin{array}{c}\text { pupils } \\
\text { motivated each } \\
\text { other }\end{array}$ & & $\begin{array}{l}\text { Pupils rated } \\
\text { their classmates, } \\
\text { they felt } \\
\text { important }\end{array}$ \\
\hline T4 & motivation & motivation & $\begin{array}{l}\text { pupils felt } \\
\text { important }\end{array}$ & $\begin{array}{l}\text { pupil felt } \\
\text { important }\end{array}$ \\
\hline T5 & $\begin{array}{l}\text { work with text } \\
\text { vocabulary } \\
\text { repetition }\end{array}$ & individual work & $\begin{array}{l}\text { pupils were } \\
\text { competitive, } \\
\text { purposeful }\end{array}$ & $\begin{array}{l}\text { expressing love } \\
\text { to family } \\
\text { members }\end{array}$ \\
\hline T6 & $\begin{array}{l}\text { team work } \\
\text { individual work } \\
\text { work with text }\end{array}$ & $\begin{array}{c}\text { praise } \\
\text { motivation by } \\
\text { means of grades }\end{array}$ & $\begin{array}{l}\text { pupils respected } \\
\text { the rules for } \\
\text { joint work, they } \\
\text { were } \\
\text { solving tasks }\end{array}$ & $\begin{array}{l}\text { pupils were } \\
\text { involved in self- } \\
\text { assessment }\end{array}$ \\
\hline T7 & $\begin{array}{l}\text { group work } \\
\text { problem } \\
\text { solving } \\
\text { situational } \\
\text { game }\end{array}$ & $\begin{array}{l}\text { situational } \\
\text { game } \\
\text { problem } \\
\text { solving }\end{array}$ & L & $\begin{array}{l}\text { pupils were } \\
\text { involved in self- } \\
\text { assessment }\end{array}$ \\
\hline T8 & & $\begin{array}{l}\text { motivation } \\
\text { role playing }\end{array}$ & $\begin{array}{c}\text { learners } \\
\text { acquired values } \\
\text { such as } \\
\text { friendship, } \\
\text { loyalty, honor, } \\
\text { responsibility } \\
\text { for work }\end{array}$ & $\begin{array}{l}\text { learners } \\
\text { acquired } \\
\text { responsibility, } \\
\text { loyalty, honor }\end{array}$ \\
\hline
\end{tabular}




\begin{tabular}{|c|c|c|c|c|}
\hline T9 & motivation & pair work & $\begin{array}{c}\text { learners created } \\
\text { sentences } \\
\text { sentence } \\
\text { creation }\end{array}$ & $\begin{array}{c}\text { learners created } \\
\text { interviews }\end{array}$ \\
\hline motivation & $\begin{array}{c}\text { motivational } \\
\text { interview }\end{array}$ & $\begin{array}{c}\text { learners } \\
\text { expressed their } \\
\text { own opinions } \\
\text { motivational } \\
\text { video }\end{array}$ & $\begin{array}{c}\text { learners } \\
\text { expressed their } \\
\text { opinions about } \\
\text { the dialogue } \\
\text { they had seen or } \\
\text { listened to }\end{array}$ \\
\hline
\end{tabular}

Item 3 in the sheets again contains a rating based on the numerical scale. In this item, respondents were asked to comment on whether the teacher took into account the differences of cultures in the multicultural environment and their impact on the personality of the pupil(s) (Figure 2). While in item 1, the evaluators were inclined to evaluate the teachers better than the teachers did in self-assessment; in item 3 we can see the opposite case. Evaluators see this aspect in a less favorable light than teachers - most of them (7) reported rating 2 (5-times) and 3 (twice) and only three of them stated rating 1, with just one better rating from the evaluator than the selfassessment of the teacher was, and 3 teachers received a worse evaluation. In the other six cases the evaluators concurred with the teacher's self-assessment.

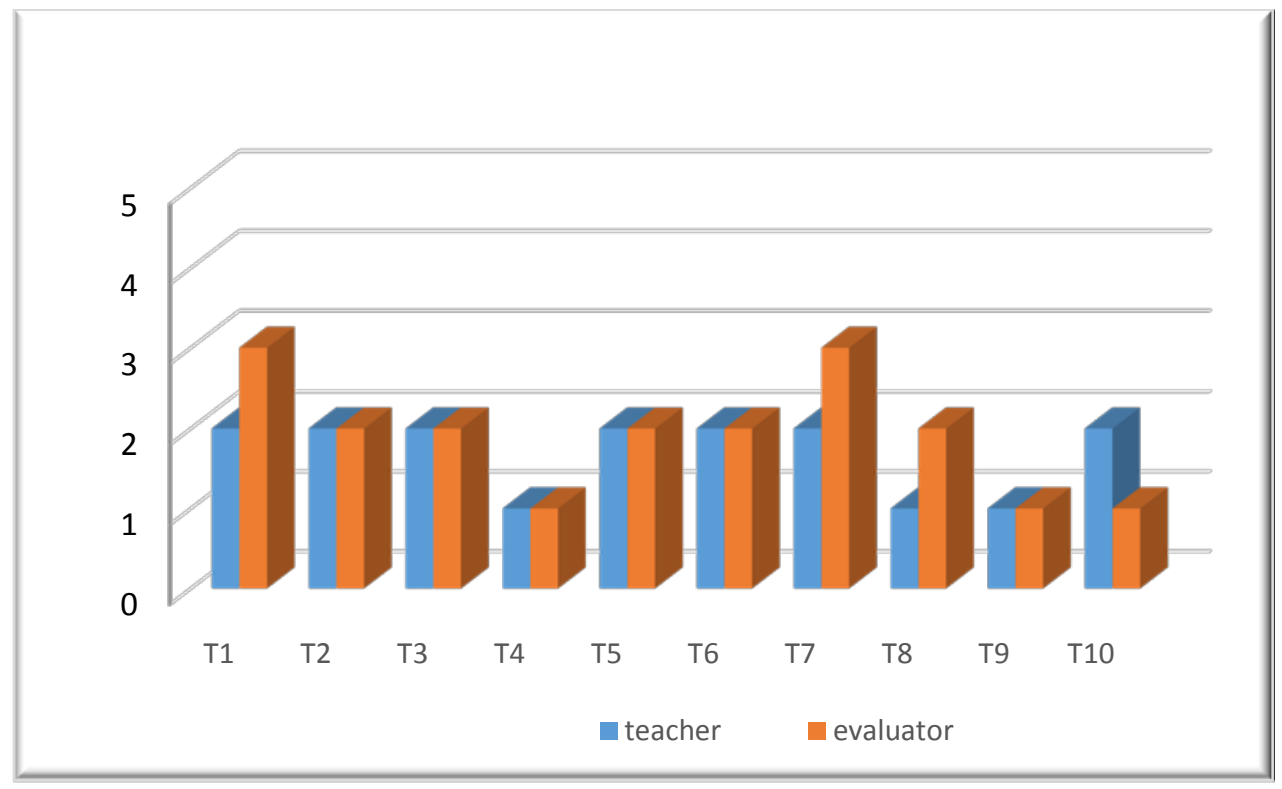

Figure 2 - Taking into account the impact of culture differences

In item 4, the respondents were asked about the teacher' ability to identify pupils from a socially disadvantaged environment (Figure 3). The answer to this question is conditioned by the prior knowledge of pupils and can be quite subjective if the evaluator is not aware about the pupils' background, as demonstrated in case of T3 (the teacher stated that in that lesson there was not such a pupil, while the evaluator 
used the rating 2) and T5 (which was the opposite case - the teacher marked in their Self-Assessment Sheet rating 2 and the evaluator thought that there was no such a pupil in the observed lesson). Disagreement, but only one degree worth occurred in the three more pairs of the respondents when the evaluator rated the teacher by a worse rating - from 2 to 3 (T1) and from 1 to 2 (T6), and only once with a better rating - from 2 to 1 (T10). In the other five cases there was an agreement in the ratings: 3 times rating 1 (T4, T8, T9), 1 rating 2 (T2) and 1 rating 3 (T7).

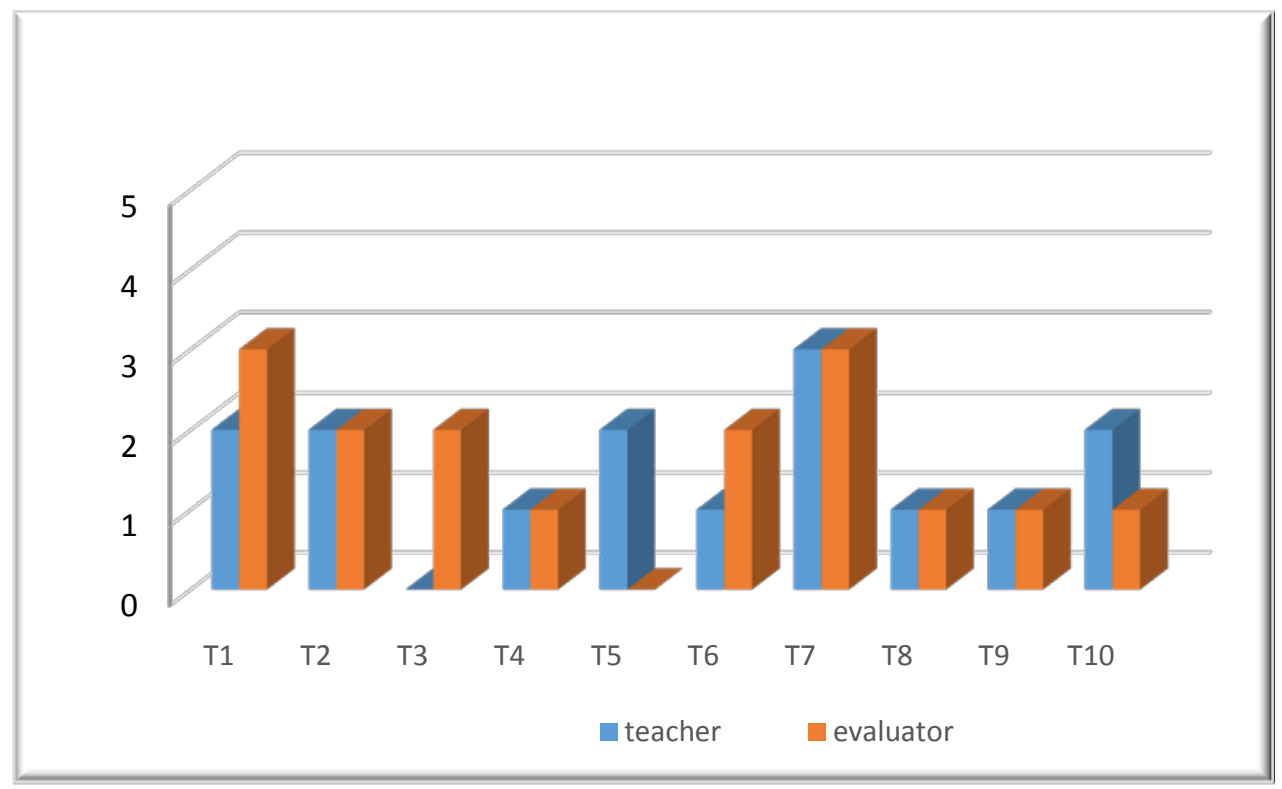

Figure 3 - Taking into account socially disadvantaged environment

In the last closed item (5) with the numerical scale, the respondents expressed their opinions on whether the teacher is able to identify and accept pupils' differences without prejudices and stereotypes (see Figure 4). This question was not enough properly stylized, since the respondents had to express their findings related to two things - the first one was, whether the teacher is able to identify pupils' differences; and the second one - whether they can also accept the differences. The selfassessment of teachers in this item is therefore very high - most teachers (6 out of 10) think they can do it (rating 1) and the remaining four think they usually can do that (rating 2). A remarkable difference is only with T7 - the teacher believes he definitely is able to do that (rating 1) and the evaluator thinks the teacher was unable to identify the differences of the pupils and accept them (rating 3). For other respondents, the differences in responses practically do not exist (responses are identical; in five cases - rating 1 and in two cases - rating 2). 


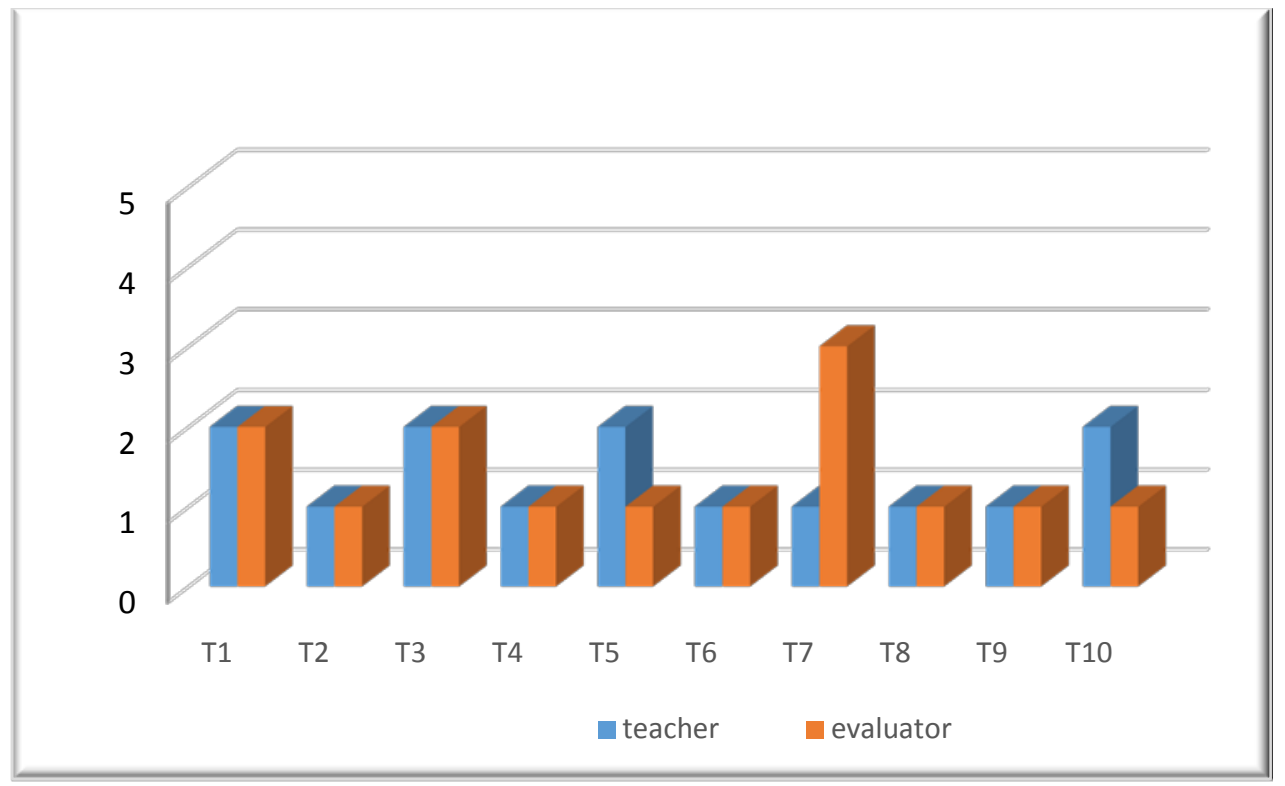

Figure 4 - Identification and acceptance of differences without prejudices and stereotypes

A part of item 5 is also an open sub-question asking how the teacher identified and accepted pupils' differences. The respondents' answers are just partial in many cases. Many times, there is missing either the way or the result that can be traced with a pupil. The counter answers are recorded at T5, where the teacher stated that in the class he was not aware about the differences between the pupils and the evaluator said that the teacher was unable to differentiate pupils. In Figure 4 we can see that the same teacher stated that he usually was able to identify and accept pupils' differences (rating 2). However, the evaluator assigned to that teacher even higher rating (1), i.e. saying that the teacher identified and accepted pupils' differences, what is in contradiction to the statement in this item sub-question that the teacher did not differentiate the pupils. The answers are also suspicious in the case of T2, where both the teacher and the evaluator expressed identical views. Counterproductive are also opinions expressed by T7, where the teacher chose to answer the closed question in previous item using the rating 1 (that he was able to identify and accept differences of pupils), and in this item he stated that there were no pupils from socially disadvantaged environment in the class. The responses of the evaluator correspond in these two issue - the evaluator assigned grading 3 to the teacher in the previous item, i.e. that the teacher had difficulties with the identification of pupils' differences and then, in item 5 , he also wrote that the teacher did not apply individual approach to pupils. Individual answers are given in Table 6 below. 


\begin{tabular}{|c|c|c|}
\hline $\begin{array}{l}\text { Number of } \\
\text { the teacher } \\
\text { and their } \\
\text { evaluator }\end{array}$ & Teacher & Evaluator \\
\hline T1 & $\begin{array}{l}\text { individual work } \\
\text { bright pupils worked, } \\
\text { the lower achievers did not want to } \\
\text { perform for the class }\end{array}$ & $\begin{array}{l}\text { (s)he had no comments on the } \\
\text { methods of the teacher's work, } \\
\text { it was harder to work with socially } \\
\text { disadvantaged pupils }\end{array}$ \\
\hline T2 & $\begin{array}{l}\text { by means of the used methods } \\
\text { during the lesson } \\
\text { teacher did not comment on the } \\
\text { achievements of their work }\end{array}$ & $\begin{array}{l}\text { by means of the used methods } \\
\text { during the lesson } \\
\text { (s)he had no comments on the } \\
\text { achievements of teacher's work }\end{array}$ \\
\hline T3 & $\begin{array}{l}\text { supplementary materials - pictures - } \\
\text { cultural differences } \\
\text { acceptance of all pupils' opinions }\end{array}$ & $\begin{array}{l}\text { (s)he had no comments on the } \\
\text { methods of the teacher's work, } \\
\text { acceptance of pupils' opinions }\end{array}$ \\
\hline T4 & $\begin{array}{l}\text { the task was repeated several times, } \\
\text { and the pupil was guided by the } \\
\text { teacher } \\
\text { accepting his speech errors }\end{array}$ & $\begin{array}{l}\text { (s)he stated that for good } \\
\text { cooperation it is necessary to accept } \\
\text { pupils' differences } \\
\text { (s)he had no comments on the } \\
\text { methods and achievements of the } \\
\text { teacher's work, }\end{array}$ \\
\hline T5 & $\begin{array}{l}\text { teacher is not aware about the } \\
\text { differences among pupils in the class }\end{array}$ & teacher did not differentiate pupils \\
\hline T6 & $\begin{array}{l}\text { in the class, there are not big } \\
\text { differences in social area }\end{array}$ & $\begin{array}{l}\text { the teacher behaved without } \\
\text { prejudices and stereotypes } \\
\text { (s)he had no comments on the } \\
\text { achievements of teacher's work }\end{array}$ \\
\hline T7 & $\begin{array}{l}\text { in the class, there are no pupils from } \\
\text { socially disadvantaged environment }\end{array}$ & teacher did not differentiate pupils \\
\hline T8 & $\begin{array}{c}\text { role playing } \\
\text { positive motivation for work }\end{array}$ & $\begin{array}{c}\text { role playing } \\
\text { and communication } \\
\text { motivation of pupils for work }\end{array}$ \\
\hline T9 & $\begin{array}{c}\text { interview } \\
\text { pupil from socially disadvantaged } \\
\text { environment positively changed their } \\
\text { opinion about studies at upper } \\
\text { secondary school }\end{array}$ & $\begin{array}{l}\text { (s)he did not comment on the } \\
\text { teacher's methods of work, } \\
\text { teacher created a friendly } \\
\text { environment, pupils could express } \\
\text { their opinions }\end{array}$ \\
\hline T10 & $\begin{array}{l}\text { method of work are not stated } \\
\text { expressing own opinions }\end{array}$ & $\begin{array}{c}\text { group work } \\
\text { all pupils were enough motivated }\end{array}$ \\
\hline
\end{tabular}


The last part of the Assessment Sheet is devoted to the evaluator's conclusions and recommendations, and teacher's opinion about them. Specific evaluator recommendations for the evaluated teacher are listed only for T1 and T4, where the evaluator recommends the teacher, in the first case, to put more emphasis on the lower achieving pupils and, in the second case, to apply individual approach to pupils. Other evaluations sound positive, but they are rather general, little specific, and one can see quite a formal, impersonal approach to the evaluation. Teachers' opinions and ideas on their assessment stated by the evaluator were in all cases consistent. Basically, they could not be other, because the evaluator did not tell them anything negative, did not recommend anything, neither highlighted any distinct positive aspects of the teacher's work in terms of the assessed competence. He stated only general statements of the type "suitably chosen method and form" - T1, T2; "methods, forms and topics in compliance with the School Education Program" T10; "positive atmosphere at the lesson" - T3, T8 and others. The respondents' answers are given in Table 7.

Table 7 - Final evaluation of the teacher and their opinion about the evaluation

\begin{tabular}{|c|c|c|c|}
\hline $\begin{array}{l}\text { Number } \\
\text { of the } \\
\text { teacher } \\
\text { and their } \\
\text { evaluator }\end{array}$ & $\begin{array}{l}\text { Conclusions and } \\
\text { recommendations of } \\
\text { the evaluator }\end{array}$ & Evaluator's opinion & Teacher's opinion \\
\hline T1 & $\begin{array}{l}\text { well-chosen methods } \\
\text { and forms } \\
\text { more attention should } \\
\text { be paid to lower } \\
\text { achievers }\end{array}$ & $\begin{array}{l}\text { apply individual } \\
\text { approach to pupils }\end{array}$ & $\begin{array}{c}\text { consent to the } \\
\text { evaluation }\end{array}$ \\
\hline T2 & $\begin{array}{l}\text { well-chosen methods } \\
\text { and forms }\end{array}$ & $\begin{array}{l}\text { well-chosen methods } \\
\text { and forms }\end{array}$ & $\begin{array}{l}\text { consent to the } \\
\text { evaluation }\end{array}$ \\
\hline T3 & $\begin{array}{c}\text { skilled teacher } \\
\text { a positive atmosphere } \\
\text { on a lesson }\end{array}$ & & $\begin{array}{l}\text { consent to the } \\
\text { evaluation }\end{array}$ \\
\hline T4 & $\begin{array}{l}\text { teaching activities of } \\
\text { the teacher contributed } \\
\text { to the development of } \\
\text { pupils' personalities } \\
\text { continue to approach } \\
\text { the pupils individually }\end{array}$ & $\begin{array}{l}\text { consent to the self- } \\
\text { evaluation of the teacher }\end{array}$ & $\begin{array}{l}\text { consent to the } \\
\text { evaluation }\end{array}$ \\
\hline T5 & $\begin{array}{l}\text { satisfaction with the } \\
\text { teacher's teaching }\end{array}$ & 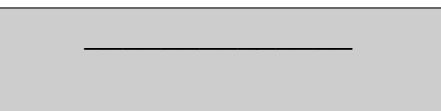 & $\begin{array}{l}\text { consent to the } \\
\text { evaluation }\end{array}$ \\
\hline T6 & $\begin{array}{l}\text { teacher appropriately } \\
\text { developed pupils' } \\
\text { personalities and their } \\
\text { competences, }\end{array}$ & $\begin{array}{l}\text { teacher appropriately } \\
\text { developed pupils' } \\
\text { personalities and their } \\
\text { competences, }\end{array}$ & $\begin{array}{l}\text { consent to the } \\
\text { evaluation }\end{array}$ \\
\hline
\end{tabular}




\begin{tabular}{|c|c|c|c|}
\hline & $\begin{array}{l}\text { gives room for self- } \\
\text { discovery of pupils }\end{array}$ & $\begin{array}{l}\text { gives room for self- } \\
\text { discovery of pupils }\end{array}$ & \\
\hline T7 & $\begin{array}{l}\text { teacher did not } \\
\text { differentiate pupils, the } \\
\text { same approach was } \\
\text { applied to all pupils }\end{array}$ & $\begin{array}{l}\text { teacher did not } \\
\text { differentiate pupils } \\
\text { the same approach was } \\
\text { applied to all pupils }\end{array}$ & $\begin{array}{c}\text { consent to the } \\
\text { evaluation }\end{array}$ \\
\hline T8 & $\begin{array}{l}\text { using the method of } \\
\text { role playing teacher } \\
\text { created positive } \\
\text { atmosphere in the class }\end{array}$ & $\begin{array}{l}\text { the course of the lesson } \\
\text { was in line with the plan } \\
\text { positive impact of the } \\
\text { teacher on pupils } \\
\text { motivation of pupils to } \\
\text { work }\end{array}$ & $\begin{array}{c}\text { consent to the } \\
\text { evaluation }\end{array}$ \\
\hline T9 & $\begin{array}{l}\text { positive impact of the } \\
\text { teacher on pupils } \\
\text { the lesson was in line } \\
\text { with the plan }\end{array}$ & $\begin{array}{l}\text { positive impact of the } \\
\text { teacher on pupils } \\
\text { the lesson was in line } \\
\text { with the plan }\end{array}$ & $\begin{array}{l}\text { consent to the } \\
\text { evaluation }\end{array}$ \\
\hline T10 & $\begin{array}{c}\text { positive and } \\
\text { motivational impact of } \\
\text { the teacher on pupils } \\
\text { methods, forms and } \\
\text { topics in line with the } \\
\text { school educational } \\
\text { program }\end{array}$ & $\begin{array}{l}\text { the lesson was in line } \\
\text { with the plan }\end{array}$ & $\begin{array}{c}\text { consent to the } \\
\text { evaluation }\end{array}$ \\
\hline
\end{tabular}

All these findings were subject to discussion in the Post-Observation Interview of the evaluator with the evaluated teacher. The most discussed issues were affective (empathy, emotions, attitudes, assertiveness, self-reflection, self-confidence, tolerance, discipline, acceptance) and social (communication, cooperation, responsibility, cohesion) factors of pupils' personality development, but also cognitive and conative (creativity) factors of their competences development and reasoning why it is important to develop them. Positive reasoning was concerned with finding pupils way in life and career choice, social communication, needs for critical thinking. Negative reasoning mentioned such things as loss of values, mentally ill kids, incomplete families, lack of adaptability, aggressiveness of pupils, intolerant behaviour. The assessed teachers focused mainly on social and affective aspects of pupils' personality development - mainly aimed at assertiveness and tolerance. The least attention they paid to conative aspects and critical thinking. As to socio-cultural context of pupils' development and ways of its identification the teachers stated that they applied differentiated approach to pupils, interest in pupils interests, discussion with individual pupil, co-operation with parents, motivation, empathy, and tactfulness. Moreover, they paid special attention to special needs pupils showing patient approach to them through personal talk or discussion, eliciting cooperation through special stimuli and more simple tasks, special 
upbringing measures - such as expression of trust and understanding, interest in pupils' opinions, motivation, influencing their attitudes.

\section{Conclusion}

The evaluation of education, schools and their staff based on so called classical complex observation of teachers' and pupils' performance in lessons has its merits but due to many changes in present society it needs to be upgraded and adapted to the current demands of society and legislation. The evaluation process is a very complex activity, which has impact on both its actors - the evaluator and the evaluated one. Through assessment, teachers gain insight into the perception of their superiors on the job they do, and based on this they should be given constructive incentives for their future work. However, teachers must also be allowed to present their needs and wishes related to their professional development. The assessment of teacher's competences should therefore create a transparent and objective framework made up of the different aspects of the teacher's work. The research team did not stay in the theoretical plane of this process. In the search for assessment options during the educational process, the teacher is drawn into evaluative and selfreflective activities and attitudes as understanding the importance of evaluation enables teachers to develop their skills and competences in a meaningful and valuable way. Current time brings new demands, which are also passed on to teachers. On the one hand, it is challenging to meet all these requirements, but on the other hand, every pupil deserves a teacher who is able to cope with all these demands and prepare them for the best possible life by means of appropriate educational activities and competent mediation of knowledge and skills.

\section{References}

Blömeke, S. et al. (2011). Kompetenzen von Lehramtsstudierenden in gering strukturierten Domänen. Erste Ergebnisse aus TEDS-LT. Münster: Waxmann.

Boboňová, I. et al. (2017). Aplikácia metodiky hodnotenia kompetencií učitel'a. Prípadové štúdie. (Applying the teacher competences assessment methodology. Case studies.) Praha: Verbum.

ETUCE (European Trade Union Committee for Education). (2008). Teacher Education in Europe. An ETUCE Policy Paper. Brussels: ETUCE.

Hagger, H., McIntyre, D. (2006). Learning teaching from teachers. Realizing the po-tential of school-based teacher eduaction. Maidenhead: Open University Press.

Jakubovská, V. et al. (2016). Učitel' a jeho kompetencie. (Teacher and their competences) Boskovice: Albert.

Kasáčová, B., Kosová, B. (2006). Kompetencie a spôsobilosti učitel'a - európske trendy a slovenský prístup. (Teacher's competences and abilities -European trends and Slovak approach) Pedagogické rozhl'ady, 22, 37-24.

Kleickmann, T. et al. (2013). Teachers' Content Knowledge and Pedagogical Content Knowledge: The Role of Structural Differences in Teacher Education. Journal of Teacher Education, 64, 90 - 106. 
Kuhn, C.H. et al. (2016). Evaluating the Pedagogical Content Knowledge of Pre- and In-service Teachers of Business and Economics to Ensure Quality of Classroom Practice in Vocational Education and Training. Empirical Research in Vocational Education and Training, 8:5, 1-18. doi: 10.1186/s40461-016-0031-2

Lomnický, I. et al. (2017). Teoretické východiská a súvislosti hodnotenia kompetencií učitel'a. (Theoretical background and context of evaluation of teacher competences.) Praha: Verbum.

Magová, L. et al. (2016). Hodnotenie kompetencií učitelov v Európskom a slovenskom kontexte. (Assessment of competences of teachers in the European and Slovak contexts.) Praha: Verbum.

Malderez, A., Bodoczky, C. (2009). Comment pratiquer un tutorat de qualité? (How to practice quality tutoring?) De Boeck Université.

Moreno-Murcia, J.A. et al. (2015). Questionnaire Evaluating Teaching Competencies in the University Environment. Evaluation of Teaching Competencies in the University. Journal of New Approaches in Educational Research, 4, 54-61.

Pavlov, I. (2013). Štandardizácia profesijných kompetencií učitelov (východiská a perspektívy). (Standardization of teachers' professional competences (background and perspectives)). Prešov: Škola plus s.r.o.

Schleicher, A. (2012). Preparing Teachers and Developing School Leaders for the 21st Century, Lessons from around the World. OECD Publishing.

Slavík, J. (1999). Hodnocení v současné škole. Vychodiska a nové metódy pro praxi. (Assessment in the current school. Background and new methods for practice). Praha: Portál.

Stranovská, E. et al. (2017). Perception of the Degree of Importance of Teacher's Professional Competences from the Perspective of Teacher and Head Teacher in the Evaluation Process of Educational Efficiency. Pedagogy, 127, 5-20. doi: $10.15823 /$ p. 2017.36

Záhorec, J. - Hašková, A. - Munk, M. (2017). Teachers` didactic technological competences: Results of the pilot research. Application of Information and Communication Technologies - AICT2017, 2, 345 - 349. doi: IEEE Catalog Number CFP1756H-PRT 


\title{
Instructional Resources and Teacher Effectiveness in Government-Aided Secondary Schools in Uganda
}

\author{
Paul Netalisile Malunda and Juliet Atwebembeire \\ Uganda Management Institute \\ Kampala, Uganda
}

\begin{abstract}
The study examines the influence of instructional resources on teacher effectiveness in government-aided secondary schools in Uganda and specifically the extent to which availability and utilization of instructional resources influence teacher effectiveness. A descriptive cross-sectional survey design was used in which 82 head teachers and 1024 teachers were selected using multistage sampling. Survey, interview, observation and document analysis methods were used to collect data. Quantitative data were analyzed using descriptive statistical analysis and ordered logistic regression, while content analysis was used to analyze qualitative data. The findings suggest that availability and utilization of instructional resources significantly contribute to teacher effectiveness although instructional resources are inadequate and not properly utilized. To enhance teacher effectiveness, the Ministry of Education and Sports (MoES) should continuously train teachers on use and improvising of instructional resources. Government should construct more libraries and science laboratories especially for Universal Secondary Education [USE] schools to promote easy accessibility of textbooks by learners and teachers and practical teaching of science subjects.
\end{abstract}

Keywords: instructional resources, teacher effectiveness and government-aided secondary schools.

\section{Introduction}

Secondary education plays a critical role in national development. Secondary education is intended to enable individuals acquire and develop knowledge and an understanding of emerging needs of society and the economy. It is also meant to provide up-to-date and comprehensive production in theoretical and practical aspects of innovative production, modern management methods in the field of commerce and industry and their application in the context of the social economic development of Uganda (National Development Programme [NDP], 2015). However, quality of education in Uganda's government-aided secondary schools does not meet the expectations of the public (MoES, 2015). Teachers in most 
secondary schools in Uganda lack the necessary instructional resources to teach effectively; where the resources are available, the teachers hardly make proper use of these resources (MoES, 2015, Uganda National Examinations [UNEB], 2015). Availability of instructional resources in a school significantly determines the teaching methods used by teachers (Musaazi, 1982). In situations where instructional materials such as textbooks, laboratory and display materials, and plant and animal specimens are lacking, teacher- centered methods of delivery tend to dominate (UNESCO, n.d). In addition, where the instructional materials are available, wellqualified and motivated teachers will skillfully use the available resources to engage learners in practical activities that give the learners the opportunity to experiment, solve problems, discuss with each other, thereby stimulating curiosity, critical thinking and innovativeness (Smith, Wood, Adams, Wieman, Knight \& Guild, 2009). Therefore, the significance of instructional materials in any learning and teaching environment cannot be underestimated. For effective learning to occur, the teacher has to make proper use of the instructional resources and appropriately guide learners by employing a variety of teaching methods such as demonstration, experimental or discussion (Raw, 2006).

Uganda's education system was one of the best on the African continent before the wars and civil strife of the seventies and eighties (Government of Uganda, 1992). Teaching focused on developing learners' competencies and students were taught in a way that fostered higher order thinking skills (Ssekamwa \& Lugumba, 2010). In order to re-establish the quality of education, government carried out major reforms in education in line with the Education White Paper (MoES, 2009) including implementation of Universal Primary and Secondary Education. The introduction of Universal Secondary Education increased access to secondary education; however, teacher effectiveness deteriorated due to the mismatch between the inputs and the increased enrollment (MoES, 2015; Nannyonjo, 2007). In the recent times, there have been reports of increasing examinations malpractices, levels of rote learning, coaching of students and examination-oriented teaching (MoES, 2015). This is happening at a time the Government of Uganda is struggling to develop initiatives aimed at equipping her people with skills needed by the employment sector.

The study is anchored on the constructivism theory, which stipulates that through active learning, students are able to use their prior knowledge in context to promote learning (Golightly \& Roath 2015). In other words, the students' prior knowledge or experience coupled with results from the activities carried out during the lesson aid the learners to construct new knowledge. According to the theory, ownership of learning in the classroom is shifted from the teacher to the learner. Since understanding is crucial to constructive learning, use of instructional resources provides students with the opportunity to learn as the teacher plays the role of a facilitator to engage and guide the students in discovering information, constructing their own learning and solving problems (Balogun, Okon, Musaazi, Thakur, 1981). Therefore, the study focuses on two key concepts: instructional resources and teacher effectiveness. 
Teacher effectiveness is complex to define given the complex nature of teaching. There is no agreed position on whether teacher effectiveness should be defined using teacher qualifications, pedagogical practices or learner achievement (Stronge, Ward \& Grant, 2011). According to Walls (1999), teacher effectiveness entails the following:

(i) clearly spelling out the learning outcomes to focus learners on the learning goals;

(ii) making the content as clear as possible as the teacher builds on existing knowledge;

(iii)engaging learners in activities during the teaching and learning process; and

(iv) display of high level of enthusiasm that reflects professional competence and confidence.

Popoola and Haliso (2009) define teacher effectiveness as the ability of a teacher to instill knowledge and skills in students, as well as positively influencing the learners' behavior for a better living. Ismaila (1999) cited in Adeoye and Popoola (2011) link teacher effectiveness to the teacher's knowledge of subject matter, expertise and resourcefulness that enhance students' academic performance. Teacher effectiveness in this study is conceptualized as the teacher using a variety of resources to plan, and present content and skills that enhance the opportunities for students to learn and attain the desired learning outcomes.

Instructional resources are support materials used by teachers in the classroom to enhance the learning process (Abdullahhi, 2010; Agun \& Okunrotifa, 1977). These help teachers to make their lessons explicit, more interesting and understandable to learners. These resources range from textbooks, display materials such as wall charts, chalkboard to pictures and diagrams, laboratory apparatus and plant and animal specimens. These make it possible to teach lessons that involve objects that cannot be brought to the classroom or which the learners cannot experience directly (Balogun, Okon, Musaazi \& Thakur, 1981). The instructional resources are intended to stimulate, motivate and focus the learners' attention during the teaching and learning process to enhance constructive learning (Shabiralyani, Hassan, Hamad \& Iqbal, 2015). In this study, instructional resources include textbooks, laboratory materials and teaching aids.

There is growing concern about teacher effectiveness in Government secondary schools in Uganda (MoES, 2016). The way teachers teach is not in consonance with the expectations of various stakeholders in the education arena (Curriculum Assessment and Examination [CURASSE], 2007; MoES, 2012; UNEB, 2016; MoES, 2016). Teacher-centered method of delivery remains the predominant form of teaching making leaners passive listeners in the classroom; science concepts are not practically taught and teachers have difficulty in relating science concepts to daily life experiences (MoES, 2015). According to the National Assessment of Progress in Education [NAPE] report of 2016 (UNEB, 2016), the teaching in secondary schools encourages rote-learning rather than imparting skills. The teaching is more examination driven and does not consider the learning of competencies. Despite government's effort to remedy the situation through provision of instructional resources, these resources are underutilized or inadequate in most governmentaided secondary schools (MoES, 2016). The purpose of the study therefore is to 
establish the extent to which instructional resources explained variations in teacher effectiveness in government-aided secondary schools in Uganda. Specifically, the study focuses on investigating the extent to which; availability and utilization of instructional resources explain variations in teacher effectiveness in public secondary schools in Uganda.

A number of scholars (Busingye \& Najjuma, 2015; Bizimana \& Oradho, 2014; Goloba, Wokadala \& Bategeka, 2010; Abdullahhi, 2010; Raw, 2006; Orji, 2000) have attempted to explore the linkage between availability of instructional resources and teacher effectiveness in the classroom. For instance, Busigye and Najjuma (2015) investigated the influence of the teaching and learning resources on the learning outcomes in Uganda's primary schools. Findings of their study revealed that availability of teaching and learning resources had minimal influence on the learning outcomes of Mathematics and English pupils. They further observed that teaching and learning resources are only effective when teachers have the necessary skills to use them. Relatedly, an earlier study by Goloba, Wokadala and Bategeka (2010), revealed that it was not actually availability of resources that majorly explained quality of teaching in schools, but supervision of the teachers. According to Goloba et al (2010), administrators were not sufficiently supervising teachers to ensure that they used instructional materials to promote the highly recommended learnercentered methods of teaching. As a result, it is imperative for the schools administrators to supervise the teachers' utilization of the available resources for effective teaching in schools.

However, Bizimana and Oradho (2014) in their study on teaching and learning resources availability and teachers' effective classroom management and content delivery in Rwanda established a significant positive relationship between availability of instructional resources and effective teaching. Bizimana and Oradho concluded that teachers should be innovative to improvise those necessary instructional materials that could be lacking in schools. Relatedly, Okwara, Shiundu and Indoshi (2009) observed that availability of instructional resources significantly contributes to effective pedagogical practices. They hence urged teachers to make instructional materials an integral part of the teaching and learning process. Mwanamukubi (2013) established that inadequate instructional resources greatly influenced teacher performance. She pointed out inadequacy of instructional resources as a deterrent to effective teaching.

Musaazi (1982) basing on Taylor's scientific management theory emphasize the critical role of instructional resources in enhancing teacher effectiveness. According to the theory, a workman should be given the appropriate tools and materials to effectively accomplish his/her work with efficiency. Musaazi argues that teachers and students need to be provided with the necessary and up-to date resources such as textbooks, science equipment, laboratories, libraries, visual aids and many others for effective teaching and learning. Despite the relevancy of instructional materials in enhancing effective teaching, most secondary schools in Uganda lack the basic teaching and learning resources. According to the NAPE report, the instructional 
materials provided by government do not match the robust increase in enrolment, most especially in USE schools (UNEB, 2015).

For the instructional resources to contribute effectively to teaching and learning, they must be put to proper use (Nannyonjo, 2007; Adeleke, 2005; Okiy, 2000). However, the Education and Sports sector Annual Performance Report [ESAPR] of 2015/16 (MoES, 2016) revealed that the few instructional materials provided by government to secondary schools, such as textbooks, chemicals/reagents and science kits were not well utilized. According to the report, teachers did not engage learners in hands-on activities that enhance cognitive and manipulative skills to interpret scientific and mathematical concepts. The earlier report of the Directorate of Education Standards of 2015 (MoES, 2015) attributed the failure of teachers to utilize available instructional materials in Uganda's secondary schools to poor lesson planning for teaching and learning aids. Balogun, Okon, Musaazi and Thakur (1981) advise that instructional materials can only be properly used, when teachers through good lesson planning; identify, prepare the necessary teaching and learning materials, and determine the order in which these materials will be used. According to Smith et al (2009), when teachers skillfully use instructional materials, it will not only facilitate interaction among learners, but it will equally engage higher order cognitive strategies of analysis, synthesis and evaluation.

Raw (2006) advises that to promote learner participation and hence enhance effective teaching, students should be allowed to manipulate the provided materials. The teacher should give the learners a chance for practical work and allow them to make their own conclusion from their findings. The teachers should let the learners discover knowledge and answers to challenges in their daily lives. This motivates learners to create their knowledge through exploring, analyzing and understanding. Raw asserts that learner participation will not only make the teaching and learning process more interesting, but will also enhance the memory level of the learners. In congruence with Raw, Orji (2000) emphasizes that instructional materials in the teaching and learning process, motivate and arouse student's desire to learn. In the same vein, Brown et al (2005) assert that instructional resources promote interaction amongst learners, effective communication and learning which enhances retention; thereby making learning more permanent. Other studies (Armbruster, Patel, Johnson, and Weiss, 2009; Armstrong, Chang, Brickman, 2007; Deslauriers, Schelew, Wieman, 2011) concur with Brown et al's underscoring the fact that when interactive methods of teaching are used, students usually demonstrate better understanding of concepts, greater participation and increased persistence to learn.

\section{Method}

\section{Participants}

The study sample consisted of 1024 teachers from government-aided secondary schools who were selected using multi-stage sampling technique, 82 head teachers, and two officials from the Directorate of Education Standards who were purposively selected. 


\section{Design}

A descriptive cross-sectional survey design was used, in which data was collected from a representative sample at one point in time to make inferences about the target population (Borg \& Gall, 1989). The design was useful in gathering factual information and data on attitudes and preferences, beliefs and behaviour and experiences of respondents in reference to instructional resources and teacher effectiveness as they naturally occurred (Cohen, Manion \& Morrison, 2007). The design facilitated the collection and analysis of data using both quantitative and qualitative methods with the purpose of providing a better understanding of the research problem and question (Creswell \& Plano Clark, 2011).

\section{Materials}

Given the large number of respondents, the questionnaire method of data collection was preferred, which helped to save time and costs. A 32-item questionnaire whose questions were acquired and revised from the teaching and learning assessment instrument of DES was used to collect data from teachers. The questionnaire comprised questions pertaining to respondents' background information, respondents' views on availability of instructional resources, utilization of instructional resources and teacher effectiveness. The questions were measured on a 5-point Likert scale: Strongly Agree (5), Agree (4), Non-committal (3), Disagree (2) and Strongly Disagree (1).

The interview method was further used to seek the views of DES officials and head teachers of the selected schools on the influence of instructional resources on teacher effectiveness. The method enabled further probing on concerns that were being studied. The document review method was also used to collect data. It adopted a checklist from DES's teaching and learning quality instrument. This method made it possible to validate the information obtained using the questionnaire and interview methods.

The tools used were pre-tested before the actual data collection was conducted. Descriptive and inferential statistical methods were used to analyze quantitative data. Specifically, the logistic regression model was used to determine the extent to which availability and utilization of instructional resources influence teacher effectiveness. The tests of significance were performed at the significance level of $p<$ 0.05. Qualitative data were analyzed using content analysis method.

\section{Procedure}

Given the vast amount of work, five research assistants were appointed and trained to assist in the data collection process. The research assistants underwent a two-days training on the following: personal presentation; how to seek permission from head teachers to access school; how to conduct interviews with the head teachers, selection of teachers and collection of data using questionnaires. 
The head teachers were interviewed from their offices using the semi-structured interview schedule. Through the head teacher, teachers to participate in the study were identified. The consent of the teachers was sought through an introduction letter that explained the purpose of the study, why they had been selected to participate in the study and highlighted the treatment of information provided. The teachers were neither expected to provide their names nor the names of their schools

\section{Results}

\section{Profile of Respondents}

The background characteristics of the respondents are presented in Table 1.

Table 1 - Background information on the Respondents

\begin{tabular}{|l|l|c|c|}
\hline Variable & Category & Frequency & Percentage \\
\hline Age & 20 to less than 30 years & 358 & 35 \\
\cline { 2 - 4 } & 30 to less than 40 years & 453 & 44.2 \\
\cline { 2 - 4 } & 40 years and above & 213 & 20.8 \\
\hline \multirow{5}{*}{ Gender } & Male & 702 & 68.6 \\
\cline { 2 - 4 } & Female & 322 & 31.4 \\
\hline \multirow{5}{*}{ Number of years in the school } & Diploma & 215 & 21 \\
\cline { 2 - 4 } & Graduate & 635 & 62 \\
\cline { 2 - 4 } & Post-graduate & 174 & 17 \\
\cline { 2 - 4 } & 3 to less than 10 years & 604 & 60 \\
\cline { 2 - 4 } & 10 years above & 227 & 21.9 \\
\hline
\end{tabular}

The findings in Table 1 indicate that $79.2 \%$ of the teachers were aged between 20 and 40 , showing that majority were young and energetic to discharge instructional tasks. Results also demonstrate a gender disparity in employment of teachers in public secondary schools with more male teachers (68.6\%) employed. Results also suggest that all the teachers have the prerequisite qualification of at least a Diploma to teach at a secondary school level. In relation to numbers of years spent in the schools, findings show that $81.9 \%$ of the teachers who participated in the study had adequate experience of more than three years of teaching. 


\section{Availability of Instructional Resources}

Table 2 - Distribution of the Teachers' Views on Availability of instructional Resources in Government-Aided Secondary Schools in Uganda

\begin{tabular}{|l|c|c|l|}
\hline Availability of resources & Disagree & $\begin{array}{l}\text { Non- } \\
\text { committal }\end{array}$ & Agree \\
\hline $\begin{array}{l}\text { The government provides the necessary } \\
\text { teaching and learning resources }\end{array}$ & $102(9.9 \%)$ & $13(1.3 \%)$ & $909(88.8 \%)$ \\
\hline $\begin{array}{l}\text { The school regularly procures relevant } \\
\text { textbooks }\end{array}$ & $658(64.3 \%)$ & $54(5.3 \%)$ & $312(30.5 \%)$ \\
\hline $\begin{array}{l}\text { The relevant text books in our school are } \\
\text { adequate for the learners }\end{array}$ & $712(69.5 \%)$ & $68(6.6 \%)$ & $244(23.8 \%)$ \\
\hline $\begin{array}{l}\text { Recommended text books are easily accessed in } \\
\text { my school }\end{array}$ & $453(44.2 \%)$ & $97(9.5 \%)$ & $474(46.3 \%)$ \\
\hline $\begin{array}{l}\text { Our science laboratories have the necessary } \\
\text { equipment, chemicals and reagents }\end{array}$ & $146(14.3 \%)$ & $215(21 \%)$ & $663(64.7 \%)$ \\
\hline Our school has a functional library & $567(55.4 \%)$ & $24(2.3 \%)$ & $433(42.3 \%)$ \\
\hline $\begin{array}{l}\text { I improvise teaching and learning materials } \\
\text { where the conventional materials are lacking to } \\
\text { facilitate my teaching }\end{array}$ & $335(32.7 \%)$ & $14(1.4 \%)$ & $675(65.9 \%)$ \\
\hline $\begin{array}{l}\text { The time allocated on the timetable is adequate } \\
\text { for use of teaching and learning resources. }\end{array}$ & $681(66.5 \%)$ & $34(3.3 \%)$ & $309(30.2 \%)$ \\
\hline $\begin{array}{l}\text { We have sufficient laboratories for effective } \\
\text { teaching of sciences }\end{array}$ & $534(52.1 \%)$ & $42(4.1 \%)$ & $448(43.8 \%)$ \\
\hline
\end{tabular}

Results in Table 2 show that, largely, government provided the necessary teaching and learning resources; however, schools hardly procured the relevant textbooks. The majority of the respondents $(64.3 \%)$ indicated the relevant textbooks were not adequate for the learners. Results in the table also show that majority of the respondents $(55.4 \%)$ indicated that their schools did not have functional libraries, and only $46.3 \%$ of the respondents agreed that the recommended textbooks were easily accessible. Although $64.7 \%$ of the respondents indicated that their science laboratories had the necessary equipment such as chemicals and reagents, only $43.8 \%$ agreed that the schools had sufficient laboratories for effective teaching of sciences. The results further suggest that there was an effort by majority of the teachers $(65.9 \%)$ to improvise teaching and learning materials to promote effective teaching.

Interviews with the head teachers revealed that the government provided laboratory equipment, chemicals and textbooks for Mathematics, Biology, Physics and Geography subjects to USE schools under IDA support. However, the teaching and learning resources were still insufficient. According to the head teachers, USE schools lacked funds to procure instructional resources to supplement what the government provided and only a few of their students could afford instructional materials for their personal use. One head teacher illustrated:

As a USE school, we do not have funds to stock the necessary teaching and learning materials. Our students come from poor families; they can 
hardly afford the required instructional materials such as textbooks for their personal use. We entirely rely of provisions of government...

The head teachers also revealed that teachers were not reaching out for the locally available materials to improvise when need arose. For example, with regard to teachers' failure to improvise instructional materials, a head teacher from a non-USE school in Ankole sub-region explained, “.....most teachers are either inadequately trained or lack the innovativeness to improvise the instructional materials. Our teachers rely on what is provided by the school."

According to the head teachers, USE schools had a big challenge of storage and utilization of the textbooks and the science equipment provided. It was observed that USE schools mainly in the Elgon and West Nile sub-regions lacked libraries and laboratories to stock these instructional resources; as a result, these resources were kept in the offices of head teachers.

\section{Utilization of Instructional Resources}

Table 3 - Distribution of Teachers' Views on Utilization of Instructional Resources in Governmentaided Secondary Schools in Uganda

\begin{tabular}{|l|c|c|c|}
\hline Utilization of the instructional resources & Disagree & $\begin{array}{l}\text { Non- } \\
\text { committal }\end{array}$ & Agree \\
\hline $\begin{array}{l}\text { I regularly use conventional teaching aids for } \\
\text { my lessons }\end{array}$ & $297(29 \%)$ & $53(5.2 \%)$ & $\begin{array}{c}674 \\
(65.8 \%)\end{array}$ \\
\hline Students use textbooks to do their class exercises & $157(15.3 \%)$ & $87(8.5 \%)$ & $\begin{array}{c}780 \\
(76.2 \%)\end{array}$ \\
\hline $\begin{array}{l}\text { I always give my students homework that } \\
\text { require use of the available instructional } \\
\text { materials }\end{array}$ & $356(34.8 \%)$ & $38(3.7 \%)$ & $\begin{array}{c}630 \\
(61.5 \%)\end{array}$ \\
\hline $\begin{array}{l}\text { Practical sessions are usually used to teach } \\
\text { sciences }\end{array}$ & $473(46.2 \%)$ & $52(5.1 \%)$ & $\begin{array}{c}499 \\
(48.7 \%)\end{array}$ \\
\hline $\begin{array}{l}\text { Students always are allowed to operate the } \\
\text { science apparatus/equipment during the science } \\
\text { lessons }\end{array}$ & $524(51.2 \%)$ & $49(4.8 \%)$ & $451(44 \%)$ \\
\hline $\begin{array}{l}\text { I complete my lessons within the allocated time } \\
\text { whenever I use teaching and learning resources }\end{array}$ & $639(62.4 \%)$ & $23(2.2 \%)$ & 362 \\
\hline $\begin{array}{l}\text { It is proper to always use teaching and learning } \\
\text { resources }\end{array}$ & $338(33 \%)$ & $29(2.8 \%)$ & $\begin{array}{c}657 \\
(64.2 \%)\end{array}$ \\
\hline
\end{tabular}

Results in Table 3 suggest that majority of the teachers use conventional teaching aids for their lessons, give class exercises that call for use of textbooks and give homework that demands the use of the available instructional materials. Results further show that only $48.7 \%$ of the respondents agreed that sciences were practically taught in their schools and $51.2 \%$ of the respondents indicated that students were not allowed to operate the science apparatus and equipment during science lessons. Although $64.2 \%$ of the teachers agreed that it was proper to always use teaching and learning resources, only $35.4 \%$ of the respondents indicated that 
they were able to complete their lessons within the allocated time whenever they used teaching and learning resources.

Information from the head teachers suggested that the government policy of placing textbooks in the hands of learners was not working. The head teachers preferred to "safely" keep the books in the libraries and their offices. One head teacher of a USE school from the west Nile sub region explained, "... government does not know the category of students we have in our USE schools. If you give them books, they destroy or even lose them. It is safer keeping them in the libraries and given to students when need arises". However, during lesson observations, of the 76 lessons observed, it was only in only $22(29 \%)$ of the lessons where the textbooks provided by government were used. In majority $(71 \%)$ of the lessons, teachers used lecture method of teaching, spent most of the lesson time dictating notes and/or writing notes on the blackboards for the learners to copy. Several students in these lessons appeared bored and some sleepy!

With regard to utilization of science equipment provided by government, head teachers of USE schools revealed that lack of space to conduct practical sessions partly explained why the science apparatus provided by government was not fully utilized. The available space was used to prepare candidates for the national examinations. Concerning practical teaching of sciences, one head teacher explained,

Practical teaching of sciences is deliberately delayed because of the limited time that is available to complete the syllabus. We are forced to start practical teaching of science subjects at Senior 4; however, teachers usually carry out demonstrations during science lessons at the lower levels

\section{Teacher effectiveness}

This sub-section presents a description of the teachers' responses on teacher effectiveness in government-aided secondary schools in Uganda. Table 4 provides the descriptive statistics, showing the frequencies and percentages of the teachers' opinions on teacher effectiveness.

Table 4 - Distribution of Teachers' Views on Teacher Effectiveness in Government-aided Secondary Schools in Uganda

\begin{tabular}{|l|c|c|c|}
\hline Teacher effectiveness & Disagree & $\begin{array}{l}\text { Non- } \\
\text { committal }\end{array}$ & Agree \\
\hline $\begin{array}{l}\text { I always adhere to National Curriculum Development } \\
\text { Centre [NCDC] guidelines when making the schemes } \\
\text { of work }\end{array}$ & $183(17.9 \%)$ & $27(2.6 \%)$ & $\begin{array}{c}814 \\
(79.5 \%)\end{array}$ \\
\hline I make lesson plans for all my lessons & $621(60.6 \%)$ & $41(4.0 \%)$ & $\begin{array}{c}362 \\
(35.4 \%)\end{array}$ \\
\hline I usually plan to use a variety of teaching aids & $411(40.1 \%)$ & $58(5.7 \%)$ & $\begin{array}{c}555 \\
(54.2 \%)\end{array}$ \\
\hline $\begin{array}{l}\text { I always prepare class exercises for students before the } \\
\text { lessons. }\end{array}$ & $233(22.7 \%)$ & $68(6.6 \%)$ & $\begin{array}{c}723 \\
(70.6 \%)\end{array}$ \\
\hline
\end{tabular}




\begin{tabular}{|l|c|c|c|}
\hline I regularly use a variety of teaching methods & $368(35.9 \%)$ & $18(1.8 \%)$ & $\begin{array}{c}638 \\
(62.3 \%)\end{array}$ \\
\hline I usually use teaching aids to enhance my teaching & $296(28.9 \%)$ & $77(7.5 \%)$ & $\begin{array}{c}651 \\
(63.6 \%)\end{array}$ \\
\hline $\begin{array}{l}\text { I usually use the demonstration method to enhance } \\
\text { learning of my learners }\end{array}$ & $305(29.8)$ & $80(7.8 \%)$ & $\begin{array}{c}639 \\
(62.4 \%)\end{array}$ \\
\hline $\begin{array}{l}\text { I encourage students to discover new knowledge on } \\
\text { their own }\end{array}$ & $402(39.3 \%)$ & $33(3.2 \%)$ & $\begin{array}{c}589 \\
(57.5 \%)\end{array}$ \\
\hline I always give class exercises during my lessons & $456(44.5 \%)$ & $24(2.3 \%)$ & $\begin{array}{c}544 \\
(53.1 \%)\end{array}$ \\
\hline I usually mark class exercises & $429(41.9 \%)$ & $32(3.1 \%)$ & $563(55 \%)$ \\
\hline $\begin{array}{l}\text { I always make constructive comments as I mark the } \\
\text { given exercises }\end{array}$ & $112(10.9 \%)$ & $19(1.8 \%)$ & $\begin{array}{c}893 \\
(87.2 \%)\end{array}$ \\
\hline I usually engage my students during lessons & $106(10.4 \%)$ & $67(6.5 \%)$ & $\begin{array}{c}851 \\
(83.1 \%)\end{array}$ \\
\hline
\end{tabular}

Results in Table 4 show that whereas $79.5 \%$ of the teachers agreed that they made schemes of work in adherence of NCDC guidelines, $60.6 \%$ indicated that they did not always make lesson plans, while $40.1 \%$ did not plan to use a variety of teaching aids and $62.3 \%$ used a variety of teaching methods. The majority of the respondents $(62.4 \%)$ agreed that they used demonstration methods to enhance their teaching, while $57.5 \%$ encouraged students to discover new knowledge on their own. Barely half of the respondents gave and marked class exercises. The results further suggest that teachers usually made constructive comments and engaged students during lessons.

Although the majority of the teachers (79.5\%) agreed that they made schemes of work to in line with NCDC guidelines, review of the schemes of work revealed that a great portion of teachers did not adhere to NCDC guidelines that emphasized learner-based approaches of teaching. The head teachers associated this failure to adhere to guidelines the teachers' fear to complete the syllabi in time for the national examinations. Findings of lesson observations revealed that only 33.9\% used learnerbased methods. A review of the students' exercise books revealed that only $53.5 \%$ teachers administered and assessed class exercises. These findings were in congruence with descriptive results in Table 3 where $55 \%$ of the teachers indicated that they gave and marked class exercises.

\section{Verification of the Hypotheses}

The ordered logistic regression was conducted to test the following null hypotheses:

I. Availability of instructional resources does not significantly influence teacher effectiveness.

II. Utilization of instructional resources does not significantly influence teacher effectiveness.

The results of the hypothesis tests are presented in Table 5. 
Table 5 - Ordered logistic regression results on teacher effectiveness

\begin{tabular}{|l|l|l|l|l|}
\hline Teacher Effectiveness & Coefficient & $\mathbf{P}>|\mathbf{Z}|$ & \multicolumn{2}{|l|}{$\mathbf{9 5 \% \text { confidence interval }}$} \\
\hline $\begin{array}{l}\text { Availability of instructional } \\
\text { resources }\end{array}$ & 5.632 & $\mathbf{0 . 0 0 0}$ & 4.76 & 6.43 \\
\hline $\begin{array}{l}\text { Utilization of instructional } \\
\text { resources }\end{array}$ & 3.231 & $\mathbf{0 . 0 0 0}$ & 2.58 & 3.64 \\
\hline Age & 1.013 & 0.803 & -0.89 & 1.15 \\
\hline Gender & -0.187 & 0.434 & -0.72 & 0.37 \\
\hline Education level & -0.438 & 0.068 & -0.71 & 0.04 \\
\hline Duration & -0.253 & 0.121 & -0.46 & 0.07 \\
\hline
\end{tabular}

Pseudo $R^{2}=0.685$, Number of respondents $=1024, \operatorname{LR} \chi^{2}(10)=1416.73$, Prob $>\chi^{2}=0.00$

The results in Table 5 show that 1024 observations were used in the analysis. The likelihood ratio chi-square of 1416.73 with $p$-value of $0.000(p<0.05)$ showed that the model overall was statistically significant compared to the null model with no predictors. Pseudo $\mathrm{R}^{2}=0.685$ means that the explanatory variables in the model explained $68.5 \%$ variability in the quality of teacher effectiveness $(p=0.000,<0.05)$, whereas, the demographic variables did not $(\mathrm{p}>0.05)$. The results also indicate that a unit increase in availability of instructional resources resulted in a 6-unit change in teacher effectiveness, while a unit change in utilization of instructional resources explained a 3-unit change in teacher effectiveness. The null hypotheses I and II were therefore rejected, implying that:

(i) Availability of instructional resources significantly influences teacher effectiveness in government-aided secondary schools in Uganda

(ii) Utilization of instructional resources significantly influences teacher effectiveness in government-aided secondary schools in Uganda.

The overall frequency of dilemmas coded as justice, care or justice-care mixtures for both genders were collated and put to SPSS.

\section{Discussion}

The purpose of the study was to establish the extent to which availability and utilization of instructional resources explained variations in teacher effectiveness in government-aided secondary schools in Uganda. Findings of the study revealed that, other factors held constant, availability and utilization of instructional resources significantly $(p<0.05)$ influenced teacher effectiveness. These finding were in congruence with findings of Bizimana and Oradho (2014), Mwanamukubi (2013) and Okwara et al. (2009). However, the results contradict findings of Goloba, Wokadala and Bategeka (2010). 
Although availability and use of instructional resources significantly contributes to improved teacher effectiveness, findings of the study revealed scarcity of these resources especially in USE schools where resources were available but utilization was low. The most affected USE schools were in the Elgon and West Nile subregions of Uganda. The findings suggested that the non-USE schools were not only better equipped with teaching and learning resources but the teaching and learning materials in these schools were better utilized than in USE schools.

The inadequacy of instructional resources in USE schools is explained by the mismatch between the resources the Government provides and robust increase in enrollment in these schools (UNEB, 2015). The study established that teachers in USE schools who sometimes carry out moonlight teaching in private schools took and used the instructional resources provided to USE schools in these schools, thereby creating scarcity of the teaching and learning resources. The study also revealed that several teachers lacked the necessary training or innovativeness to improvise teaching materials; this partly explained the insufficiency of instructional materials. This insufficiency has contributed to failure of a large proportion of learners to apply concepts learnt in class to daily life (UNEB, 2016) because most teachers continued to use teacher-centered methods of teaching that promote rote learning for examinations rather than imparting skills or enhancing learning competencies. This could also explain the poor performance of the USE schools in the Elgon and West-Nile sub-regions.

Although availability of instructional materials is critical in the teaching and learning of students, utilization of these resources is fundamental in ensuring quality-learning outcomes (Nannyonjo, 2007; Adeleke, 2005; Okiy, 2000). The study established that instructional resources provided by Government under the IDA support such as textbooks and laboratory equipment were not easily accessible to the learners. These findings contradict what was reported in the Education and Sports Sector Annual Performance Report of 2014/15 that provision of textbooks for the core subjects, science kits and chemicals had improved learner access to instructional resources (MoES, 2015). It was observed that most rural USE schools lacked libraries to stock the books provided; as a result, the textbooks were kept in the offices of the head teachers, which were not easily accessible to the learners.

The study further established that the policy of placing textbooks in the hands of the learners was not working in several of the USE schools because of the administrators' negative perception of the leaners' level of responsibility in maintaining the textbooks. The administrators preferred to have the textbooks safely kept in the libraries or their offices and only had the books used when a teacher distributed and supervised learners using them. Generally, utilization of the textbooks provided by government was significantly low. However, the study established that use of the textbooks to enhance teaching was more common among teachers of English and Mathematics because the curriculum of these subjects is designed in a manner that requires teachers to give class exercises from specific textbooks recommended by the NCDC. 
The study also established that the science equipment provided by government was not fully utilized due to lack of space to conduct practical sessions. The findings demonstrated that a number of schools specifically the USE schools lacked functional laboratories due to lack of apparatus, chemicals and reagents. In these schools, the laboratory materials remained locked up in the head teachers' offices or the Ministry of Education lockable cupboards. These findings are in agreement with New Vision article on wastage of laboratory chemicals worth billions of Uganda shillings because the schools lack laboratories to utilize the materials provided by the Ministry of Education and Sports (Nakajubi, 2017). Furthermore, findings revealed that failure to utilize the available resources was due to the lack of the teachers' competence resulting from the inadequate pre-service training (UNESCO, n.d).

Furthermore, the study found that teachers shunned using available resources arguing that the allotted time was not sufficient to engage learners on practical activities. These findings on the attitude of teachers using the resources to involve learners in activities during lessons conform to the ESAPR report of 2015/16 (MoES, 2016). The findings suggested that teachers think practical sessions delay completion of the syllabus; therefore, practical teaching of science subjects in majority of the schools across the sub-regions were mainly conducted in the candidate classes to prepare their candidates for the practical papers in the national examinations. This explains the theoretical teaching of science concepts in the lower classes despite availability of science kits and laboratories (MoES, 2015). Hands on activities call for time to plan, practice, set the necessary materials and clear the classroom after the lesson; those teachers who are not motivated, find this rather demanding and cannot spare time for such a process (MoES, 2015; UNESCO, n.d).

\section{Conclusion}

The study established that availability and utilization of instructional resources play a significant role in enhancing teacher effectiveness. The findings generally suggest that proper use of instructional resources helps teachers to gain and hold the attention of students. It also stimulates and attracts learners' attention during the teaching and learning process thereby promoting teacher effectiveness. However, in Uganda's government-aided secondary schools, instructional resources are inadequate, while the few available ones are not well utilized. The results further suggest that teachers hardly improvise the teaching and learning materials where the commercial or conventional materials are lacking.

To improve teacher effectiveness in government-aided secondary schools, the Ministry of Education and Sports and school administrators should avail teachers with instructional resources and supervise to ensure that the teachers appropriately use these resources. Government should construct more libraries and science laboratories especially for USE schools to promote easy accessibility of textbooks by learners and teachers and practical teaching of science subjects. The Ministry and 
schools should provide training to teachers on how use and improvise instructional resources to improve their effectiveness in the teaching and learning process.

\section{References}

Adeleke, A.A. (2005). Use of Library Resources by Academic Staff of the Nigerian Polytechnics, Journal of Library Science, 12(2) 15-24.

Armbruster P, Patel M, Johnson E, Weiss M. Active learning and student-centered pedagogy improve student attitudes and performance in introductory biology. CBE Life Sciences Education. 2009; 8:203-213.

Armstrong N, Chang SM, Brickman M. (2007). Cooperative learning in industrialsized biology classes. CBE Life Sciences Education. 6:163-171.

Agun, I \& Okunrotifa, P (1977). Educational Technology in Nigerian Teacher Education. NERDDC Press, Lagos.

Balogun, D. A., Okon, S.E., Musaazi, J.C.S, Thankur, A.S. (1981). Principles and Practices of Practice of Education. Lagos, Macmillan Nigeria Publishers Ltd.

Bizimana, B. \& Orodho, J. A., (2014). Teaching and Learning Resource Availability and Teachers' Effective Classroom Management and Content Delivery in Secondary Schools in Huye District, Rwanda. Journal of Education and Practice, Vol 5 (9), 111-122

Busingye, J.D. \& Najjuma, R. (2015). Do Learning And Teaching Materials Influence Learning Outcomes Amidst High Enrolments? Lessons from Uganda's Universal Primary Education, Africa Education Review, Vol 12 (1), 109-126,

Cohen, L., Manion, L. \& Morrison, K. (2007).Research Methods in Education (6 ${ }^{\text {th }}$ ed.). Routledge, Taylor \&Francis group, London

Creswell, J. W., \& Plano Clark, V. L. (2011). Designing and conducting mixed methods research (second Ed.). Thousand Oaks, CA: Sage.

Deslauriers L, Schelew E, Wieman C. (2011). Improved learning in a large-enrollment physics class. Retrieved from www.sciencemag.org on 03 ${ }^{\text {rd }}$ September 2017

Golightly, A. \& Raath. S. (2015). Problem-based learning to foster Deep Learning in Pre-service Geography Teacher Education. Journal of Geography. 114 (2), 5668 ,

Guloba, M., Wokadala, J. \& Bategeka, L. (2010). Does teachers ${ }^{\text {ee }}$ method and availability of teaching resources influence pupils"e performance: Evidence from four districts in Uganda. Economics Policy Research Centre, Uganda. Research Series 77

Ismaila, B.A. (1999). The relationship between teachers characteristics and students' academic achievement in secondary schools in Adamawa State, Nigeria. Unpublished M.Ed Dissertation, University of Maiduguri.

Ministry of Education and Sports (2012). Annual report of the Directorate of Education Standards. Kampala: The Government of Uganda.

Ministry of Education, Science, Technology and Sports (2014). The Education and Sports Sector. Annual Performance Report (ESAPR) (FY 2014/15). Kampala: The Government of Uganda. 
Ministry of Education and Sports (2015). Annual report of the Directorate of Education Standards. Kampala: The Government of Uganda.

Ministry of Education and Sports (2016). The Education and Sports Sector Annual Performance Report (ESAPR) (FY 2014/15). Kampala, The Government of Uganda.

Musaazi, J.C.S. (1982). The Theory and Practice of Educational Administration. Oxford: Macmillan Publishers Ltd.

Nakajubi, G. (2017, July 19). Laboratory Chemicals Worth Sh540b Dumped in Schools. The New Vision p. 22

Nannyonjo, H. (2007). Education Inputs in Uganda. An Analysis of Factors Influencing Learning Achievements in Grade Six. Working paper 98. Washington D.C, World Bank

Okwara, M.O., Shiundu, J. O. \& Indoshi, F. C. (2009). Towards a Model of Integrated English Language Curriculum for Secondary Schools in Kenya. Educational Research and Review. Retrieved from http:/ / academicjournals.org on $3^{\text {rd }}$ September 2017

Orji, A. B. (2000). Comparability of two problem-solving models in facilitating students' learning outcomes in physics. Journal of the Science Teachers Association of Nigeria. 35 (1 \& 22), 25 - 30.

Popoola, S.O., \& Haliso, Y. (2009). Use of library information resources and services as predator of teaching effectiveness of social scientists in Nigerian universities. AJLAIS Vol 19 (1): 65-77.

Ssekamwa, J.C., \& Lugumba S.M.E. (2010). Development and Administration of Education in Uganda, (2nd edition). Kampala: Fountain Publishers.

Smith MK, Wood WB, Adams, W. K., Wieman C, Knight J. K., Guild N, et al. (2009). Why peer discussion improves student performance on in-class concept questions. Science. (323) 122-124.

Uganda National Examination Board. (2015). The Achievement of S2 Students and Teachers in English Language, Mathematics and Biology. Kampala: Uganda National Examination Board.

Walls, R.T. (1999). Psychological foundations of learning. Morgantown, WV: WVU International Center for Disability Information.

Walls, R.T., \& Cather, W.L. (1987). Principles of instruction. Emittsburg, MD: National Emergency Training Center. 


\title{
Assessing Senior Engineering Student's Ability to Identify Graduate Attributes
}

\author{
Maraka Lefera \\ Central University of Technology Free State, \\ Bloemfontein South Africa \\ James Arthur Swart \\ Central University of Technology Free State, \\ Bloemfontein South Africa
}

\begin{abstract}
Universities around the world are responding to industry and accreditation body requests by embedding graduate attributes into their curricula. The Central University of Technology in South Africa has followed suit by stipulating 10 graduate attributes that academics need to cover in their curricula. It is important that engineering students be able to identify and understand these graduate attributes, as they need to demonstrate them through their entire educational career. The purpose of this paper is to demonstrate which graduate attributes senior engineering students can easily identify based on their understanding of the definition. A questionnaire was used as the data-collection instrument in a descriptive case study. Senior engineering students could easily identify 8 of the 10 graduate attributes that has a word that can be linked to the definition. The graduate attribute "communication" was identified by $92 \%$ of the students while $66 \%$ identified "entrepreneurship". However, the majority of students struggled to identify two graduate attributes, which are numeracy (6\% identified it) and technical and conceptual competence ( $40 \%$ identified it). A possible reason for this could be that there are no words in the definition that may be linked to the name of the attribute. It is therefore recommended that all graduate attributes be defined is such a way that a synonym or antonym of the attribute be used in the actual definition. This may assist students to better identify and understand vital graduate attributes that they need to acquire before entering Industry.
\end{abstract}

Keywords: assessment, graduates, universities, curriculum.

\section{Introduction}

"At the heart of self-regulated formative assessment is the capability to critically selfreflect when utilising feedback for improving learning towards reaching desired standards," was noted by Sin \& Mcguigan, (2013). Assessment of graduate attributes 
reflects on student's capability to identify graduate attributes. Students should be able to identify graduate attributes in order to meet industry standards and attributes ensures students ability to be effective members of the industry.

Universities around the globe are responding to the call by industry and professional bodies that graduates attributes be embedded into the curriculum, so that students may be able to demonstrate them (Meda \& Swart, 2017). Different authors agree that universities have to cultivate required skills and abilities amongst their graduates in order for them to meet the needs of industry. According to Hughes \& Barrie (2010), universities must pay attention to the delivery and assessment of graduates attributes. Graduate's attributes have not been considered as learning outcomes that are integrated into the curriculum, but rather as a set of generic outcomes that need to be implemented outside of the learning environment. Furthermore, Green, Hammer \& Star (2009), illustrate that global trends have prompted universities worldwide to do more for their students than just teaching disciplinary content. They emphasize that these trends have driven the agenda of developing personal, generic and transferable skills to improve the employability of graduates in different countries.

Herok, Chuck \& Miller (2013), indicate that the assessment of graduates attributes is fraught with challenges. One stated challenge is that it may not always be open to an objective evaluation. This claims are based on outcomes which feature verbs that are difficult to quantify or measure, such as understand (Meda \& Swart, 2017). Moreover, the number of years learning, rather than of weeks based on contextdependent. On the other hand, Sin \& McGuigan (2013), relate that there are challenges associated with developing graduate attributes. They are limited to traditional assessment approaches for assessing the learning outcomes of modules and courses. In addition, this challenge is typically diverse, indeterminate and requires judgement of quality from multiple perspectives (Sin \& McGuigan, 2013). This means that if it is difficult to assess graduate attributes, it may also be difficult to identify or understand them. This may be true for both academics and students in higher education.

The purpose of this paper is to determine which graduate attributes senior engineering students can easily identify based on their understanding of the definition. A descriptive case study is used where quantitative data was gathered from a pre-workshop questionnaire. The paper begins with defining graduate attributes as adopted by the Central University of Technology (CUT), followed by the context of the study. The research methodology, results and discussions then follow. Succinct conclusions are finally presented.

\section{Graduate attributes adopted by CUT}

The International Engineering Alliance (IEA) (Alliance, 2013) defines graduate attributes as clear, succinct statements of the expected capability, qualified, if necessary, by a range indication appropriate to the type of programme. These graduate attributes are stipulated for engineers (Washington Accord), engineering 
technologists (Sydney Accord) and engineering technicians (Dublin Accord). These Accords list a number of graduate attribute profiles that engineering student must gain. The Washington Accord focuses on solving complex engineering problems and results in a professional engineering graduate. The Sydney Accord focuses on the application of engineering procedures, processes, systems or methodologies and results in an engineering technologist graduate. The Dublin Accord focuses on wide practical procedures and practices, resulting in an engineering technician graduate. Moreover, graduate attributes serve to identify the distinctive characteristics as well as the areas of commonality between the expected outcomes of different modules within a given curriculum.

CUT has responded to the needs of industry and professional bodies by trying to implement 10 graduate attributes into their curricula. According to CUT's strategic plan (often called Vision 2020 (Strategic Plan, 2016-20)), these 10 graduate attributes have been mandated for inclusion into all university curricula. Table 1 illustrates the 10 CUT graduate attributes, with their accepted definitions.

Table 1: Graduate attributes at CUT

\begin{tabular}{|c|c|}
\hline $\begin{array}{l}\text { Graduate } \\
\text { Attribute }\end{array}$ & Definition \\
\hline 1. Numerate & $\begin{array}{l}\text { Use basic mathematics, budgeting and financial management } \\
\text { skills. }\end{array}$ \\
\hline 2. Teamwork & $\begin{array}{l}\text { Work independently and in teams, to manage your own } \\
\text { learning, work and take responsibility for self while } \\
\text { contributing to teams such as learning communities. }\end{array}$ \\
\hline 3. Communication & $\begin{array}{l}\text { Communicate proficiently, in oral, written, presentation, } \\
\text { information searching and listening skills. Be assertive and } \\
\text { articulate, be able to negotiate responsibly and persuade } \\
\text { others. }\end{array}$ \\
\hline $\begin{array}{l}\text { 4.Innovation and } \\
\text { Problem Solving }\end{array}$ & $\begin{array}{l}\text { Be innovative, think creatively and critically and apply a } \\
\text { range of strategies to solve/find solutions for real world } \\
\text { problems. Demonstrate the ability to apply theoretical } \\
\text { knowledge that will lead to development of new ideas, } \\
\text { methods, techniques, practices, products and services in a } \\
\text { variety of contexts (technology, commerce, social systems). }\end{array}$ \\
\hline $\begin{array}{l}\text { 5.Community } \\
\text { Engagement }\end{array}$ & Be socially engaged in your communities. \\
\hline $\begin{array}{l}\text { 6.Sustainable } \\
\text { Development }\end{array}$ & $\begin{array}{l}\text { Be environmentally sensitive and recognize your role as a } \\
\text { socially responsible citizen who care for the common good of } \\
\text { others, the country and environment. }\end{array}$ \\
\hline $\begin{array}{l}\text { 7.Technologically } \\
\text { Literacy }\end{array}$ & Use information and communication technologies effectively. \\
\hline $\begin{array}{l}\text { 8.Citizenship and } \\
\text { Global Leadership }\end{array}$ & $\begin{array}{l}\text { Make a meaningful and positive contribution to society, be } \\
\text { ethical and visionary leaders who can show leadership. }\end{array}$ \\
\hline
\end{tabular}




\begin{tabular}{|l|l|}
\hline $\begin{array}{l}\text { 9. } \\
\text { Entrepreneurship }\end{array}$ & $\begin{array}{l}\text { Be entrepreneurial, industrious and be able to recognize } \\
\text { opportunities; turn them into ideas for enterprises. You shall } \\
\text { have business acumen and display basic business skills. }\end{array}$ \\
\hline $\begin{array}{l}\text { 10.Technical and } \\
\text { Conceptual } \\
\text { Competence }\end{array}$ & $\begin{array}{l}\text { Demonstrate depth of specialized disciplinary knowledge } \\
\text { and skills and be able to apply them in different contexts to } \\
\text { solve problems. }\end{array}$ \\
\hline
\end{tabular}

The implementation of graduate attributes commenced in January 2014, underpinning CUT's academic learning programmes. They have since been integrated into the teaching and learning process, into the curricula and into other activities, such as co-curricular, cultural and sporting activities and work-integrated learning (WIL). It is important to note that ALL these graduate attributes need to be demonstrated by ALL students over their entire educational career within one qualification or programme. This suggests that some graduate attributes would be found in the first year of study, with others in the second or third year of study. This means that the sum of ALL the graduate attributes would be assessed over the total period of the entire programme.

It is often said that engineering students must be able to solve problems and use technology effectively (Phang, et al., 2016). One could say that these graduate attributes should be found in every module within a given study programme or engineering qualification. They are listed as number 4 and 7 by CUT, and as number 2 and 5 by the IEA who use the terms Problem Analysis and Modern Tool Usage (Wendel, Minichiello, Graham, Graham, \& Zundel, 2017). Other two graduate attributes that were easily identified by students are sustainable development and teamwork. Sustainable development features the word "environment" in its definition, while team work features the word "groups" in its definition. This makes it easier for student to identify the graduate attribute form a given definition.

\section{Context of the study}

CUT has four main faculties on its main campus in the Free State Province of South Africa. The four faculties cover Engineering and Information Technology, Health and Environmental Sciences, Humanities and Management Sciences. Table 2 presents the number of registered students in the four faculties for 2015 and the number of full time staff members working at CUT. This shows that Engineering and Information Technology has the highest number of students and staff members requiring more workshops to create awareness of the importance of graduate attributes.

A Student Peer Mentorship programme commenced in 2013 in order to better help first-year student's transition into higher education. This programme was initially funded by the Department of Higher Education to address low success rates, graduation rates and throughput rates in order to enhance student learning. This Student Peer Mentorship Programme was implemented in all four faculties at CUT, where senior engineering students were appointed as mentors to a group of 10 firstyear students (mentees). These mentors are required to welcome first-year students 
to the University, assisting them with orientation and information regarding basic student support and information about the faculty. All faculties have their own Student Peer Mentorship co-ordinator who is responsible for organizing workshops and training for their appointed mentors. However, the context of this study is limited to mentors who were appointed in 2017 in the Faculty of Engineering and Information Technology at CUT. The main reason for this is due to the fact that the authors only had access to these mentors.

Table 2: Number of registered students in the four faculties in 2015

\begin{tabular}{|l|l|l|}
\hline Faculties & $\begin{array}{l}\text { Number of } \\
\text { students } \\
\text { registered }\end{array}$ & $\begin{array}{l}\text { Number of } \\
\text { full time staff } \\
\text { members }\end{array}$ \\
\hline $\begin{array}{l}\text { Faculty of Engineering and Information } \\
\text { Technology }\end{array}$ & 4213 & 88 \\
\hline $\begin{array}{l}\text { Faculty of Health and Environmental } \\
\text { Sciences }\end{array}$ & 1642 & 54 \\
\hline Faculty of Humanities & 2358 & 74 \\
\hline Faculty of Management Sciences & 3390 & 80 \\
\hline
\end{tabular}

There are five departments in this faculty, namely Electrical engineering, Mechanical engineering, Civil engineering, the Built environment and Information Technology. Senior students from second year to fourth year level who are performing well academically are appointed as mentors. These students must be able to work well with fellow students and academics, displaying good leadership, interpersonal and organisational skills. Mentors in the faculty were given training on graduate attributes in January of 2017, in order for them to better convey the meaning and importance of them to their mentees. Mentors should therefore be able to correctly identify the 10 graduate attributes of CUT.

\section{Method}

\section{Participants}

The target population for this study comprised all 72 mentors in the faculty, thereby negating a sampling technique. These mentors were invited to a follow-up workshop on how to assess graduate attributes, which was held in May 2017. However, only 51 attended the workshop, which becomes the sample size of this research. The sample size is acceptable as it represents $75 \%$ of all mentors in the faculty.

\section{Design}

A descriptive case study is used with quantitative data. Yin (2009), points out that a case study is an empirical inquiry that investigates a contemporary phenomenon in depth and within its real-life context, especially when the boundaries between phenomenon and context are not evident. Yin (2009), states that there are three types of case studies, which are exploratory, descriptive, and multiple case studies. A descriptive case study is used where a situation (identifying graduate attributes) is described within a real life context (mentors who are mentoring first-year students). 


\section{Data collection instrument}

Data was collected through a questionnaire that was distributed to the participants before the workshop commenced. Mentors were asked to complete the questionnaire and return it to the facilitator. The questionnaire was anonymous, being compiled using the institutions strategic plan document and graduate attributes information brochure. The 10 graduate attributes and their definitions were extracted from the brochure and from the strategic plan document. This contributes to the content validity and reliability of the questionnaire. Content validity (McMillan \& Schumarcher, 2010) refers to the study's aim, scope and relevance to the value being measured and the accuracy of the measurements to be made. The reliability of a research is related to the repeatability of measurements and the similarity of results for measurements at different times.

\section{Descriptive statistics}

Descriptive data is presented in bar graphs, where the demographics of the participants and the questionnaires results are shown. It is important to include participant's demographic information when presenting findings because they provide credible information of the study. According to Kohler, Londis, \& Cortina, (2017), reviewers often rely on the demographics of the sample size. The researchers should collect and provide all the relevant information about the demographic attributes of the sample that may be related to how individuals might respond to the selected measures. These include, but are not limited to gender, age, nationality, and tenure in the organization and university programme. Furthermore, the purpose of the demographic information is to assist the reader to be able to assess the degree to which sample of the study may have driven the findings and limit potential of generalising (Kohler, Lundis \& Cortina, 2017).

\section{Results and discussions}

The demographics of the mentors is shown in Figure 1. The findings show that most of the students are male (71\%). The majority of students are aged between 21 and 25 years of age (male students comprise $80 \%$ of this group), with the dominant home language being Sesotho. The findings compare well with the national head count by Case, Marshall and Grayson (Case, Marshall, \& Grayson, 2013) who found that the student population in South Africa are primarily between 20-24 years of age. Hodges and Park (Hodges \& Park, 2013) research findings show that the majority of $77 \%$ of students in Engineering are male. 


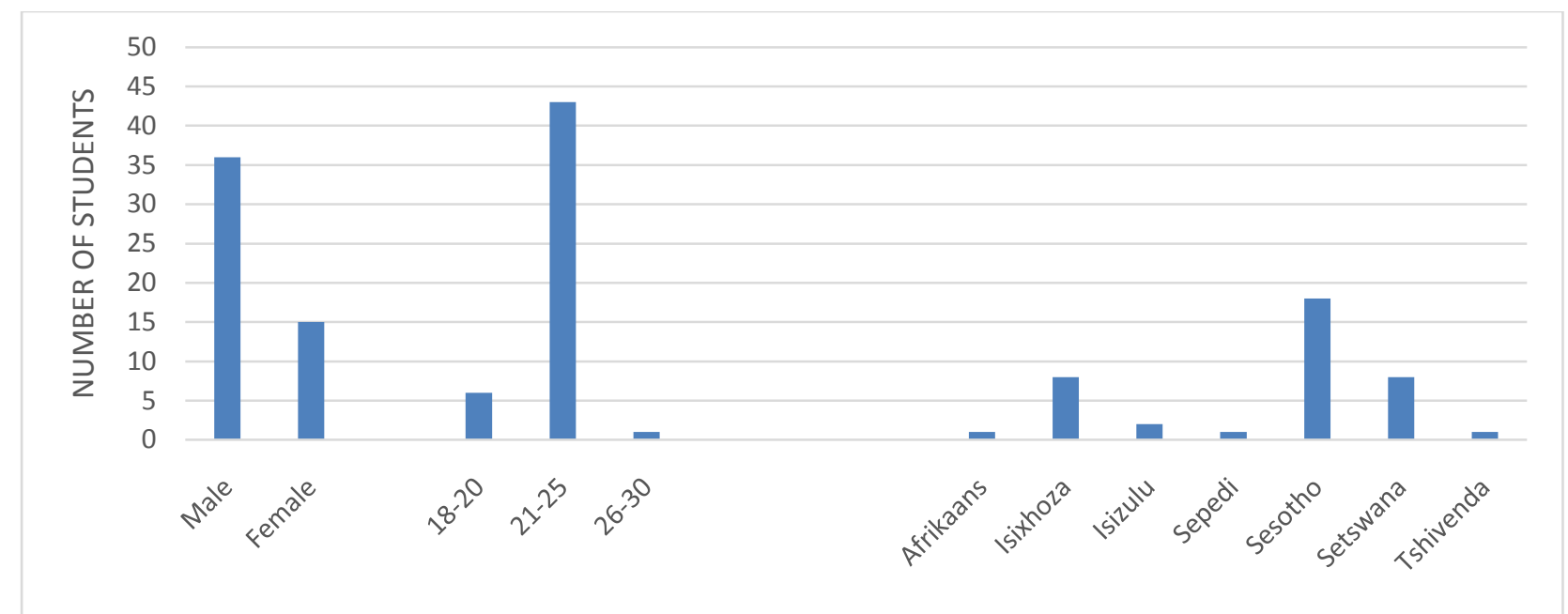

Figure 1: Demographic Information of students

The results of the graduate attributes assessment are illustrated in Figure 2. The results indicate two graduate attributes that the mentors struggled to identify correctly, namely technical and conceptual competency and numeracy. The other eight graduate attributes (teamwork, communication, problem solving, community engagement, sustainable development technological literacy, entrepreneurship, citizenship and global leadership) were correctly identified by more than $65 \%$ of the mentors. It is important to note that the correctly identified graduate attributes have a synonym in their definition that may be traced back to the attribute name. For example, problem-solving attributes is linked with challenges, communication is linked with the word talk and community engagement attribute is linked with the word communities.

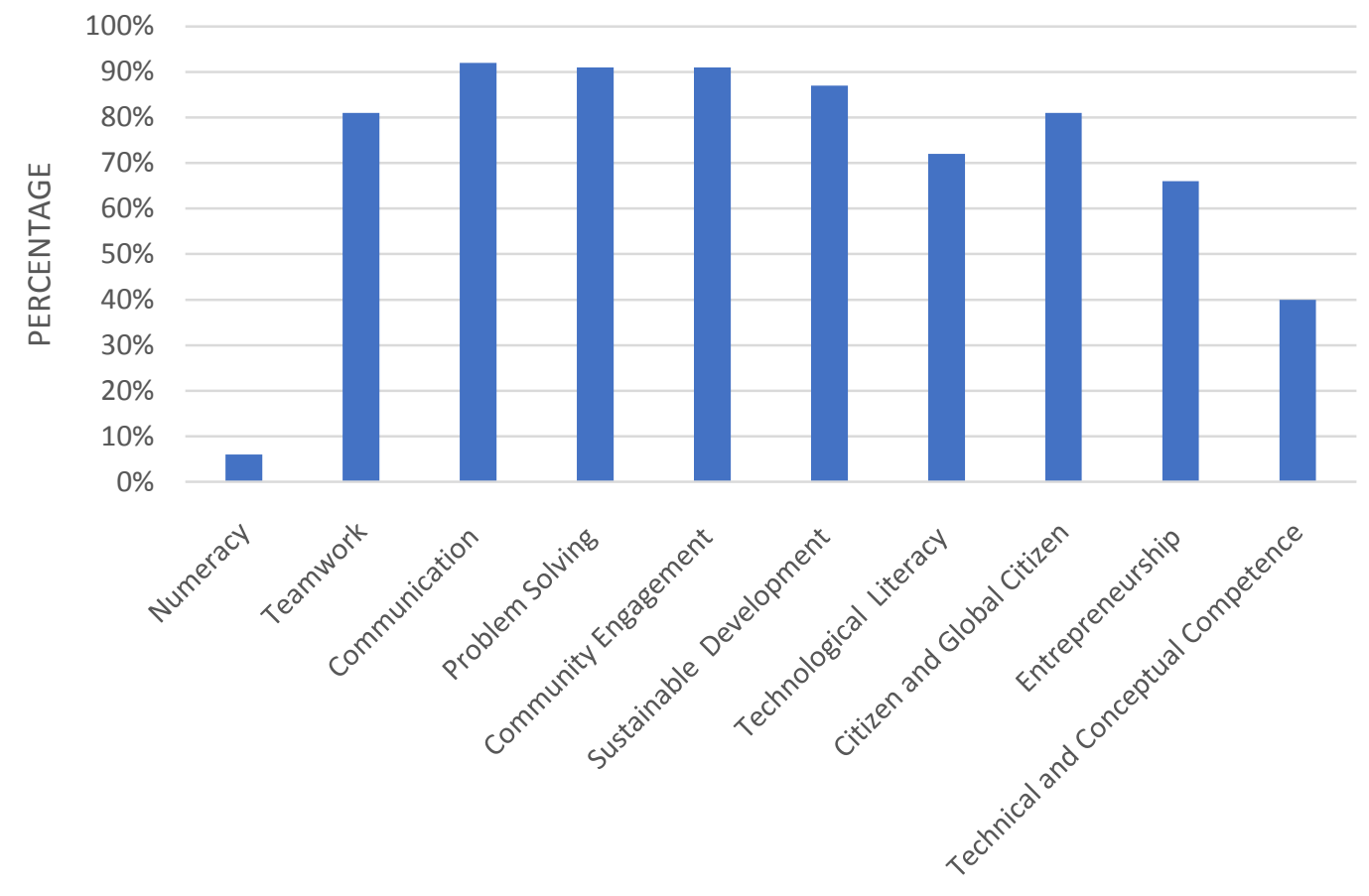

GRADUATE ATTRIBUTES

Figure 2: Results of Graduate Attributes Assessment 


\section{Conclusion}

The purpose of this paper was to determine which graduate attribute senior engineering students can easily identify based on their understanding of the definition. Although the majority of the students who are mentors could identify most of graduate attributes, it was difficult for them to identify and understand two graduate attributes, being numeracy and technical and conceptual competency. Therefore, a lack of understanding of these graduate attributes may result from the inability to correctly identify them. When mentors are not able to understand and identify graduate attributes, it reinforces the need for more workshops to create awareness of what these attributes are and what they really entail. What is not known is whether all senior engineering students in the faculty can easily identify each graduate attributes based on their understanding of the definition. Further research is needed in this regard.

Mentors were assessed on graduate attributes in a form of a questionnaire. Results illustrates that the mentors could easily identify eight out of the ten attributes. However, they struggled to identify technical and conceptual competence and numeracy, as there is no word in the definition that may be linked (synonym or antonym) to the name of the attribute. It is recommended that the definitions of the graduate attribute include a synonym or antonym related to the name of the attribute, which may help students to better identify and understand them. This may also assist engineering students to better demonstrate these graduate attributes through their educational career, as they prepare for their work career in industry.

\section{References}

Alliance, I. E. (2013). Graduate Attributes and Professional Competencies. IEA website: http://www.ieagreements.org., 3, 1-16.

Case, J., Marshall, D., \& Grayson, D. (2013). Mind the gap:Science and engineering eduaction at the secondary-tertiary interface. South African Journal of Science, 109(8), 2-5.

Green, W., Hammer, S., \& Star, C. (2009). Facing up to the challenge: Why is it so hard to develop graduate attributes? Higher Education Research and Development, 28(1), 1729.

Herok, G. H., Chuck, J., \& Miller, T. J. (2013). Teaching and Evaluating Graduate Attributes in Science Based Disciplines. Creative Education, 4(7A2), 42-49.

Hodges, A. J., \& Park, B. (2013). Oppositional Identities: Dissimilarities in How Women and Men Experience Parent Versus Professional Roles. Journal of Personality and Social Psychology, 105(2), 193-216.

Hughes, C., \& Barrie, S. (2010). Influences on the assessment of graduate attributes in higher education. Routledge Taylor and Francis Group, 35(3), 325-334. 
Kohler, T., Lundis, R., \& Cortina, J. M. (2017). From the Editors:Establishing Methodological Rigor in Quantitative Management Learning and Education Research: The Role of Design, Statistical Methods, and Reporting Standards. Academy of Management Learning and Education, 16(2), 173-192.

McMillan, S., \& Schumarcher, J. H. (2010). Research in education: Evidence based inquiry. London: Pearson.

Meda, L., \& Swart, A. J. (2017). Graduate Attributes in an Electrical Engineering. International Journal of Engineering Education, 33(2), 653-661.

Meda, L., \& Swart, A. J. (2017). Analysing learning outcomes in an Electrical Engineering curriculum using illustrative verbs derived from Bloom's Taxonomy. European Journal of Engineering Education, 1-14.

Phang, F. A., Anuar, A. N., Aziz, A. A., Yusof, K. M., Hassan, S. A., \& Ahmad, Y. (2016). "Perception of Complex Engineering Problem Solving Among Engineering Educators". In Engineering Education for a Smart Society (pp. 215-224). Cham: Springer.

Sin, S., \& McGuigan, N. (2013). Fit for Purpose: A Framework for Developing and Assessing Complex Graduate Attributtes in a changing Higher Education Environment. Accounting Education: an international journal,, 22(6), 522-543.

Strategic Plan. (2016-20). GRADUATE ATTRIBUTES. BLOEMFONTEIN AND WELKOM: Central University of Technology,Free State.

Wendel, S., Minichiello, A., Graham, K., Graham, J., \& Zundel, J. (2017). "Developing an Introduction to Mechanical Engineering Course at Utah State University Based Upon National Foudation. 2017 ASSE Rocky Mountain Section Conference. Denver.

Yin, R. K. (2009). Case Study research. Los Angels: CA: Sage.

Yin, R. K. (2009). Case Study Research: Design and Methods. California: Thousand Oaks. 


\title{
Gendered Influence of Artisanal and Small Scale Mining on Primary School Pupils' Learning Achievements in Western Tanzania
}

\author{
Boaz Kamugisha \\ Nyakahura Secondary School, \\ Biharamulo District Council, \\ P.O. Box 193, Biharamulo, Tanzania
}

Theodora A.L. Bali

St John's University of Tanzania, Faculty of Humanities and Education, P.O. Box 47, Dodoma, Tanzania

\author{
Abich Dah Omollo \\ University of Dodoma, \\ College of Education, \\ P.O. 523, Dodoma, Tanzania
}

\begin{abstract}
This study explored a critical step towards improving quality of education in ASM area by addressing the problems concerning children's rights and gendered child rearing. It was guided by the social cognitive theory in explaining the role of environment in moulding children's behaviour. It measured three objectives: examining the kinds of artisanal and small scale mining activities contribution to development of adjacent primary schools; assessing the influence of artisanal and small scale mining activities on primary school pupils' learning achievements; and exploring the influence of ASM activities on pupils' learning achievements by gender. It employed a cross sectional survey design and was conducted in Biharamulo District located Western Tanzania. The respondents were head teachers, primary school teachers, primary school pupils, and ASM leaders, obtained through purposive sampling techniques. Data were collected through observation, interviews, questionnaire, documentary review, and focus group discussion. Data were analysed and presented through tables, figures and thematic analysis. Chisquare test was performed to assess the influence of ASM on pupils' learning achievement. It was found that the influence of ASM activities on primary school pupils' learning achievements was significant $-\mathrm{X}^{2}=7.133(1), a=0.008$, with a symmetric measure coefficient (Phi $=-.501, \alpha=.003$ ). The negative coefficient value
\end{abstract}


of .50 indicates that ASM activities have large negative effect on pupils learning. Gender-wise, ASM activities affected boys more compared to girls - X $X^{2}=5.819(1)$, a $=0.016$, with a symmetric measure coefficient $(\mathrm{Phi}=-0.69, \mathrm{a}=0.003)$.

Keywords: Child artisans, pupils and small scale mining, gendered child neglect.

\section{Introduction}

The concept of Artisanal and small scale mining (ASM) refers to labour intensive mining with low technological methods and belongs to the informal sector of the economy. The influence of small scale mining activities on education is not a new phenomenon in the World history. It goes as far back as 1716 when the first school was built at Joachimstal, Czechoslovakia as the output of mining activities (Choshi, 2001). The mining projects have contributed to social developments, for instance, in Malawi where primary schools were built in different mining places, E.g. the primary school at Mchenga coal mines in Rumphi and at Namadidi lime-making factory, serving the entire community. These primary schools help children living adjacent to the mines to access education, hence reducing illiteracy in the country (Dreschler, 2001).

For years the international community has been commenting on ASM's potential for playing an important role in economic and social development in developing and rural contexts. A 2002 UN-organized conference in Yaoundé, Cameroon emphasized through its "Yaoundé Vision" 50 that if properly harnessed, ASM and partner institutions can contribute to the UN Millennium Development Goals (MDGs). They suggested that strategic support and interventions in ASM could help in achieving universal primary education (UPE) by decreasing the involvement of children in the ASM sector through a process of eliminating the economic benefits of child labour, substituting children's jobs with technology and making school a viable, affordable and accessible alternative to children living in ASM communities (Villegas, et al., 2012). Yet evidence of ASM persistent use of child labour still exists (Thorsen, 2012; Gyamfi, 2014). Although no specific theory has addressed this phenomenon, scholars in different disciplines have tried to highlight the relationship between ASM activities and children's schooling (Jimenez, 2011; Noronha et al., 1998).

The extraction of minerals and precious stones in ASM is governed by complex social hierarchies of land owners, license holders, buyers, watchmen, employers, miners, auxiliary workers, and others who can make a living off the sites. Children, whether directly involved in mining or indirectly through a range of auxiliary services, are usually at the bottom of this hierarchy due to their age, gender and body size which shape the work they do (Thorsen, 2012). However, the International Labour Organisation (ILO) estimates that nearly 1 million children between the age of 5 and 17 years work in the mines and quarries (Gyamfi, 2014).

Dreschler (2002) cites the Tanzania employment ordinance (1995, Section 2), which defines a child as a person under the apparent age of fifteen (15) years. The ordinance emphasizes (in Section 77) that children of apparent age of twelve (12) 
years shall not be employed in any capacity of whatsoever. It provides further that no child shall be employed in any employment which is injurious to health, dangerous, or otherwise unsuitable. Yet the persistence of ASM-related child labour is common.

On the other hand, child education in ASM areas has attracted debate all over the world because access to education is generally very poor in the artisanal mining areas. In 2009-2010, the net enrolment rate was 60.6 per cent, and only 56.3 percent of children completed primary school (Jimenez, 2011). For instance, in India, the majority of people living in ASM areas lack education despite of the modern education systems and adult literacy programs, as skills are regarded to be more lucrative than education. This appears to be resulting in adolescent girls deciding to assist their mothers at the quarries rather than pursuing education (Noronha, Khalwadekar, \& Mahambre, 1998). In Mali, child labour in artisanal mining is attributed to lack of access to education. In Tanzania, child labour issues were raised in several studies of mining, particularly those led by the ILO. As has been noted elsewhere, children engage in ASM related activities, particularly in the gold mining areas. Many school children undertake what are regarded as 'petty chores' in the ASM industry. School boys, far more than girls, in Nyarugusu and Mgusu mines were found in the processing tasks such as carrying ore to washing places, washing the ore, amalgamation, and manual crushing of tailings (Mwaipopo et al., 2004).

Many children fit ASM-related work around schooling, while others leave primary school for mine work (Mwaipopo et al., 2004). This highlights an important point on the potential influence of ASM activities on students' learning. The reviewed studies however, did not discuss the influence of ASM on primary school pupils' learning achievement. Hence our study sought to fill-in the gap by:

1. Examining the kinds of contribution made by artisanal and small scale mining activities to development of adjacent primary schools in Biharamulo District.

2. Assessing the influence of artisanal and small scale mining activities on primary school pupils' learning achievements in Biharamulo District.

3. Exploring the influence of ASM activities on pupils' learning achievements in Biharamulo District by gender.

\section{Method}

\section{Participants}

Fifty eight participants were recruited for this study, using simple random and purposive techniques. Out of those, 12 teachers were selected randomly, while 36 pupils, six head teachers and four ASM leaders were purposively selected. Participants' age range was 10 to above 50 years. Gender representation was observed in recruiting pupil participants.

\section{Design}

This study was designed to assess two categorical variables, namely gender difference in the influence of ASM activities (independent variables) and primary school pupils' learning achievements (dependent variable). Chi-square tests were performed to test if there was significant difference between the learning 
achievements of pupils from ASM areas and from non-ASM areas. Also the test of no significant difference in the influence of ASM activities on boys' and girls' learning achievements was performed. This design was deemed appropriate for categorical variables and small sample.

\section{Materials}

The study employed questionnaire for primary school teachers, interview guide for head teachers and ASM leaders, and focus group discussion guide for pupils. Observation and documentary review checklists as well as a camera and phone recorder were also used to collect data. Specifically, observation field notes and photographs of children's involvement in ASM activities were taken.

\section{Procedure}

Questionnaires were distributed to teachers at schools and collected as soon as the participants finished filling them. Head teachers and ASM leaders were interviewed in their respective offices. Focus group discussion (FGD) was held with six to seven pupils from ASM areas per school. FGDs were held in exclusive classrooms on premises, moderated by the researcher. A question, "How do ASM activities affect your studies" was posed to participants in each FGD. They were encouraged to be frank and to feel free to decline at every point. Participants' informed consents were obtained continously, while ensuring confidentiality of their private information. Prior to their participation in the study, pupils were given letters explaining the purpose of the study to seek permission from their parents and guardians. Only children 10 years of age and above with signed permission from their parents/guardians participated in FGDs. During the data collection, the researcher explained the reasons of the study to respondants and personal identifiers were not used on piences of data collected. That's why photographs were artistically reconstructed in order to secure pupils' faces from being identified. In the report, the researcher acknowledged sources of ideas whereever they have been used and discribed the surveyed school using letters, instead of real names.

\section{Results and Discussion}

\section{ASM Contribution to Adjacent Primary Schools}

Slightly more than half $(59 \%)$ of the respondents said that the ASM sites contribution to development of adjacent primary schools was not much. Table 1 presents participants' responses on ASM sites' contribution to adjacent primary schools.

Table 1: Respondents' Views on Kinds of Contribution from ASM Sites to Adjacent Primary Schools $(\mathrm{N}=58)$

\begin{tabular}{lcc}
\hline Kind of Contributions & Frequency & Percentage \\
\hline Pupil Porridge & 9 & 15.5 \\
Pupil Uniform & 5 & 8.6 \\
Pupils' Stationary & 5 & 8.6 \\
Classroom \&Teachers' house Building & 2 & 3.4 \\
Others not Listed & 3 & 5.1 \\
None & 34 & 59 \\
Grand Total & $\mathbf{5 8}$ & $\mathbf{1 0 0}$ \\
\hline
\end{tabular}

Source: (Field data, 2015) 
Among other reasons for ASM miners' failures to contribute something for to the development of the adjacent primary schools include their unwillingness. According to respondents, sometimes school administrators and village authorities had to exert pressure on miners in order to get some contribution. These findings somehow contradict the existing literature. Studies in the Republic of South Africa and Malawi have indicated that, ASM projects had positive social impact of skills development and the potential to uplift rural and sometimes poorer communities (Dreschler, 2002). It has been documented that in partnership with the Department of Education, they built primary schools in different mining places and provided infrastructure to schools including classrooms, teaching and learning materials, administration blocks, water provision, boarding facilities for children with special needs, and a security guard house (Dreschler, 2002). No evidence indicates that ASM miners were pressurized to give these contributions.

Unfortunately, the reasons as to why the Beharamulo ASM miners were unwilling to contribute could not be established in this study. At best, participants reported that some miners lacked awareness on the importance of contributing to development of their primary schools. It was lamented that ASM miners do not realize that they should give back to the community in terms of contribution to social services for the treasures they accumulate from the area. E.g. miners also don't seem to understand that building classrooms, teacher houses, and making desks for pupils can be productive in the long run.

\section{Influence of ASM Activities on Primary School Pupils' Learning Achievements}

Data on this theme are presented in three parts: engagement of pupils in ASM activities, effects of ASM activities on pupils' learning outcome and academic performance of pupils from ASM areas and non-ASM areas in that order.

\section{Engagement of Pupils in ASM Activities}

Findings have shown ways the school children involve themselves in ASM activities. Particularly in the gold mining areas, many school children undertake ASM-related activities regarded as Nyoka wa shimoni, literally 'pit snakes'. As nyoka wa shimoni, they do all sorts of odd jobs from spying to stealing mineral particles. School boys and girls, in mining sites are found in the processing tasks such as carrying sand containing mineral particles, washing or cleaning minerals, extracting minerals, selling food and taking care of siblings during ASM activities.

Table 2: Respondents' Views on Pupils' Engagement in ASM Activities ( $N=58)$

\begin{tabular}{clcc}
\hline S/N & ASM Activities & Frequency & Percent $\%$ \\
\hline $\mathbf{1}$ & Carrying sand with minerals to washing areas & 11 & 18.9 \\
$\mathbf{2}$ & Washing minerals & 12 & 20.6 \\
$\mathbf{3}$ & Taking care of sibling during ASM activities & 8 & 13.7 \\
$\mathbf{4}$ & Selling foods and drinks & 2 & 3.4 \\
$\mathbf{5}$ & Mineral extracting & 4 & 6.8 \\
$\mathbf{6}$ & All of the above mentioned & 3 & 5.1 \\
$\mathbf{7}$ & None of the above mentioned & 18 & 31 \\
$\mathbf{8}$ & Grand Total & $\mathbf{5 8}$ & $\mathbf{1 0 0}$ \\
\hline
\end{tabular}

Source: (Field data, 2015). 
One of the standard seven pupils, aged 15 years bragged in FGD that he is mwoshaji (Mineral cleaner) because he has been working as mineral cleaner for the past four years (See Figure 2). During the fieldwork, the researcher came across some children who were engaging in ASM activities due to their parents' involvement in mining as shown in Figure 1.

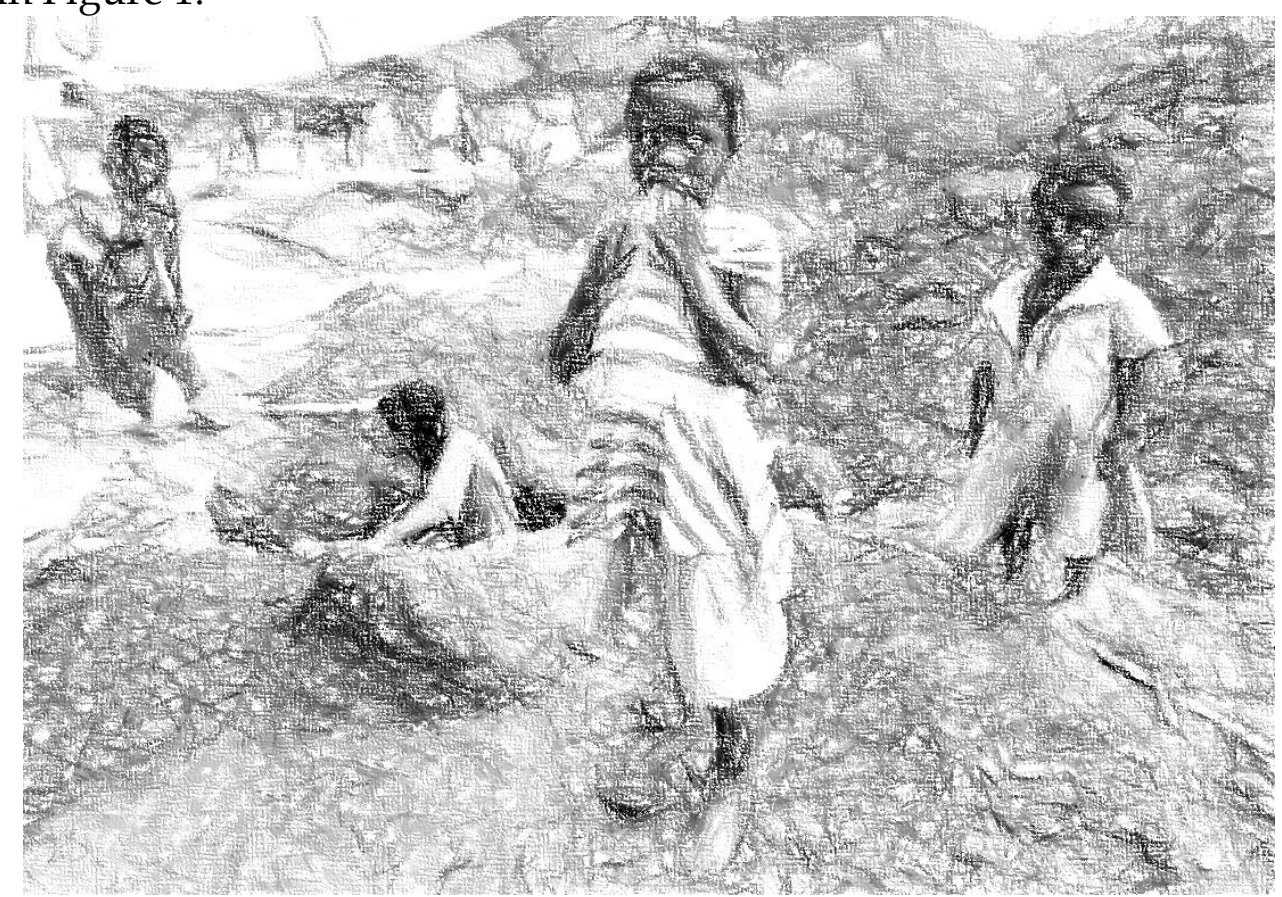

Figure 1: A Picture of Children Engaging in ASM Activities

Source: (Reconstructed from field data photographic information, 2015)

The observation around the mining sites during fieldwork revealed that children were carrying out ASM activities including carrying sand with mineral particles, extracting minerals during school time (See Figure 1 and Figure 2). When they were asked if they were pupils, they responded affirmatively and admittedly confessed that they skipped school for two days. This finding was corroborated by pupils' own accounts in the FGD where they disclosed having regularly involved in ASM activities. 


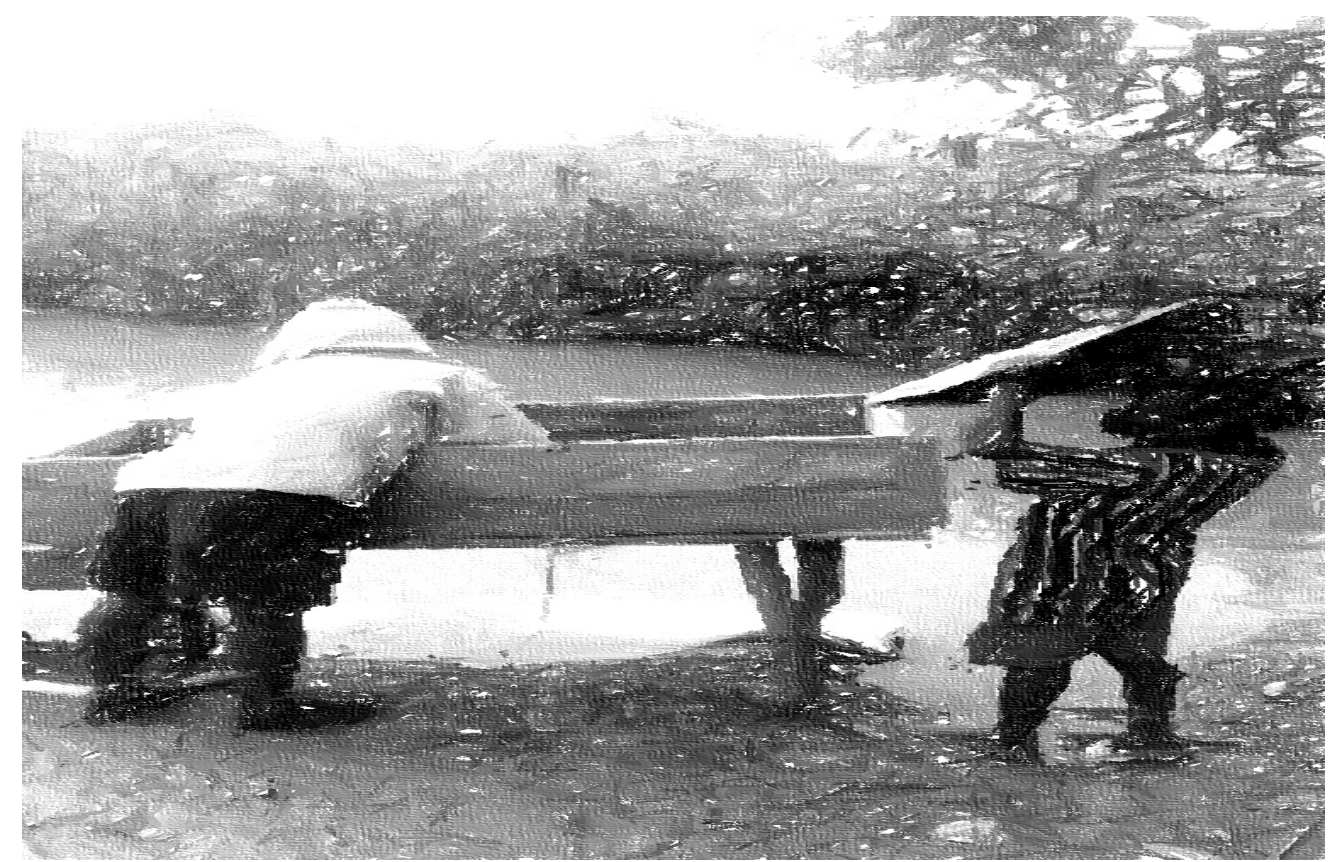

Figure 2: A Picture of Pupils Washing Minerals at a Mining Site.

Source: (Reconstructed from field data photographic information, 2015).

One of the head teachers lamented that this practice is fostering the persistence of child labour in ASM areas:

School administration has no full support from ASM leaders in reproaching and stopping pupils' involvement in ASM activities. Instead, they protect truant pupils in order to continue working with them (Translated from Kiswahili, 2015)

The study also revealed that most children in ASM activities work on contract basis whereby parents or elders pay them immediately after completion of an activity. This shows that ASM families depend on child labour in some areas in order to earn living. This observation concurs with Kuramoto (2001) who observed that the mining families conserve cultural patterns that justify child labour, especially in the areas where migrant labour from agricultural communities is concentrated. The involvement of children in ASM activities affects their schooling in terms of attendance and completion. Those children whom the researcher came across washing minerals during schooling hours justified missing school in order to earn money to support their family:

We have not gone to school today because our mother is sick and at home there is no money for her treatment, even for buying our basic needs. Our father passed away so we have to struggle for life (Researcher's translation, 2015).

Some children are involved in ASM activities due to involvement of their parents (reference is made to Figure 1). In a casual conversation with children during fieldwork observation, one of the children aged seven years disclosed: 
Whenever I ask my mother when I will start schooling, she says next year because there is nobody to take care of my young sister (Researcher's translation, 2015).

\section{Effects of ASM Activities to Pupils' Learning Outcome}

The implication of ASM activities for pupils' learning outcome was the gist of the matter for this study. Table 3 shows respondents' accounts on the implications of ASM activities to pupils' participation in learning.

Table 3: Respondents' Response on Implications of ASM Activities to Pupils' Learning Outcome (N=58)

\begin{tabular}{llcc}
\hline S/N & Outcomes & Frequency & Percent $\%$ \\
\hline 1 & Poor attendance & 15 & 25.8 \\
2 & Moral decay & 5 & 8.6 \\
3 & Late coming & 6 & 10.1 \\
5 & Early marriage & 8 & 13.7 \\
6 & School Dropouts & 10 & 17.2 \\
7 & All above mentioned & 6 & 10.1 \\
8 & Disagreed & 8 & 13.7 \\
& Grand Total & $\mathbf{5 8}$ & $\mathbf{1 0 0}$ \\
\hline
\end{tabular}

Source: (Field Data, 2015)

Poor attendance and school dropouts were the leading effects of ASM activities on pupils' learning. One of the head teachers reported that ASM activities affect pupils school attendance and completion as many pupils who come from ASM areas do not complete standard VII due to influence of former school dropout pupils who seem to be earning some money from ASM activities. In the FGD, one standard seven girl lamented on how ASM activities affected her studies and her sister's who got married early:

I could have been in form two now. In the year 2009 my parents shifted from Ushirombo to Busili purposely to engage in ASM activities. By then, I was in standard three and my elder sister was in standard six. When we arrived we did not continue with studies until 2011 when I repeated standard three. But my sister couldn't; instead she got married in 2013 (Researcher's translation, 2015). 
Table 4: Registered Pupils from ASM Areas for Current Standard Seven Pupils since year 2012 to March 2015

\begin{tabular}{cccccccccc}
\hline Schools & \multicolumn{3}{c}{ Std Four } & \multicolumn{3}{c}{ Std Seven } & \multicolumn{3}{c}{ Dropouts } \\
\hline & Boys & Girls & Total & Boys & Girls & Total & Boys & Girls & Total \\
\hline A & 15 & 7 & 22 & 9 & 3 & 12 & 6 & 4 & 10 \\
B & 8 & 5 & 13 & 5 & 3 & 8 & 3 & 2 & 5 \\
C & X & X & X & X & X & X & X & X & X \\
D & 10 & 16 & 26 & 8 & 12 & 20 & 2 & 4 & 6 \\
E & 7 & 2 & 9 & 5 & 0 & 5 & 2 & 2 & 4 \\
F & 9 & 6 & 15 & 6 & 3 & 9 & 3 & 3 & 6 \\
Grand & & & & & & & & & \\
Total & $\mathbf{4 9}$ & $\mathbf{3 6}$ & $\mathbf{8 5}$ & $\mathbf{3 3}$ & $\mathbf{2 1}$ & $\mathbf{5 4}$ & $\mathbf{1 6}$ & $\mathbf{1 5}$ & $\mathbf{3 1}$ \\
\hline
\end{tabular}

Source: (Field Data, 2015)

$\mathbf{X}=$ school $\mathbf{C}$ by 2012 had no standard Four.

Records of attendance and registration of pupils from ASM areas were reviewed. Table 4 shows the pupils from ASM areas who were registered in all five primary schools between 2012 and 2015. School C was established in 2010 that's why in year 2012 it had no standard four pupils. The data show a total of 85 registered pupils (49 boys and 36 girls) in 2012. In 2015, the number of pupils had dropped to 54 registered pupils (33 boys and 21 girls). In other words, 31 pupils (16 boys and 15 girls) had dropped out of schools. Among other reasons for school dropout for were ASM activities and girls were early pregnancy. Only two pupils died.

\section{Academic Performance of Pupils from ASM Areas and Non - ASM Areas}

The academic performance of pupils who came from ASM areas and those from non ASM areas were compared (See Table 5). Annual examination results of 36 pupils (18 who came from ASM areas and 18 from non ASM areas) were analyzed using Chisquare test to assess significant difference in the influence of ASM activities on pupils' learning achievement. Table 6 shows the Chi-Square test results, while Table 7 shows the symmetric measure coefficient.

Tables 5 and 6 reveal that pupils who come from non-ASM activities had good performance academically. Only five (05) out of 18 had low academic performance, while 14 out 18 of those from ASM areas performed poorly. Table 6 shows that the corrected value of 7.133 , with an associated significance level of 0.008 , which is smaller than the alpha value of .05. This indicates that the result is statistically significant, indicating that ASM activities have significant influence on pupils' learning achievement. The Phi coefficient value of symmetric measure shown in Table 7 is -.50, suggesting a large effect on pupils' learning achievement that leads to pupils in mining areas not studying well. Indeed, ASM activities lead to pupils' poor classroom attendance, moral decay, school dropout and early marriage. They are bound to affect pupils' learning achievement negatively. 
Table 5: Response of Pupils on the involvement in ASM Activities in Relation to their Academic Performance (from documentary review $\mathrm{N}=36$ )

\begin{tabular}{|c|c|c|c|c|c|}
\hline & \multicolumn{2}{|c|}{$\begin{array}{l}\text { Pupils' Academic } \\
\text { Performance }\end{array}$} & \multirow[b]{2}{*}{ Total } \\
\hline & & & \begin{tabular}{|c|} 
Low \\
Academic \\
Performance
\end{tabular} & $\begin{array}{c}\text { Good } \\
\text { Academic } \\
\text { Performance }\end{array}$ & \\
\hline \multirow{4}{*}{$\begin{array}{l}\text { Are you involved } \\
\text { in ASM activities? }\end{array}$} & \multirow[t]{2}{*}{ No } & Count & 5 & 13 & 18 \\
\hline & & $\begin{array}{l}\text { Expected } \\
\text { Count }\end{array}$ & 9.5 & 8.5 & 18.0 \\
\hline & \multirow[t]{2}{*}{ Yes } & Count & 14 & 4 & 18 \\
\hline & & $\begin{array}{l}\text { Expected } \\
\text { Count }\end{array}$ & 9.5 & 8.5 & 18.0 \\
\hline \multirow[t]{2}{*}{ Total } & & Count & 19 & 17 & 36 \\
\hline & & $\begin{array}{l}\text { Expected } \\
\text { Count }\end{array}$ & 19.0 & 17.0 & 36.0 \\
\hline
\end{tabular}

Source: (Field data, 2015).

Table 6: Chi-Square Test of Results of Table 5

\begin{tabular}{|c|c|c|c|c|c|}
\hline & Value & Df & $\begin{array}{l}\text { Asymp. Sig. } \\
\text { (2-sided) }\end{array}$ & $\begin{array}{l}\text { Exact Sig. (2- } \\
\text { sided) }\end{array}$ & $\begin{array}{l}\text { Exact Sig. (1- } \\
\text { sided) }\end{array}$ \\
\hline $\begin{array}{l}\text { Pearson Chi- } \\
\text { Square }\end{array}$ & $9.028^{a}$ & 1 & .003 & \multirow{6}{*}{.007} & \multirow{6}{*}{.003} \\
\hline $\begin{array}{l}\text { Continuity } \\
\text { Correction }^{\mathrm{b}}\end{array}$ & 7.133 & 1 & .008 & & \\
\hline Likelihood Ratio & 9.456 & 1 & .002 & & \\
\hline $\begin{array}{l}\text { Fisher's Exact } \\
\text { Test }\end{array}$ & & & & & \\
\hline $\begin{array}{l}\text { Linear-by-Linear } \\
\text { Association }\end{array}$ & 8.777 & 1 & .003 & & \\
\hline $\begin{array}{l}\mathrm{N} \text { of Valid } \\
\text { Cases }^{\mathrm{b}}\end{array}$ & 36 & & & & \\
\hline
\end{tabular}

a. 0 cells $(.0 \%)$ have expected count less than 5 . The minimum expected count is 8.50 .

b. Computed only for a $2 \times 2$ table

Source: (Field data, 2015) 
Table 7: The Symmetric Measure Coefficient of Tables 5 and 6

\begin{tabular}{|l|l|c|c|}
\hline & & Value & Approx. Sig. \\
\hline $\begin{array}{l}\text { Nominal by } \\
\text { Nominal }\end{array}$ & Phi & -.501 & .003 \\
\cline { 2 - 4 } & Cramer's V & .501 & .003 \\
\hline N of Valid Cases & 36 & \\
\hline
\end{tabular}

Source: (Field data, 2015)

These findings are consistent with Fuller et al., (cited in UNICEF, 2000) who revealed that to achieve academically, children must attend school consistently. A child's exposure to curriculum - his or her 'opportunity to learn' - significantly influences achievement, and exposure to curriculum comes from being in school. Similarly, a study of village-based schools in Malawi and many others found that students with higher rates of attendance had greater learning gains and lower rates of repetition (Miske, Dowd et al., cited in UNICEF, 2000).

\section{Influence of ASM Activities on Pupils' Learning Achievements by Gender}

A significant gender difference in the influence of ASM activities on pupils' learning achievement was explored. This question was posed to two groups of respondents namely, head teachers and teachers. Documentary review was also used to verify the difference and relationship between boys' and girls' learning achievements. Figure 3 shows respondents' accounts on how boys and girls are affected differently by ASM activities.

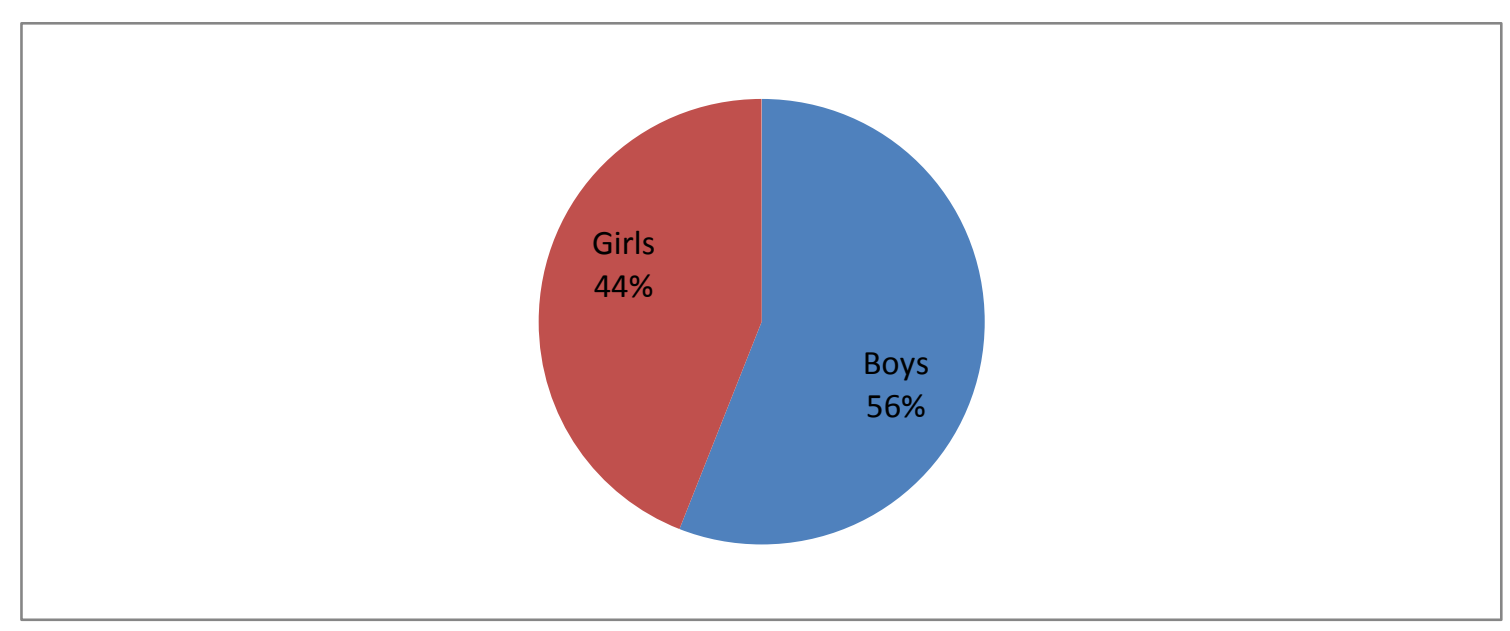

Figure 3: ASM Activities Effects to Pupils by Gender ( $N=18)$

Source: (Field data, 2015). 
During interviews with the head teachers, they reported that the effect of ASM activities on pupils' learning achievements varies with gender. Head teachers said that most pupils who engage in ASM activities are boys and academically they do not perform well compared to girls. This is because boys spend much time on ASM activities hence being are absent from school more. From the documents reviewed, namely registration book, attendance book and previous annual examination results of 2014, it was found that 38 pupils (21 boys and 17 girls) dropped out of schools between 2012 and 2015. This suggests that boys were more likely to be affected by ASM activities than girls. A chi-square test of no significant difference by gender was performed to examine the relationship between ASM activities and pupils' academic achievement (See Table 8, 9 and 10).

Table 8 shows 18 head teachers and teachers' responses to the question: "is there significant difference in the influence of ASM activities on academic performance of pupils by gender?" Respondents correctly said boys are affected more by ASM activities than girls and so they perform poorly. Only three girls out of eight performed poorly, all 10 boys performed poorly. There is no down in this case that ASM activities influence on boys' learning achievement than girls'. Table 9 shows the corrected value of 5.819, with an associated significance level of 0.016 , which is smaller than the alpha value of .05 . This means that the null hypothesis of no significant difference in the influence of ASM activities on academic achievement of boys and girls is not retained. Boys are more affected academically than girls. The phi coefficient value of "symmetric measure" is -.69, suggesting a large effect of the influence of ASM activities on boys' academic achievementas a result of boys in mining areas not studying well. It is thus, recommended that the government and other education stakeholders should prevent the involvement of children in ASM activities, regardless of their gender. 
Table 8: The Influence of ASM Activities on Pupils' Academic Performance by Gender

\begin{tabular}{|c|c|c|c|c|c|}
\hline \multicolumn{6}{|c|}{$(\mathrm{N}=18)$} \\
\hline & & & \multicolumn{2}{|c|}{$\begin{array}{l}\text { Academic performance of } \\
\text { pupils basing on gender }\end{array}$} & \multirow{2}{*}{ Total } \\
\hline & & & $\begin{array}{c}\text { Low } \\
\text { performance }\end{array}$ & $\begin{array}{c}\text { High } \\
\text { performance }\end{array}$ & \\
\hline \multirow{4}{*}{$\begin{array}{l}\text { Gender which is } \\
\text { mostly involved } \\
\text { in ASM activities }\end{array}$} & Girls & Count & 3 & 5 & 8 \\
\hline & & $\begin{array}{l}\text { Expected } \\
\text { Count }\end{array}$ & 5.8 & 2.2 & 8.0 \\
\hline & Boys & Count & 10 & 0 & 10 \\
\hline & & $\begin{array}{l}\text { Expected } \\
\text { Count }\end{array}$ & 7.2 & 2.8 & 10.0 \\
\hline \multirow[t]{2}{*}{ Total } & & Count & 13 & 5 & 18 \\
\hline & & $\begin{array}{l}\text { Expected } \\
\text { Count }\end{array}$ & 13.0 & 5.0 & 18.0 \\
\hline
\end{tabular}

Source: (Field data, 2015)

Table 9: The Chi-Square Test of Results on the Influence of ASM Activities on Pupils' Academic Performance by Gender.

\begin{tabular}{|c|c|c|c|c|c|}
\hline & Value & Df & $\begin{array}{c}\text { Asymp. Sig. } \\
\text { (2-sided) }\end{array}$ & $\begin{array}{l}\text { Exact Sig. } \\
\text { (2-sided) }\end{array}$ & $\begin{array}{l}\text { Exact Sig. } \\
\text { (1-sided) }\end{array}$ \\
\hline Pearson Chi-Square & $8.654^{a}$ & 1 & .003 & \multirow{6}{*}{.007} & \multirow{6}{*}{.007} \\
\hline $\begin{array}{l}\text { Continuity } \\
\text { Correction }^{b}\end{array}$ & 5.819 & 1 & .016 & & \\
\hline Likelihood Ratio & 10.685 & 1 & .001 & & \\
\hline Fisher's Exact Test & & & & & \\
\hline $\begin{array}{l}\text { Linear-by-Linear } \\
\text { Association }\end{array}$ & 8.173 & 1 & .004 & & \\
\hline $\mathrm{N}$ of Valid Cases ${ }^{\mathrm{b}}$ & 18 & & & & \\
\hline
\end{tabular}

a. 2 cells (50.0\%) have expected count less than 5 . The minimum expected count is 2.22 .

b. Computed only for a $2 \times 2$ table

Source: (Field Data, 2015) 
Table 10: The Symmetric Measure Coefficient from Tables 8 and 9

\begin{tabular}{|ll|c|c|}
\hline & & Value & Approx. Sig. \\
\hline Nominal by & Phi & -.693 & .003 \\
Nominal & Cramer's V & .693 & .003 \\
Nof Valid Cases & 18 & \\
\hline
\end{tabular}

Source: Field Data (February, 2015)

\section{Conclusion}

A number of conclusions are drawn regarding the research objectives: First, ASM activities have negligible positive contribution to primary school pupil's learning achievements given that the owners of ASM sites barely contribute to social service projects unless pressured in some ways. Unintended negative contribution include school dropout among pupils due to failure to get school contributions such as token money for school feeding- i.e. porridge, uniforms, school infrastructure building, among others. Second, poor attendance and school dropout as a result of involvement in ASM activities mostly contribute to the negative influence on pupils' learning achievement as exemplified by Chi-square test $-X^{2}=7.133(1), a=0.008$, with asymmetric measure coefficient (Phi $=-.501, \mathrm{a}=.003)$. Third, the most negatively affected pupils' gender performance-wise as a result of ASM activities is male $-X^{2}=5.819(1), \alpha=0.016$ with the asymmetric measure coefficient value (Phi $=-$ $0.69, a=0.003)$. This indicates that ASM activities exert negative influence more on academic achievement of boys than girls'. Nonetheless, children should be protected from involvement in ASM activities, regardless of their gender.

\section{References}

Ary, D. Jacob S.C and Sorensen, C (2010) Introduction to Research in Education $8^{\text {th }}$ edition; Wadsworth Cengage learning Canada.

Bandura, A. (1986). Social Foundations of Thought and Action. Englewood Cliffs, NJ: Prentice-Hall.

Bandura, A. (1989). Human Agency in Social Cognitive Theory. American Psychologist, 44 (9), 1175-1184.

Choshi, S. (2001). Mining Minerals Sustainable Development Southern Africa: MMSDSA Regional Research. Africa Institute of Corrporation Citizenship.

Gyamfi, A.E (2014) Journal of Education \& Human Development; New York

Jimenez, R. (2011). A poisonous Mix: Child Labour, Mercury, and Artisanal Gold Minig in Mali: Human Rights Watch.

Kuramoto, J. (2001). Artisanal and Informal Mining in Peru: Country Study commissioned by MMSD,53p. http://www.iied.org/mmsd/.

Mwaipopo, R., Mutagwaba, W., Nyange, D and Fisher, E., (2004). Increasing the Contribution of Artisanal and Small - scale Mining to Poverty Reduction in Tanzania: Based on an Analysis of Mining Livelihoods in Misungwi and Geita Districts, Mwanza Region. A Report Prepared for the Department for International Development. Available online at www.swan.ac.uk/cds/pdffiles/TANZANIA20ASM20 REPORT.pdf. $21 / 11 / 2014$. 
Noronha, L., Khalwadekar, S. \& Mahambre, S. (1998). The Small Scale Indian Laterite Stone Quarry and Working Sector: A case Study of Goa. New Delhi: Tata Energy Research Institute.

Ormrod, J. E. (1999). Human learning (3rd ed.). Upper Saddle River, NJ: Prentice-Hall Thorsen, D (2012). Children Working in Mines and Quarries; UNICEF.

UNICEF. (2000). Defining Quality in Education. Working paper series. New York: UNICEF. Acessed on 30 th October, 2017 from https://www.unicef.org/education/files/QualityEducation.PDF

Villegas, C., Weinbergerg R., Levin, E., and Hund, K. (2012).Artisanal and Small Scale Mining in Protected Areas and Critical Ecosystems Programme (ASM $P A C E)$ : A Global Solution Study, Estelle Levin, Ltd and WWF Nairobi Kenya. 


\title{
The Relationship between Contingencies of Self- Worth and Happiness with Academic Achievement of High School Male Students
}

\author{
Professor Firouzeh Sepehrianazar \\ Urmia University \\ Urmia, Iran
}

\author{
Abolfazl Heidari \\ Urmia University Urmia, Iran
}

\author{
Roshank Fallah \\ Azad university, \\ Tabriz, Iran
}

\begin{abstract}
Academic achievement has been one of the major issues in psychology and affiliated sciences over a century. There are many factors involved with that, one of them is a sense of happiness among students. And, the other one is self- esteem of students. So, the purpose of this study was to examine the relationship between contingencies of self-worth and happiness with academic achievement in male students of second high school. The research method was descriptive and correlations study. The statistical population of research consisted of all the male students of second high school Shahriyar City, Iran (3800 people). First of all, according to Morgan's suggested table, 340 students were selected by multistage cluster random sampling from this population. Second, all participants were asked to complete contingencies of self-worth tests of Crocker et al., and Oxford Happiness Questionnaire. The final academic semester GPA was considered as a measure of academic achievement. Then, the data were analyzed with descriptive and inferential statistics such as frequency, percentage, mean, standard deviation, Pearson's correlation coefficient and regression analyses. The results of Pearson correlation revealed that there were significant relation between the contingencies of self -worth and happiness with academic achievement. And, the correlation between happiness and contingencies of self- worth were negative and significant. In general, the result of regression analysis indicated that happiness is the best predicator of academic achievement. Educational specialists should pay attention to psychological variables along with formal education.
\end{abstract}


Keywords: Academic Achievement, Contingencies of Self-Worth, Happiness.

\section{Introduction}

Academic performance is one of the top priorities for schools and academic achievement refers to the learner's acquired abilities in school subjects that are measured through standardized learning tests or teacher-made tests (Navidi, 2003). Some psychological factors play an important role to promote or decline academic performance (Sepehrianazar, 2013). It can be studied in two general categories of factors: individual factors and the educational system (Soleymannejad \& Shahraray, 2001). One of individual factors is Contingent self-worth. Cracker (2001) introduced this new concept in psychology by studying the origin of self-esteem. The meaning of Contingent self-worth is self-esteem based on the approval of others or on social comparisons. It is certain events will shape one's self-esteem when the individual bases their self-worth on the outcome of those events (Chip, Canevello, Bush, Cook, 2008). Relationship contingent self- worth is an unhealthy form of self-esteem that depends on one's relationship and represents a particular kind of relationship investment (Cross \& Morris, 2003 cited by Chip \& colleagues). Contingent selfworth is a fragile component of self-esteem and refers to the extent to which selfesteem is contingent upon outcomes and achievement (Kernis, 2002 cited by Chen, 2011). Jennifer Crocker and Connie Wolfe (2001) argue that how and why events affect the self- centers on the notion of contingent self-esteem. And they believe that we can understand the relationship between self-esteem and behavior by examining specific sources of self-esteem. Crocker and Wolfe (2001) studied the concept of contingent self-esteem within specific domains. They claimed that major domains of contingent self-esteem among college students are appearance, competition, family support, perception of God's love, approval from others, school competence and behavior. Students who base their self-esteem on their academic successes use their school work to prove their intelligence. Since failures in domains of contingency have negative effects on one's self-esteem, students will avoid failure by increasing their effort. Jennifer Crocker and Riia K Luhtanen (2003) in a longitudinal study of 642 college students show that academic competence contingency predicted academic problems.

The other variable that studied in this research was happiness. It is one of the important variables that effects on the students' lifestyles and academic achievement (Headarzadegan \& Mashdiabbas, 2011). Happiness has three components; emotional, social and cognitive components. The emotional component makes the person happy. The social component enhances social relations and social support. The cognitive component causes a person to think and process his particular information (Myes \& Diener ,1995, cited by Carr, 2004). Tabbodi, Rahgozar, Mozaffari Makki Abadi (2015) studied on 320 students and showed that There is a significant positive correlation between happiness and the progress of students. Lyubomirsky, King, \& Diener, et al., 2005, cited by Marie Spice (2011) found that people with high levels of happiness have good performance job than those who are less happy. Thelwell, Lane \& Weston, (2007) with studied on 57 undergraduate 
students, showed that mood states is one of their variable that predicted examination performance.

In this article, we describe a program of research that examining contingencies of self-worth and happiness associated with academic achievement to get a better picture of contingent self-esteem and happy in academic domains among high school students. We tested three hypotheses: There is a meaningful relationship between happiness and achievement academic. There is a negative meaningful relationship between contingencies of self-worth and happiness. Does contingencies of self-worth and happiness predict academic achievement? What is the contribution of each in predicting academic achievement?

\section{Method}

\section{Participants}

The statistical population of the present study are all of the male students who, studying in second grade of Shahriar city, Iran (3800 people). Their age range was 15 to 16 years.

Study samples of the study were 340 students. They were selected by multistage cluster random sampling and based on Morgan's table.

\section{Design}

The method of this study is a descriptive (non-experimental) one. The design of study is correlational.

\section{Materials}

In the present study, two instruments have been used. Semester GPA of the academic year was considered as a measure of academic achievement.

Contingencies of self-worth tests of Crocker et al.:

The questionnaire was designed by Cracker and her colleagues in 2003. They reported the reliability of this scale in terms of Cronbach's alpha equal to 0.73 (Cracker and colleague, 2003).

Contingencies of self-worth tests is self-report instrument and consisted of 35 items. This scale has seven subscales of approval from others, physical appearance, outdoing others in competition, family love and support, being a virtuous or moral person, and God's love (Crocker, Luhtanen, Cooper, \& Bouvrette, 2003). Cronbach's alpha of this study was 0.80 . In the present study, the reliability coefficient of subtests was 0.35 to 0.80 .

\section{Oxford Happiness Questionnaire (OHQ):}

The Oxford Happiness Questionnaire was developed by Argyle, M., \& Crosland, J. at Oxford University in 1989. This scale has 29 items. Each item was scored on a 4 point Likert scale ranging from 'not at all' (0) to 'too much' (3). For example:

I am not particularly optimistic about the future

I feel optimistic about the future. 
I feel I have so much to look forward to.

I feel that the future is overflowing with hope and promise.

Hills and Argyle (2002) reported the reliability of the questionnaire equal to 0.91 . Cronbach's alpha of this study was 0.89. In the present study, the reliability coefficient was 0.40, 0.55, 0.65, 0.67 and 0.81 for all subtests, Self-esteem, Efficiency, the health, Positive mood and Satisfaction respectively.

\section{Procedure}

The students responded simultaneously to the contingencies of self-worth tests of Crocker et al., and Oxford Happiness Questionnaire. Subjects responded to the tests in group class within 20 minutes at the classroom.

\section{Results}

First, Data obtained through the questionnaires were submitted to SPSS version 18. Then, the descriptive indices of variables (mean, standard deviation, Skewness and Kurtosis) have been presented in order to check the normal distribution of the data. Skewness and Kurtosis indices suggest the normality of data distribution. The data by using statistical Pearson correlation test and multivariable regression analysis analyzes were performed. Before examining the Regression test, all of regression assumptions were examined.

Table 1 represents the descriptive statistics including means, SDs as well as the low, and high.

Table 1 - Descriptive statistics of Variables $(n=340)$

\begin{tabular}{|l|r|r|c|l|}
\hline Variable & Mean & SD & Low & High \\
\hline family support & 26.30 & 4.97 & 5 & 35 \\
\hline competition & 25.37 & 5.33 & 5 & 35 \\
\hline appearance & 20.14 & 4.66 & 8 & 33 \\
\hline God 's love & 28.87 & 4.80 & 9 & 35 \\
\hline school competence & 24.36 & 5.48 & 5 & 35 \\
\hline virtue & 24.65 & 5.66 & 5 & 35 \\
\hline approval from others & 17.37 & 6.47 & 5 & 35 \\
\hline contingencies of self-worth & 166.79 & 22.44 & 89 & 222 \\
\hline Satisfaction & 20.99 & 6.60 & 1 & 32 \\
\hline Positive mood & 14.40 & 4.53 & 0 & 23 \\
\hline the health & 11.24 & 3.50 & 1 & 18 \\
\hline Efficiency & 5.95 & 2.59 & 0 & 12 \\
\hline Self-esteem & 3.54 & 1.51 & 0 & 6 \\
\hline Happiness & 56.12 & 15.97 & 12 & 85 \\
\hline GPA & 16.68 & 2.33 & 10 & 20 \\
\hline
\end{tabular}

The correlations between contingencies of self-worth, happiness with academic achievement were calculated using Pearson's correlation coefficients (see Table 2). 
Table 2: Correlation between contingencies of self-worth, happiness with academic achievement

\begin{tabular}{|l|l|l|}
\hline Variable & $\mathbf{R}$ & $\mathbf{P}$ \\
\hline family support & 0.15 & 0.005 \\
\hline competition & 0.158 & 0.004 \\
\hline appearance & 0.076 & 0.16 \\
\hline God 's love & 0.107 & 0.49 \\
\hline school competence & 0.198 & 0.001 \\
\hline virtue & 0.206 & 0.001 \\
\hline approval from others & 0.119 & 0.29 \\
\hline contingencies of self-worth & 0.244 & 0.001 \\
\hline Satisfaction & 0.255 & 0.001 \\
\hline Positive mood & 0.171 & 0.002 \\
\hline the health & 0.200 & 0.001 \\
\hline Efficiency & 0.277 & 0.001 \\
\hline Self-esteem & 0.177 & 0.001 \\
\hline Happiness & 0.260 & 0.001 \\
\hline
\end{tabular}

According to results (see Table 2), there is a significant correlation between contingencies of self-worth, happiness with academic achievement. The correlations between contingencies of self-worth with happiness were calculated using Pearson's correlation coefficients. The result of Pearson's correlation coefficients shows that there is negative relationship between contingencies of selfworth and happiness.

A multiple regression analysis was carried out to find which of the variables predicts academic achievement. Results of the analysis have been summarized in Table 3.

Table 3: Summary of Regression Analysis of Academic Performance According to Variables

\begin{tabular}{|l|l|l|l|l|l|l|l|l|l|l|l|}
\hline Predictor variables & $B$ & Beta & SE & $T$ & $P$ & $F$ & $P$ & $R$ & $R^{2}$ & $\begin{array}{l}\text { Adjusted } \\
R \text { square }\end{array}$ \\
\hline Constant & 12.18 & - & .914 & 13.33 & .001 & 16.90 & .001 & .302 & .091 & .086 \\
happiness & 0.028 & .193 & .008 & 3.42 & .001 & & & & & \\
contingencies of & 0.017 & .168 & .006 & 2.97 & .003 & & & & & \\
self-worth & & & & & & & & & &
\end{tabular}

The result of multiple regression analysis revealed that happiness (Beta $=0.193, \mathrm{P}<0$. 001) was the best predicators of academic achievement. Contingencies of self-worth $($ Beta $=0.168, \mathrm{P}<0.001)$ is a significant predictor of academic achievement (see Table 3). They explanation $.091 \%$ variance of academic achievement. There are some other factors which have roles in predicting academic achievement, which have not been investigated in this research.

\section{Discussion}

As mentioned above, the aim of the present research was to examine the relationship between contingencies of self-worth and happiness with academic achievement. In response to the research hypothesis number one, the result of Pearson correlation test showed that there is a positive and significant correlation between all subscales 
of happiness and academic achievement (table2). This result is consistent with some studies such as Thelwell, Lane \& Weston (2007) and Tabbodi and Colleagues (2015). In explaining of this finding, we can say that, students with higher positive affect have more energetic, and more focused on their goals. As a result, they gain more success in their education.

In response to the research hypothesis number two; The result of Pearson correlation test showed that there is a negative and significant correlation between happiness and contingencies of self-worth $(\mathrm{r}=-0.34, \mathrm{P}=0.001)$. In explaining of this finding, we can say that, students with contingencies of self-worth are constantly trying to gain approval from others. They may be constantly worried about their appearance or school competence. So, they have less happiness in school and social activities.

In response to the research hypothesis number three, the results from multi-variable regression indicated that happiness and contingencies of self-worth predicted of academic achievement of students. These findings are similar with the findings of many previous international studies, such as Crocker and Luhtanen (2003). In this study happiness was the best predictor of academic achievement $(\beta=0.193)$. Selfefficacy was the best predictor of academic achievement among the components of happiness $(\beta=0.207)$. And virtue was the best predictor of academic achievement among the components of contingencies of self-worth $(\beta=0.205)$. In explaining of this finding we can say that, students with contingencies of self-worth try to gain approval from others and prove their competence. They use their school work to prove their own value. So, they will do more to get academic achievement, But they may not be happy. students with happiness and Self-efficacy have a positive mood, so they work to succeed with higher energy in the educational environment.

\section{Conclusion}

The results of this research show that those who have a sense of happiness have higher academic achievement. This finding show that Education officials should pay attention to the feelings of students. The findings of this study would also help teachers know and understand that their student's happiness and contingencies of self-worth have significant roles in students' academic achievement, and help teachers knows that there is negative relationship between contingencies of selfworth and happiness. Consequently, increasing student happiness in school is essential for improving academic outcomes. Furthermore, this study is recommended to improve real self- esteem in students without making their selfworth contingencies to domains.

On the limitations of this research, it can be said that this research was only conducted in a small city of Iran with the second grade of male high school students. So it is impossible to generalize the findings to students of other schools of the country. In spite of the mentioned limitations and according to the findings, the present research is recommended that future research study the relationship between academic acheivement with other variables. We suggest that researcher conduct this study with female students. 


\section{References}

Argyle, M. \& Crossland, J. (1987). Dimensions of positive emotions. British Journal of Social Psychology, 26, 127-37.Gilligan, C. F. (1982): In a different voice: Psychological theory and women's development. Cambridge, MA: Harvard University Press.

Chen, L. (2011). Contingent Self-esteem in Chinese Early and Late Adolescents. Submitted to the graduate degree program in Psychology, University of Kansas

Crocker, J., Luhtanen, R. K., Cooper, M. L., \& Bouvrette, A. (2003). Contingencies of self-worth in college students: Theory and measurement. Journal of Personality and Social Psychology, 85, 894-908.

Crocker, J. \& Wolfe, C. T. (2001). Contingencies of self-worth. Psychological Review, $108,593-623$.

Crocker,J. and Luhtanen, R K. (2003). Effects on Academic, Social, and Financial Problems in College Students. Personality and Social Psychology Bulletin. 29(6):701-12

Duckworth, A. (2007). Happiness and Academic Achievement: Evidence for Reciprocal Causality. https://www.researchgate.net/publication/237751866

Hills, P. \& Argyle, M. (2002). The Oxford Happiness Questionnaire: a compact scale for the measurement of psychological well -being. Personality and Individual Differences, 33, 1073-1082.

Heidarzadegan, A. \& Mashhadiabbas, F. (2011). Relationship of happiness and happiness with academic success of students of Sistan-Baluchestan University. Quarterly of Culture at Islamic University. 1, 83-69. (Persian)

Knee, CR, Canevello, A., Bush, M L. \& Cook,A. (2008). Relationship-Contingent SelfEsteem and the Ups and Downs of Romantic Relationships. Journal of Personality and Social Psychology. 95 (3): 608-627. doi:10.1037/00223514.95.3.608.

Lawrence J S. \& Crocker J. (2009). Academic contingencies of self-worth impair positively- and negatively-stereotyped students' performance in performancegoal settings. Journal of Research in Personality. 43(5):868-874

Marie Spice, L. (2011). The Effect of Induced Happiness Levels on Academic performance. A Thesis Presented to the Department of Psychology College of Liberal Arts and Sciences, Butler University ,25

Sepehrianazar F. (2013). Self- efficacy, achievement motivation and academic procrastination as predictors of academic performance, Journal of US-China Education Review B, USA, 3(11), 847-858

Solymonnejad, A. \& Shahrara, M. (2001). Relationship self-regulation with academic achievement. Journal of Psychology and Education. 31( 2), 176. (Persian)

Tabbodi,M., Rahgozar,H. \& Mozaffari makkiabadi, M. (2015). The Relationship between Happiness and Academic Achievements. European Online Journal of Natural and Social Sciences. 240-246

Thelwell, R.C., Lane, A.M., \& Weston, N.W.(2007). Mood states, self-set goals, selfefficacy and performance in academic examinations. Personality and Individual Differences, 42 (3), 573-583. 


\title{
Gender and Differences in School Performance in Reading and in Mathematics in Italian Schools
}

\author{
Francesca Sartori and Carlo Buzzi \\ University of Trento \\ Italy
}

\begin{abstract}
The paper takes gender into consideration in assessing if and how much this ascribed factor impacts school performance. The effects of gender connected to the type of school attended and to the geographical area of residence are analysed.

Although there are gender gaps in literacy resulting from the OECD PISA Programme for International Student Assessment' study - with greater female proficiency in text comprehension and greater male ability in conceptual skill in mathematics - it seems, however, that these trends can be significantly mitigated by the type of school attended and by the geographical area of residence. The paper takes into consideration the Italian case.
\end{abstract}

Keywords: learning and equal opportunity - gender segregation - ranking of performance - reading and mathematics - Italian school.

\section{Foreword}

In an egalitarian, meritocratic education system that ensures equal opportunities, any differences in learning, in the choice of training courses and in educational outcomes should be linked solely to the individual's talent, ability, preferences and commitment.

In actual fact, in Italy and in most educational systems in general, some characteristics have a significant impact on successful learning.

In detail, the studies continue to confirm how socio-cultural and geographical provenance, gender and ethnic group significantly correlate with performance and success in school and in training (Schizzerotto, 2002; Schizzerotto \& Barone, 2006). Focusing on gender ${ }^{1}$, we see that in Italy, in the last few decades, the process of

\footnotetext{
${ }^{1}$ At birth, a male or a female is defined by the possession of specific and differentiated sexual attributes. Later on, through socialisation, individuals acquire ways of feeling, of interacting and of communicating, rules of behaviour and roles that interpret the gender they belong to from a social and cultural viewpoint. Gender, therefore, is the social construction of the sex. It is based on the biological characteristics but is not something natural, innate and therefore inevitable. However, it
} 
female emancipation is linked to a growth of education level of women who were once excluded, or almost, from classrooms and above all from university. This transformation has been so strong that today we are faced with a switch: amongst new generations, the percentage of students who achieve their school leaving certificate or a university degree is higher among the females than the males. In addition, in upper secondary schools, compared to their male peers, girls achieve better scores on average, they incur in less irregular paths (Gasperoni, 2007; Argentin, 2007; Argentin \& Cavalli, 2007) and are less prone to dropping out. Investment in training and in job careers are the behavioural contexts that have most significantly brought substantial change to the existential opportunities of present day female generations.

On the other hand, this is not linked to a growth in visibility of women inside the labour market, since discrimination against the female segment is still persistent, to the point that in order to achieve career positions (and wages) similar to those of the men, women must acquire higher credentials ${ }^{2}$.

Going back to the educational system, another element of inequality in opportunities is found in the paths followed by the two genders within the upper secondary school system. If female are over-represented in the 'licei' when compared to the technical schools, it is equally true they mainly choose humanistic courses (classic studies, languages, humanities, art) while male students tend more towards scientific and technological contents. Furthermore in education and vocational training girls prefer services while boys choose paths related to the industrial and crafts sectors. (Pisati, 2002; Schizzerotto \& Barone, 2006) (Table 1).

does condition and limit the subjects within codified avenues, reducing their opportunity to express intentions, projects, desires, choices, to play roles that may differ from the canons defined by the society they belong to (Ruspini, 2013; Sartori, 2009). Representations of gender through time have undergone continuous changes and appear different in the various cultures and traditions. The basic principles have remained stable, however, to the point of having structured and confirmed the very same perception that individuals have of themselves and of having produced a "sex gender system" (Rubin, 1975) that encompasses all of the existential ambits, situations, places and institutions inside which to be a boy or a girl, man or woman has a different meaning. One cannot but remark, however, that, especially in the western world that in the last decades has seen veritable revolutions as regards sexuality, the role of women in society and the relationship between sexes and between generations, the ways of interpreting gender are becoming less and less fixed and rigid.

${ }^{2}$ Gender, today, is still an important explicative category - although obviously not the only one and not even univocally interpreted - especially when analysing the imparity persisting in societies and when seeking a political perspective in order to reduce it or even eliminate it. This concept is increasingly being considered an essential variable, possessing a strong heuristic value that cuts across social phenomena. One speaks of gender mainstreaming because we are increasingly realising that equal opportunity must not be considered an issue per se but an integral part of democracy and of social equity. 
Table 1 - Students enrolled in the first year of upper secondary school by gender in Italy: composition based on gender (s.y. 2015-2016; values in percentages)

\begin{tabular}{|c|c|c|}
\hline & \multicolumn{2}{|c|}{ Italy } \\
\hline Liceo (Upper Secondary High-school) type & Males & Females \\
\hline - Classic studies & 31 & $\mathbf{6 1}$ \\
\hline - Languages & 20 & 69 \\
\hline$-\quad$ Science & 53 & 47 \\
\hline - Humanities & 10 & 90 \\
\hline - Art & 30 & 70 \\
\hline Technical Schools & $\mathbf{6 9}$ & $\mathbf{3 1}$ \\
\hline - Business & 45 & 55 \\
\hline - Technology & 84 & 16 \\
\hline Vocational Training & $\mathbf{5 7}$ & $\mathbf{4 3}$ \\
\hline$-\quad$ Professional industry and crafts & 75 & 25 \\
\hline - Professional services & 51 & 49 \\
\hline
\end{tabular}

Source: MIUR Ministero Istruzione, Università e Ricerca 2015

Therefore, if the vertical disparities (relative to the qualifications achieved) have been attenuated until overtaking by the female component, the horizontal ones (relative to the internal paths at the same level of education), remain significantly (Schizzerotto \& Barone, 2006) ${ }^{3}$. This phenomenon, in itself, would not be worthy of evidence if not because it will lead to different allocations in terms of degree courses and / or access to the labor market, as already mentioned (Pisati, 2002).

These are just some of the basic indicators. In this paper we will use the data from the PISA study4 ("Programme for International Student Assessment" launched by the OECD - Organisation for Economic Co-operation and Development) regarding the performance of males and females linked, more specifically, to the knowledge levels acquired as regards competences in native language and in mathematics, so as to verify the impact of gender on learning processes.

\footnotetext{
${ }^{3}$ To this end, one must not overlook the social and cultural factors that have an even greater impact on male and female students' choices in the first and the second transition, namely in the transition from primary to secondary and tertiary studies. The feminisation rates of several upper secondary schools and university curricula are proof of this (MIUR 2016). This results today, although less so than in past decades, in significant educational segregation the effects of which are strongly to the detriment of women.

${ }^{4}$ As everyone knows, the three-yearly PISA study involves young 15-year old students from all of the member states of the OECD (35 nations) plus from many other countries (in 2015, another 34 nations plus several Chinese provinces and regions) amounting to a total of 72 different territorial units. The two subjects analysed in this paper are Reading literacy (i.e. the student's capacity to understand, use and reflect on written texts in order to achieve one's goals, to develop one's knowledge and potential, and to participate effectively in society) and Mathematics literacy (i.e. the student's capacity to formulate, use and interpret mathematics in a variety of contexts. It includes mathematical reasoning and the use of mathematical concepts, procedures, facts and tools to describe, explain and forecast phenomena. It allows people to recognise the role that mathematics plays in the world and to make well-founded judgements and grounded decisions as constructive, concerned and reflective citizens).
} 


\section{A first look at the PISA results by gender}

Several comparative results of the PISA study are shown in Table 2 and Figure 1 below. We have limited ourselves to listing the average scores for Reading literacy and Mathematics literacy in G7 member states, i.e. the group that includes the seven largest advanced economies ${ }^{5}$. In displaying these data by gender, we find that females consistently have better average scores than males in skills related to understanding the text in the mother tongue but worse as regards mathematical skills. This seems to be a universal phenomenon and found to be highly common in all of the geographical areas that participated in the latest edition of the PISA study as well as in those of previous years 6 .

Table 2 - Average scores in the G7 member states by gender

\begin{tabular}{|l|c|c|c|c|c|c|c|c|}
\hline & \multicolumn{4}{|c|}{ Reading literacy } & \multicolumn{4}{c|}{ Mathematics literacy } \\
\cline { 1 - 4 } & \multicolumn{3}{|c|}{ Average score } & \multirow{2}{*}{$\Delta$} & \multicolumn{3}{c|}{ Average score } & $\Delta$ \\
\cline { 1 - 4 } & Total & Males & Females & M - F & Total & Males & Females & M - F \\
\hline Italy & 485 & 477 & 493 & -16 & 490 & 500 & 480 & +20 \\
\hline USA & 497 & 487 & 507 & -20 & 470 & 474 & 465 & +9 \\
\hline Japan & 516 & 509 & 523 & -14 & 532 & 539 & 525 & +14 \\
\hline Germany & 509 & 499 & 520 & -21 & 506 & 514 & 498 & +16 \\
\hline Canada & 527 & 514 & 540 & -26 & 516 & 520 & 511 & +9 \\
\hline UK & 498 & 487 & 509 & -22 & 492 & 498 & 487 & +11 \\
\hline France & 499 & 485 & 514 & -29 & 493 & 496 & 490 & +6 \\
\hline & & & & & & & & \\
\hline OECD & 487 & 479 & 506 & -27 & 490 & 494 & 496 & +8 \\
\hline
\end{tabular}

Source. OECD, Database PISA 2015

\footnotetext{
${ }^{5}$ The G7 nations feature very high scores but except for rare exceptions they do not belong to the group of absolute excellence. In the Reading literacy test, only Canada (average score $=527$ ) ranks among the leading nations, preceded by Singapore (535), on a par with Hong Kong (527) and followed by Finland (526) and Ireland (521). In the Mathematics literacy test, of the G7 nations only Japan (532) is amongst the top performers, preceded by Singapore (564), Hong Kong (548), Macao (544), China Taipei (542) and followed by the Chinese provinces of Beijing, Shanghai, Jiangsu and Guangdong (531), The Republic of Korea (524), Switzerland (521) and Estonia (520).

${ }^{6}$ In the Reading literacy test, the dominance of girls is total in all 72 territorial units participating in the study, while in the Mathematics literacy test the dominance of boys is very high but not total, since there are 21 nations in which the girls outperform the boys by 2-9 points in the average score given and by 12-18 points in 4 cases (Trinidad Tobago, Georgia, Qatar and Jordan).
} 


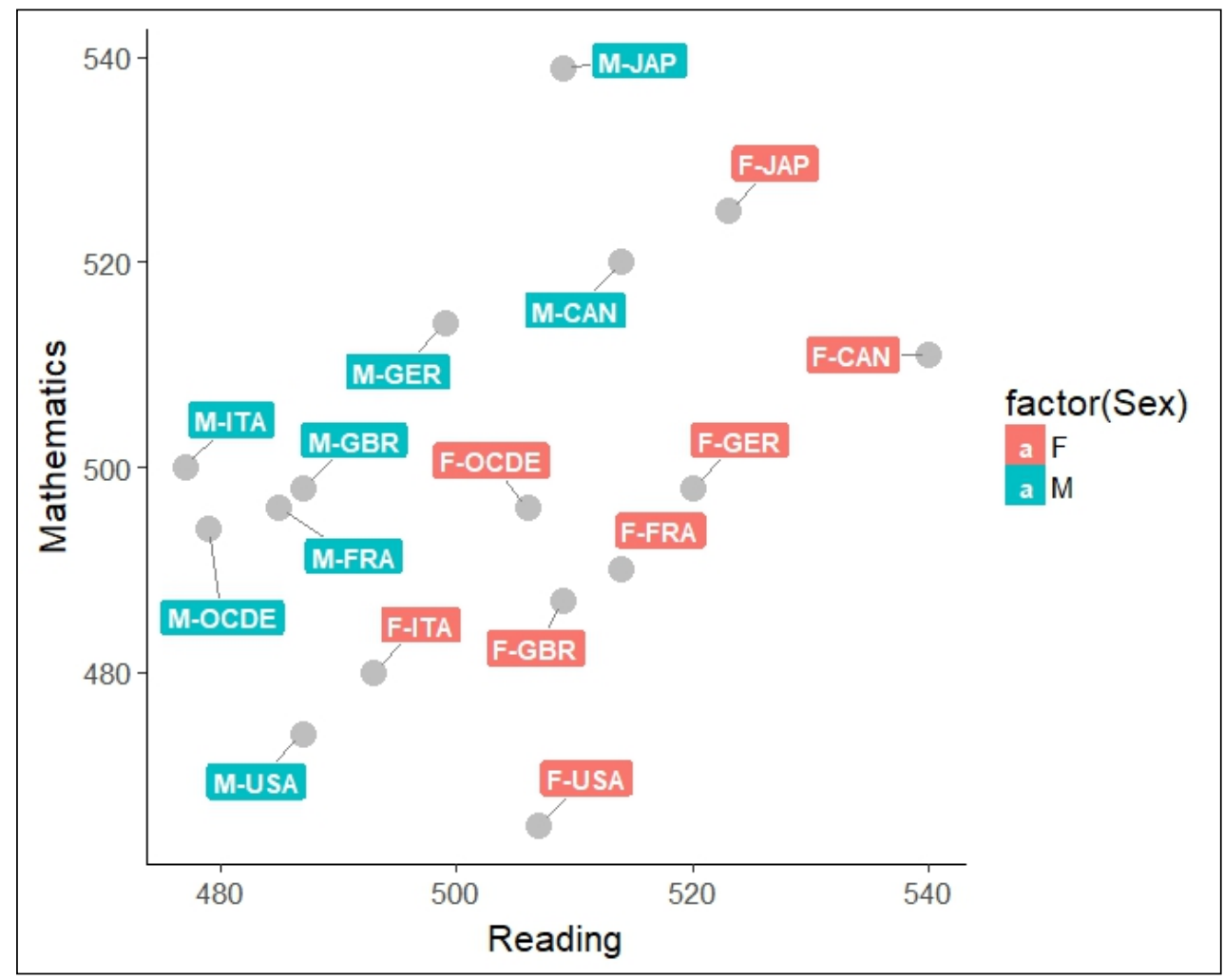

Figure 1 - Average scores in G7 countries and OECD by gender

These scores would be meaningful only if the gender was not influenced by other variables. Therefore let's consider them only as trend evidences with a purely explorative value and try to insert the performances of males and females within a broader context of influences.

Figure 2 shows the influences hypothesised and therefore is the conceptual model on which to base the analysis.

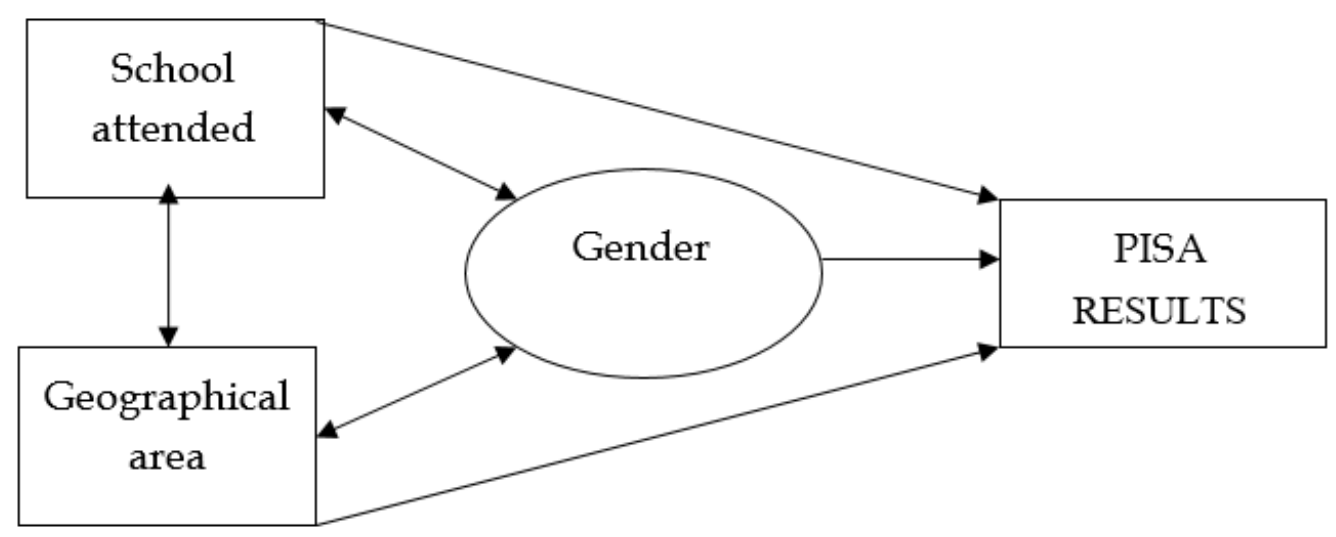

Figure 2 - The gender-based conceptual model 
The conceptual model assumes that the results of Reading literacy and Mathematics literacy tests are directly affected by three factors:

a) gender;

b) geographical area of residence;

c) type of school attended;

and also that:

d) the general effect depends in turn on the school attended (and therefore on the different preparation obtained, it being a well-known fact that the post-mandatory school choices in studies of males and females are not homogeneous and differ considerably;

and finally that:

e) the gender effect also depends on the geographical area of residence (and therefore on the school system prevailing in the various territories).

In short, the conceptual model shown in Figure A prove the effect of gender must not be confused with that of the school attended or with that dictated by the area of provenance.

\section{The role of gender in defining the PISA results in the Italian case}

Once the overall picture of the analysis has been clarified we can now focus on the Italian case by analysing the scores obtained by boys and girls and using as fixed parameters the geographical area (Italy features very large geographical differences) and the type of school attended (the various types of schools also differ greatly as regards gender-based composition). The latter data can be further analysed considering that the groups of schools considered consist of institutes that prepare their students in highly different ways. In the 'licei' (upper secondary schools), the curricula range from classical studies to scientific studies, from art to humanities. In the technical schools, there is an even larger disparity between economic/commercial and technological/industrial programmes and approaches, and the same applies to vocational training. The four groups are, however, representative of the various scholastic levels existing in Italy. As regards the geographical subdivision, the sectors considered (North-West, North-East, Center, South, Islands) effectively describe the cultural, economic and social reality of Italy.

When analysing the results (Table 3), the following phenomena stand out:

- As regards reading, girls outperform boys in all Italian geographical areas and in almost all kinds of schools; the gap in favour of the girls narrows with the increase in prestige of the type of school, and therefore in the 'licei' the boys outperform girls, albeit by very little, while in the technical and professional schools the difference in favour of the girls is significant and peaks in the vocational training schools;

- $\quad$ As regards Mathematical literacy, boys are always ahead by geographical area, although the gap narrows when we look at school type, and diminishes in parallel with the decrease in level of prestige of the school: the gap is widest in the 'licei', consistent in the technical schools and 
limited in the professional schools, shrinking to almost no gap in the vocational training centers.

Gender differences with regard to literacy actually exist and the data show greater female ability in understanding the texts and greater male ability in mathematical conceptualisation. The geographical area, however, that coincides with different levels of school organisation quality, can significantly mitigate these trends. Within the same territory, for example, girls outperform boys in reading, but the boys living in the North-Eastern regions (the area in Italy where the schools are absolutely the best) are just a few points behind the girls of the North-Western regions and far above the girls of the Southern regions and the Islands. This is also true with regard to mathematical skills in which the boys outperform the girls although the girls living in the North-Eastern regions score just a few points below their male peers in the North-Western regions and score above the boys living in Italy's central regions, and definitely outperform the boys living in the South and in the Islands.

Similarly, the type of school attended also has a significant impact in gender dynamics. Even though the girls greatly outperform the boys in Reading literacy in the technical and professional schools and in the vocational training centers, the same cannot be said for the 'licei' where the boys score slightly above the girls (this is most probably due to the greater self-selection operated by the males enrolling in a liceo, to the point where less than two fifths of all students are male). The boys of the technical schools, moreover, outperform the girls of the professional schools and of the vocational training centers. This is also true for Mathematics literacy in which the less performing gender (the girls) anyhow scores higher than the boys attending a lower level school.

These data allow us to conclude that in defining the scores, gender plays a significant role within the same geographical areas (and therefore having the same school quality levels) and when attending the same school type but that, at the same time, geographical area and school type appear to be equally decisive when assessing the performance level achieved beyond gender too. 
Table 3 - Average scores by gender (geographical area and type of school attended in Italy being equal)

\begin{tabular}{|c|c|c|c|c|c|c|c|c|}
\hline & \multicolumn{4}{|c|}{ Reading literacy } & \multicolumn{4}{|c|}{ Mathematics literacy } \\
\hline \multirow{2}{*}{$\begin{array}{l}\text { Geographical } \\
\text { area }\end{array}$} & \multicolumn{3}{|c|}{ Average score } & \multirow{2}{*}{$\begin{array}{c}\Delta \\
\mathrm{M}-\mathrm{F}\end{array}$} & \multicolumn{3}{|c|}{ Average score } & \multirow{2}{*}{$\begin{array}{c}\Delta \\
M-F\end{array}$} \\
\hline & Total & Males & Females & & Total & Males & Females & \\
\hline $\mathrm{N}-\mathrm{W}$ & 503 & 494 & 511 & -18 & 505 & 517 & 495 & +22 \\
\hline N-E & 515 & 509 & 521 & -12 & 525 & 535 & 514 & +21 \\
\hline Centre & 488 & 485 & 492 & -7 & 497 & 509 & 486 & +23 \\
\hline South & 461 & 452 & 471 & -19 & 468 & 476 & 458 & +18 \\
\hline Islands & 451 & 437 & 462 & -25 & 446 & 453 & 440 & +13 \\
\hline Italy & 485 & 477 & 493 & -16 & 490 & 500 & 480 & +20 \\
\hline \multirow[t]{2}{*}{ School type } & \multicolumn{3}{|c|}{ Average score } & $\Delta$ & \multicolumn{3}{|c|}{ Average score } & $\Delta$ \\
\hline & Total & Males & Females & $M-F$ & Total & Males & Females & $M-F$ \\
\hline CFP & 417 & 405 & 433 & -28 & 416 & 417 & 415 & +2 \\
\hline Ist.Prof. & 410 & 405 & 415 & -10 & 424 & 431 & 417 & +14 \\
\hline Ist.Tecn. & 473 & 471 & 479 & -8 & 490 & 497 & 474 & +23 \\
\hline Liceo & 526 & 528 & 524 & +4 & 521 & 547 & 505 & +42 \\
\hline Italy & 485 & 477 & 493 & -16 & 490 & 500 & 480 & +20 \\
\hline & & & & & & & & \\
\hline
\end{tabular}

Source: OECD, PISA 2015 Database, INVALSI processing

Note: $\mathrm{CFP}=$ Vocational Training Centers; Ist.Prof. $=$ Professional Schools; Ist.Tecn $=$ Technical Schools .

In addition to the awareness that school type and geographical area have a strong impact, it is also interesting to analyse the way in which performance is distributed among the genders by using the scores that define the low and top performers based on the general parameters defined by the whole set of results collected in the PISA study.

The consistency of the groups by competence within genders is not surprising because it confirms all of the trends seen up to this point (Tables 4 and 5).

Table 4 - Percentage of low and top performer students by gender in Reading literacy in Italy

\begin{tabular}{|l|c|c|c|}
\hline & Males & Females & Total \\
\hline $\begin{array}{l}\text { Low performers } \\
\text { (less than 407.47 points) }\end{array}$ & 24.1 & 17.9 & 21.0 \\
\hline $\begin{array}{l}\text { Average performers } \\
\text { (between 407.47 and } \\
\text { 625.60 points) }\end{array}$ & 71.0 & 75.7 & 73.3 \\
\hline $\begin{array}{l}\text { Top performers } \\
\text { (more than 625.60 points) }\end{array}$ & 4.9 & 6.4 & 5.7 \\
\hline Total & 100.0 & 100.0 & 100.0 \\
\hline
\end{tabular}

Source: OECD, PISA 2015 Database 
Table 5 - Percentage of low and top performer students by gender in Mathematics literacy in Italy

\begin{tabular}{|l|c|c|c|}
\hline & Males & Females & Total \\
\hline $\begin{array}{l}\text { Low performers } \\
\text { (less than 420.07 points) }\end{array}$ & 20.7 & 25.8 & 23.3 \\
\hline $\begin{array}{l}\text { Average performers } \\
\text { (between 420.07 and } \\
\text { 606.98 points) }\end{array}$ & 66.1 & 66.4 & 66.2 \\
\hline $\begin{array}{l}\text { Top performers } \\
\text { (more than 606.99 points) }\end{array}$ & 13.2 & 7.8 & 10.5 \\
\hline Total & 100.0 & 100.0 & 100.0 \\
\hline
\end{tabular}

Source: OECD, PISA 2015 Database

\section{Conclusions}

The gender perspective in reading social phenomena, now widely accepted by scholars and institutions, leads to the overcoming of the nativism that traditionally assigns skills and competences in different disciplines to males and females and interprets the difference in their choice of school and university - and consequently of job careers (Bettio 2008; Triventi et al. 2015) - as natural and therefore connected to biological factors. Up until the 1990's, there was a shared conviction that girls and women had fewer skills in mathematics compared to boys and men (Hyde, Fennema \& Lamon, 1990). The statistics show, instead, that in recent years the scoring gap between male and female students resulting from national and international tests is closing (Hyde \& Linn, 2006; Stoet \& Geary, 2013).

The increase, albeit slowly growing, in choices outside the stereotypical genderrelated secondary and tertiary paths seen among girls (including the enrolment in technical schools and in technical, scientific and statistical university curricula) confirms the prospect of a more balanced distribution of jobs, both horizontally and vertically, among men and women (the MIUR data confirm this for Italy).

The latest studies have shown that the factors acquired have a significant impact on learning and on school performance. The main factors are the culture of belonging (Guiso et al, 2008), the role attributed to women in different societies, and especially in the in the labour market, the education and the social class of parents, the distribution of domestic and care duties within the parental couple, the home learning environment (Melhuish et al., 2008) and the proposals of number-based games and activities more frequently addressed to males. After all, a rigid planned gender division can be seen in every toy store (Jacobs \& Eccles, 1992; Jacobs \& Bleeker, 2004; Sartori, 2010).

Moreover, as regards the school, a greater focus on overcoming the traditional models of male and female representation in the textbooks at primary school level (Biemmi, 2009; Biemmi, 2010) and the dissemination of teaching methodologies (Cooperative learning and Peer education) aimed at integration and cooperation between all students, are thought to be an important booster in a gender equality oriented school education model. 
Special attention is paid to the impact of sexist stereotypes and prejudice against women that are widely common even in the most advanced societies. Particular attention is given to the mothers' endorsement and reproduction of these stereotypes, as mothers usually play a leading role in the development of their children's school performance starting from early childhood and have a significant influence (Bleeker \& Jacobs, 2004; Lindberg, Hyde \& Hirsch, 2008; González de San Román \& De la Rica Goiricelaya, 2012).

The variables interacting with gender are many and not always assessable with similar and neutral tools. This complex analysis and the assessments of learning are therefore not always comparable (DIES, 2007). The results of the studies are therefore contradictory (Tomasetto et al., 2011).

Anyway, the sensitisation of parents about stereotype-free educational models, the training of teacher in gender equality, the new guidance teaching methods sensitive to gender disparity will favour the opening up of prospects for girls towards less typified and not coherent choices with the expectations of the female role. The reinforcement of their self-esteem and the belief in their potential with regard to subjects that are considered more suitable for males will help them choose their educational and occupational future based on their abilities, interests and dreams.

\section{References}

Argentin, G. \& Cavalli, A. (2007). Giovani a scuola. [Young people at school]. Bologna: il Mulino.

Argentin, G. (2007). Come funziona la scuola oggi: esperienze e opinioni dei giovani italiani. [How the school works today: experiences and opinions of young Italians]. In Buzzi, C., Cavalli, A., de Lillo, A. (Eds.) Rapporto giovani. Sesta indagine dell'Istituto IARD sulla condizione giovanile in Italia. [Young people report. Sixth survey by the IARD Institute on the condition of youth in Italy]. Bologna: il Mulino.

Bettio, F. (2008). Occupational Segregation and Gender Wage Disparities in Developed Economies: Should We Still Worry? In Bettio F. \& Verashchagina A. (Eds.) Frontiers in the economics of gender. Siena Studies in Political Economy, 267-285.

Biemmi, I. (2009). Genere e processi formativi. Sguardi femminili e maschili sulla professione di insegnante. [Gender and training processes. Female and male looks on the profession of teacher]. Pisa: ETS.

Biemmi, I. (2010). Educazione sessista. Stereotipi di genere nei libri delle elementari. [Sexist education. Gender stereotypes in textbooks of the primary school. Torino: Rosenberg \& Sellier.

Bleeker, M. M., \& Jacobs, J. E. (2004). Achievement in math and science: Do mothers' beliefs matter 12 years later? Journal of Educational Psychology, 96, 97-109.

DIES (Department for Education and Skills (UK)) (2007). Gender and Education: the evidence on pupils in England. London: Department for Education and Skills.

Gasperoni, G. (2007). Percorsi e prestazioni nella scuola secondaria superiore. [Paths and performances in upper secondary school]. In Buzzi, C. (Ed.) Crescere a scuola. Il profilo degli studenti italiani. [Grow up at school. The profile of Italian 
Students]. I Quaderni, 8. Torino: Fondazione per la Scuola.

González de San Román, A. \& De la Rica Goiricelaya, S. (2012), Gender Gaps in PISA Test Scores: The Impact of Social Norms and the Mother's Transmission of Role Attitudes, Bonn: Discussion Paper 6338 IZA.

Guiso, L., Monte F., Sapienza P., Zingales L. (2008). Culture, Gender, and Math. Science, 320, 1164-1179.

Hyde, J. S., Fennema, E., Lamion, S.J. (1990). Gender differences in mathematics performance: a meta-analysis. Psycological Bulletin, 107, 139-155.

Hyde, J. S. \& Linn, M. C. (2006). Gender similarities in mathematics and science. Science, 314, 599-600.

Jacobs, J. E. \& Bleeker, M. M. (2004). Girls' and boys' developing interests in math and science: Do parents matter? New Directions for Child and Adolescent Development, 106, 5-21.

Jacobs, J. E. \& Eccles, J. S. (1992). The impact of mothers' gender-role stereotypic beliefs on mothers' and children's ability perceptions. Journal of Personality and Social Psychology, 63, 932-944.

Lindberg, S. M., Hyde, J. S., Hirsch, L. M. (2008). Gender and mother-child interactions during mathematics homework: The importance of individual differences. Merrill-Palmer Quarterly, 54, 232-255.

Melhuish et al. (2008). Effects of the Home Learning Environment and Preschool Center. Experience upon Literacy and Numeracy Development. In Early Primary School. Journal of Social Issues, 1, 95-114.

Pisati, M. (2002). La partecipazione al sistema scolastico [Participation in the school system]. In Schizzerotto, A. (Ed.) Vite ineguali [Uniqual lives]. Bologna: il Mulino.

Rubin, G. (1975). The traffic in Women, Notes on the Political economy of sexim. In Reiter, R. Toward an Anthropology of women. New York-London: Monthly Review Press.

Ruspini, E. (2013). Le identità di genere [Gender identities]. Milano: Feltrinelli.

Sartori, F. (2010). Politiche giovanili in una prospettiva di genere [Youth policies in a gender perspective]. In RicercAzione, 2, 237-251.

Sartori, F. (2009). Differenze e disuguaglianze di genere [Gender differences and inequalities]. Bologna: il Mulino.

Schizzerotto, A. (Ed.) (2002). Vite ineguali [Uniqual lives]. Bologna: il Mulino.

Schizzerotto, A. \& Barone, C. (2006). Sociologia dell'istruzione [Sociology of education]. Bologna: Il Mulino.

Stoet, G. \& Geary, C. (2013). Sex Differences in Mathematics and Reading Achievement Are Inversely Related: Within- and Across-Nation Assessment of 10 Years of PISA Data. Tel Aviv: Eshel Ben-Jacob Editor.

Tomasetto, C., Alparone, F., Cadinu, M. (2011). Girls' math performance under stereotype threat. The moderating role of mothers' gender stereotypes. Developmental Psychology, 47, 943-947.

Triventi, M., Skopek, J., Kosyakova, Y., Buchholz, S., \& Blossfeld, H. P. (2015). Gender 33 inequalities at labour market entry: A comparative view from the edu LIFE project. In C. Imdorf, K. Hegna, \& L. Reisel (Eds.). Gender segregation in vocational education. Bingley: Emerald Insight. 


\title{
Final Pass Rate Fluctuations of Power Engineering Students May Be Related to Load Shedding, and not Load Shifting
}

\author{
Arthur James Swart \\ Department of Electrical, Electronic and Computer Engineering, \\ Central University of Technology, \\ Private Bag X20539, Bloemfontein, South Africa, 9300
}

\begin{abstract}
Power engineering students need to register for and successfully complete a capstone module within the Baccalaureus Technologiae qualification in Electrical Engineering in South Africa. These students need to submit six different assignments over a period of 10 months, comprising a proposal, a progress report, a poster, a power point presentation, a conference paper and a final report. However, it has been observed that the final grades of working students have fluctuated dramatically over the past three years, leading to the following research question "What may be contributing to these fluctuations?" The purpose of this paper is to discuss the fluctuations and provide possible reasons for them based on literature applicable to this field. An exploratory case study is used to provide quantitative data of the student final grades from 2014 - 2016. Results show that the pass rate varied from $71 \%$ to $77 \%$ to $56 \%$. Furthermore, some 18 students submitted their proposal in 2016, but never submitted the other five assignments. This concern may be related to load shedding by both students and academics, rather than load shifting. A key recommendation of this research is to share its findings with top management of student employers and the university, thereby hoping to create awareness of the importance of lightening the workloads of employed students and academics during important submission periods of capstone modules.
\end{abstract}

Keywords: capstone, technology, workloads, working students.

\section{Introduction}

"Success is no accident. It is hard work, perseverance, learning, studying, sacrifice and most of all, love of what you are doing or learning to do" (Brainy Quote, 2017). These words, by the well-known Brazilian athlete Pele, well emphasise that success in life does not come automatically. This especially holds true for students in Higher Education, who have to put forth much hard work and perseverance to complete 
their studies, often sacrificing time from other endeavours. However, ever-changing personal circumstances of both students and academics may result in the wrong sacrifices being made.

Load shedding may be seen as a sacrifice, where power from one area is turned-off (or sacrificed) to keep the power in another area on. Load shedding is defined as a coordinated sets of controls that decrease the electric load in one part of the system to restore the overall system back to its normal operating condition (Mageshvaran et al., 2015). This instantaneous removal of power from the system therefore helps to keep the remaining section of the system operational (Lim et al., 2011). Applying this same principle to students in higher education means that some students prioritize their responsibilities of working and studying in a way that their studies are turn-off, so as to keep their work on. In other words, their studies are often sacrificed in behalf of other responsibilities. This is in contrast to load shifting, which is defined as the process of shifting loads from peak periods to off-peak periods (Hamidi et al., 2012). Some students are just not afforded the opportunity to shift their responsibilities around, as they are kept engaged by other commitments. For example, when they may need to study at night (off-peak period) they may be called to work to care for an emergency. In such cases, load shedding rather than load shifting occurs. Research has shown that students who study and work at the same time (often called working students) is a phenomenon that entails risks and may be detrimental to the quality of education, as it often prolongs the period of study, preventing students from finishing their qualification in a timely manner (Chavdarova, 2006; Radojković \& Milojević, 2016). This usually has a harmful effect on academic performance (Davletov \& Bishkek, 2014; Swart, 2016).

These same principles may be applied to academics. Academics may be given such a high workload, that they may sacrifice the quality of their feedback to students in order to just get through the amount of work which they have. They are thus shedding quality, and not shifting their responsibilities around, so as to maintain the standard of education (or the normal operation of the system). Research has shown that, in general, the main problems students report on with regard to academic feedback, is not on the amount they receive, but rather on its timeliness and perceived relevance (Cann, 2014). As academic workloads increase, these issues worsen, and the quality deteriorates.

It was observed, in a power engineering module, termed Industrial Projects IV (IP4), that the final grades of working students have fluctuated dramatically over the past three years, leading to the following research question "What may be contributing to these fluctuations?" The purpose of this paper is to discuss the fluctuations and provide possible reasons for them based on literature applicable to this field. An exploratory study is used to provide quantitative data of the student final grades from 2014 through 2016. Plausible reasons for such fluctuations are firstly presented based on published literature. The context of the study is then presented along with the research methodology. Results and discussions then follow. 


\section{Possible reasons for pass rate fluctuations deduced from existing literature}

A number of plausible reasons may be deduced from the literature for the abnormal fluctuations in the success rates of working students in the same module over a period of time. These may include unsympathetic employers, demanding work responsibilities, or changing family circumstances. However, it must be noted that students who work and study at the same time demonstrate a desire to take charge of their careers (Supangco, 2014). This is excellent, as a higher degree may lead to promotional opportunities at work and subsequently to a higher quality of life, both for the student and for his or her family. In fact, research shows that individuals who obtain a higher degree have between a $28 \%$ and $40 \%$ higher chance of becoming managers (Georgellis \& Sankae, 2016), as compared to those who do not obtain a higher degree. Furthermore, students who work and study simultaneously are able to relate all their learning assignments to their work, while the employer can develop its business and processes by applying the state-of-the art theoretical knowledge acquired by the student (Mikkelsen et al., 2015).

However, there are challenges that exist for those who work and study at the same time. Students are of the opinion that working and studying at the same time can be difficult (Kori et al., 2016) with many reporting that they feel very tired from doing this (Ahmed et al., 2015). This is especially so when the employer is unsympathetic to the student's academic career. Feelings of frustration with non-performing colleagues, anger about irate customers and interaction with unsympathetic managers are all contributing factors that cause emotion in the workplace (Jonker \& Van der Merwe, 2013). These unhealthy emotions may, in turn, lead to higher levels of stress or anxiety, reduced performance and low feelings of self-esteem or efficacy. Dealing with unsympathetic managers or employers who place excessive demands on employee time can cause working students to sacrifice their study time, in order to maintain a reasonable working relationship with their supervisors.

Work responsibilities have also increased over the past decade, which is largely attributed to the advances in technology. Mobile technology has enabled managers and clients to contact staff at all times so that work responsibilities are extended to the home, during holidays and even during sleep time (Rubery, 2015). Working students are therefore not really cut-off from their employers, who may demand more time from them at various times of the week, no matter where they are.

Family circumstances may also change rapidly, affecting both the emotional and physical stability of working students. Relevant changes of family circumstances include marriage, divorce, the death of a love one, the death of relatives and the birth of children (Gill \& Allatt, 2011). All these aspects may cause more stress or anxiety to the working student, who then loses focus on his or her academic studies. This is understandable, as family is often perceived to be more important than work (Gill \& Allatt, 2011), not to mention academic studies.

These external factors relating to family and work responsibilities have been shown to influence student persistence, especially among at-risk and minority populations 
(Kruse, 2013). Furthermore, for non-traditional learners to be successful students they must have the availability of time to engage in academic studies after family and work responsibilities have been satisfied (Campbell, 2015). However, if family and work responsibilities are taking all the time, then nothing is left for the academic studies, which have in effect been sacrificed. Load shedding of academic studies has occurred.

Depending on the number of hours worked, working and studying at the same time has mixed effects on student's achievement at higher educational institutions (Lee et al., 2013). However, this is also applicable to academics in higher education. Massive expansion has resulted in class sizes ballooning, academics being overloaded, resources declining, teaching activities being trimmed and facilities deterioratingall leading to the perfect storm for a quality crisis (Teferra, 2015). Quality education includes all the processes, procedures, and programmes drafted by institutions to fulfil the demands of the stakeholders (Deshpande, 2015). This includes effective academic feedback to students who have submitted assignments or completed assessments. This may suffer when academic workloads increase due to more student enrolments or due to the resignation of fellow academics from the faculty.

In the last 10-20 years, academic workloads have become increasingly demanding and complex in the UK and North America, with Australia being no exception (Hemer, 2014). This is the case in many countries, where the current "normal" workload is so heavy that academics can only complete all their teaching, research and administration duties by working beyond the contracted number of working hours - at night, during the weekend and even when on holiday (Hemer, 2014). Performing at this level over an extended period of time may prove exhausting and stressful. In this way, academic load shedding may occur, as academics seek to sacrifice various aspects of teaching just to be able to complete the assigned work (keep the rest of the system afloat).

\section{Study context}

IP4 is a compulsory capstone module in the Baccalaureus Technologiae: Engineering: Electrical qualification (better known as the BTech). The course structure (highlighting six different submission requirements) used by the Electrical, Electronic and Computer Engineering Department at the Central University of Technology (CUT) for this module is shown in Table 1, which must be completed over a 10-month period (registration is completed in February with the final summative report submitted in October). The Department's objective is to provide quality education to students with regard to both electronic and power engineering, where the principles and application of electricity, electronics, electrostatics and electromagnetism are discussed (Hertzog \& Swart, 2015). No formal electrical or electronic based circuits are required from these students who often work on high voltage systems (up to $132 \mathrm{kV}$ ). Their final report or dissertation is usually based on a real life case study which was identified in industry. 
The purpose of the project proposal is to identify the topic, formulate the problem and present the time frame for completing the study. It must include numerous sketches with two highlighted areas in the sketch which are discussed in the text. Students often struggle to formulate the problem, or to engage with problem-based learning (Swart \& Toolo, 2015). The exact phrase "The problem is..." is often missing from the proposal and is very difficult to discern from the text provided by the student. Students also struggle to follow the correct referencing style, and often just copy and paste from the Internet. Here the academic needs to intervene and provide effective feedback to address these concerns.

The purpose of the progress report is to rectify any concerns from the project proposal, requiring students to act on the comments and feedback given by the academic (Swart \& Hertzog, 2017). It must also include three possible solutions to the identified problem in the progress report, again presenting numerous highlighted sketches to support their solutions. Students often struggle to explain their solutions in text format, and usually just provide a number of sketches which have not been highlighted or explained in the text. This is to be commented on by the academic when giving written feedback to the students.

Table 1 - Industrial Projects 4 (IP4) structure

\begin{tabular}{|l|c|c|c|}
\hline \multicolumn{1}{|c|}{ Requirement } & Assignment & Due period & Weight \\
\hline Project proposal (formative) & 1 & End April & $10 \%$ \\
\hline Progress report (formative) & 2 & End July & $10 \%$ \\
\hline Conference paper or article (summative) & 3 & Mid-August & $5 \%$ \\
\hline Poster presentation (summative) & 4 & End August & $5 \%$ \\
\hline Oral defense or colloquium (summative) & 5 & Mid-Sept. & $10 \%$ \\
\hline Final written report (summative) & 6 & End October & $60 \%$ \\
\hline & & TOTAL & $100 \%$ \\
\hline
\end{tabular}

The purpose of the conference article or paper is to create awareness among students about the importance of publishing their research results. The official IEEE template is prescribed where students need to focus on their results and preferred solution (recall they suggested three possible solutions in the progress report) (Swart \& Hertzog, 2016). Students often struggle to keep to the page limit, while failing to provide an adequate introduction and conclusion to their paper. These deficiencies must be noted by the academic in the written feedback.

The purpose of the poster is to create awareness among the students of its required structure, which should focus primarily on the preferred solution, having as little text as possible (Swart \& Hertzog, 2017). It must include the title of the project, problem statement and student particulars. All sketches in the poster must feature at least three highlighted sections, and have a concise meaningful caption or label attached to it. Students often struggle to get the right balance between text and sketches, with many inserting lengthy paragraphs and many references. Feedback comments from the academic should focus on these concerns. 
The purpose of the oral defense is to afford students the opportunity to engage with peer assessment, as their peers form the audience. Students need to compile a power point presentation highlighting their problem statement, three possible solutions and preferred solution, drawing on simulated results to substantiate their preferred solution (Swart \& Hertzog, 2016). This furthermore helps to prepare students for conference presentations, which follows the same format and often includes questions from the audience. Students often struggle to effectively articulate the problem and preferred solution, with many students simply reading each slide word for word. This is often commented on by the academic who is present to assess the presentation.

The purpose of the final report is to draw all the previous assignments together, where the student should have acted on a number of written feedback concerns. This final report usually follows the structure of a dissertation or thesis required for postgraduate degrees. Students often struggle to be consistent in the use of fonts, spacing and terms, especially in the front and back matter. $40 \%$ of the final report is awarded to these two sections of the report (declaration, expression of thanks, abstract, table of contents, references and annexures) (Swart \& Toolo, 2015). This is the only assignment where the students will not view the feedback from the academic, as it is a summative assessment. However, the academic needs to include comments on these reports, as they will be made available for scrutiny to the Engineering Council of South Africa (ECSA), when they do their accreditation visits. ECSA representatives will want to see why a certain grade was awarded, and that the academics actually assessed each section of the final report.

\section{Research methodology}

An exploratory case study is used in this study as it describes fluctuating student pass rates over a three-year period, where no clear explanation of the causes may be identified. Yin (2009) describes three types of case studies, being exploratory (examines a situation where an intervention produces no single clear result), descriptive (describes a situation within a real life context) and multiple case studies (discover the differences between and within cases).

Quantitative data is used to highlight the pass rates of power engineering students over a three-year period (2014 - 2016). These results cover the six individual assignments listed in Table 1, as well as the final pass rates of the students in the module, IP4. The target population is restricted to all the students $(n=243)$ enrolled in this module for the three-year period, thereby negating the use of a sampling technique. More than $95 \%$ of the target population are working students.

Quantitative data also includes the number of academics that were involved for the three-year period, so as to indicate any possible academic workload increase during this time period. The number of students who only submitted their proposal is contrasted to the students who submitted all their assignments (see Table 1), so as to 
indicate the fluctuating submissions which may have been caused by a change in the circumstances of these students.

\section{Results and discussions}

Figure 1 highlights the percentage of students who were assigned to different academics for the three-year period. In 2014, three academics where involved with IP4, with each mentoring around 30 students (equals around $33 \%$ of the 98 registered students). In 2015, two academic staff members were involved with IP4, where each mentored around 40 students (approximately 50\% of the 81 registered students). Then in 2016, only one academic was assigned to IP4 who had to mentor 64 registered students. This indicates a drastic shift in workload, as that one academic still had the original workload of the previous two years. That one academic in 2016 had to, in effect, do the work of the two academics of 2015 or of the three academics of 2014. It must be noted that this one academic has been involved with IP4 for more than 5 consecutive years, and is thus well-equipped to deal with the module's content. However, to mentor 64 students on your own and then also complete all your other responsibilities may well prove challenging and overwhelming. Effective academic feedback may also have suffered during the 2016 period.

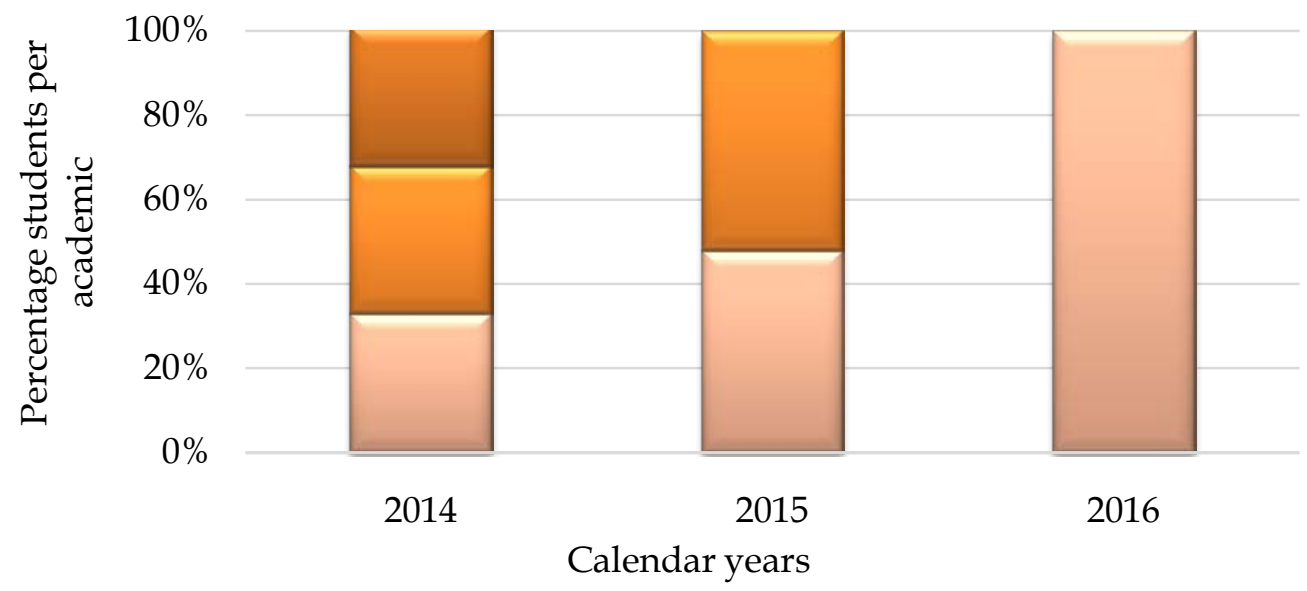

Figure 1 - Percentage of students assigned to different academics over the 3-year period

Figure 2 presents the pass rates of the 243 students for the three-year period, when considering the six assignments outlined in Table 1. It is evident in 2014 and 2015, that the overall pass rate (right-hand side) was relatively high $(71 \%$ and $77 \%$ respectively). The majority of students consistently handed in their six assignments, with an upward trend in 2015 for the final report (highest weighting towards the final mark). However, this trend is not observed for 2016, where a significant decline in the submissions after the progress report is evident $(100 \%$ submitting the proposal and only 55\% the progress report). 2016 had an all-time low pass rate for the threeyear period of only $56 \%$. 


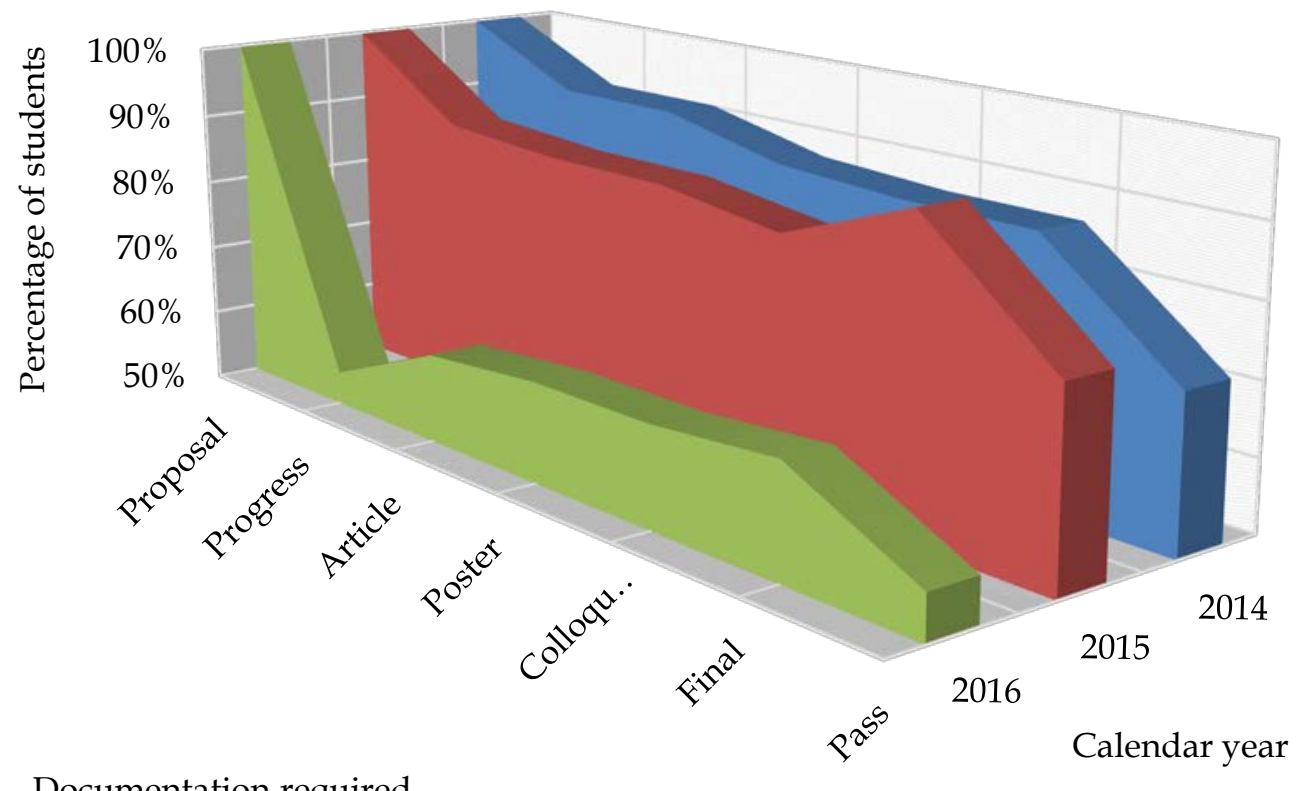

Documentation required

Figure 2 - Percentage of students submitting the required documentation for the three calendar years

Figure 3 portrays the breakdown of the six assignments (see Table 1) according to three criteria. First, students only submitting a proposal was found to be $7 \%$ and $6 \%$ in 2014 and 2015 respectively. However, in 2016 (outer circle), it jumped to 28\%. This means that 18 students registered and submitted their proposal, and then disappeared. A drastic change in the personal circumstances (family or work) of these students may have contributed to this phenomenon. One such drastic change may be an increase in their work responsibilities (as they are studying and working at the same time), thereby preventing them from dedicating time to their studies. In 2016 , only $48 \%$ of all the registered students submitted all six assignments.

Proposal only
with NO othe
documents
Proposal and
less than 5
documents
Proposal and
all 5 required
documents

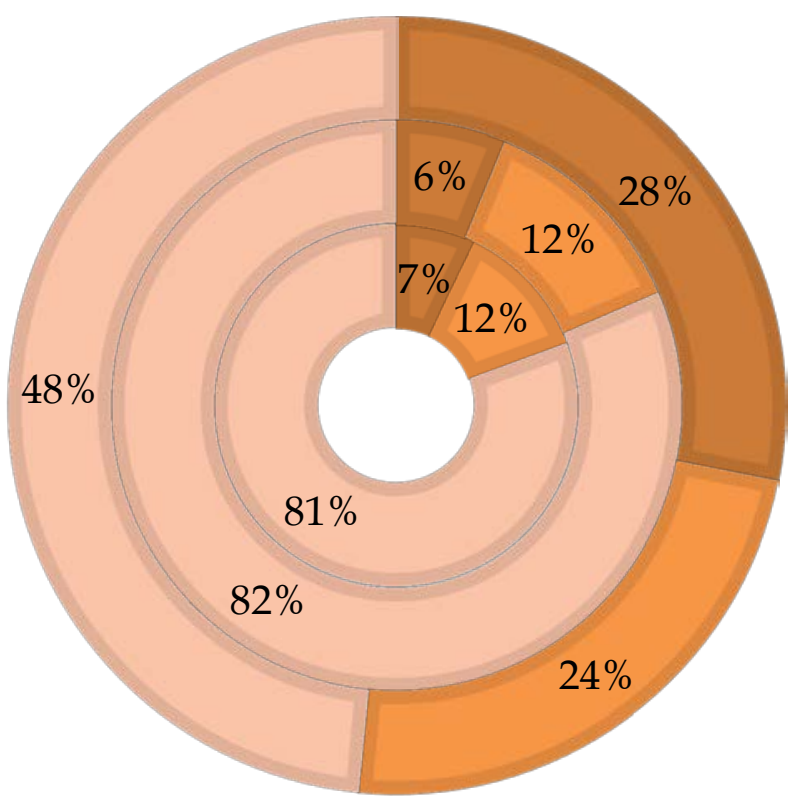

Figure 3 - Percentage of students submitting the proposal for different conditions for 2014 (inner circle), 2015 (middle circle) and 2016 (outer circle) 


\section{Conclusion}

The purpose of this paper was to discuss the fluctuations of final grades of working students in a compulsory capstone module, termed IP4, and to provide possible reasons for these fluctuations based on literature applicable to this field. Results show that the pass rate for IP4 varied from $71 \%$ to $77 \%$ to $56 \%$ over the three-year period. Three academics mentored 98 students in 2014, while only one academic mentored 64 students in 2016. Furthermore, in 2016, 18 students (28\% of all registered students) only submitted their proposal, while never completing the other five assignments.

This concern may be related to the load shedding of both students and academics, rather than load shifting. Load shedding is seen in that other responsibilities of both the students and academic may have taken precedence over the six assignments required in IP4, thereby effectively sacrificing IP4 to keep their other responsibilities intact. These other responsibilities may be linked to changing family circumstances or demanding work responsibilities.

A comparison of student numbers registered for a capstone module at a University of Technology has been given, along with the number of students that completed the module. The numbers are only given for a 3-year period, which does make it difficult to draw inferential conclusions. However, it must be noted that this is an exploratory case study, where no clear explanation of the causes exists. Case studies may also be used to set the stage for future research (Yin, 2013). There are indeed many variables, or contributing factors, to student performance that have not been explored in this paper. However, its purpose was to present plausible reasons from published literature for significant pass rate fluctuations for working students.

Research on additional contributing factors to working student success rates will add to this study. For example, these working students may be interviewed to obtain their perceptions on the study-work relationship. Furthermore, the 18 students who only submitted the proposal could be contacted in an effort to try and understand why they never submitted the other five assignments. Finally, the academics who mentored the students may be interviewed to determine their perceptions of why the abnormal pass rate fluctuation exists. A key recommendation of this research is to make top management of student employers and of universities aware of the importance of lightening the workloads of students and academics during important time periods stipulated in compulsory capstone modules (for this study, it is July through October according to Table 1). 


\section{References}

Ahmed, M. Z., Touama, H., \& Rayan, A. (2015). Students' Perspectives about Nursing Education. American Journal of Educational Research, 3(3), 288-291.

Brainy Quote. (2017). Homepage. Retrieved from http://www.brainyquote.com/quotes/

Campbell, B. G. (2015). Diffusion of Innovations of Videoconference Technology: An Instrumental Case Study Concerning Undergraduate Degree-Seeking Nontraditional Learners. Nova Southeastern University.

Cann, A. (2014). Engaging students with audio feedback. Bioscience Education, 22(1), 31-41.

Chavdarova, T. (2006). Young people-those New Bulgarians: what do they think about Bulgaria? South-East Europe Review for Labour and Social Affairs, 3(2006), 57-67.

Davletov, C., \& Bishkek, K. (2014). Determinants of Academic Performance: The Case of American University of Central Asia.

Deshpande, B. (2015). Application of Balanced Score Card in Higher Education with special emphasis in a Business School. Paper presented at the International Conference on Technology and Business Management March.

Georgellis, Y., \& Sankae, N. (2016). The personality of managers in Britain: gender and sector differences. Paper presented at the Evidence-based HRM: a Global Forum for Empirical Scholarship.

Gill, A., \& Allatt, J. (2011). Statutory wills. Social Care and Neurodisability, 2(1), 48-53.

Hamidi, N., Abdullah, M., Hassan, M., \& Hussin, F. (2012). Load shifting and retrofitting strategy for reducing electricity bill. Paper presented at the Research and Development (SCOReD), 2012 IEEE Student Conference on.

Hemer, S. R. (2014). Finding time for quality teaching: An ethnographic study of academic workloads in the social sciences and their impact on teaching practices. Higher Education Research \& Development, 33(3), 483-495.

Hertzog, P. E., \& Swart, A. J. (2015). The Use of An Innovative Jig to Stimulate Awareness of Sustainable Technologies Among Freshman Engineering Students. Sustainability, 7, 9100-9117. doi:DOI:10.3390/su7079100

Jonker, C. S., \& Van der Merwe, A. (2013). Emotion episodes of Afrikaans-speaking employees in the workplace. SA Journal of Industrial Psychology, 39(1), 00-00.

Kori, K., Pedaste, M., Leijen, Ä., \& Tõnisson, E. (2016). The role of programming experience in ICT students' learning motivation and academic achievement. International Journal of Information and Education Technology, 6(5), 331.

Kruse, T. L. (2013). The intersection of social capital and finances on intentions to transfer in STEM fields: A study of community college students in a rural midwestern state. Iowa State University.

Lee, K. A., Almonte, J. L. J., \& Youn, M.-J. (2013). What to do next: an exploratory study of the post-secondary decisions of American students. Higher Education, $66(1), 1-16$. 
Lim, Y., Kim, H.-M., Park, J., \& Kinoshita, T. (2011). A load-shedding scheme using optimization for proportional fairness in the islanded microgrid. Control and Automation, and Energy System Engineering, 235-241.

Mageshvaran, R., Jayabarathi, T., Shah, V., \& Sood, S. (2015). Optimum steady state load shedding using shuffled frog leaping algorithm to avert blackout in power systems during overload and generation contingencies. Journal of Engineering Science and Technology, 10(10), 1239-1260.

Mikkelsen, B. E., Justesen, L., Wendin, K., Olsson, V., Yngve, A., Scander, H., . . . Mertanen, E. (2015). Research based educations for future foodscapes: the New NordicFood4Many experience. Paper presented at the 9th International Conference on Culinary Arts and Sciences, Montclair State University.

Radojković, M., \& Milojević, A. (2016). Serbia: A Market Pushed By Digital Media-A Challenge For Curricula. Requirements for Modern Journalism Education, 160, 136.

Rubery, J. (2015). Change at work: feminisation, flexibilisation, fragmentation and financialisation. Employee Relations, 37(6), 633-644.

Supangco, V. T. (2014). To Leave or to Stay in the Current Organization After an MBA. Philippine Management Review, 21.

Swart, A. J. (2016). Distance Learning Engineering Students Languish Under ProjectBased Learning, But Thrive in Case Studies and Practical Workshops. IEEE Transactions on Education, 59(2), 98-104.

Swart, A. J., \& Hertzog, P. E. (2016). Contrasting three different academic's assessments of a compulsory capstone module in power engineering indicates reliability! Paper presented at the SAUPEC 2016, Vaal University of Technology, Three Rivers Lodge. ISBN: 978-1-77012-386 retrieved from

Swart, A. J., \& Hertzog, P. E. (2017). Are power engineering students really using their formative assessments to achieve academic success? - A case study. Paper presented at the SAUPEC 2017, Stellenbosch University, South Africa. ISBN 978-0-62074503-1.

Swart, A. J., \& Toolo, L. E. (2015). Fundamental problem-solving skills are found across the board in education: Are our power engineering students on-board? Paper presented at the ICEE 2015, Zagreb, Croatia.

Teferra, D. (2015). Africa's Troika conundrums: Expansion, consolidation, and un (der) employment. International Higher Education, 80, 18.

Yin, R. K. (2009). Case study research - Design and Methods. Thousand Oaks: SAGE publications Inc.

Yin, R. K. (2013). Case study research: Design and methods: Sage publications. 


\title{
Learning Hypothesis Test the Flipped Way: What Do Students Feel?
}

\author{
Saras Krishnan \\ INTI International University, \\ Nilai, Malaysia
}

\begin{abstract}
The blended approach has been found to be more effective in enhancing learning because this method increases student engagement and involvement, and thus improves their performance. This approach is also more favored among the students of the twenty-first century because by leveraging on technology, blended learning gives more flexibility and autonomy to the students. This paper discusses students' preference towards one of the blended learning techniques that is the flipped classroom method, in learning the hypothesis test. It was found that students preferred the flipped classroom method compared to the traditional teaching primarily because it enhances their understanding. However, a number of students have mixed feelings towards the flipped classroom because they feel the effectiveness of this technique depends on their group members and the contents of the lesson.
\end{abstract}

Keywords: classroom activity, rotational model, student engagement.

\section{Introduction}

Flipped classroom is a type of blended learning model whereby the autonomy of learning is on the students. In specific, flipped classroom is a type of rotational model, the other being station rotation, lab rotation and individual rotation (Staker \& Horn, 2012) as shown in Figure 1. The autonomy of learning is on the students whereby most of the learning takes place outside the classroom at their own pace and time. This student-centered approach has been found to increase student engagement and involvement (e.g., Gilboy, Heinerichs \& Pazzaglia, 2015) and thus improves students' performance and success rates (e.g., Tune, Sturek \& Basile, 2013). 


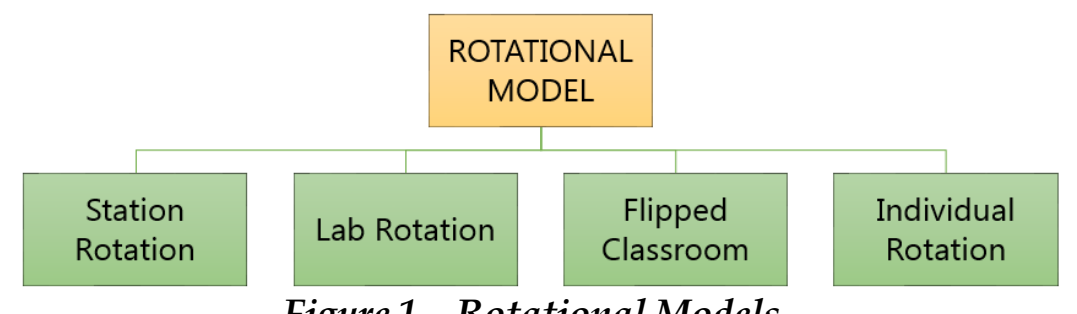

Figure 1 - Rotational Models

Flipped classroom method has generated favorable responses from the students. For example, a study by Dove (2013) found that students enjoyed and preferred the flipped classroom method in learning statistics. Clark's (2015) study also showed evidence of positive response towards this technique as well as evidence of increased engagement and communication among students in a mathematics classroom. Students in this study also attested to the improved quality of instruction and improved use of their class time. Likewise, greater motivation towards learning was observed among students in the flipped environment in the study by Bhagat, Chang and Chang (2016).

Flipped classroom method has been used in different courses and programs of study such as biology (e.g., Jensen, Kummer \& Godoy, 2015), chemistry (e.g., Schultz, Duffield, Rasmussen \& Wageman, 2014) and in nursing education (e.g., Critz \& Knight, 2013). Flipped classroom method has also been used in the teaching of statistics (e.g., Strayer, 2012; Butt, 2014). In mathematics classrooms in particular, flipped technique has been used for example in algebra lessons (e.g., Clark, 2015), arithmetic and geometry lessons (e.g., Lo \& Hew, 2017) and trigonometry lessons (e.g., Bhagat, Chang \& Chang, 2016).

Studies concerning flipped classroom method have ranged from secondary school students (e.g., Clark, 2015; Lo \& Hew, 2017) to undergraduates (e.g., Casasola, Nguyen, Warschauer \& Schenke, 2017) as well as involving the teachers (e.g., de Araujo, Otten \& Birisci, 2017). In this study, flipped classroom method has been used to teach hypothesis test to a group of engineering students in an undergraduate program at a higher education institution.

\section{Background}

As the name implies, flipped classroom flips or swaps what is done in a traditional classroom and what follows afterwards as homework or assignments. As shown in Figure 2, learning first takes place in the online modality followed by the face-to-face classroom activity. Students learn the content materials online before they come to class and then engage in classroom activities facilitated by the teacher (Herreid \& Schiller, 2013). In this sense, the teacher takes the role of a guide and does not actually teach. If at all, the teaching is done online with the aid of technological tools such as using self-recorded videos. 


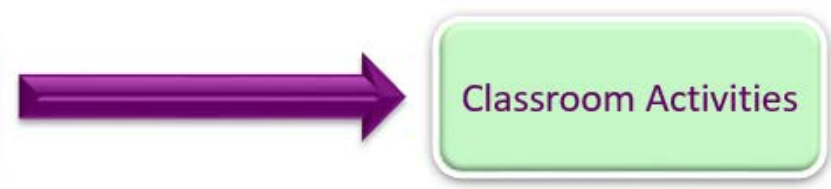

Figure 2 - Flipped Classroom

Flipped classroom method gives the students more control of the learning process and thus enhances student engagement and motivation. Students feel a sense of empowerment when they know that they are the one in control of the learning process. Herreid and Schiller (2013) mention some of the advantages of the flipped classroom as: (i) students learn at their own pace, (ii) teacher understands students' learning better, in particular through classroom activities, (iii) lessons can be more easily customized, (iv) more effective and creative use of classroom time, (iv) increases students' interest, engagement and involvement, (v) flexible and relevant use of technology in enhancing twenty-first century skills and (vi) promotes students' thinking both inside and outside of the classroom.

Dove (2013) find that having access to the content materials online gives students the time and flexibility to learn these materials well prior to the classroom activities. Flipped classroom method gives students more time for practice, exploration and interaction with the teacher compared to doing these activities as homework in the traditional lecture format. More importantly, while the flipped technique allows the better students to work independently, the teacher can spend more time on the weaker students as the students work through the problems in class (Tucker, 2012). As Bhagat, Chang and Chang (2016) found the low achievers in their experimental group who were taught using the flipped classroom method performed better than the low achievers in the control group who were taught using the traditional teaching method.

On the other hand, some of the challenges faced in the implementation of the flipped classrooms are: (i) students' resistance towards this method because they are required to do more work, (ii) students are not serious about the online learning resulting in an unsuccessful classroom activity and subsequently unsuccessful learning, (iii) difficulty faced by the teacher in finding suitable resources for the online learning, (iv) time and effort required by the teacher to design and prepare the online learning materials and instructions (Herreid \& Schiller, 2013).

\section{Learning Hypothesis Test the Flipped Way}

This study purports to investigate students' perceptions towards the flipped classroom method in an undergraduate mathematics program. The subjects of this study are twenty-three students from the mechanical engineering and civil engineering backgrounds. The online learning involved use of power point slides while the classroom activity involved small group discussion and presentation. 


\section{Online Learning}

Two sets of power point slides were uploaded on Blackboard learning management system for the students to learn the hypothesis test at their pace independently prior to the classroom activity. The first set of slides are voiced over and contained explanation about hypothesis test and the hypothesis testing procedure (see Figure 3). Each step is explained in detail and so the duration of the first set is six minutes. The second set of the power point slides discusses three examples of hypothesis test situations, showing detailed working steps (see Figure 4). The examples include both the one-tailed tests and the two-tailed test.
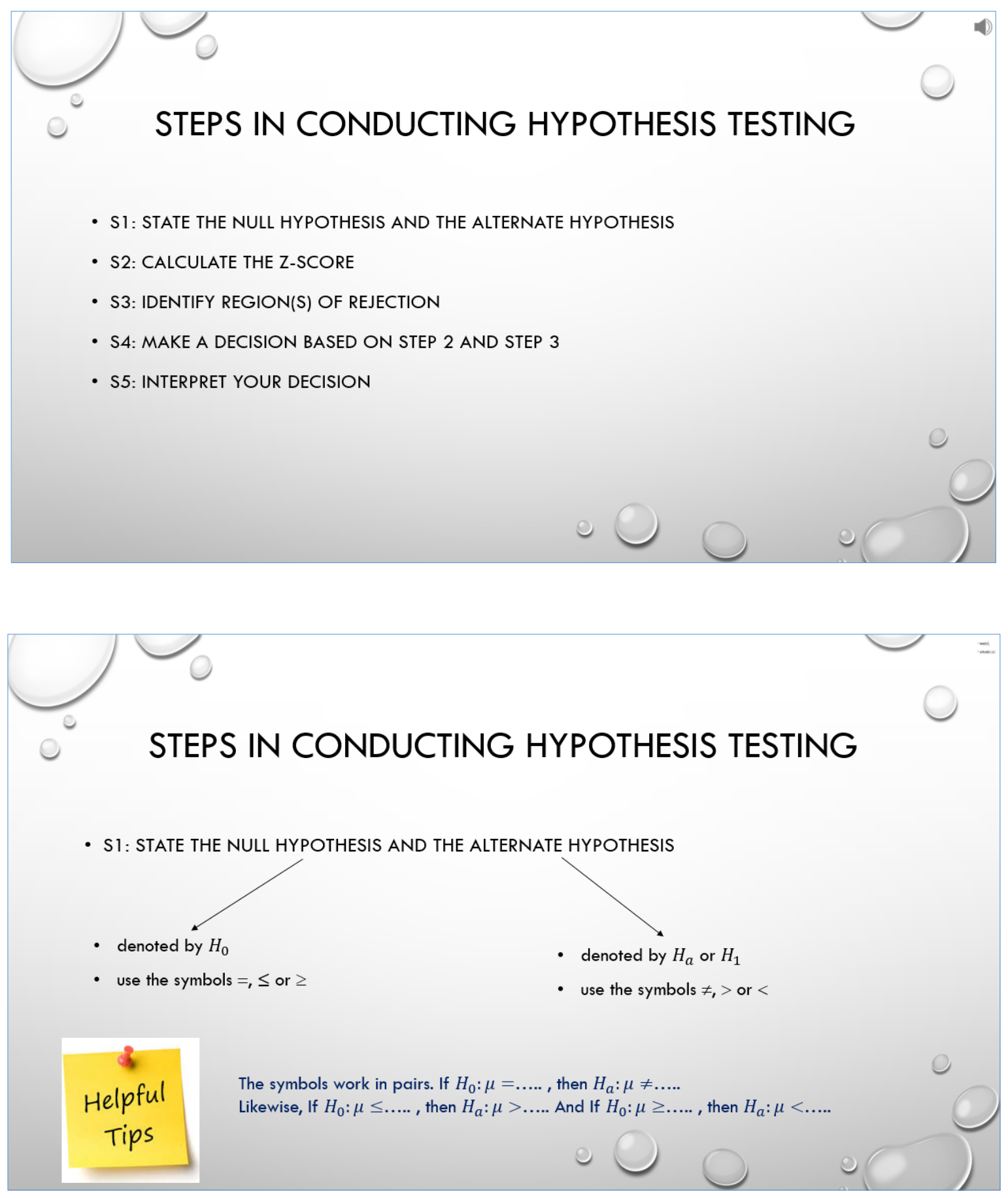

Figure 3 - Examples of the first set of slides 


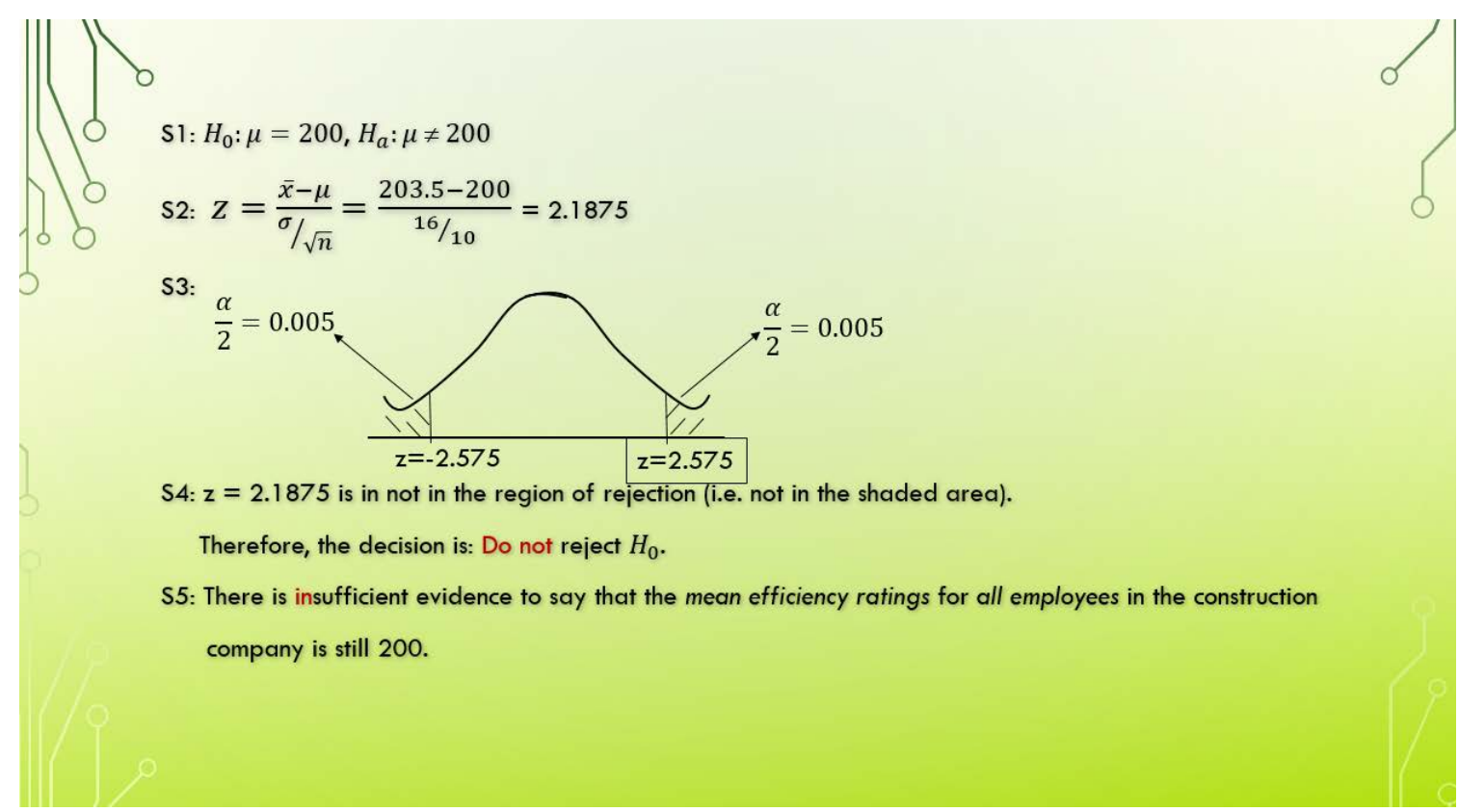

Figure 4 - Example of the second set of slides

\section{Classroom Activity}

The students were divided into groups of four by first segregating them according to their English language proficiency. Before the class begun, three placards labelled "Good English", "Mediocre English" and "Poor English" were pasted on different parts of the classroom walls and students were asked to decide for themselves which category they belong to. This is particularly because it is important for the students to be reasonably proficient in English to be able to understand and interpret the hypothesis test questions especially those questions in contextual form. All the students in this class categorized themselves into the "Mediocre English" and "Poor English" groups. Students from the "Mediocre English" group were asked to choose three other students from the "Poor English" group. All together there were eight groups and they each chose one hypothesis question to work on.

The students were provided with papers and colored markers and were given about thirty minutes to write their solution steps on the piece of paper. It was observed that the students collaborated well and every one of them was actively involved in the group discussion. Then, they put up their answers on the walls of the classroom and took turns in presenting them. Most of them provided the correct answers and presented them in a clear and concise manner suggesting that learning has taken place effectively and successfully. In fact, it appeared that students learned hypothesis test better and faster in this way compared to the traditional chalk-andtalk method. Figure 5 shows an example of the hypothesis test question and Figure 6 shows the students' solution steps for this question. 
A light bulb manufacturer has established that the life of a bulb has mean 95.2 days with standard deviation 10.4 days. Following a change in the manufacturing process which is intended to increase the life of a bulb, a random sample of 96 bulbs has mean life 96.6 days. Assuming that the population standard deviation is unchanged, test whether there is significant evidence, at the $1 \%$ level, of an increase in life.

Figure 5 - Sample Question

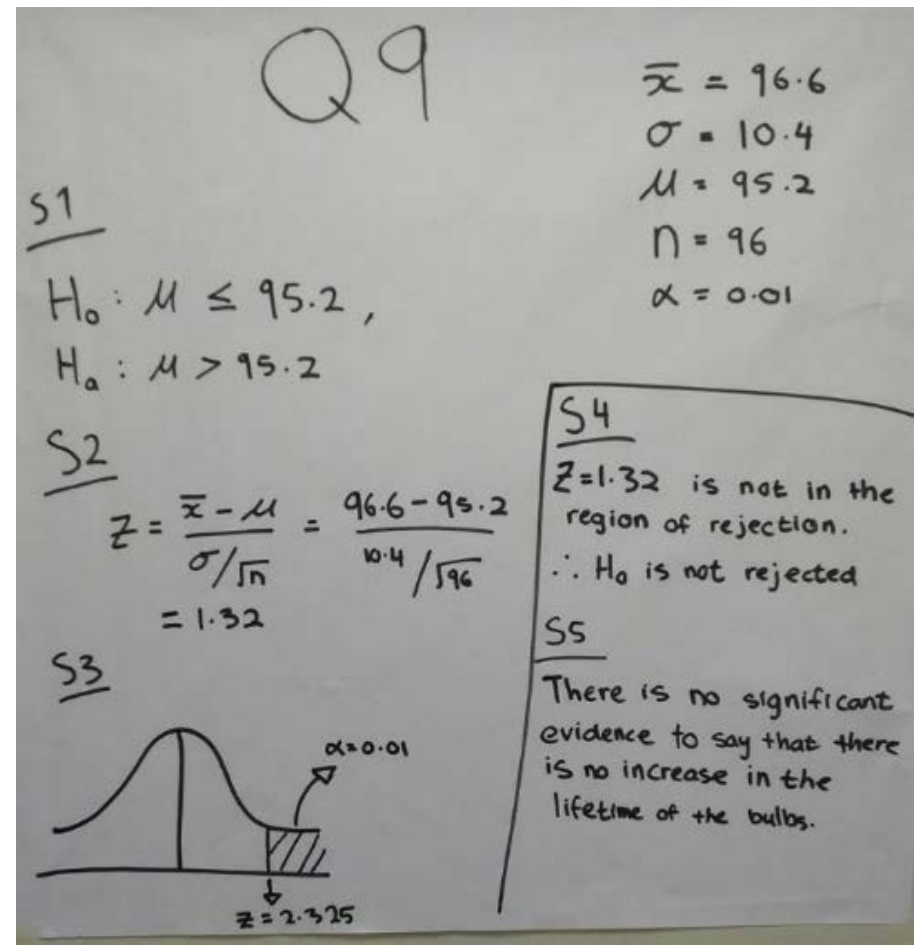

Figure 6 - Students' Work

\section{Students' Perceptions}

\section{STUDENT'S PREFERENCE}

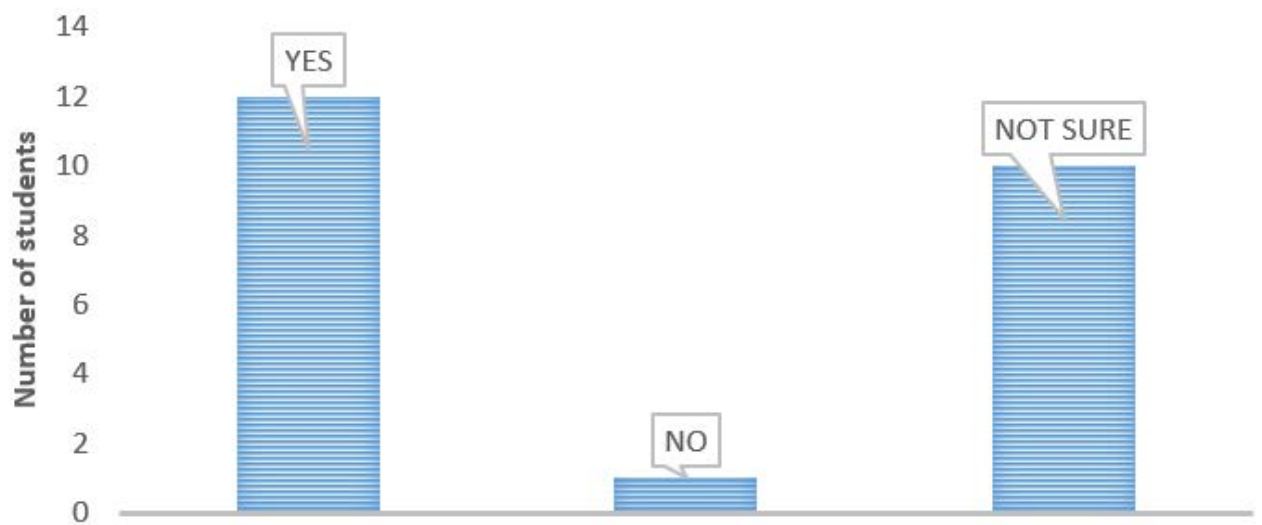

Figure 7 - Students' preference of the learning method 
Students' perceptions towards the flipped way of learning were investigated through a simple questionnaire that asked them if they preferred this method of learning and to give reasons for their preference. As shown in Figure 7, twelve students $(52.17 \%)$ said they prefer they flipped classroom method while one student $(4.35 \%)$ said he/she prefers the traditional way of learning. The remaining ten students $(43.48 \%)$ were not sure of their preference.

Table 1 - Students' feedback on the flipped classroom

We are more actively involved

Able to work with classmates

Make students think on their own

Able to learn from mistakes instantly

Different teaching method makes the lesson more interesting

We can discuss together and teach each other

Positive responses to
flipped classroom

\section{Mixed responses to flipped classroom}

We are actually doing instead of just copying

Forces us to study on our own

This method forces students to understand

Allows us to understand the topic

Students can better understand

Makes students remember easily and understand the topic

Students can learn faster and learn from mistake

Easier and better understanding

It makes me understand clearly

Depends on the topic

Difficult to keep up with the smarter students in the group

Group members have different approach

Both methods are interesting

Some members do not contribute towards the group work

I like the method but I feel working in group slows down the pace of the lesson

We are forced to learn it ourselves but the instructor is the best to teach us

Some topics are better explained by my friends while for some other topics, it is better for the lecturer to explain

I like the group work but it is difficult to complete the task when all members are equally lost

Table 1 displays the students' feedback on their positive and mixed responses towards the flipped classroom technique. Students who responded positively towards the flipped classroom said that they have better understanding of the lesson using this method of learning. They also like the flipped classroom method because they are actively involved in the problem solving and is able to learn from each other's mistakes. On the other hand, although some students agree that flipped classroom can be effective, they still feel that the teacher is the best person to teach the topic. Besides, they feel the effectiveness of the group work depends a lot on team work which can be compromised if there are members who do not contribute towards the discussion. 


\section{Conclusion}

In conclusion, students' positive responses towards the flipped classroom method in this study are consistent with earlier studies (e.g., Butt, 2014). The successfulness of any flipped classroom will depend on the amount of work and effort the students invest in their online learning. As such, it is important that the online learning materials are interesting and motivating. Students are unlikely to find reading typed-out notes to be stimulating. Online videos instead of online reading materials have been found to be preferred by the teachers and students (Herreid \& Schiller, 2013; Overmyer, 2012). In this study, voiced over power point slides have been used instead of uploading the lecture materials in word or pdf format.

Although initial observation during the classroom discussion and participation suggested successful learning took place, there is small difference between the percentage of students who favored flipped classroom and who still feels traditional teaching has its advantages. The primary reason for students to favor the flipped classroom is because they feel that they can understand the lesson better through active involvement and participation. On the other hand, group dynamics and topic chosen are reasons for the mixed responses towards the flipped classroom in this study.

As pointed out by Tucker (2012), teachers should focus on how the instructional tools are integrated into the overall curriculum instead of just focusing on the tool itself. For instance, the power point slides used in this study demonstrated detailed working steps of the hypothesis test and so students were able to easily understand the procedure and apply it in the class on their own. In addition, although students take ownership and responsibility of learning during the classroom activities, teachers should not assume a passive role. Instead, teachers must be committed in guiding, directing and motivating the students towards effective learning.

\section{References}

Bhagat, K. K., Chang, C. N., \& Chang, C. Y. (2016). The Impact of the Flipped Classroom on Mathematics Concept Learning in High School. Educational Technology \& Society, 19(3), 124-132.

Butt, A. (2014). Student views on the use of a flipped classroom approach: Evidence from Australia. Business Education \& Accreditation, 6(1), 33.

Casasola, T., Nguyen, T., Warschauer, M., \& Schenke, K. (2017). Can Flipping the Classroom Work? Evidence from Undergraduate Chemistry. International Journal of Teaching and Learning in Higher Education, 29(3), 421-435.

Clark, K. R. (2015). The effects of the flipped model of instruction on student engagement and performance in the secondary mathematics classroom. The Journal of Educators Online, 12(1), 91-114.

Critz, C. M., \& Knight, D. (2013). Using the flipped classroom in graduate nursing education. Nurse educator, 38(5), 210-213. 
de Araujo, Z., Otten, S., \& Birisci, S. (2017). Mathematics teachers' motivations for, conceptions of, and experiences with flipped instruction. Teaching and Teacher Education, 62, 60-70.

Dove, A. (2013, March). Students' perceptions of learning in a flipped statistics class. In Society for Information Technology \& Teacher Education International Conference (pp. 393-398). Association for the Advancement of Computing in Education (AACE).

Gilboy, M. B., Heinerichs, S., \& Pazzaglia, G. (2015). Enhancing student engagement using the flipped classroom. Journal of nutrition education and behavior, 47(1), 109-114.

Herreid, C. F., \& Schiller, N. A. (2013). Case studies and the flipped classroom. Journal of College Science Teaching, 42, 62-66.

Jensen, J. L., Kummer, T. A., \& Godoy, P. D. D. M. (2015). Improvements from a Flipped Classroom May Simply Be the Fruits of Active Learning. CBE Life Sciences Education, 14(1).

Lo, C. K., \& Hew, K. F. (2017). Using “First Principles of Instruction" to Design Secondary School Mathematics Flipped Classroom: The Findings of Two Exploratory Studies. Educational Technology E Society, 20(1), 222-236.

Overmyer, J. (2012). Flipped classrooms 101. Principal (September/October), 46-47.

Schultz, D., Duffield, S., Rasmussen, S. C., \& Wageman, J. (2014). Effects of the flipped classroom model on student performance for advanced placement high school chemistry students. Journal of chemical education, 91(9), 1334-1339.

Staker, H. \& Horn, M. B. (2012). Classifying K-12 Blended Learning. Innosight Institute.

Strayer, J. (2012). How learning in an inverted classroom influences cooperation, innovation and task orientation. Learning Environments Research, 15, 171-193.

Tucker, B. (2012). The Flipped Classroom: Online instruction at home frees class time for learning, Education Next, 12(1), 82-83.

Tune, J. D., Sturek, M., \& Basile, D. P. (2013). Flipped classroom model improves graduate student performance in cardiovascular, respiratory, and renal physiology. Advances in physiology education, 37(4), 316-320. 


\section{Abstract}

\section{Track 1: STEM}

\section{(Science, Technology, Engineering \& Mathematics)}

Engineering disciplines (Civil, Electrical, Mechanical, Ocean, etc) Green and Renewable Energy

Design \& Technology

Image Processing \& Remote Sensing in Geography

Information and Communication Technologies

Artificial Intelligence \& Robotics

Innovations in Mathematics \& Statistics

Climate Change and Environmental Issues

Agriculture, Forestry \& Food Science

Diversity in STEM

STEM Education 


\title{
Mathematical Model Reduction using Inertial Manifold Concept Applied to Digitalization of Laser Processing
}

\section{Wolfgang Schulz}

RWTH Aachen University

Aachen, Germany

\begin{abstract}
Implementing a virtual production system is challenging due to machine specific interactions, uncertainties and unknowns. The theory of design-oriented thinking adapted for manufacturing favours fast iteration in digitised design cycles instead of optimising the model quality in one step. A virtual production system is seen to become a set of "digital shadows" emulating relevant properties of the underlying techno-physical systems. Different model reduction techniques are demonstrated resulting in "digital shadows" able to generate dense data by millions of runs within acceptable calculation time.
\end{abstract}

Keywords: mathematical model reduction, inertial manifold, time scale separation, digitalisation, agile production systems, laser processing. 


\section{Abstract}

Track 2: Arts, Humanities \&

Social Sciences

Sociology

Psychology

Political Science

Religion \& Philosophy

Language \& Literature

Law, Justice \& Human Rights

History

Art, Music \& Design

Communication \& Media 


\title{
Interpreting Zigi from the Perspective of Anku's Set Theory: An Analytical Inquiry
}

\author{
Moses Adzei \\ University of Education Winneba, Ghana \\ Michael Kwasi Adomina \\ University of Education Winneba, Ghana
}

\begin{abstract}
The paper presents Zigi, a traditional music of the people of Ziavi in the Volta region of Ghana through the lenses of Aku's analytical theory. It interrogates both contextual as well as the structural architecture of Zigi, as a traditional musical form while leveraging on the procedures employed in this theoretical framework for interpretation. The master drummer concept which is at the core of Anku's theory was examined against the inherent rhythmic dynamics of Zigi while probing the intrigues of the music's background ostinato. The study is an analytical work that focused on the validity of such basic concepts espoused as shifting-set orientation and successive sets in Anku's model. The study identifies the existence of these theoretical concepts in Zigi upon thorough analyses. The study also reveals that some of the procedural concepts in this theory do not only happen at the master drummer level of rhythmic manipulation but are also pervasive in the background ostinato. The study also finds Anku's framework as a useful one not only for its analytical insight but also as a credible paradigm for interpreting African traditional music from the perspective of aesthetic appreciation.
\end{abstract}

Keywords: Zigi, Manipulation, Extrapolation, Set-Interpolation, Ostinato. 


\title{
Sensationalism in Argumentation: A Case of the Zimbabwean Parliament Debates
}

\author{
Jakaza Ernest \\ Department of English and Communication \\ Midlands State University, Zimbabwe
}

\begin{abstract}
Parliamentary debates are an argumentative interaction in which Members of Parliament (MPs) employ varied language devices in order to win debates in their favour. However, in an effort to win the debate at all cost, parliamentarians seem to sensationalise their arguments in order to win favour or support from both internal and external audience- the rationale judge. Sensationalism in argumentation is a presentation of an argument in a specific way in order to appeal to the hearer or the other participant's emotions or feelings. The study examines the role of sensationalism in argumentation drawing its examples from the Zimbabwean parliamentary debates. The research is qualitative in nature utilising a case study research design. Debates from the Zimbabwean parliament are purposively sampled and the analysis is couched within the argumentation theoretic framework. The research concludes citing the impact that sensationalism has in argumentation, policies passed and development of a country.
\end{abstract}

Keywords: sensationalism, argumentation, parliamentary discourse, Zimbabwe. 


\title{
Cross-Cultural Differences in Attitudes to Workplace Relationships in Russia and in the United States
}

\author{
Olga Tararukhina and Svetlana Gurieva \\ St Petersburg State University \\ St Petersburg, Russian Federation
}

\begin{abstract}
In the global context of cross-cultural development, work automation, accelerating pace of change, the ability be effective (quickly and with satisfaction for both parties) in those interactions and long-term relationships, the ability to develop, sustain, improve, deal with tensions and conflicts, as well as to help others deal with them, is increasingly becoming an asset for organizations and individuals.
\end{abstract}

The study included theoretical and empirical cross-cultural research of workplace relationships in Russia and the United States, revealing hallmark aspects of organizational cultures in those countries through elements of workplace relationships. The main hypothesis of this study is that the attitudes to workplace relationships are determined by belonging to a national culture.

Employees of organizations in United States and in Russia who took part in the research were individual contributors, team leads, and middle managers: 229 Russian speaking persons living and working in Russia, and 279 English speaking persons living and working in the United States.

The following methods were used to determine attitudes of respondents towards workplace relationships:

- Projective method "Incomplete Sentences" by J. Sacks and S. Levy (author's version) to determine mindsets and attitudes of the respondents for participants of the existing system of relationships in an organization, as well as the content thereof;

- Content analysis, which was employed for qualitative analysis of the data obtained using the projective method;

- Quantitative methods (analysis of mean values, correlation analysis, contingency tables analysis, analysis of variance, one-factor ANOVA analysis, Mann-Whitney $\mathrm{U}$ criterion for independent selections, Pearson's chi-squared $\left(\chi^{2}\right)$ criterion for comparison of nominal selections, Spearman's rho for detection of non-parametric 
correlations) were used for statistical solution of the certain research objectives. Statistical analysis performed with IBM SPSS Statistics 20.0.

Keywords: workplace relationship, organizational culture, belonging, attitude, cross-cultural

\section{Results and Discussion}

Analysis revealed a statistically significant difference between samples of employees in Russia and employees in the United States, in seven out of ten sales of the questionnaire: "Attitude to Work" $(p<0,001)$, "Attitude to Self at Work" $(p<0,001)$, "Attitude to Senior Leaders" ( $p=0,002)$, "Attitude to Attachment" ( $p=0,017)$, "Attitude to Others at Work" $(p=0,003)$, "Expectations" $(p=0,015)$ and "Openness" $(p<0,045)$. The scores on scales "Attitude to Tension", "Principles", and "Attitude to Coworkers" did not reveal statistically significant differences.

\section{Content Analysis}

Ten incomplete statements about belonging and attachment were further processed with frequency content analysis, and revealed:

- Employees in the United States like people who they share values more than employees in Russia do, which is an illustration of uncertainty avoidance intrinsic to American culture;

- Belonging in their organization is twice as strong among employees in Russia than among employees in the United States; this may again be an illustration of collectivist and rather feminine aspects of the culture in Russia. The sense of belonging is probably experienced differently in both cultures: in Russia it is based on mutual liking and shared interests, and in the United States it is based on common values.

\section{Correlations overall}

- Positive attitudes, expectations and attitude to the immediate manager, coworkers, others at work, and positive attitude to work are connected.

- Attitude to senior leaders positively directly and significantly correlates to the attitude to work.

- Positive attitude to others at work is negatively correlates to the sense of belonging with the immediate team or work group.

- Statistically significant differences by gender were not established. This can mean that cultural determinants are stronger than gender.

Correlation analysis results for the sample of employees in Russia only, suggest that in situations of tension, positive relationships with their leaders and coworkers, who are expecting good relationships at work overall, and who are positively assessing integrity and principles, may have an easier time resolving interpersonal tensions. Aspiration of employees in Russia to appear strong and stoic, as well as their underestimating of the positive impact their vulnerability can have on satisfaction with workplace relationships. 
Correlation analysis results separately for the sample in the United States suggests that in situations of interpersonal tension employees in United States primarily lean on the relationships with their coworkers. Positive correlation between "Principles" and "Expectations" can be interpreted as the aspiration of employees in the United States for integrity and trust.

In general, Russian and employees in the United States are significantly different from one another, while each demonstrating a strong identity.

\section{Conclusion}

The results of the study demonstrated that there are more differences between the workplace cultures in Russia and the United States, than there are commonalities. It appears that each culture has a strong identity in the workplace, despite the fact that each country is multicultural, multinational, multi religious, multilingual that constitute their respective identities.

\section{References}

Gibb J. (1991): Trust. A New Vision of Human Relationships for Business, Education, Family, and Personal Living. CA: Newcastle Publishing.

Gurieva S., Tararukhina O. (2015). Interpersonal Relationships In Oganizations: Dynamics of Development. Lotto I., Tarasenko F., Dragavtsev V., Spirin S., Kozlov A. (Eds.) Science - XXI century. Proceeding of Internaltional Scientific Conference. Moscow.

Hostede G., Hofstede G., Minkov M. (2010): Cultures and Organizations: Software of the Mind. McGraw-Hill.

Leary T. (2004): Interpersonal Diagnosis of Personality: A Functional Theory and Methodology for Personality Evaluation. Wipf \& Stock Pub.

Lewin K. (1951): Field Theory in Social Science. Selected Theorical Papers. Harper and Brothers.

Sias P.M. (2009): Organizing Relationships: Traditional and Emerging Perspectives on Workplace Relationships. Los Angeles, CA: Sage.

Soldatova G.U. (1998): Psychology of Inter-Ethncal Tension. Moscow, Russia: Smysl.

Tararukhina O. (2010). Perceptions of Entrepreneurs About Themselves, in the Context of Russian Business Situation. Thesis for Diploma on Professional Requalification on "Pshychological Counseling". St. Petersburg, St Petersburg State University.

Trompenaars F., Hampden-Turner C. (1998): Riding the Waves of Culture. Understanding Diversity in Global Business. McGrawHill 


\section{Abstract}

Track 3:

Business,

Management \&

Accounting

Economics

Business Models for Economic Growth

Management \& Leadership

Forensic \& Financial Accounting

Knowledge Economy

Marketing \& Digital Marketing

Actuarial Science

Human Resource Management

Transport \& Logistics

Travel \& Tourism 


\title{
Creating a Knowledge Economy: What Can Mauritius Learn from U.S. Models of Innovation and Entrepreneurship?
}

\author{
Rajeev Sooreea \\ Associate Professor of International Business \\ Director, MBA Healthcare Leadership Program \\ Dominican University of California \\ Barowsky School of Business \\ San Francisco, California, USA \\ Jaydoyal Sooreea \\ Managing Director \\ Focus Global Ltd \\ Port-Louis, Mauritius
}

\section{Extended Abstract}

There is general consensus that Mauritius has passed the stages of the agricultural and industrial economy and is rapidly transforming into a knowledge economy. With a Global Competitiveness rank of $45^{\text {th }}$ among 137 countries (World Economic Forum, 2017-18), Mauritius seems to be on the right track. However, Executive Opinion Surveys conducted by the same organization indicate that "Insufficient capacity to innovate" and "Inadequately educated workforce" are among the top three "most problematic factors for doing business in Mauritius." In order for Mauritius to become a knowledge economy, it imperatively needs to address these issues.

One model that knowledge societies like the U.S., Switzerland, and Singapore have used is to create a well-integrated and an effectively-managed Triple Helix model. The triple helix model initiated in the 1990s by Etzkowitz (1993) proposes shifting the industry-government dyad that defines an Industrial Society to a triad that consists of industry-government-academia in the Knowledge-based Society. The Triple Helix model postulates that innovation and economic development in the Knowledge-based Society relies primarily on the role of academia (university) and the hybridization of components from university, industry and government to create new formats for producing, transferring and applying knowledge.

Since Schumpeter's (1942) theory of creative destruction where innovation occurs as a natural dynamics, theoretical and empirical research has evolved into two main 
complementary directions. The (neo) institutional perspective examines the rising prominence of the university as an innovation actor whose 'third mission' (besides teaching and research) is to commercialize academic research and take leadership in socio-economic development by partnering with industry and government (Etzkowitz and Leydesdorff, 2000). The (neo) evolutionary perspective views the university, industry and government as "sub-sets of social systems" that interact and communicate by creating networks that generate new innovation landscapes (Dolfsma and Leydesdorff, 2009).

The result of a Triple Helix model from both perspectives is that the university embarks on a "third mission" as Ranga, Perälampi and Kansikas (2016) puts it and becomes the Entrepreneurial University. It goes beyond its traditional mission of teaching and research to put knowledge to use and take leadership in socioeconomic development by partnering with industry and government (Etzkowitz and Leydesdorff, 2000). The university takes a pro-active stance to create and put knowledge to use.

Firms engage in higher levels of training and knowledge sharing because of improved technological levels. In addition to its traditional role of providing regulation, the government now becomes a public entrepreneur as well as a venture capitalist. Academic institutions, which classically were believed to not have a direct role in innovation, now take the lead. Greater diversification of knowledge production does not reduce the role of universities in producing knowledge.

Entrepreneurial universities extend their roles and capabilities of educating people to educating organizations, for instance, through collaborative leadership development, entrepreneurship, consulting, and hosting incubation programs. Rather than just producing static knowledge and being a source of human resources, universities combine research and teaching capabilities to generate new technology transfer and become a source of new firm formation and collaborative leadership. Technology clusters such as Silicon Valley in California and Boston in Massachusetts are prime examples of regions where universities are redefining their role in society. These clusters are creating such massive spillover and demonstration effects that several universities in the San Francisco Bay Area, for example, are taking the leadership to transform themselves into innovative and entrepreneurial universities.

The purpose of this research is to demonstrate what Mauritius may learn from models of innovation and entrepreneurship in U.S. academia to devise an effective Triple Helix framework that will push Mauritius further on to the path to the knowledge economy.

We use newly developed human capital datasets and borrow methodological concepts from the trade policy literature to empirically show the state of education policy and the standpoint of economic policy as regards to education in Mauritius. We also use empirical facts to highlight where Mauritius position itself on a global scale in terms of its educational system and innovation capabilities. In addition, we 
empirically demonstrate how education policy is impacting economic development in Mauritius. We then use the results to motivate our research that Mauritius needs to strategically enhance its Triple Helix model. We propose a framework that captures this model and provide examples of innovation and entrepreneurship models that are currently being implemented in the U.S. and how Mauritius can possibly learn from these to create a distinctive knowledge economy. The strength of our model is that it is not restricted to business schools alone; both business and nonbusiness schools and departments can successfully launch and benefit from such innovative and entrepreneurial models of higher learning.

Keywords: Knowledge Economy, Triple Helix, Innovation and Entrepreneurship, Education.

\section{References}

Etzkowitz, H. (1993). Technology transfer: The second academic revolution. Technology Access Report, 6, 7-9.

Etzkowitz, H., Leydesdorff, L. (2000). The dynamics of innovation: from National Systems and "Mode 2" to a Triple Helix of university-industry-government relations. Research Policy, 29, 109-123.

Dolfsma W. \& Leydesdorff, L. (2009). Lock-in and break-out from technological trajectories: Modelling and policy implications. Technological Forecasting and Social Change, 76, 932- 941.

Ranga, M., Perälampi, J. and Kansikas, J. (2016). The new face of university-business cooperation in Finland. Science \& Public Policy, 43 (5), 601-612.

Schumpeter, J. A. (1942). Capitalism, Socialism and Democracy. George Allen \& Unwin, New York.

World Economic Forum. (2017). The Global Competitiveness Report 2017-2018. 


\title{
The Microfinance Institutions Development in Morocco
}

\author{
Oxana Wieland \\ University of Minnesota Crookston \\ Crookston, USA
}

\begin{abstract}
The microfinance institution is a relatively modern innovation, with dominating growth compared with the old age banking sector globally. The institutions have attained a profound recognition across various continents in the recent past. In 2007, as part of the implementation of the Green Morocco Plan, the Moroccan government emphasized the need to provide financing benefiting medium size businesses and smallholders' families. The development of the microfinance institution has continued perhaps due to its ability of serving as a poverty alleviation tool as well as a potential new sector of participatory (non- conventional) lending practice. The fundamental objective of this paper is to explore the issue of the microfinance development in Morocco, comparing conventional and participatory forms of microfinance. The research paper embarks on an effort to provide background information on microfinance institutional development, outlining specific financial needs of the rural population as well as providing policy recommendations to remedy the challenges.
\end{abstract}

Keywords: Microfinance, Rural Development, Participatory Financing, Green Morocco Plan. 


\title{
Economic Growth and the Linkage Effects Between Foreign Direct Investment and Domestic Investment: The Case of Mauritius
}

\author{
Brinda Sooreea-Bheemul \\ University of Mauritius \\ Reduit, Mauritius
}

\begin{abstract}
Policy makers in developing countries are often concerned about whether they should concentrate their efforts either in improving the investment climate of their countries in order to enhance domestic investment or in boosting investment promotion efforts to attract more Foreign Direct Investment (FDI). Theoretically, both domestic investment and FDI can have growth enhancing effects and consequently one needs not choose between them.
\end{abstract}

This paper argues that this relationship holds subject to the condition that aggregate investment increases with FDI inflows. The objective of this paper is to uncover the degree of substitutability or complementarity between these two types of investment. This is closely linked to the idea of crowding-in or out effects (Agosin and Mayer, 2005). One might expect that the complementarity and substitutability between FDI and domestic investment alternate over the life-cycle of FDI-related products (de Mello, 1997). Initially, the inflows of FDI into competitive industries are thought to crowd out domestic investors. However, there may eventually be a crowding-in effect as domestic investors are attracted in the less competitive intermediate goods industries, thus creating a backward linkage effect. Therefore, the impact of FDI on domestic investment may not stop at the first generation effect. It would, therefore, be crucial to understand the long-run effects of FDI on domestic investment to undertake appropriate policy measures to achieve economic growth.

The second objective of the paper is to empirically measure the contributions of these two types of investment to economic growth in Mauritius and understand the implications. If FDI is thought of as the pathway to global value chains, domestic firms need to be empowered to be able to integrate into them (World Bank Group Report, 2017).

In Mauritius, inward Foreign Direct Investment (FDI) has experienced an increasing trend since the establishment of the Export Processing Zones in 1970. Yet, the 
volume of FDI that the island economy receives is highly volatile and fluctuates significantly on a year-to-year basis and is thought be project-based. Capital flows have been more volatile for developing countries since the mid-1980s (United Nations Conference on Trade And Development, 2016), giving rise to concerns over their long-run economic growth implications for these capital-scarce host economies. Such volatility may be negatively correlated to economic and political uncertainties as well as a high cost of investment, which may undermine domestic investment, trade and financial stability.

The third objective of the paper will therefore contribute to the literature by uncovering the determinants of the volatility of FDI in Mauritius and explore whether they are due to country specific factors or push factors. A qualitative research approach is adopted linking major domestic policy changes and landmark changes.

The methodology to explore the empirical relationships between FDI inflows, its volatility, domestic investment and economic growth in Mauritius is based on a standard growth model over the time span of 1980-2015. Differing measures of volatility of FDI are used to ensure the robustness of the results. Policy conclusions are drawn from the main findings.

Keywords: Foreign Direct Investment (FDI), Volatility of FDI, Domestic Investment, Economic Growth, Policy Recommendations.

\section{References}

Agosin, M. \& Mayer, R. (2000). Foreign Investment in Developing Countries: Does It Crowd in Domestic Investment?. United Nations Conference on Trade And Development Discussion Paper, No. 146.

World Bank. (2017). Investment Policy And Promotion Diagnostics And Tools : Maximizing The Potential Benefits Of Foreign Direct Investment (FDI) For Competitiveness And Development (English). Washington, D.C. : World Bank Group Report 117475.

de Mello, L. R. (1997), 'Foreign Direct Investment in Developing Countries and Growth: A Selective Survey', Journal of Development Studies, Vol. 34, No. 1, pp. 1-34.

United Nations Conference on Trade And Development (2006), Trade and Development Report: Structural transformation for inclusive and sustained growth, United Nations Conference on Trade And Development, Geneva. 


\title{
The Effect of Organisational Culture and Leadership on Employees' Job Satisfaction at a Private Commercial Bank in Mauritius
}

\author{
Roshan Oodally \\ Lecturer \\ Université des Mascareignes, \\ Pamplemousses, Mauritius
}

\section{Extended Abstract}

The success of any organisation depends on a satisfied workforce. This is why it has received substantial consideration in conducting empirical research on the workplace. Several determinants of employee job satisfaction have been identified in previous studies (Lok \& Crawford, 2004). Leadership is regarded as one of the most significant antecedents of job satisfaction (Hamidifar, 2009). Besides, it has also been found that organisational culture is fundamental to the improvement of employee job satisfaction (Hosseinkhanzadeh, Hosseinkhanzadeh, \& Yeganeh, 2013). The aim of this study is to analyse what effect leadership and organisational culture have on job satisfaction in a Mauritian private commercial bank which has been established in Mauritius since forty-five years. Since then it has mushroomed wide and far, precisely in the Indian sub-continent and African continent.

The research philosophy underpinnings this study is pragmatism while the approach used is an amalgamation of inductive and deductive research. While conducting the field research, I have used the cross-sectional study to reflect and analyse the effect of leadership and organisational culture have on employee job satisfaction. This empirical study involves both quantitative and quantitative methods of research. A census has been used for the former while a stratified nonrandom sampling comprises of 13 informants is used for the latter. A structured questionnaire consisting of 126 items has been administered. It has been regrouped under four sections namely: leadership style, job satisfaction and organisational culture and demographics, respectively. Leadership items have been taken from Multifactor Leadership Questionnaire (Avolio \& Bass, 2004), job satisfaction items from Minnesota Satisfaction Questionnaire (Weiss, Dawis, England, \& Lofquist, 1967) and organisational culture items from Organisation Culture Questionnaire (Harrison, 1995). As for demographics, one question has been designed on gender of respondents based on prior theoretical and empirical studies. Cronbach alpha tests have been performed and it has been proved that the questionnaire is a reliable one 
(MLQ, MSQ, and OCQ are $0.891,0.783$ and 0.804 respectively). To test validity of the questionnaire, a pilot test has been conducted and necessary adjustments have been made accordingly. For the qualitative part, the research strategy devised is the grounded theory and the research instrument used is a semi-structured in-depth interview for collecting data (Nunkoo \& Ramkissoon, 2013). I have purposively recruited staff in using convenience and snowball sampling. Theoretical Saturation has been reached on interviewing the thirteenth respondent. Interview transcripts have been analysed using the principles of the grounded theory method whereby three levels of coding has been used namely, open coding, axial coding and selective coding (Nunkoo \& Ramkissoon, 2013).

The current study which I undertook, reveals that all three types of leadership styles exist at the bank. Transformational leadership suggests that leaders are charismatic and motivate employees by inspiring them, consider them individually and stimulate their intellectual needs. Transactional leadership specifies tasks and monitor performance to achieve tasks by providing a reward system. Laissez-faire leadership is a passive style which avoids involvement (Bodla \& Nawaz, 2010). However, transformational leadership has been found to be the most prevailing one. Results also show that in general, staff are overall satisfied with their jobs. Moreover, this study discloses that extrinsic factors such as company polices, compensation, coworkers, and quality of working conditions have much more influence on employees' job satisfaction level. It is quite interesting to note that facets of job satisfaction that participants are satisfied with differ. For some, it is their job performance; for others, it is their supervisors' satisfaction with their jobs; for another group, it is their working hours. Besides, respondents explained that there are two aspects of their jobs they are dissatisfied with namely, working conditions and opportunity for advancement. Furthermore, the prevailing organisational culture is role-orientation followed by task-orientation. Nevertheless, the least prevailing cultures are power orientation and self-orientation. In terms of relationship between leadership styles and job satisfaction, it has been found that there is a weak, positive and non-significant correlation between these two variables. It has also been observed that there is a weak, positive and non-significant relationship between organisational culture and job satisfaction. Our findings contradict other studies due to contextual differences. This study is the first one which has been conducted in Mauritius, a small African island state whereas the majority of studies have been conducted in Northern Hemisphere countries (mainly Western countries).

The present study demonstrates that leadership and organisational culture do not share a strong relational components among the employees' job satisfaction in a bank setting in Mauritius. Hence, there is an urgent need to further this type of study in other fields of research in Mauritius to confirm or contradict the current findings. This research problematic in itself can give food for thought for a more comprehensive and reflective analysis among academics. 
Keywords: employees' job satisfaction, organisational culture, leadership style, private commercial bank, Mauritius.

\section{References}

Avolio, B. J., \& Bass, B. M. (2004). Multifactor Leadership Questionnaire Manual. Redwood City: Mind Garden.

Bodla, M. A., \& Nawaz, M. M. (2010). Comparative Study of Full Range Leadership Model among Faculty Members in Public and Private Sector Higher Education Institutes and Universities. International Journal of Business and Management, 208-214.

Hamidifar, F. (2009). A Study of the Relationship between Leadership Styles and Employee Job Satisfaction at Islamic Azad University Branches in Tehran, Iran. AU-GSB-e-Journal, 1-13.

Harrison, R. (1995). Understanding Your Organisation's Character. The Collected Papers of Roger Harrison, 149-164.

Hosseinkhanzadeh, A. A., Hosseinkhanzadeh, A., \& Yeganeh, T. (2013). Investigate Relationship Between Job Satisfaction and Organizational Culture Among Teachers. Procedia - Social and Behavioral Sciences, 832 - 836.

Lok, P., \& Crawford, J. (2004, September). The effect of organisational culture and leadership style on job satisfaction and organisational commitment. Journal of Management Development, 321-338.

Nunkoo, R., \& Ramkissoon, H. (2013). Stakeholders' Views of Enclave Tourism: A Grounded Theory Approach. Journal of Hospitality $\mathcal{E}$ Tourism Research, 1-32.

Weiss, D. J., Dawis, R. V., England, G. W., \& Lofquist, L. H. (1967). Manual for the Minnesota Satisfaction Questionnaire. Minnesota: University of Minnesota, Industrial Relations Center. 


\title{
Are Small Businesses Being Sustainable? Is Cash Management the Key to Profitability and Sustainability?
}

\author{
Avika Mungal \\ Durban University of Technology \\ Durban, South Africa
}

\begin{abstract}
South Africa falls behind other third world countries with regards to promoting the development and sustainability of small businesses. Small businesses are vital for employment and job creation in South Africa. The implementation of sound cash management practices is essential to ensure to profitability and sustainability of any successful business. The commonly used expression, "Cash is king" cannot be contested as its validity was more prevalent in this research study. Foster (2012) indicated that, since the year 2008, business failures have risen by 30\%. Businesses with less than five employees were hit the hardest. $57 \%$ of those small businesses failed in a year. Businesses that had employed six to nineteen employees faced a $40 \%$ increase in bankruptcy. Three quarters of businesses did not plan what their expected cash inflow and cash outflow would be. This directly affects the potential survival and sustainability of businesses (Nick ,2009). Nick (2009) also noted that those businesses who budgeted for the future at least once a year had only a $36 \%$ chance of survival. Those that budgeted once a month for the future had increased their chance of survival to $80 \%$. Many small businesses face liquidity problems and eventually fail due to the lack of cash management knowledge and implementation. It is vital to have viable small businesses in developing communities as these businesses provide the necessities of the community.
\end{abstract}

The aim of the study was to identify the current cash management practices of small retail businesses in a developing community and identify the impact of such practices on their profitability and sustainability. It will look at the underlying reasons if or why small businesses are not managing their cash inflow and outflow. This study will directly benefit these communities in becoming more viable in our tough economy. This study will also equip business owners with sound management practices, which will ensure the profitability and sustainability of their businesses. The lack of support in terms of cash management knowledge and services for small businesses forms part of the motivation for the study. 
This descriptive, cross sectional study, using a quantitative research paradigm and a non-probability sampling method targeted a sample of 69 businesses in the chosen area. The sample structure consisted of small retail businesses with less than 5 employees.

There was a significant relationship between drawing budgets and sustainability. This finding suggests that the more often the business draws up cash budgets, the more viable and sustainable the business is. $78.3 \%$ of the respondents acknowledged the importance of keeping records in the business, however, only $29.9 \%$ drew up cash budgets.

The findings of this study could be useful to potential, emerging and established owners of all types of businesses since effective and efficient cash management is an integral component of any successful business. There should be more emphasis placed on the impact of how proper cash management practices can affect profitability and sustainability of a small retail business.

A recommendation that adds value to the study was to engage the final-year, National Diploma: Cost and Management Accounting students in an integrated project, where they can provide cash management advice to these businesses. This will enhance the small businesses' knowledge of cash management and encourage implementation of these procedures to assist in increasing profitability and sustainability in their business. The engagement by students would not only expose them to some hands on, experiential learning to prepare them for the world of work, but, also allow them to provide a service to the community.

Keywords: Cash Management, Small businesses, Profitability, Sustainability, lack of cash management implementation.

\section{References}

Foster, S. (2012). Epidemic of Failures hits small busineses. Retrieved from http://web.b.ebscohost.com/ehost/detail?vid=2\&sid=b9ce9436-1cbf-4381$8792-$ 972220e8265b\%40sessionmgr112\&hid=126\&bdata=JnNpdGU9ZWhvc3QtbG12 ZQ\%3d\%3d\#db=nfh\&AN=201202221011919011 (Accessed 30 April 2014).

Nick, S. (2009, March 27). Cashflow ignorance is big problem for firms. Domimion Post, pp. 3. 


\section{Abstract}

\section{Track 4: \\ Education}

Early childhood education

Primary \& secondary education

Curriculum design \& development

eLearning, distance \& blended learning

Massive Open Online Courses (MOOCs)

Higher Education

Special Education, Learning Difficulties and Learner Diversity

Adult and lifelong learning

Education for sustainable development

Education for interdisciplinary thinking

Emerging trends in Education 


\title{
Factors Affecting the Participation of Rural Male Students in Fashion and Fabrics and Food and Nutrition in Secondary Schools in Zimbabwe
}

\author{
Anna Gudyanga, Josephine Gora and Lydia Moyo \\ Midlands State University, Faculty of Education, \\ Department of Educational Foundations, \\ Management and Curriculum Studies, Gweru, Zimbabwe
}

\begin{abstract}
This study focused on the factors that affect rural male students participation in Fashion and Fabrics and Food and Nutrition in secondary schools in Zimbabwe. The qualitative research design grounded in the interpretive paradigm was used to generate data relevant, to provide a sound detailed picture of isolated variables in relation to the participation of male students. Three data generating instruments were used namely interview and observation guides as well as document analysis. Purposive sampling was used to select two school heads, two Fashion and Fabric and two Food and Nutrition teachers, ten O-level male students doing the two subjects and ten male students not participating in the subjects. Data were analyzed using the thematic analysis approach. The data revealed that male students have negative perceptions which emanate from peer pressure. They also lack interest in the subject because the way they are socialised force them to think that these practical subjects are feminine; there are no male teachers who teach the subject who would act as role models to the students and that parents and siblings do not support them as they consider the subjects to be expensive. Some of the recommendations are that responsible authorities should erect suitable infrastructure for practical subjects and carry out fund raising activities in order to raise money to purchase practical subject materials. Workshops, seminars and carrier guidance should be carried out to educate the students on the importance of these practical subjects in order to increase male students' participation.
\end{abstract}

Keywords: factors, participation, fashion and fabric, food and nutrition. 


\title{
English or Communication: Engineering Students' Dilemma
}

\author{
Faiza Haddam sp Bouabdallah
}

Tlemcen University (Abou Baker Belkaid ),

Tlemcen, Algeria

\begin{abstract}
Nowadays, it is undeniable that the communicative competence plays a leading role in professional setting especially in the context where the mastery of foreign languages is the ultimate goal to improve the communicative sphere for technical and engineering fields. ESP learners can significantly improve their communicative competence by developing their ability to use communication strategies (CSs) for coping with face-to-face oral interaction issues. The present research, which is an exploratory case study was conducted at the University of Tlemcen, faculty of Mechanical engineering. The participants are the first professional licence students in the field of welding.
\end{abstract}

The aim of the study is to explore engineering students' issues in face to face communication in professional setting whether in French or in English since those students are supposed to do their training period within companies during the six semesters of their studies (Licence Degree). The data collected through a semistructured interview and a classroom observation were analysed to identify various strategies to cope and overstep such dilemma between the interlocutor and the receiver. Linguistically speaking, Algeria is still influenced by the French colonisation for more than 130 years; so any workplace situation need the use of French and any technical field need the mastery of English as well. Here's comes the question: what should they/we do to improve both levels?

In 2018, our students belong to the digital generation and it's really no secret that we spend most of our time in front of our screen to exchange via any social networks or emails. Electronic devices speed up the means of communication and left behind face to face communication which is a primary aspect to consider, mainly in any workplace context. Meanwhile, the sample population under study were selected to highlight students' communication issues to express themselves at least in the classroom with the teachers either in French or in Arabic; so, what about English? Welding is the first professional licence in the University of Tlemcen which is a project launched with an European cooperation programme. The course for that ESP programme has been designed for only 6 semesters considering English and 
communication as primary majors to improve in this short period of time. Starting from September 2017, the 20 students enrolled in this new professional field (different from academic one) use to communicate using their electronic devices either in Arabic or some of them in English between their classmate and teachers, but when they come to communicate directly within the classroom, they face a real dilemma even in their mother tongue. When we communicate primarily through a screen, we miss out on the ability to form lasting connections with people. In order to maintain and grow personal and professional relationships, face-to-face communication is absolutely imperative.

Keywords: Engineering students, professional licence, communication, ESP, dilemma. 


\title{
Integrated Assessments the Key to Developing Critical Thinking Skills of First-Year University Students
}

\author{
Melanie Cloete \\ Durban University of Technology \\ Durban, South Africa
}

Lombard and Grosser (2008, p. 561), the South African economy can only remain competitive with a workforce of critical, creative thinkers, who can solve problems and make sound decisions. Institutions of higher learning are required to instill critical thinking skills within their graduates. However, in order to achieve this, teaching and, in particular, assessment practices would need to be rethought and transformed (Boud \& Associates, 2010).

Higher education institutions are not achieving this directive. This has been revealed by numerous empirical studies, which have highlighted the inability of graduates to cope with the workplace requirements (Jansen, 2012, p. 1; McPhun \& McZoom Ltd consultancy, 2010, pp. 1-2). Osborne et al. (2013, p. 1) argued that assessment is the key that will enhance students' employability and close the gap between higher education and the workplace requirements. Integrated assessments that mirror real life situations are particularly useful in the development of critical thinking skills.

The purpose of this study was to investigate whether the integrated assessment conducted with the first-year students at a University of Technology has enhanced the critical thinking skills of these students.

This descriptive and inferential study employed a quantitative strategy, with a quasi-experimental, pre-test, post-test, non-equivalent group design and it was longitudinal in nature. The target population consisted of two groups an experimental and a control group. All five categories of the Watson-Glaser Critical Thinking Appraisal United Kingdom edition were administered to both the control and experimental groups in a pre-test and post-test measure. However, only the experimental group was exposed to the integrated assessment. The aim was to measure the change, if any, in the students' critical thinking skills over a period of time.

The findings revealed a statistical significance in the overall post-test scores in favour of the experimental group. The norm group comparisons also revealed that, after the completion of the integrated assessment, the experimental group's post-test mean scores were higher than the United States grade 12 students and were closely 
matched to the United States first year of 4 year colleges. In addition, the experimental group's pre-and post-test mean scores were closely matched to the South African norm group. These results suggested that the experimental group experienced gains in their critical thinking ability in the post-integrated assessment.

It is, therefore, recommended that integrated assessments, which are based on real world problems, should be conducted in the first year and in subsequent years. It is not always possible for universities of technology to offer cooperative education/ inservice training to all students. Contextualized integrated assessments, therefore, bridge this gap by allowing students to experience workplace requirements without physically being in the work environment.

Keywords: critical thinking; integrated assessments; workplace requirements; employability; linking theory and practice. 


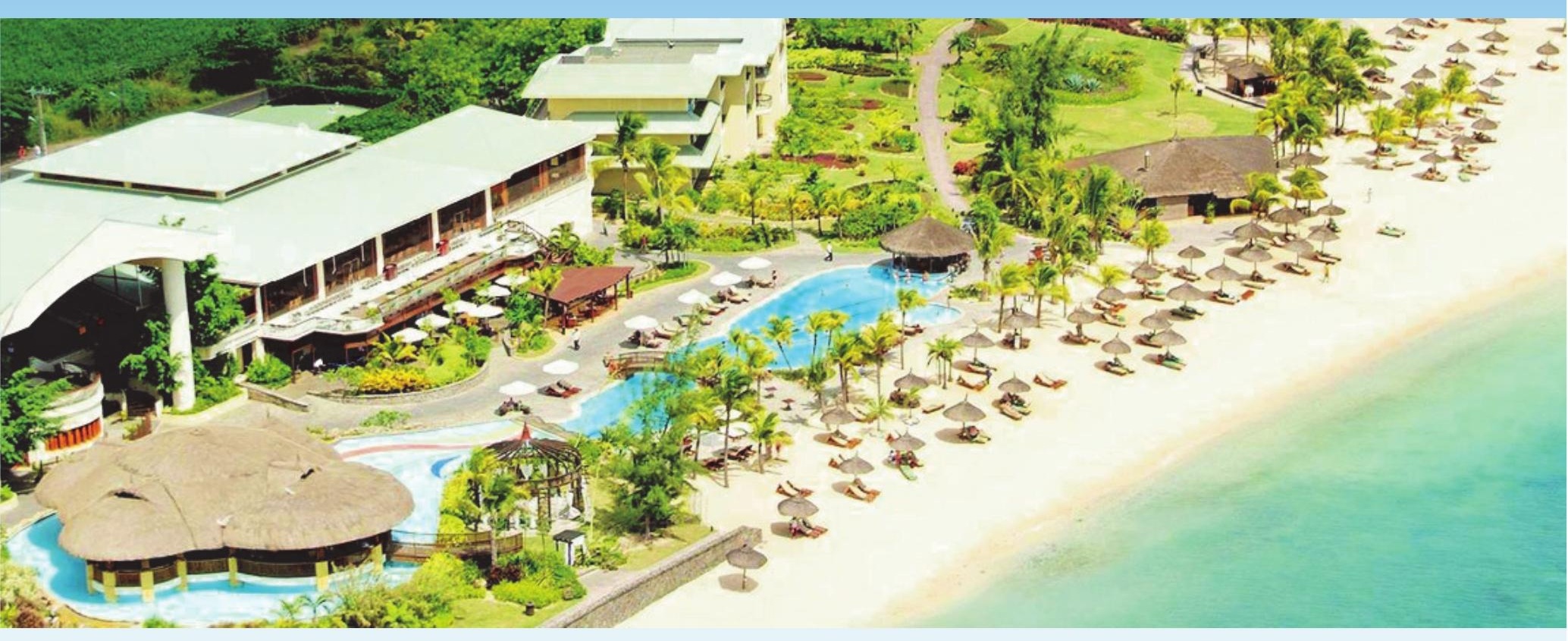

2 myresnet $\mathrm{S}|\mathrm{R}| \mathrm{A}|\mathrm{K}| \mathrm{M} \quad$ LeMERIDIEN 